\title{
WestVirginiaUniversity
}

THE RESEARCH REPOSITORY @ WVU

Graduate Theses, Dissertations, and Problem Reports

2009

\section{Asymmetric synthesis of SL0101, cyclitol and jadomycin}

Mingde Shan

West Virginia University

Follow this and additional works at: https://researchrepository.wvu.edu/etd

\section{Recommended Citation}

Shan, Mingde, "Asymmetric synthesis of SL0101, cyclitol and jadomycin" (2009). Graduate Theses, Dissertations, and Problem Reports. 2899.

https://researchrepository.wvu.edu/etd/2899

This Dissertation is protected by copyright and/or related rights. It has been brought to you by the The Research Repository @ WVU with permission from the rights-holder(s). You are free to use this Dissertation in any way that is permitted by the copyright and related rights legislation that applies to your use. For other uses you must obtain permission from the rights-holder(s) directly, unless additional rights are indicated by a Creative Commons license in the record and/ or on the work itself. This Dissertation has been accepted for inclusion in WVU Graduate Theses, Dissertations, and Problem Reports collection by an authorized administrator of The Research Repository @ WVU.

For more information, please contact researchrepository@mail.wvu.edu. 


\title{
Asymmetric Synthesis of SL0101, Cyclitol and Jadomycin
}

\author{
Mingde Shan
}

\author{
Dissertation submitted to the \\ Eberly College of Arts and Sciences \\ at West Virginia University \\ in partial fulfillment of the requirements \\ for the degree of \\ Doctor of Philosophy \\ In \\ Organic Chemistry
}

George A. O'Doherty, Ph.D., Chair

Patrick S. Callery, Ph.D.

Jeffrey L. Petersen, Ph.D.

X. Michael Shi, Ph.D.

Björn C. G. Söderberg, Ph.D.

C. Eugene Bennett Department of Chemistry

Morgantown, West Virginia

2009

Keywords: SL0101, Cyclitol and Jadomycin

Copyright 2009 Mingde Shan 


\section{ABSTRACT \\ Asymmetric Synthesis of SL0101, Cyclitol and Jadomycin}

\section{Minde Shan}

The carbohydrate portion of natural products plays a crucial role in biology, such as target binding, solubility, tissue targeting, and membrane transportation. The O'Doherty group has developed a de novo methodology to build the desired functionality and stereochemistry within each sugar, in contrast to the traditional approach using known carbohydrates as starting materials. This methodology relies on a highly diastereoselective palladium(0)-catalyzed glycosylation reaction to control the stereochemistry at the anomeric center and postglycosylation transformation to introduce the corresponding functionality in the sugar moiety.

Continuing our investigations on the utility of this strategy, we turned our attention to the syntheses of the bioactive carbohydrate-based natural product analogues. The targets we chose are the p90 ribosomal S6 kinase (RSK) inhibitors SL0101 and its analogues. We developed a practical 7-10 step diastereoselective route for the syntheses of six different analogues of SL0101 in either enantiomeric form without using any protecting groups in the sugar moieties.

$C$-glycosides are carbohydrates where one of the acetal oxygens has been replaced with a methylene group. These non-hydrolyzable sugar mimics have long been recognized as an important pharmacophore. Cyclitols/Carbasugars are an important subset of the $C$-glycoside structural motif where the endo-acetal ring oxygen has been replaced with a methylene group. In particular, polyhydroxylated cyclohexane cyclitols that mimic pyrano-sugars are ubiquitous in many biologically important natural products. We successfully developed a pratical route to achieve rhamno-carbasugars in either enantiomeric and diastereomeric form (e.g., $\alpha-\mathrm{L}, \beta-\mathrm{L}, \alpha-\mathrm{D}$, $\beta$-D) starting with quinic acid. Three cyclitol/carbasugar monosaccharides and five carbasugar $C$ 1 phosphates have been synthesized.

In order to further facilitate the medicinal structure-activity relationship (SAR) study of SL0101, we also turned our attention to the synthesis of carbsugar glycoside analogues of 
SL0101. A practical palladium(0)-catalyzed cyclitolization/C-in ring-glycosylation and a postcyclitolization transformation has been successfully developed. This methodology has been utilized to synthesize six carbasugar analogues of SL0101 in either enantiomeric form.

Efforts have been made toward the synthesis of biologically important jadomycin A and B. A practical approach has been successfully developed to achieve $\alpha$-digitoxose, the sugar moiety of jadomycin B by employing a new post-glycosylation reactions following palladium(0)-catalyzed glycosylation reaction. Jadomycin A, the aglycone part of jadomycin B, has been successfully synthesized. The key transformations included an oxidative ring closure of dihydropyridine followed by acid induced lactonization to form an oxazolone ring. 


\section{DEDICATED TO}

\section{My wife Huihua Lu, My son Kevin L. Shan \\ My mother Xinying Wu}

\section{IN MEMORY OF}

My father Yuanxi Shan 


\section{ACKNOWLEDGEMENTS}

First of all, I would like to give my sincere gratitude to my advisor, Dr. George A. O'Doherty, for his great support, patience, and encouragement throughout my graduate studies. His mentorship was essential to the completion of this dissertation.

My thanks also go to Dr. Patrick S. Callery, Dr. Jeffrey L. Petersen, Dr. X. Michael Shi, Dr. Björn C. G. Söderberg for their kindness for being my committee members and their valuable comments that improved the contents of this dissertation.

I would like to thank the entire faculty and staff for their teaching and help during my graduate study in past five years.

I am thankful to Dr. Weixiang Dai, who has helped me a lot during my early life at Morgantown. I would also like to thank the former and present group members, Dr. Md. Moinuddin Ahmed, Dr. Satheesh Babu Ravula, Dr. Joseph Dougherty, Dr. Dennis Tomcik, Dr. Dong Gao, Dr. Miaosheng Li, Dr. Sanjeeva Guppi, Dr. Maoquan Zhou, Dr. Matthew Mortensen, Dr. Haibing Guo, Philip Harsh, Xiaomei Yu, Wenjun Xin, Bulan Wu, Yalan Xing, Leo Wang, Ehesan Sharif, Melvin Rajatanan, Qi Zhang, Michael Mcuccarese, Joshua Osbourn, Natasha Smith, Jason Coral, Shan Chen, Ian Townsend for their help and all other postdocs and graduate students in this department for their friendship and help in a variety of ways.

I would also like to thank Dr. Jeffrey L. Petersen for his help in the X-ray crystallographic analysis of my research samples, Dr. Novruz Akhmedov for his help during NMR experiments and my fellow Brent Reschke, Heather Glover for their help in finding the mass spectra.

Financial support from the National Institute of Health, C. Eugene Bennett Department of Chemistry at West Virginia University, the National Science Foundation, NSF-EPSCoR (0314742) for a $600 \mathrm{MHz}$ NMR at WVU are gratefully acknowledged. A dissertation fellowship from Professor Jonathan Cumming, Assistant Vice President for Graduate Education, Office of Graduate Education and Life, West Virginia University are highly appreciated.

Finally, I would like to thank all my family members for their love and support for my education through the past many years. 


\section{TABLE OF CONTENTS}

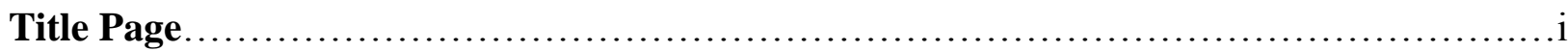

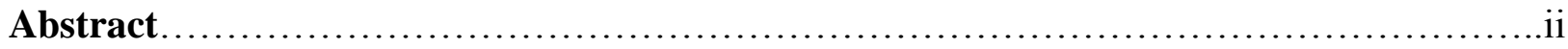

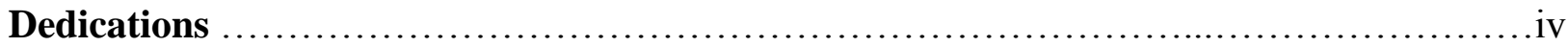

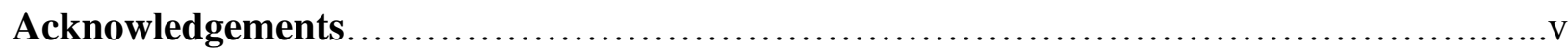

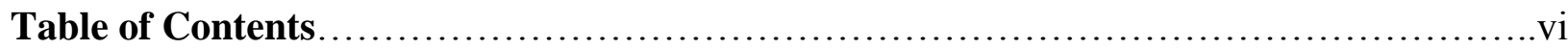

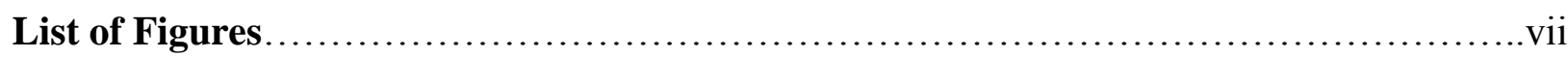

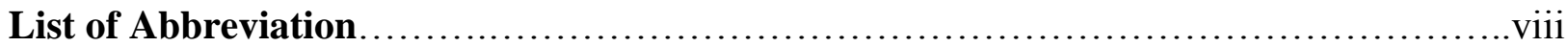

Chapter I. De Novo Asymmetric Syntheses of SL0101 and Its Analogues.................

Chapter II. Asymmetric Synthesis of Cyclitol/Carbasugar...........................11

II.1. Cyclitol/carbasugar monosaccharide......................................11

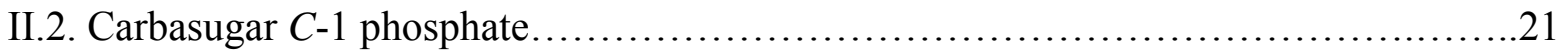

Chapter III. Methodology \& Synthesis of Carbasugar Analogues of SL0101.............27

III.1. Methodology development of carbasugar glycoside...........................27

III.2. Synthesis of carbasugar glycoside analogues of SL0101 .......................33

Chapter IV. Efforts toward the Total Synthesis of Jadomycin A \& B....................38

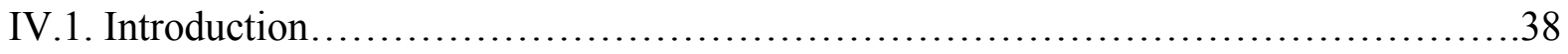

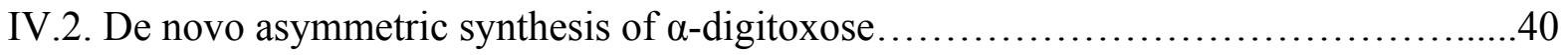

IV.3. Synthesis of jadomycin A............................................... 46 
V.1. General methods and materials.............................................52

V.2. Chapter I experimental................................................53

V.3. Chapter II experimental................................................. 73

V.4. Chapter III experimental............................................... 118

V.5. Chapter IV experimental................................................. 144

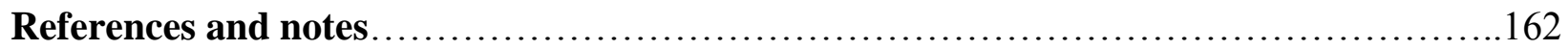

\section{List of Figures}

Figure 1. Rsk2 inhibitory activities of SL0101 (I-1a) and its analogues (I-1b to I-1e)..........1

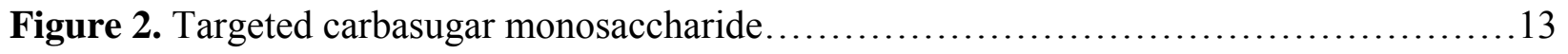

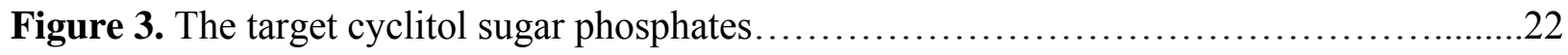

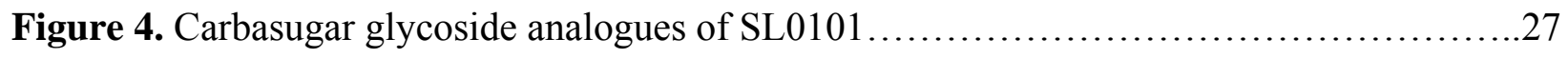

Figure 5. Structural features of Jadomycin A and Jadomycin B.......................... 


\section{List of Abbreviations}

Ac

AIBN

Anal.

$\mathrm{Bn}$

$\mathrm{Bz}$

Boc

bp

$\mathrm{Bu}$

$\mathrm{BuLi}$

Calcd

$\mathrm{Cbz}$

CI

$m \mathrm{CPBA}$

CSA

d

DBA

DBU

DCC

de

DEAD

$\delta$

DIAD

DIBAL-H

DIEPA

DMAP

DMDO

DMF

DMSO

ee
Acetyl

Azobisisobutyronitrile

Analysis

Benzyl

Benzoyl

t-Butoxycarbonyl

Boiling point

Butyl

n-Butyllithium

Calculated

Benzyloxyl carbonyl

Chemical Ionization

m-Chloroperbenzoic acid

Camphorsulfonic acid

Doublet

trans, trans-dibenzylideneacetone

1,8-Diazabicyclo[5.4.0]undec-7-ene

Dicyclohexylcarbodiimide

Diastereomeric excess

Diethyl azodicarboxylate

Chemical shift (ppm)

Diisopropylazodicarboxylate

Diisobutylaluminum hydride

Diisopropylethylamine

4-Dimethylaminopyridine

Dimethyldioxirane

Dimethylformamide

Dimethyl sulfoxide

Enantiomeric excess 


\begin{tabular}{|c|c|}
\hline EI & Electron ionization \\
\hline ent & Enantiomer \\
\hline equiv & Equivalent(s) \\
\hline ESI & Electron spray ionization \\
\hline Et & Ethyl \\
\hline EtOAc & Ethyl acetate \\
\hline g & $\operatorname{Gram}(\mathrm{s})$ \\
\hline $\mathrm{h}$ & Hour(s) \\
\hline HRMS & High resolution mass spectrum \\
\hline $\mathrm{Hz}$ & Hertz (cycles per second) \\
\hline IR & Infrared \\
\hline$J$ & Spin-spin coupling constant \\
\hline LAH & Lithium aluminum hydride \\
\hline LDA & Lithium diisopropylamide \\
\hline L-selectride & Lithium tri-sec-butylborohydride \\
\hline mol & Mole(s) \\
\hline $\mathrm{m}$ & Multiplet \\
\hline $\mathrm{Me}$ & Methyl \\
\hline $\mathrm{MHz}$ & Megahertz \\
\hline $\min$ & Minute(s) \\
\hline mmol & Millimole(s) \\
\hline MOM & Methoxy methyl \\
\hline $\mathrm{mp}$ & Melting point \\
\hline MS & Mass spectrum \\
\hline NBS & N-Bromosuccinimide \\
\hline NIS & N-Iodosuccinimide \\
\hline NBSH & $o$-Nitrobenzenesulfonylhydrazide \\
\hline NMO & N-Methylmorpholine N-oxide \\
\hline NMR & Nuclear magnetic resonance \\
\hline $\mathrm{Ph}$ & Phenyl \\
\hline
\end{tabular}




$\begin{array}{ll}\text { PMB } & p \text {-Methoxybenzyl } \\ \text { PNBz } & p \text {-Nitrobenzoyl } \\ \text { ppm } & \text { Parts per million } \\ \text { Py } & \text { Pyridine } \\ \mathrm{q} & \text { Quartet } \\ R_{f} & \text { Ratio to front } \\ \mathrm{rt} & \text { Room temperature } \\ \mathrm{t} & \text { Triplet } \\ \text { TBAF } & \text { Tetrabutylammonium fluoride } \\ \text { TBS } & t \text {-Butyldimethylsilyl } \\ \text { TFA } & \text { Trifluoroacetic acid } \\ \text { THF } & \text { Tetrahydrofuran } \\ \text { TLC } & \text { Thin layer chromatography } \\ \text { TTMSS } & \text { Tris(trimethylsilyl)silane } \\ p \text {-TsOH } & p \text {-toluene sulfonic acid }\end{array}$




\section{Chapter I. De Novo Asymmetric Syntheses of SL0101 and Its Analogues ${ }^{*, 1}$}

In an effort to find specific inhibitors of p90 Ribosomal S6 Kinase (RSK), Smith and Hecht screened an extensive collection of botanical extracts derived from rare plants. ${ }^{2}$ Using a dual high-throughput screen they found only one extract, which inhibited the RSK2 isoform (RSK2) without inhibiting the tyrosine kinase (FAK). The active extract was from a South American dogbane plant named Forsteronia refracta. More detailed bioactive fractionization revealed the active constituent to be a kaempferol glycoside, which was given the name SL0101 (Figure 1). ${ }^{2}$ SL0101 (I-1a) is a member of a class of acylated kaempferol L-rhamnosides which occur naturally with various degrees of acylation (e.g., I-1a to I-1e). ${ }^{3}$ SL0101 sans acetyl groups (I-1b) is also known as afzelin. ${ }^{3 a}$ The kaempferol glycosides, like most flavonoids, have received a great deal of attention because they are believed to induce many positive biological effects. ${ }^{4}$

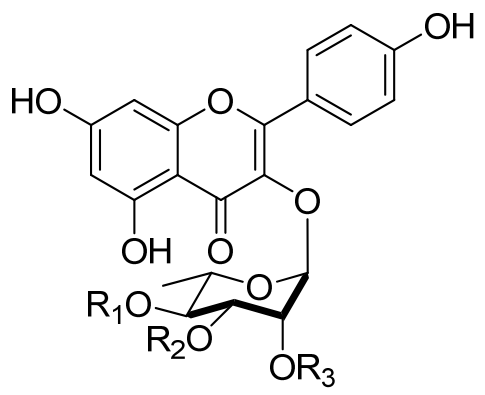

\begin{tabular}{ccccc}
\hline & $\mathrm{R}_{1}$ & $\mathrm{R}_{2}$ & $\mathrm{R}_{3}$ & $\mathrm{IC}_{50}(\mathrm{RSK} 2)$ \\
\hline I-1a & $\mathrm{Ac}$ & $\mathrm{Ac}$ & $\mathrm{H}$ & $89 \mathrm{nM}$ \\
I-1b & $\mathrm{H}$ & $\mathrm{H}$ & $\mathrm{H}$ & ------- \\
I-1c & $\mathrm{Ac}$ & $\mathrm{Ac}$ & $\mathrm{Ac}$ & ------ \\
I-1d & $\mathrm{Ac}$ & $\mathrm{H}$ & $\mathrm{H}$ & $189 \mathrm{nM}$ \\
I-1e & $\mathrm{Ac}$ & $\mathrm{H}$ & $\mathrm{Ac}$ & $580 \mathrm{nM}$
\end{tabular}

Figure 1. Rsk2 inhibitory activities of SL0101 (I-1a) and its analogues (I-1b to I-1e)

In addition to this unique activity, our interest in SL0101 (I-1a) was peaked by the report that it displayed some 150 times greater activity than the simple aglycon, kaempferol. Similarly, we

\footnotetext{
* Reproduced with permission from Org. Lett. 2006, 8, 5149-5152. Copyright 2006, American Chemical Society.
} 
were intrigued by the importance of the specific placement of acetyl groups on the L-rhamnose and its effect on the structure-activity relationship (SAR) of SL0101 (Figure 1). ${ }^{5}$ As part of an effort to elucidate the role of the sugar and acetyl portion of SL0101 to its activity, we decided to prepare both enantiomers of SL0101 (I-1a) and its analogues I-1b to I-1e (Figure 1).

Not long after the isolation and structure elucidation of SL0101 (I-1a), its first synthesis was reported by Professor Hecht. ${ }^{5}$ The Hecht synthesis derived the absolute and relative stereochemistry from L-rhamnose. In contrast, we were interested in the possibility of preparing all five members of this class of kaempferol glycosides (I-1a to I-1e) via asymmetric catalysis. This de novo approach would have the additional advantage of preparing both the D- and the Lenantiomers for biological testing.

Recently we reported a diastereoselective palladium-catalyzed glycosylation reaction that used alcohols as nucleophiles and Boc-pyranones such as $\mathbf{I}-\mathbf{4} \boldsymbol{\alpha}$ as glycosyl donors. ${ }^{6}$ We have also found several post glycosylation transforms, which subsequently install the desired sugar stereochemistry. ${ }^{7}$ This methodology also works well for other $N$ - and $O$-nucleophiles, such as 6chloropurine/benzimidizole and phenol. ${ }^{8}$ In order to produce this class of interesting compounds for activity studies, we decided to apply this methodology toward the syntheses of the flavone glycosides, SL0101 (I-1a) and its analogues (I-1b to I-1e). In addition to providing material for biological study, this effort should also allow us to study flavon-3-ol as a nucleophile in the palladium-catalyzed glycosylation. 
Scheme 1. Retrosynthetic analysis of kaempferol rhamnosides (I-1a to I-1e)

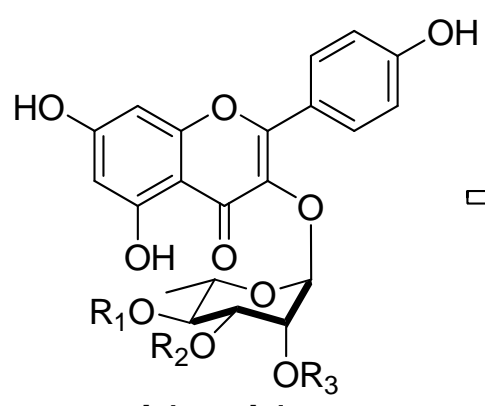

I-1a to I-1e<smiles>C=CCc1ccccc1</smiles>

$\mathrm{I}-2$<smiles>O=c1c(O)c(-c2ccc(OCc3ccccc3)cc2)oc2cc(OCc3ccccc3)cc(OCc3ccccc3)c12</smiles>

I-3

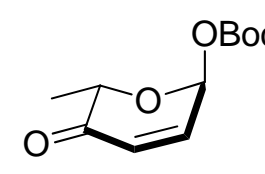

$\mathrm{I}-4 \alpha$

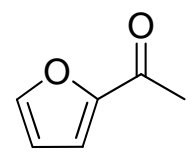

I-5

Retrosynthetically, we envisioned that pyranone $\mathbf{I}-\mathbf{2}$ could be derived from a palladium(0)catalyzed glycosylation between flavonol I-3 and pyranone I-4a (Scheme 1). Subsequent application of $\mathrm{NaBH}_{4}$ reduction and Upjohn dihydroxylation $\left(\mathrm{OsO}_{4} / \mathrm{NMO}\right)^{9}$ would install the manno-stereochemistry. ${ }^{10}$ The selective introduction of the C-4 acetyl group should occur by introducing an acylation reaction between the $\mathrm{NaBH}_{4}$ reduction and dihydroxylation. All that would remain would be to differentiate the C-2 hydroxyl group from the C-3 hydroxyl group. For this, we planned to use a combination of selective orthoester hydrolysis ${ }^{11}$ and acyl migration reactions. ${ }^{12}$ Since pyranone $\mathbf{I}-\mathbf{4} \boldsymbol{\alpha}$ has been prepared in either enantiomeric form, this procedure should be amenable to the preparation of both enantiomers of I-1a to I-1e. In this chapter, we will describe our successful efforts at the implementation of this strategy to this class of kaempferol glycosides (I-1a to I-1e), which is noteworthy in that the various acetyl groups in I1a to I-1e are installed without any hydroxyl protecting groups on the sugar. 
Scheme 2. Synthesis of aglycone

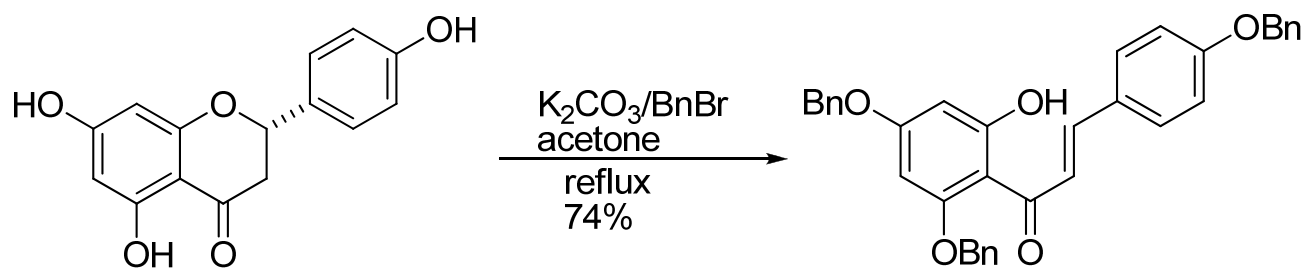

I-6<smiles>COc1cc(O[13CH3])c2c(=O)cc(-c3ccc(OCC(C)(C)C)cc3)oc2c1</smiles>

I-7

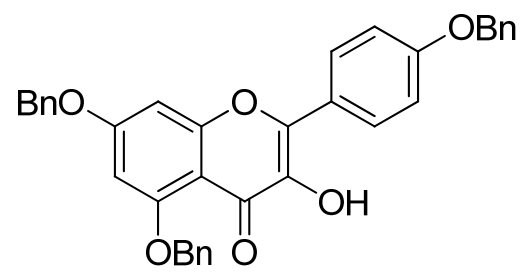

I-3

Our synthesis started with the known perbenzylated kaempferol I-3, which was synthesized from narringin I-6 in three steps (Scheme 2). ${ }^{5}$ Benzylation of narrigin I-6 by $\mathrm{BnBr}$ in the $\mathrm{K}_{2} \mathrm{CO}_{3}$ /acetone reflux condition with concomitant $\beta$-elimination afforded enone $\mathbf{I}-\mathbf{7}$, which was subjected to oxidative cyclization to enone $\mathbf{I - 8}$ under $\mathrm{I}_{2} / \mathrm{DMSO}$ condition. The cyclic enone was oxidized to epoxide by in situ produced DMDO in a basic buffer, which underwent acid-induced rearrangement to give aglycone flavonol I-3.

Scheme 3. Synthesis of Boc-pyranones as sugar donors

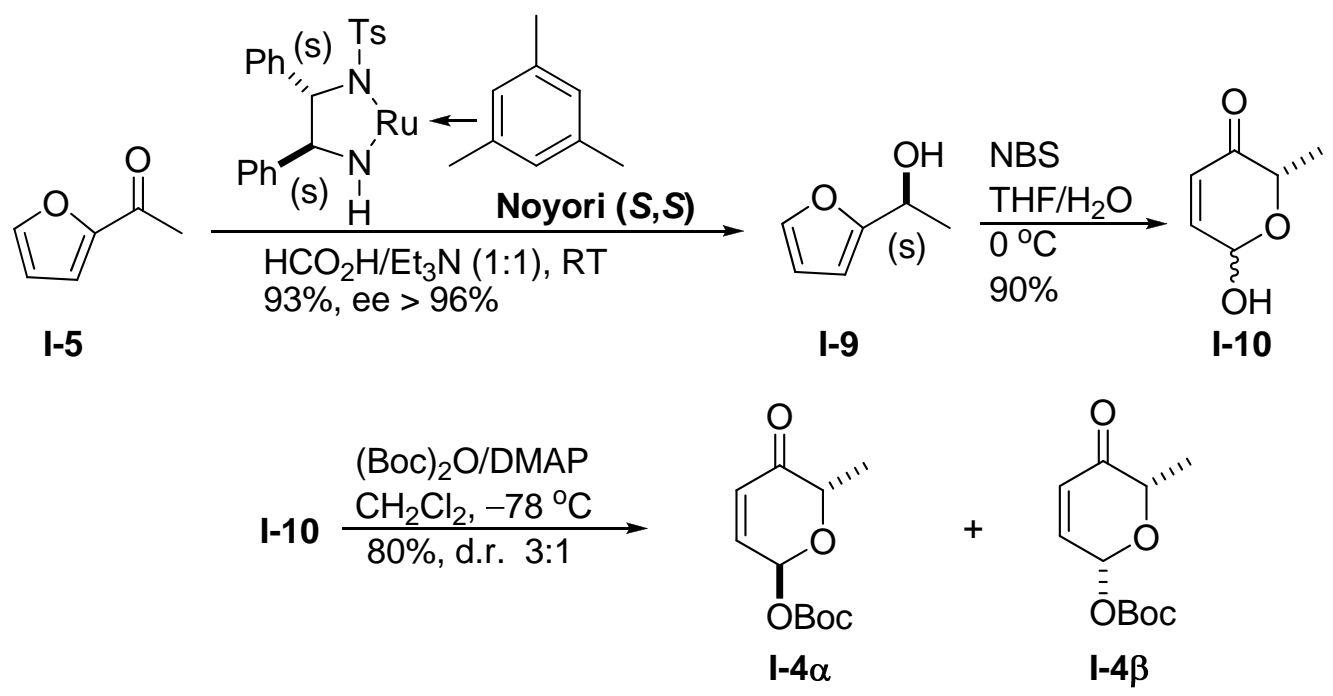


For the synthesis of the Boc-pyranone, we started with the achiral acylfuran (Scheme 3). Noyori reduction of acetylfruan I-5 by using Noyori $(S, S)$ catalyst in the presence of complex of formic acid and triethylamine as hydride source produced the furfuryl alcohol I-9 in 93\% yield with high enantiomeric excess (>96\% ee). An Achmatowiz rearrangement transformed the furfuryl alcohol to hemi-acetal I-10 in 91\% yield. The hemiacetal was then protected as $t$-butyl carbonate $\mathbf{I}-\mathbf{4} \boldsymbol{\alpha}$ and $\mathbf{I}-\mathbf{4} \boldsymbol{\beta}$ at low temperature in $78 \%$ yield with $3: 1(\alpha / \beta)$ diastereomeric ratio, which could be easily separated to give the L-sugar precursor. It is worth noting that by simply switching the catalyst Noyori $(S, S)$ to Noyori $(R, R)$, we are able to synthesize the D-sugar precursor.

Scheme 4. Synthesis of kaempferol-3- $\alpha$-L-rhamnoside (I-1b)

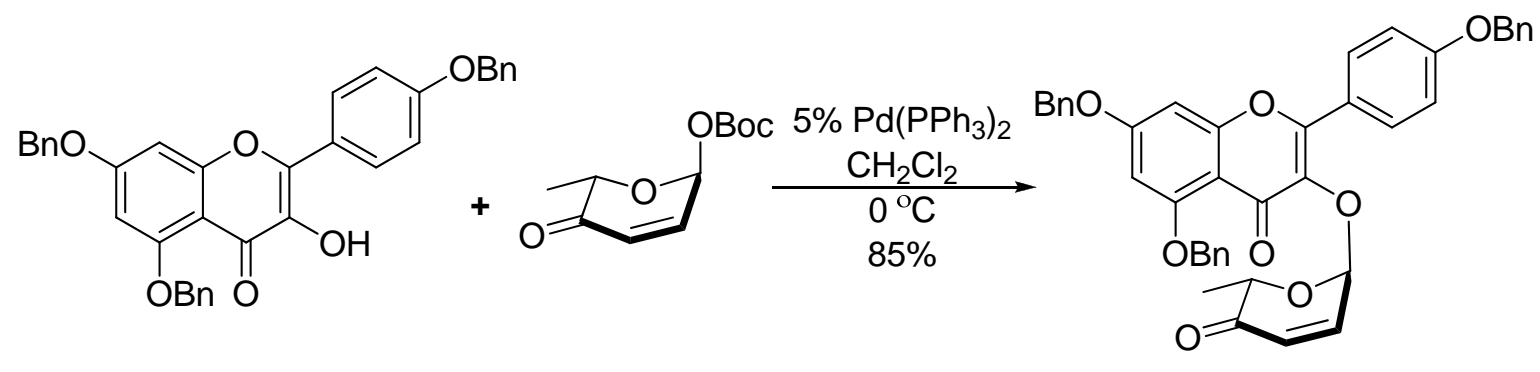

I-3

I- $4 \alpha$

I-2

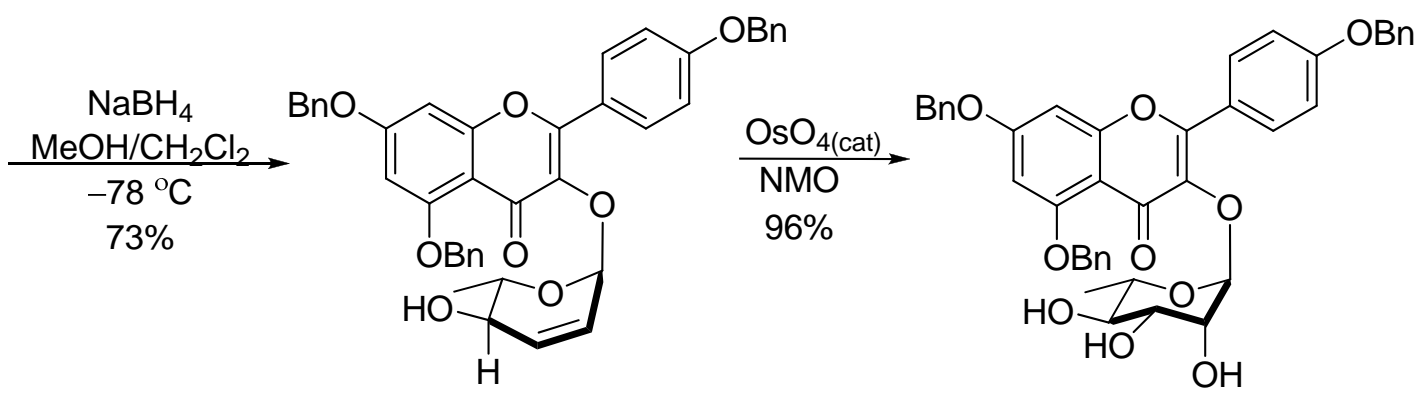

I-11

I-12<smiles></smiles> 
The glycosylation was carried out with flavonol I-3 and L-Boc-pyranone I-4 $\boldsymbol{\alpha}$ under catalysis of $2.5 \mathrm{~mol}_{0} \mathrm{Pd}_{2}(\mathrm{dba})_{3}{ }^{\circ} \mathrm{CHCl}_{3}$ and $10 \mathrm{~mol} \%$ of $\mathrm{PPh}_{3}$ in $\mathrm{CH}_{2} \mathrm{Cl}_{2}$ at $0{ }^{\circ} \mathrm{C}$, which afforded glycosylated pyranone I-2 in $85 \%$ yield with complete $\alpha$-selectivity (Scheme 4). Reduction of the enone $\mathbf{I - 2}$ by $\mathrm{NaBH}_{4}$ at $-78{ }^{\circ} \mathrm{C}$ in $\mathrm{CH}_{2} \mathrm{Cl}_{2} / \mathrm{MeOH}$ resulted in allylic alcohol I-11 in $73 \%$ yield with excellent diastereoselectivity ( $\mathrm{dr}>20: 1)$. The rhamno-stereochemistry in I-1b was diastereoselectively introduced upon exposure of $\mathbf{I}-\mathbf{1 1}$ to the Upjohn conditions $\left(\mathrm{OsO}_{4} / \mathrm{NMO}\right.$, 96\%). Debenzylation of resulted I-12 using Pearlman's catalyst (10\% $\mathrm{Pd} / \mathrm{C})$ in the presence of hydrogen gave kaempferol-3- $\alpha$-L-rhamnoside (I-1b) in $80 \%$ yield.

In addition to the unacylated rhamno-sugar I-1b, the peracylated sugar I-1c could also be easily prepared in two steps from triol I-12 (Scheme 5). Exhaustive acylation of the triol I-12 with the excess acetic anhydride in presence of pyridine and 10 mol\% DMAP gave triacetate I13 in $86 \%$ yield. Debenzylation of triacetate $\mathbf{I}-\mathbf{1 3}$ by hydrogen using Pearlman's catalyst ( $10 \%$ $\mathrm{Pd} / \mathrm{C}$ ) again produced kaempferol-3- $\alpha$-L-2",3",4"-O-triacetylrhamnoside (I-1c) in $86 \%$ yield.

Scheme 5. Synthesis of kaempferol-3- $\alpha-L-2 ", 3$ ", 4"-O-triacetylrhamnoside (I-1c)

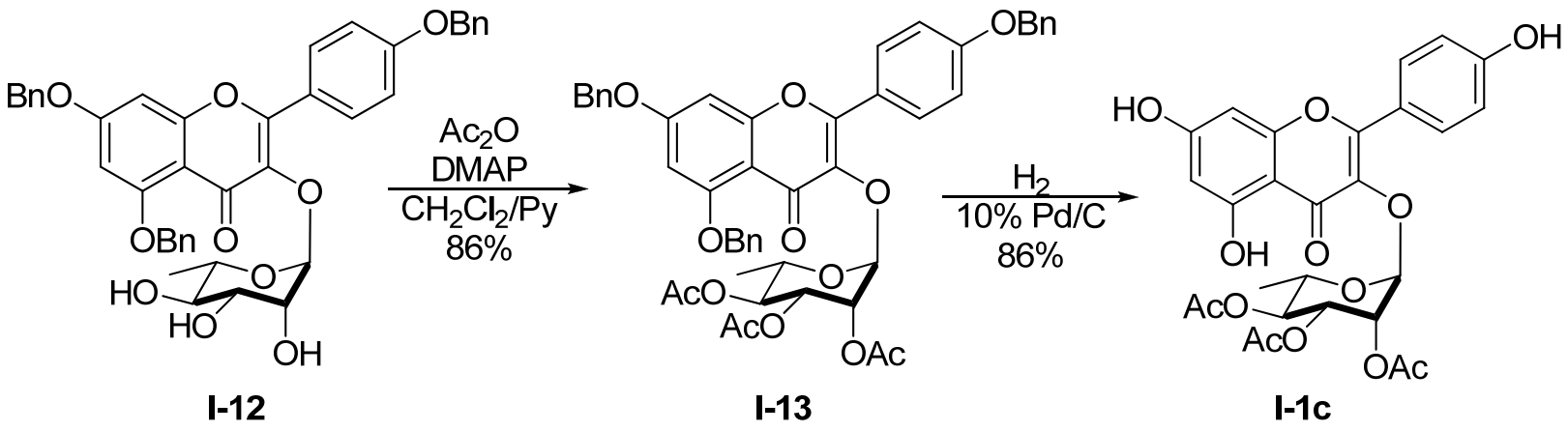

The selective installation of the C-4 acetyl group in I-1d was easily achieved without the need of additional protecting groups from the previously described allylic alcohol I-11 (Scheme 4). In this route (Scheme 6), the $\mathrm{NaBH}_{4}$ reduction of enone $\mathbf{I - 2}$ was followed by an acylation of the resulting allylic alcohol with acetic anhydride in the presence of pyridine and DMAP. This two-step procedure afforded acetate $\mathbf{I - 1 4}$ in $70 \%$ overall yield. Once again, dihydroxylation using the Upjohn condition stereoselectively converted allylic acetate I-14 into the rhamno-diol I-15 in 77\% yield. Global deprotection was accomplished under hydrogenolysis conditions by 
exposure of diol I-15 to 1 atm of $\mathrm{H}_{2}$ in the presence of Pearlman's catalyst $(\mathrm{Pd} / \mathrm{C})$, which furnished kaempferol-3- $\alpha$-L-4"'-O-acetylrhamnoside (I-1d) in 87\% yield.

Scheme 6. Synthesis of kaempferol-3- $\alpha-L-4$ "'-O-acetylrhamnoside (I-1d)

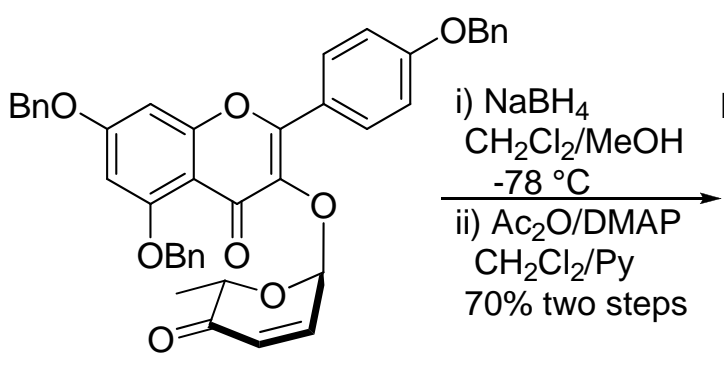

I-2

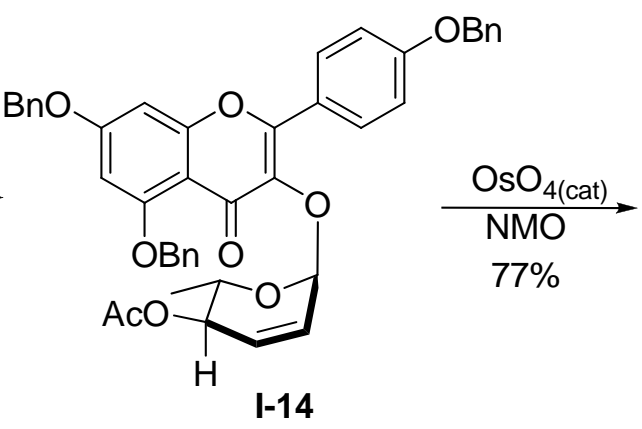

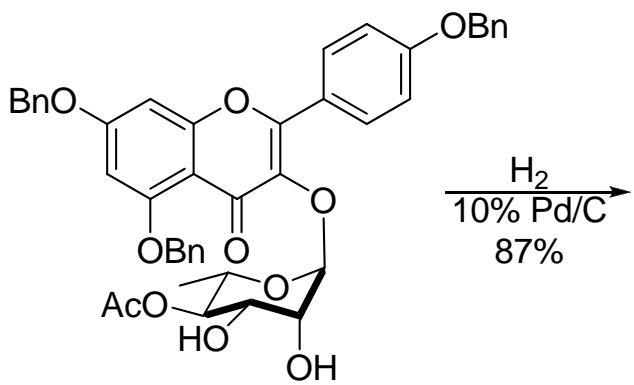

I-15<smiles></smiles>

I-1d

Acylation of the C-2 axial hydroxyl group of diol I-15 was selectively achieved by using orthoester chemistry in excellent yield (99\%) (Scheme 7). ${ }^{11}$ Exposure of diol I-15 to trimethyl orthoacetate in the presence of $10 \% p-\mathrm{TsOH}$ in $\mathrm{CH}_{2} \mathrm{Cl}_{2}$ followed by hydrolysis with excess $90 \%$ $\mathrm{HOAc} / \mathrm{H}_{2} \mathrm{O}$ afforded diacetate I-16 (Scheme 5). Once again, reductive debenzylation of I-16 with hydrogen and 10\% $\mathrm{Pd} / \mathrm{C}$ produced kaempferol-3- $\alpha-\mathrm{L}-2$ ",4"-O-diacetylrhamnoside (I-1e) in $88 \%$ yield. 
Scheme 7. Synthesis of kaempferol-3- $\alpha$-L-2",4"-O-diacetylrhamnoside (I-1e)

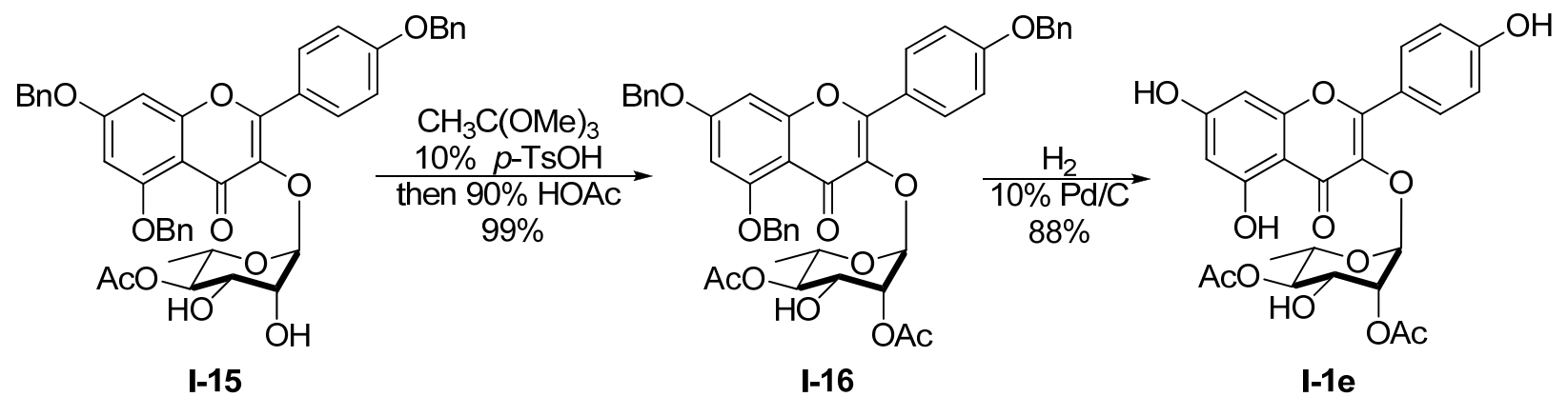

In contrast to the selective C-2 acylation of the axial alcohol in I-15 (Scheme 7), our synthesis of SL0101 required the selective acylation of the C-3 equatorial alcohol (Scheme 8). Unfortunately, all of our attempts with $\mathrm{Ac}_{2} \mathrm{O} / \mathrm{Py}$ at various temperature only furnished mixtures of diacetate I-17 and I-16, as well as triacetate I-13 ( 1:1:1). Since we could achieve I-16 with high regioselectivity, we turned our attention to the acetyl group shift from the less stable axial C-2 acetate in I-16 to the more stable equatorial C-3 acetate in I-17.

All attempts to shift the axial acetyl group of diacetate I-16 to the equatorial position by using $10 \mathrm{~mol} \%$ p-TsOH in $\mathrm{CH}_{2} \mathrm{Cl}_{2}$ at room temperature failed to give desired diacetate $\mathbf{I}-\mathbf{1 7}$ (Scheme 8). In contrast, more promising results were seen with basic conditions. For instance, analysis of crude ${ }^{1} \mathrm{H}$ NMR of dilute toluene solutions of I-16 with one equiv of DBU showed clean conversion to a 2:1 mixture of I-17 and I-16. In practice good yields of I-17 (62\%) could be obtained along with recovered starting material (34\%) after $\mathrm{SiO}_{2}$ chromatography. Finally, debenzylation of I-17 under the similar condition as before produced SL0101 (I-1a) in 91\% yield. 
Scheme 8. Synthesis of SL0101 (I-1a)

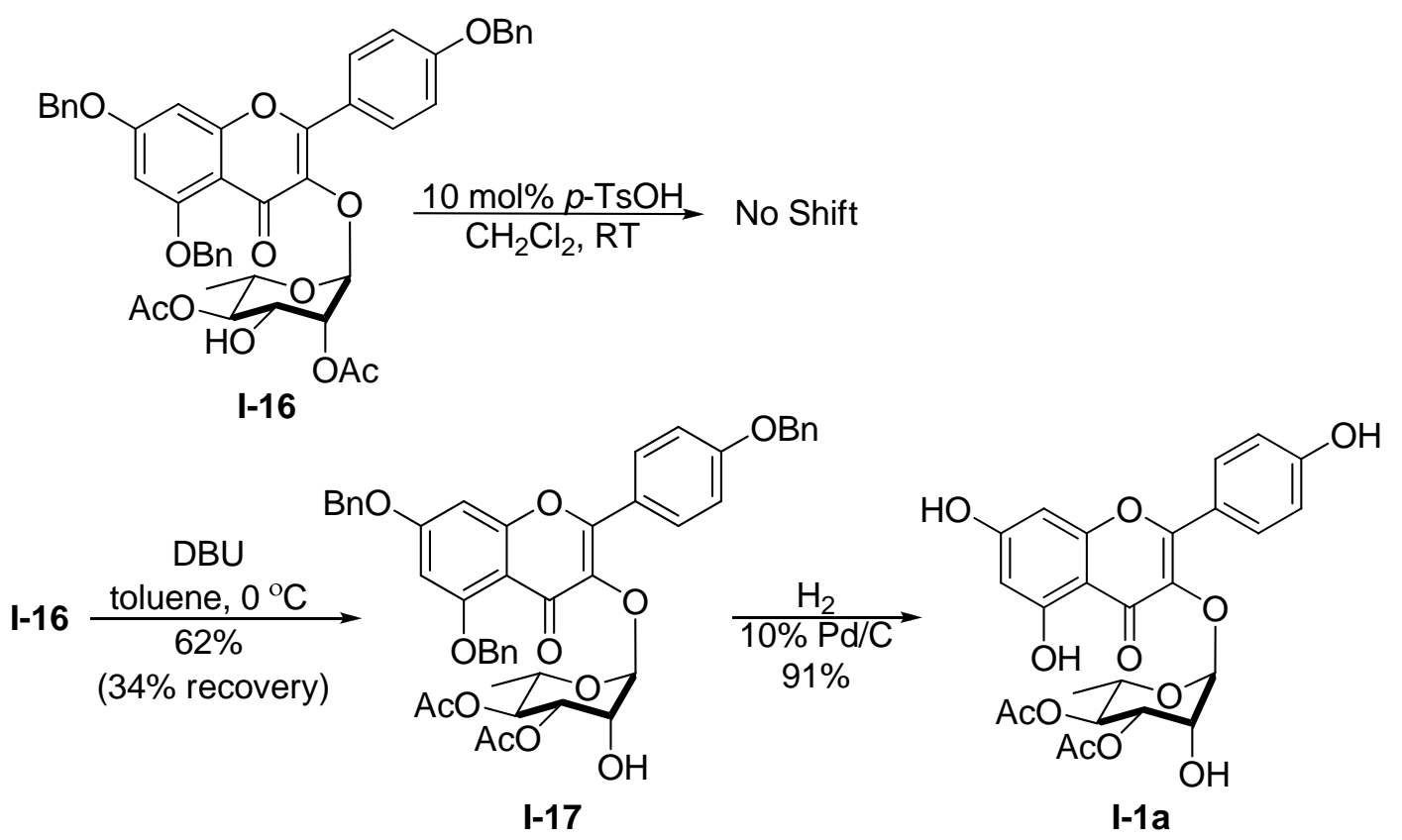

To synthesize the D-sugar analogue of SL0101, we started our synthesis by simply switching the $\alpha$-L-Boc-pyranone to $\alpha$-D-Boc-pyranone (Scheme 9). Palladium(0)-catalyzed glycosylation of perbenzylated aglycone I-3 with $\alpha$-D-Boc-pyranone (ent)-I-4a afforded glycosylated pyranone (ent)-I-2 in $71 \%$ yield and absolute stereocontrol. $\mathrm{NaBH}_{4}$ reduction of (ent)-I-2 followed by the dihydroxylation of the resulted allylic alcohol upon Upjohn condition furnished triol (ent)-I-8 in $60 \%$ yield over two steps. Debenzylation of the triol (ent)-8 under $\mathrm{Pd} / \mathrm{C}$ hydrogenation condition produced SL0101 $\alpha$-D-sugar analogue (ent)-1b in 90\% yield. Our synthetic products (I-1a to I-1e) were physically and spectroscopically identical to the isolated natural materials in terms of melting point, optical rotation, $R_{f},{ }^{1} \mathrm{H} \mathrm{NMR},{ }^{13} \mathrm{C} \mathrm{NMR}$ and MS. ${ }^{3,5}$ 
Scheme 9. Synthesis of SL0101 D-sugar analogue

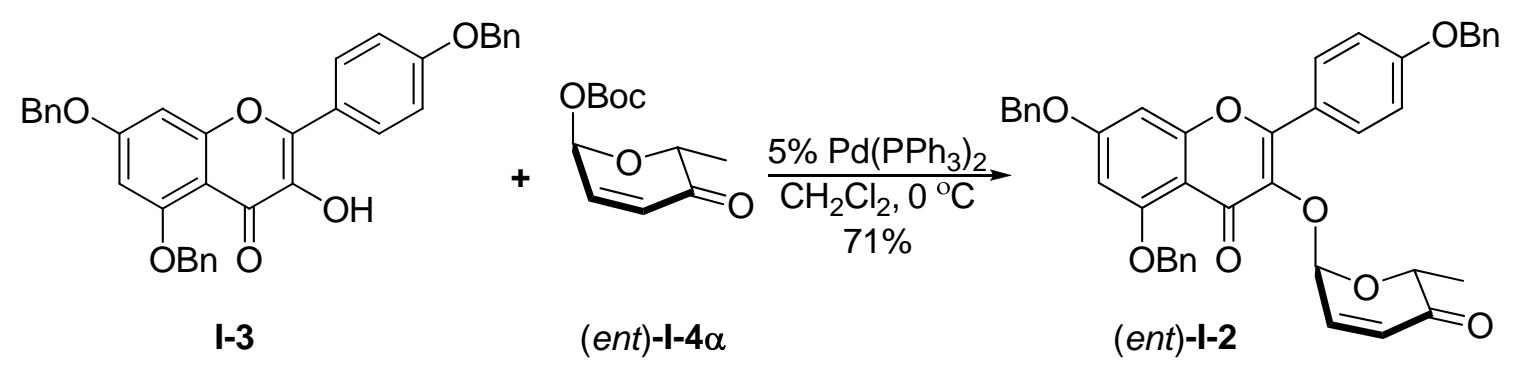

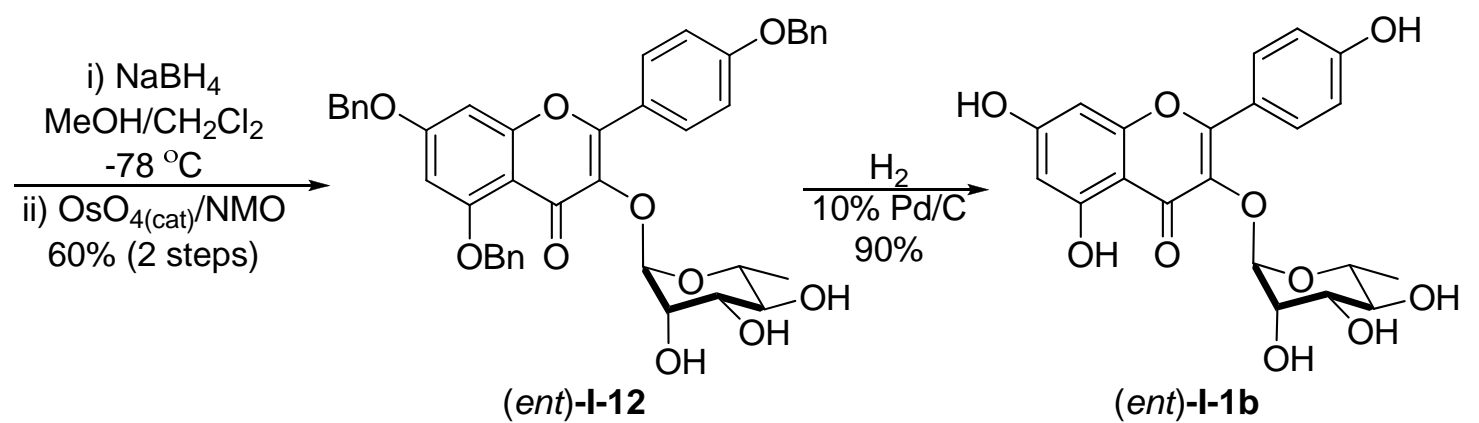

In conclusion, a divergent and highly enantio- and diastereoselective procedures for the preparation of naturally occurring SL0101 (I-1a) as well as four kaempferol rhamnoside analogues (I-1b to I-1e) and one enantiomeric analogue ((ent)-1b) has been developed. This approach provides all the kaempferol glycosides without protecting any of the sugar hydroxyl groups. Our approach relied upon a diastereoselective palladium(0)-catalyzed glycosylation followed by a sequence of reduction/dihydroxylation reaction, as well as acylation. An acetyl group shift from an axial position to an equatorial position provided an alternative way for selective acylation of the equatorial hydroxyl group of a cis-diol in carbohydrate chemistry. 


\section{Chapter II. Asymmetric Synthesis of Cyclitol/Carbasugar}

\section{II.1. Cyclitol/Carbasugar monosaccharide ${ }^{\dagger, 13}$}

$C$-glycosides are carbohydrates where one of the acetal oxygens has been replaced with a methylene group. ${ }^{14}$ These non-hydrolyzable sugar mimics have long been recognized as an important pharmacophore. ${ }^{15}$ Cyclitols are an important subset of the $C$-glycoside structural motif where the endo-acetal ring oxygen has been replaced with a methylene group. ${ }^{16}$ In particular, polyhydroxylated cyclohexane cyclitols that mimic pyrano-sugars are ubiquitous in biologically important natural products (e.g., aminoglycosides, validamycins, acarbose, pyralomicins, pancratistatin). ${ }^{16}$

Scheme 10. Existing routes to cyclitol pyranose sugar

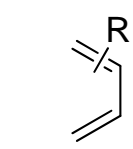

butadienes

dienes

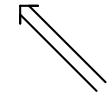<smiles>[R]c1ccccc1</smiles>

arenes

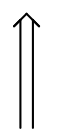<smiles>[R]C1CC2C=CC1C2=O</smiles>
bicycloheptenones<smiles>OC[C@H]1O[C@H](O)[C@@H](O)[C@H](O)[C@H]1O</smiles>

sugars

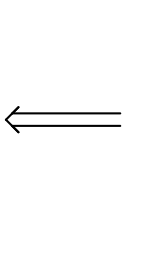<smiles>[R]C1C[C@H](O)[C@H](O)[C@H](O)[C@H]1O</smiles>

pyranose sugar<smiles>C=CC=C</smiles>

D-(-)-quinic acid

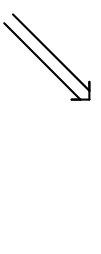

furans<smiles>O=C1C=CC(=O)C=C1</smiles>

quinones

\footnotetext{
${ }^{\dagger}$ Reproduced in part with permission from Synthesis, 2008, 3171-3179. Copyright 2008, Georg Thieme Verlag KG Stuttgart New York.
} 
Because of their pharmacological importance, there has been a myriad of approaches to the cyclitols (Scheme 10). ${ }^{16,17}$ These various routes have used both chiral (e.g., sugars and quinic acid) and achiral (e.g., arenes, furans, dienes) molecules as starting materials, with carbohydrates dominating this list of starting materials. ${ }^{16,17}$

Scheme 11. Our approach to normal pyranose carbohydrates

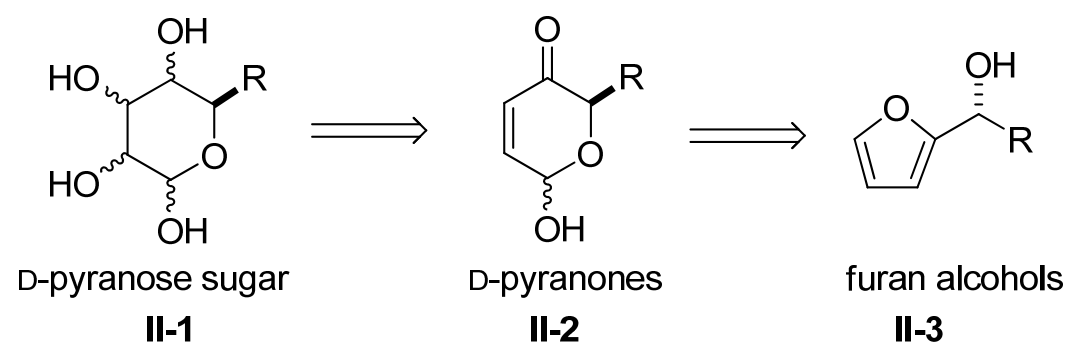

For the last ten years, we have been interested in developing de novo approaches to pyranose sugars using dienoates and furans as starting materials. ${ }^{18}$ The dienoate route uses an iterative Sharpless asymmetric dihydroxylation to prepare galacto-lactones. The furan route uses asymmetric catalysis to a furan alcohol II-3, followed by an Achmatowicz rearrangement to a pyranone II-2, which in turn can be converted into various pyranose sugars II-1 (Scheme 11). ${ }^{19}$ Of these two approaches, the route that uses furans as starting materials has the advantage of being more flexible in terms of substitution pattern and stereochemistry.

Scheme 12. Pseudoenantiomeric enones from quinic acid<smiles>CC1(C)O[C@H]2CC(=O)C=C[C@H]2O1</smiles>

II-4

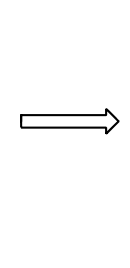

D-(-)-quinic acid

II-5

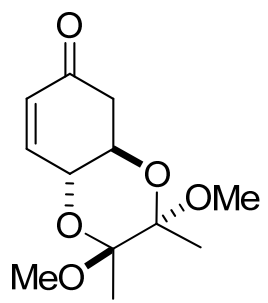

II-6

As part of our continuing efforts to develop new approaches to biologically important carbohydrates, we desired a new approach to cyclitol sugars. In particular, we were interested in developing an approach to the cyclitols that had the same structural flexibility as our furan 
approach. In particular, we desired a route that uses enones as cyclitol pyranones analogues that could similarly be converted into various cyclitol stereoisomers. While we ultimately want to develop a de novo asymmetric approach, we chose to use quinic acid II-5 as our starting material, because it was already known to be easily converted into pseudoenantiomeric enones II-4 and II6 (Scheme 12). ${ }^{20}$ Herein, we describe our approach to cyclitol sugars II-7, II-8, (ent)-II-7 from known enones II-4 and II-6 (Figure 2).

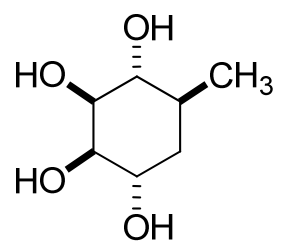

II-7

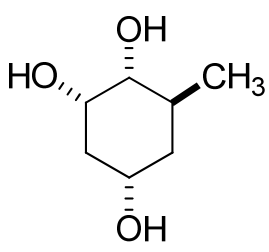

II-8<smiles>C[C@H]1CC(O)[C@H](O)[C@H](O)C1O</smiles>

(ent)-II-7

Figure 2. Targeted carbasugar monosaccharide

Our initial plan to install the methyl group for these target molecules was to use enolate chemistry to introduce a methyl group at the $\alpha$-position of $\alpha, \beta$-unsaturated ketone either through in situ generated enolate or silylenol ether. We envisioned the stereochemistry of methylation could be controlled by the other substituents in the ring. Finally, the desired sugar stereochemistry could be established by subsequent transformation, akin to our pyranone to sugar chemistry. ${ }^{18,19}$

Our synthesis started with enone II-4, which was easily prepared from D-quinic acid II-5 in 4 steps with $60 \%$ overall yield. ${ }^{20}$ Unfortunately, all attempts at 1,4-reduction and direct alkylation of enone II-4 were unsuccessful. For instance, the Tsuda-Saegusa catalytic copper hydride 1,4reduction proceeded smoothly, but in situ alkylation of the enolate intermediate with methyl iodide failed to cleanly give the desired $\alpha$-methyl ketones II-11a/II-11b (Scheme 13). ${ }^{21}$ The in situ use of Stryker's reagent for 1,4-hydrosilyation of II-4 also proved to be problematic. ${ }^{22}$

Alternatively, we envisioned that a cyclopropanation and ring opening process could lead to the formation of $\alpha$-methyl ketone. ${ }^{23}$ Thus, one pot hydrosilylation of enone II-4 $\left(\mathrm{RhCl}\left(\mathrm{PPh}_{3}\right)_{3} / \mathrm{PhMe}_{2} \mathrm{SiH}, \quad 60 \quad{ }^{\circ} \mathrm{C}\right){ }^{24}$ followed by Simmons-Smith cyclopropanation $\left(\mathrm{ZnEt}_{2} / \mathrm{CH}_{2} \mathrm{I}_{2}\right)^{25}$ of the resulting silylenol ether at $0{ }^{\circ} \mathrm{C}$ gave an inseparable mixture of 
cyclopropanol silyl ethers, which when desilylated under acidic conditions ( $p-\mathrm{TsOH} / \mathrm{MeOH})$ furnished two readily separable diastereoisomers II-9a/II-9b in $83 \%$ overall yield with $\mathrm{dr} \sim 1: 1$ (Scheme 13). With the cyclopropanol in hand, we next turned our attention to the ring opening of cyclopropanol.

Scheme 13. Cyclopropanation and attempts to ring-opening

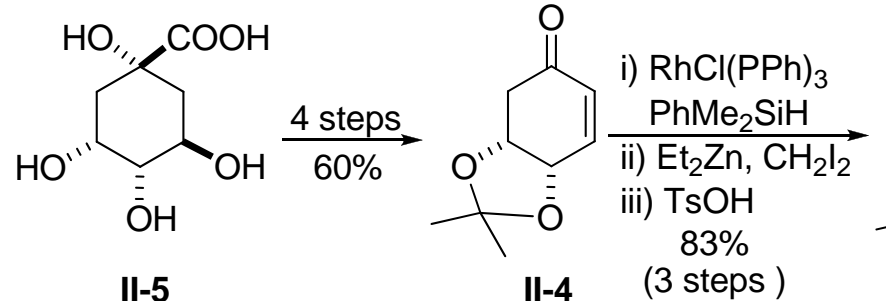<smiles>C=C1C[C@H]2OC(C)(I)O[C@@H]2CC(=O)C1=O</smiles><smiles>C[C@@H]1C[C@@H]2OC(C)([14CH3])O[C@H]2CC1=O</smiles><smiles>C[C@H]1C[C@H](O)C=CC1=O</smiles>

Unfortunately, all attempts to open the three membered ring using acid or base failed to give the desired $\alpha$-methyl ketone. ${ }^{26}$ The ring opening promoted by $\operatorname{Pd}(\mathrm{OAc})_{2}$ in the presence of pyridine at $80{ }^{\circ} \mathrm{C}$ resulted in a mixture of separable exo- and endo- $\alpha, \beta$-unsaturated ketone (II10a/II-10b $1: 2$ ) in $86 \%$ yield. ${ }^{27}$ When this reaction was carried out under similar conditions with 
the addition of two equivalents of quinone, II-10a was the only enone product produced in $70 \%$ yield along with $10 \%$ recovered starting material, which could prevent palladium(0) catalyzed isomerization of II-10a to II-10b. Interestingly, the cyclopropanol could also be opened by stoichiometric $\mathrm{Pd} / \mathrm{C}$ at RT to afford II-10a exclusively. With access to enones 10a and 10b, we next explored their hydrogenation to prepare the $\alpha$-methyl ketones II-11a/II-11b. Thus, hydrogenation of II-10a under typical conditions $\left(\mathrm{H}_{2}, \mathrm{Pd} / \mathrm{C}\right)$ afforded an inseparable diastereomeric mixture of $\alpha$-methyl ketone II-11a/II-11b in $88 \%$ yield (dr 3:1), while hydrogenation of II-10b under the same conditions produced II-11a/II-11b in $89 \%$ yield with opposite diastereoselectivity ( $\mathrm{dr}$ 1:10). In order to skirt the separation problem at this point, we continued our synthesis with the diastereomeric mixture of II-11a/II-11b. Base promoted $\beta$ elimination of II-11a/II-11b $(\mathrm{dr} 3: 1)\left(0.5 \mathrm{M} \mathrm{NaOH} / \mathrm{THF}, 0{ }^{\circ} \mathrm{C}\right)$ furnished a mixture of allylic alcohol II-12a/II-12b (dr 2:3) in $85 \%$ yield, ${ }^{28}$ which were also difficult to separate. These results forced us to reinvestigate the cyclopropanol ring opening process.

\section{Scheme 14. Synthesis of $\alpha$-D-5a-carba-rhamnose}
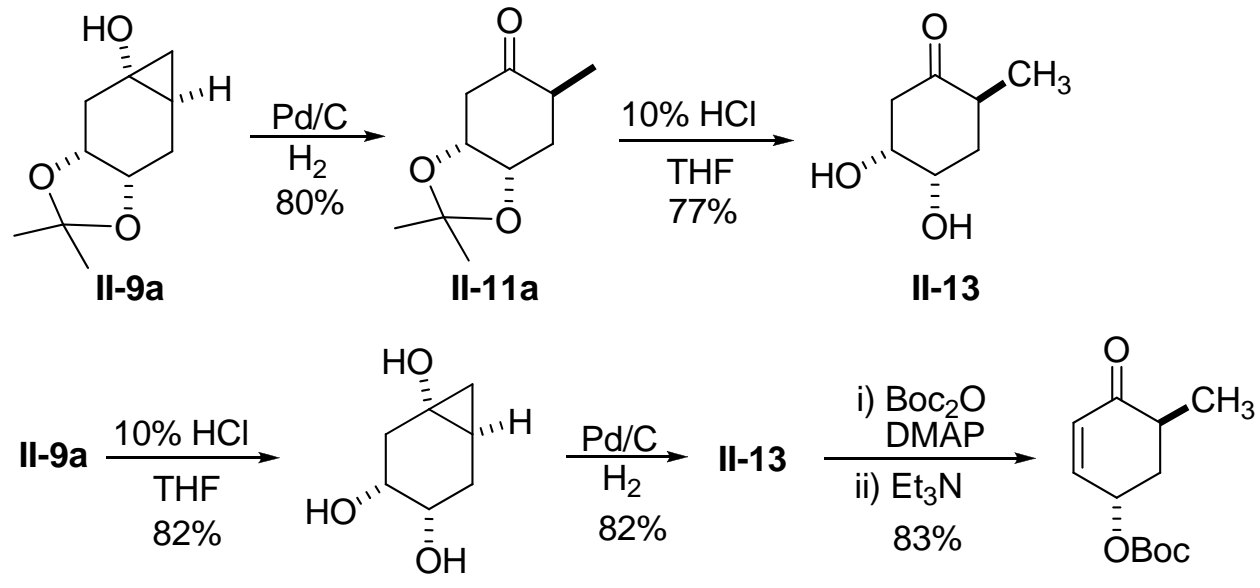

II-14

II-15

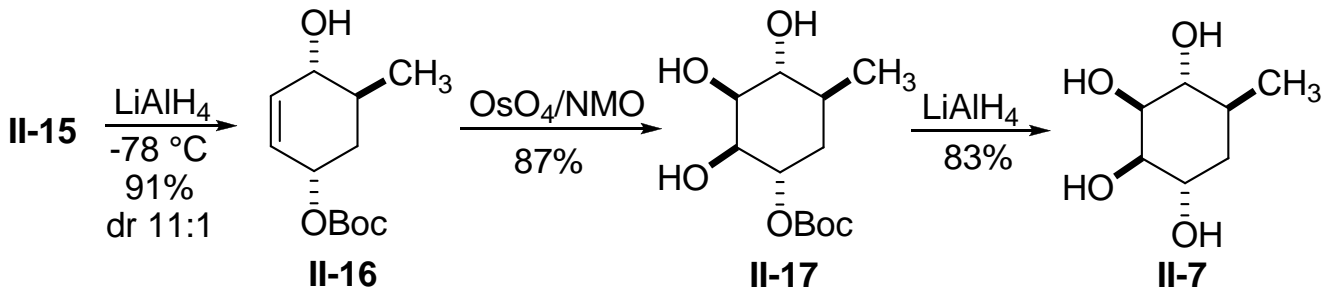

We thought a direct hydrogenation with $\mathrm{Pd} / \mathrm{C}$ of the cyclopropanol ring could avoid 
formation of the enone intermediate II-10a by preventing $\beta$-hydride elimination. That is to say, we hoped the Pd-homoenolate intermediate might be trapped by the Pd-bound hydrogen. ${ }^{29}$ To our delight, hydrogenation of II-9a with $\mathrm{Pd} / \mathrm{C}$ under balloon pressure of $\mathrm{H}_{2}$ resulted in II-11a as a single diastereomer $(\mathrm{dr}>100: 1)$ in $80 \%$ yield (Scheme 14). Deprotection of II-11a under acidic conditions (10\% HCl/THF) produced diol II-13. Alternatively, the diol could be achieved as a single diastereomer by first deprotection of acetonide in $9 \mathbf{a}(10 \% \mathrm{HCl} / \mathrm{THF}, 82 \%)$, then followed by the isomerization of the resulted triol $\mathbf{1 4}$ under the same hydrogenation condition ( $\left.\mathrm{Pd} / \mathrm{C}, \mathrm{H}_{2}, 82 \%\right)$.

With the methyl group having been set, we next tried to install the $\alpha$-D-rhamnose stereochemistry. Thus, the diol II-13 was acylated with Boc-anhydride followed by $\beta$ elimination to afford enone II-15 in 83\% yield over two steps. The carbonyl group of II-15 was diastereoselectively reduced with $\mathrm{LiAlH}_{4}$ at $-78{ }^{\circ} \mathrm{C}$ to provide allylic alcohol II-16 in $91 \%$ yield (dr 11:1) with easy removal of the minor diastereomer by column chromatography. Dihydroxylation of cyclohexene II-16 under Upjohn conditions produced triol II-17 as a single diastereomer with good yield $(87 \%)$. Reductive removal of the $t$-butyl carbonate group with $\mathrm{LiAlH}_{4}$ resulted in 5a-carba- $\alpha$-D-rhamnopyranose II-7 in $83 \%$ yield.

In order to prepare the $\beta$-D-carbasugar, we switched the starting material to enone II-6 (Scheme 15). Enone II-6 could also be readily prepared from D-quinic acid II-5 in 5 steps in $50 \%$ yield. ${ }^{30}$ As with enone II-4 (c.f. Scheme 13), a one pot hydrosilylation and cyclopropanation of II-6, followed by desilylation under acidic conditions gave a diastereomeric mixture of cyclopropanol II-18a/ II-18b $(\mathrm{dr} \sim 1: 1)$ in $85 \%$ overall yield. Although these two cyclopropanol isomers were inseparable, a direct cyclopropanol ring opening of this mixture by $\mathrm{Pd} / \mathrm{C}$ under hydrogenation conditions (balloon pressure of $\mathrm{H}_{2}, 6 \mathrm{~h}, \mathrm{RT}$ ) afforded a diastereomeric mixture of $\alpha$-methyl ketones II-19a/II-19b (63\% yield, dr 2:1) along with some recovered cyclopropanol II-18b (20\%, as a single diastereomer). The mixture of II-19a and II-19b could be separated by a combination of crystallization and silica gel chromatography resulting in a diastereomerically enriched mixture of II-19a $(\mathrm{dr}>13: 1)$ and diastereomerically pure II-19b. Xray crystallography analysis of II-19b confirmed its stereochemistry. 
Scheme 15. Synthesis of $\beta$-D-5a-carba-digitoxose

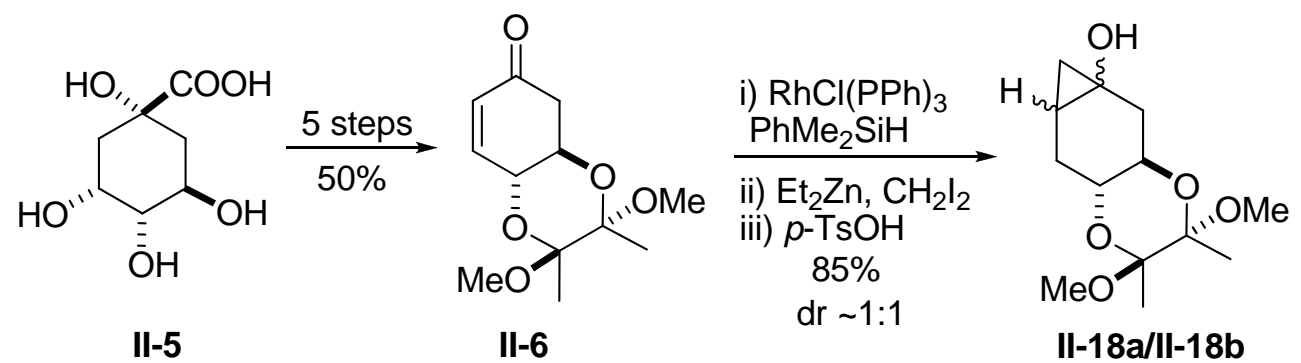

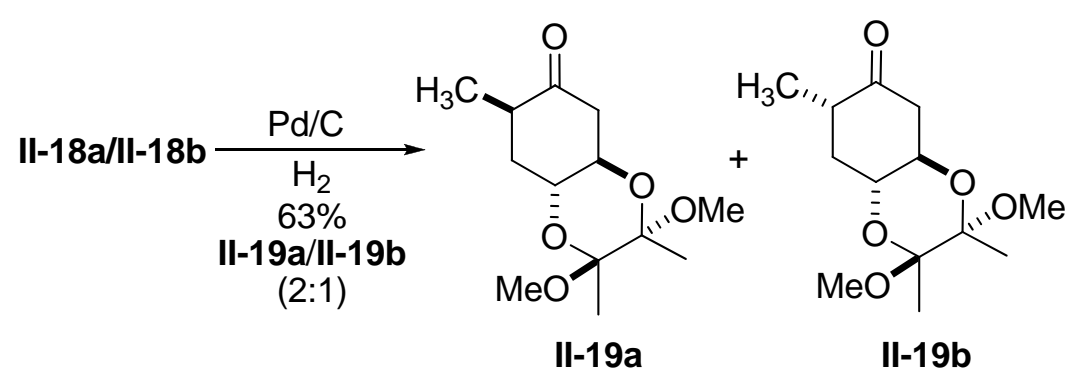

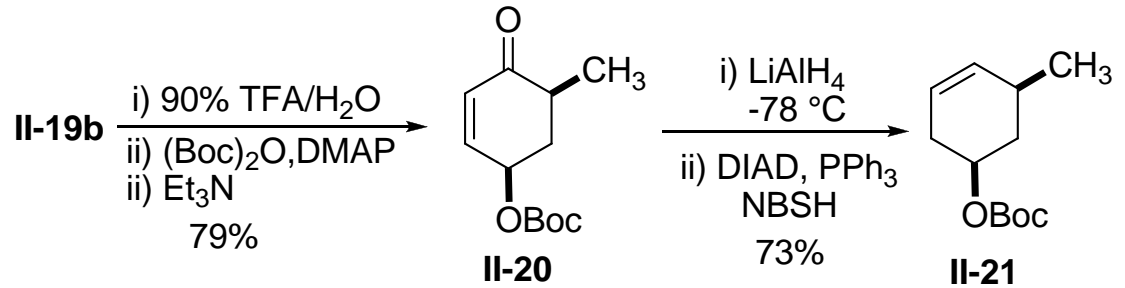<smiles>CC1CC(O)C[C@@H](O)[C@H]1O</smiles>

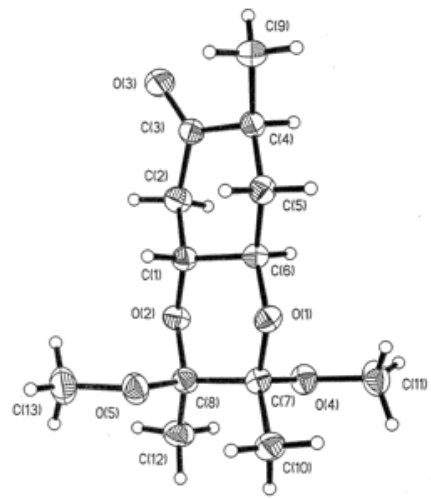


With the II-19b in hand, we next turned our attention to install the $\beta$-D-digitoxose stereochemistry. Thus, deprotection of II-19b in acidic condition $\left(90 \% \mathrm{TFA} / \mathrm{H}_{2} \mathrm{O}\right)$ gave crude diol (albeit with some C-5 epimerization, $\mathrm{dr} \sim 15: 1$ ), which underwent acylation with Bocanhydride, and subsequent $\beta$-elimination to afford enone II-20 (79\% yield from II-19b, dr 15:1). While pure isomer II-20 could be obtained by careful chromatography, the diastereomeric mixture of II-20 could be directly used in the diastereoselective reduction $\left(\mathrm{LiAlH}_{4}\right.$ at $\left.-78{ }^{\circ} \mathrm{C}\right)$, resulting in an allylic alcohol in $82 \%$ yield, with the minor diastereomers readily removed by chromatography, which was then treated in Meyer's reductive rearrangement condition to provide olefin II-21 in good yield (73\%, two steps). ${ }^{31}$ Dihydroxylation of the olefin II-21 under the Upjohn dihydroxylation conditions $\left(\mathrm{OsO}_{4} / \mathrm{NMO}\right)$ afforded an inseparable diastereomeric mixture of diol (dr 11:1), which was derivatized as an acetonide II-22 (81\% for two steps), with the minor diastereomer removed by chromatography. Finally, reduction of the Boc-carbonate by $\mathrm{LiAlH}_{4}$ and removal of acetonide in acid condition $(10 \% \mathrm{HCl} / \mathrm{THF})$ afforded $5 \mathrm{a}$-carba- $\beta$-Ddigitoxopyranose II-8 in $85 \%$ yield.

Our efforts toward the L-carbasugars, commenced with the synthesis of $\alpha$-methyl ketone II19a (Scheme 16). Acid hydrolysis of the bis-ketal in II-19a (dr 10:1) afforded diol II-23 in $70 \%$ yield. It was difficult to prevent some epimerization of the axial methyl group in II-23 under the reaction conditions ( $\mathrm{dr} \sim 8: 1$ for crude $\mathbf{I I - 2 3}$ ). However, the minor diastereomer could be removed by silica gel chromatography. Then the diol II-23 subsequently underwent acylation with Boc-anhydride and $\beta$-elimination under basic condition to furnish enone (ent)-II-15 (90\% overall yield, two steps). Thus, this enantiomer of II-15 could be converted into 5a-carba- $\alpha$-Lrhamnopyranose (ent)-II-7 using the same 3-step reaction sequence for the conversion of II-15 to II-7. 
Scheme 16. Synthesis of $\alpha$-L-5a-carba-rhamnose

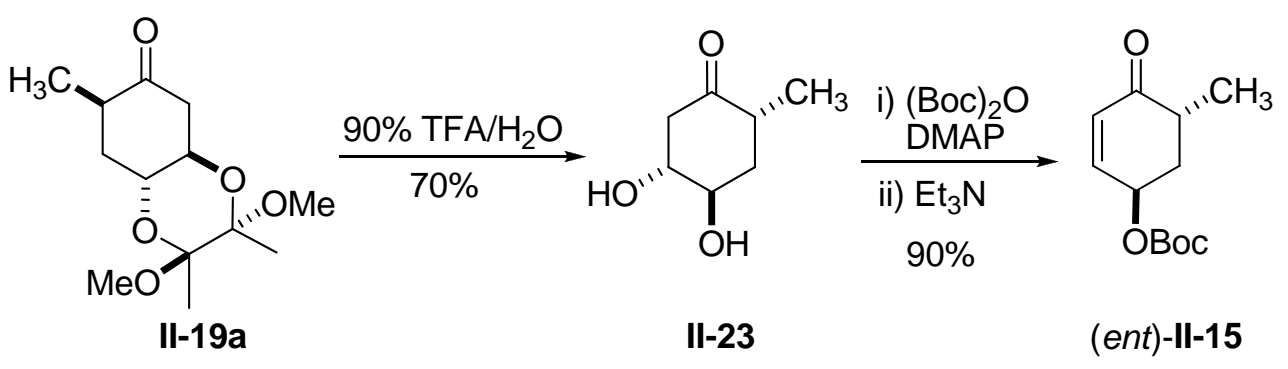

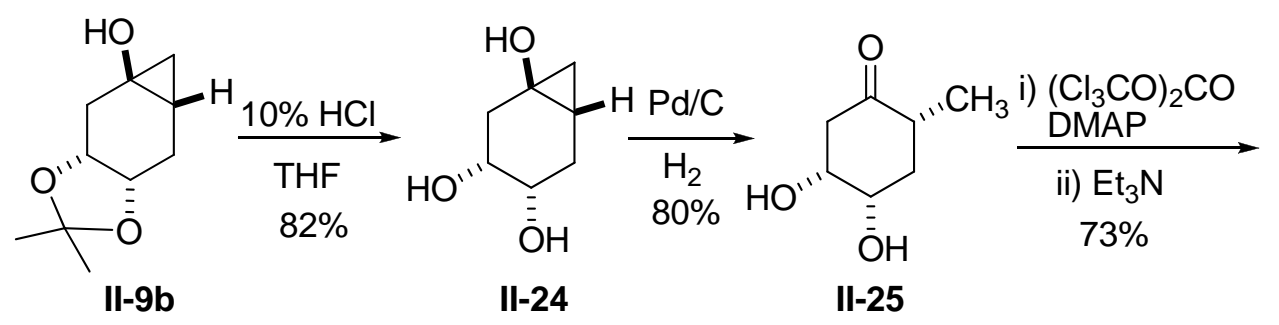<smiles>C[C@@H]1C[C@@H](O)C=CC1=O</smiles>

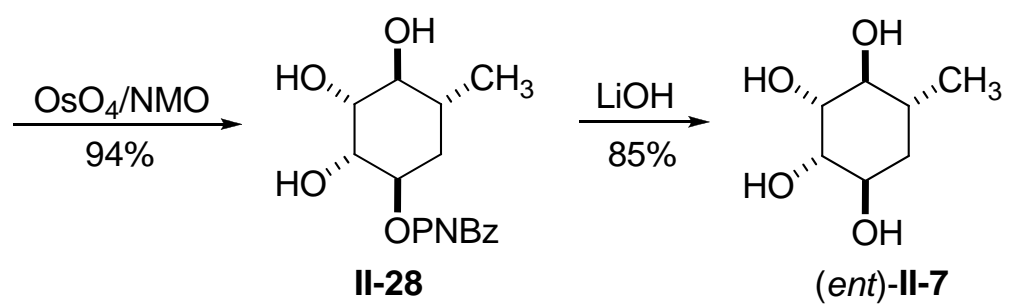

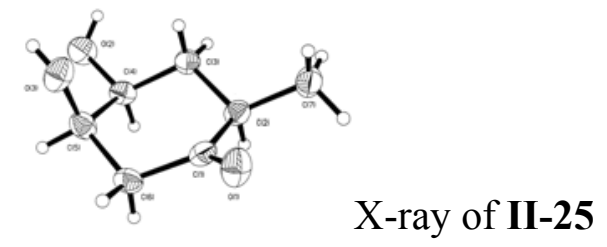

Alternatively, the L-series of carbasugar could be prepared from cyclopropanol II-9b (Scheme 4). We first deprotected the acetonide of II-9b under acidic conditions $(10 \% \mathrm{HCl} / \mathrm{THF})$ to give triol II-24 in 82\% yield (Scheme 16). Exposure of the cyclopropanol II-24 to similar hydrogenation conditions $\left(\mathrm{Pd} / \mathrm{C}, \mathrm{H}_{2}\right)$ as before afforded the $\alpha$-methyl ketone II-25 in $80 \%$ yield 
as a single diastereomer. An X-ray crystallography analysis of II-25 clearly confirmed the formation of $\alpha$-methyl ketone and its stereochemistry. Dehydration of diol II-25 through carbonate formation and $\beta$-elimination furnished allylic alcohol II-12b in 73\% yield over two steps. In order to prepare the $\alpha$-sugar, we need to invert the stereochemistry of the C-1 hydroxyl group. Thus, Mitsunobu reaction of II-12b with $p$-nitrobenzoic acid in the presence of DIAD and $\mathrm{PPh}_{3}$ gave the ester II-26 in excellent yield (95\%) with desired inverted stereochemistry. ${ }^{32}$ Diastereoselective reduction of the enone II-26 $\left(\mathrm{LiAlH}_{4},-78{ }^{\circ} \mathrm{C}\right)$ afforded allylic alcohol II-27 in $90 \%$ yield ( $\mathrm{dr}$ 11:1) with the minor isomer easily removed by chromatography. Dihydroxylation of the allylic alcohol II-27 produced triol II-28 in 94\% yield with complete stereocontrol. Saponification of ester II-28 under basic conditions $(\mathrm{LiOH} / \mathrm{MeOH})$ afforded $5 \mathrm{a}-$ carba- $\alpha$-L-rhamnopyranose (ent)-II-7 in $85 \%$ yield. ${ }^{33}$

In conclusion, three carbasugars in $\mathrm{D} / \mathrm{L}$ and $\alpha / \beta$ forms have been successfully synthesized starting with D-(-)-quinic acid. This synthesis highlights a novel diastereospecific Pd/C catalyzed cyclopropanol ring opening, diastereoselective reduction and dihydroxylation. Mitsunobu reaction and Myers' reductive rearrangement were also used to install the desired carbasugar stereochemistry. 


\section{II.2. Carbasugar $C-1$ Phosphate ${ }^{\ddagger, 34}$}

Carbohydrates are a recurring structural motif in many bioactive natural products (e.g., antimicrobial and anticancer agents). ${ }^{35}$ Often the sugar portion of the molecule is a crucial element for the activities of these compounds; for instance, many aglycons of biologically active natural products are void of activity. ${ }^{36}$ A cursory structure activity relationship analysis of the carbohydrate containing natural products points to the use of rare and deoxysugars (Scheme 17). ${ }^{37}$ The removal or stereochemical inversion of the various sugar hydroxyl groups can impart significant in vitro-stability by lessening the abilities of naturally occurring glycosidase enzymes to degrade the structure. In fact, our ongoing interest in the de novo synthesis of carbohydrates is aimed at being able to provide new glycosylated natural products for medicinal chemistry studies. ${ }^{38}$ An alternative approach is to alter the biosynthesis of the glycosylated natural products. $^{39}$

Scheme 17. Biosynthesis of glycosyltransferase inhibitors

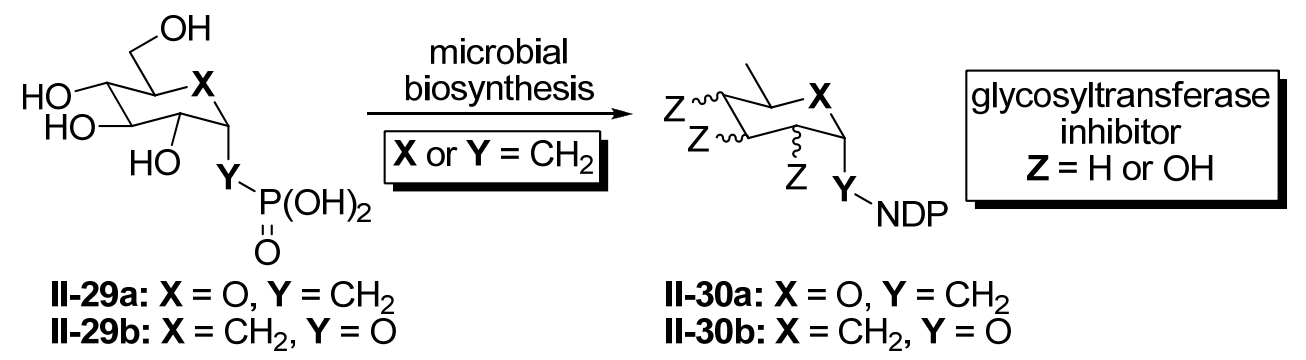

In an effort to understand and ultimately alter the microbial biosynthesis of rare/deoxy-sugar containing natural products various $C$-glycoside analogues of the NDP-sugars have been prepared. $^{40}$ Most of these efforts have been focused on the synthesis and use of $C$-glycoside analogues with exo-acetal oxygen replaced with a methylene group $\left(\mathbf{Y}=\mathrm{CH}_{2}\right) .{ }^{40}$ In contrast, there is only one report that describes the use of the endo-acetal oxygen $\mathrm{C}$-glycoside $\left(\mathbf{X}=\mathrm{CH}_{2}\right.$, cyclitol). ${ }^{41}$ To these ends, we have initiated an effort to develop an approach to these cyclitol sugar phosphates $\left(\mathbf{X}=\mathrm{CH}_{2}\right)$ in a manner that would mimic our de novo approach to carbohydrates (i.e., one that is amenable to both enantiomers of either $\alpha$ - or $\beta$-isomers). Herein

\footnotetext{
${ }^{\ddagger}$ Reproduced in part with permission from Org. Lett. 2008, 10, 3381-3384. Copyright 2008, American Chemical Society.
} 
we described our successful efforts towards these aims.<smiles>CC1C[C@H](OCOC(=O)O)CCC1=O</smiles>

II-31<smiles>CC1C[C@@H](C(=O)O)CC[C@@H]1O</smiles>

II-32<smiles></smiles>

II-33<smiles>CC1CC([O+]=O)C[C@@H](O)[C@@H]1O</smiles>

II-34<smiles></smiles>

(ent)-II-33

Figure 3. The target cyclitol sugar phosphates

To address these aims, we targeted the synthesis of four $\alpha$-sugars and one $\beta$-sugar. These structures are outlined in Figure 3: 2,3-dideoxy-4-oxo-5a-carba- $\alpha$-D-rhamnopyanose phosphate (II-31), 2,3-dideoxy-5a-carba- $\alpha$-D-rhamnopyranose phosphate (II-32), 5a-carba- $\alpha$-Drhamnopyranose phosphate (II-33), 5a-carba- $\beta$-D-digitoxopyranose phosphate (II-34) and 5acarba- $\alpha-L-r h a m n o p y r a n o s e ~ p h o s p h a t e ~((e n t)-\mathbf{I I}-33)$.

Scheme 18. Retrosynthetic analysis

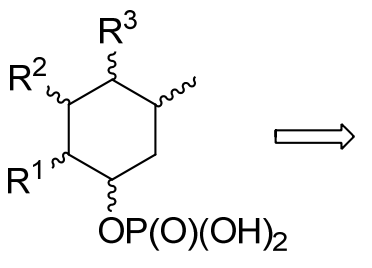

II-35

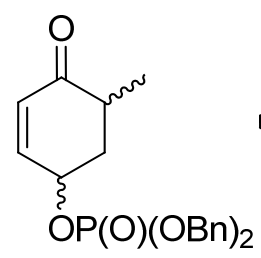

II-36<smiles>C[C@@H]1C[C@H](O)C=CC1=O</smiles>

II-37

Retrosynthetically, we envisioned that the desired carbasugar phosphates stereoisomers II-35 could be achieved from both enantiomers of the cis- and trans-diastereomers of the enone $\gamma$ phosphate II-36 (Scheme 18). We thought II-36 could be derived from corresponding allylic alcohol II-37 which are readily available or could be easily synthesized from suitable intermediates we already achieved during the synthesis of carbasugar monosaccharide. 
Scheme 19. Synthesis of 2,3-dideoxy-4-oxo-5a-carba- $\alpha$-D-rhamnopyanose 1-phosphate (II-32)<smiles>C[C@@H]1C[C@@H](O)[C@@H](O)CC1=O</smiles>

II-13

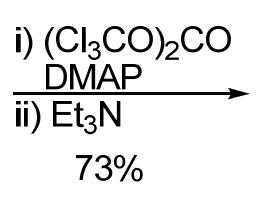

$73 \%$<smiles>CC1C[C@H](O)C=CC1=O</smiles>

II-38

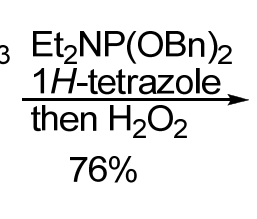

$76 \%$

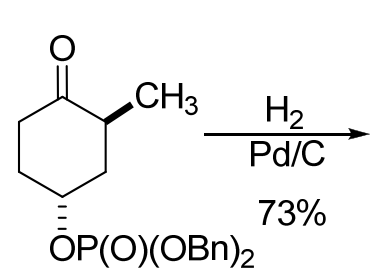

II-41

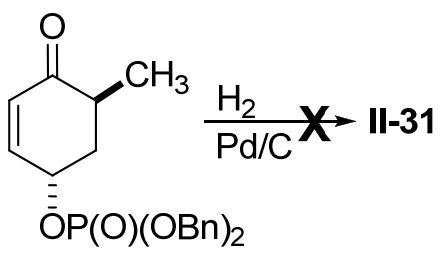

II-39<smiles>C[C@@H]1CC(=O)C[C@H](O)C1</smiles>

II-40

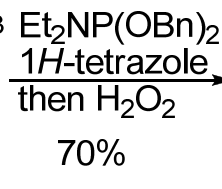

$70 \%$

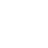

We started the synthesis of the D-carbasugar phosphates with diol II-13. Carbonate formation of II-13 using triphosgene followed by $\beta$-elimination in basic condition resulted in allylic alcohol II-38 in 73\% yield (Scheme 19). Phosphorylation of the alcohol II-38 with $N, N$-diethyl dibenzylphosphoramidite in the presence of $1 \mathrm{H}$-tetrazole gave a phosphite intermediate which was oxidized by addition of $30 \% \mathrm{H}_{2} \mathrm{O}_{2}$ to form dibenzyl phosphate II-39 in $76 \%$ yield. Debenzylation and reduction of double bond under hydrogenation condition $\left(\mathrm{H}_{2}, \mathrm{Pd} / \mathrm{C}\right)$ failed to give the desired dihydrogen phosphate II-31, presumably due to hydrogenolysis of the allylic phosphate. Attempts at hydrogenation of $\mathbf{I I - 3 9}$ with diimide $\left(\mathrm{NBSH} / \mathrm{Et}_{3} \mathrm{~N}\right)$ proved unsuccessful. Thus we decided reduce the double bond before introduction of the phosphate functional group. Hydrogenation of II-38 $\left(\mathrm{H}_{2}, \mathrm{Pd} / \mathrm{C}\right)$ gave saturated alcohol II-40 in 72\% yield. Phosphorylation of II-40 in the same condition described before gave dibenzyl phosphate II-41 in 70\% yield. Then debenzylation under typical hydrogenation condition $\left(\mathrm{H}_{2}, \mathrm{Pd} / \mathrm{C}\right)$ afforded the 2,3-dideoxy4-oxo-5a-carba- $\alpha$-D-rhamnopyanose phosphate (II-31) in 73\% yield. While analysis of the crude product showed diastereomerically clean material, upon sitting the product slowly epimerized to $\mathrm{a} \sim 2: 1$ of methyl diastereomers. 
Scheme 20. Synthesis of 2,3-dideoxy-5a-carba- $\alpha$-D-rhamnopyranose 1-phosphate (II-32), 5acarba- $\alpha$-D-rhamnopyranose 1-phosphate (II-33)

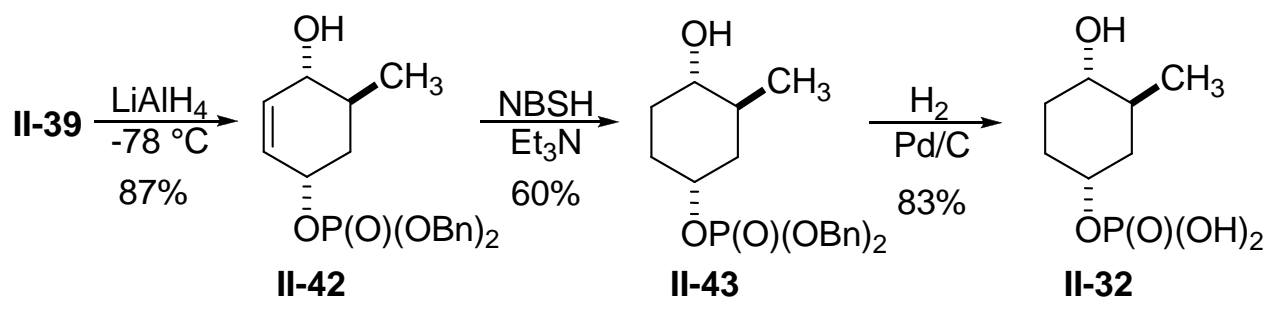

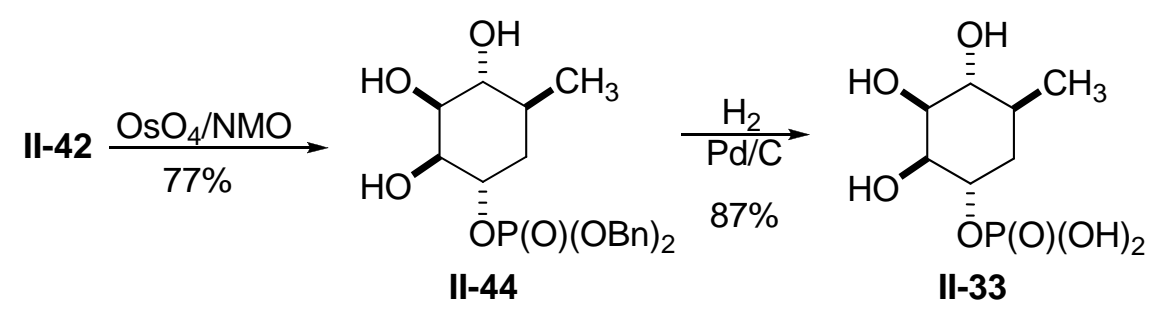

To synthesize the phosphate II-32 and II-33, we returned to enone II-39 (Scheme 20). A diastereoselective reduction with $\mathrm{LiAlH}_{4}$ at $-78{ }^{\circ} \mathrm{C}$ gave the equatorial alcohol II-42 in good yield (87\%) and diastereoselectivity (dr 11:1). Diimide reduction $\left(\mathrm{NBSH} / \mathrm{Et}_{3} \mathrm{~N}\right)$ of II-42 resulted in saturated alcohol II-43 in 60\% yield with 20\% starting material back. Hydrogenation of II-43 on $\mathrm{Pd} / \mathrm{C}$ furnished 2,3-dideoxy-5a-carba- $\alpha$-D-rhamnopyranose phosphate (II-32) in $83 \%$ yield. Alternatively, dihydroxylation of allylic alcohol II-42 under Upjohn condition ${ }^{42}$ resulted in a single diastereomeric triol II-44 in $86 \%$ yield, which similarly underwent hydrogenolysis to afford 5a-carba- $\alpha$-D-rhamnopyranose phosphate (II-33) in $87 \%$ yield. 
In order to achieve the $\beta$-D-digitoxose phosphate II-34, we started with Boc-carbonate II-22 (Scheme 21). Reduction of Boc-carbonate II-22 by $\mathrm{LiAlH}_{4}$ gave alcohol II-45 in 93\% yield which was then phosphorylated to give dibenzyl phosphate II-46 in 74\% yield. The acetonide deprotection of II-46 in acid condition produced diol II-47 with excellent yield (95\%), which under typical hydrogenation conditions $\left(\mathrm{H}_{2}, \mathrm{Pd} / \mathrm{C}\right)$ resulted in 5a-carba- $\beta$-D-digitoxopyranose phosphate (II-34) in 78\% yield.

Scheme 21. Synthesis of 5a-carba- $\beta$-D-digitoxopyranose 1-phosphate (II-34)

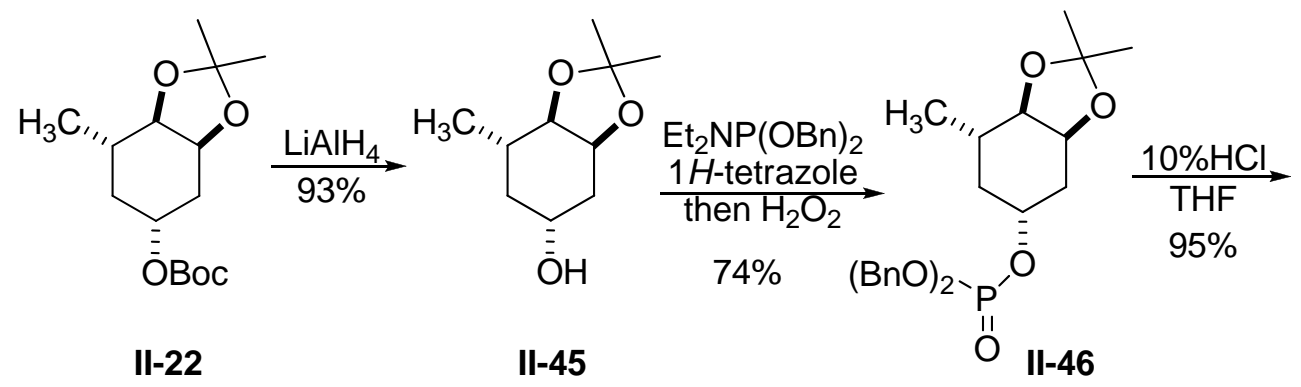

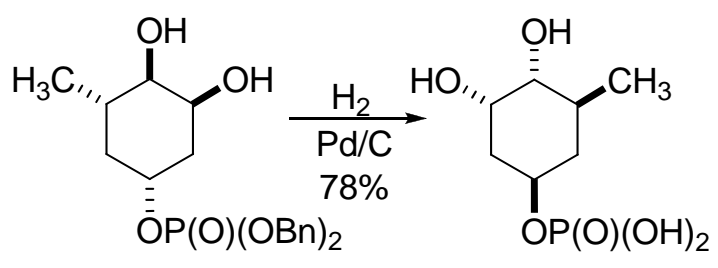

II-47

II-34

In order to access the L-series of carbasugar phosphate, we started with the intermediate II27. TBS-protection and ester hydrolysis of II-27 gave alcohol allylic alcohol II-48 in good overall yield (85\% for 2 steps) (Scheme 22). Phosphorylation of alcohol II-48 afforded dibenzyl phosphate II-49 in 66\% yield. Dihydroxylation of II-49 under Upjohn conditions produced diol in $81 \%$ yield as a single diastereomer, which after acid deprotection $(p-\mathrm{TsOH} / \mathrm{MeOH})$ provided triol II-50 in excellent overall yield (79\% for two steps). Finally hydrogenation of dibenzylphosphate II-50 resulted in 5a-carba- $\alpha$-L-rhamnopyranose phosphate ((ent)-II-33) in $81 \%$ yield. 
Scheme 22. Synthesis of 5a-carba- $\alpha$-L-rhamnopyranose 1-phosphate ((ent)-5)

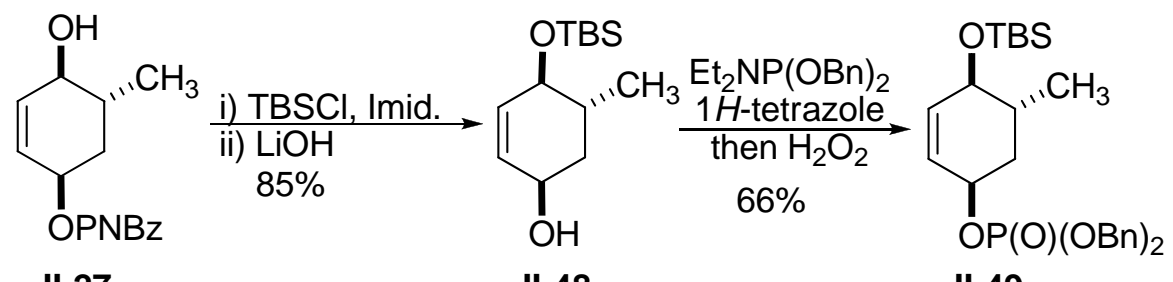

$$
\text { II-27 }
$$

II-48

II-49

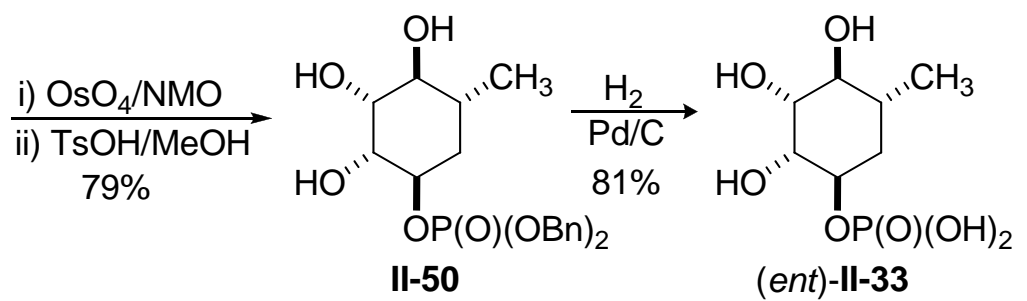

In conclusion, five carbasugar phosphates have been successfully synthesized in either D or L, $\alpha$ or $\beta$ form from $\mathrm{D}-(-)$-quinic acid. This synthesis includes a novel highly diastereospecific $\mathrm{Pd} / \mathrm{C}$ catalyzed cyclopropanol ring opening, which may be a useful alternative for the introduction of methyl groups alpha to ketones. Diastereoselective reduction, dihydroxylation, Mitsunobu reaction and Myers' reductive rearrangement were also used to install the desired sugar stereochemistry. The biological evaluation of these cyclitol phosphates is ongoing. 


\section{Chapter III. Methodology and Synthesis of Carbasugar Analogues of SL0101}

\section{III.1. Methodology development of carbasugar glycoside}

In order to facilitate the medicinal structure-activity relationship (SAR) study of SL0101, we turned our attention to the carbasugar glycoside analogues of SL0101 (Figure 4). The advantage is that because these carbasugar analogues are not hydrolyzable because the ring oxygen has been replaced with a methylene group, and the biological activities of these carbasugar analogues will provide very important information to the structure-activity relationship (SAR) study of SL0101, particularly it may help us to understand the function of the sugar moiety.

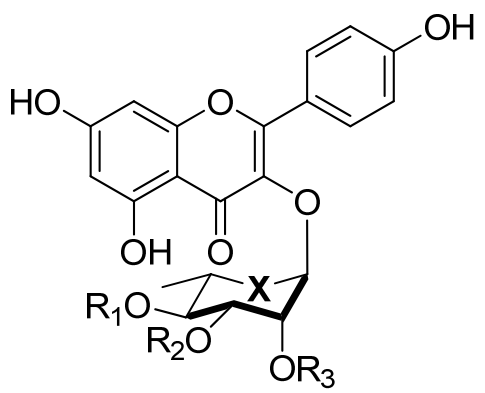

III-1 $R_{1}=A c, R_{2}=H, R_{3}=H$ III-2 $R_{1}=A c, R_{2}=H, R_{3}=A c$ III-3 $R_{1}=A c, R_{2}=A c, R_{3}=H$

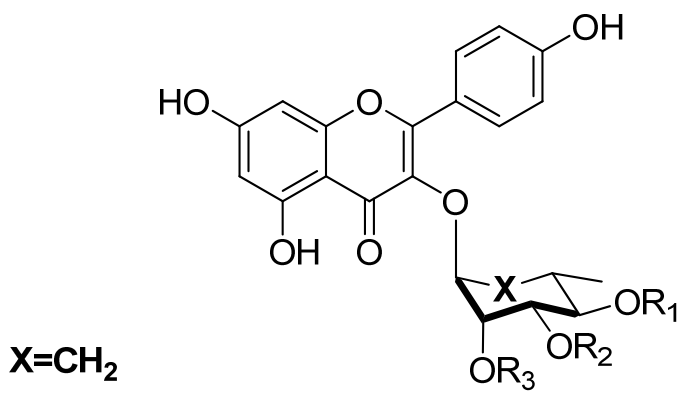

(ent)-III-1 $\mathrm{R}_{1}=\mathrm{Ac}, \mathrm{R}_{2}=\mathrm{H}, \mathrm{R}_{3}=\mathrm{H}$ (ent)-III-2 $\mathrm{R}_{1}=\mathrm{Ac}, \mathrm{R}_{2}=\mathrm{H}, \mathrm{R}_{3}=\mathrm{Ac}$ (ent)-III-3 $\mathrm{R}_{1}=\mathrm{Ac}, \mathrm{R}_{2}=\mathrm{Ac}, \mathrm{R}_{3}=\mathrm{H}$

Figure 4. Carbasugar glycoside analogues of SL0101

Over these years, O'Doherty group has successfully developed a de novo approach to synthesize carbohydrates by using a palladium(0)-catalyzed glycosylation to introduce the glycosidic bond and post-glycosylation transformations to install corresponding stereochemistry of all kinds of glycosides. ${ }^{43}$ We want to develop a practical method to synthesize carbasugar or cyclitol glycoside, which is compatible to our method in the synthesis of normal carbohydrates (Scheme 23). To do this, we have two problems that need to be addressed. First, we want to know whether or not the palladium(0)-catalyzed glycosylation (i.e. Palladium(0)-catalyzed cyclitolization) from III-4 to III-5 would still work well without a ring oxygen. Second, we want to know whether or not a post-glycosylation (i.e. post-cyclitolization) could be developed to convert enone III-5 to the corresponding carbasugar III-6 accordingly as we did for normal 
carbohydrates, since the anomeric effect no longer exists when the ring oxygen was replaced by a methylene group.

Scheme 23. Proposed carbasugar synthesis

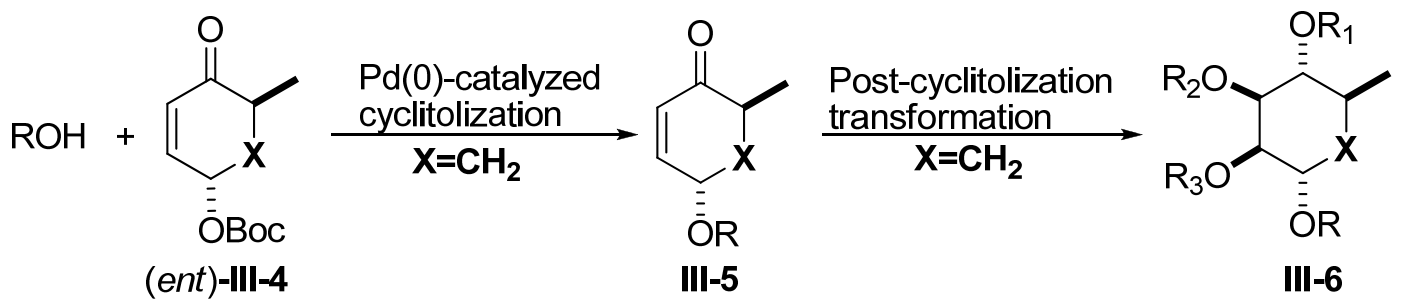

As we mentioned in chapter II of this dissertation, we already synthesized both $\alpha$-L-Bocenone (III-4) and $\alpha$-D-Boc-enone ((ent)-III-4) from quinic acid. Our carbasugar synthesis was carried out by first testing the palladium(0)-catalyzed cyclitolization. In practice, $\alpha$-D-Boc-enone ((ent)-III-4) was reacted with $\mathrm{BnOH}$ in $\mathrm{CH}_{2} \mathrm{Cl}_{2}$ in the presence of $10 \mathrm{~mol} \% \mathrm{Pd}\left(\mathrm{PPh}_{3}\right)_{2}$ at $0{ }^{\circ} \mathrm{C}$ for 10 hours. As a result, the reaction afforded glycosylated enone III-7 in a reasonable $60 \%$ yield (Scheme 24).

Scheme 24. Palladium(0)-catalyzed cyclitolization or C-in ring glycosylation

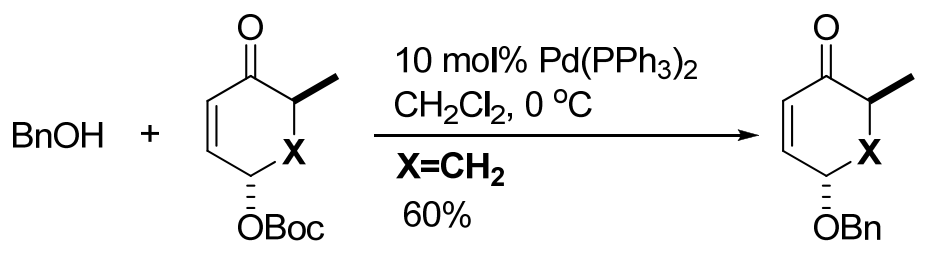
(ent)-III-4 III-7

In order to produce SL0101 carbasugar analogues we next examined the cyclitolization reation of SL0101 aglycone (Scheme 25). Reaction between flavonol aglycone III-8 and $\alpha$-DBoc-enone ((ent)-III-4) went smoothly in $\mathrm{CH}_{2} \mathrm{Cl}_{2}$ in the presence of $\mathrm{Pd}\left(\mathrm{PPh}_{3}\right)_{2}$ catalyst to afford desired glycosylated enone (ent)-III-9 in 84\% yield. In reality, this reaction finished in half an hour at $0{ }^{\circ} \mathrm{C}$ with only $5 \mathrm{~mol} \%$ Pd loading. 
Scheme 25. Palladium(0)-catalyzed cyclitolization of the SL0101 aglycone

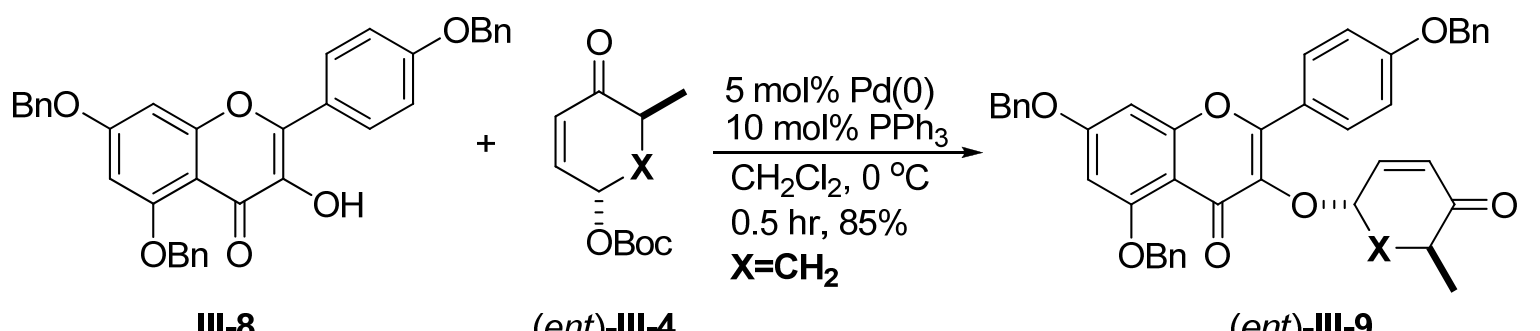

Since the palladium(0)-catalyzed cyclitolization reaction between flavonol III-8 and the Bocenone (ent)-III-4 worked very well, we believe a testing of the substrate scope will be useful for us to understand this reaction in terms of the electronic and steric effect from the stubstrates, which also would broaden its application to the synthesis of carbasugar glycoside analogues of many biologically important natural glycosides. For this purpose, a number of phenols and enols with different substitution patterns were chosen for testing (Scheme 26). From the experiments, the palladium(0)-catalyzed cyclitolization overall gave reasonable to excellent yields including phenols such as III-10 to III-17 and coumarin III-19, enols such as naphthaquinone-2-ol (III-20) and flavonol III-8. Tentatively, we could conclude that (1) Phenols with electron donating groups tend to give higher yield for the cyclitolization than those with electron withdrawing groups, and for the extreme case, highly electron deficient $p$-nitrophenol did not react at all; (2) Steric hinderance did not greatly affect those phenols with electron donating groups even with a $t$-butyl group in ortho position such as III-14; but for those with electron withdrawing groups such as III-16, the steric hinderance might be responsible for the lower yield; (3) Highly electron deficient enol such as III-20 also gave high yield of cyclitolization, which we did not expect that before. 
Scheme 26. Palladium(0)-catalyzed cyclitolization for phenols and enols

III-10 to III-20, III-8 (ent)-III-4 III-21 to III-30, (ent)-III-9

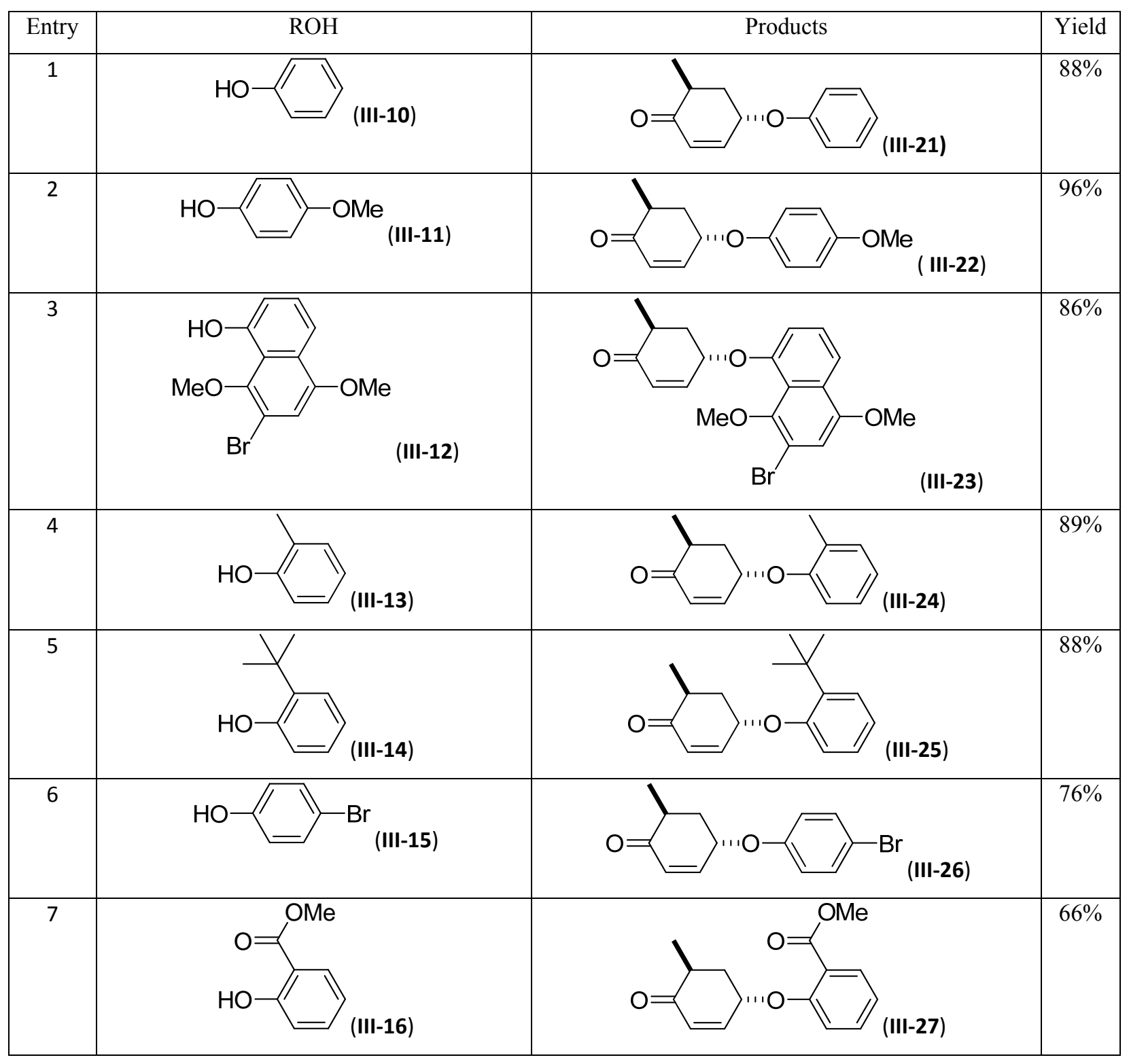




(II)

Once the palladium(0)-catalyzed cyclitolization has been developed, we next turned our attention to the post-cyclitolization transformation to install desired stereochemistry of the carbasugars. In particularly, we hope to develop a practical way to the rhmannose stereochemistry since the sugar moiety in SL0101 and its analogues are rhmannose. The first thing we wanted to do was the reduction of the glycosylated enone such as III-7 to allylic alcohol with high diastereoselectivity (Scheme 27). $\mathrm{NaBH}_{4}$ reduction with or without $\mathrm{CeCl}_{3}$ at $78{ }^{\circ} \mathrm{C}$, which are usual conditions we used to reduce pyranones in our normal carbohydrate synthesis, had some selectivity of III-31 over III-32 but not that satisfying. We next examined DIBAL-H, which gave a 1:1 ratio of these two allylic alcohols. The more bulky lithium tri-tbutoxyaluminohydride did not increase the selectivity too much. Fortunately, $\mathrm{LiAlH}_{4}$ reduction at $-78^{\circ} \mathrm{C}$ resulted in a reasonable diastereoselectiviy of $11: 1$ to afford allylic alcohol III-31 with $85 \%$ yield, when the temperature was lowered down to $-100{ }^{\circ} \mathrm{C}$, the selectivity slightly increased to $12: 1$. 
Scheme 27. Reduction of glycosylated enone

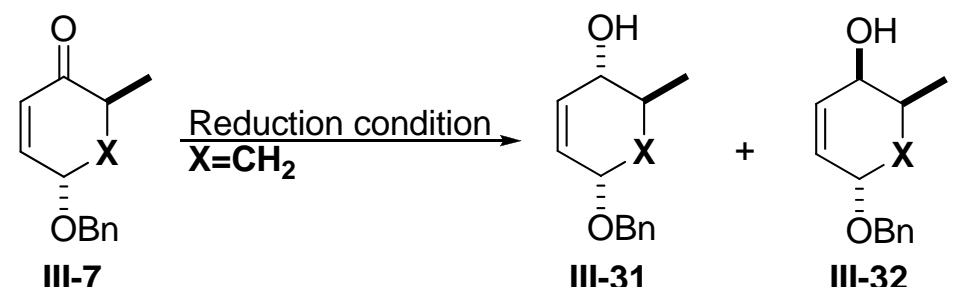

\begin{tabular}{|c|c|ccc|}
\hline Entry & Reduction condition & \multicolumn{3}{|c|}{ Ratio of III-31/ III-32 } \\
\hline 1 & $\mathrm{NaBH}_{4} /-78^{\circ} \mathrm{C}$ & 3 & $:$ & 1 \\
\hline 2 & $\mathrm{NaBH}_{4} / \mathrm{CeCl}_{3} /-78^{\circ} \mathrm{C}$ & 1.5 & $:$ & 1 \\
\hline 3 & $\mathrm{DIBAL}-\mathrm{H} /-78^{\circ} \mathrm{C}$ & 1 & $:$ & 1 \\
\hline 4 & $\mathrm{LiAlH}(t-\mathrm{BuO})_{3} /-78^{\circ} \mathrm{C}$ & 3.5 & $:$ & 1 \\
\hline 5 & $\mathrm{LiAlH} / 4-78^{\circ} \mathrm{C}$ & 11 & $:$ & 1 \\
\hline 6 & $\mathrm{LiAlH}_{4} /-100^{\circ} \mathrm{C}$ & 12 & $:$ & 1 \\
\hline
\end{tabular}

To install the rhmannose stereochemistry, olefin III-31 was then converted to diol III-33 (Scheme 28). In practice, dihydroxylation of the allylic alcohol III-31 upon Upjohn condition $\left(\mathrm{OsO}_{4} / \mathrm{NMO}\right)$ condition at $0{ }^{\circ} \mathrm{C}$ afforded triol III-33 in $90 \%$ yield with complete stereocontrol.

Scheme 28. Dihydroxylation of allylic alcohol III-31

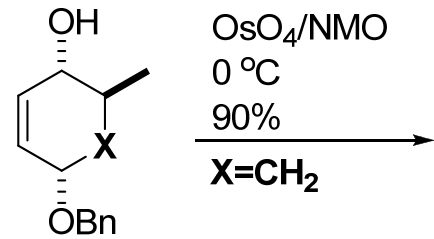

III-31<smiles>CC1[X]C(Br)C(O)C(O)[C@@H]1O</smiles>

III-33 


\section{III.2. Synthesis of carbasugar glycoside analogues of SL0101}

Once the methodology of palladium(0)-catalyzed cyclitolization and post-cyclitolization transformation had been successfully developed, we next started the synthesis of carbasugar analogues of SL0101. Treatment of tribenzylated kaempferol III-8 with $\alpha$-L-Boc-enone III-4 in the presence of $5 \mathrm{~mol} \% \mathrm{Pd}$ catalyst in $\mathrm{CH}_{2} \mathrm{Cl}_{2}$ at $0{ }^{\circ} \mathrm{C}$ for 30 minutes furnished the glycosylated enone III-9 with 84\% yield (Scheme 29).

Scheme 29. Palladium(0)-catalyzed cyclitolization of SL0101 aglycone<smiles></smiles>

III-8<smiles>C[C@H]1CC(OC(C)(C)C)C=CC1=O</smiles>

III-4<smiles>C[C@@H]1CC(Oc2c(-c3ccc(OCc4ccccc4)cc3)oc3cc(OCc4ccccc4)cc(OCc4ccccc4)c3c2=O)C=CC1=O</smiles>

III-9

Reduction of the enone III-9 by $\mathrm{LiAlH}_{4}$ at $-78{ }^{\circ} \mathrm{C}$ afforded allylic alcohol III-34 in $89 \%$ yield with the diastereoselectivity of 11:1 (Scheme 30). Acylation of the resulted allylic alcohol gave allylic acetate IV-24 in 93\% yield. Dihydroxylation of the olefin in IV-24 furnished the diol IV25 in 73\% yield as a single diastereomer. The diol IV-25 was then converted to one of our desired carbasugar analogues of SL0101, C-4 monoacetate IV-1 after debenzylation on Pd/C hydrogenation condition in $68 \%$ yield. 
Scheme 30. Synthesis of $\alpha$-L carbasugar analogue of SL0101 (C-4 monoacetate)<smiles>CC(=O)OC1C=CC(Oc2c(-c3ccc(OCc4ccccc4)cc3)oc3cc(OCc4ccccc4)cc(OCc4ccccc4)c3c2=O)C[C@@H]1C</smiles>

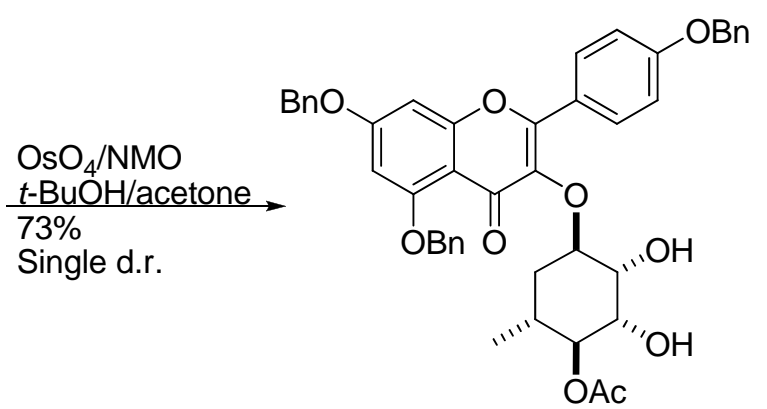

III-36

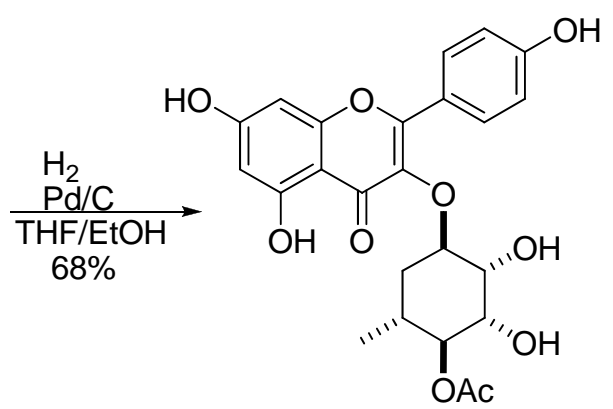

III-1

In order to achieve the diacetate carbasugar analogues of SL0101, we thought a selective acylation could be carried out on intermediate diol III-36 by an orthoacetate formation and kinetic hydrolysis just like what we did in the synthesis of SL0101 (Scheme 31). Treatment of diol with trimethyl orthoacetate in the presence of catalytical amount of $p$-toluenesulfonic acid followed by the hydrolysis by using $90 \%$ aqueous acetic acid furnished a mixture of 2,4diacetate III-37 and 3,4-diacetate III-38 in a 1.5:1 ratio, which was in contrast to the completely regioselective acylation of the axial hydroxy group in the synthesis of SL0101. This result clearly indicated the ring flip of cyclohexane ring is much easier due to the loss of anomeric effect which helps control the sugar conformation. Fortunately, we could separate these two regioisomers on silica gel column chromatrography. Then, debenzylation of III-37 and III-38 was carried out by hydrogenation to produce SL0101 C-2,C-4-diacetate carbasugar analogue III2 and $C$-3,C-4-diacetate carbasugar analogue III-3 respectively. 
Scheme 31. Synthesis of diacetate carbasugar analogues of SL0101

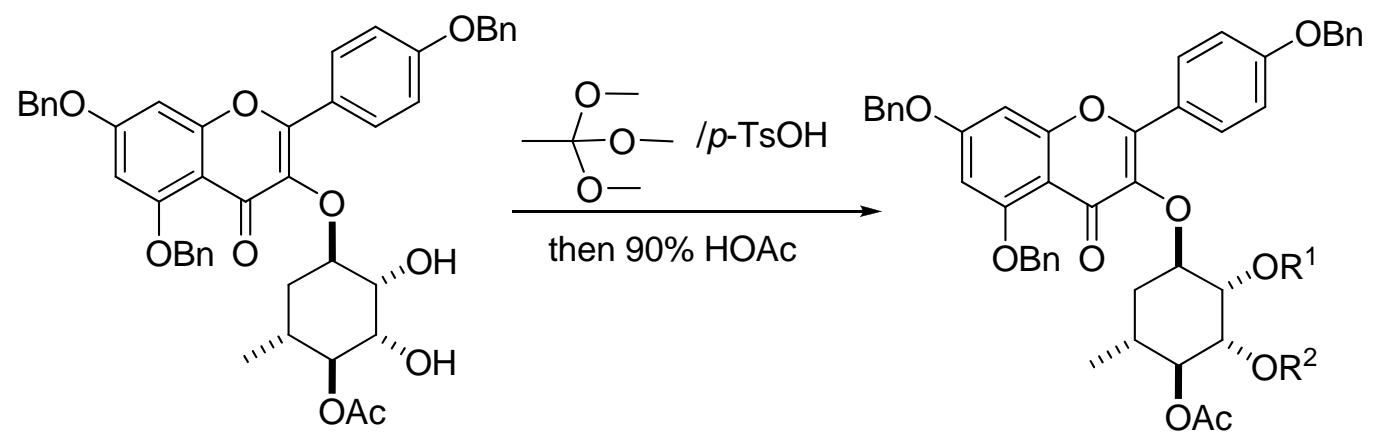

III-36

III-37 $\mathrm{R}^{1}=\mathrm{Ac}, \mathrm{R}^{2}=\mathrm{H} \quad 54 \%$

III-38 $R^{1}=H, \quad R^{2}=A C 34 \%$<smiles>CC(=O)O[C@H]1C(Oc2c(-c3ccc(O)cc3)oc3cc(O)cc(O)c3c2=O)C[C@H](C)[C@@H](OC(C)=O)[C@@H]1O</smiles>

III-2<smiles>CC(=O)O[C@H]1[C@@H](C)CC(Oc2c(-c3ccc(O)cc3)oc3cc(O)cc(O)c3c2=O)[C@H](O)[C@@H]1OC(C)=O</smiles>

III-3

SL0101 carbasugar analogue

In order to access D-series of carbasugar analogues of SL0101, we first coupled the aglycone kaempferol III-8 with the $\alpha$-D-Boc-enone (ent)-III-4 by using palladium(0)-catalyzed cyclitolization condition to furnish glycosylated enone (ent)-III-9 in 85\% yield (Scheme 32).

Scheme 32. Palladium(0)-catalyzed cyclitolization of SL0101 aglycone with $\alpha$-D-Boc-enone

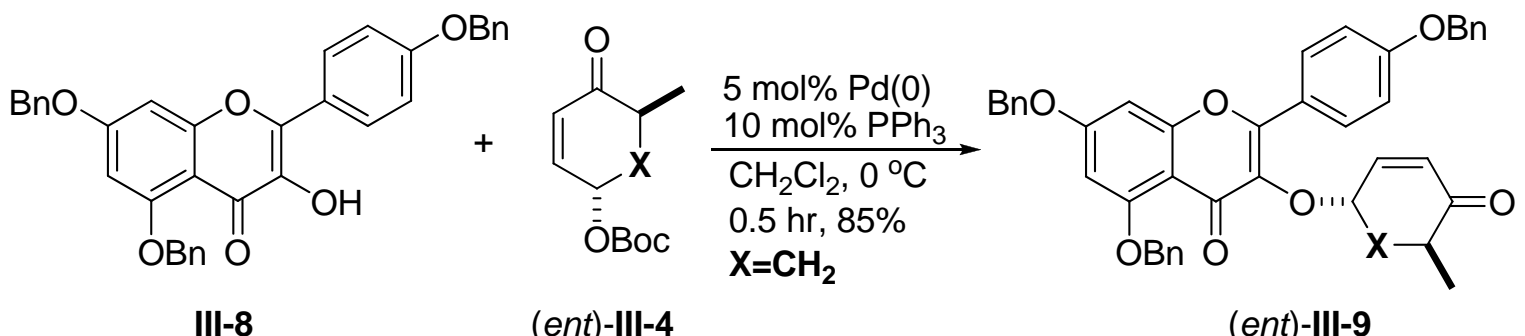

Reduction of enone (ent)-III-9 by $\mathrm{LiAlH}_{4}$ at $-78{ }^{\circ} \mathrm{C}$ afforded allylic alcohol (ent)-III-34 in $89 \%$ yield with the diastereoselectivity 11:1 (Scheme 33). Acylation of the resulted allylic 
alcohol gave allylic acetate (ent)-III-35 in 98\% yield. Dihydroxylation of the olefin (ent)-III-35 upon Upjohn condition furnished the diol (ent)-III-36 in $82 \%$ yield as a single diastereomer. The diol (ent)-III-36 was then converted to one of $\alpha$-D carbasugar analogues of SL0101, C-4 monoacetate (ent)-III-1 after debenzylation on Pd/C hydrogenation condition in 73\% yield.

Scheme 33. Synthesis of monoacetate of $\alpha$-D-carbasugar analogue of SL0101

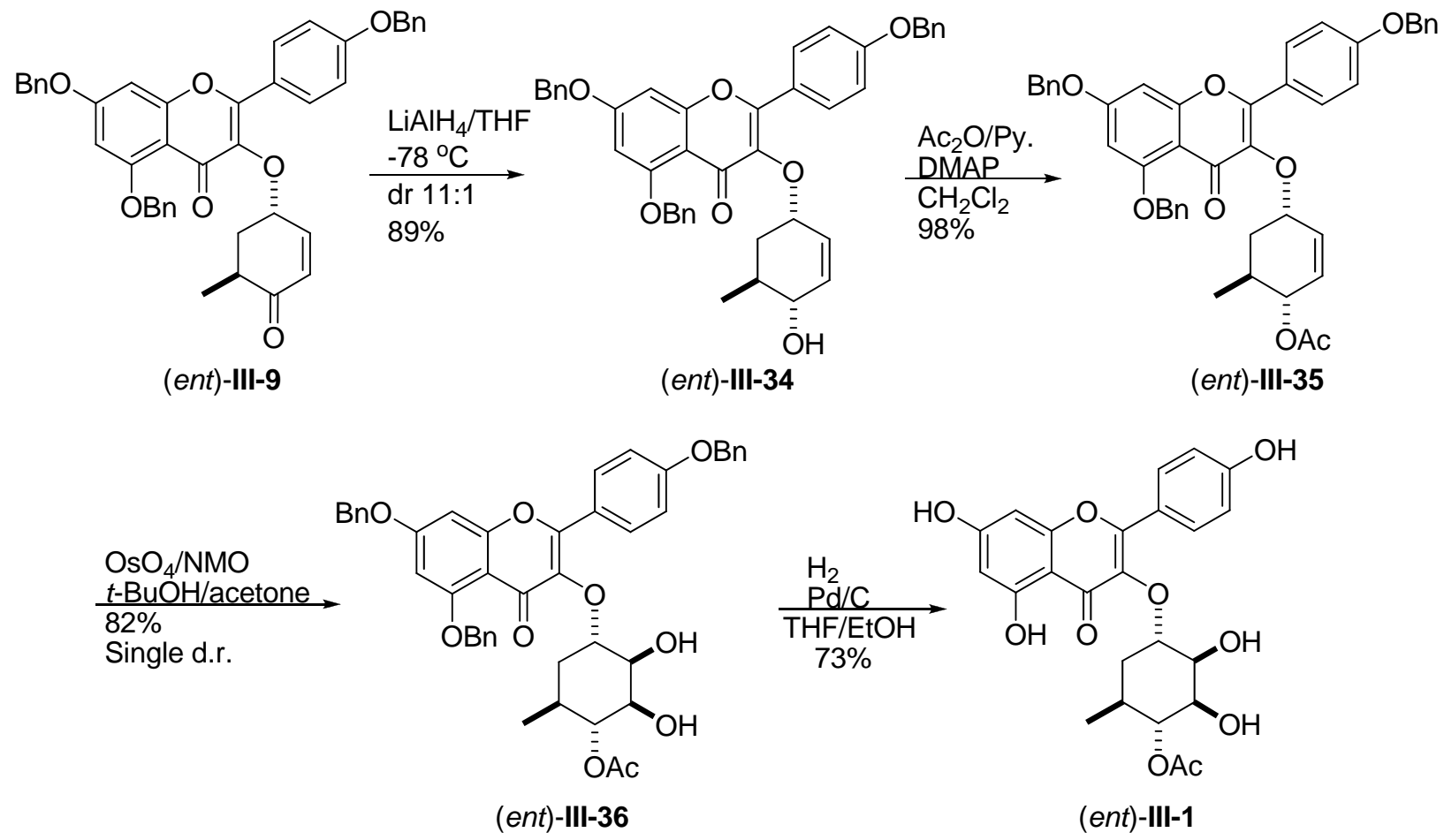

To synthesize the diacetate of $\alpha$-D carbasugar analogues of SL0101, an orthoacetate formation and kinetic hydrolysis was performed on the diol intermediate (ent)-III-36 (Scheme 34). Treatment of diol with trimethyl orthoacetate in the presence of catalytic amount of $p$ toluenesulfonic acid followed by hydrolysis using 90\% aqueous acetic acid furnished 2,4diacetate (ent)-III-37 and 3,4-diacetate (ent)-III-38 in about 1:1 ratio. After separation of these two regioisomers, (ent)-III-37 and (ent)-III-38 were debenzylated by the hydrogenation to produce SL0101 $C$-2,C-4-diacetate of $\alpha$-D carbasugar analogue (ent)-III-2 and $C$-3,C-4-diacetate of $\alpha$-D carbasugar analogue (ent)-III-3, respectively. 
Scheme 34. Synthesis of diacetate of $\alpha$-D-carbasugar analogues of SL0101

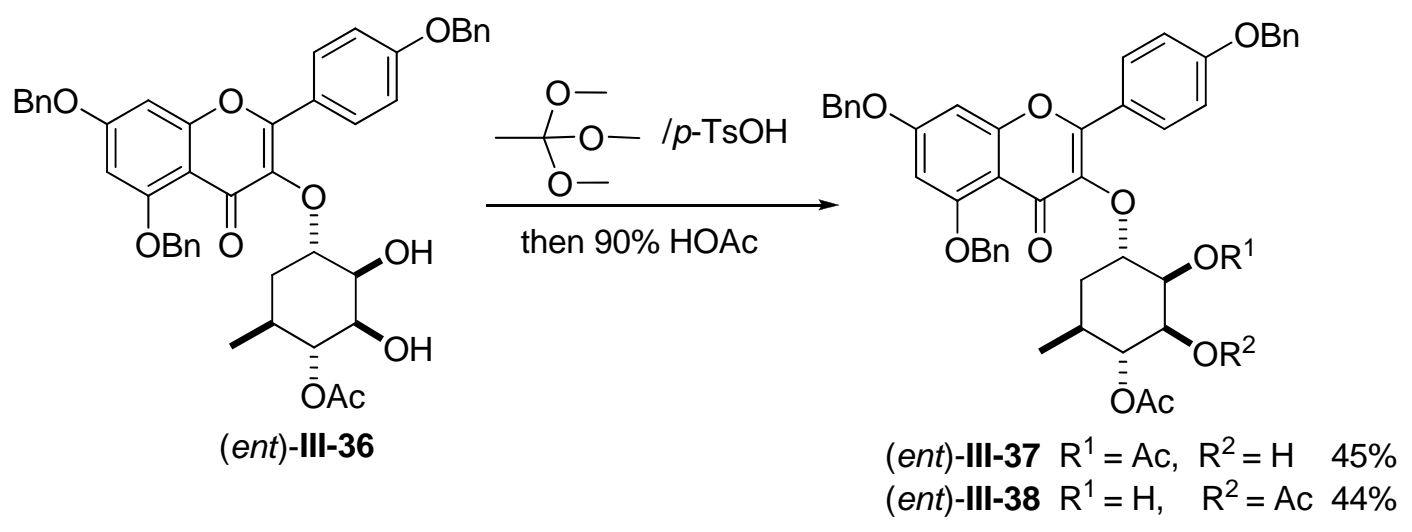

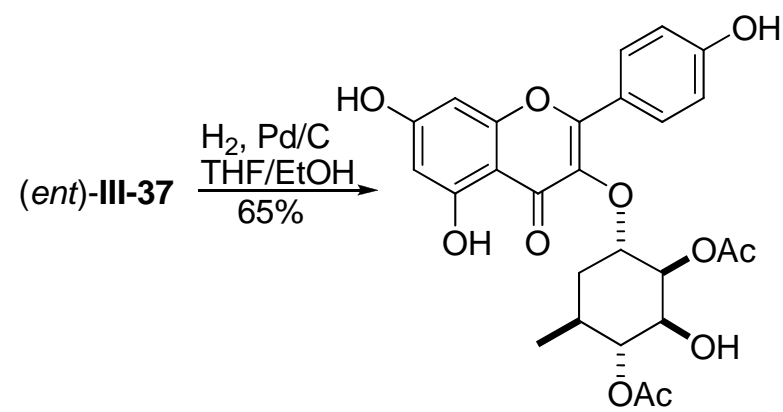

(ent)-III-2<smiles>CC(=O)OC1C(C)C[C@@H](Oc2c(-c3ccc(O)cc3)oc3cc(O)cc(O)c3c2=O)C(O)[C@@H]1OC(C)=O</smiles>

(ent)-III-3

In conclusion, a palladium(0)-catalyzed cyclitolization and post-cyclitolization transformation have been successfully developed. Six carbasugar analogues of SL0101 including three $\alpha-\mathrm{L}$ and three $\alpha$-D forms have been synthesized by utilizing this methodology. 


\section{Chapter IV. Efforts toward the Total Synthesis of Jadomycin A and B}

\section{IV.1. Introduction}

Jadomycins are a group of angucycline-derived antibiotics that were produced by Streptomyces venezuelae following heat shock, ethanol shock or phage infection. ${ }^{44}$ The unique structural feature of these class of antibiotics is the pentacyclic $8 H$-benz[b]oxazolo[3,2- $f]$ phenanthridine backbone, which consists of a dihydropyridine ring and an oxazolone ring. The formation of these two unique rings are believed to arise from the condensation of a biosynthetic aldehyde intermediate, which is derived from anguclinone, with amino acid followed by a series of transformations. ${ }^{45}$ The jadomycins exhibited a multitude of activities such as antibacterial, antiyeast, cytotoxicity against cancer cell lines, antivirus, and enzyme inhibitory activities. ${ }^{44,46}$

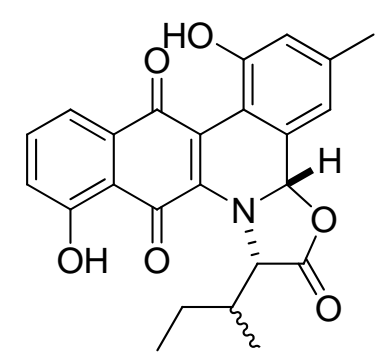

Jadomycin A (IV-1)

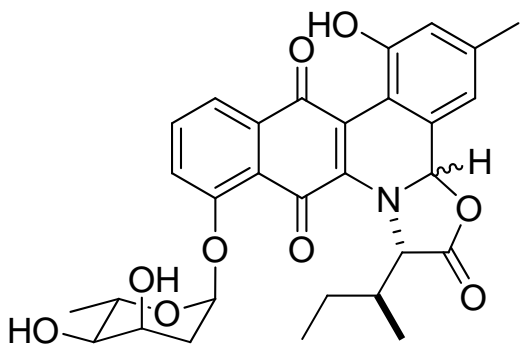

Jadomycin B (IV-2)

Figure 5. Structural Features of Jadomycin A and Jadomycin B

Jadomycin A and B (Figure 5) were the first jadomycins that were isolated from Streptomyces venezulae ISP5230 when grown in an isoleucine containing medium under stress

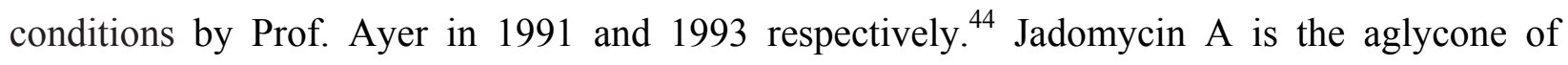
jadomycin B, which is an $\alpha$-L-digitoxoside. The sugar moiety, $\alpha$-L-digitoxose in jadomycin B is believed of great importance for some biological activities such as anti-yeast, which jadomycin A does not have. ${ }^{44 c, 47}$ Since the discovery of jadomycin A and B, many analogues of jadomycin $\mathrm{B}$ have been produced by the fermentation in medium with variant non-natural and natural aminoacids. All these jadomycin analogues were called jadomycins and their biological activities, as well as their biosynthetic pathway have been broadly investigated. ${ }^{45,48,49}$ 
Although they are structurally unique and biologically important, so far no total synthesis of jadomycins including jadomycin A, B and their analogues have been reported. We believe a practical synthetic approach to these structures as well as the ease to incorporate different aminoacid and sugar moiety would significantly enhance the medicinal structure-activity relationship (SAR) studies of this class of antibiotics.

Scheme 35. Retrosynthetic analysis of jadomycin $B$<smiles>C=C=CC1OC2C=CC1C(=O)OC2C(C)(C)OC(C)(C)C</smiles>

Jadomycin B (IV-2)

Jadomycin A (IV-1)
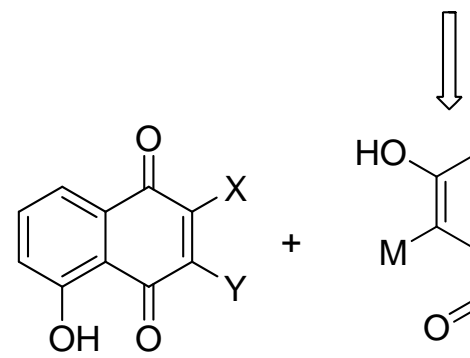

IV-4 $\mathrm{X}, \mathrm{Y}=\mathrm{H}$ or halogen<smiles>[M]c1c(O)cc(C)cc1C=O</smiles>

IV-5 $\mathrm{M}=\mathrm{H}$ or metal

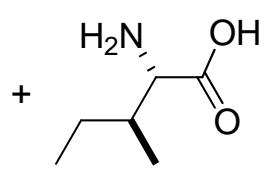

IV-6

Retrosynthetically, we think the jadomycin B (IV-2) could be disconnected on the glycosidic bond between the sugar moiety and aglycone (Scheme 35). The successful synthesis of the aglycone of jadomycin B could be used to determine the structure of jadomycin A (IV-1), which was not clearly identified in the isolation paper in terms of the stereochemistry of the methyl group in the aminoaicd. We envisioned that the glycosidic bond could be installed via our palladium(0)-catalyzed glycosylation reaction between jadomycin A (IV-1) and Boc-pyranone (IV-3), while a post-glycosylation transformation from glycosylated pyranone to $\alpha$-digitoxose need to be developed. We believed the aglycone part could be achieved from three components juglone IV-4, benzaldehyde IV-5, and the aminoacid IV-6. The connection between juglone part and benzaldehyde part could be achieved by using some sort of cross coupling reactions or direct 
nucleophilic addition reactions. The aminoacid part could be introduced at due course, although we don't know the stereochemistry of the methyl group, it is reasonable for us to start with natural isoleucine.

\section{IV.2. De novo asymmetric synthesis of $\alpha$-digitoxose}

Scheme 36. Limitation of our current methodology to digitoxose
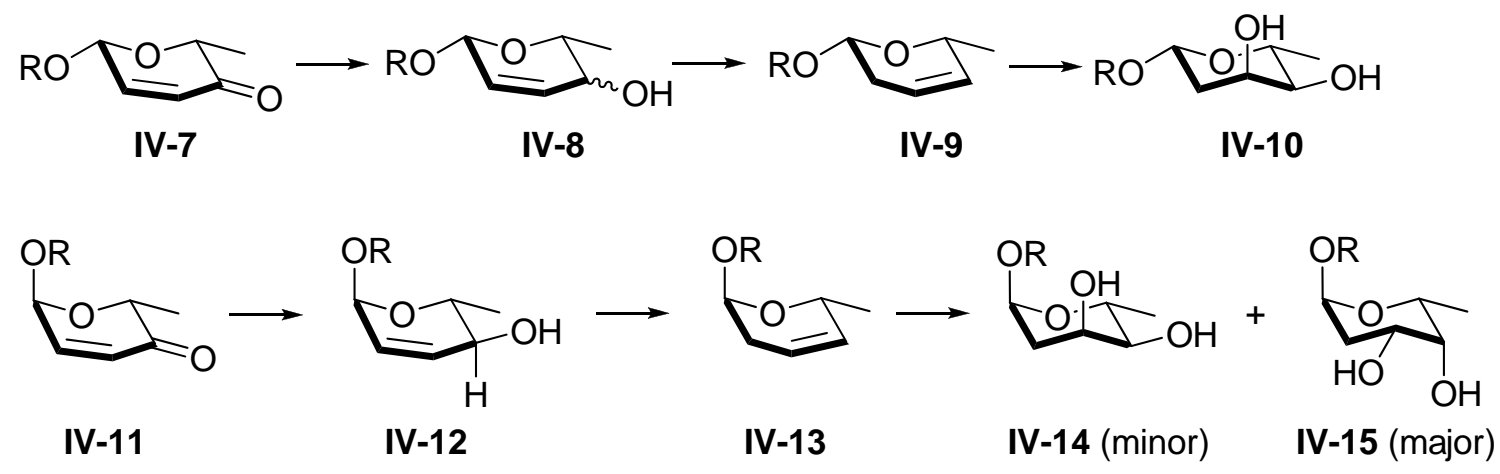

In order to synthesize the sugar moiety of jadomycin B, first we need to develop a practical method to achieve $\alpha$-digitoxose in terms of installation of the correct stereochemistry. As part of efforts to search for more potent digitoxin analogues for the anti-cancer agents, we successfully developed a route to $\beta$-digitoxose (Scheme 36 ). ${ }^{50}$ The approach features a palladium(0)glycosylation to a $\beta$-pyranone IV-7, which subsequently underwent reduction to allylic alcohol IV-8, Myers' 1,3-transposition to olefin IV-9, and dihydroxylation of the resulted olefin. This approach afforded exclusively the desired $\beta$-digitoxose IV-10. When it comes to the $\alpha$-digitoxose case, after reduction of an $\alpha$-pyranone IV-11 to allylic alcohol IV-12, 1,3-transposition of the double bond and dihydroxylation of the resulted olefin IV-13 will produce a diastereomeric mixture of desired $\alpha$-digitoxose IV-14 and undesired sugar IV-15 with the undesired diastereomer as the major product. This limitation clearly indicated that a new practical postglycosylation transformation should be needed to prepare $\alpha$-digitoxose. 
Scheme 37. Proposed approach to $\alpha$-digitoxose

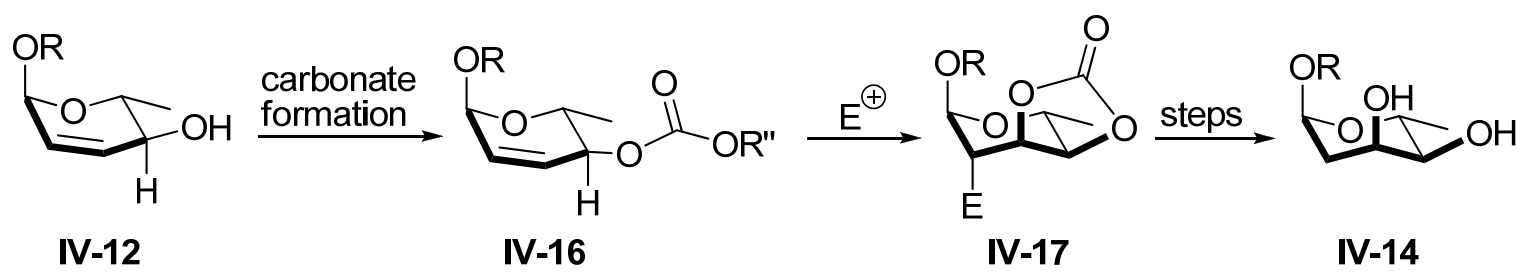

We envisioned that since the reduction of the $\alpha$-pyranone gave an allylic alcohol IV-12 which has the same stereochemistry as required in jadomycin B (IV-2), the hydroxyl group could possibly direct the introduction of adjacent hydroxy group by a cyclic carbonate formation from IV-16 to IV-17 to set up the second hydroxy group which after suitable transformation could be converted to $\alpha$-digitoxose (scheme 37). After searching in the literature, similar transformations could be seen. ${ }^{51}$ But all these transformations from allyl carbonate to cyclic carbonate involved the use of very strong electrophile such as $\mathrm{I}(\mathrm{Collidine}){ }_{2} \mathrm{ClO}_{4}$, which limited the compatibility of this approach. We started to investigate more general electrophiles that could promote the formation of cyclic carbonate IV-17 from a mixed carbonate IV-16, at the same time it might be compatible with the aromatic ring such as aglycone in jadomycin B.

Scheme 38. Asymmetric Synthesis of Boc-pyranone

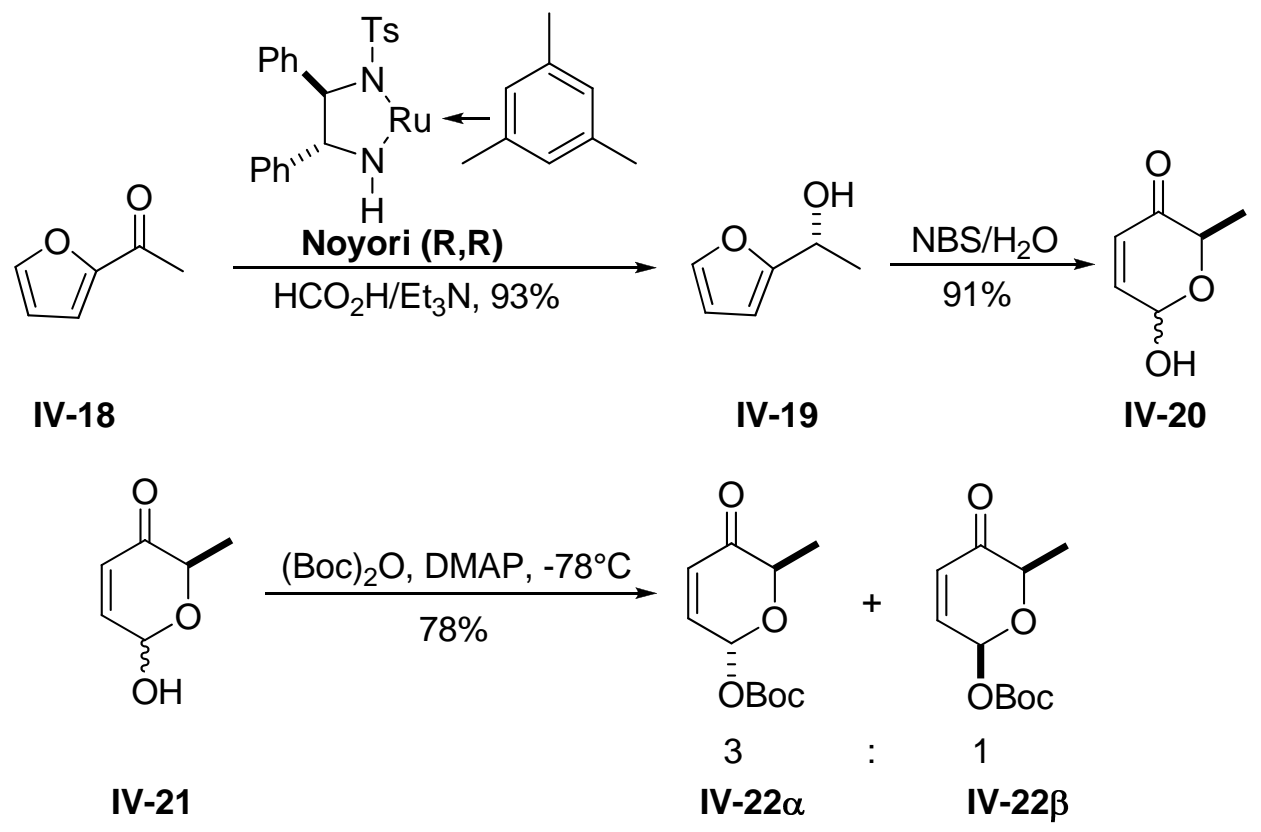


We started the synthesis with the achiral acylfuran (Scheme 38). Noyori reduction of acylfruan IV-18 by using Noyori $(R, R)$ catalyst in the presence of complex of formic acid and triethylamine as hydride source produced the furyl alcohol IV-19 in 93\% yield with high enantiomeric excess ( $>96 \%$ ee). An Achmatowiz rearrangement transformed the furfyl alcohol to hemicacetal IV-20 in $91 \%$ yield. The hemiacetal was then protected as $t$-butyl carbonate IV$\mathbf{2 2} \alpha$ and IV-22 $\beta$ at low temperature in 78\% yield with 3:1 $(\alpha / \beta)$ diastereomeric ratio, which could be easily separated to give the D-sugar precursor. ${ }^{52}$ It worth noting that by simply switching the catalyst Noyori $(R, R)$ to Noyori $(S, S)$, we are able to synthesize the L-sugar precursor.

Scheme 39. Synthesis of the allyl carbonate

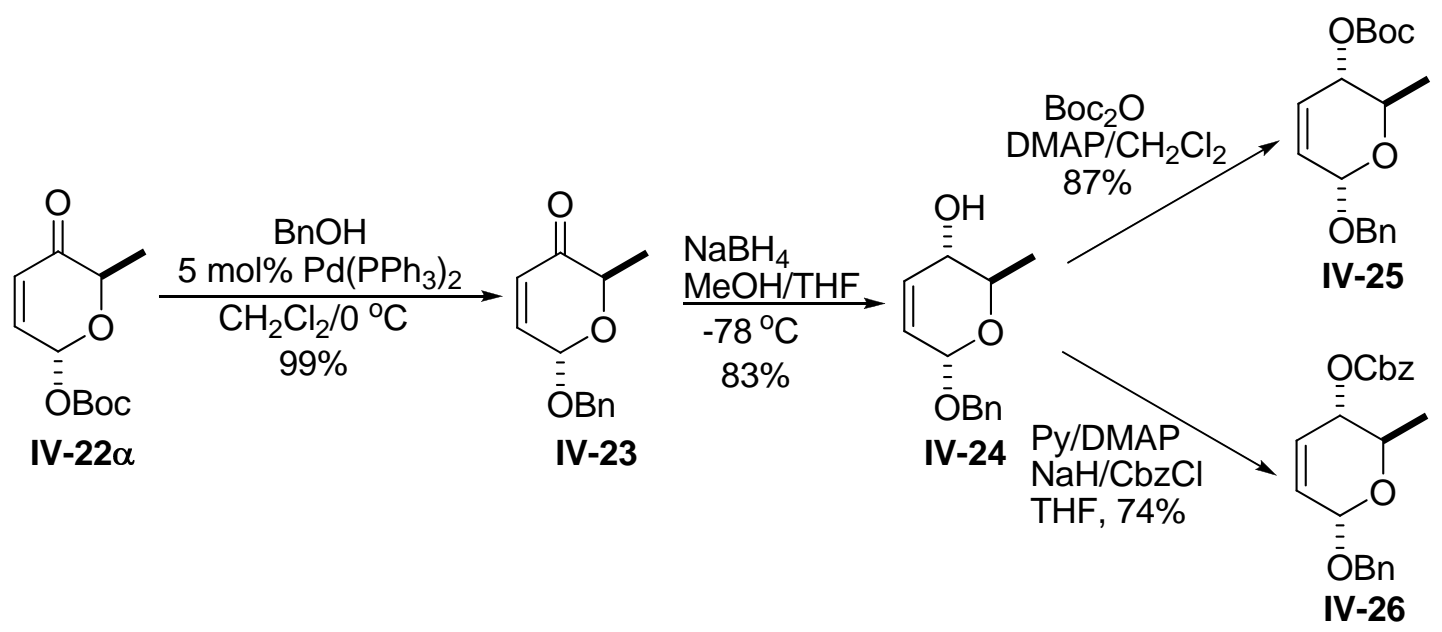

Palladium(0)-catalyzed glycosylation reaction developed in our lab diastereoselectively installed glycosidic bond at the anomeric center with complete $\alpha$ selectivity (IV-22 $\alpha$ to IV-23) (Scheme 39). $\mathrm{NaBH}_{4}$ reduction of the enone $\mathbf{I V - 2 3}$ at $-78^{\circ} \mathrm{C}$ resulted in the allylic alcohol IV-24 with high diastereoselectivity $(\mathrm{dr}>20: 1)$. Acylation of the allylic alcohol IV-24 with either $\mathrm{CbzCl}$ or $\mathrm{Boc}_{2} \mathrm{O}$ afforded carbonate $\mathbf{I V - 2 5}$ and $\mathbf{I V - 2 6}$ respectively, which are ready for the screening of iodo-carbonation. 
Scheme 40. Screening for the halo-carbonation of allyl carbonate

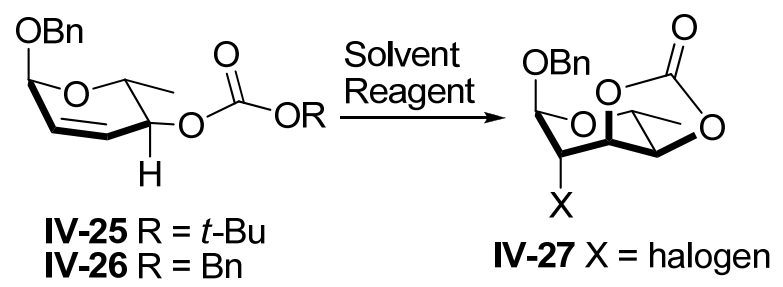

\begin{tabular}{|c|c|c|c|c|c|}
\hline Entry & $\mathrm{R}$ & Solvent & Electrophile & Lewis Acid & Yield \\
\hline 1 & Boc & $\mathrm{CH}_{2} \mathrm{Cl}_{2}$ & NBS & & NR \\
\hline 2 & Boc & $\mathrm{CH}_{2} \mathrm{Cl}_{2}$ & NIS & & NR \\
\hline 3 & Boc & $\mathrm{CH}_{2} \mathrm{Cl}_{2}$ & $\mathrm{I}_{2}$ & & NR \\
\hline 4 & Boc & $\mathrm{CH}_{2} \mathrm{Cl}_{2}$ & $\mathrm{PhNMe}_{3} \mathrm{Br}_{3}$ & & NR \\
\hline 5 & Boc & $\mathrm{CH}_{2} \mathrm{Cl}_{2}$ & NIS & $\mathrm{LiClO}_{4}$ & Trace amount \\
\hline 6 & Boc & $\mathrm{CH}_{2} \mathrm{Cl}_{2}$ & NIS & $\mathrm{MgBr}_{2} \cdot \mathrm{Et}_{2} \mathrm{O}$ & $26 \%$ \\
\hline 7 & Boc & $\mathrm{CH}_{2} \mathrm{Cl}_{2}$ & NIS & $\mathrm{ZnI}_{2}$ & NR \\
\hline 8 & Boc & $\mathrm{CH}_{2} \mathrm{Cl}_{2}$ & NIS & $\mathrm{Zn}(\mathrm{OTf})_{2}$ & decompose \\
\hline 9 & Boc & $\mathrm{CH}_{2} \mathrm{Cl}_{2}$ & NIS & $\mathrm{Sc}(\mathrm{OTf})_{3}$ & decompose \\
\hline 10 & Boc & $\mathrm{CH}_{2} \mathrm{Cl}_{2}$ & NIS & $\mathrm{BF}_{3} \cdot \mathrm{Et}_{2} \mathrm{O}$ & decompose \\
\hline 11 & $\mathrm{Cbz}$ & $\mathrm{CH}_{2} \mathrm{Cl}_{2}$ & NIS & $\mathrm{LiClO}_{4}$ & NR \\
\hline 12 & $\mathrm{Cbz}$ & $\mathrm{CH}_{2} \mathrm{Cl}_{2}$ & NIS & $\mathrm{MgBr}_{2} \cdot \mathrm{Et}_{2} \mathrm{O}$ & NR \\
\hline 13 & $\mathrm{Cbz}$ & $\mathrm{CH}_{2} \mathrm{Cl}_{2}$ & NIS & $\mathrm{ZnI}_{2}$ & NR \\
\hline 14 & $\mathrm{Cbz}$ & $\mathrm{CH}_{2} \mathrm{Cl}_{2}$ & NIS & $\mathrm{Zn}(\mathrm{OTf})_{2}$ & NR \\
\hline 15 & $\mathrm{Cbz}$ & $\mathrm{CH}_{2} \mathrm{Cl}_{2}$ & NIS & $\mathrm{Sc}(\mathrm{OTf})_{3}$ & Not clean reaction \\
\hline 16 & $\mathrm{Cbz}$ & $\mathrm{CH}_{2} \mathrm{Cl}_{2}$ & NIS & $\mathrm{BF}_{3} \cdot \mathrm{Et}_{2} \mathrm{O}$ & decompose \\
\hline 17 & Boc & HOAc & NIS & & $96 \%$ \\
\hline 18 & $\mathrm{Cbz}$ & HOAc & NIS & & $51 \%$ \\
\hline
\end{tabular}

At the beginning, we screened this cyclic carbonate formation in $\mathrm{CH}_{2} \mathrm{Cl}_{2}$ by using halogen sources such as NBS, NIS, $\mathrm{I}_{2}$, and $\mathrm{PhNMeBr}_{3}$ as electrophiles and $t$-butyl allyl mixed carbonate IV-25 as substrate (Scheme 40). No reaction was observed by TLC as shown in entry 1-4. Next, we tried to use Lewis acid to promote the cyclization reactions. Therefore, a number of Lewis acid such as $\mathrm{LiClO}_{4}, \mathrm{MgBr}_{2} \bullet \mathrm{Et}_{2} \mathrm{O}, \mathrm{ZnI}_{2}, \mathrm{Zn}(\mathrm{OTf})_{2}, \mathrm{Sc}(\mathrm{OTf})_{3}, \mathrm{BF}_{3} \bullet \mathrm{Et}_{2} \mathrm{O}$ were used as the 
promoters in term of their attenuated Lewis acidity. The screening was carried out in a $0.05 \mathrm{M}$ solution of substrate in $\mathrm{CH}_{2} \mathrm{Cl}_{2}$ with one eqivalent NIS as electrophile and $30 \mathrm{~mol} \%$ Lewis acid as promoter. As a result, $\mathrm{MgBr}_{2} \cdot \mathrm{Et}_{2} \mathrm{O}$ was found having some activity in promoting the conversion of mixed carbonate $\mathbf{I V - 2 5}$ to cyclic carbonate $\mathbf{I V - 2 7}$, while $\mathrm{LiClO}_{4}$ case also gave trace amount of desired product, although both conversions were not complete, and most substrate remained untouched. $\mathrm{ZnI}_{2}$ showed no reactivity probably due to its poor Lewis acidity. The stronger Lewis acid such as $\mathrm{Zn}(\mathrm{OTf})_{2}, \mathrm{Sc}(\mathrm{OTf})_{3}, \mathrm{BF}_{3} \cdot \mathrm{Et}_{2} \mathrm{O}$ caused the decomposition of the substrate. We also examinated the substrate of benzyl allyl mixed carbonate IV-26. This time the $\mathrm{LiClO}_{4}, \mathrm{MgBr}_{2} \bullet \mathrm{Et}_{2} \mathrm{O}, \mathrm{ZnI}_{2}, \mathrm{Zn}(\mathrm{OTf})_{2}$ did not give any conversion, instead, $\mathrm{Sc}(\mathrm{OTf})_{3}$ afforded some products but the reaction was not clean. Once again, the $\mathrm{BF}_{3} \cdot \mathrm{Et}_{2} \mathrm{O}$ caused the decomposition of substrate.

Fortunately, when we switched the solvent from $\mathrm{CH}_{2} \mathrm{Cl}_{2}$ to $\mathrm{HOAc}$ without any Lewis acid, the $t$-butyl allyl mixed carbonate IV-25 was converted to the desired iodo-carbonate IV-27 cleanly with high yield. When the benzyl allyl mixed carbonate IV-26 was used as the substrate, the reaction also had reasonable conversion in the same condition as used for $\mathbf{I V - 2 5}$.

Scheme 41. Synthesis of $\alpha$-digitoxose<smiles>CC1OC(Cc2ccccc2)C=CC1OC(C)(C)C</smiles>

IV-25

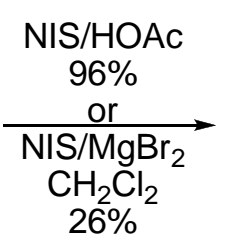

$26 \%$

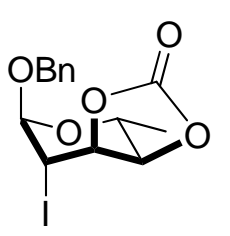

IV-27
$\frac{\mathrm{NIS} / \mathrm{HOAC}}{51 \%}$<smiles>CC(=O)OC1C=CC(OCc2ccccc2)OC1C(C)(F)F</smiles>

IV-26

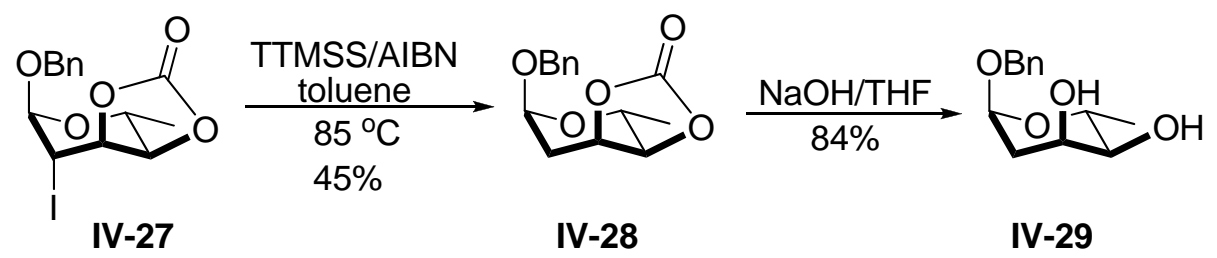

Encouraged by the preliminary results, we next carried out our synthesis of $\alpha$-digitoxose (Scheme 41). The iodocarbonate IV-27 could be obtained from carbonate IV-25 with NIS/HOAc 
condition in $96 \%$ yield or NIS/ $\mathrm{MgBr}_{2} / \mathrm{CH}_{2} \mathrm{Cl}_{2}$ condition in $25 \%$. And also the carbonate IV-26 could be converted to IV-27 in 51\% yield. Iodine in iodocarbonate IV-27 was reductively removed by tris(trimethylsilyl)silane upon heating in the presence of AIBN as a radical initiator to afford cyclic carbonate $\mathbf{I V}-\mathbf{2 8},{ }^{53}$ which was subjected to basic hydrolysis to furnish benzyl $\alpha$ digitoxoside (IV-29)

To sum up this part, a highly diastereoselective route to $\alpha$-digitoxose has been developed. The route relies on a palladium(0)-catalyzed glycosylation reaction and post-glycosylation transformation. The asymmetry of $\alpha$-digitoxose originated from Noyori reduction of acylfuran and subsequent introduction of other stereogenic centers. An iodo-carbonate formation is the key step for the construction of $\alpha$-digitoxose stereochemistry. 


\section{IV.3. Synthesis of Jadomycin A}

With the approach to $\alpha$-digitoxose having been successfully developed, we next turned our attention to synthesize jadomycin A, the aglycone part of the jadomycin B. Our first strategy to the jadomycin A (IV-1) was shown in Scheme 42, which features an oxazolone ring formation as the key step to connect the three building blocks together (i.e. from IV-32 or IV-33 and IV-34 to IV-30 or IV-31 respectively). Once the oxazolone ring was formed, the dihydropyridine ring formation could be achieved via direct Michael addition or Heck reaction (i.e. from IV-30 or IV31 to $\mathbf{I V - 1 ) .}$

Scheme 42. First strategy to jadomycin A<smiles>CC[C@H](C)[C@H]1C(=O)O[C@]2(C)c3cc(C)cc(O)c3C3=C(C(=O)c4c(O)cccc4C3=O)N12</smiles>

Jadomycin A (IV-1)

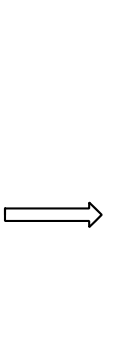<smiles>[X]C1=C(N2[C@@H](c3cc(C)cc(O)c3)OC(=O)[C@H]2[C@H](C)CC)C(=O)c2c(O)cccc2C1=O</smiles>

IV-30 $X=\mathrm{H}$ IV-31 $X=\mathrm{Br}$<smiles>C=CCCC</smiles>

IV-32 X = H IV-33 X = Br<smiles>Cc1cc(O)cc(C=O)c1</smiles>

IV-34

We started the synthesis with the benzaldehyde part IV-34 (Scheme 43). By a modification of the reported method in the literature, ${ }^{54}$ benzoate $\mathbf{I V - 3 7}$ was produced in $56 \%$ overall yield in three steps from acetopyruvate (IV-35). Protection of phenol IV-37 as TBS ether followed by $\mathrm{LiAlH}_{4}$ reduction delivered benzyl alcohol IV-38 in 89\% yield over two steps, which upon $\mathrm{MnO}_{2}$ oxidation to aldehyde IV-39 (84\%) and deprotection of TBS ether in acid condition afforded 3-hydroxyl-5-methyl benzaldehyde (IV-34) in 93\% yield. 
Scheme 43. Synthesis of 3-hydroxyl-5-methyl benzaldehyde<smiles>CCOC(=O)C(=O)O[N+](=O)[O-]</smiles>

IV-35<smiles>CCOC(=O)C1(CC(C)=O)OC(=O)C(O)=C1C(C)=O</smiles>

IV-36 i) $\mathrm{Mg}(\mathrm{OH})_{2} / \mathrm{H}_{2} \mathrm{O}$ reflux

ii) $\mathrm{MeOH}, \mathrm{H}_{2} \mathrm{SO}_{4}$ $56 \%$ (three steps)<smiles>COC(=O)c1cc(C)cc(O)c1</smiles>

IV-37

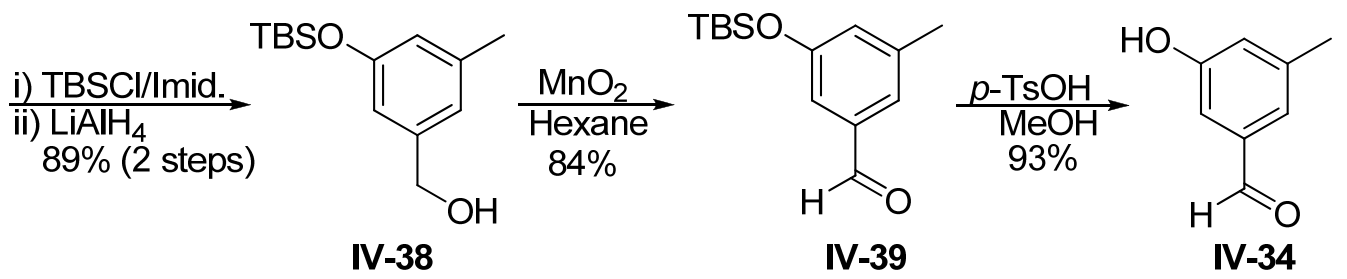

Our next synthetic intermediate is the juglone part IV-43 (Scheme 44). Isoleucine (IV-6) was first protected as $t$-butyl ester IV-40. 3-bromojuglone (IV-42) was synthesized from juglone IV41 according to the reported method in $80 \%$ yield. ${ }^{55}$ Introducing the aminoacid into the 3 position of the juglone was successfully achieved in 67\% yield by mixing IV-40 and IV-42 in $\mathrm{CH}_{2} \mathrm{Cl}_{2}$ in the presence of triethylamine. ${ }^{56}$ The resulted aminojuglone IV-43 was then brominated by $\mathrm{Br}_{2}$ in ethanol to afford 2-bromo-3-aminojuglone IV-44 in $87 \%$ yield. $^{57}$

Scheme 44. Synthesis of bromojuglone IV-44<smiles>CCC(C)[C@H](N)C(=O)O[Mg]</smiles>

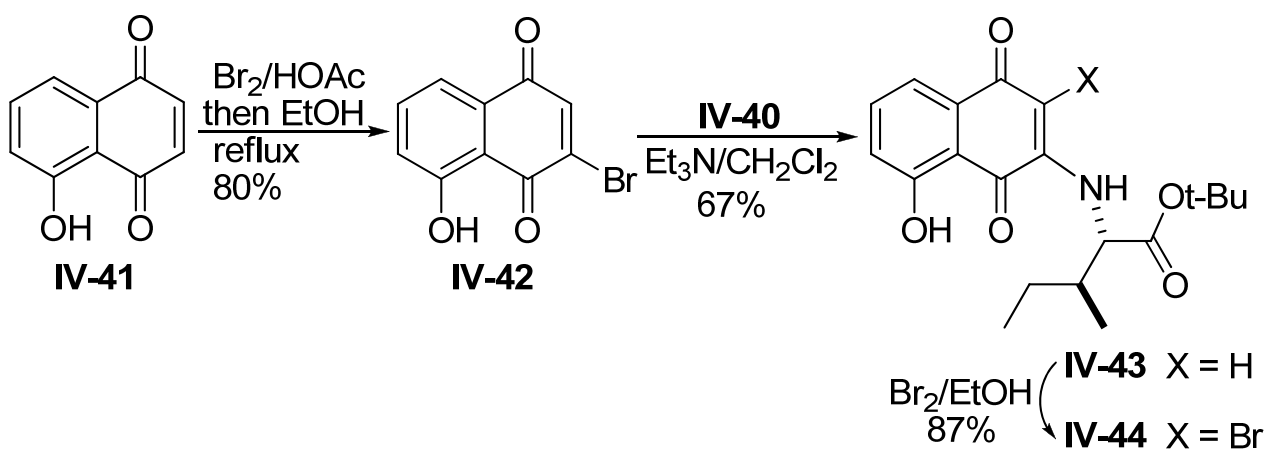


With the two building blocks in hand, we turned our attention to the investigation of the oxazolone ring formation (Scheme 45). Bromoaminojuglone IV-44 was first chosen to test this reaction. The $t$-butyl ester was deprotected by exposure to trifluoroacetic acid, which afforded free carboxylic acid IV-33. The carboxylic acid was then heated with benzaldehyde IV-34 in benzene in the presence of $p$-toluenesulfonic acid by using Dean-Stark trap technique, or direct distillation. Unfortunately, no desired oxazolone was formed. We thought it might be due to the steric hinderance from the bromine. Therefore, we examinated the reaction between benzaldehyde IV-34 and the free acid IV-32, which came from the deprotection of $t$-butyl ester IV-43, by using the same technique of Dean-Stark trap. Once again, no reaction was observed.

Scheme 45. Tries on oxazolone ring formation
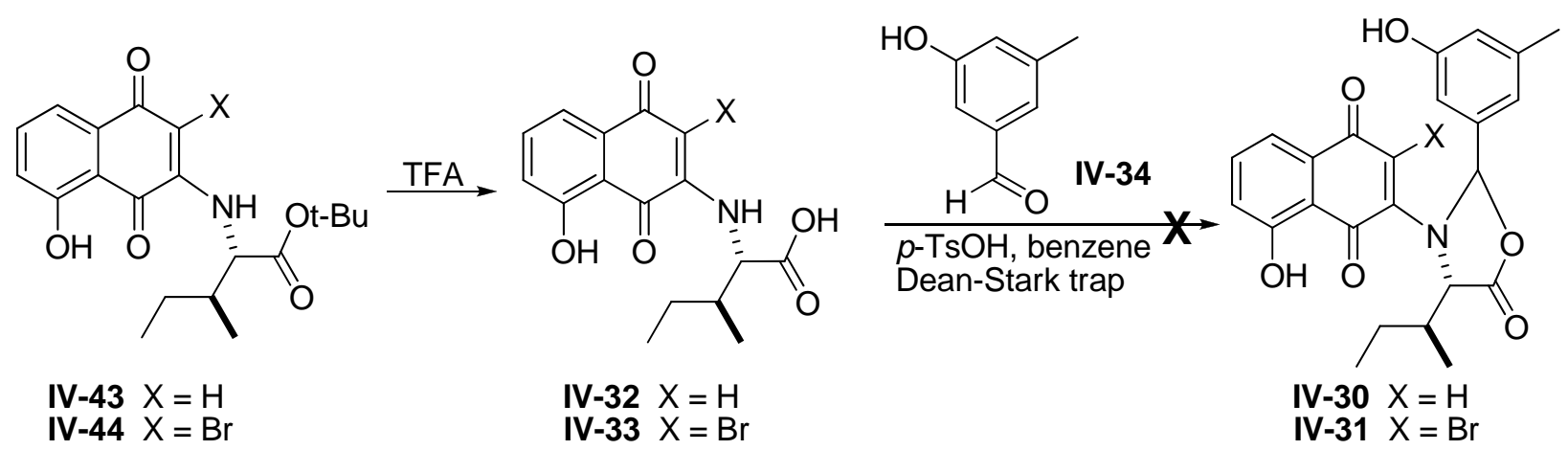

Due to the difficulty we ran into during the synthesis of the aglycone by forming oxozalone ring, we next examined the possibility of direct coupling of the two components, which will significantly facilitate the formation of the dihdropyridine ring and oxazolone ring as well (Scheme 46). Therefore, benzaldehyde IV-34 was first protected as acetal by 1,3-propanediol in the presence of acid (84\%), which was then further protected as MOM ether in the condition of MOMCl/DIPEA (68\%). The MOM ether IV-45 was converted to stannane IV-46 by a directed lithiation followed by reacting the anion intermediate with tributyltin chloride in $49 \%$ yield with the recovery of $35 \%$ starting material. A Stille coupling was carried out between bromojuglone IV-44 and stannane IV-46 in the presence of palladium(0) and cocatalyst $\mathrm{CuI},{ }^{58}$ but unfortunately, no desired product was observed. 
Scheme 46. First try of Stille coupling<smiles>Cc1cc(O)cc(C=O)c1</smiles>

IV-34 i) 1,3-propanediol p-TsOH, 84\% $68 \%$

IV-44

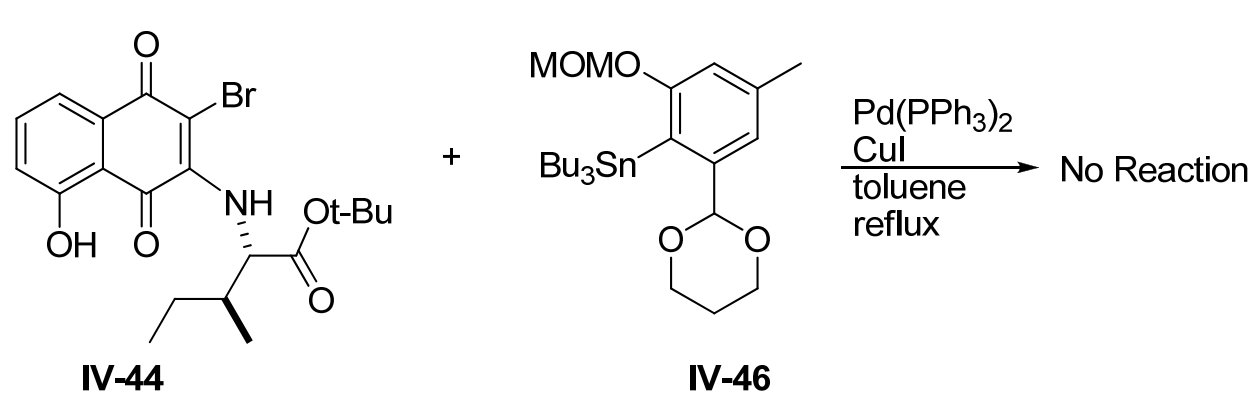<smiles>COc1cc(C)cc(C2OCCCO2)c1</smiles>

IV-45

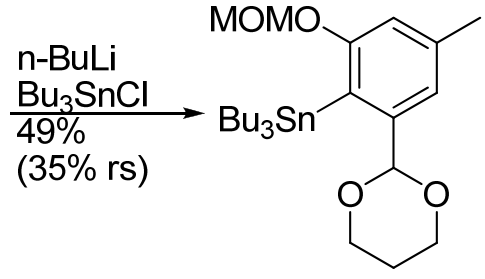

IV-46

The failure of the Stille coupling between aminobromojuglone IV-44 and stannane IV-46 suggested that the steric hinderance should be an important factor here. We now believed that a coupling between the juglone part and benzaldehyde should be needed before the introduction of the aminoacid part. Therefore, we turned our attention to the synthesis of 2-bromojuglone (IV-50) by following the reported method. ${ }^{59}$ Naphthalenediol IV-47 was converted to diacetate IV-48 in the presence of acetic chloride/pyridine in 92\% yield (Scheme 47). Diacetate IV-48 was oxidized by NBS to 2-bromojuglone acetate IV-49 in $80 \%$ yield, which was hydrolyzed in acid condition to produce 2-bromojuglone (IV-50) in $75 \%$ yield. With the 2-bromojuglone in hand, the coupling between it and stannane IV-46 was then tested by following the conditions reported in the literature. ${ }^{58}$ In practice, the coupling was carried out in the presence of $5 \mathrm{~mol} \%$ palladium $(0)$ and $20 \mathrm{~mol} \% \mathrm{CuI}$ as cocatalyst in THF at elevated temperature, which afforded the coupled product IV-51 in 76\% yield. 
Scheme 47. Stille coupling between 2-bromojuglone and stannane<smiles>Oc1cccc2c(O)cccc12</smiles>

IV-47<smiles></smiles>

IV-48<smiles>[R]Oc1cccc2c1C(=O)C=C(Br)C2=O</smiles>

$\mathrm{H}_{2} \mathrm{SO}_{4}$
$\mathrm{EtOH}$
$75 \%$$\left(\begin{array}{l}\text { IV-49 R }=\mathrm{AC} \\ \text { IV-50 R }=\mathrm{H}\end{array}\right.$<smiles>O=C1C=C(Br)C(=O)c2c(O)cccc21</smiles>

IV-50<smiles>COc1cc(C)cc(C2OCCCO2)c1[SnH2]C(C)(C)C</smiles>

IV-46<smiles>COc1cc(C)cc(C2OCCCO2)c1C1=CC(=O)c2c(O)cccc2C1=O</smiles>

IV-51

With the juglone part and benzaldehyde having been coupled. We then investigated the introduction of the aminoacid (Scheme 48). A direct aza-Michael addition to achieve IV-52 proved to be not successful by mixing the juglone part IV-51 and aminoacid IV-40. We next tried to knock off the protecting group to produce aldehyde, which then possibly help introduction of the aminoacid part. To our surprise, deprotection of the acetal and MOM ether by treatment of IV-51 with TFA caused the decomposition of the starting material. Upon a short time heating of IV-51 in aqueous HCl/THF, around 20\% yield of aldehyde IV-53 was obtained. Mixing the aldehyde IV-53 with aminoacid IV-40 in toluene at RT, over two days, the starting material disappeared. After passing the mixture through a short column, a crude product was obtained, which was then treated with TFA furnished jadomycin A (IV-1). The crude NMR data showed that the product was a mixture of diastereomers at a ratio of 6 to 1 with the major isomer matching very well with the reported data of jadomycin A. This also confirmed that the stereochemistry of the methyl group is the same as it is in natural isoleucine. 
Scheme 48. Synthesis of jadomycin A

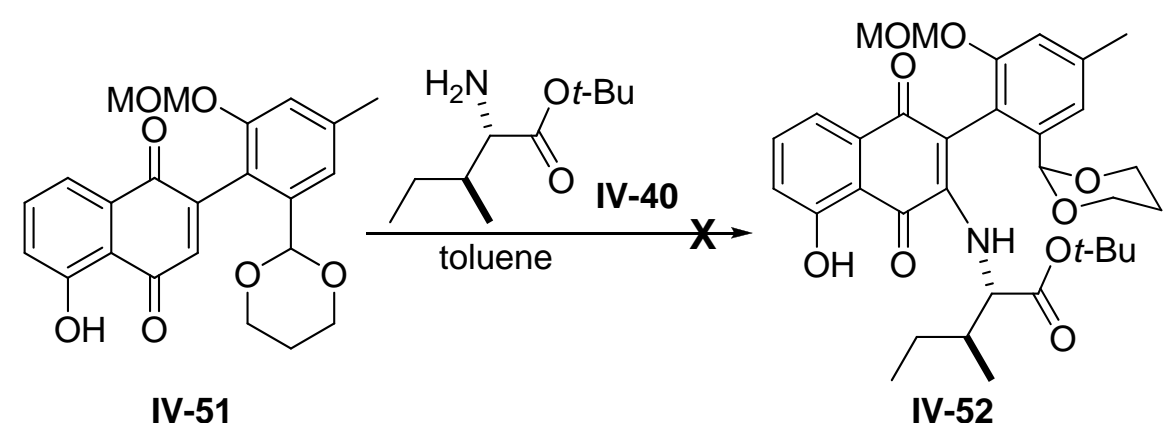

IV-51 $\stackrel{\text { TFA }}{\longrightarrow}$ decompose

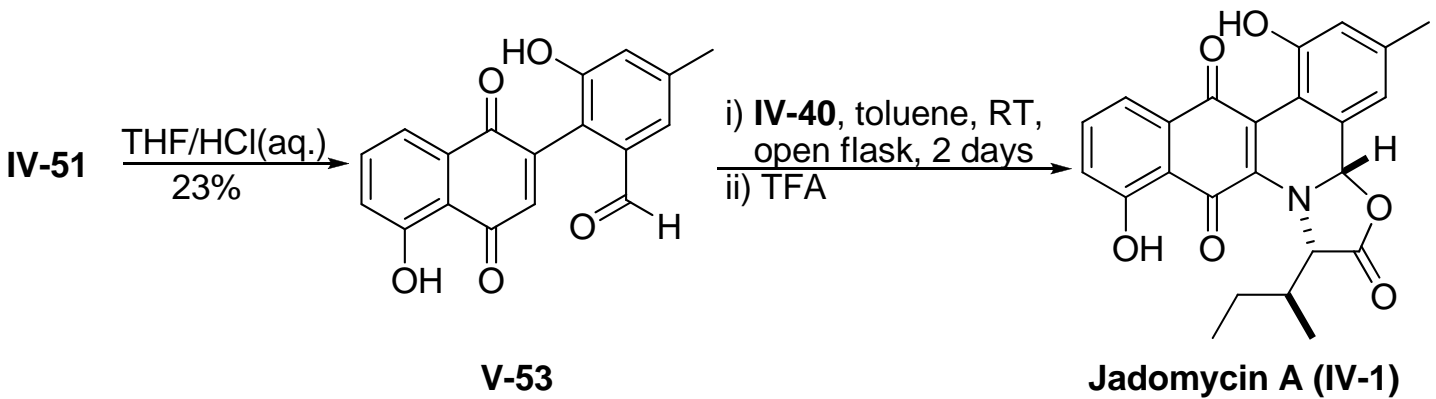

In conclusion, a model study for synthesis of $\alpha$-digitoxose, the sugar moiety of jadomycin B has been successfully carried out by preparing benzyl $\alpha$-digitoxoside, which relies upon a palladium(0) catalyzed glycosylation, post-glycosylation featuring an iodo-carbonation and corresponding transformations. Jadomycin A, the aglycone part of jadomycin $\mathrm{B}$, has also been successfully synthesized and structurally defined by the comparison of our spectral data with the reported one from isolation. This synthesis features a Stille cross coupling reaction, a tandem imine formation/dihydropyridine ring formation during the incorporation of the aminoacid. Efforts toward the synthesis of the jadomycin B will be continued by applying the newly developed methodology for installation of $\alpha$-digitoxose to the jadomycin A. 


\section{Chapter V. Experimental Section}

\section{V.1. General methods and materials}

${ }^{1} \mathrm{H}$ and ${ }^{13} \mathrm{C}$ spectra were recorded on $270 \mathrm{MHz}$ and $600 \mathrm{MHz}$ spectrometers. Chemical shifts were reported relative to benzene- $\mathrm{d}_{6}(\delta 7.16 \mathrm{ppm}), \mathrm{CDCl}_{3}(\delta 7.26 \mathrm{ppm}), \mathrm{CD}_{3} \mathrm{OD}(\delta 3.31 \mathrm{ppm})$, acetone- $\mathrm{d}_{6}(\delta 2.05 \mathrm{ppm}), \mathrm{D}_{2} \mathrm{O}(\delta 4.80 \mathrm{ppm})$ for ${ }^{1} \mathrm{H}$, and benzene- $\mathrm{d}_{6}(\delta 127.68 \mathrm{ppm}), \mathrm{CDCl}_{3}(\delta$ $77.0 \mathrm{ppm}), \mathrm{CD}_{3} \mathrm{OD}(\delta 49.15 \mathrm{ppm})$, acetone- $\mathrm{d}_{6}(\delta 29.92 \mathrm{ppm})$ for ${ }^{13} \mathrm{C}$, and $\mathrm{H}_{3} \mathrm{PO}_{4}$ in $\mathrm{D}_{2} \mathrm{O}(\delta 0.00$ $\mathrm{ppm}$ ) as external reference for ${ }^{31} \mathrm{P}$. Optical rotations were measured with a digital polarimeter in the solvent specified. Infrared (IR) spectra were obtained on a FT-IR spectrometer. Flash column chromatography was performed on 60-200 mesh silica gel. Analytical thin-layer chromatography was performed with precoated glass-backed plates and visualized by quenching of fluorescence and by charring after treatment with panisaldehyde or phosphomolybdic acid or potassium permanganate stain. $R_{f}$ values were obtained by elution in the stated solvent ratios $(\mathrm{v} / \mathrm{v})$. Ether, THF, methylene chloride, toluene and triethylamine were dried by passing through activated alumina ( $8 \times 14$ mesh) column with argon gas pressure. Commercial reagents were used without purification unless otherwise noted. Air and/or moisture-sensitive reactions were carried out under an atmosphere of argon/nitrogen using oven/flamed-dried glassware and standard syringe/septum techniques. 


\section{V.2. Chapter I experimental}

(E)-1-(2,4-bis(benzyloxy)-6-hydroxyphenyl)-3-(4-(benzyloxy)phenyl)prop-2-en-1-one (I-7) ${ }^{5}$<smiles>O=C(/C=C/c1ccc(OCc2ccccc2)cc1)c1c(O)cc(OCc2ccccc2)cc1OCc1ccccc1</smiles>

To a solution of $4.0 \mathrm{~g} \mathrm{4}$, 5, 7-trihydroxyflavanone $\mathbf{I - 6}(14.7 \mathrm{mmol})$ in $50 \mathrm{~mL}$ acetone was added $5.3 \mathrm{~mL} \mathrm{BnBr}(44.4 \mathrm{mmol})$ and $12.2 \mathrm{~g} \mathrm{~K}_{2} \mathrm{CO}_{3}(88.2 \mathrm{mmol})$. The reaction mixture was heated under reflux for 6 hours, then cooled down to room temperature. The mixture was concentrated under reduced pressure to give a residue which was then partitioned between $150 \mathrm{~mL}$ EtOAc and $80 \mathrm{~mL} \mathrm{H} \mathrm{H}_{2} \mathrm{O}$. The water layer was extracted by EtOAc $(100 \mathrm{~mL} \times 3)$. The organic layer was pooled, then washed by saturated brine, dried over $\mathrm{Na}_{2} \mathrm{SO}_{4}$ and concentrated under reduced pressure to give a crude product. Recrystallization and further chromatography for the mother liquid eluting with hexane-EtOAc (6:1) gave pure phenol I-7 (6 g, 75\%): Yellow solid; $R_{f}=0.51$ (3:1 (v/v) hexane/EtOAc); ${ }^{1} \mathrm{H}$ NMR $(\mathrm{CDCl} 3,270 \mathrm{MHz}) \delta 7.75(\mathrm{dd}, J=15.6,15.3 \mathrm{~Hz}, 2 \mathrm{H}), 7.53$ $7.30(\mathrm{~m}, 15 \mathrm{H}), 7.00(\mathrm{~d}, J=8.9 \mathrm{~Hz}, 2 \mathrm{H}), 6.78(\mathrm{~d}, J=8.7 \mathrm{~Hz}, 2 \mathrm{H}), 6.22(\mathrm{~d}, J=2.5 \mathrm{~Hz}, 1 \mathrm{H}), 6.17$ $(\mathrm{d}, J=2.5 \mathrm{~Hz}, 1 \mathrm{H}), 6.13(\mathrm{~d}, J=10.2 \mathrm{~Hz}, 1 \mathrm{H}), 5.10(\mathrm{~s}, 2 \mathrm{H}), 5.08(\mathrm{~s}, 2 \mathrm{H}), 5.05(\mathrm{~s}, 2 \mathrm{H}) ;{ }^{13} \mathrm{C}$ NMR $(\mathrm{CDCl} 3,67.5 \mathrm{MHz}) \delta 192.6,168.8,165.1,161.7,160.3,142.7,136.5,135.9,135.5,130.1,128.9$, $128.8,128.7,128.6,128.6,128.5,128.3,128.1,127.7,127.4,125.2,115.0,106.3,95.0,92.5$, $71.4,70.3,70.0$.

\section{5,7-bis(benzyloxy)-2-(4-(benzyloxy)phenyl)-4H-chromen-4-one (I-8) ${ }^{5}$}<smiles>O=c1cc(-c2ccc(OCc3ccccc3)cc2)oc2cc(OCc3ccccc3)cc(OCc3ccccc3)c12</smiles> 
$2.76 \mathrm{~g}$ phenol I-7 $(5.09 \mathrm{mmol})$ was dissolved in $6 \mathrm{~mL}$ dry DMSO, and a catalytic amount of $\mathrm{I}_{2}$ (41 mg, $0.16 \mathrm{mmol}$ ) was added into the solution. The reaction mixture was heated to $110^{\circ} \mathrm{C}$ and kept stirring at this temperature for 24 hours. Then it was cooled down to the room temperature. $10 \mathrm{~mL}$ saturated aqueous $\mathrm{NaHCO}_{3}$ was added to quench the reaction. The water layer was extracted with EtOAc $(30 \mathrm{~mL} \times 3)$. The organic layer was pooled, then washed by $10 \mathrm{~mL}$ saturated brine, dried over $\mathrm{Na}_{2} \mathrm{SO}_{4}$, and concentrated the organic layer under reduced pressure to give a crude product. Chromatography eluting with hexane-EtOAc (2:1) afforded desired flavone I-8 (2.10 g, 76\%): Yellow solid; $R_{f}=0.20\left(2: 1(\mathrm{v} / \mathrm{v})\right.$ Hexane/EtOAc); ${ }^{1} \mathrm{H}$ NMR $(\mathrm{CDCl} 3,600$ MHz) $\delta 7.81(\mathrm{~d}, J=8.4 \mathrm{~Hz}, 2 \mathrm{H}), 7.62(\mathrm{~d}, J=8.4 \mathrm{~Hz}, 2 \mathrm{H}), 7.46-7.28(\mathrm{~m}, 13 \mathrm{H}), 7.07(\mathrm{~d}, J=8.4$ $\mathrm{Hz}, 2 \mathrm{H}), 6.64(\mathrm{~d}, J=1.8 \mathrm{~Hz}, 1 \mathrm{H}), 6.58(\mathrm{~s}, 1 \mathrm{H}), 6.49$ (d, $J=2.4 \mathrm{~Hz}, 1 \mathrm{H}), 5.23(\mathrm{~s}, 2 \mathrm{H}), 5.14(\mathrm{~s}$, 2H), $5.11(\mathrm{~s}, 2 \mathrm{H}) ;{ }^{13} \mathrm{C} \mathrm{NMR}(\mathrm{CDCl} 3,67.5 \mathrm{MHz}) \delta 177.3,162.8,161.1,160.6,159.6,136.4$, 136.2, 135.7, 128.7, 128.6, 128.5, 128.4, 128.2, 127.6, 127.4, 126.5, 124.0, 118.0, 115.2, 109.7, $107.7,98.3,94.2,70.7,70.4,70.1$.

\section{5,7-bis(benzyloxy)-2-(4-(benzyloxy)phenyl)-3-hydroxy-4H-chromen-4-one (I-3) ${ }^{5}$}

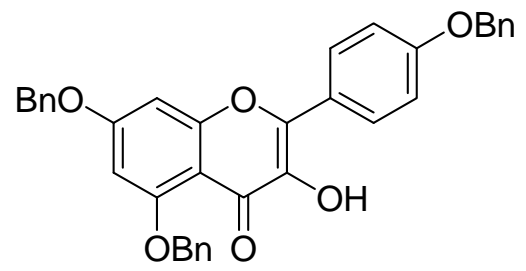

$150 \mathrm{~mL}$ solution of freshly generated DMDO in acetone $(\sim 0.1 \mathrm{M})$ was dried by $3 \AA$ MS at $20^{\circ} \mathrm{C}$ under the protection of argon, and into this solution was added a solution of $2.3 \mathrm{~g}$ flavone I-8 (4.26 mmol) in $20 \mathrm{~mL}$ dry $\mathrm{CH}_{2} \mathrm{Cl}_{2}$ at this temperature via a cannula. Then allow it warm up to $0{ }^{\circ} \mathrm{C}$ and keep stirring at $0{ }^{\circ} \mathrm{C}$ overnight. The solvent was evaporated under reduced pressure to give brown-yellowish solid, which was then redissolved in $20 \mathrm{~mL} \mathrm{CH}_{2} \mathrm{Cl}_{2}$. Into this solution was added $2 \mathrm{mg} \mathrm{TsOH} \cdot \mathrm{H}_{2} \mathrm{O}$ and it was then kept stirring at room temperature for 1 hour. The reaction mixture was concentrated under reduced pressure to a residue, which was then subjected to chromatography. Elution with hexane-EtOAc (5:1) gave perbenzylated flavonol I-3 (1.2 g, $50 \%)$ and elution with hexane-EtOAc (2:1) recovered $0.7 \mathrm{~g}$ starting material. 
Alternative procedure:

A solution of $1.5 \mathrm{~g}(2.77 \mathrm{mmol})$ enone $\mathbf{I - 8}$ in $54 \mathrm{~mL} \mathrm{CH}_{2} \mathrm{Cl}_{2}$ and $27 \mathrm{~mL}$ acetone was added a buffer solution of $3.6 \mathrm{~g} \mathrm{NaHCO}_{3}$ and $7.5 \mathrm{~g} \mathrm{Na}_{2} \mathrm{CO}_{3}$ in $189 \mathrm{~mL} \mathrm{H}_{2} \mathrm{O}$ at RT. When vigorous stirring, the mixture was slowly added a solution of $16.2 \mathrm{~g}(26.4 \mathrm{mmol})$ oxone $\left(2 \mathrm{KHSO}_{5} \cdot \mathrm{KHSO}_{4} \cdot \mathrm{K}_{2} \mathrm{SO}_{4}\right.$ ) in $189 \mathrm{~mL} \mathrm{H}_{2} \mathrm{O}$ over 2 hour. Then it was stirred for another $10 \mathrm{~min}$. Then it was separated and the aqueous layer was extracted with $\mathrm{CH}_{2} \mathrm{Cl}_{2} 80 \mathrm{~mL} \mathrm{X} \mathrm{2.} \mathrm{The}$ combined organic layer was washed subsequently with $120 \mathrm{~mL}$ aqueous solution of $\mathrm{Na}_{2} \mathrm{~S}_{2} \mathrm{O}_{3}(30$ $\mathrm{mL}$ saturated aqueous $\mathrm{Na}_{2} \mathrm{~S}_{2} \mathrm{O}_{3}$ plus $90 \mathrm{~mL} \mathrm{H} \mathrm{H}_{2} \mathrm{O}$ ), $120 \mathrm{~mL}$ saturated brine and then was dried over $\mathrm{Na}_{2} \mathrm{SO}_{4}$. Into the solution, $9 \mathrm{mg}(0.05 \mathrm{mmol}) p-\mathrm{TsOH} \cdot \mathrm{H}_{2} \mathrm{O}$ was added at $\mathrm{RT}$, and the mixture was stirred for $20 \mathrm{~min}$. It was then diluted with $200 \mathrm{~mL} \mathrm{Et}_{2} \mathrm{O}$ followed by washing with saturated aqueous $\mathrm{NaHCO}_{3}$, saturated brine, and dried over $\mathrm{Na}_{2} \mathrm{SO}_{4}$. After removal of the solvent, a viscous residue was obtained. Then addition of several $\mathrm{mL}$ of $\mathrm{CH}_{2} \mathrm{Cl}_{2}$ into the mixture and stirring resulted in solid formation, which was filtered and rinsed with hexane-EtOAc $(1: 1, \mathrm{v} / \mathrm{v})$ to afford $380 \mathrm{mg}$ product I-3 (25\%). The mother liquid was concentrated and once again addition of $\mathrm{CH}_{2} \mathrm{Cl}_{2}$ induced the solid formation, which after filtration delivered another $140 \mathrm{mg}$ product $\mathbf{I}-$ $3(9 \%)$.

Perbenzylated flavonol I-3: Yellow solid; $R_{f}=0.35$ (3:1 (v/v) Hexane/EtOAc); ${ }^{1} \mathrm{H}$ NMR $(\mathrm{CDCl}$, $600 \mathrm{MHz}) \delta 8.17(\mathrm{~d}, J=9.6 \mathrm{~Hz}, 2 \mathrm{H}), 7.61(\mathrm{~d}, J=7.2 \mathrm{~Hz}, 2 \mathrm{H}), 7.47-7.30(\mathrm{~m}, 13 \mathrm{H}), 7.10(\mathrm{~d}, J=$ $9.0 \mathrm{~Hz}, 2 \mathrm{H}), 6.64$ (d, $J=1.8 \mathrm{~Hz}, 1 \mathrm{H}), 6.49$ (d, $J=1.8 \mathrm{~Hz}, 1 \mathrm{H}), 5.24$ (s, 2H), 5.15 (s, 2H), 5.12 (s, $2 \mathrm{H}) ;{ }^{13} \mathrm{C}$ NMR $(\mathrm{CDCl} 3,67.5 \mathrm{MHz}) \delta 171.8,163.2,159.8,159.4,158,7,142.2,137.6,136.5$, 136.2, 135.6, 128.9, 128.8, 128.6, 128.5, 128.1, 127.8, 127.6, 127.5, 126.7, 123.8, 114.9, 106.8, 97.6, 93.7, 70.7, 70.6, 70.0.

(S)-1-(furan-2-yl)ethanol (I-9) ${ }^{60}$

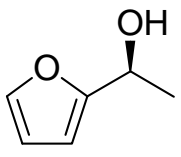


To a solution of $15 \mathrm{~g}$ acylfuran $\mathbf{I - 5}(136.4 \mathrm{mmol})$ in $20 \mathrm{~mL} \mathrm{CH}_{2} \mathrm{Cl}_{2}$ was added a prepared solution of formic acid/triethylamine $(40 \mathrm{~mL}, 2: 1(\mathrm{~mol} / \mathrm{mol}))$ and Noyori asymmetric transfer hydrogenation catalyst $(R)-\operatorname{Ru}\left(\eta^{6}\right.$-mesitylene)-( $\left.S, S\right)$-TsDPEN $(0.2 \mathrm{~g}, \quad 0.25 \mathrm{~mol} \%) .{ }^{61}$ The resulting solution was stirred at room temperature for $24 \mathrm{~h}$. Then it was diluted with water $(90$ $\mathrm{mL})$ and extracted with $\mathrm{Et}_{2} \mathrm{O}(200 \mathrm{~mL} \times 3)$. The pooled organic layer was washed with $50 \mathrm{~mL}$ saturated aqueous $\mathrm{NaHCO}_{3}, 50 \mathrm{~mL}$ saturated brine, dried over $\mathrm{Na}_{2} \mathrm{SO}_{4}$ and then concentrated under reduced pressure to give a residue. Flash chromatography on silica gel eluting with hexane- $\mathrm{Et}_{2} \mathrm{O}$ (1:1) gave furan alcohol I-9 (14 g, 92\%): Colorless oil; $R_{f}=0.45(7: 3(\mathrm{v} / \mathrm{v})$ Hexane/EtOAc); $[\alpha]_{\mathrm{D}}{ }^{25}=-21\left(\mathrm{c}=1.0, \mathrm{CH}_{2} \mathrm{Cl}_{2}\right) ;{ }^{1} \mathrm{H}$ NMR $\left(600 \mathrm{MHz} \mathrm{CDCl}_{3}\right) \delta 7.30(\mathrm{~d}, J=1.8$, $1 \mathrm{H}), 6.26(\mathrm{dd}, J=3.0,1.8 \mathrm{~Hz}, 1 \mathrm{H}), 6.15(\mathrm{~d}, J=3.0,1 \mathrm{H}), 4.78$ (dq, $J=6.6,6.6 \mathrm{~Hz}, 1 \mathrm{H}), 3.11$ (s, 1H), $1.46(\mathrm{~d}, J=6.6 \mathrm{~Hz}, 3 \mathrm{H}) ;{ }^{13} \mathrm{C} \mathrm{NMR}\left(150 \mathrm{MHz}, \mathrm{CDCl}_{3}\right) \delta 157.7,141.6,109.9,104.9,63.3$, 21.1.

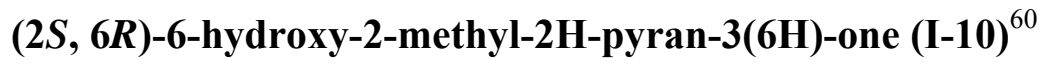<smiles>C[C@H]1O[C@H](O)C=CC1=O</smiles>

To a solution of $14 \mathrm{~g}$ furan alcohol $\mathbf{I - 9}(125 \mathrm{mmol})$ in $416 \mathrm{~mL}$ THF- $\mathrm{H}_{2} \mathrm{O}$ (3:1) was added $21 \mathrm{~g}$ $\mathrm{NaHCO}_{3}(250 \mathrm{mmol}), 17 \mathrm{~g} \mathrm{NaOAc} \cdot 3 \mathrm{H}_{2} \mathrm{O}(125 \mathrm{mmol})$, and $22.3 \mathrm{~g} \mathrm{NBS}(125 \mathrm{mmol})$ at $0{ }^{\circ} \mathrm{C}$. The reaction mixture was kept stirring at this temperature for 1 hour, then at $0^{\circ} \mathrm{C} 200 \mathrm{~mL}$ saturated aqueous $\mathrm{NaHCO}_{3}$ was added to quench the reaction. The reaction mixture was directly extracted with $\mathrm{Et}_{2} \mathrm{O}(300 \mathrm{~mL} \times 3)$ and the organic layer was pooled, washed by $100 \mathrm{~mL}$ saturated brine, dried over $\mathrm{Na}_{2} \mathrm{SO}_{4}$ and then concentrated reduced pressure to give a residue, which was rapidly subjected to flash chromatography on silica gel. Elution with hexane-EtOAc $(1: 1)$ afforded pyranone alcohol I-10 (14.4 g, 90\%, $\alpha: \beta=2.8: 1)$ : White solid; $R_{f}=0.25$ (7:3 (v/v) hexane/EtOAc); $[\alpha]_{\mathrm{D}}{ }^{25}=+44\left(\mathrm{c}=1.0, \mathrm{CH}_{2} \mathrm{Cl}_{2}\right) ;{ }^{1} \mathrm{H}$ NMR $\left(600 \mathrm{MHz}, \mathrm{CDCl}_{3}, \alpha\right.$ isomer $) \delta 6.82(\mathrm{dd}, J=10.2,3.0$ 
$\mathrm{Hz}, 1 \mathrm{H}), 5.96(\mathrm{~d}, J=10.2,1 \mathrm{H}), 5.48(\mathrm{~d}, J=3.0 \mathrm{~Hz}, 1 \mathrm{H}), 3.99(\mathrm{q}, J=7.2 \mathrm{~Hz}, 1 \mathrm{H}), 1.23(\mathrm{~d}, J=$ $7.2 \mathrm{~Hz}, 3 \mathrm{H}) ;{ }^{13} \mathrm{C} \mathrm{NMR}\left(150 \mathrm{MHz}, \mathrm{CDCl}_{3}, \alpha\right.$ isomer $) \delta$ 197.6, 145.3, 126.6, 87.2, 74.8, 15.1.

(2S,6S)-t-butyl -5,6-dihydro-6-methyl-5-oxo-2H-pyran-2-yl carbonate (I-4a $)^{60}$<smiles>C[C@@H]1OC(OC(C)(C)C)C=CC1=O</smiles>

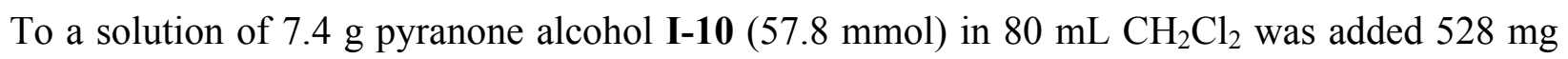
DMAP $(4.33 \mathrm{mmol})$ at $-78{ }^{\circ} \mathrm{C}$. A pre-cooled solution of $25.2 \mathrm{~g}(\text { Boc })_{2} \mathrm{O}(115.6 \mathrm{mmol})$ in $30 \mathrm{~mL}$ $\mathrm{CH}_{2} \mathrm{Cl}_{2}$ was dropwised into the reaction mixture via a cannula. The reaction mixture was stirred at $-78{ }^{\circ} \mathrm{C}$ for 12 hours. The reaction was quenched by $100 \mathrm{~mL}$ saturated aqueous $\mathrm{NaHCO}_{3}$ and then extracted with $\mathrm{Et}_{2} \mathrm{O}(300 \mathrm{~mL} \times 3)$. The organic layers were pooled, then washed by $70 \mathrm{~mL}$ saturated aqueous $\mathrm{NaCl}$, dried over $\mathrm{Na}_{2} \mathrm{SO}_{4}$ and concentrated under reduced pressure to give a residue. Flash chromatography on silica gel eluting with hexane- $\mathrm{Et}_{2} \mathrm{O}$ (100:7) gave Boc protected $\alpha$-pyranone $\mathbf{I}-4 \boldsymbol{\alpha}(7.8 \mathrm{~g}, 60 \%)$. Elution with hexane-Et ${ }_{2} \mathrm{O}(10: 1)$ gave $\beta$-isomer $\mathbf{I}-\mathbf{4} \boldsymbol{\beta}$ $(2.5 \mathrm{~g}, 19 \%)$.

$\alpha$-pyranone I-4a: $R_{f}=0.60\left(7: 3(\mathrm{v} / \mathrm{v})\right.$ hexane/EtOAc); $[\alpha]_{\mathrm{D}}{ }^{25}=+98\left(c \quad 1.0, \mathrm{CH}_{2} \mathrm{Cl}_{2}\right) ;{ }^{1} \mathrm{H}$ NMR $\left(600 \mathrm{MHz}, \mathrm{CDCl}_{3}\right) \delta 6.78(\mathrm{dd}, \mathrm{J}=10.2,3.6 \mathrm{~Hz}, 1 \mathrm{H}), 6.22(\mathrm{~d}, \mathrm{~J}=3.6 \mathrm{~Hz}, 1 \mathrm{H}), 6.09(\mathrm{~d}, \mathrm{~J}=10.2$ $\mathrm{Hz}, 1 \mathrm{H}), 4.53(\mathrm{q}, \mathrm{J}=6.6 \mathrm{~Hz}, 1 \mathrm{H}), 1.40(\mathrm{~s}, 9 \mathrm{H}), 1.28(\mathrm{~d}, \mathrm{~J}=6.6 \mathrm{~Hz}, 3 \mathrm{H}) ;{ }^{13} \mathrm{C} \mathrm{NMR}(150.8 \mathrm{MHz}$, CDCl3) $\delta 195.5,151.7,140.9,128.2,89.1,83.3,72.0,27.5,15.1$;

3-((2S,6S)-5,6-dihydro-6-methyl-5-oxo-2H-pyran-2-yloxy)-5,7-bis(benzyloxy)-2-(4(benzyloxy)phenyl)-4H-chromen-4-one (2) 


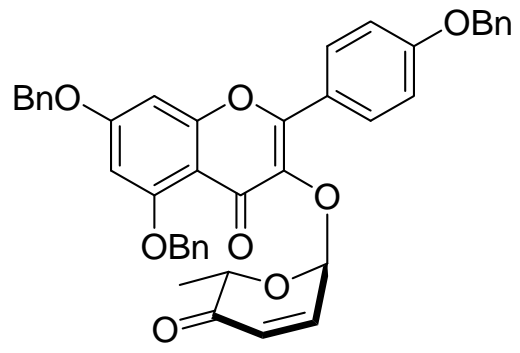

To a solution of $875 \mathrm{mg}$ perbenzylated flavonol $\mathbf{I}-3$ (1.6 mmol) and $537 \mathrm{mg}$ pyranone $\mathbf{I}-4 \boldsymbol{\alpha}(2.4$ mmol) in $8 \mathrm{~mL} \mathrm{CH}_{2} \mathrm{Cl}_{2}$ was added a solution of $41 \mathrm{mg} \mathrm{Pd}_{2}(\mathrm{DBA})_{3} \cdot \mathrm{CHCl}_{3}(0.04 \mathrm{mmol})$ and 41 mg $\mathrm{PPh}_{3}(0.16 \mathrm{mmol})$ in $1 \mathrm{~mL} \mathrm{CH}_{2} \mathrm{Cl}_{2}$ at $0{ }^{\circ} \mathrm{C}$. The reaction mixture was stirred at $0{ }^{\circ} \mathrm{C}$ for 3 hours and then quenched by $15 \mathrm{~mL}$ saturated aqueous $\mathrm{NaHCO}_{3}$, followed by extraction with $\mathrm{Et}_{2} \mathrm{O}(25 \mathrm{~mL} \times 3)$. The organic layer was pooled, then washed by $15 \mathrm{~mL}$ saturated aqueous $\mathrm{NaCl}$, dried over $\mathrm{Na}_{2} \mathrm{SO}_{4}$ and concentrated under reduced pressure to give crude product. Chromatography on silica gel, eluting with hexane-EtOAc (3:1) gave glycosylated pyranone I-2 (888 mg, 85\%): Yellow solid, mp: $75-77{ }^{\circ} \mathrm{C} ; R_{f}=0.50$ (2:1 (v/v) hexane/EtOAc); $[\alpha]_{\mathrm{D}}{ }^{25}-63(c$ 0.93, CHCl3); IR (thin film, $\mathrm{cm}^{-1}$ ) 3063, 2921, 1699, 1636, 1607, 1574, 1509, 1454, 1375, 1355, 1300, 1252, 1198, 1178, 1162, 1103, 1013, 937. ${ }^{1} \mathrm{H}$ NMR (CDCl3, $\left.600 \mathrm{MHz}\right) \delta 7.87$ (d, $J=8.4$ $\mathrm{Hz}, 2 \mathrm{H}), 7.57$ (d, $J=7.8 \mathrm{~Hz}, 2 \mathrm{H}), 7.46-7.29$ (m, 13H), 7.27 (dd, $J=10.2,3.6 \mathrm{~Hz}, 1 \mathrm{H}), 7.06$ (d, $J$ $=9.0 \mathrm{~Hz}, 2 \mathrm{H}), 6.58(\mathrm{~d}, J=1.8 \mathrm{~Hz}, 1 \mathrm{H}), 6.47(\mathrm{~d}, J=1.8 \mathrm{~Hz}, 1 \mathrm{H}), 6.13(\mathrm{~d}, J=10.2 \mathrm{~Hz}, 1 \mathrm{H}), 5.92$ $(\mathrm{d}, J=3.6 \mathrm{~Hz}, 1 \mathrm{H}), 5.27(\mathrm{~s}, 2 \mathrm{H}), 5.15(\mathrm{~s}, 2 \mathrm{H}), 5.08(\mathrm{~d}, J=12 \mathrm{~Hz}, 1 \mathrm{H}), 5.08(\mathrm{~d}, J=12 \mathrm{~Hz}, 1 \mathrm{H})$, $4.02(\mathrm{q}, J=6.0 \mathrm{~Hz}, 1 \mathrm{H}), 0.93(\mathrm{~d}, J=6.6 \mathrm{~Hz}, 3 \mathrm{H}) ;{ }^{13} \mathrm{C} \mathrm{NMR}(\mathrm{CDCl} 3,150 \mathrm{MHz}) \delta 196.7,173.3$, 163.0, 160.4, 159.8, 159.0, 154.9, 143.1, 137.6, 136.3, 136.2, 135.6, 130.4, 128.8, 128.7, 128.6, 128.4, 128.2, 127.7, 127.6, 127.4, 126.6, 123.2, 114.7, 109. 9, 98.3, 94.3, 94.0, 71.5, 70.8, 70.5, 70.1, 14.6; HRMS (CI): calcd. for $\left[\mathrm{C}_{42} \mathrm{H}_{34} \mathrm{O}_{8}+\mathrm{Na}\right]^{+}:$689.2146, Found: 689.2153 .

3-((2S,5R,6S)-5,6-dihydro-5-hydroxy-6-methyl-2H-pyran-2-yloxy)-5,7 bis(benzyloxy)-2-(4(benzyloxy)phenyl)-4H-chromen-4-one (I-11) 


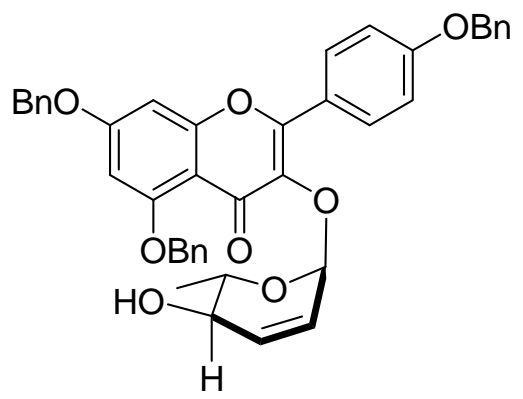

A solution of $87 \mathrm{mg}$ pyranone $\mathbf{I}-\mathbf{2}(0.13 \mathrm{mmol})$ in $3.5 \mathrm{~mL} \mathrm{CH} \mathrm{Cl}_{2}-\mathrm{MeOH}(5: 1)$ was cooled down to $-78{ }^{\circ} \mathrm{C}$, then $10 \mathrm{mg} \mathrm{NaBH} 4(0.26 \mathrm{mmol})$ was added in. The reaction mixture was kept stirring at $-78{ }^{\circ} \mathrm{C}$ for 3 hours. The reaction was then quenched by $2 \mathrm{~mL}$ saturated aqueous $\mathrm{NaHCO}_{3}$, followed by extraction with $\mathrm{Et}_{2} \mathrm{O}(10 \mathrm{~mL} \times 3)$. The organic layer was washed by $2 \mathrm{~mL}$ saturated aqueous $\mathrm{NaCl}$, dried over $\mathrm{Na}_{2} \mathrm{SO}_{4}$, and concentrated under reduced pressure to give crude product. Crude ${ }^{1} \mathrm{H}$ NMR showed a $\mathrm{dr}>20: 1$. Chromatography on silica gel eluting with hexaneEtOAc (2:1) gave allylic alcohol I-11 (63 mg, 73\%): Amorphous yellow solid; $R_{f}=0.29$ (1:1 (v/v) hexane/EtOAc); $[\alpha]_{\mathrm{D}}^{25}-83$ (c 0.85, CHCl3); IR (thin film, $\mathrm{cm}^{-1}$ ) 3361, 2927, 1604, 1509, 1453, 1354, 1298, 1251, 1176, 1098, 1013, 916; ${ }^{1} \mathrm{H}$ NMR (CDCl3, $\left.600 \mathrm{MHz}\right) \delta 7.95$ (d, $J=8.4$ $\mathrm{Hz}, 2 \mathrm{H}), 7.55$ (d, $J=7.8 \mathrm{~Hz}, 2 \mathrm{H}), 7.45-7.27$ (m, 13H), 7.06 (d, $J=9.0 \mathrm{~Hz}, 2 \mathrm{H}), 6.56$ (d, $J=2.4$ $\mathrm{Hz}, 1 \mathrm{H}), 6.44$ (d, $J=2.4 \mathrm{~Hz}, 1 \mathrm{H}), 6.20$ (ddd, $J=10.2,2.4,2.4 \mathrm{~Hz}, 1 \mathrm{H}), 5.97$ (d, $J=10.2 \mathrm{~Hz}, 1 \mathrm{H}$ ), $5.60(\mathrm{~s}, 1 \mathrm{H}), 5.26(\mathrm{~s}, 2 \mathrm{H}), 5.15(\mathrm{~s}, 2 \mathrm{H}), 5.07(\mathrm{~d}, J=12 \mathrm{~Hz}, 1 \mathrm{H}), 5.07$ (d, $J=12 \mathrm{~Hz}, 1 \mathrm{H}), 3.74(\mathrm{dd}$, $J=7.2,6.6 \mathrm{~Hz}, 1 \mathrm{H}), 3.31(\mathrm{dq}, J=8.4,6.0 \mathrm{~Hz}, 1 \mathrm{H}), 0.71(\mathrm{~d}, J=6.6 \mathrm{~Hz}, 3 \mathrm{H}) ;{ }^{13} \mathrm{C} \mathrm{NMR}(\mathrm{CDCl} 3$, $67.5 \mathrm{MHz}) \delta 173.8,162.8,160.3,159.9,159.0,154.8,138.3,136.5,136.4,135.7,133.9,130.7$, $128.8,128.7,128.6,128.5,128.2,127.8,127.7,127.5,126.7,126.6,123.8,114.6,110.2,98.3$, 95.7, 94.0, 70.8, 70.5, 70.1, 69.6, 69.4, 17.0; HRMS (CI): calcd. for $\left[\mathrm{C}_{42} \mathrm{H}_{36} \mathrm{O}_{8}+\mathrm{Na}\right]^{+}$: 691.2302, Found: 691.2296.

5,7-bis(benzyloxy)-2-(4-(benzyloxy)phenyl)-3-( $\alpha$-L-rhamnopyranosyloxy)-4H-chromen-4one (I-12) 


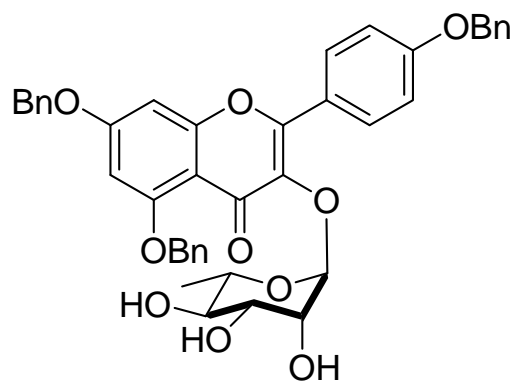

A solution of $80 \mathrm{mg}$ allylic alcohol $\mathbf{I}-\mathbf{1 1}(0.12 \mathrm{mmol})$ in $2 \mathrm{~mL}$ acetone-t-BuOH (1:1) was cooled down to $0{ }^{\circ} \mathrm{C}$. To the solution was added $0.5 \mathrm{~mL} \mathrm{NMO}-\mathrm{H}_{2} \mathrm{O}(\mathrm{w} / \mathrm{w} 1: 1)$, and then catalytic amount of crystalline $\mathrm{OsO}_{4}$ was added into the mixture when stirring. The reaction mixture was kept stirring at $0{ }^{\circ} \mathrm{C}$ for 3 hours. The reaction mixture was then quenched by $0.5 \mathrm{~mL}$ saturated aqueous $\mathrm{Na}_{2} \mathrm{~S}_{2} \mathrm{O}_{3}$. After stirring for another 5 hours, all the staff was passed through a small pad of celite and silica gel, washed by EtOAc-MeOH $(1: 1)(10 \mathrm{~mL} \times 3)$. The organic effluent was dried by $\mathrm{Na}_{2} \mathrm{SO}_{4}$, and concentrated under reduced pressure to give crude prodcut. Chromatography on silica gel eluting with $\mathrm{Et}_{2} \mathrm{O}-\mathrm{MeOH}$ (20:1) gave triol I-12 (80 mg, 96\%): White solid, mp: $105-107^{\circ} \mathrm{C} ; R_{f}=0.56\left(10: 1(\mathrm{v} / \mathrm{v}) \mathrm{Et}_{2} \mathrm{O} / \mathrm{MeOH}\right) ;[\alpha]_{\mathrm{D}}{ }^{25}-112(c 1.3, \mathrm{MeOH}) ; \mathrm{IR}$ (thin film, $\mathrm{cm}^{-1}$ ) 3418 (broad), 2924, 1606, 1506, 1453, 1354, 1299, 1255, 1177, 1141, 1102, 1058, 998, 953; ${ }^{1} \mathrm{H}$ NMR (acetone-d $\left.\mathrm{d}_{6}, 600 \mathrm{MHz}\right) \delta 7.91$ (d, $\left.J=9.0 \mathrm{~Hz}, 2 \mathrm{H}\right), 7.68$ (d, $J=7.8 \mathrm{~Hz}$, 2H), 7.50 (d, $J=7.8 \mathrm{~Hz}, 4 \mathrm{H}) 7.43-39$ (m, 6H), 7.38-7.29 (m, 3H), 7.19 (d, $J=8.4 \mathrm{~Hz}, 2 \mathrm{H}), 6.80$ $(\mathrm{d}, J=1.8 \mathrm{~Hz}, 1 \mathrm{H}), 6.67(\mathrm{~d}, J=2.4 \mathrm{~Hz}, 1 \mathrm{H}), 5.57$ (d, $J=1.8 \mathrm{~Hz}, 1 \mathrm{H}), 5.29(\mathrm{~d}, J=13.2 \mathrm{~Hz}, 1 \mathrm{H})$, $5.28(\mathrm{~d}, J=13.2 \mathrm{~Hz}, 1 \mathrm{H}), 5.24(\mathrm{~s}, 2 \mathrm{H}), 5.23(\mathrm{~s}, 2 \mathrm{H}), 4.28(\mathrm{dd}, J=3.6,1.8 \mathrm{~Hz}, 1 \mathrm{H}), 3.70(\mathrm{dd}, J=$ 9.0, 3.6 Hz, 1H), 3.33 (dd, $J=9.6,9.6 \mathrm{~Hz}, 1 \mathrm{H}$ ), 3.21 (dq, $J=9.6,6.0 \mathrm{~Hz}, 1 \mathrm{H}$ ), 2.91 (brs, $1 \mathrm{H}$ ), $0.87(\mathrm{~d}, J=6.0 \mathrm{~Hz}, 3 \mathrm{H}) ;{ }^{13} \mathrm{C}$ NMR (acetone- $\left.\mathrm{d}_{6}, 150 \mathrm{MHz}\right) \delta 173.5,164.1,161.5,160.8,159.8$, 154.8, 138.4, 138.1, 138.0, 137.4, 131.4, 129.5, 129.4, 129.3, 129.1, 128.9, 128.7, 128.5, 128.4, $127.9,124.4,115.7,110.6,102.2,98.9,95.1,73.2,72.2,71.5,71.4,71.3,71.2,70.8,17.9$; HRMS (ESI) calcd. for $\left[\mathrm{C}_{42} \mathrm{H}_{38} \mathrm{O}_{10}+\mathrm{H}\right]^{+}:$703.2543, Found: 703.2537.

Kaempferol-3-a-L-rhamnoside (I-1b) ${ }^{62}$ 


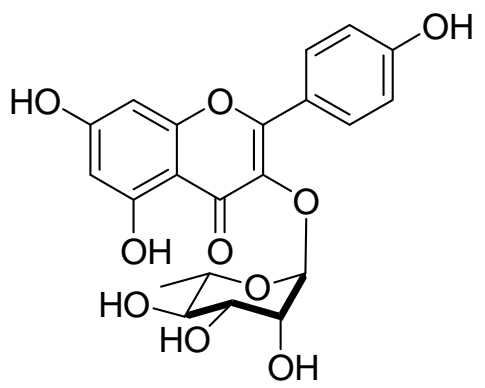

A solution of $50 \mathrm{mg}$ triol I-12 $(0.07 \mathrm{mmol})$ in $2 \mathrm{~mL}$ THF-EtOH (1:1) was added $20 \mathrm{mg}$ Pearlman's catalyst (Pd-C, 10\%). The solution was degassed using vacuum at $-90{ }^{\circ} \mathrm{C}$ and refilling with $\mathrm{H}_{2}$. This procedure was repeated three times, and then the bath was removed. The reaction was warmed up to room temperature and stirred under a $\mathrm{H}_{2}$ atmosphere for 3 hours. The reaction mixture was loaded onto silica gel and elution with $\mathrm{Et}_{2} \mathrm{O}-\mathrm{MeOH}$ (20:1) gave kaempferol-3- $\alpha$-L-rhamnoside (I-1b) (24mg, 80\%): Pale yellow solid, mp: $140-142{ }^{\circ} \mathrm{C} ; R_{f}=0.71$ (5:1 (v/v) Et $2 \mathrm{O} / \mathrm{MeOH}) ;[\alpha]_{\mathrm{D}}{ }^{25}-96$ (c 1.3, MeOH); IR (thin film, $\left.\mathrm{cm}^{-1}\right) 3259$ (broad), 2924, 2855, $1653,1607,1442,1360,1251,1207,1172,1057,996 ;{ }^{1} \mathrm{H}$ NMR $\left(\mathrm{CD}_{3} \mathrm{OD}, 600 \mathrm{MHz}\right) \delta 7.72(\mathrm{~d}, J$ $=8.4 \mathrm{~Hz}, 2 \mathrm{H}), 6.89(\mathrm{~d}, J=9.0 \mathrm{~Hz}, 2 \mathrm{H}), 6.30(\mathrm{~d}, J=2.4 \mathrm{~Hz}, 1 \mathrm{H}), 6.13(\mathrm{~d}, J=1.8 \mathrm{~Hz}, 1 \mathrm{H}), 5.33$ $(\mathrm{d}, J=1.8 \mathrm{~Hz}, 1 \mathrm{H}), 4.18(\mathrm{dd}, J=3.0,1.8 \mathrm{~Hz}, 1 \mathrm{H}), 3.67$ (dd, $J=9.0,2.4 \mathrm{~Hz}, 1 \mathrm{H}), 3.30-3.27$ (m, $4 \mathrm{H}), 0.88(\mathrm{~d}, J=6.0 \mathrm{~Hz}, 3 \mathrm{H}) ;{ }^{13} \mathrm{C} \mathrm{NMR}\left(\mathrm{CD}_{3} \mathrm{OD}, 150 \mathrm{MHz}\right) \delta 179.5,163.2,161.6,159.1,158.7$, 136.1, 131.9, 122.7, 116.6, 105.5, 103.5, 100.4, 95.2, 73.2, 72.2, 72.2, 71.9, 17.6; HRMS (ESI) calcd. for $\left[\mathrm{C}_{21} \mathrm{H}_{20} \mathrm{O}_{10}+\mathrm{Na}\right]^{+}: 455.0954$, Found: 455.0949 .

5,7-bis(benzyloxy)-2-(4-(benzyloxy)phenyl)-3-(2,3,4-O-triacetyl-a-L-rhamnopyranoyloxy)4H-chromen-4-one (I-13)

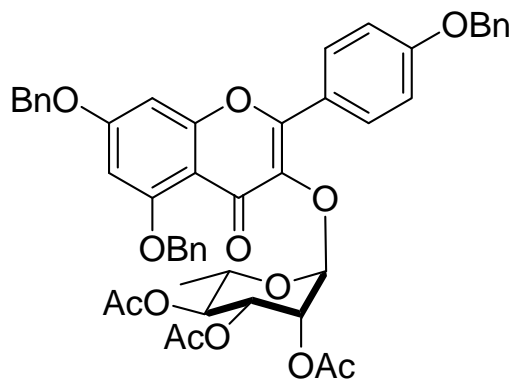




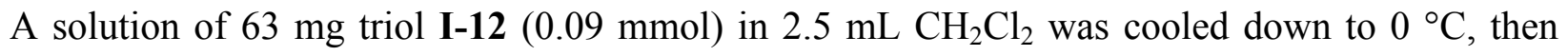
sequentially $0.4 \mathrm{~mL}$ pyridine, catalytic amount of DMAP and $0.4 \mathrm{~mL} \mathrm{Ac}_{2} \mathrm{O}$ were added. The reaction mixture was stirred at $0{ }^{\circ} \mathrm{C}$ overnight. The reaction mixture was diluted by $15 \mathrm{~mL} \mathrm{Et}_{2} \mathrm{O}$ and then the reaction was quenched by $1.5 \mathrm{~mL} \mathrm{H}_{2} \mathrm{O}$, the organic layer was washed by $2 \mathrm{~mL}$ saturated aqueous $\mathrm{NaHCO}_{3}, 2 \mathrm{~mL}$ saturated aqueous $\mathrm{NaCl}$, dried over $\mathrm{Na}_{2} \mathrm{SO}_{4}$, and concentrated under reduced pressure to give a residue which was subjected to chromatography on silica gel. Elution with hexane-EtOAc (2:1) afforded triacetate I-13 (63 mg, 86\%): White solid, mp: 90$92{ }^{\circ} \mathrm{C} ; R_{f}=0.58\left(1: 1(\mathrm{v} / \mathrm{v})\right.$ hexane/EtOAc); $[\alpha]_{\mathrm{D}}{ }^{25}-175\left(c 1.3, \mathrm{CHCl}_{3}\right) ; \mathrm{IR}\left(\right.$ thin film, $\left.\mathrm{cm}^{-1}\right) 3035$, 2982, 2938, 1746, 1632, 1605, 1574, 1509, 1498, 1486, 1453, 1432, 1368, 1298, 1247, 1217, 1174, 1136, 1101, 1044, 1019, 968; ${ }^{1} \mathrm{H}$ NMR $\left(\mathrm{CDCl}_{3}, 600 \mathrm{MHz}\right) \delta 7.86(\mathrm{~d}, J=9.0 \mathrm{~Hz}, 2 \mathrm{H}), 7.56$ (d, $J=7.2 \mathrm{~Hz}, 2 \mathrm{H}), 7.46-7.27$ (m, 13H), 7.14 (d, $J=9.0 \mathrm{~Hz}, 2 \mathrm{H}), 6.56$ (d, $J=2.4 \mathrm{~Hz}, 1 \mathrm{H}), 6.45$ $(\mathrm{d}, J=2.4 \mathrm{~Hz}, 1 \mathrm{H}), 5.73(\mathrm{~d}, J=1.8 \mathrm{~Hz}, 1 \mathrm{H}), 5.72(\mathrm{dd}, J=3.6,1.8 \mathrm{~Hz}, 1 \mathrm{H}), 5.32(\mathrm{dd}, J=10.2$, $3.0 \mathrm{~Hz}, 1 \mathrm{H}), 5.26(\mathrm{~s}, 2 \mathrm{H}), 5.15(\mathrm{~s}, 2 \mathrm{H}), 5.08(\mathrm{~s}, 2 \mathrm{H}), 4.92(\mathrm{dd}, J=10.2,9.6 \mathrm{~Hz}, 1 \mathrm{H}), 3.37$ (dq, $J=$ 10.2, 6.6 Hz, 1H), 2.11 (s, 3H), 1.99 (s, 3H), $1.96(\mathrm{~s}, 3 \mathrm{H}), 0.85$ (d, $J=6.0 \mathrm{~Hz}, 3 \mathrm{H}) ;{ }^{13} \mathrm{C} \mathrm{NMR}$ $\left(\mathrm{CDCl}_{3}, 150 \mathrm{MHz}\right) \delta 172.9,169.9,169.8,169.5,162.9,160.6,159.9,158.8,154.2,136.5,136.4$, 136.3, 135.6, 130.4, 128.8, 128.7, 128.6, 128.4, 128.2, 127.7, 127.5, 127.4, 126.6, 123.1, 114.9, $110.1,98.4,97.9,94.0,70.8,70.7,70.5,70.2,69.3,69.1,68.1,20.9,20.8,20.7,17.1$; HRMS (ESI) calcd. for $\left[\mathrm{C}_{48} \mathrm{H}_{44} \mathrm{O}_{13}+\mathrm{Na}\right]^{+}:$: 851.2679, Found: 851.2678.

\section{Kaempferol-3- $\alpha-L-3 ", 4$ ",5"'-O-triacetylrhamnoside (I-1c) ${ }^{63}$}

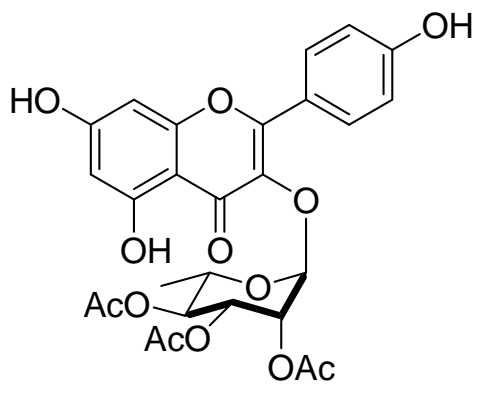

To a solution of $60 \mathrm{mg}$ triacetate $\mathbf{I - 1 3}(0.07 \mathrm{mmol})$ in $3 \mathrm{~mL}$ THF-EtOH $(1: 1)$ was added $30 \mathrm{mg}$ Pearlman's catalyst (Pd-C, 10\%). The reaction mixture was degassed using vacuum at $-90{ }^{\circ} \mathrm{C}$ 
and refilling with $\mathrm{H}_{2}$. This procedure was repeated three times, then the bath was removed and the reaction was warmed up to room temperature. The reaction mixture was stirred under a $\mathrm{H}_{2}$ atmosphere for 3 hours. The reaction mixture was loaded onto silica gel and elution with hexaneEtOAc (1:1) gave kaempferol-3- $\alpha$-L-3",4",5"-O-triacetylrhamnoside (I-1c) (35 mg, 86\%): Yellow solid, mp: $152-154{ }^{\circ} \mathrm{C} ; R_{f}=0.42\left(1: 2(\mathrm{v} / \mathrm{v})\right.$ hexane/EtOAc); $[\alpha]_{\mathrm{D}}{ }^{25}-128\left(c 1.4, \mathrm{CHCl}_{3}\right)$; IR (thin film, $\mathrm{cm}^{-1}$ ) 3372 (broad), 2982, 1749, 1654, 1609, 1504, 1364, 1207, 1174, 1085, 1043, 971; ${ }^{1} \mathrm{H}$ NMR $\left(\mathrm{CDCl}_{3}, 600 \mathrm{MHz}\right) \delta 12.5(\mathrm{~s}, 1 \mathrm{H}), 7.77(\mathrm{~d}, J=9.0 \mathrm{~Hz}, 2 \mathrm{H}), 6.98(\mathrm{~d}, J=8.4 \mathrm{~Hz}$, 2H), 6.35 (d, $J=1.8 \mathrm{~Hz}, 1 \mathrm{H}), 6.25$ (d, $J=2.4 \mathrm{~Hz}, 1 \mathrm{H}), 5.62$ (dd, $J=3.6,1.8 \mathrm{~Hz}, 1 \mathrm{H}), 5.55$ (d, $J$ $=1.8 \mathrm{~Hz}, 1 \mathrm{H}), 5.32(\mathrm{dd}, J=10.2,3.6 \mathrm{~Hz}, 1 \mathrm{H}), 4.97(\mathrm{dd}, J=10.2,9.6 \mathrm{~Hz}, 1 \mathrm{H}), 3.54(\mathrm{dq}, J=10.2$, $6.0 \mathrm{~Hz}, 1 \mathrm{H}), 2.13$ (s, 3H), 2.02 (s, 3H), 2.01 (s, 3H), 0.94 (d, $J=6.6 \mathrm{~Hz}, 3 \mathrm{H}) ;{ }^{13} \mathrm{C} \mathrm{NMR}\left(\mathrm{CDCl}_{3}\right.$, $150 \mathrm{MHz}) \delta 177.9,170.8,170.3,170.1,162.5,162.1,158.6,157.4,156.9,134.1,130.7,122.1$, 115.7, 105.7, 99.3, 98.2, 94.1, 70.4, 69.4, 69.2, 68.4, 20.9, 20.8, 20.7, 17.1; HRMS (ESI) calcd. for $\left[\mathrm{C}_{27} \mathrm{H}_{26} \mathrm{O}_{13}+\mathrm{Na}\right]^{+}: 581.1271$, Found: 581.1266 .

\section{6-(5,7-bis(benzyloxy)-2-(4-(benzyloxy)phenyl)-4-oxo-4H-chromen-3-yloxy)-3,6-dihydro-2- methyl-2H-pyran-3-yl acetate (I-14)}

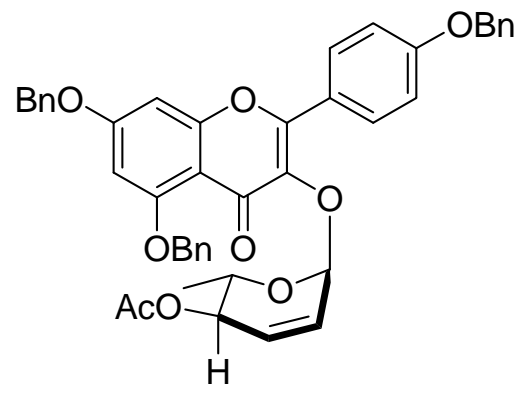

A solution of $210 \mathrm{mg}$ pyranone $\mathbf{I}-\mathbf{2}(0.32 \mathrm{mmol})$ in $3.5 \mathrm{~mL} \mathrm{CH} \mathrm{Cl}_{2}-\mathrm{MeOH}$ (5:1) was cooled down to $-78{ }^{\circ} \mathrm{C}$, then $25 \mathrm{mg} \mathrm{NaBH} 4(0.64 \mathrm{mmol})$ was added. The reaction mixture was stirred at $-78{ }^{\circ} \mathrm{C}$ for 3 hours. Then the reaction was quenched by $3 \mathrm{~mL}$ saturated aqueous $\mathrm{NaHCO}_{3}$, followed by extraction with $\mathrm{Et}_{2} \mathrm{O}(15 \mathrm{~mL} \times 3)$. The organic layer was washed by $3 \mathrm{~mL}$ saturated aqueous $\mathrm{NaCl}$, dried over $\mathrm{Na}_{2} \mathrm{SO}_{4}$, and concentrated under reduced pressure to give a residue. All residual solvent was removed by applying a high vacuum produced by oil pump for half an 
hour. The dry residue was redissolved in $\mathrm{CH}_{2} \mathrm{Cl}_{2}$ and cooled down to $0{ }^{\circ} \mathrm{C}$. To the solution was added $0.4 \mathrm{~mL}$ pyridine, catalytic amount of DMAP, and $0.3 \mathrm{~mL} \mathrm{Ac} 2 \mathrm{O}$. After stirring for 1 hour, the mixture was diluted by $20 \mathrm{~mL} \mathrm{Et}_{2} \mathrm{O}$. The organic layer was sequentially washed with $2 \mathrm{~mL}$ $\mathrm{H}_{2} \mathrm{O}, 2 \mathrm{~mL}$ saturated aqueous $\mathrm{NaHCO}_{3}, 2 \mathrm{~mL}$ saturated brine, dried over $\mathrm{Na}_{2} \mathrm{SO}_{4}$ and then concentrated under reduced pressure to afford crude product. Chromatography on silica gel eluting with hexane-EtOAc (5:1) gave pure acetate I-14 (156 mg, 70\%): Amorphous yellow solid; $R_{f}=0.52\left(3: 2(\mathrm{v} / \mathrm{v})\right.$ hexane/EtOAc); $[\alpha]_{\mathrm{D}}^{25}-146(c 1.1, \mathrm{CHCl})$ ); IR (thin film, $\left.\mathrm{cm}^{-1}\right)$ 3052, 2927, 1736, 1636, 1608, 1509, 1454, 1375, 1355, 1298, 1240, 1178, 1104, 1023, 936; ${ }^{1} \mathrm{H}$ NMR $(\mathrm{CDCl} 3,600 \mathrm{MHz}) \delta 7.96(\mathrm{~d}, J=9.0 \mathrm{~Hz}, 2 \mathrm{H}), 7.55(\mathrm{~d}, J=7.2 \mathrm{~Hz}, 2 \mathrm{H}), 7.47-7.27(\mathrm{~m}, 13 \mathrm{H}), 7.09$ (d, $J=8.4 \mathrm{~Hz}, 2 \mathrm{H}), 6.56(\mathrm{~d}, J=2.4 \mathrm{~Hz}, 1 \mathrm{H}), 6.44$ (d, $J=2.4 \mathrm{~Hz}, 1 \mathrm{H}), 6.20$ (ddd, $J=10.2,2.4$, $2.4 \mathrm{~Hz}, 1 \mathrm{H}), 5.90(\mathrm{~d}, J=10.2 \mathrm{~Hz}, 1 \mathrm{H}), 5.60(\mathrm{brs}, 1 \mathrm{H}), 5.27$ (s, 2H), 5.17 (s, 2H), 5.08 (d, $J=$ $11.4 \mathrm{~Hz}, 1 \mathrm{H}), 5.07$ (d, $J=11.4 \mathrm{~Hz}, 1 \mathrm{H}), 4.96$ (ddd, $J=9.6,1.8,1.8 \mathrm{~Hz}, 1 \mathrm{H}), 3.60$ (dq, $J=9.6$, $6.6 \mathrm{~Hz}, 1 \mathrm{H}), 2.08(\mathrm{~s}, 3 \mathrm{H}), 0.61(\mathrm{~d}, J=6.0 \mathrm{~Hz}, 3 \mathrm{H}) ;{ }^{13} \mathrm{C} \mathrm{NMR}\left(\mathrm{CDCl}_{3}, 150 \mathrm{MHz}\right) \delta 173.5,170.3$, $162.8,160.2,159.8,154.5,138.4,136.5,136.4,135.7,130.6,130.3,128.7,128.6,128.6,128.5$, $128.4,128.1,127.6,127.5,127.3,126.6,123.8,114.5,110.0,98.2,95.8,94.0,70.8,70.7,70.4$, 70.1, 66.5, 21.1, 17.0; HRMS (CI): calcd. for $\left[\mathrm{C}_{44} \mathrm{H}_{38} \mathrm{O}_{9}+\mathrm{Na}\right]^{+}:$733.2408, Found: 733.2405.

\section{5,7-bis(benzyloxy)-2-(4-(benzyloxy)phenyl)-3-(4-O-acetyl- $\alpha$-L-rhamnopyranoyloxy)-4H- chromen-4-one (I-15)}

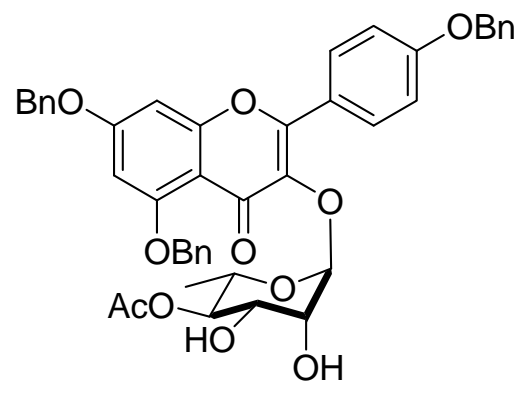

A solution of $142 \mathrm{mg}$ acetate $\mathbf{I - 1 4}(0.20 \mathrm{mmol})$ in $3 \mathrm{~mL}$ acetone- $t$-BuOH (1:1) was cooled down to $0{ }^{\circ} \mathrm{C}$. To the solution was added $1 \mathrm{~mL} \mathrm{NMO}-\mathrm{H}_{2} \mathrm{O}$ (w/w 1:1), and a catalytic amount $(\sim 5 \mathrm{mg})$ of crystalline $\mathrm{OsO}_{4}$ was added into the mixture. The reaction mixture was stirred at $0{ }^{\circ} \mathrm{C}$ for 24 
hours. The reaction was quenched with $1 \mathrm{~mL}$ saturated aqueous $\mathrm{Na}_{2} \mathrm{~S}_{2} \mathrm{O}_{3}$ (aq.) and stirred overnight. Then the mixture was passed through a small pad of celite and silica gel, washed with EtOAc-MeOH (1:1) $(15 \mathrm{~mL} \times 3)$, the organic solution was dried over $\mathrm{Na}_{2} \mathrm{SO}_{4}$, and concentrated under reduced pressure to give a residue. Chromatography on silica gel eluting with hexaneEtOAc (2:3) gave diol I-15 (115 mg, 77\%): Pale yellow solid, mp: 90.5-92.5 ${ }^{\circ} \mathrm{C} ; R_{f}=0.67(10: 1$

(v/v) $\left.\mathrm{Et}_{2} \mathrm{O} / \mathrm{MeOH}\right) ;[\alpha]_{\mathrm{D}}{ }^{25}-116\left(c 0.8, \mathrm{CHCl}_{3}\right.$ ); IR (thin film, $\mathrm{cm}^{-1}$ ) 3424 (broad), 3066, 3035 , 2928, 1741, 1605, 1574, 1509, 1498, 1486, 1454, 1375, 1354, 1299, 1251, 1199, 1177, 1144, 1102, 1049, 1004, 955; ${ }^{1} \mathrm{H}$ NMR (acetone- $\left.\mathrm{d}_{6}, 600 \mathrm{MHz}\right) \delta 7.82(\mathrm{~d}, J=9.0 \mathrm{~Hz}, 2 \mathrm{H}), 7.53(\mathrm{~d}, J=$ $7.8 \mathrm{~Hz}, 2 \mathrm{H}), 7.46-7.27(\mathrm{~m}, 13 \mathrm{H}), 7.09(\mathrm{~d}, J=9.0 \mathrm{~Hz}, 2 \mathrm{H}), 6.55(\mathrm{~d}, J=2.4 \mathrm{~Hz}, 1 \mathrm{H}), 6.45(\mathrm{~d}, J=$ $2.4 \mathrm{~Hz}, 1 \mathrm{H}), 5.51(\mathrm{~d}, J=1.8 \mathrm{~Hz}, 1 \mathrm{H}), 5.27(\mathrm{~s}, 2 \mathrm{H}), 5.15(\mathrm{~s}, 2 \mathrm{H}), 5.07(\mathrm{~s}, 2 \mathrm{H}), 4.78(\mathrm{dd}, J=9.6$, $9.0 \mathrm{~Hz}, 1 \mathrm{H}), 4.51(\mathrm{ddd}, J=3.6,3.6,2.4 \mathrm{~Hz}, 1 \mathrm{H}), 3.93(\mathrm{ddd}, J=8.4,7.8,3.0 \mathrm{~Hz}, 1 \mathrm{H}), 3.45(\mathrm{~d}, J=$ $4.2 \mathrm{~Hz}, 1 \mathrm{H}), 3.40$ (dq, $J=9.6,6.6 \mathrm{~Hz}, 1 \mathrm{H}), 2.91$ (d, $J=7.8 \mathrm{~Hz}, 1 \mathrm{H}), 2.05$ (s, 3H), 0.81 (d, $J=$ $6.6 \mathrm{~Hz}, 3 \mathrm{H}$ ); ${ }^{13} \mathrm{C}$ NMR (acetone-d $\left.6,150 \mathrm{MHz}\right) \delta 173.5,171.2,163.0,160.5,159.8,158.9,154.6$, $137.8,136.4,136.3,135.6,130.5,128.8,128.7,128.6,128.4,128.2,127.8,127.6,127.3,126.8$, 123.3, 114.7, 109.9, 101.4, 98.4, 94.0, 74.7, 70.8, 70.7, 70.5, 70.2, 69.9, 67.8, 21.0, 17.1; HRMS (ESI) calcd. for $\left[\mathrm{C}_{44} \mathrm{H}_{40} \mathrm{O}_{11}+\mathrm{H}\right]^{+}:$745.2649, Found: 745.2642.

\section{Kaempferol-3- $\alpha-L-4$ '’-O-acetylrhamnoside (1d) ${ }^{62 \mathrm{c}}$}

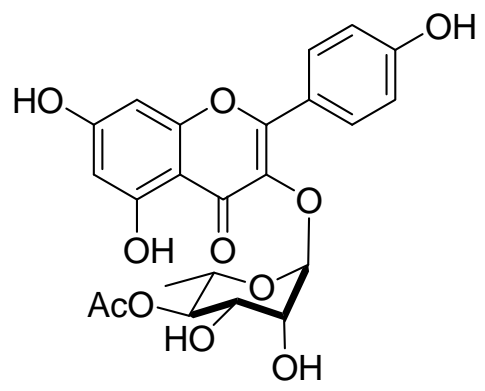

To a solution of $54.8 \mathrm{mg}$ diol $\mathbf{I}-15(0.07 \mathrm{mmol})$ in $3 \mathrm{~mL}$ THF-EtOH (1:1) was added $30 \mathrm{mg}$ Pearlman's catalyst (Pd-C, 10\%). The reaction mixture was degassed using vacuum at $-90{ }^{\circ} \mathrm{C}$ and refilling with $\mathrm{H}_{2}$. This procedure was repeated three times, then the bath was removed and the reaction was warmed up to room temperature. The reaction mixture was stirred under a $\mathrm{H}_{2}$ 
atmosphere for 3 hours. The reaction mixture was loaded onto silica gel and elution with hexaneEtOAc (1:2) to give kaempferol-3- $\alpha$-L-4"-O-acetylrhamnoside (I-1d) (30 mg, 87\%): Yellow solid, mp: $150-152^{\circ} \mathrm{C} ; R_{f}=0.18\left(1: 3(\mathrm{v} / \mathrm{v})\right.$ hexane/EtOAc); $[\alpha]_{\mathrm{D}}{ }^{25}-145(c 1.3, \mathrm{MeOH})$; IR (thin film, $\mathrm{cm}^{-1}$ ) 3317 (broad), 2927, 1721, 1651, 1607, 1500, 1446, 1359, 1253, 1207, 1172, 1141, 1085, 1047, 1004, 970, 952; ${ }^{1} \mathrm{H}$ NMR (acetone-d 6 , $\left.600 \mathrm{MHz}\right) \delta 7.84$ (d, $\left.J=9.0 \mathrm{~Hz}, 2 \mathrm{H}\right), 7.05$ (d, $J=9.0 \mathrm{~Hz}, 2 \mathrm{H}), 6.47(\mathrm{~d}, J=1.8 \mathrm{~Hz}, 1 \mathrm{H}), 6.28(\mathrm{~d}, J=1.8 \mathrm{~Hz}, 1 \mathrm{H}), 5.53(\mathrm{~d}, J=1.2 \mathrm{~Hz}, 1 \mathrm{H}), 4.82$ (dd, $J=9.6,9.6 \mathrm{~Hz}, 1 \mathrm{H}), 4.23(\mathrm{dd}, J=3.6,1.8 \mathrm{~Hz}, 1 \mathrm{H}), 3.85(\mathrm{dd}, J=9.6,3.6 \mathrm{~Hz}, 1 \mathrm{H}), 3.40(\mathrm{dq}$, $J=9.6,6.0 \mathrm{~Hz}, 1 \mathrm{H}), 2.87$ (brs, $1 \mathrm{H}), 1.97$ (s, 3H), 0.78 (d, $J=6.6 \mathrm{~Hz}, 3 \mathrm{H}) ;{ }^{13} \mathrm{C}$ NMR (acetone- $\mathrm{d}_{6}$, $150 \mathrm{MHz}) \delta 179.3,170.9,165.1,163.1,161.1,158.7,158.2,135.7,131.8,122.5,116.4,105.9$, 102.4, 99.6, 94.7, 74.6, 71.5, 69.8, 69.1, 21.0, 17.6; HRMS (ESI) calcd. for $\left[\mathrm{C}_{23} \mathrm{H}_{22} \mathrm{O}_{11}+\mathrm{Na}\right]^{+}$: 497.1060, Found: 497.1054.

\section{5,7-bis(benzyloxy)-2-(4-(benzyloxy)phenyl)-3-(2,4-O-triacetyl-a-L-rhamnopyranoyloxy)- 4H-chromen-4-one (I-16)}

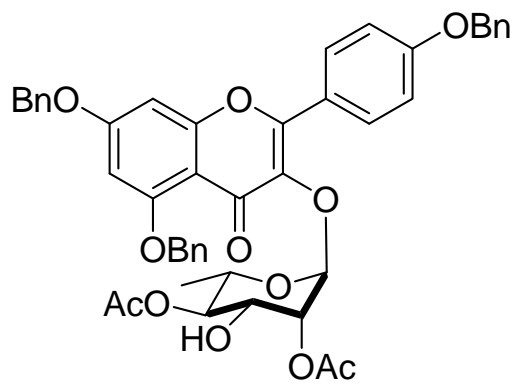

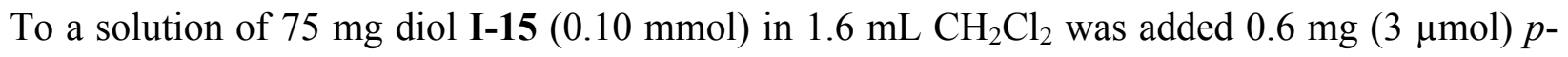
$\mathrm{TsOH} \cdot \mathrm{H}_{2} \mathrm{O}, 38 \mu \mathrm{L}$ trimethylorthoacetate $(0.30 \mathrm{mmol})$ at $0{ }^{\circ} \mathrm{C}$. After stirring at $0{ }^{\circ} \mathrm{C}$ for $15 \mathrm{~min}$, $0.5 \mathrm{~mL} \mathrm{90 \%} \mathrm{HOAc} \mathrm{(aq.)} \mathrm{was} \mathrm{added} \mathrm{and} \mathrm{the} \mathrm{reaction} \mathrm{mixture} \mathrm{was} \mathrm{stirred} \mathrm{for} \mathrm{another} 15 \mathrm{~min}$. The reaction mixture was diluted with $25 \mathrm{~mL}$ EtOAc and washed at $0{ }^{\circ} \mathrm{C}$ by $3 \mathrm{~mL}$ saturated aqueous $\mathrm{NaHCO}_{3}$ two times, then it was washed by $3 \mathrm{~mL}$ saturated brine, and dried over $\mathrm{Na}_{2} \mathrm{SO}_{4}$. The organic layer was concentrated under reduced pressure to give crude product, which was then subjected to chromatography on silica gel. Elution with hexane-EtOAc (1:1) gave diacetate 12 (78 mg, 99\%): White solid, mp: 99.5-101.5 ${ }^{\circ} \mathrm{C} ; R_{f}=0.58$ (1:2 (v/v) hexane/EtOAc); $[\alpha]_{\mathrm{D}}{ }^{25}-115$ (c 1.1, $\mathrm{CHCl}_{3}$ ); IR (thin film, $\mathrm{cm}^{-1}$ ) 3430 (broad), 3032, 2927, 1741, 1628, 1604, 1575, 1509, 1499, 1486, 1453, 1433, 1373, 1299, 1225, 1196, 1174, 1136, 1099, 1047, 1017, 963; ${ }^{1} \mathrm{H}$ NMR 
$\left(\mathrm{CDCl}_{3}, 600 \mathrm{MHz}\right) \delta 7.81(\mathrm{~d}, J=9.0 \mathrm{~Hz}, 2 \mathrm{H}), 7.56(\mathrm{~d}, J=7.2 \mathrm{~Hz}, 2 \mathrm{H}), 7.46-7.27(\mathrm{~m}, 13 \mathrm{H}), 7.11$ $(\mathrm{d}, J=9.0 \mathrm{~Hz}, 2 \mathrm{H}), 6.55(\mathrm{~d}, J=2.4 \mathrm{~Hz}, 1 \mathrm{H}), 6.45(\mathrm{~d}, J=2.4 \mathrm{~Hz}, 1 \mathrm{H}), 5.58(\mathrm{~d}, J=1.2 \mathrm{~Hz}, 1 \mathrm{H})$, 5.57 (dd, $J=3.6,1.8 \mathrm{~Hz}, 1 \mathrm{H}), 5.26$ (s, 2H), 5.16 (s, 2H), 5.07 (s, 2H), 4.76 (dd, $J=10.2,9.6 \mathrm{~Hz}$, $1 \mathrm{H}), 4.11(\mathrm{ddd}, J=9.6,6.6,3.0 \mathrm{~Hz}, 1 \mathrm{H}), 3.50(\mathrm{dq}, J=10.2,6.6 \mathrm{~Hz}, 1 \mathrm{H}), 2.28(\mathrm{~d}, J=6.6 \mathrm{~Hz}$, $1 \mathrm{H}), 2.12(\mathrm{~s}, 3 \mathrm{H}), 2.06(\mathrm{~s}, 3 \mathrm{H}), 0.89(\mathrm{~d}, J=6.0 \mathrm{~Hz}, 3 \mathrm{H}) ;{ }^{13} \mathrm{C} \mathrm{NMR}\left(\mathrm{CDCl}_{3}, 150 \mathrm{MHz}\right) \delta 173.0$, 171.2, 170.5, 162.9, 160.6, 159.9, 158.8, 154.3, 136.9, 136.3, 135.6, 130.3, 128.8, 128.7, 128.6, $128.4,128.2$, 127.7, 127.6, 127.3, 126.7, 123.1, 114.9, 110.0, 98.4, 98.3, 94.0, 74.1, 72.1, 70.8, 70.5, 70.2, 68.8, 67.9, 21.0, 20.9, 17.1; HRMS (ESI) calcd. for $\left[\mathrm{C}_{46} \mathrm{H}_{42} \mathrm{O}_{12}+\mathrm{Na}\right]^{+}: 809.2574$, Found: 809.2567.

\section{Kaempferol-3- $\boldsymbol{\alpha}-\mathrm{L}-2$ ", 4 "'-O-diacetylrhamnoside (I-1e $)^{64}$}

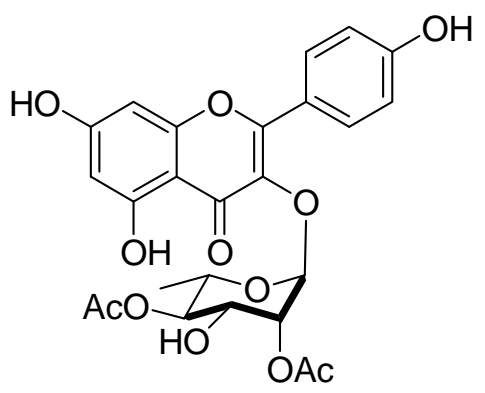

To a solution of $66.8 \mathrm{mg}$ diacetate $\mathbf{I - 1 6}(0.08 \mathrm{mmol})$ in $3 \mathrm{~mL}$ THF-EtOH $(1: 1)$ was added $30 \mathrm{mg}$ Pearlman's catalyst (Pd-C, 10\%). The reaction mixture was degassed using vacuum at $-90{ }^{\circ} \mathrm{C}$ and refilling with $\mathrm{H}_{2}$. This procedure was repeated three times, then the bath was removed and the reaction was warmed up to room temperature. The reaction mixture was stirred under a $\mathrm{H}_{2}$ atmosphere for 3 hours. The reaction mixture was loaded onto silica gel and elution with hexaneEtOAc (2:3) to give kaempferol-3- $\alpha$-L-2",4"-O-diacetylrhamnoside (I-1e) (38 mg, 88\%): Yellow solid, mp: $127-129^{\circ} \mathrm{C} ; R_{f}=0.24\left(1: 2(\mathrm{v} / \mathrm{v})\right.$ hexane/EtOAc); $[\alpha]_{\mathrm{D}}{ }^{25}-93(c$ 0.96, MeOH); IR (thin film, $\mathrm{cm}^{-1}$ ) 3311 (broad), 2925, 1721, 1653, 1608, 1502, 1445, 1362, 1205, 1171, 1144, 1129, 1085, 1047, 1007, 970; ${ }^{1} \mathrm{H}$ NMR (acetone-d $\left.6,600 \mathrm{MHz}\right) \delta 7.85(\mathrm{~d}, J=9.0 \mathrm{~Hz}, 2 \mathrm{H}), 7.06(\mathrm{~d}, J=$ $8.4 \mathrm{~Hz}, 2 \mathrm{H}), 6.48(\mathrm{~d}, J=1.8 \mathrm{~Hz}, 1 \mathrm{H}), 6.28(\mathrm{~d}, J=1.8 \mathrm{~Hz}, 1 \mathrm{H}), 5.60$ (s, 1H), 5.47 (dd, $J=3.6,1.8$ $\mathrm{Hz}, 1 \mathrm{H}), 4.76$ (dd, $J=10.2,9.6 \mathrm{~Hz}, 1 \mathrm{H}), 4.04$ (dd, $J=9.6,3.6 \mathrm{~Hz}, 1 \mathrm{H}), 3.38$ (dq, $J=10.2,6.0$ 
$\mathrm{Hz}, 1 \mathrm{H}), 2.06(\mathrm{~s}, 3 \mathrm{H}), 1.99(\mathrm{~s}, 3 \mathrm{H}), 0.81(\mathrm{~d}, J=6.0 \mathrm{~Hz}, 3 \mathrm{H}) ;{ }^{13} \mathrm{C}$ NMR (acetone- $\left.\mathrm{d}_{6}, 150 \mathrm{MHz}\right) \delta$ 179.0, 170.8, 170.3, 165.2, 163.0, 161.2, 158.8, 158.1, 134.8, 131.8, 122.3, 116.5, 105.8, 99.7, 99.3, 94.7, 74.4, 72.4, 69.2, 68.0, 21.0, 20.9, 17.7; HRMS (ESI) calcd. for $\left[\mathrm{C}_{25} \mathrm{H}_{24} \mathrm{O}_{12}+\mathrm{Na}\right]^{+}$: 539.1166, Found: 539.1159.

\section{5,7-bis(benzyloxy)-2-(4-(benzyloxy)phenyl)-3-(3,4-O-triacetyl-a-L-rhamnopyranoyloxy)- 4H-chromen-4-one (I-17)}

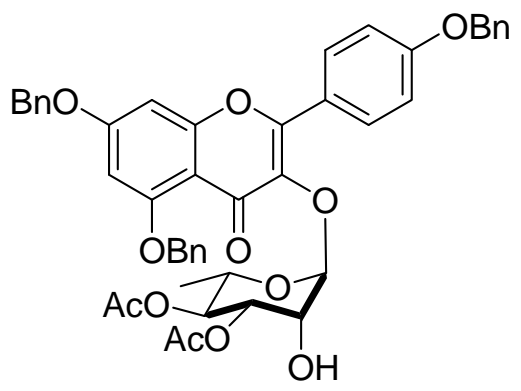

To a solution of $50 \mathrm{mg}$ diacetate $\mathbf{I - 1 6}(0.064 \mathrm{mmol})$ in $4 \mathrm{~mL}$ toluene was added a solution of 10 $\mu \mathrm{DBU}$ in $1 \mathrm{~mL}$ toluene at $0{ }^{\circ} \mathrm{C}$. The reaction mixture was stirred at $0{ }^{\circ} \mathrm{C}$ for 1 hour, then diluted with $20 \mathrm{~mL} \mathrm{Et}{ }_{2} \mathrm{O}-\mathrm{EtOAc}(1: 1)$. The mixture was sequentially washed with $2 \mathrm{~mL}$ saturated aqueous $\mathrm{NH}_{4} \mathrm{Cl}, 2 \mathrm{~mL}$ saturated aqueous $\mathrm{NaHCO}_{3}$, dried over $\mathrm{Na}_{2} \mathrm{SO}_{4}$. The organic layer was concentrated under reduced pressure to give a residue. Crude ${ }^{1} \mathrm{H}-\mathrm{NMR}$ showed this residue contains $64 \%$ diacetate I-17 vs. 36\% diacetate I-16. Chromatography on silica gel eluting with hexane-EtOAc (1.6:1) gave desired diacetate I-17 (31 mg, 62\%) and further elution with hexaneEtOAc (1.4:1) gave $17 \mathrm{mg}$ (34\%) starting material I-16 back. Diacetate I-17 : White solid, mp: 93-95 ${ }^{\circ} \mathrm{C} ; R_{f}=0.59\left(2: 3(\mathrm{v} / \mathrm{v})\right.$ hexane/EtOAc); $[\alpha]_{\mathrm{D}}{ }^{25}-151\left(c 1.3, \mathrm{CHCl}_{3}\right) ; \mathrm{IR}$ (thin film, $\left.\mathrm{cm}^{-1}\right)$ 3394 (broad), 3064, 2924, 1741, 1604, 1509, 1453, 1432, 1369, 1353, 1296, 1249, 1222, 1172, 
1100, 1043, 1004, 958; ${ }^{1} \mathrm{H}$ NMR $\left(\mathrm{CDCl}_{3}, 600 \mathrm{MHz}\right) \delta 7.86(\mathrm{~d}, J=8.4 \mathrm{~Hz}, 2 \mathrm{H}), 7.55(\mathrm{~d}, J=7.8$ $\mathrm{Hz}, 2 \mathrm{H}), 7.46-7.27$ (m, 13H), 7.12 (d, $J=9.0 \mathrm{~Hz}, 2 \mathrm{H}), 6.56$ (d, $J=2.4 \mathrm{~Hz}, 1 \mathrm{H}), 6.46$ (d, $J=2.4$ $\mathrm{Hz}, 1 \mathrm{H}), 5.53(\mathrm{~d}, J=2.4 \mathrm{~Hz}, 1 \mathrm{H}), 5.31(\mathrm{dd}, J=9.0,3.0 \mathrm{~Hz}, 1 \mathrm{H}), 5.26(\mathrm{~s}, 2 \mathrm{H}), 5.15(\mathrm{~s}, 2 \mathrm{H}), 5.08$ (s, 2H), $4.98(\mathrm{dd}, J=9.6,9.0 \mathrm{~Hz}, 1 \mathrm{H}), 4.57(\mathrm{ddd}, J=6.6,3.6 \mathrm{~Hz}, 1 \mathrm{H}), 3.46(\mathrm{dq}, J=9.6,6.6 \mathrm{~Hz}$, 1H), 2.87 (brs, $1 \mathrm{H}$ ), 2.07 (s, 3H), 1.99 (s, 3H), 0.82 (d, $J=6.0 \mathrm{~Hz}, 3 \mathrm{H}) ;{ }^{13} \mathrm{C} \mathrm{NMR}\left(\mathrm{CDCl}_{3}, 150\right.$ MHz) $\delta 173.3,169.9,169.8,163.0,160.5,159.9,158.9,154.5,137.6,136.4,136.3,135.6,130.5$, $128.8,128.7,128.6,128.4,128.2,127.7,127.6,127.3,126.7,123.2,114.8,109.9,101.9,98.3$, 94.0, 74.7, 71.2, 70.8, 70.5, 70.1, 69.5, 67.9, 20.9, 20.8, 17.2; HRMS (ESI) calcd. for $\left[\mathrm{C}_{46} \mathrm{H}_{42} \mathrm{O}_{12}+\mathrm{Na}\right]^{+}: 809.2574$, Found: 809.2569.

\section{SL0101 (I-1a) $)^{3 \mathrm{~d}, 5}$}

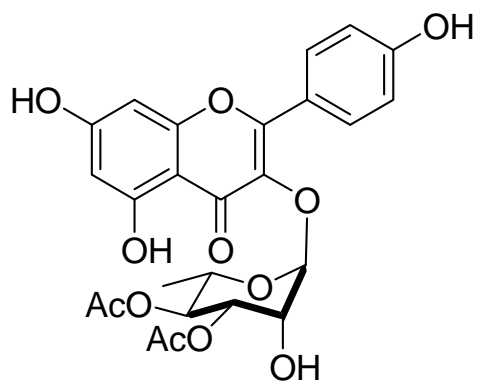

To a solution of $46 \mathrm{mg}$ diacetate $\mathbf{I}-17(0.058 \mathrm{mmol})$ in $2.5 \mathrm{~mL}$ THF-EtOH (1:1) was added 25 mg Pearlman's catalyst (Pd-C, 10\%). The reaction mixture was degassed using vacuum at $-90{ }^{\circ} \mathrm{C}$ and refilling with $\mathrm{H}_{2}$. This procedure was repeated three times, then the bath was removed and the reaction was warmed up to room temperature. The reaction mixture was stirred under a $\mathrm{H}_{2}$ atmosphere for 3 hours. The reaction mixture was loaded onto silica gel and elution with hexaneEtOAc (2:3) to give SL0101 (I-1a) (28 mg, 91\%): Yellow solid, mp: 147-149 ${ }^{\circ} \mathrm{C} ; R_{f}=0.26(1: 2$ (v/v) hexane/EtOAc); $[\alpha]_{\mathrm{D}}^{25}-163$ (c 1.6, MeOH); IR (thin film, $\left.\mathrm{cm}^{-1}\right) 3211$ (broad), 2980, 2930, 1722, 1650, 1607, 1502, 1444, 1361, 1206, 1171, 1045, 1005, 970; ${ }^{1} \mathrm{H}$ NMR (acetone- $\mathrm{d}_{6}, 600$ MHz) $\delta 7.86(\mathrm{~d}, J=9.0 \mathrm{~Hz}, 2 \mathrm{H}), 7.05(\mathrm{~d}, J=9.0 \mathrm{~Hz}, 2 \mathrm{H}), 6.48(\mathrm{~d}, J=1.8 \mathrm{~Hz}, 1 \mathrm{H}), 6.28(\mathrm{~d}, J=$ $1.8 \mathrm{~Hz}, 1 \mathrm{H}), 5.56(\mathrm{~d}, J=1.8 \mathrm{~Hz}, 1 \mathrm{H}), 5.17(\mathrm{dd}, J=10.2,3.0 \mathrm{~Hz}, 1 \mathrm{H}), 5.07$ (dd, $J=10.2,9.6 \mathrm{~Hz}$, 1H), 4.42 (dd, $J=3.0,1.8 \mathrm{~Hz}, 1 \mathrm{H}), 3.49$ (dq, $J=9.6,6.0 \mathrm{~Hz}, 1 \mathrm{H}), 2.02$ (s, 3H), 1.96 (s, 3H), 0.82 
$(\mathrm{d}, J=6.0 \mathrm{~Hz}, 3 \mathrm{H}) ;{ }^{13} \mathrm{C}$ NMR (acetone- $\left.\mathrm{d}_{6}, 150 \mathrm{MHz}\right) \delta 179.2,170.7,170.4,165.1,163.0,161.1$, 158.7, 158.2, 135.5, 131.8, 122.4, 116.5, 105.8, 102.2, 99.7, 99.6, 94.7, 72.3, 71.2, 69.3, 69.2, 21.0, 20.8, 17.6; HRMS (ESI) calcd. for $\left[\mathrm{C}_{25} \mathrm{H}_{24} \mathrm{O}_{12}+\mathrm{Na}\right]^{+}: 539.1166$, Found: 539.1161.

3-((2R,6R)-5,6-dihydro-6-methyl-5-oxo-2H-pyran-2-yloxy)-5,7-bis(benzyloxy)-2-(4(benzyloxy)phenyl)-4H-chromen-4-one ((ent)-I-2)

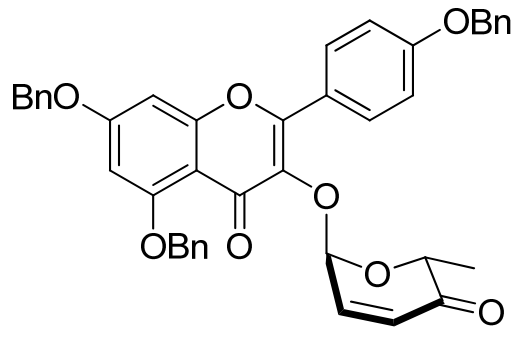

To a solution of $44 \mathrm{mg}(0.079 \mathrm{mmol})$ perbenzylated flavonol I-3 and $45 \mathrm{mg}(0.197 \mathrm{mmol}) \alpha-\mathrm{D}-$ pyranone (ent)-I-4 $\boldsymbol{\alpha}$ in $1 \mathrm{~mL} \mathrm{CH}_{2} \mathrm{Cl}_{2}$ was added a solution of $3 \mathrm{mg}(0.0029 \mathrm{mmol})$ $\mathrm{Pd}_{2}(\mathrm{DBA})_{3} \cdot \mathrm{CHCl}_{3}$ and $3 \mathrm{mg} \mathrm{PPh}(0.011 \mathrm{mmol})$ in $0.3 \mathrm{~mL} \mathrm{CH}_{2} \mathrm{Cl}_{2}$ at $0{ }^{\circ} \mathrm{C}$. The reaction mixture was stirred at $0{ }^{\circ} \mathrm{C}$ for 3 hours and loaded directly onto silica gel column, eluting with hexaneEtOAc (2.5:1) gave glycosylated pyranone (ent)-I-2 (37.4 mg, 71\%): Light yellow solid, mp: 75$77^{\circ} \mathrm{C} ; R_{f}=0.34\left(2: 1(\mathrm{v} / \mathrm{v})\right.$ hexane/EtOAc); $[\alpha]_{\mathrm{D}}{ }^{20}+72(c 2.22, \mathrm{CHCl}) ;$ IR (thin film, $\left.\mathrm{cm}^{-1}\right) v$ 3064, 3032, 2985, 2918, 2871, 1698, 1634, 1604, 1573, 1509, 1452, 1374, 1353, 1299, 1250, 1196, 1175, 1161, 1100, 1002, 934. ${ }^{1} \mathrm{H} \mathrm{NMR}\left(\mathrm{CDCl}_{3}, 600 \mathrm{MHz}\right) \delta 7.86(\mathrm{~d}, J=9.0 \mathrm{~Hz}, 2 \mathrm{H}), 7.57$ (d, $J=7.8 \mathrm{~Hz}, 2 \mathrm{H}), 7.47-7.29(\mathrm{~m}, 13 \mathrm{H}), 7.27$ (dd, $J=10.2,3.6 \mathrm{~Hz}, 1 \mathrm{H}), 7.06(\mathrm{~d}, J=9.0 \mathrm{~Hz}, 2 \mathrm{H})$, $6.58(\mathrm{~d}, J=1.8 \mathrm{~Hz}, 1 \mathrm{H}), 6.47$ (d, $J=2.4 \mathrm{~Hz}, 1 \mathrm{H}), 6.13(\mathrm{~d}, J=10.2 \mathrm{~Hz}, 1 \mathrm{H}), 5.91$ (d, $J=3.6 \mathrm{~Hz}$, 1H), 5.28 (s, 2H), 5.15 (s, 2H), 5.09 (s, 2H), 4.01 (q, $J=7.2 \mathrm{~Hz}, 1 \mathrm{H}), 0.92$ (d, $J=6.6 \mathrm{~Hz}, 3 \mathrm{H})$; ${ }^{13} \mathrm{C} \mathrm{NMR}\left(\mathrm{CDCl}_{3}, 150 \mathrm{MHz}\right) \delta 196.7,173.3,163.0,160.4,159.9,159.0,154.9,143.1,137.6$, 
136.3, 136.2, 135.6, 130.4, 128.8, 128.7, 128.6, 128.4, 128.2, 127.7, 127.6, 127.4, 127.4, 126.7, 123.2, 114.7, 110.0, 98.3, 94.3, 94.1, 71.5, 70.8, 70.5, 70.1, 14.6; HRMS (ESI): calcd. for $\left[\mathrm{C}_{42} \mathrm{H}_{34} \mathrm{O}_{8}+\mathrm{Na}\right]^{+}:$689.2151, Found: 689.2149 .

\section{5,7-bis(benzyloxy)-2-(4-(benzyloxy)phenyl)-3-((2R,3S,4S,5S,6R)-3,4,5-trihydroxy-6- methyltetrahydro-2H-pyran-2-yloxy)-4H-chromen-4-one ((ent)-I-12)}

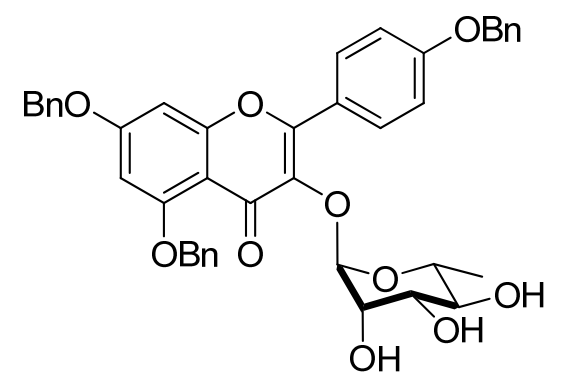

A solution of $135.5 \mathrm{mg}$ pyranone (ent)-I-2 $(0.203 \mathrm{mmol})$ in $2.5 \mathrm{~mL} \mathrm{CH} \mathrm{Cl}_{2} \mathrm{Cl}_{2}-\mathrm{MeOH}$ (5:1) was cooled down to $-78^{\circ} \mathrm{C}$, then $19 \mathrm{mg}(0.50 \mathrm{mmol}) \mathrm{NaBH}_{4}$ was added in. The reaction mixture was stirred at $-78{ }^{\circ} \mathrm{C}$ for 3 hours. The reaction was then quenched by $2 \mathrm{~mL}$ saturated aqueous $\mathrm{NaHCO}_{3}$, followed by extraction with EtOAc $(20 \mathrm{~mL} \times 3)$. The organic layer was washed by 2 $\mathrm{mL}$ saturated aqueous $\mathrm{NaCl}$, dried over $\mathrm{Na}_{2} \mathrm{SO}_{4}$, and concentrated under reduced pressure to give crude product. The crude product was redissolved in a mixture of $1 \mathrm{~mL} t-\mathrm{BuOH}, 1 \mathrm{~mL}$ acetone and $1 \mathrm{~mL} \mathrm{NMO}\left(50 \% \mathrm{w} / \mathrm{w}\right.$ aqueous) at $0{ }^{\circ} \mathrm{C}$. Then catalytic amount of crystalline $\mathrm{OsO}_{4}$ was added into the mixture. The reaction mixture was stirred at $0{ }^{\circ} \mathrm{C}$ overnight, then was quenched by addition of $0.5 \mathrm{~mL}$ saturated aqueous $\mathrm{Na}_{2} \mathrm{~S}_{2} \mathrm{O}_{3}$. After stirring for another 0.5 hour at $0{ }^{\circ} \mathrm{C}$, all the staff was passed through a small pad of celite and silica gel, washed by EtOAc-MeOH (10:1) (10 $\mathrm{mL} \times 3$ ). The organic effluent was dried by $\mathrm{Na}_{2} \mathrm{SO}_{4}$, and concentrated under reduced pressure to give crude prodcut. Chromatography on silica gel column, eluting with $\mathrm{Et}_{2} \mathrm{O}-\mathrm{MeOH}$ (20:1), gave 
triol (ent)-I-12 (85 mg, $60 \%$ over two steps): White solid, mp: $103-105{ }^{\circ} \mathrm{C} ; R_{f}=0.27(20: 1(\mathrm{v} / \mathrm{v})$ $\mathrm{Et}_{2} \mathrm{O} / \mathrm{MeOH}$ ); $[\alpha]_{\mathrm{D}}^{20}+121\left(c\right.$ 1.0, $\mathrm{MeOH}$ ); IR (thin film, $\left.\mathrm{cm}^{-1}\right) v 3386$ (broad), 3063, 2931, 1602, 1509, 1453, 1352, 1299, 1252, 1198, 1174, 1102, 1098, 1055, 998, 993; ${ }^{1} \mathrm{H}$ NMR (acetone-d $\mathrm{d}_{6}$, $600 \mathrm{MHz}) \delta 7.91(\mathrm{~d}, J=9.0 \mathrm{~Hz}, 2 \mathrm{H}), 7.69(\mathrm{~d}, J=7.8 \mathrm{~Hz}, 2 \mathrm{H}), 7.50$ (d, $J=8.4 \mathrm{~Hz}, 4 \mathrm{H}) 7.43-7.39$ (m, 6H), 7.38-7.29 (m, 3H), 7.19 (d, $J=8.4 \mathrm{~Hz}, 2 \mathrm{H}), 6.80(\mathrm{~d}, J=1.8 \mathrm{~Hz}, 1 \mathrm{H}), 6.67$ (d, $J=2.4$ Hz, 1H), 5.57 (d, $J=1.8 \mathrm{~Hz}, 1 \mathrm{H}), 5.29(\mathrm{~d}, J=13.2 \mathrm{~Hz}, 1 \mathrm{H}), 5.28(\mathrm{~d}, J=13.2 \mathrm{~Hz}, 1 \mathrm{H}), 5.24(\mathrm{~s}$, 2H), $5.23(\mathrm{~s}, 2 \mathrm{H}), 4.28(\mathrm{dd}, J=3.6,1.8 \mathrm{~Hz}, 1 \mathrm{H}), 3.70(\mathrm{dd}, J=9.0,3.6 \mathrm{~Hz}, 1 \mathrm{H}), 3.33(\mathrm{dd}, J=9.6$, $9.6 \mathrm{~Hz}, 1 \mathrm{H}), 3.21(\mathrm{dq}, J=9.6,6.0 \mathrm{~Hz}, 1 \mathrm{H}), 2.91(\mathrm{brs}, 1 \mathrm{H}), 0.87(\mathrm{~d}, J=6.0 \mathrm{~Hz}, 3 \mathrm{H}) ;{ }^{13} \mathrm{C} \mathrm{NMR}$ (acetone- $\left.\mathrm{d}_{6}, 150 \mathrm{MHz}\right) \delta 173.5,164.1,161.5,160.8,159.8,154.8,138.4,138.1,138.0,137.4$, 131.4, 129.5, 129.4, 129.3, 129.1, 128.8, 128.8, 128.5, 128.4, 127.9, 124.4, 115.7, 110.6, 102.2, 98.9, 95.1, 73.2, 72.2, 71.5, 71.4, 71.3, 71.2, 70.8, 17.9; HRMS (ESI) calcd. for $\left[\mathrm{C}_{42} \mathrm{H}_{38} \mathrm{O}_{10}+\mathrm{H}\right]^{+}$: 703.2543, Found: 703.2541.

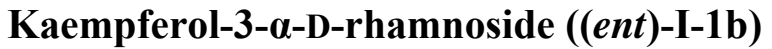

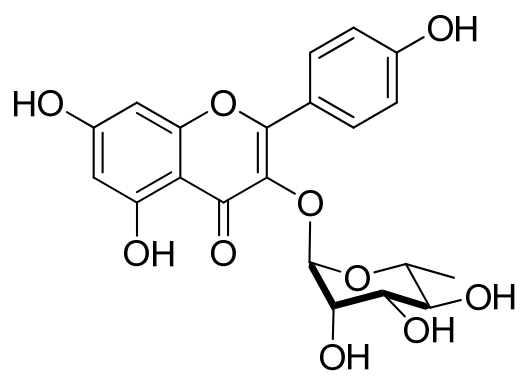

A solution of $24 \mathrm{mg}$ triol (ent)-I-12 (0.034 mmol) in $2 \mathrm{~mL}$ THF-EtOH (1:1) was added $14 \mathrm{mg}$ $\mathrm{Pd} / \mathrm{C}(10 \% \mathrm{w} / \mathrm{w})$. The solution was degassed using vacuum at -50 to $-40{ }^{\circ} \mathrm{C}$ and refilling with $\mathrm{H}_{2}$. This procedure was repeated three times, and then the bath was removed. The reaction was warmed up to room temperature and stirred under a $\mathrm{H}_{2}$ atmosphere for 5 hours. The reaction mixture was loaded onto silica gel and elution with hexane-EtOAc (1:3) gave kaempferol-3- $\alpha-D-$ rhamnoside ((ent)-I-1b) $(14 \mathrm{mg}, 93 \%)$ : Pale yellow solid, mp: $148-150{ }^{\circ} \mathrm{C} ; R_{f}=0.71(5: 1(\mathrm{v} / \mathrm{v})$ $\left.\mathrm{Et}_{2} \mathrm{O} / \mathrm{MeOH}\right) ;[\alpha]_{\mathrm{D}}^{20}+118$ (c 1.0, MeOH); IR (thin film, $\left.\mathrm{cm}^{-1}\right)$ v 3290 (broad), 2922, 2853, 1654, $1607,1500,1448,1359,1261,1207,1173,1056,947 ;{ }^{1} \mathrm{H}$ NMR (acetone- $\left.\mathrm{d}_{6}, 600 \mathrm{MHz}\right) \delta 7.85(\mathrm{~d}$, 
$J=9.0 \mathrm{~Hz}, 2 \mathrm{H}), 7.01(\mathrm{~d}, J=8.4 \mathrm{~Hz}, 2 \mathrm{H}), 6.47$ (d, $J=1.8 \mathrm{~Hz}, 1 \mathrm{H}), 6.27(\mathrm{~d}, J=2.4 \mathrm{~Hz}, 1 \mathrm{H}), 5.53$ (d, $J=1.8 \mathrm{~Hz}, 1 \mathrm{H}), 4.21(\mathrm{dd}, J=3.0,1.8 \mathrm{~Hz}, 1 \mathrm{H}), 3.68(\mathrm{dd}, J=9.0,3.6 \mathrm{~Hz}, 1 \mathrm{H}), 3.33(\mathrm{dd}, J=$ 9.6, 8.4 Hz, 1H), $3.30(\mathrm{dd}, J=9.6,6.0 \mathrm{~Hz}, 1 \mathrm{H}), 0.89(\mathrm{~d}, J=6.0 \mathrm{~Hz}, 3 \mathrm{H}) ;{ }^{13} \mathrm{C}$ NMR (acetone- $\mathrm{d}_{6}$, $150 \mathrm{MHz}) \delta 179.4,165.1,163.0,161.0,158.5,158.1,135.9,131.8,122.6,116.3,105.8,102.8$, 99.6, 94.6, 73.0, 72.2, 71.5, 71.4, 17.9; HRMS (ESI) calcd. for $\left[\mathrm{C}_{21} \mathrm{H}_{20} \mathrm{O}_{10}+\mathrm{Na}\right]^{+}: 455.0954$, Found: 455.0949 .

\section{V.3. Chapter II Experimental}

(3aR,4aS,5aR,6aS)-hexahydro-2,2-dimethyl-3aH-bicyclo[4.1.0]hept-1(6)-eno[3,4d][1,3]dioxol-4a-ol (II-9a)

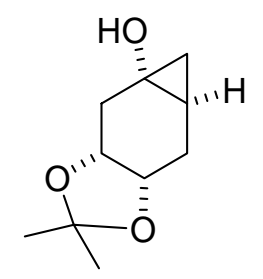

(3aR,4aR,5aS,6aS)-hexahydro-2,2-dimethyl-3aH-bicyclo[4.1.0]hept-1(6)-eno[3,4d][1,3]dioxol-4a-ol (II-9b)

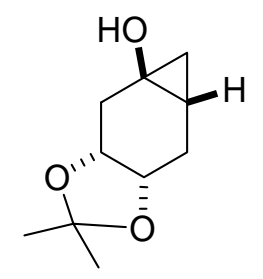

To a solution of $17.0 \mathrm{~g}$ enone II-4 $(101.2 \mathrm{mmol})$ in $185 \mathrm{~mL}$ toluene was added $155 \mathrm{mg}$ $\mathrm{RhCl}\left(\mathrm{PPh}_{3}\right)_{3}(0.34 \mathrm{mmol})$ and $17.0 \mathrm{~mL}$ phenyldimethylsilane $(111.3 \mathrm{mmol})$, the mixture was stirred at $60{ }^{\circ} \mathrm{C}$ for 1 hour. Then the reaction mixture was cooled down to $0{ }^{\circ} \mathrm{C}$, into which a 
solution of $18.7 \mathrm{~g} \mathrm{ZnEt}_{2}(151.8 \mathrm{mmol})$ was slowly added followed by addition of $18.4 \mathrm{ml} \mathrm{CH}_{2} \mathrm{I}_{2}$ (228.0 mmol). The reaction mixture was stirred at $0{ }^{\circ} \mathrm{C}$ overnigtht. Then it was diluted with 800 $\mathrm{mL}$ hexane-ether ( $\mathrm{v} / \mathrm{v}$ 1:1) and quenched with $150 \mathrm{~mL}$ saturated aqueous $\mathrm{NH}_{4} \mathrm{Cl}$. The aqueous layer was extracted with $\mathrm{Et}_{2} \mathrm{O}$ with hexane-ether (v/v 1:1) $500 \mathrm{~mL}$ X 2. The combined organic layer was washed wih $100 \mathrm{~mL}$ saturated aqueous $\mathrm{Na}_{2} \mathrm{~S}_{2} \mathrm{O}_{3}, 100 \mathrm{~mL}$ saturated brine and dried over $\mathrm{Na}_{2} \mathrm{SO}_{4}$. The solution was then concentrated to a residue, which was redissolved in $360 \mathrm{~mL}$ $\mathrm{MeOH}$ at $0{ }^{\circ} \mathrm{C}$ and $546 \mathrm{mg}(2.87 \mathrm{mmol}) p$-TsOH was added in portions. It was stirred at $0{ }^{\circ} \mathrm{C}$ for $15 \mathrm{~min}$, then quenched with $50 \mathrm{~mL}$ saturated aqueous $\mathrm{NaHCO}_{3}$. The mixture was concentrated under reduced pressure to remove most of the $\mathrm{MeOH}$, and then extracted with hexane-EtOAc (v/v 1:5) $800 \mathrm{~mL}$ X 3. The organic layer was washed with saturated brine $300 \mathrm{~mL}$ and dried over $\mathrm{Na}_{2} \mathrm{SO}_{4}$. Concentration of the solution gave a residue, which was subjected to chromatography on silica gel column. Elution with hexane-EtOAc (3:1) afforded cyclopropanol II-9a (9.3 g, 50 \%) and elution with hexane-EtOAc (3:2) afforded II-9b (3.7 g, 20\%).

II-9a: Colorless oil; $R_{f}=0.44\left(1: 1(\mathrm{v} / \mathrm{v})\right.$ hexane/EtOAc); $[\alpha]_{\mathrm{D}}{ }^{20}+19\left(\right.$ c $\left.2.50, \mathrm{CHCl}_{3}\right)$; IR (thin film, $\left.\mathrm{cm}^{-1}\right) v 3433,2987,2933,1453,1371 ;{ }^{1} \mathrm{H}$ NMR $\left(600 \mathrm{MHz}, \mathrm{CDCl}_{3}\right) \delta 4.48$ (ddd, $J=7.8$, 3.0, $2.4 \mathrm{~Hz}, 1 \mathrm{H}), 4.32$ (ddd, $J=7.8,3.0,2.4 \mathrm{~Hz}, 1 \mathrm{H}), 2.72(\mathrm{~s}, 1 \mathrm{H}), 2.50$ (dd, $J=15.6,3.0 \mathrm{~Hz}$, 1H), 2.30 (dddd, $J=15.6,4.8,2.4,1.8 \mathrm{~Hz}, 1 \mathrm{H}$ ), $\delta 1.48$ (s, 3H), 1.33 (s, 3H), 1.25 (dd, $J=15.6$, $2.4 \mathrm{~Hz}, 1 \mathrm{H}), 1.08(\mathrm{~m}, 2 \mathrm{H}), 0.62(\mathrm{~m}, 1 \mathrm{H}) ; 0.18(\mathrm{~m}, 1 \mathrm{H}) ;{ }^{13} \mathrm{C} \mathrm{NMR}\left(150 \mathrm{MHz}, \mathrm{CDCl}_{3}\right) \delta 107.1$, 74.0, 73.6, 52.0, 34.4, 29.9, 26.4, 23.4, 20.6, 14.8. HRMS (ESI): calcd. for $\left[\mathrm{C}_{10} \mathrm{H}_{16} \mathrm{O}_{3}+\mathrm{Na}\right]^{+}$: 207.09917, Found: 207.09916.

II-9b: Colorless oil; $R_{f}=0.26\left(1: 1(\mathrm{v} / \mathrm{v})\right.$ hexane/EtOAc); $[\alpha]_{\mathrm{D}}{ }^{20}+11\left(c\right.$ 2.36, $\left.\mathrm{CHCl}_{3}\right)$; IR (thin film, $\left.\mathrm{cm}^{-1}\right) v 3371,2988,2915,1463,1371 ;{ }^{1} \mathrm{H}$ NMR $\left(600 \mathrm{MHz}, \mathrm{CDCl}_{3}\right) \delta 4.45$ (ddd, $J=7.8$, 6.0, $6.0 \mathrm{~Hz}, 1 \mathrm{H}$ ), 4.29 (ddd, $J=8.4,6.0,6.0 \mathrm{~Hz}, 1 \mathrm{H}), 2.35$ (dd, $J=15.0,6.0 \mathrm{~Hz}, 1 \mathrm{H}$ ), 2.24 (ddd, $J=15.0,5.4,5.4,1 \mathrm{H}), 2.05$ (brs, $1 \mathrm{H}), 1.82$ (dd, $J=15.0,6.0 \mathrm{~Hz}, 1 \mathrm{H}), \delta 1.44$ (s, 3H), 1.39 (ddd, $J=15.0,6.0,6.0 \mathrm{~Hz}, 1 \mathrm{H}), 1.30$ (s, 3H), 1.06 (dddd, $J=9.6,6.0,6.0,5.4 \mathrm{~Hz}, 1 \mathrm{H}), 0.92$ (dd, $J=$ 9.6, $5.4 \mathrm{~Hz}, 1 \mathrm{H}), 0.88(\mathrm{dd}, J=6.0,5.4 \mathrm{~Hz}, 1 \mathrm{H}) ;{ }^{13} \mathrm{C} \mathrm{NMR}\left(150 \mathrm{MHz}, \mathrm{CDCl}_{3}\right) \delta 107.2,72.0,71.8$, 53.4, 34.6, 27.6, 26.4, 23.1, 20.4, 17.1. HRMS (ESI): calcd. for $\left[\mathrm{C}_{10} \mathrm{H}_{16} \mathrm{O}_{3}+\mathrm{H}\right]^{+}$: 185.11722, Found: $185.11719 .{ }^{1} \mathrm{H}-{ }^{1} \mathrm{H}$ COSY and NOE as follow: 


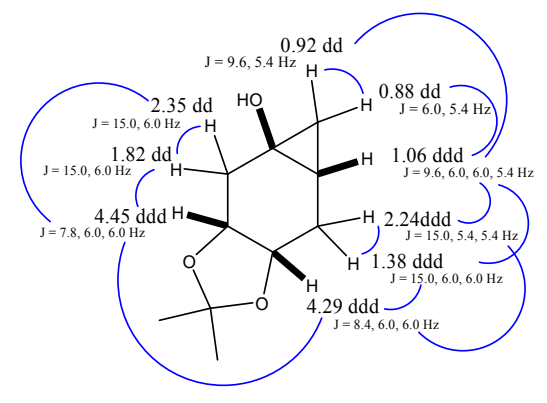

${ }^{1} \mathrm{H}-{ }^{1} \mathrm{H} \operatorname{COSY}$ of $\mathbf{1 3 b}$

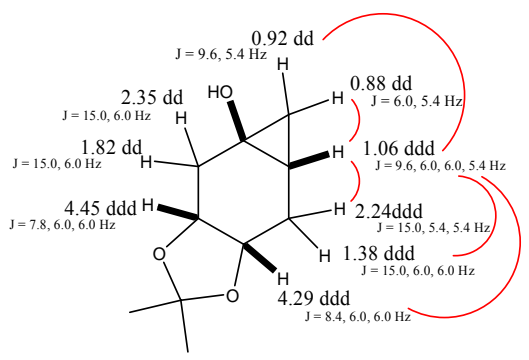

NOE of 13b<smiles>C=C1C[C@H]2OC(C)(C)O[C@@H]2CC1=O</smiles>

(3aR,7aS)-3a,4-dihydro-2,2,6-trimethylbenzo [d][1,3]dioxol-5(7aH)-one (II-10b)<smiles>CC1=C[C@H]2OC(C)(C)O[C@@H]2CC1=O</smiles>

To a solution of $139 \mathrm{mg}$ II-9a $(0.76 \mathrm{mmol})$ in $6 \mathrm{~mL}$ toluene was added $128 \mu \mathrm{L}$ pyridine $(1.59$ $\mathrm{mmol})$ and $178 \mathrm{mg} \mathrm{Pd}(\mathrm{OAc})_{2}(0.79 \mathrm{mmol})$. The reaction mixture was heated up to $80{ }^{\circ} \mathrm{C}$ and kept stirring for $3 \mathrm{~h}$. Then it was cooled to RT, and was filtered through filter paper, rinsing with $\mathrm{Et}_{2} \mathrm{O}$-hexane $(1: 1, \mathrm{v} / \mathrm{v}) 50 \mathrm{~mL}$. The solution was washed with $1 \mathrm{~mL} 0.5 \mathrm{M} \mathrm{NaHSO}_{4}$ at $0{ }^{\circ} \mathrm{C}$, then was washed with $1 \mathrm{~mL}$ saturated aqueous $\mathrm{NaHCO}_{3}$, and $1 \mathrm{~mL}$ saturated aqueous $\mathrm{NaCl}$, dried over $\mathrm{Na}_{2} \mathrm{SO}_{4}$. The solution was concentrated under reduced pressure to give a residue, which was 
purified by silica gel column. Elution with hexane-EtOAc (7:1) afforded two pure compounds II10a (42 mg, 31\%) and II-10b (75 mg, 55\%).

To a solution of $10 \mathrm{mg}$ II-9a $(0.05 \mathrm{mmol})$ in $0.5 \mathrm{~mL}$ toluene was added $8.7 \mu \mathrm{L}$ pyridine $(0.11$ $\mathrm{mmol}), 12 \mathrm{mg}$ quinone $(0.10 \mathrm{mmol})$ and $14 \mathrm{mg} \mathrm{Pd}(\mathrm{OAc})_{2}(0.06 \mathrm{mmol})$. The reaction mixture was heated up to $80^{\circ} \mathrm{C}$ and kept stirring for $3 \mathrm{~h}$. Then it was cooled to RT, and was filtered through filter paper, rinsing with $\mathrm{Et}_{2} \mathrm{O}$-hexane $(1: 1, \mathrm{v} / \mathrm{v}) 5 \mathrm{~mL}$. The solution concentrated under reduced pressure and directly loaded onto a silica gel column. Elution with hexane-EtOAc $(5: 1)$ afforded compounds II-10a (7 mg, 70\%) and II-9a (1 mg, 10\% recovery).

To a solution of $1.6 \mathrm{mg}$ II-9a $(0.009 \mathrm{mmol})$ in $0.6 \mathrm{~mL}$ THF-EtOH $(1: 1 \mathrm{v} / \mathrm{v})$ was added $11 \mathrm{mg}$ $10 \% \mathrm{Pd} / \mathrm{C}(0.01 \mathrm{mmol} \mathrm{Pd})$. The reaction mixture was stirred at RT for $24 \mathrm{~h}$ and the mixture was then filtered through filter paper. The solution was concentrated under reduced pressure to give a residue, which was shown by crude ${ }^{1} \mathrm{H}$ NMR that it was exclusively II-10a.

II-10a: White solid; mp: $58.3-60.3{ }^{\circ} \mathrm{C} ; R_{f}=0.45\left(1: 1(\mathrm{v} / \mathrm{v})\right.$ hexane/EtOAc); $[\alpha]_{\mathrm{D}}{ }^{20}+16(c 0.33$, $\mathrm{CHCl}_{3}$ ); IR (thin film, $\mathrm{cm}^{-1}$ ) v 2991, 2917, 1709, 1671, 1627, 1431, 1384, 1363, 1286, 1274 , 1243, 1207, 1163, 1081, 1062, 1038, 950; ${ }^{1} \mathrm{H} \mathrm{NMR}\left(\mathrm{CDCl}_{3}, 270 \mathrm{MHz}\right) \delta 6.17(\mathrm{dd}, J=2.0,2.0$ $\mathrm{Hz}, 1 \mathrm{H}), 5.32(\mathrm{dd}, J=2.2,1.7 \mathrm{~Hz}, 1 \mathrm{H}), 4.69$ (ddd, $J=6.7,3.2,3.2 \mathrm{~Hz}, 1 \mathrm{H}), 4.60$ (ddd, $J=7.4$, 3.2, $3.2 \mathrm{~Hz}, 1 \mathrm{H}), 2.79(\mathrm{dd}, J=15.3,2.7 \mathrm{~Hz}, 1 \mathrm{H}), 2.77$ (dd, $J=15.8,3.0 \mathrm{~Hz}, 1 \mathrm{H}), 2.64$ (dddd, $J=$ 15.3, 3.7, 2.2, $2.2 \mathrm{~Hz}, 1 \mathrm{H}), 2.58(\mathrm{dd}, J=15.8,3.5 \mathrm{~Hz}, 1 \mathrm{H}), 1.34$ (s, $3 \mathrm{H}), 1.33$ (s, 3H); ${ }^{13} \mathrm{C} \mathrm{NMR}$ $\left(\mathrm{CDCl}_{3}, 67.5 \mathrm{MHz}\right) \delta 197.3,138.9,123.5,108.0,73.1,72.4,42.0,34.9,26.0,24.1$; HRMS (ESI) calcd. for $\left[\mathrm{C}_{10} \mathrm{H}_{14} \mathrm{O}_{3}+\mathrm{H}\right]^{+}:$: 183.1016 , Found: 183.1016 .

II-10b: Colorless oil; $R_{f}=0.55\left(1: 1(\mathrm{v} / \mathrm{v})\right.$ hexane/EtOAc); $[\alpha]_{\mathrm{D}}{ }^{20}+71\left(c 1.89, \mathrm{CHCl}_{3}\right)$; IR (thin film, $\left.\mathrm{cm}^{-1}\right) v 2986,2926,1679,1450,1380,1368,1243,1223,1155,1063,1038,1012,967 ;{ }^{1} \mathrm{H}$ NMR $\left(\mathrm{CDCl}_{3}, 270 \mathrm{MHz}\right) \delta 6.39(\mathrm{dq}, J=4.7,1.5 \mathrm{~Hz}, 1 \mathrm{H}), 4.69$ (dddd, $J=4.5,3.0,3.0,1.5 \mathrm{~Hz}$, 1H), 4.63 (ddq, $J=4.9,3.0,2.0 \mathrm{~Hz}, 1 \mathrm{H}), 2.92$ (dd, $J=17.3,2.7 \mathrm{~Hz}, 1 \mathrm{H}), 2.65$ (dd, $J=17.3,3.9$ $\mathrm{Hz}, 1 \mathrm{H}), 1.80(\mathrm{dd}, J=1.5,1.5 \mathrm{~Hz}, 3 \mathrm{H}), 1.36(\mathrm{~s}, 3 \mathrm{H}), 1.34(\mathrm{~s}, 3 \mathrm{H}) ;{ }^{13} \mathrm{C} \mathrm{NMR}\left(\mathrm{CDCl}_{3}, 67.5 \mathrm{MHz}\right)$ 
$\delta$ 195.7, 140.6, 135.5, 109.6, 73.6, 71.6, 39.1, 27.8, 26.6, 15.8; HRMS (ESI) calcd. for $\left[\mathrm{C}_{10} \mathrm{H}_{14} \mathrm{O}_{3}+\mathrm{H}\right]^{+}:$183.1016, Found: 183.1016 .

\section{Hydrogenation of II-10a and II-10b}

To a solution of $40 \mathrm{mg}$ enone II-10a (or II-10b) $(0.22 \mathrm{mmol})$ in $3 \mathrm{~mL}$ THF-EtOH $(1: 1 \mathrm{v} / \mathrm{v})$ was added $20 \mathrm{mg} 10 \% \mathrm{Pd} / \mathrm{C}$. The reaction mixture was degassed using vacuum and refilled with $\mathrm{H}_{2}$ at $\sim-30{ }^{\circ} \mathrm{C}$ for three times and then was stirred under balloon pressure of $\mathrm{H}_{2}$ at RT for $2 \mathrm{~h}$. The mixture was filtered through filter paper and the solution was concentrated under reduced pressure to give a residue. A crude ${ }^{1} \mathrm{H}$ NMR showed that it was a diastereomeric mixture of $\alpha$ -

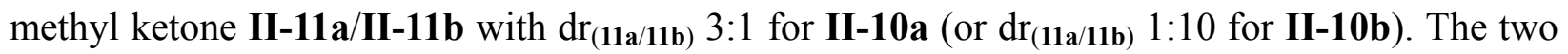
isomers II-11a and II-11b were inseparable on silica gel column and the mixture of isomers II11a/II-9b were obtained in $88 \%$ yield for II-10a (or $89 \%$ for II-10b).

\section{$\beta$-elimination of II-11a/II-11b}

To a solution of $18 \mathrm{mg}$ mixture of II-11a/II-11b (dr 3:1) $(0.1 \mathrm{mmol})$ in $6 \mathrm{~mL}$ THF at $0{ }^{\circ} \mathrm{C}$ was added 5 drops of $0.5 \mathrm{M} \mathrm{NaOH}_{\text {(aq.) }}$ and the mixture was stirred at $0{ }^{\circ} \mathrm{C}$ for $30 \mathrm{~h}$. Then into the mixture was added 10 drops of saturated aqueous $\mathrm{NH}_{4} \mathrm{Cl}$ and diluted with $20 \mathrm{~mL}$ EtOAc. The organic layer was washed with $1 \mathrm{~mL}$ saturated aqueous $\mathrm{NH}_{4} \mathrm{Cl}, 1 \mathrm{~mL}$ saturated aqueous $\mathrm{NaCl}$ and dried over $\mathrm{Na}_{2} \mathrm{SO}_{4}$. The solution was concentrated under reduced pressure to give a residue which by crude ${ }^{1} \mathrm{H}$ NMR was shown that it was a mixture of II-12a/II-12b $\left(\operatorname{dr}_{(\mathbf{1 2 a} / \mathbf{1 2 b})} 2: 3\right)$. The two isomers II-12a and II-12b were inseparable on silica gel column and the mixture of II12a/II-12b was obtained in $85 \%$ yield.

(3aR,6S,7aS)-tetrahydro-2,2,6-trimethylbenzo[d][1,3]dioxol-5(6H)-one (II-11a) 


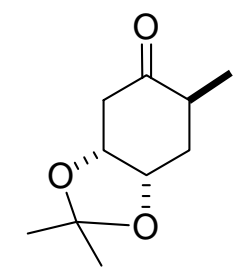

To a solution of $660 \mathrm{mg}$ cyclopropanol II-9a $(3.6 \mathrm{mmol})$ in $30 \mathrm{~mL}$ THF-EtOH (v/v 1:1) was added $350 \mathrm{mg} 10 \% \mathrm{Pd} / \mathrm{C}$. The solution was degassed using vacuum and refilled with $\mathrm{H}_{2}$ three times at low temperature $\left(\sim-30^{\circ} \mathrm{C}\right)$. Then at room temperature, the solution was stirred overnight under balloon pressure of $\mathrm{H}_{2}$. It was filtered through a filter paper and rinse with ethyl acetate. The solution was concentrated under reduced pressure to a small volume, which was then subjected to chromatography on silica gel column. Elution with Hexane-EtOAc (7:2) gave product II-11a (530 mg, 80\%).

II-11a: Colorless oil; $R_{f}=0.55\left(1: 1(\mathrm{v} / \mathrm{v})\right.$ hexane/EtOAc); $[\alpha]_{\mathrm{D}}{ }^{20}+105(c$ 2.7, CHCl3); IR (thin film, $\left.\mathrm{cm}^{-1}\right)$ v 2989, 2937, 1702, 1458, 1378, 1204, 1164, 1017; ${ }^{1} \mathrm{H}$ NMR (CDCl3, $\left.600 \mathrm{MHz}\right) \delta$ $4.63(\mathrm{ddd}, J=7.2,4.2,3.0 \mathrm{~Hz}, 1 \mathrm{H}) 4.52(\mathrm{ddd}, J=7.2,3.0,2.4 \mathrm{~Hz}, 1 \mathrm{H}), 2.67$ (dd, $J=17.4,3.0$ $\mathrm{Hz}, 1 \mathrm{H}), 2.53$ (dqd, $J=13.8,6.6,4.2 \mathrm{~Hz}, 1 \mathrm{H}), 2.44$ (dd, $J=16.8,3.6 \mathrm{~Hz}, 1 \mathrm{H}), 2.06$ (ddd, $J=$ 15.0, 4.2, $2.4 \mathrm{~Hz}, 1 \mathrm{H}), 1.65$ (ddd, $J=16.2,13.2,3.0 \mathrm{~Hz}, 1 \mathrm{H}), 1.44$ (s, 3H), 1.35 (s, 3H), 1.09 (d, $J=7.2 \mathrm{~Hz}, 3 \mathrm{H}) ;{ }^{13} \mathrm{C} \mathrm{NMR}(\mathrm{CDCl} 3,150 \mathrm{MHz}) \delta 211.7,107.6,72.9,71.9,41.1,37.4,34.3,26.0$, 23.8, 14.8; HRMS (ESI): calcd. for $\left[\mathrm{C}_{10} \mathrm{H}_{16} \mathrm{O}_{3}+\mathrm{H}\right]^{+}:$185.11722, Found: 185.11723.

\section{(2S,4S,5R)-4,5-dihydroxy-2-methylcyclohexanone (II-13)}<smiles>CC1C[C@H](O)[C@H](O)CC1=O</smiles>

$1.0 \mathrm{~g}$ acetonide II-11a $(5.4 \mathrm{mmol})$ was placed in $250 \mathrm{~mL}$ flask at $0{ }^{\circ} \mathrm{C}$, a pre-cooled $40 \mathrm{~mL}$ solution of THF-10\% $\mathrm{HCl}(\mathrm{v} / \mathrm{v}$ 1:1) was added into the flask when stirring. Then the ice bath was removed and the mixture was allowed to warm up to room temperature over 1 hour when stirring. 
The mixture was cooled down to $0{ }^{\circ} \mathrm{C}$ and diluted with $120 \mathrm{~mL}$ EtOAc, into which $10 \mathrm{~g} \mathrm{NaHCO}_{3}$ (s) was slowly added to quench the reaction. The mixture was extracted with EtOAc $200 \mathrm{~mL}$ X 3 and the organic layer was washed with $40 \mathrm{~mL}$ saturated aqueous $\mathrm{NaCl}$, dried over $\mathrm{Na}_{\mathrm{S}} \mathrm{O}_{4}$. The solution was concentrated under reduced pressure to a residue which was subjected to chromatography on silica gel column. Elution with hexane-EtOAc (1:3) gave product diol II-13 (600 mg, 77\%).

II-13: Colorless oil; $R_{f}=0.53(10: 1(\mathrm{v} / \mathrm{v}) \mathrm{EtOAc} / \mathrm{MeOH}) ;[\alpha]_{\mathrm{D}}{ }^{20}+35$ (c 1.32, CHCl3); IR (thin film, $\left.\mathrm{cm}^{-1}\right) v$ 3391, 2969, 2932, 1701, 1455, 1430, 1376, 1355, 1243, 1215, 1105, 1066, 1031, 963, 915; ${ }^{1} \mathrm{H} \mathrm{NMR}(\mathrm{CDCl} 3,270 \mathrm{MHz}) \delta 4.15(\mathrm{ddd}, J=2.7,2.7,2.7 \mathrm{~Hz}, 1 \mathrm{H}), 3.94$ (ddd, $J=11.6$, 5.4, 3.0 Hz, 1H), $2.78(\mathrm{~m}, 2 \mathrm{H}), 2.55(\mathrm{dd}, J=13.1,5.2 \mathrm{~Hz}, 1 \mathrm{H}), 2.22(\mathrm{ddd}, J=14.4,5.9,4.0 \mathrm{~Hz}$, 1H), 1.42 (ddd, $J=14.6,13.1,2.5 \mathrm{~Hz}, 1 \mathrm{H}), 1.01(\mathrm{~d}, J=6.4 \mathrm{~Hz}, 3 \mathrm{H}) ;{ }^{13} \mathrm{C} \mathrm{NMR}(\mathrm{CDCl} 3,150$ $\mathrm{MHz}) \delta 210.1,72.0,68.3,45.1,37.6,36.2,13.6$; HRMS (ESI): calcd. for $\left[\mathrm{C}_{7} \mathrm{H}_{12} \mathrm{O}_{3}+\mathrm{Na}\right]^{+}$: 167.06787, Found: 167.06781.

(1S,3R,4S,6R)-bicyclo[4.1.0]heptane-1,3,4-triol (II-14)<smiles>OC1[C@@H](O)C[C@@H]2C[C@H]2C[C@@H]1O</smiles>

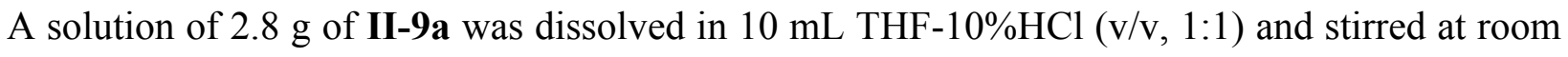
temperature for $20 \mathrm{~min}$. The mixture was cooled down to $0{ }^{\circ} \mathrm{C}$, and diluted with $100 \mathrm{~mL}$ EtOAc, then $\sim 7 \mathrm{~g} \mathrm{NaHCO}_{3}$ was slowly added to quench the reaction. The mixture was filtered through a glass wool and the liquid was concentrated to a residue which was loaded to a silica gel column. Elution with EtOAc-MeOH (10:1 v/v) gave triol II-14 (1.8g, 82\%).

II-14: White solid; $\mathrm{mp}: 148-149{ }^{\circ} \mathrm{C} ; R_{f}=0.19(10: 1(\mathrm{v} / \mathrm{v}) \mathrm{EtOAc} / \mathrm{MeOH}) ;[\alpha]_{\mathrm{D}}{ }^{20}+26(c 4.99$, $\mathrm{MeOH}$ ); IR (thin film, $\mathrm{cm}^{-1}$ ) $v$ 3368, 3245, 2928, 2911, 1434, 1363, 1276, 1213, 1172; ${ }^{1} \mathrm{H}$ NMR $\left(\mathrm{CD}_{3} \mathrm{OD}, 600 \mathrm{MHz}\right) \delta 3.56(\mathrm{ddd}, J=6.6,4.8,1.8 \mathrm{~Hz}, 1 \mathrm{H}), 3.38(\mathrm{ddd}, J=9.0,4.8,1.8 \mathrm{~Hz}, 1 \mathrm{H})$, 
$2.23(\mathrm{ddd}, J=13.2,13.2,5.4 \mathrm{~Hz}, 1 \mathrm{H}), 2.19(\mathrm{~m}, 2 \mathrm{H}), 1.56(\mathrm{ddd}, J=15.0,4.8,1.8 \mathrm{~Hz}, 1 \mathrm{H}), 1.03$ (dddd, $J=10.2,7.8,6.0,1.8 \mathrm{~Hz}, 1 \mathrm{H}$ ), 0.84 (ddd, $J=10.2,5.4,1.2 \mathrm{~Hz}, 1 \mathrm{H}$ ), 0.18 (dd, $J=6.0,6.0$ $\mathrm{Hz}, 1 \mathrm{H}) ;{ }^{13} \mathrm{C} \mathrm{NMR}\left(\mathrm{CD}_{3} \mathrm{OD}, 150 \mathrm{MHz}\right) \delta 70.1,69.7,55.4,36.2,31.5,20.0,18.1$; HRMS (ESI): calcd. for $\left[\mathrm{C}_{7} \mathrm{H}_{12} \mathrm{O}_{3}+\mathrm{Na}\right]^{+}:$167.06787, Found: 167.06783 .

\section{Alternatively preparation of II-13 from II-14}

Into a solution of $300 \mathrm{mg}(2.08 \mathrm{mmol})$ cyclopropanol II-14 in $13 \mathrm{~mL}$ THF-EtOH $(1: 1 \mathrm{v} / \mathrm{v})$ was added $150 \mathrm{mg} 10 \% \mathrm{Pd} / \mathrm{C}$. The solution was degassed using vacuum and refilled with $\mathrm{H}_{2}$ three times at low temperature $\left(\sim-30{ }^{\circ} \mathrm{C}\right)$. Then at room temperature, the solution was stirred $4 \mathrm{hr}$ under balloon pressure of $\mathrm{H}_{2}$. It was filtered through a filter paper and rinsed with ethyl acetate. The solution was concentrated under reduced pressure to a small volume (some of the solution was taken out and concentrated to dry, then a crude NMR was taken to show that NO diastereomer was seen). The residue was subjected to chromatography on silica gel column. Elution with Hexane-EtOAc (1:3) gave product II-13 (246 mg, 82\%).

\section{t-Butyl (1S,5S)-5-methyl-4-oxocyclohex-2-enyl carbonate (II-15)}<smiles>CC1CC(OC(C)(C)C)C=CC1=O</smiles>

Diol II-13 (250 mg, $1.74 \mathrm{mmol}$ ) was dissolved in $15 \mathrm{~mL} \mathrm{CH}_{2} \mathrm{Cl}_{2}$ at RT, into which $24 \mathrm{mg}$ DMAP $(0.2 \mathrm{mmol})$ was added, followed by addition of a solution of $1.2 \mathrm{~g} \mathrm{Boc}_{2} \mathrm{O}(5.6 \mathrm{mmol})$ in 5 $\mathrm{mL} \mathrm{CH} \mathrm{CH}_{2} \mathrm{Cl}_{2}$. The mixture was stirred at $\mathrm{RT}$ for $2 \mathrm{~h}$, then it was cooled down to $0{ }^{\circ} \mathrm{C}$, diluted with $\mathrm{Et}_{2} \mathrm{O} 30 \mathrm{~mL}$ and was then quenched with $10 \mathrm{~mL}$ saturated aqueous $\mathrm{NaHCO}_{3}$. The mixture was stirred at $0{ }^{\circ} \mathrm{C}$ for another $1 \mathrm{~h}$ and then was extracted with Hexane-Et $2 \mathrm{O}(1: 2 \mathrm{v} / \mathrm{v}) 50 \mathrm{~mL}$ x 2 . The pooled organic layer was washed with $15 \mathrm{~mL}$ saturated aqueous $\mathrm{NaCl}$ and dried over $\mathrm{Na}_{2} \mathrm{SO}_{4}$. 
The solution was concentrated under reduced pressure to afford a residue. The residue was redissolved in $9 \mathrm{~mL} \mathrm{CH} \mathrm{Cl}_{2}$ at $0{ }^{\circ} \mathrm{C}$. Into the solution $1.8 \mathrm{~mL} \mathrm{Et}_{3} \mathrm{~N}$ (12.9 mmol) was slowly added. The mixture was then stirred at $0{ }^{\circ} \mathrm{C}$ overnight and continued to be stirred at $\mathrm{RT}$ for another $2 \mathrm{~h}$. Then it was cooled back to $0{ }^{\circ} \mathrm{C}$, diluted with hexane- $\mathrm{Et}_{2} \mathrm{O}(1: 1 \mathrm{v} / \mathrm{v}) 50 \mathrm{~mL}$ and it was then quenched by $30 \mathrm{~mL} 0.5 \mathrm{M} \mathrm{NaHSO}_{4}$ at $0{ }^{\circ} \mathrm{C}$. The aqueous layer was extracted with hexane-Et $\mathrm{t}_{2} \mathrm{O}(1: 1 \mathrm{v} / \mathrm{v}) 100 \mathrm{~mL} \times 2$. The pooled organic layer was washed with $20 \mathrm{~mL}$ saturated aqueous $\mathrm{NaHCO}_{3}, 20 \mathrm{~mL}$ saturated aqueous $\mathrm{NaCl}$ and dried over $\mathrm{Na}_{2} \mathrm{SO}_{4}$. The solution was concentrated under reduced pressure to afford a residue, which was purified by silica gel column. Elution with hexane-EtOAc (10:1 v/v) gave enone II-15 (325 mg, 83\% for two steps) as white solid; mp: $36.8-38.2{ }^{\circ} \mathrm{C} ; R_{f}=0.49\left(4: 1(\mathrm{v} / \mathrm{v})\right.$ hexane/EtOAc); $[\alpha]_{\mathrm{D}}{ }^{20}-175$ (c 1.54, $\left.\mathrm{CHCl}_{3}\right)$; IR (thin film, $\mathrm{cm}^{-1}$ ) $v$ 2991, 2969, 2930, 2879, 1731, 1686, 1627, 1454, 1369, 1343, 1274, 1252, $1195,1156,1121,1079,1067,1046,965 ;{ }^{1} \mathrm{H}$ NMR $\left(\mathrm{CDCl}_{3}, 270 \mathrm{MHz}\right) \delta 6.86(\mathrm{dd}, J=10.2,4.2$ $\mathrm{Hz}, 1 \mathrm{H}), 6.04$ (dd, $J=9.9,1.0 \mathrm{~Hz}, 1 \mathrm{H}), 5.29$ (ddd, $J=4.5,4.5,4.2 \mathrm{~Hz}, 1 \mathrm{H}), 2.77$ (dqd, $J=9.6$, 7.2, $4.9 \mathrm{~Hz}, 1 \mathrm{H}$ ), 2.25 (dddd, $J=13.9,4.9,4.9,1.0 \mathrm{~Hz}, 1 \mathrm{H}$ ), 2.06 (ddd, $J=13.9,9.4,4.5 \mathrm{~Hz}, 1 \mathrm{H}$ ), $1.49(\mathrm{~s}, 9 \mathrm{H}), 1.15(\mathrm{~d}, J=6.9 \mathrm{~Hz}, 3 \mathrm{H}) ;{ }^{13} \mathrm{C} \mathrm{NMR}\left(\mathrm{CDCl}_{3}, 67.5 \mathrm{MHz}\right) \delta 201.0,152.8,143.3,131.2$, $82.9,68.1,37.7,35.4,27.7,14.9$;

\section{t-Butyl (1S,4R,5S)-4-hydroxy-5-methylcyclohex-2-enyl carbonate (II-16)}

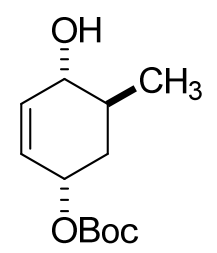

To a solution of $69 \mathrm{mg}$ enone II-15 $(0.30 \mathrm{mmol})$ in $6 \mathrm{~mL}$ THF at $-78{ }^{\circ} \mathrm{C}$ was added $40 \mathrm{mg}$ $\mathrm{LiAlH}_{4}(0.92 \mathrm{mmol})$ in several portions. The reaction mixture was stirred at $-78{ }^{\circ} \mathrm{C}$ for $30 \mathrm{~min}$, then $2 \mathrm{~mL}$ acetone was added dropwise at $-78^{\circ} \mathrm{C}$ and the mixture was stirred for another $20 \mathrm{~min}$ to quench the reaction. Then it was diluted with $30 \mathrm{~mL}$ EtOAc and $6 \mathrm{~mL}$ saturated aqueous $\mathrm{NH}_{4} \mathrm{Cl}$ was added. The mixture was warmed up and stirred at $0{ }^{\circ} \mathrm{C}$ for $15 \mathrm{~min}$ and then aqueous layer was extracted with EtOAc $30 \mathrm{~mL}$ x 2. The pooled organic layer was washed with $5 \mathrm{~mL}$ 
saturated aqueous $\mathrm{NH}_{4} \mathrm{Cl}, 5 \mathrm{~mL}$ saturated brine, dried over $\mathrm{Na}_{2} \mathrm{SO}_{4}$. The solution was concentrated under reduced pressure to give a residue, which was purified by chromatography on silica gel column. Elution with hexane-EtOAc (5:1) afforded allylic alcohol II-16 (60 mg, 90\%) as white solid; $\mathrm{mp}: 81.6-83.6^{\circ} \mathrm{C} ; R_{f}=0.27\left(4: 1 \mathrm{v} / \mathrm{v}\right.$ hexane/EtOAc); $[\alpha]_{\mathrm{D}}{ }^{20}-131\left(c 1.05, \mathrm{CHCl}_{3}\right)$; IR (thin film, $\mathrm{cm}^{-1}$ ) v 3354, 2974, 1732, 1455, 1397, 1370, 1340, 1281, 1256, 1155, 1058, 1035, 945; ${ }^{1} \mathrm{H}\left(\mathrm{CDCl}_{3}, 600 \mathrm{MHz}\right) \delta 5.95$ (ddd, $J=9.6,1.8 \mathrm{~Hz}, 1 \mathrm{H}$ ), 5.85 (dddd, $J=10.2,4.2,1.8,1.8$ Hz, 1H), 4.99 (m, 1H), 3.70 (ddd, $J=9.0,3.6,1.8 \mathrm{~Hz}, 1 \mathrm{H}$ ), 1.91 (dddd, $J=14.4,3.0,3.0,1.8 \mathrm{~Hz}$, 1H), 1.82 (ddqd, $J=12.0,9.6,6.6,3.0 \mathrm{~Hz}, 1 \mathrm{H}), 1.57$ (ddd, $J=15.0,12.0,4.8 \mathrm{~Hz}, 1 \mathrm{H}), 1.48$ (s, 9H), $1.07(\mathrm{~d}, J=6.6 \mathrm{~Hz}, 3 \mathrm{H}) ;{ }^{13} \mathrm{C} \mathrm{NMR}\left(\mathrm{CDCl}_{3}, 150 \mathrm{MHz}\right) \delta 153.2,136.9,125.4,82.1,73.2$, 69.1, 34.2, 32.8, 27.8, 18.0; HRMS (ESI) calcd. for $\left[\mathrm{C}_{12} \mathrm{H}_{20} \mathrm{O}_{4}+\mathrm{Na}\right]^{+}:$251.1254, Found: 251.1254.

\section{t-Butyl (1S,2S,3S,4R,5S)-2,3,4-trihydroxy-5-methylcyclohexyl carbonate (II-17)}

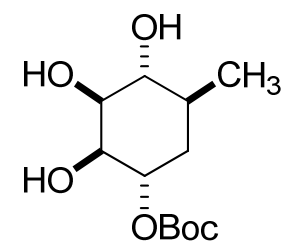

To a solution of $37 \mathrm{mg}$ olefin II-16 $(0.16 \mathrm{mmol})$ in $1.5 \mathrm{~mL} t$-BuOH-actone- $50 \% \mathrm{NMO}_{(\text {aq.) }}(1: 1: 1$ $\mathrm{v} / \mathrm{v} / \mathrm{v}$ ) was added a small piece of crystalline $\mathrm{OsO}_{4}$ at $0{ }^{\circ} \mathrm{C}$. The reaction mixture was stirred at $0{ }^{\circ} \mathrm{C}$ for overnight, then $1.5 \mathrm{~mL}$ saturated aqueous $\mathrm{Na}_{2} \mathrm{~S}_{2} \mathrm{O}_{4}$ was added and it was stirred at $0{ }^{\circ} \mathrm{C}$ for another $2 \mathrm{~h}$. The reaction mixture was passed through a pad of sand-silica gel-celite, rinsing with EtOAc-MeOH $(10: 1, \mathrm{v} / \mathrm{v})$. The effluent was dried over $\mathrm{Na}_{2} \mathrm{SO}_{4}$ and concentrated under reduced pressure to give a residue, which was purified by silica gel column. Elution with hexaneEtOAc (1:2) gave triol II-17 (37 mg, 87\%) as colorless oil: $R_{f}=0.27(1: 3(\mathrm{v} / \mathrm{v})$ hexane/EtOAc); $[\alpha]_{\mathrm{D}}^{20}+10(c 1.9, \mathrm{MeOH})$; IR (thin film, $\left.\mathrm{cm}^{-1}\right) v 3388,2933,1740,1457,1369,1277,1255,1157$, 1051, 960; ${ }^{1} \mathrm{H}$ NMR $\left(\mathrm{CD}_{3} \mathrm{OD}, 600 \mathrm{MHz}\right) \delta 4.69(\mathrm{ddd}, J=3.6,3.0,1.8 \mathrm{~Hz}, 1 \mathrm{H}), 3.92$ (ddd, $J=$ 3.6, 3.6, $1.2 \mathrm{~Hz}, 1 \mathrm{H}), 3.48$ (dd, $J=9.6,3.6 \mathrm{~Hz}, 1 \mathrm{H}), 3.29$ (ddd, $J=9.0,9.0,1.8 \mathrm{~Hz}, 1 \mathrm{H}), 1.67(\mathrm{~m}$, $3 \mathrm{H}), 1.46(\mathrm{~s}, 9 \mathrm{H}), 1.02(\mathrm{~d}, J=5.4 \mathrm{~Hz}, 3 \mathrm{H}) ;{ }^{13} \mathrm{C} \mathrm{NMR}\left(\mathrm{CD}_{3} \mathrm{OD}, 150 \mathrm{MHz}\right) \delta 154.4,83.1,76.4$, 
76.3, 74.5, 72.2, 33.8, 33.2, 28.2, 18.4; HRMS (ESI) calcd. for $\left[\mathrm{C}_{12} \mathrm{H}_{22} \mathrm{O}_{6}+\mathrm{Na}\right]^{+}: 285.1309$, Found: 285.1309 .

\section{5a-Carba- $\alpha$-D-rhamnopyranose (II-17)}<smiles>CC1C[C@H](O)C(O)C(O)C1O</smiles>

To a solution of $26 \mathrm{mg}$ carbonate $\mathbf{I I - 1 7}(0.10 \mathrm{mmol})$ in $2 \mathrm{~mL}$ THF at $0{ }^{\circ} \mathrm{C}$ was added $26 \mathrm{mg}$ $\mathrm{LiAlH}_{4}(0.68 \mathrm{mmol})$. The reaction mixture was warmed up and stirred at RT for $1 \mathrm{~h}$, then it was diluted with $10 \mathrm{~mL}$ EtOAc and $2 \mathrm{~mL}$ saturated aqueous $\mathrm{NaHCO}_{3}$ was added. It was stirred for another 20 min to quench the reaction and then the mixture was passed through a pad of sandsilica gel-celite, eluting with EtOAc-MeOH $(10: 1 \mathrm{v} / \mathrm{v})$. The effluent was dried over $\mathrm{Na}_{2} \mathrm{SO}_{4}$. The solution was concentrated under reduced pressure to give a residue, which was purified by silica gel column. Elution with EtOAc-MeOH (10:1) afforded cyclitol II-7 (13 mg, 83\%) as colorless oil; $R_{f}=0.24\left(5: 1(\mathrm{v} / \mathrm{v})\right.$ EtOAc/MeOH); $[\alpha]_{\mathrm{D}}^{20}-17(c 0.58, \mathrm{MeOH})$; IR (thin film, $\left.\mathrm{cm}^{-1}\right) v 3315$, 2922, 1404, 1377, 1281, 1240, 1186, 1154, 1052, 1003, 964; ${ }^{1} \mathrm{H}$ NMR $\left(\mathrm{CD}_{3} \mathrm{OD}, 600 \mathrm{MHz}\right) \delta$ $3.87(\mathrm{dd}, J=3.6,3.0 \mathrm{~Hz}, 1 \mathrm{H}), 3.83(\mathrm{ddd}, J=3.6,3.0,3.0 \mathrm{~Hz}, 1 \mathrm{H}), 3.61(\mathrm{dd}, J=9.6,3.0 \mathrm{~Hz}, 1 \mathrm{H})$, $3.26(\mathrm{dd}, J=9.6,9.6 \mathrm{~Hz}, 1 \mathrm{H}), 1.78(\mathrm{ddqd}, J=10.2,9.6,6.6,6.6 \mathrm{~Hz}, 1 \mathrm{H}), 1.59(\mathrm{~m}, 2 \mathrm{H}), 1.01(\mathrm{~d}$, $J=6.6 \mathrm{~Hz}, 3 \mathrm{H}) ;{ }^{13} \mathrm{C} \mathrm{NMR}\left(\mathrm{CD}_{3} \mathrm{OD}, 150 \mathrm{MHz}\right) \delta 76.9,75.0,74.3,71.1,35.9,33.1,18.6$;

(2S,3S,4aR,5aS,7aR)-octahydro-2,3-dimethoxy-2,3-dimethyl-2H-bicyclo[4.1.0]hept-1(6)eno[3,4-b][1,4]dioxin-5a-ol (II-18b) 


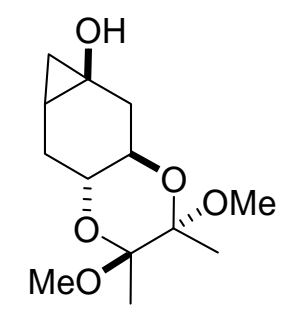

To a solution of $5.2 \mathrm{~g}$ enone II-6 (21.5 mmol) in $74 \mathrm{~mL}$ toluene was added $30 \mathrm{mg} \mathrm{RhCl}\left(\mathrm{PPh}_{3}\right)_{3}$ $(0.065 \mathrm{mmol})$ and $4.1 \mathrm{~mL}$ phenyldimethylsilane $(26.9 \mathrm{mmol})$, the mixture was stirred at $60{ }^{\circ} \mathrm{C}$ for 1 hour. Then the reaction mixture was cooled down to $0{ }^{\circ} \mathrm{C}$, into which a solution of $5.96 \mathrm{~g}$ $\mathrm{ZnEt}_{2}(48.3 \mathrm{mmol})$ was slowly added followed by addition of $4.9 \mathrm{~mL} \mathrm{CH}_{2} \mathrm{I}_{2}(60.7 \mathrm{mmol})$. The reaction mixture was stirred at $0{ }^{\circ} \mathrm{C}$ overnight. Then it was diluted with $300 \mathrm{~mL}$ hexane-ether (v/v 1:1) and quenched with $120 \mathrm{~mL}$ saturated aqueous $\mathrm{NH}_{4} \mathrm{Cl}$. The aqueous layer was extracted with hexane-ether (v/v 1:1) $300 \mathrm{~mL} \mathrm{X} \mathrm{2.} \mathrm{The} \mathrm{combined} \mathrm{organic} \mathrm{layer} \mathrm{was} \mathrm{washed} \mathrm{with} 10 \mathrm{~mL}$ saturated aqueous $\mathrm{Na}_{2} \mathrm{~S}_{2} \mathrm{O}_{3}, 100 \mathrm{~mL}$ saturated brine, dried over $\mathrm{Na}_{2} \mathrm{SO}_{4}$. The solution was then concentrated to a residue, which was redissolved in $215 \mathrm{~mL} \mathrm{MeOH}$ at $0{ }^{\circ} \mathrm{C}$ and $326 \mathrm{mg}(1.72$ mmol) $p$ - TsOH was added in portions. It was stirred at $0{ }^{\circ} \mathrm{C}$ for $15 \mathrm{~min}$, and then quenched with $10 \mathrm{~mL}$ saturated aqueous $\mathrm{NaHCO}_{3}$ and $1 \mathrm{~g} \mathrm{NaHCO}_{3}$. The mixture was concentrated under reduced pressure to remove most of solvent, then $50 \mathrm{~mL}$ EtOAc was added and concentrated again to remove more solvent. The residue $(\sim 10 \mathrm{~mL})$ was directly loaded onto a silica gel column. Elution with hexane-EtOAc $(3: 2, \mathrm{v} / \mathrm{v})$ afforded cyclopropanol $(4 \mathrm{~g}, 73 \%)$ as diastereomeric mixture $(\mathrm{dr} \sim 1: 1)$.

To a solution of $2.0 \mathrm{~g}$ diastereomeric mixture $(7.75 \mathrm{mmol}, \mathrm{dr} \sim 1: 1)$ in $300 \mathrm{~mL}$ EtOAc was added $700 \mathrm{mg} 10 \% \mathrm{Pd} / \mathrm{C}$. The solution was degassed using vacuum and refilled with $\mathrm{H}_{2}$ three times at low temperature $\left(\sim-30^{\circ} \mathrm{C}\right)$. Then at room temperature, the solution was stirred for $6 \mathrm{hr}$ under balloon pressure of $\mathrm{H}_{2}$. It was then filtered through a filter paper and rinse with ethyl acetate. The solution was washed with $30 \mathrm{~mL}$ saturated aqueous $\mathrm{NaHCO}_{3}, 30 \mathrm{~mL}$ saturated aqueous $\mathrm{NaCl}$, dried over $\mathrm{Na}_{2} \mathrm{SO}_{4}$ and then concentrated under reduced pressure to a small volume, which was subjected to chromatography on silica gel column. Elution with hexane-EtOAc (2.5:1) gave product diastereomeric mixture of II-19 $\left(1.26 \mathrm{~g}, 63 \%, \mathrm{dr}_{(\mathrm{II-19} / \mathrm{a} / \mathrm{I}-19 \mathrm{~b})} \sim 2: 1\right)$ and elution with hexane-EtOAc (1:1) gave pure cyclopropanol II-18b (420 mg, 21\%). II-18b: Colorless oil; $R_{f}=$ $0.48\left(1: 2(\mathrm{v} / \mathrm{v})\right.$ hexane/EtOAc); $[\alpha]_{\mathrm{D}}{ }^{20}=+159\left(c 1.0, \mathrm{CHCl}_{3}\right) ;$ IR (thin film, $\left.\mathrm{cm}^{-1}\right) v 3405,2992$, 
2933, 2833, 1456, 1377, 1218, 1200, 1123, 1050, 1038; ${ }^{1} \mathrm{H}$ NMR $\left(600 \mathrm{MHz}, \mathrm{CDCl}_{3}\right) \delta 3.61(\mathrm{ddd}$, $J=11.4,10.8,7.2 \mathrm{~Hz}, 1 \mathrm{H}), 3.25$ (s, $3 \mathrm{H}), 3.23$ (s, 3H), 3.22 (ddd, $J=11.4,10.2,5.4 \mathrm{~Hz}, 1 \mathrm{H})$, 2.51 (ddd, $J=12.6,5.4,0.6 \mathrm{~Hz}, 1 \mathrm{H}$ ), 2.31 (ddd, $J=16.2,9.6,7.2 \mathrm{~Hz}, 1 \mathrm{H}$ ), 2.03 (ddd, $J=12.0$, 10.8, $1.2 \mathrm{~Hz}, 1 \mathrm{H}), 1.36$ (ddd, $J=13.8,11.4,2.4 \mathrm{~Hz}, 1 \mathrm{H}), 1.28$ (s, 3H), 1.26 (s, 3H), 1.12 (dddd, $J$ $=9.6,9.6,6.6,1.2 \mathrm{~Hz}, 1 \mathrm{H}), 1.01(\mathrm{ddd}, J=10.8,6.0,1.8 \mathrm{~Hz}, 1 \mathrm{H}), 0.38(\mathrm{dd}, J=6.0,5.4 \mathrm{~Hz}, 1 \mathrm{H})$; ${ }^{13} \mathrm{C}$ NMR $\left(150 \mathrm{MHz}, \mathrm{CDCl}_{3}\right) \delta 99.2,99.0,68.9,67.1,55.9,47.9,47.8,36.0,29.6,20.9,18.0$, 17.8. HRMS (ESI): calcd. for $\left[\mathrm{C}_{13} \mathrm{H}_{22} \mathrm{O}_{5}+\mathrm{Na}\right]^{+}: 281.13594$, Found: 281.13599 .

$(2 S, 3 S, 4 a R, 7 R, 8 a R)$-hexahydro-2,3-dimethoxy-2,3,7-trimethylbenzo[b][1,4]dioxin-6(7H)one (II-19a)

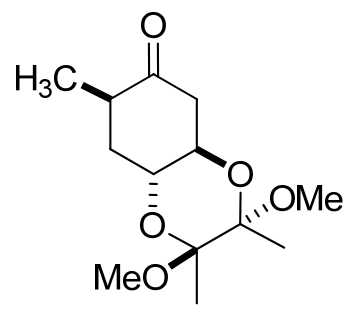

(2S,3S,4aR,7S,8aR)-hexahydro-2,3-dimethoxy-2,3,7-trimethylbenzo[b][1,4]dioxin-6(7H)one (II-19b)

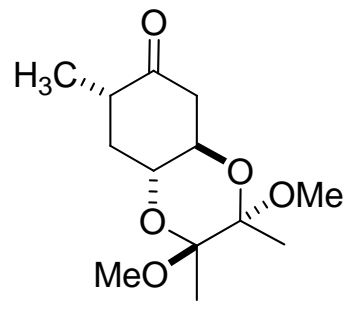




\section{Separation of II-19a and II-19b}

Diastereomeric mixture of II-19a/II-19b $(6.5 \mathrm{~g}, \mathrm{dr} \sim 2: 1)$ was recrystallized from hexane-ether multiple times, which resulted in diastereomerically enriched II-19a $\left(\sim 3.3 \mathrm{~g}, \mathrm{dr}_{(\mathbf{1 9 a} / \mathbf{1 9 b})} \sim 10: 1\right)$ from the mother solutions and II-19b $\left(\sim 2.0 \mathrm{~g}, \mathrm{dr}_{(\mathbf{I I}-19 \mathbf{b} / \mathbf{I I}-19 \mathrm{a})}>20: 1\right)$ from the crystal form, as well as other small amount of mixtures (dr ratio range from 4:1 to 1:4). The II-19a enriched part and the II-19b enriched part were further purified respectively by silica gel column chromatography, eluting with hexane-EtOAc (6:1) to give more enriched II-19a (2.6 g, dr $\sim 18: 1,40 \%$ recovery from original mixture) and the pure II-19b (1.8g, 28\% recovery from original mixture).

II-19a $\left(\operatorname{dr}_{(\mathrm{II}-19 \mathrm{~b} / I I-19 a)}\right)$ 18:1): White solid; mp: 92.0-93.0 ${ }^{\circ} \mathrm{C} ; R_{f}=0.31(4: 1(\mathrm{v} / \mathrm{v})$ hexane/EtOAc); IR (thin film, $\mathrm{cm}^{-1}$ ) v 3016, 2949, 2911, 2840, 1731, 1461, 1430, 1364, 1277, 1204, 1108, 1073, 1039, 1025, 971; ${ }^{1} \mathrm{H} \mathrm{NMR}\left(\mathrm{CDCl}_{3}, 600 \mathrm{MHz}\right) \delta 4.00(\mathrm{ddd}, J=12.0,9.6,4.8 \mathrm{~Hz}, 1 \mathrm{H}), 3.74(\mathrm{ddd}$, $J=13.2,10.2,5.4 \mathrm{~Hz}, 1 \mathrm{H}), 3.28(\mathrm{~s}, 3 \mathrm{H}), 3.21(\mathrm{~s}, 3 \mathrm{H}), 2.61(\mathrm{dd}, J=15.0,12.6 \mathrm{~Hz}, 1 \mathrm{H}), 2.58(\mathrm{~m}$, 1H), 2.48 (ddd, $J=15.0,5.4,1.2 \mathrm{~Hz}, 1 \mathrm{H}), 1.84$ (ddd, $J=13.2,12.0,6.6 \mathrm{~Hz}, 1 \mathrm{H}), 1.74$ (ddd, $J=$ 13.8, 6.6, 3.0 Hz, 1H), 1.29 (s, 3H), $1.28(\mathrm{~s}, 3 \mathrm{H}), 1.20(\mathrm{~d}, J=7.2 \mathrm{~Hz}, 3 \mathrm{H}) ;{ }^{13} \mathrm{C} \mathrm{NMR}\left(\mathrm{CDCl}_{3}, 150\right.$ MHz) $\delta 210.7,99.7,99.3,68.8,66.3,48.0$, 47.9, 43.3, 41.5, 32.2, 17.9, 17.7, 17.6; HRMS (ESI) calcd. for $\left[\mathrm{C}_{13} \mathrm{H}_{22} \mathrm{O}_{5}+\mathrm{Na}\right]^{+}: 281.1359$, Found: 281.1360 .

II-19b: White crystal; mp: $174-175{ }^{\circ} \mathrm{C} ; R_{f}=0.33\left(4: 1(\mathrm{v} / \mathrm{v})\right.$ hexane/EtOAc); $[\alpha]_{\mathrm{D}}{ }^{20}=+145(c$ 0.37, CHCl3); IR (thin film, $\mathrm{cm}^{-1}$ ) v 2987, 2951, 2913, 2884, 2833, 1703,1468, 1444, 1431, 1377, 1277, 1231, 1220, 1202, 1156, 1112, 1081, 1054, 1037, 928; ${ }^{1} \mathrm{H}$ NMR (600 MHz, $\left.\mathrm{CDCl}_{3}\right)$ $\delta 3.96(\mathrm{ddd}, J=12.0,9.6,4.8 \mathrm{~Hz}, 1 \mathrm{H}), 3.71(\mathrm{ddd}, J=13.2,9.6,5.4 \mathrm{~Hz}, 1 \mathrm{H}), 3.32(\mathrm{~s}, 3 \mathrm{H}), 3.23$ (s, $3 \mathrm{H}), 2.60(\mathrm{dd}, J=13.8,5.4 \mathrm{~Hz}, 1 \mathrm{H}), 2.53(\mathrm{ddd}, J=13.2,13.2,1.2 \mathrm{~Hz}, 1 \mathrm{H}), 2.41$ (ddqd, $J=12.6$, 6.6, 6.6, 1.2 Hz, 1H), 2.08 (ddd, $J=12.6,6.6,4.2 \mathrm{~Hz}, 1 \mathrm{H}), 1.43$ (ddd, $J=13.2,12.6,12.0 \mathrm{~Hz}$, $1 \mathrm{H}), 1.32(\mathrm{~s}, 3 \mathrm{H}), 1.31(\mathrm{~s}, 3 \mathrm{H}), 1.07(\mathrm{~d}, J=6.0 \mathrm{~Hz}, 3 \mathrm{H}) ;{ }^{13} \mathrm{C} \mathrm{NMR}\left(150 \mathrm{MHz}, \mathrm{CDCl}_{3}\right) \delta 208.0$, 99.6, 99.4, 69.6, 69.4, 48.0, 47.9, 44.5, 43.0, 34.5, 17.7, 17.6, 14.0. HRMS (ESI): calcd. For $\left[\mathrm{C}_{13} \mathrm{H}_{22} \mathrm{O}_{5}+\mathrm{H}\right]^{+}: 259.1540$, Found: 259.1540 . 


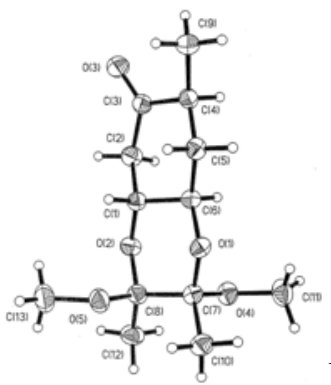

X-ray crystal data: Empirical formula $\mathrm{C}_{13} \mathrm{H}_{22} \mathrm{O}_{5}$; formula weight 258.31; Temperature 293(2) K; wavelength $0.71073 \AA$; crystal system monoclinic; space group $\mathrm{P} 2{ }_{1}$; uni cell dimensions $a=6.934(1) \AA, b=6.997(1) \AA, c=14.613(2) \AA, \alpha=90^{\circ}, \beta=99.312(2)^{\circ}, \gamma=90^{\circ}$; volume 699.66(17) $\AA^{3} ; \mathrm{Z}=2$; density (calculated) $1.226 \mathrm{~g} / \mathrm{cm}^{3}$; absorption coefficient $0.93 \mathrm{~cm}^{-1} ; \mathrm{F}(000)$ 280 ; crystal size $0.08 \times 0.18 \times 0.26 \mathrm{~mm} ; \theta$ range for data collection 2.83 to $27.55^{\circ}$; index ranges $-7 \leq \mathrm{h} \leq 9, \quad-9 \leq \mathrm{k} \leq 9, \quad-17 \leq \mathrm{l} \leq 18$; reflections collected 4849; independent reflections 3075 $[\mathrm{R}$ (int) $=0.0325]$; completeness to $\theta=27.55^{\circ} 97.3 \%$; refinement method Full-matrix least-squares on $\mathrm{F}^{2}$; data/restraints/parameters 3075/1/168; goodness-of-fit on $\mathrm{F}^{2} 1.040$; final $\mathrm{R}$ indices $[\mathrm{I}>2 \sigma(\mathrm{I})] \mathrm{R} 1=0.0497, \mathrm{wR} 2=0.1245 ; \mathrm{R}$ indices (all data) $\mathrm{R} 1=0.0649$, $\mathrm{wR} 2=0.1347$; absolute structure parameter -0.3(12); largest diff. peak and hole 0.243 and $-0.158 \mathrm{e} / \AA^{3}$.

\section{Epimerization of II-19a to II-19b}

Ketone II-19a enriched diastereomeric mixture $\left(\mathrm{dr}_{(\mathrm{II-19} / / \mathrm{I}-19 \mathrm{~b})} \sim 2: 1\right)$ was treated in a THF-conc. $\mathrm{HCl}$ (aq.) (v/v 2:1) at $0{ }^{\circ} \mathrm{C}$ for $30 \mathrm{~min}$ at $0.1 \mathrm{M}$ concentration. The solution was then neutralized with saturated aqueous $\mathrm{NaHCO}_{3}$ and extracted with EtOAc. The organic layer was washed with saturated aqueous $\mathrm{NaCl}$, dried over $\mathrm{Na}_{2} \mathrm{SO}_{4}$. Concentration of the solution under reduced pressure gave a residue. Crude ${ }^{1} \mathrm{H}$ NMR show that the reaction epimerized most of II-19a to II$19 b$, resulting in a II-19b enriched mixture $\left(\operatorname{dr}_{(\mathbf{I I - 1 9 b / I - 1 9 a )}}>13: 1\right)$. Chromatography on silica gel afforded pure II-19b ( 90\%).

\section{t-butyl (1R,5S)-5-methyl-4-oxocyclohex-2-enyl carbonate (II-20)}




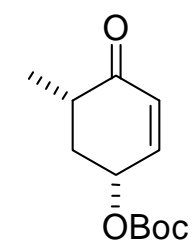

A $2.03 \mathrm{~g}$ sample of bisketal II-19b $(7.86 \mathrm{mmol})$ was placed in $250 \mathrm{~mL}$ flask, a $5 \mathrm{~mL}$ solution of $\mathrm{TFA} / \mathrm{H}_{2} \mathrm{O}(5: 1 \mathrm{v} / \mathrm{v})$ was added into the flask at $\mathrm{RT}$ when stirring. The mixture was continued to be stirred at RT for $15 \mathrm{~min}$, then cooled down to $0{ }^{\circ} \mathrm{C}$ and diluted with $50 \mathrm{~mL}$ EtOAc, into which $20 \mathrm{~mL}$ saturated aqueous $\mathrm{NaHCO}_{3}$, as well as $10 \mathrm{~g} \mathrm{NaHCO}_{3}$ (s) was slowly added to quench the reaction. The mixture was run through a pad of sand-silica gel-celite, rinsing with EtOAc-MeOH $(10: 1, \mathrm{v} / \mathrm{v})$, and the effluent was dried over $\mathrm{Na}_{2} \mathrm{SO}_{4}$. The solution was concentrated under reduced pressure to give a residue. Crude ${ }^{1} \mathrm{H}$ NMR showed that a little isomerization on the $\alpha$ methyl group happened with dr ratio 25:1. The residue was then subjected to chromatography on silica gel column. Elution with hexane-EtOAc (1:3) gave product diol (930 mg, 88\%, dr 15:1).

$930 \mathrm{mg}$ diol (6.46 mmol, dr 15:1) was dissolved in $32 \mathrm{~mL} \mathrm{THF-} \mathrm{CH}_{2} \mathrm{Cl}_{2}$ (v/v 1:1) at RT, into which $73 \mathrm{mg}$ DMAP was added in, followed by addition of a solution of $5.0 \mathrm{~g} \mathrm{Boc}_{2} \mathrm{O}(22.9$ $\mathrm{mmol}$ ) in $5 \mathrm{~mL} \mathrm{CH}_{2} \mathrm{Cl}_{2}$. The mixture was stirred at RT for $2 \mathrm{hr}$, then it was cooled down to $0{ }^{\circ} \mathrm{C}$, diluted with $\mathrm{Et}_{2} \mathrm{O} 30 \mathrm{~mL}$ and quenched by $15 \mathrm{~mL}$ saturated aqueous $\mathrm{NaHCO}_{3}$. The mixture was stirred at $0{ }^{\circ} \mathrm{C}$ for another $2 \mathrm{hr}$ and then was extracted with Hexane-Et $2 \mathrm{O}(1: 2 \mathrm{v} / \mathrm{v}) 150 \mathrm{~mL} \mathrm{X} 2$. The pooled organic layer was washed with $15 \mathrm{~mL}$ saturated aqueous $\mathrm{NaCl}$ and dried over $\mathrm{Na}_{2} \mathrm{SO}_{4}$. The solution was concentrated under reduced pressure to afford a residue.

The residue was redissolved in $35 \mathrm{~mL} \mathrm{CH}_{2} \mathrm{Cl}_{2}$ at $0{ }^{\circ} \mathrm{C}$. Into the solution $5.5 \mathrm{~mL} \mathrm{Et}_{3} \mathrm{~N}$ was slowly added in. The mixture was then stirred at $0{ }^{\circ} \mathrm{C}$ overnight and continued to be stirred at RT for another $2 \mathrm{hr}$. Then it was cooled down to $0{ }^{\circ} \mathrm{C}$, diluted with hexane- $\mathrm{Et}_{2} \mathrm{O}(1: 1 \mathrm{v} / \mathrm{v}) 130 \mathrm{~mL}$ and quenched by $50 \mathrm{~mL} 0.5 \mathrm{M} \mathrm{NaHSO}_{4}$. The aqueous layer was then extracted with hexane-Et $2 \mathrm{O}$ $(1: 1 \mathrm{v} / \mathrm{v}) 150 \mathrm{~mL} \mathrm{X} 2$. The pooled organic layer was washed with $20 \mathrm{~mL} 0.5 \mathrm{M} \mathrm{NaHSO}_{4}, 20 \mathrm{~mL}$ saturated aqueous $\mathrm{NaHCO}_{3}, 20 \mathrm{~mL}$ saturated aqueous $\mathrm{NaCl}$ and dried over $\mathrm{Na}_{2} \mathrm{SO}_{4}$. The solution was concentrated under reduced pressure to afford a residue, which was subjected to chromatography on silica gel column. Elution with hexane-EtOAc $(10: 1 \mathrm{v} / \mathrm{v})$ gave enone II-20 (1.23g, $84 \%$ overall for two steps, $40 \%$ with $\mathrm{dr}>25: 1$ and $44 \%$ with $\mathrm{dr} \sim 8: 1$ ). 
II-20: White solid; mp: $64.7-66.1^{\circ} \mathrm{C}, R_{f}=0.45\left(4: 1(\mathrm{v} / \mathrm{v})\right.$ hexane/EtOAc); $[\alpha]_{\mathrm{D}}{ }^{20}+42(c 4.23$, $\mathrm{CHCl}_{3}$ ); IR (thin film, cm ${ }^{-1}$ ) v 2982, 2948, 1733, 1683, 1618, 1479, 1457, 1366, 1336, 1275 , 1252, 1153, 1098, 1082, 974; ${ }^{1} \mathrm{H} \mathrm{NMR}\left(\mathrm{CDCl}_{3}, 270 \mathrm{MHz}\right) \delta 6.81(\mathrm{ddd}, J=10.2,2.4,1.8 \mathrm{~Hz}$, 1H), 5.99 (dd, $J=9.0,2.4 \mathrm{~Hz}, 1 \mathrm{H}), 5.46$ (dddd, $J=10.8,5.4,2.4,2.4 \mathrm{~Hz}, 1 \mathrm{H}), 2.43$ (m, 2H), $1.84(\mathrm{ddd}, J=14.4,12.6,10.4 \mathrm{~Hz}, 1 \mathrm{H}), 1.5(\mathrm{~s}, 9 \mathrm{H}), 1.15(\mathrm{~d}, J=6.0 \mathrm{~Hz}, 3 \mathrm{H}),{ }^{13} \mathrm{C} \mathrm{NMR}\left(\mathrm{CDCl}_{3}\right.$, $67.5 \mathrm{MHz}) \delta 200.0,152.7,147.9,130.0,82.9$, 71.7, 39.9, 37.5, 27.7, 14.8; HRMS (ESI) calcd. for $\left[\mathrm{C}_{12} \mathrm{H}_{18} \mathrm{O}_{4}+\mathrm{H}\right]^{+}: 227.12779$, Found: 227.12780 .

\section{t-Butyl (1R,4R,5S)-4-hydroxy-5-methylcyclohex-2-enyl carbonate (II-20a)}

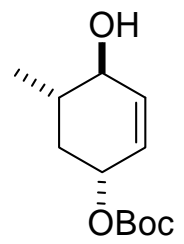

To a solution of $545 \mathrm{mg}$ enone II-20 (2.41mmol, dr 10:1) in $35 \mathrm{~mL}$ THF at $-78{ }^{\circ} \mathrm{C}$ was added $200 \mathrm{mg} \mathrm{LiAlH} 4(5.26 \mathrm{mmol})$ in several portions. The reaction mixture was stirred at $-78^{\circ} \mathrm{C}$ for $30 \mathrm{~min}$, then $2 \mathrm{~mL}$ acetone was added dropwise in at $-78{ }^{\circ} \mathrm{C}$ and the mixture was stirred for another $20 \mathrm{~min}$ to quench the reaction. Then it was diluted with $\mathrm{Et}_{2} \mathrm{O} 150 \mathrm{~mL}$ and $50 \mathrm{~mL}$ saturated aqueous $\mathrm{NH}_{4} \mathrm{Cl}$ was added. The mixture was stirred at $0{ }^{\circ} \mathrm{C}$ for 15 min then aqueous layer was extracted with $\mathrm{Et}_{2} \mathrm{O} 200 \mathrm{~mL}$ X 2. The pooled organic layer was washed with $20 \mathrm{~mL}$ saturated aqueous $\mathrm{NH}_{4} \mathrm{Cl}, 20 \mathrm{~mL}$ saturated brine, dried over $\mathrm{Na}_{2} \mathrm{SO}_{4}$. The solution was concentrated under reduced pressure to give a residue. Crude ${ }^{1} \mathrm{H}$ NMR showed that there was three isomers with diastereomeric ratio 11:1:1.1. The two minor isomers were easily removed by chromatography on silica gel column, elution with hexane-EtOAc (5:1) afforded allylic alcohol II-20a (450 mg, 82\%).

II-20a: White solid; mp: $100.2-101.7{ }^{\circ} \mathrm{C} ; R_{f}=0.15(4: 1 \mathrm{v} / \mathrm{v}$ hexane/EtOAc $) ;[\alpha]_{\mathrm{D}}{ }^{20}+95(c 1.90$, $\mathrm{CHCl}_{3}$ ); IR (thin film, cm ${ }^{-1}$ ) v 3515, 2977, 2941, 2878, 1713, 1458, 1396, 1351, 1366, 1334, 1284, 1257, 1153, 1076, 1054, 956; ${ }^{1} \mathrm{H}\left(\mathrm{CDCl}_{3}, 600 \mathrm{MHz}\right) \delta 5.80$ (ddd, $J=10.2,2.4,1.8 \mathrm{~Hz}$, 1H), 5.73 (ddd, $J=10.2,3.6,1.8 \mathrm{~Hz}, 1 \mathrm{H}), 5.04$ (dddd, $J=9.6,7.2,2.4,1.8 \mathrm{~Hz}, 1 \mathrm{H}), 3.85$ (ddd, $J$ 
$=9.0,4.8,2.4 \mathrm{~Hz}, 1 \mathrm{H}), 2.13(\mathrm{dddd}, J=12.6,5.4,2.4,1.8 \mathrm{~Hz}, 1 \mathrm{H}), 1.65$ (dddd, $J=12.0,9.6,6.6$, $3.0 \mathrm{~Hz}, 1 \mathrm{H}), 1.49$ (s, 9H), 1.45 (ddd, $J=13.2,13.2,10.2 \mathrm{~Hz}, 1 \mathrm{H}), 1.12(\mathrm{~d}, J=6.6 \mathrm{~Hz}, 3 \mathrm{H}) ;{ }^{13} \mathrm{C}$ NMR $\left(\mathrm{CDCl}_{3}, 150 \mathrm{MHz}\right) \delta 153.1,134.4,128.4,82.2,73.3,72.8,37.3,35.8,27.8,18.2$; HRMS (ESI) calcd. for $\left[\mathrm{C}_{12} \mathrm{H}_{20} \mathrm{O}_{4}+\mathrm{Na}\right]^{+}: 251.12538$, Found: 251.12550 .

\section{t-Butyl (1S,5S)-5-methylcyclohex-3-enyl carbonate (II-21)}

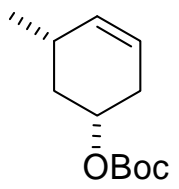

To a solution of $312 \mathrm{mg}$ allylic diol II-20a $(1.37 \mathrm{mmol})$ in $6 \mathrm{~mL} N$-methylmorpholine at $-25{ }^{\circ} \mathrm{C}$ was added $1.07 \mathrm{~g}(4.1 \mathrm{mmol}) \mathrm{PPh}_{3}$, followed by dropwise addition of $0.81 \mathrm{~mL} \mathrm{DIAD,} \mathrm{the}$ mixture was stirred at $-25{ }^{\circ} \mathrm{C}$ for $10 \mathrm{~min}$, then $889 \mathrm{mg} \mathrm{NBSH}(4.1 \mathrm{mmol}, \quad o-$ nitrobenzenesulfonylhydrazide) was added in. It was stirred at $-25{ }^{\circ} \mathrm{C}$ for $2 \mathrm{hr}$ and then was warmed up and stirred at RT for another $2 \mathrm{hr}$. The reaction mixture was diluted with $120 \mathrm{~mL}$ hexane-Et ${ }_{2} \mathrm{O}(1: 1 \mathrm{v} / \mathrm{v})$ and washed at $0{ }^{\circ} \mathrm{C}$ with $30 \mathrm{~mL} 0.5 \mathrm{M} \mathrm{NaHSO}_{4}$. The aqueous layer was extracted with hexane-Et $2 \mathrm{O}(1: 1 \mathrm{v} / \mathrm{v}) 120 \mathrm{~mL} \mathrm{X} 2$. The pooled organic layer was washed with 30 $\mathrm{mL} 0.5 \mathrm{M} \mathrm{NaHSO}_{4}$ again, then with $20 \mathrm{~mL}$ saturated aqueous $\mathrm{NaHCO}_{3}, 20 \mathrm{~mL}$ saturated brine, dried over $\mathrm{Na}_{2} \mathrm{SO}_{4}$. The organic layer was concentrated under reduced pressure to give crude product, which was then subjected to chromatography on silica gel. Elution with hexane-EtOAc $(20: 1 \mathrm{v} / \mathrm{v})$ gave olefin II-21 (260 mg, 89\%).

II-21: Colorless oil; $R_{f}=0.69\left(4: 1 \mathrm{v} / \mathrm{v}\right.$ hexane/EtOAc); $[\alpha]_{\mathrm{D}}{ }^{20}-55\left(c 0.89, \mathrm{CHCl}_{3}\right)$; IR (thin film, $\mathrm{cm}^{-1}$ ) v 3024, 2958, 2934, 2873, 1736, 1476, 1457, 1393, 1368, 1313, 1300, 1275, 1249, 1159, 1097, 1066, 983; ${ }^{1} \mathrm{H} \mathrm{NMR}\left(\mathrm{CDCl}_{3}, 600 \mathrm{M}\right): 5.53$ (dddd, $\left.J=9.6,4.8,2.4,2.4 \mathrm{~Hz}, 1 \mathrm{H}\right), 5.47$ (dddd, $J=10.2,3.0,3.0,1.2 \mathrm{~Hz}, 2 \mathrm{H}), 4.77$ (dddd, $J=12.0,9.6,6.0,3.6 \mathrm{~Hz}, 1 \mathrm{H}), 2.41$ (m, 2H), $2.10(\mathrm{~m}, 1 \mathrm{H}), 1.49(\mathrm{~s}, 9 \mathrm{H}), 1.29(\mathrm{ddd}, J=12.0,12.0,12.0 \mathrm{~Hz}, 1 \mathrm{H}), 1.02(\mathrm{~d}, J=7.2 \mathrm{~Hz}, 3 \mathrm{H}) ;{ }^{13} \mathrm{C}$ NMR $\left(\mathrm{CDCl}_{3}, 150 \mathrm{MHz}\right) \delta 153.1,133.2,122.7,81.8,73.4,37.2,31.0,30.9,27.8,21.3$; HRMS (ESI) calcd. for $\left[\mathrm{C}_{12} \mathrm{H}_{20} \mathrm{O}_{3}+\mathrm{Na}\right]^{+}: 235.13047$, Found: 235.13049 . 
t-Butyl (3aR,4S,6R,7aS)-hexahydro-2,2,4-trimethylbenzo[d][1,3]dioxol-6-yl carbonate (II22)

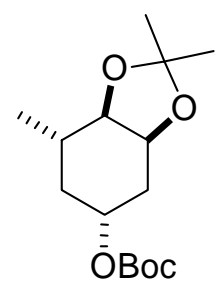

To a solution of $138 \mathrm{mg}$ olefin II-21 $(0.65 \mathrm{mmol})$ in $3 \mathrm{~mL} \mathrm{t-BuOH-Actone-50 \%} \mathrm{NMO} \mathrm{(aq.)}$ $(1: 1: 1 \mathrm{v} / \mathrm{v} / \mathrm{v})$ was added a small piece of crystalline $\mathrm{OsO}_{4}$ at $0{ }^{\circ} \mathrm{C}$. The reaction mixture was stirred at $0{ }^{\circ} \mathrm{C}$ for overnight, then $3 \mathrm{~mL}$ saturated aqueous $\mathrm{Na}_{2} \mathrm{~S}_{2} \mathrm{O}_{4}$ was added and it was stirred at $0{ }^{\circ} \mathrm{C}$ for another $3 \mathrm{hr}$. The reaction mixture was passed through a pad of sand-silica gel-celite, rinsing with EtOAc. The effluent was dried over $\mathrm{Na}_{2} \mathrm{SO}_{4}$ and concentrated under reduced pressure to give a residue. Crude ${ }^{1} \mathrm{H}-\mathrm{NMR}$ showed that the diastereoselectivity is $11: 1$. The residue was subjected to chromatography on silica gel column, eluting with hexane-EtOAc (1:1.5) gave inseparable mixture of diol (156 mg, 95\%, dr 11:1).

A $160 \mathrm{mg}$ sample of diol $(0.65 \mathrm{mmol}$, dr 11:1) was treated with $1 \mathrm{~mL}$ dimethoxypropane in the presence of $6.3 \mathrm{mg} p$-TsOH $(0.033 \mathrm{mmol})$ at $0{ }^{\circ} \mathrm{C}$ for $2 \mathrm{hr}$. It was diluted with $40 \mathrm{~mL}$ hexane$\mathrm{Et}_{2} \mathrm{O}(1: 1 \mathrm{v} / \mathrm{v})$ and quenched with $3 \mathrm{~mL}$ saturated aqueous $\mathrm{NaHCO}_{3}$ at $0{ }^{\circ} \mathrm{C}$. The aqueous layer was extracted with hexane-Et ${ }_{2} \mathrm{O}(1: 1 \mathrm{v} / \mathrm{v}) 40 \mathrm{~mL}$ X 2. The pooled organic layer was washed with $3 \mathrm{~mL}$ saturated brine, dried over $\mathrm{Na}_{2} \mathrm{SO}_{4}$. The solution was concentrated under reduced pressure to give a residue. The minor diastereomer was easily removed by chromatography on silica gel column, elution with hexane-EtOAc (25:1) afforded acetonide II-22 (155 mg, 83\%).

II-22: Colorless oil; $R_{f}=0.51\left(4: 1(\mathrm{v} / \mathrm{v})\right.$ hexane-EtOAc); $[\alpha]_{\mathrm{D}}{ }^{20}+23\left(c\right.$ 1.74, $\left.\mathrm{CHCl}_{3}\right)$; IR (thin film, $\left.\mathrm{cm}^{-1}\right) v$ 2982, 2933, 2874, 1736, 1459, 1368, 1276, 1242, 1218, 1156, 1101, 1065, 1042, 975; ${ }^{1} \mathrm{H} \mathrm{NMR}\left(\mathrm{CDCl}_{3}, 600 \mathrm{MHz}\right) \delta 4.84$ (dddd, $\left.J=10.8,10.8,4.2,4.2 \mathrm{~Hz}, 1 \mathrm{H}\right), 4.31$ (ddd, $J=$ 4.8, 4.8, 2.4 Hz, 1H), 3.55 (dd, $J=9.0,4.8 \mathrm{~Hz}, 1 \mathrm{H}), 2.44$ (dddd, $J=14.4,5.4,3.0,3.0 \mathrm{~Hz}, 1 \mathrm{H})$, 1.96 (ddd, $J=12.6,6.6,3.6 \mathrm{~Hz}, 1 \mathrm{H}), 1.78$ (ddd, $J=15.0,10.8,4.8 \mathrm{~Hz}, 1 \mathrm{H}), 1.73$ (ddqd, $J=10.2$, 
10.2, 6.6, 3.6 Hz, 1H), 1.48 (s, 9H), 1.45 (s, 3H), 1.33 (s, 3H), 1.09 (ddd, $J=12.6,12.6,10.2 \mathrm{~Hz}$, $1 \mathrm{H}), 1.03(\mathrm{~d}, J=6.6 \mathrm{~Hz}, 3 \mathrm{H}) ;{ }^{13} \mathrm{C}$ NMR $\left(\mathrm{CD}_{3} \mathrm{OD}, 150 \mathrm{MHz}\right) \delta 152.9,108.4,82.1,80.4,73.9$, $71.8,36.5,33.5,32.6,28.5,27.8,26.1,18.7$; HRMS (ESI) calcd. for $\left[\mathrm{C}_{15} \mathrm{H}_{26} \mathrm{O}_{5} \mathrm{P}+\mathrm{Na}\right]^{+}$: 309.16725, Found: 309.16729.

\section{5a-Carba- $\beta$-D-digitoxopyranose (II-8)}

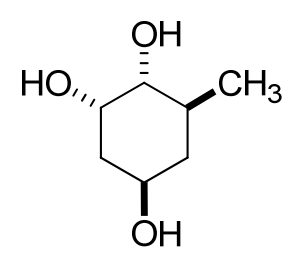

To a solution of $16 \mathrm{mg}$ carbonate II-22 $(0.06 \mathrm{mmol})$ in $2 \mathrm{~mL}$ THF at $0{ }^{\circ} \mathrm{C}$ was added $16 \mathrm{mg}$ $\mathrm{LiAlH}_{4}(0.42 \mathrm{mmol})$. The reaction mixture was warmed up and stirred at RT for $1 \mathrm{~h}$, then it was diluted with $5 \mathrm{~mL}$ EtOAc and $1.5 \mathrm{~mL}$ saturated aqueous $\mathrm{NaHCO}_{3}$ was added. It was stirred for another 20 min to quench the reaction and then the mixture was passed through a pad of sandsilica gel-celite, eluting with EtOAc. The effluent was dried over $\mathrm{Na}_{2} \mathrm{SO}_{4}$. The solution was concentrated under reduced pressure to give a residue. The residue was redissolved in $1.5 \mathrm{~mL}$ THF- $10 \% \mathrm{HCl}(1: 1 \mathrm{v} / \mathrm{v})$ and the mixture was stirred at room temperature for $30 \mathrm{~min}$. It was then cooled down to $0{ }^{\circ} \mathrm{C}$, and diluted with $5 \mathrm{~mL}$ EtOAc, then $1 \mathrm{~mL}$ saturated aqueous $\mathrm{NaHCO}_{3}$ and $250 \mathrm{mg} \mathrm{NaHCO}$ (s) was slowly added to quench the reaction. The mixture was concentrated to a residue, which was directly loaded to a silica gel column. Elution with EtOAc-MeOH (20:1) gave cyclitol II-8 (7 mg, 85\%) as colorless oil; $R_{f}=0.44\left(5: 1(\mathrm{v} / \mathrm{v})\right.$ EtOAc-MeOH); $[\alpha]_{\mathrm{D}}{ }^{20}+33(c$ 0.64, $\mathrm{CHCl}_{3}$ ); IR (thin film, $\mathrm{cm}^{-1}$ ) v 3353, 2975, 2950, 2930, 1454, 1417, 1370, 1307, 1184, 1141, 1094, 1047, 980; ${ }^{1} \mathrm{H}$ NMR $\left(\mathrm{CD}_{3} \mathrm{OD}, 600 \mathrm{MHz}\right) \delta 3.95$ (ddd, $\left.J=3.0,3.0,2.4 \mathrm{~Hz}, 1 \mathrm{H}\right), 3.94$ (dddd, $J=11.4,11.4,4.2,4.2 \mathrm{~Hz}, 1 \mathrm{H}), 3.06(\mathrm{dd}, J=10.2,3.0 \mathrm{~Hz}, 1 \mathrm{H}), 2.11$ (dddd, $J=13.2,3.6,3.6$, $3.0 \mathrm{~Hz}, 1 \mathrm{H}$ ), 1.88 (dddd, $J=12.0,3.6,3.6,2.4 \mathrm{~Hz}, 1 \mathrm{H}), 1.83$ (ddqd, $J=10.2,10.2,6.6,3.6 \mathrm{~Hz}$, 1H), 1.39 (ddd, $J=13.2,11.4,2.4 \mathrm{~Hz}, 1 \mathrm{H}), 1.01$ (ddd, $J=12.6,12.6,11.4 \mathrm{~Hz}, 1 \mathrm{H}), 1.00$ (d, $J=$ $6.6 \mathrm{~Hz}, 3 \mathrm{H}) ;{ }^{13} \mathrm{C}$ NMR $\left(\mathrm{CD}_{3} \mathrm{OD}, 150 \mathrm{MHz}\right) \delta 78.3,71.7,66.1,43.4,41.5,32.4,19.2$; HRMS (ESI) calcd. for $\left[\mathrm{C}_{7} \mathrm{H}_{14} \mathrm{O}_{3}-\mathrm{H}\right]^{-}: 145.0870$, Found: 145.0871 . 
(2R,4R,5R)-4,5-dihydroxy-2-methylcyclohexanone (II-23)<smiles>C[C@H]1CC(O)[C@H](O)CC1=O</smiles>

A $500 \mathrm{mg}$ sample of bisketal II-19a $(1.94 \mathrm{mmol}$, dr $\sim 10: 1)$ was placed in $250 \mathrm{~mL}$ flask at $0{ }^{\circ} \mathrm{C}$, and $3.7 \mathrm{~mL}$ solution of TFA $/ \mathrm{H}_{2} \mathrm{O}(5: 1 \mathrm{v} / \mathrm{v})$ was added into the flask at $0{ }^{\circ} \mathrm{C}$ when stirring. The mixture was stirred at $0{ }^{\circ} \mathrm{C}$ for $20 \mathrm{~min}$, then was diluted with $30 \mathrm{~mL}$ EtOAc, into which $10 \mathrm{~mL}$ saturated aqueous $\mathrm{NaHCO}_{3}$, as well as $6 \mathrm{~g} \mathrm{NaHCO}_{3}(\mathrm{~s})$ was slowly added to quench the reaction. The mixture was run through a pad of sand-silica gel-celite, rinsing with EtOAc-MeOH (10:1, $\mathrm{v} / \mathrm{v}$ ), and the effluent was dried over $\mathrm{Na}_{2} \mathrm{SO}_{4}$. The solution was concentrated under reduced pressure to give a residue. Crude ${ }^{1} \mathrm{H}$ NMR showed that a little isomerization on the $\alpha$-methyl group happened, which lower the dr to $\sim 8: 1$. The residue was then purified by silica gel column. Elution with hexane-EtOAc (1:3) gave pure diol II-23 (195 mg, 70\%) with the minor isomer removed.

II-23: White solid; mp: 67.9-69.2 ${ }^{\circ} \mathrm{C} ; R_{f}=0.50\left(10: 1(\mathrm{v} / \mathrm{v})\right.$ EtOAc/MeOH); $[\alpha]_{\mathrm{D}}{ }^{20}-13(c$ 2.6, $\mathrm{CHCl}_{3}$ ); IR (thin film, cm ${ }^{-1}$ ) v 3373, 3280, 2969, 2924, 1694, 1437, 1421, 1399, 1380, 1276, 1229, 1165, 1123, 1094, 1071, 1032, 967; ${ }^{1} \mathrm{H} \mathrm{NMR}\left(\mathrm{CDCl}_{3}, 600 \mathrm{MHz}\right) \delta 4.19$ (dddd, $J=4.8,4.2$, 3.6, 3.2 Hz, 1H), 4.02 (dddd, $J=4.2,3.6,3.6,3.6 \mathrm{~Hz}, 1 \mathrm{H}), 2.91$ (ddd, $J=14.4,3.6,0.6 \mathrm{~Hz}, 1 \mathrm{H}$ ), 2.78 (ddq, $J=7.8,7.2,6.61 \mathrm{H}), 2.42(\mathrm{dd}, J=14.4,4.8 \mathrm{~Hz}, 1 \mathrm{H}), 2.14$ (d, $J=3.0 \mathrm{~Hz}, 1 \mathrm{H}), 2.07$ (d, $J=3.6 \mathrm{~Hz}, 1 \mathrm{H}), 2.00(\mathrm{dd}, J=8.4,4.21 \mathrm{H}), 1.99(\mathrm{dd}, J=8.4,3.61 \mathrm{H}), 1.08(\mathrm{~d}, J=6.6 \mathrm{~Hz}, 1 \mathrm{H})$; ${ }^{13} \mathrm{C} \mathrm{NMR}\left(\mathrm{CDCl}_{3}, 150 \mathrm{MHz}\right) \delta 211.9,74.0,69.0,44.5,40.1,36.9,14.7$; HRMS (ESI) calcd. for $\left[\mathrm{C}_{7} \mathrm{H}_{12} \mathrm{O}_{3}+\mathrm{H}\right]^{+}:$145.0859, Found: 145.0859 .

\section{t-Butyl (1R,5R)-5-methyl-4-oxocyclohex-2-enyl carbonate ((ent)-II-15)}




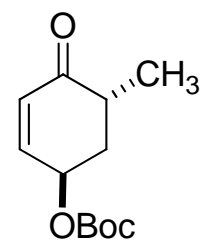

$142 \mathrm{mg}$ diol II-23 (1.0 mmol) was dissolved in $7.5 \mathrm{~mL}$ THF- $\mathrm{CH}_{2} \mathrm{Cl}_{2}(1: 2 \mathrm{v} / \mathrm{v})$ at RT, into which $12 \mathrm{mg}$ DMAP $(0.1 \mathrm{mmol})$ was added in, followed by addition of a solution of $870 \mathrm{mg} \mathrm{Boc} 2 \mathrm{O}(4$ mmol) in $2.5 \mathrm{~mL} \mathrm{CH}_{2} \mathrm{Cl}_{2}$. The mixture was stirred at $\mathrm{RT}$ for $2 \mathrm{~h}$, then it was cooled down to $0{ }^{\circ} \mathrm{C}$, diluted with $\mathrm{Et}_{2} \mathrm{O} 30 \mathrm{~mL}$ and was then quenched with $7 \mathrm{~mL}$ saturated aqueous $\mathrm{NaHCO}_{3}$. The mixture was stirred at $0{ }^{\circ} \mathrm{C}$ for another $2 \mathrm{~h}$ and then was extracted with Hexane-Et $\mathrm{O}_{2} \mathrm{O}(1: 2 \mathrm{v} / \mathrm{v}) 30$ $\mathrm{mL} \times 2$. The pooled organic layer was washed with $7 \mathrm{~mL}$ saturated aqueous $\mathrm{NaCl}$ and dried over $\mathrm{Na}_{2} \mathrm{SO}_{4}$. The solution was concentrated under reduced pressure to afford a residue. The residue was redissolved in $6.5 \mathrm{~mL} \mathrm{CH}_{2} \mathrm{Cl}_{2}$ at $0{ }^{\circ} \mathrm{C}$. Into the solution $1.0 \mathrm{~mL} \mathrm{Et}_{3} \mathrm{~N} \mathrm{(5} \mathrm{mmol)} \mathrm{was} \mathrm{slowly}$ added in. The mixture was then stirred at $0{ }^{\circ} \mathrm{C}$ overnight and continued to be stirred at RT for another $2 \mathrm{~h}$. Then it was cooled back to $0{ }^{\circ} \mathrm{C}$, diluted with hexane- $\mathrm{Et}_{2} \mathrm{O}(1: 1 \mathrm{v} / \mathrm{v}) 40 \mathrm{~mL}$ and it was then quenched by $10 \mathrm{~mL} 0.5 \mathrm{M} \mathrm{NaHSO}_{4}$ at $0{ }^{\circ} \mathrm{C}$. The aqueous layer was extracted with hexane- $\mathrm{Et}_{2} \mathrm{O}(1: 1 \mathrm{v} / \mathrm{v}) 40 \mathrm{~mL} \times 2$. The pooled organic layer was washed with $10 \mathrm{~mL} 0.5 \mathrm{M}$ $\mathrm{NaHSO}_{4}$ at $0{ }^{\circ} \mathrm{C}$, then was washed with $10 \mathrm{~mL}$ saturated aqueous $\mathrm{NaHCO}_{3}, 10 \mathrm{~mL}$ saturated aqueous $\mathrm{NaCl}$ and dried over $\mathrm{Na}_{2} \mathrm{SO}_{4}$. The solution was concentrated under reduced pressure to afford a residue, which was purified by silica gel column. Elution with hexane-EtOAc $(10: 1 \mathrm{v} / \mathrm{v})$ gave enone (ent)-II-15 (200 mg, 90\%) as white solid; mp: 37.5-38.9 ${ }^{\circ} \mathrm{C} ; R_{f}=0.44(4: 1(\mathrm{v} / \mathrm{v})$ hexane/EtOAc); $[\alpha]_{\mathrm{D}}{ }^{20}+168\left(c 1.0, \mathrm{CHCl}_{3}\right)$; IR (thin film, $\left.\mathrm{cm}^{-1}\right) v 2980,2935,2875,1735,1684$, $1459,1369,1341,1272,1252,1155,1080,1044,965 ;{ }^{1} \mathrm{H} \mathrm{NMR}\left(\mathrm{CDCl}_{3}, 600 \mathrm{MHz}\right) \delta 6.86(\mathrm{dd}, J$ $=10.2,4.2 \mathrm{~Hz}, 1 \mathrm{H}), 6.03(\mathrm{~d}, J=10.2 \mathrm{~Hz}, 1 \mathrm{H}), 5.29(\mathrm{ddd}, J=4.8,4.8,4.2 \mathrm{~Hz}, 1 \mathrm{H}), 2.76(\mathrm{dqd}, J=$ 10.2, 7.2, $5.4 \mathrm{~Hz}, 1 \mathrm{H}$ ), 2.25 (dddd, $J=14.4,4.8,4.8,0.6 \mathrm{~Hz}, 1 \mathrm{H}$ ), 2.06 (ddd, $J=14.4,9.6,4.8$ $\mathrm{Hz}, 1 \mathrm{H}), 1.49$ (s, 9H), 1.15 (d, $J=7.2 \mathrm{~Hz}, 3 \mathrm{H}) ;{ }^{13} \mathrm{C} \mathrm{NMR}\left(\mathrm{CDCl}_{3}, 150 \mathrm{MHz}\right) \delta 200.9,152.9$, 143.3, 131.2, 82.9, 68.1, 37.7, 35.4, 27.7, 14.9; HRMS (ESI) calcd. for $\left[\mathrm{C}_{12} \mathrm{H}_{18} \mathrm{O}_{4}+\mathrm{Na}\right]^{+}$: 249.1097, Found: 249.1097.

(1R,3R,4S,6S)-bicyclo[4.1.0] heptane-1,3,4-triol (II-24) 


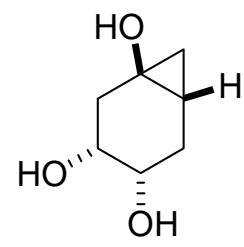

A solution of $1.39 \mathrm{~g}(7.5 \mathrm{mmol})$ of II-9b was dissolved in $6 \mathrm{~mL}$ THF- $10 \% \mathrm{HCl}(1: 1 \mathrm{v} / \mathrm{v})$ and stirred at room temperature for $15 \mathrm{~min}$. The mixture was cooled down to $0{ }^{\circ} \mathrm{C}$, and diluted with $70 \mathrm{~mL}$ EtOAc, then $\sim 5 \mathrm{~g} \mathrm{NaHCO}_{3}$ (s) was slowly added to quench the reaction. The mixture was filtered through a glass wool and the liquid was concentrated to a residue which was loaded to a silica gel column. Elution with EtOAc-MeOH (10:1 v/v) gave triol II-24 (1.0 g, 82\%).

II-24: Colorless oil; $R_{f}=0.38(5: 1(\mathrm{v} / \mathrm{v}) \mathrm{EtOAc} / \mathrm{MeOH}) ;[\alpha]_{\mathrm{D}}^{20} 0.71(c$ 7.03, MeOH); IR (thin film, $\left.\mathrm{cm}^{-1}\right) v$ 3304, 3009, 2924, 1432, 1362, 1276, 1218, 1183, 1093, 1045, 914; ${ }^{1} \mathrm{H}$ NMR $\left(\mathrm{CD}_{3} \mathrm{OD}, 600 \mathrm{MHz}\right) \delta 3.82(\mathrm{ddd}, J=5.4,5.4,2.4 \mathrm{~Hz}, 1 \mathrm{H}), 3.70$ (ddd, $\left.J=7.8,5.4,1.8 \mathrm{~Hz}, 1 \mathrm{H}\right)$, 2.36 (ddd, $J=14.4,6.0 \mathrm{~Hz}, 1 \mathrm{H}$ ), 2.11 (dd, $J=14.4,5.4 \mathrm{~Hz}, 1 \mathrm{H}$ ), 2.09 (ddd, $J=13.8,8.4,6.0 \mathrm{~Hz}$, 1H), 1.64 (ddd, $J=14.4,8.4,1.8 \mathrm{~Hz}, 1 \mathrm{H}$ ), 0.97 (dddd, $J=10.2,8.4,8.4,2.4 \mathrm{~Hz}, 1 \mathrm{H}$ ), 0.80 (m, $2 \mathrm{H}) ;{ }^{13} \mathrm{C}$ NMR $\left(\mathrm{CD}_{3} \mathrm{OD}, 150 \mathrm{MHz}\right) \delta 70.5,70.3,53.5,38.3,29.7,21.9,18.9$; HRMS (ESI): calcd. for $\left[\mathrm{C}_{7} \mathrm{H}_{12} \mathrm{O}_{3}+\mathrm{Na}\right]^{+}:$167.06787, Found: 167.06785 .

\section{(2R,4S,5R)-4,5-dihydroxy-2-methylcyclohexanone (II-25)}<smiles>C[C@H]1C[C@H](O)[C@H](O)CC1=O</smiles>

Into a solution of $200 \mathrm{mg}(1.39 \mathrm{mmol})$ cyclopropanol II-24 in $4.5 \mathrm{~mL}$ THF-EtOH $(1: 1 \mathrm{v} / \mathrm{v})$ was added $80 \mathrm{mg} 10 \% \mathrm{Pd} / \mathrm{C}$. The solution was degassed using vacuum and refilled with $\mathrm{H}_{2}$ three times at low temperature $\left(\sim-30^{\circ} \mathrm{C}\right)$. Then at room temperature, the solution was stirred $5 \mathrm{hr}$ under balloon pressure of $\mathrm{H}_{2}$. It was filtered through a filter paper and rinsed with ethyl acetate. The solution was concentrated under reduced pressure to a small volume (some of the solution 
was taken out and concentrated to dry, then a crude NMR was taken to show that NO diastereomer was seen). The residue was subjected to chromatography on silica gel column. Elution with hexane-EtOAc (1:4) gave product II-25 (160 mg, 80\%).

II-25: White solid; mp: $76-78^{\circ} \mathrm{C} ; R_{f}=0.55\left(5: 1(\mathrm{v} / \mathrm{v})\right.$ EtOAc/MeOH); $[\alpha]_{\mathrm{D}}{ }^{20}-19(c 1.47, \mathrm{MeOH})$; IR (thin film, $\mathrm{cm}^{-1}$ ) v 3366, 2940, 2918, 2863, 1696, 1447, 1405, 1380, 1315, 1244, 1213, 1160, 1097, 1036, 1008, 973, 937; ${ }^{1} \mathrm{H}$ NMR (CDCl3, $\left.600 \mathrm{MHz}\right) \delta 4.26$ (ddd, $\left.J=5.4,3.0,3.0 \mathrm{~Hz}, 1 \mathrm{H}\right)$, 4.13 (ddd, $J=11.4,4.2,2.4 \mathrm{~Hz}, 1 \mathrm{H}$ ), 3.83 (brs, $1 \mathrm{H}$ ), 3.66 (brs, 1H), 2.55 (ddd, $J=14.4,3.0,0.6$ Hz, 1H), 2.51 (dd, $J=15.0,3.6 \mathrm{~Hz}, 1 \mathrm{H}), 2.43$ (dqd, $J=12.6,6.6,6.0 \mathrm{~Hz}, 1 \mathrm{H}), 2.03$ (dddd, $J=$ 12.0, 6.0, 4.8, $1.8 \mathrm{~Hz}, 1 \mathrm{H}), 1.82(\mathrm{ddd}, J=12.6,12.6,12.0 \mathrm{~Hz}, 1 \mathrm{H}), 0.99(\mathrm{~d}, J=6.0 \mathrm{~Hz}, 3 \mathrm{H}) ;{ }^{13} \mathrm{C}$ NMR (CDCl3, $150 \mathrm{MHz}) \delta 211.3,72.1,70.0,45.8,41.8,36.3,14.2$; HRMS (ESI): calcd. for $\left[\mathrm{C}_{7} \mathrm{H}_{12} \mathrm{O}_{3}+\mathrm{H}\right]^{+}:$145.08592, Found: 145.08589 .

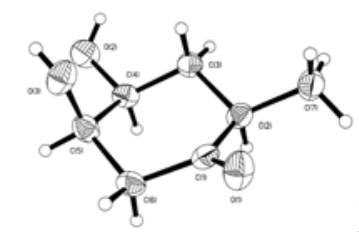

X-Ray Crystal data: Empirical formula $\mathrm{C}_{7} \mathrm{H}_{12} \mathrm{O}_{3}$; formula weight 144.17; Temperature 293(2) K; wavelength $0.71073 \AA$; crystal system monoclinic; space group P2 ${ }_{1}$; uni cell dimensions $a=5.7707(9) \AA, b=8.6125(13) \AA, c=7.7163(12) \AA, \alpha=90^{\circ}, \beta=105.997(3)^{\circ}, \gamma=90^{\circ}$; volume 368.6(1) $\AA^{3} ; Z=2$; density (calculated) $1.299 \mathrm{~g} / \mathrm{cm}^{3}$; absorption coefficient $1.01 \mathrm{~cm}^{-1}$; $\mathrm{F}(000)$ 156; crystal size $0.16 \times 0.18 \times 0.50 \mathrm{~mm}$; $\theta$ range for data collection 3.63 to $27.50^{\circ}$; index ranges $-7 \leq \mathrm{h} \leq 7,-11 \leq \mathrm{k} \leq 10,-9 \leq 1 \leq 10$; reflections collected 2356; independent reflections 1230 $[\mathrm{R}$ (int) $=0.0318]$; completeness to $\theta=27.50^{\circ} 96.7 \%$; refinement method Full-matrix least-squares on $\mathrm{F}^{2}$; data/restraints/parameters 1230/1/94; goodness-of-fit on $\mathrm{F}^{2} 1.022$; final R indices [I $\left.>2 \sigma(\mathrm{I})\right]$ $\mathrm{R} 1=0.0460, \mathrm{wR} 2=0.1213 ; \mathrm{R}$ indices (all data) $\mathrm{R} 1=0.0494, \mathrm{wR} 2=0.1253$; absolute structure parameter -0.3(15); largest diff. peak and hole 0.364 and $-0.178 \mathrm{e} / \AA^{3}$.

(4S,6R)-4-hydroxy-6-methylcyclohex-2-enone (II-12b) 


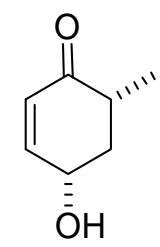

A solution of $1.88 \mathrm{~g}$ diol $\mathbf{I I - 2 5}(13.1 \mathrm{mmol})$ in $70 \mathrm{~mL} \mathrm{CH}_{2} \mathrm{Cl}_{2}$ was added $159 \mathrm{mg}(1.31 \mathrm{mmol})$ DMAP at $0{ }^{\circ} \mathrm{C}$, then $7.9 \mathrm{~mL}(9.80 \mathrm{mmol})$ pyridine was injected in. Then a solution of $3.8 \mathrm{~g} \mathrm{(13.1}$ mmol) triphosgene in $20 \mathrm{~mL} \mathrm{CH}_{2} \mathrm{Cl}_{2}$ was slowly injected in at $0{ }^{\circ} \mathrm{C}$. The reaction mixture was stirred at $0{ }^{\circ} \mathrm{C}$ for $1 \mathrm{hr}$. The solution was then diluted with $150 \mathrm{~mL}$ hexane-EtOAc $(1: 2 \mathrm{v} / \mathrm{v})$, and added $25 \mathrm{~mL} 0.5 \mathrm{M} \mathrm{NaHSO}_{4}$, it was stirred for $\sim 10 \mathrm{~min}$ at $0{ }^{\circ} \mathrm{C}$. The aqueous layer was then extracted with hexane-EtOAc (1:2 v/v) $150 \mathrm{~mL} \mathrm{X} \mathrm{2.} \mathrm{The} \mathrm{pooled} \mathrm{organic} \mathrm{layer} \mathrm{was} \mathrm{washed} \mathrm{with}$ $25 \mathrm{~mL} 0.5 \mathrm{M} \mathrm{NaHSO}_{4}$ at $0{ }^{\circ} \mathrm{C}$, then washed with $20 \mathrm{~mL}$ saturated aqueous $\mathrm{NaHCO}_{3}, 20 \mathrm{~mL}$ saturated aqueous $\mathrm{NaCl}$, dried over $\mathrm{Na}_{2} \mathrm{SO}_{4}$. Concentration of the solution under reduced pressure gave a residue. Then the residue was redissolved in $50 \mathrm{~mL} \mathrm{CH}_{2} \mathrm{Cl}_{2}$ at $0{ }^{\circ} \mathrm{C}$, into which $7.2 \mathrm{~mL}(52.2 \mathrm{mmol}) \mathrm{Et}_{3} \mathrm{~N}$ was added slowly. The mixture was stirred overnight at $0{ }^{\circ} \mathrm{C}$ and continued to be stirred at room temperature for another 1 hour. Then the mixture was diluted with hexane-EtOAc $(1: 1 \mathrm{v} / \mathrm{v}) 200 \mathrm{~mL}$ and cooled down to $0{ }^{\circ} \mathrm{C}$, then was quenched with $25 \mathrm{~mL}$ $0.5 \mathrm{M} \mathrm{NaHSO}_{4}$ at $0{ }^{\circ} \mathrm{C}$. The aqueous layer was then extracted with hexane-EtOAc $(1: 1 \mathrm{v} / \mathrm{v}) 150$ $\mathrm{mL} \mathrm{X} \mathrm{2.} \mathrm{The} \mathrm{pooled} \mathrm{organic} \mathrm{layer} \mathrm{was} \mathrm{washed} \mathrm{with} 25 \mathrm{~mL} 0.5 \mathrm{M} \mathrm{NaHSO}_{4}$ at $0{ }^{\circ} \mathrm{C}$ again, then it was washed with $25 \mathrm{~mL}$ saturated aqueous $\mathrm{NaHCO}_{3}, 25 \mathrm{~mL}$ saturated aqueous $\mathrm{NaCl}$, dried over $\mathrm{Na}_{2} \mathrm{SO}_{4}$. Concentration of the solution under reduced pressure gave a residue, which was purified by silica gel column. Elution with hexane-EtOAc $(2: 3, \mathrm{v} / \mathrm{v})$ gave product allylic alcohol II-12b $(1.2 \mathrm{~g}, 73 \%)$.

II-12b: Colorless oil; $R_{f}=0.40\left(1: 2(\mathrm{v} / \mathrm{v})\right.$ hexane/EtOAc); $[\alpha]_{\mathrm{D}}{ }^{20}-65\left(c\right.$ 2.38, $\left.\mathrm{CHCl}_{3}\right)$; IR (thin film, $\left.\mathrm{cm}^{-1}\right) v 3406,3036,2968,2934,2870,1668,1457,1376,1252,1214,1182,1100,1067$, 1027, 952; ${ }^{1} \mathrm{H}$ NMR $\left(\mathrm{CDCl}_{3}, 270 \mathrm{MHz}\right) \delta 6.87(\mathrm{ddd}, J=10.2,1.8,1.8 \mathrm{~Hz}, 1 \mathrm{H}), 5.96(\mathrm{dd}, J=$ 10.2, $2.4 \mathrm{~Hz}, 1 \mathrm{H}), 4.64(\mathrm{~d}, J=8.4 \mathrm{~Hz}, 1 \mathrm{H}), 2.40(\mathrm{dqd}, J=13.2,6.6,4.8 \mathrm{~Hz}, 1 \mathrm{H}), 2.37$ (dddd, $J=$ 12.0, 5.4, 4.8, $2.4 \mathrm{~Hz} 1 \mathrm{H}), 1.73$ (ddd, $J=13.8,13.2,10.8 \mathrm{~Hz} 1 \mathrm{H}), 1.16(\mathrm{~d}, J=6.6 \mathrm{~Hz}, 3 \mathrm{H}),{ }^{13} \mathrm{C}$ NMR $\left(\mathrm{CDCl}_{3}, 150 \mathrm{MHz}\right) \delta 201.2,152.9,128.7,67.4,41.4,40.1,14.9$; HRMS (ESI) calcd. for $\left[\mathrm{C}_{7} \mathrm{H}_{10} \mathrm{O}_{2}+\mathrm{H}\right]^{+}:$127.07536, Found: 127.07535 . 
(1R,5R)-5-methyl-4-oxocyclohex-2-enyl 4-nitrobenzoate (II-26)

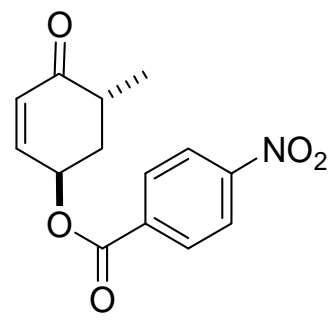

To a solution of $710 \mathrm{mg}$ allylic diol II-12b $(5.6 \mathrm{mmol})$ in $40 \mathrm{~mL}$ THF at $0{ }^{\circ} \mathrm{C}$ was injected a solution of $1.14 \mathrm{~g}(6.8 \mathrm{mmol})$ p-nitrobenzoic acid in $10 \mathrm{~mL}$ THF, followed by a injection of a solution of $1.79 \mathrm{~g} \mathrm{PPh}_{3}(6.8 \mathrm{mmol})$ in $10 \mathrm{~mL}$ THF. Then at $0{ }^{\circ} \mathrm{C} 1.65 \mathrm{~mL}(7.1 \mathrm{mmol})$ DIAD was added dropwise. The mixture was stirred at $0{ }^{\circ} \mathrm{C}$ for $1 \mathrm{hr}$. The mixture was diluted with $150 \mathrm{~mL}$ hexane- $\mathrm{Et}_{2} \mathrm{O}(1: 2 \mathrm{v} / \mathrm{v})$ and was washed with $15 \mathrm{~mL} 0.5 \mathrm{M} \mathrm{NaHSO}_{4}$ at $0{ }^{\circ} \mathrm{C}$. The aqueous layer was extracted with hexane-Et $2 \mathrm{O}(1: 2 \mathrm{v} / \mathrm{v}) 120 \mathrm{~mL} \mathrm{X} 2$ at $0{ }^{\circ} \mathrm{C}$. The pooled organic layer was washed with $20 \mathrm{~mL}$ saturated aqueous $\mathrm{NaHCO}_{3}, 20 \mathrm{~mL}$ saturated brine, dried over $\mathrm{Na}_{2} \mathrm{SO}_{4}$. The organic layer was concentrated under reduced pressure to give crude product, which was then subjected to chromatography on silica gel column. Elution with hexane-EtOAc $(7: 1 \mathrm{v} / \mathrm{v})$ gave ester II-26 (1.28g, 95\%).

II-26: Pale yellow solid; mp: $119-120.5{ }^{\circ} \mathrm{C} ; R_{f}=0.42\left(3: 1(\mathrm{v} / \mathrm{v})\right.$ hexane/EtOAc); $[\alpha]_{\mathrm{D}}{ }^{20}+265(c$ 6.24, $\mathrm{CHCl}_{3}$ ); IR (thin film, $\mathrm{cm}^{-1}$ ) v 3112, 3086, 2992, 2970, 2933, 2874, 1718, 1676, 1607, 1523, 1343, 1321, 1274, 1222, 1205, 1099, 1063, 1045, 1009, 931; ${ }^{1} \mathrm{H}$ NMR $\left(\mathrm{CDCl}_{3}, 600 \mathrm{MHz}\right) \delta 8.31$ (d, $J=9.0 \mathrm{~Hz}, 1 \mathrm{H}), 8.22(\mathrm{~d}, J=8.4 \mathrm{~Hz}, 1 \mathrm{H}), 6.95(\mathrm{dd}, J=10.2,4.2 \mathrm{~Hz}, 1 \mathrm{H}), 6.16(\mathrm{~d}, J=10.2 \mathrm{~Hz}$, 1H), 5.78 (ddd, $J=4.8,4.2,4.2 \mathrm{~Hz}, 1 \mathrm{H}), 2.85(\mathrm{dqd}, J=12.0,7.2,2.4 \mathrm{~Hz}, 1 \mathrm{H}), 2.37$ (ddd, $J=$ 13.8, 4.8, 4.8 Hz 1H), 1.73 (ddd, $J=14.4,10.2,4.2 \mathrm{~Hz} 1 \mathrm{H}), 1.25$ (d, $J=7.2 \mathrm{~Hz}, 3 \mathrm{H}),{ }^{13} \mathrm{C}$ NMR $\left(\mathrm{CDCl}_{3}, 150 \mathrm{MHz}\right) \delta 200.6,164.1,150.8,142.6,135.1,132.0,130.9,123.7,67.3,37.9,35.5$, 15.0; HRMS (ESI) calcd. for $\left[\mathrm{C}_{14} \mathrm{H}_{13} \mathrm{NO}_{5}+\mathrm{Na}\right]^{+}: 298.06859$, Found: 298.06865 .

(1R,4S,5R)-4-hydroxy-5-methylcyclohex-2-enyl 4-nitrobenzoate (II-27) 


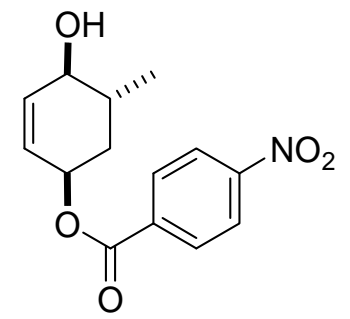

To a solution of $1.28 \mathrm{~g}$ ester II-26 (4.64 mmol) in $60 \mathrm{~mL}$ THF at $-78{ }^{\circ} \mathrm{C}$ was slowly added 330 $\mathrm{mg}(8.68 \mathrm{mmol}) \mathrm{LiAlH}_{4}$ in portions. The reaction mixture was stirred at $-78{ }^{\circ} \mathrm{C}$ for $30 \mathrm{~min}$, then $6 \mathrm{~mL}$ acetone was added dropwise in at $-78{ }^{\circ} \mathrm{C}$ to quench the reaction, which was stirred at $78{ }^{\circ} \mathrm{C}$ for $30 \mathrm{~min}$. It was then diluted with $360 \mathrm{~mL}$ hexane-EtOAc $(1: 5 \mathrm{v} / \mathrm{v})$ and slowly $30 \mathrm{~mL}$ saturated aqueous $\mathrm{NH}_{4} \mathrm{Cl}$ was added in. It was then allowed to stir at $0{ }^{\circ} \mathrm{C}$ for $20 \mathrm{~min}$. The organic layer was washed again with $30 \mathrm{~mL}$ saturated aqueous $\mathrm{NH}_{4} \mathrm{Cl}$, then washed with $30 \mathrm{~mL}$ saturated brine, dried over $\mathrm{Na}_{2} \mathrm{SO}_{4}$. The solution was concentrated under reduced pressure to give a residue. A crude ${ }^{1} \mathrm{H}$ NMR showed that the diastereoselectivity is 11:1. The residue was subjected to chromatography on silica gel column and elution with hexane-EtOAc (4:1) afforded allylic alcohol II-27 (1.16 g, 91\%).

II-27: White solid; mp: $77.2-78.5^{\circ} \mathrm{C} ; R_{f}=0.55\left(1: 1(\mathrm{v} / \mathrm{v})\right.$ hexane/EtOAc); $[\alpha]_{\mathrm{D}}{ }^{20}+197(c) 3.52$, $\mathrm{CHCl}_{3}$ ); IR (thin film, $\mathrm{cm}^{-1}$ ) $v$ 3272, 3113, 3039, 2959, 2909, 2889, 1717, 1607, 1521, 1460, $1338,1270,1116,1101,1056,1038,1009,958,903 ;{ }^{1} \mathrm{H} \mathrm{NMR}\left(\mathrm{CDCl}_{3}, 600 \mathrm{MHz}\right) \delta 8.30(\mathrm{~d}, J=$ $9.0 \mathrm{~Hz}, 1 \mathrm{H}), 8.20$ (d, $J=9.6 \mathrm{~Hz}, 1 \mathrm{H}), 6.05(\mathrm{dd}, J=10.2,2.4 \mathrm{~Hz}, 1 \mathrm{H}), 5.92$ (dddd, $J=10.2,4.2$, $1.8,1.8 \mathrm{~Hz}, 1 \mathrm{H}), 5.47$ (ddd, $J=6.0,4.2,1.8 \mathrm{~Hz}, 1 \mathrm{H}), 3.81(\mathrm{ddd}, J=9.0,3.6,2.4 \mathrm{~Hz}, 1 \mathrm{H}), 2.01$ (dddd, $J=15.0,3.0,1.8,1.8 \mathrm{~Hz} 1 \mathrm{H}$ ), 1.92 (ddqd, $J=12.0,9.0,6.6,3.0 \mathrm{~Hz} 1 \mathrm{H}$ ), 1.71 (ddd, $J$ $=14.4,12.0,4.2 \mathrm{~Hz} 1 \mathrm{H}), 1.14(\mathrm{~d}, J=6.6 \mathrm{~Hz}, 3 \mathrm{H}),{ }^{13} \mathrm{C} \mathrm{NMR}\left(\mathrm{CDCl}_{3}, 150 \mathrm{MHz}\right) \delta 164.2,150.5$, 137.5, 135.8, 130.7, 125.0, 123.5, 73.1, 68.2, 34.3, 32.9, 18.1; HRMS (ESI) calcd. for $\left[\mathrm{C}_{14} \mathrm{H}_{15} \mathrm{NO}_{5}+\mathrm{Na}\right]^{+}: 300.08424$, Found: 300.08421 .

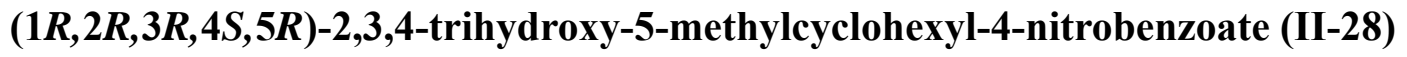




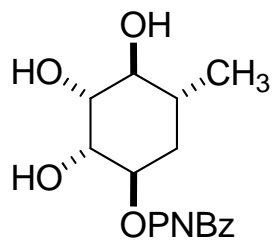

To a solution of $30 \mathrm{mg}$ olefin II-27 $(0.11 \mathrm{mmol})$ in $1.5 \mathrm{~mL} t$-BuOH-actone- $50 \% \mathrm{NMO}_{\text {(aq.) }}(1: 1: 1$ $\mathrm{v} / \mathrm{v} / \mathrm{v}$ ) was added a small piece of crystalline $\mathrm{OsO}_{4}$ at $0{ }^{\circ} \mathrm{C}$. The reaction mixture was stirred at $0{ }^{\circ} \mathrm{C}$ for overnight, then $1.5 \mathrm{~mL}$ saturated aqueous $\mathrm{Na}_{2} \mathrm{~S}_{2} \mathrm{O}_{3}$ was added and it was stirred at $0{ }^{\circ} \mathrm{C}$ for another $2 \mathrm{~h}$. The reaction mixture was passed through a pad of sand-silica gel-celite, rinsing with EtOAc-MeOH $(10: 1, \mathrm{v} / \mathrm{v})$. The effluent was dried over $\mathrm{Na}_{2} \mathrm{SO}_{4}$ and concentrated under reduced pressure to give a residue, which was purified by silica gel column. Elution with EtOAc$\mathrm{MeOH}(20: 1)$ gave triol II-28 (32 mg, 94\%) as white solid; mp: 203-204 ${ }^{\circ} \mathrm{C} ; R_{f}=0.20(1: 2(\mathrm{v} / \mathrm{v})$ hexane/EtOAc); $[\alpha]_{\mathrm{D}}{ }^{20}-33$ ( $c$ 0.54, MeOH); IR (thin film, $\left.\mathrm{cm}^{-1}\right) v$ 3351, 2925, 1715, 1608, 1525, 1408, 1346, 1314, 1275, 1159, 1122, 1102, 1078, 1052, 1009, 952; ${ }^{1} \mathrm{H}$ NMR ( $\left.\mathrm{CD}_{3} \mathrm{OD}, 600 \mathrm{MHz}\right)$ $\delta 8.33(\mathrm{~d}, J=9.0 \mathrm{~Hz}, 2 \mathrm{H}) ; 8.22(\mathrm{~d}, J=9.0 \mathrm{~Hz}, 2 \mathrm{H}) ; 5.20(\mathrm{ddd}, J=4.2,2.4,2.4 \mathrm{~Hz}, 1 \mathrm{H}), 4.05(\mathrm{dd}$, $J=3.0,3.0 \mathrm{~Hz}, 1 \mathrm{H}), 3.65(\mathrm{dd}, J=9.6,3.6 \mathrm{~Hz}, 1 \mathrm{H}), 3.39(\mathrm{dd}, J=9.6,9.6 \mathrm{~Hz}, 1 \mathrm{H}), 1.82(\mathrm{~m}, 3 \mathrm{H})$, $1.07(\mathrm{~d}, J=6.0 \mathrm{~Hz}, 3 \mathrm{H}) ;{ }^{13} \mathrm{C} \mathrm{NMR}\left(\mathrm{CD}_{3} \mathrm{OD}, 150 \mathrm{MHz}\right) \delta 165.2,152.3,137.0,131.9,124.9,76.3$, 75.4, 74.8, 72.0, 34.1, 33.1, 18.5; HRMS (ESI) calcd. for $\left[\mathrm{C}_{14} \mathrm{H}_{17} \mathrm{O}_{7}+\mathrm{Na}\right]^{+}: 334.0897$, Found: 334.0896 .

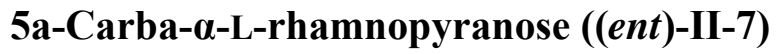<smiles>C[C@H]1CC(O)[C@H](O)[C@H](O)C1O</smiles>

To a solution of $25 \mathrm{mg}$ ester II-28 $(0.08 \mathrm{mmol})$ in $1.5 \mathrm{~mL} \mathrm{MeOH}$ was added $3.0 \mathrm{mg} \mathrm{LiOH}(0.12$ $\mathrm{mmol}$ ) at RT. The reaction mixture was stirred at RT for $4 \mathrm{~h}$. Then $0.5 \mathrm{~mL}$ saturated aqueous $\mathrm{NH}_{4} \mathrm{Cl}$ was added. This small amount of mixture was directly loaded onto a silica gel column. Elution with EtOAc-MeOH (10:1) afforded cyclitol (ent)-II-7 (11mg, 85\%) as colorless oil; $R_{f}=$ 
$0.28(5: 1(\mathrm{v} / \mathrm{v}) \mathrm{EtOAc} / \mathrm{MeOH}) ;[\alpha]_{\mathrm{D}}^{20}+11\left(c\right.$ 0.65, MeOH); IR (thin film, $\left.\mathrm{cm}^{-1}\right) v 3297,2922$, $1455,1406,1377,1296,1239,1186,1153,1050,1002,964 ;{ }^{1} \mathrm{H}$ NMR $\left(\mathrm{CD}_{3} \mathrm{OD}, 600 \mathrm{MHz}\right) \delta$ $3.87(\mathrm{dd}, J=3.6,3.6 \mathrm{~Hz}, 1 \mathrm{H}), 3.83(\mathrm{ddd}, J=3.0,3.0,3.0 \mathrm{~Hz}, 1 \mathrm{H}), 3.62(\mathrm{dd}, J=9.6,3.0 \mathrm{~Hz}, 1 \mathrm{H})$, $3.26(\mathrm{dd}, J=10.2,9.6 \mathrm{~Hz}, 1 \mathrm{H}), 1.78(\mathrm{ddqd}, J=9.6,9.6,6.6,6.6 \mathrm{~Hz}, 1 \mathrm{H}), 1.59(\mathrm{~m}, 2 \mathrm{H}), 1.01(\mathrm{~d}$, $J=6.6 \mathrm{~Hz}, 3 \mathrm{H}) ;{ }^{13} \mathrm{C} \mathrm{NMR}\left(\mathrm{CD}_{3} \mathrm{OD}, 150 \mathrm{MHz}\right) \delta 76.9,75.0,74.3,71.1,35.9,33.1,18.6$; HRMS (ESI) calcd. for $\left[\mathrm{C}_{7} \mathrm{H}_{14} \mathrm{O}_{4}+\mathrm{Na}\right]^{+}: 185.0784$, Found: 185.0784 .

\section{(4S,6S)-4-hydroxy-6-methylcyclohex-2-enone (II-38)}

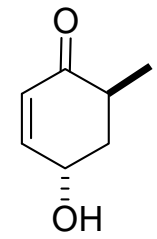

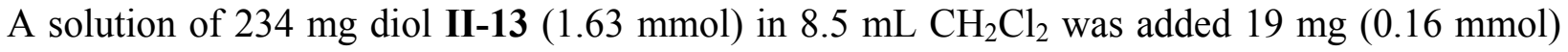
DMAP at $0{ }^{\circ} \mathrm{C}$, then $0.46 \mathrm{~mL}(5.69 \mathrm{mmol})$ pyridine was slowly added via syringe. Then a solution of $250 \mathrm{mg}(0.81 \mathrm{mmol})$ triphosgene in $2 \mathrm{~mL} \mathrm{CH}_{2} \mathrm{Cl}_{2}$ was slowly injected in at $0{ }^{\circ} \mathrm{C}$. The reaction mixture was stirred at $0{ }^{\circ} \mathrm{C}$ for $2 \mathrm{hr}$. The solution was then diluted with $80 \mathrm{~mL}$ EtOAc, and quenched with $8 \mathrm{~mL} 0.5 \mathrm{M} \mathrm{NaHSO}_{4}$. The aqueous layer was then extracted with EtOAc 50 $\mathrm{mL} \mathrm{X} \mathrm{2.} \mathrm{The} \mathrm{pooled} \mathrm{organic} \mathrm{layer} \mathrm{was} \mathrm{washed} \mathrm{with} 10 \mathrm{~mL}$ saturated aqueous $\mathrm{NaHCO}_{3}, 10 \mathrm{~mL}$ saturated aqueous $\mathrm{NaCl}$, dried over $\mathrm{Na}_{2} \mathrm{SO}_{4}$. Concentration of the solution under reduced pressure gave a residue. Then the residue was redissolved in $6.5 \mathrm{~mL} \mathrm{CH}_{2} \mathrm{Cl}_{2}$ at $0{ }^{\circ} \mathrm{C}$, into which $0.8 \mathrm{~mL}(5.8 \mathrm{mmol}) \mathrm{Et}_{3} \mathrm{~N}$ was added. The mixture was stirred overnight at $0{ }^{\circ} \mathrm{C}$ and continued to be stirred at room temperature for another 1 hour. Then the mixture was diluted with EtOAc 120 $\mathrm{mL}$ EtOAc, and cooled down to $0{ }^{\circ} \mathrm{C}$ then was quenched with $20 \mathrm{~mL} 0.5 \mathrm{M} \mathrm{NaHSO}_{4}$ at $0{ }^{\circ} \mathrm{C}$. The aqueous layer was then extracted with EtOAc $50 \mathrm{~mL} \mathrm{X} \mathrm{2.} \mathrm{The} \mathrm{pooled} \mathrm{organic} \mathrm{layer} \mathrm{was}$ washed with $15 \mathrm{~mL}$ saturated aqueous $\mathrm{NaHCO}_{3}, 15 \mathrm{~mL}$ saturated aqueous $\mathrm{NaCl}$, dried over $\mathrm{Na}_{2} \mathrm{SO}_{4}$. Concentration of the solution under reduced pressure gave a residue, which was purified by silica gel column. Elution with hexane-EtOAc $(3: 2, \mathrm{v} / \mathrm{v})$ gave product allylic alcohol II-38 (150 mg, 73\%) 
II-38: Colorless oil; $R_{f}=0.40\left(1: 2(\mathrm{v} / \mathrm{v})\right.$ hexane/EtOAc); $[\alpha]_{\mathrm{D}}{ }^{20}-145\left(c 1.72, \mathrm{CHCl}_{3}\right)$; IR (thin film, $\left.\mathrm{cm}^{-1}\right) v$ 3412, 3036, 2967, 2934, 2875, 1664, 1454, 1380, 1252, 1056; ${ }^{1} \mathrm{H}$ NMR $\left(\mathrm{CDCl}_{3}\right.$, $270 \mathrm{MHz}) \delta 6.86(\mathrm{dd}, J=10.1,3.9 \mathrm{~Hz}, 1 \mathrm{H}), 5.96(\mathrm{dd}, J=10.1,1.0 \mathrm{~Hz}, 1 \mathrm{H}), 4.55(\mathrm{ddd}, J=4.7$, $4.7,4.5 \mathrm{~Hz}, 1 \mathrm{H}), 2.76(\mathrm{ddd}, J=7.4,7.4,5.2 \mathrm{~Hz}, 1 \mathrm{H}), 2.11(\mathrm{~m}, 2 \mathrm{H}), 1.17(\mathrm{~d}, J=7.2 \mathrm{~Hz}, 3 \mathrm{H}),{ }^{13} \mathrm{C}$ NMR $\left(\mathrm{CDCl}_{3}, 67.5 \mathrm{MHz}\right) \delta 201.9,148.3,129.2,63.5,38.8,37.8,15.3$; HRMS (ESI) calcd. for $\left[\mathrm{C}_{7} \mathrm{H}_{10} \mathrm{O}_{2}+\mathrm{H}\right]^{+}:$127.07536, Found: 127.07535 .

\section{Dibenzyl (1S,5S)-5-methyl-4-oxocyclohex-2-enyl phosphate (II-39)}

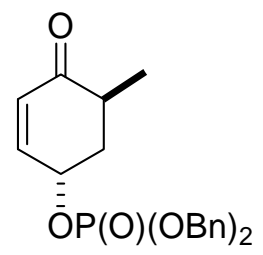

Into a solution of $54 \mathrm{mg}$ allylic alcohol $\mathbf{I I - 3 8}(0.43 \mathrm{mmol})$ in $1.8 \mathrm{~mL} \mathrm{CH}_{2} \mathrm{Cl}_{2}$ was added a solution of $0.36 \mathrm{~mL} 85 \% \mathrm{~N}, N$-diethyl dibenzylphosphoramidite $(1.1 \mathrm{mmol})$ in $1.5 \mathrm{~mL} \mathrm{CH}_{2} \mathrm{Cl}_{2}$ at room temperature followed by addition of $2.5 \mathrm{~mL} 0.45 \mathrm{M} 1 \mathrm{H}$-tetrazole in acetonitrile $(1.1 \mathrm{mmol})$. The mixture ws stirred at RT for $1 \mathrm{hr}$. Then it was cooled down to $0{ }^{\circ} \mathrm{C}$, and added $1.5 \mathrm{~mL} 30 \%$ $\mathrm{H}_{2} \mathrm{O}_{2}$. It was stirred at to $0{ }^{\circ} \mathrm{C}$ for $15 \mathrm{~min}$. Into the mixture $2 \mathrm{~mL}$ saturated aqueous $\mathrm{Na}_{2} \mathrm{~S}_{2} \mathrm{O}_{3}$ was added and it was then stirred $0{ }^{\circ} \mathrm{C}$ for $2 \mathrm{hr}$. The mixture was extracted with EtOAc $30 \mathrm{~mL} \mathrm{X} 2$. The organic layer was washed with $4 \mathrm{~mL}$ saturated aqueous $\mathrm{NaHCO}_{3}, 4 \mathrm{~mL}$ saturated aqueous $\mathrm{NaCl}$, dried over $\mathrm{Na}_{2} \mathrm{SO}_{4}$, and concentrated under reduced pressure to give a residue which was subjected to chromatography on silica gel column. Elution with hexane-EtOAc (1:1) afforded phosphate II-39 (126 mg, 76\%).

II-39: Colorless oil; $R_{f}=0.48\left(1: 2(\mathrm{v} / \mathrm{v})\right.$ hexane/EtOAc); $[\alpha]_{\mathrm{D}}{ }^{20}-43\left(\right.$ c 3.09, $\left.\mathrm{CHCl}_{3}\right)$; IR (thin film, $\left.\mathrm{cm}^{-1}\right)$ v 3035, 2965, 1683, 1498, 1456, 1381, 1269, 1215, 981; ${ }^{1} \mathrm{H}$ NMR $\left(\mathrm{CDCl}_{3}, 600 \mathrm{MHz}\right) \delta$ 7.36 (m, 10H), 6.69 (ddd, $J=10.2,4.8,1.2 \mathrm{~Hz}, 1 \mathrm{H}), 5.96$ (d, $J=10.2 \mathrm{~Hz}, 1 \mathrm{H}), 5.09$ (ddd, $J=$ $11.4,8.4,5.4 \mathrm{~Hz}, 2 \mathrm{H}$ ), 5.04 (ddd, $J=12.0,9.0,3.6 \mathrm{~Hz}, 2 \mathrm{H}$ ), 4.99 (dddd, $J=7.8,4.2,4.2,4.2 \mathrm{~Hz}$, 1H), 2.64 (dqd, $J=9.6,7.2,4.8 \mathrm{~Hz}, 1 \mathrm{H}), 2.18$ (dddd, $J=14.4,4.8,4.8,1.2 \mathrm{~Hz}, 1 \mathrm{H}$ ), 1.94 (dddd, $J=14.4,10.2,4.2,1.2 \mathrm{~Hz}, 1 \mathrm{H}), 1.08(\mathrm{~d}, J=6.6 \mathrm{~Hz}, 1 \mathrm{H}) ;{ }^{13} \mathrm{C} \mathrm{NMR}\left(\mathrm{CDCl}_{3}, 150 \mathrm{MHz}\right) \delta 200.7$, 102 
$143.2(\mathrm{~d}, J=4.6 \mathrm{~Hz}), 135.6(\mathrm{~d}, J=5.2 \mathrm{~Hz}), 131.0,128.7,128.6,128.0(\mathrm{~d}, J=5.2 \mathrm{~Hz}), 69.6$ (d, $J$ $=5.7 \mathrm{~Hz}), 69.5(\mathrm{~d}, J=5.2 \mathrm{~Hz}), 37.3,36.7(\mathrm{~d}, J=4.5 \mathrm{~Hz}), 14.7$; HRMS (ESI) calcd. for $\left[\mathrm{C}_{21} \mathrm{H}_{23} \mathrm{O}_{5} \mathrm{P}+\mathrm{Na}\right]^{+}:$:09.11753, Found: 409.11727.

\section{(2S,4R)-4-hydroxy-2-methylcyclohexanone (II-40)}

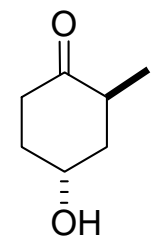

To a solution of $52 \mathrm{mg}$ allylic alcohol II-38 $(0.41 \mathrm{mmol})$ in $4 \mathrm{~mL} \mathrm{MeOH}$ was added $30 \mathrm{mg} \mathrm{10 \%}$ $\mathrm{Pd} / \mathrm{C}$. The reaction mixture was degassed using vacuum and refilled with $\mathrm{H}_{2}$ at $\sim-30{ }^{\circ} \mathrm{C}$ three times. The reaction was stirred under balloon pressure of $\mathrm{H}_{2}$ at room temperature for $3 \mathrm{hr}$. The reaction mixture was filtered and rinsed with EtOAc and concentrated under reduced pressure to give a residue, which was loaded onto silica gel column. Elution with hexane-EtOAc (3:2) gave saturated alcohol II-40 (43 mg, 72\%). II-40: Colorless oil; $R_{f}=0.43(1: 1(\mathrm{v} / \mathrm{v})$ hexane/EtOAc); $[\alpha]_{\mathrm{D}}^{20}+6\left(c 1.7, \mathrm{CHCl}_{3}\right)$; IR (thin film, $\left.\mathrm{cm}^{-1}\right) v 3407,2966,2931,1697,1436,1349,1318,1257$, $1138,1111,1011,950 ;{ }^{1} \mathrm{H} \mathrm{NMR}\left(\mathrm{CDCl}_{3}, 600 \mathrm{MHz}\right) \delta 4.25$ (dddd, $\left.J=3.6,3.0,2.4,2.4 \mathrm{~Hz}, 1 \mathrm{H}\right)$, 2.90 (m, 1H), 2.76 (ddd, $J=13.2,13.2,6.0 \mathrm{~Hz}, 1 \mathrm{H}), 2.27$ (ddd, $J=13.8,4.8,2.4 \mathrm{~Hz}, 1 \mathrm{H}), 2.15$ (m, 2H), 1.92 (dddd, $J=13.8,13.8,5.4,3.0 \mathrm{~Hz}, 1 \mathrm{H}), 1.63$ (ddd, $J=12.6,12.6,2.4 \mathrm{~Hz}, 1 \mathrm{H}), 1.03$ $(\mathrm{d}, J=6.6 \mathrm{~Hz}, 3 \mathrm{H}) ;{ }^{13} \mathrm{C} \mathrm{NMR}\left(\mathrm{CDCl}_{3}, 150 \mathrm{MHz}\right) \delta 213.1,65.3,42.5,39.2,36.2,34.3,14.3$; HRMS (ESI) calcd. for $\left[\mathrm{C}_{7} \mathrm{H}_{12} \mathrm{O}_{2}+\mathrm{H}\right]^{+}: 129.09101$, Found: 129.09102 .

\section{Dibenzyl (1R,3S)-3-methyl-4-oxocyclohexyl phosphate (II-41)}

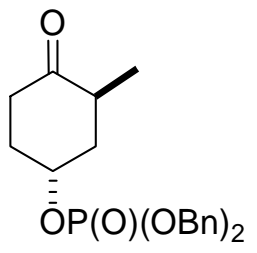

103 
Into a solution of $20 \mathrm{mg}$ alcohol II-40 $(0.16 \mathrm{mmol})$ in $0.5 \mathrm{~mL} \mathrm{CH}_{2} \mathrm{Cl}_{2}$ was added a solution of $0.14 \mathrm{~mL} 85 \% \mathrm{~N}$,N-diethyl dibenzylphosphoramidite $(0.43 \mathrm{mmol})$ in $0.5 \mathrm{~mL} \mathrm{CH}_{2} \mathrm{Cl}_{2}$ at room temperature followed by addition of $0.95 \mathrm{~mL} 0.45 \mathrm{M} 1 H$-tetrazole in acetonitrile $(0.43 \mathrm{mmol})$. The mixture ws stirred at RT for $1 \mathrm{hr}$. Then it was cooled down to $0{ }^{\circ} \mathrm{C}$, and slowly added 0.75 $\mathrm{mL} 30 \% \mathrm{H}_{2} \mathrm{O}_{2}$. It was stirred at to $0{ }^{\circ} \mathrm{C}$ for $15 \mathrm{~min}$. Into the mixture, $2 \mathrm{~mL}$ saturated aqueous $\mathrm{Na}_{2} \mathrm{~S}_{2} \mathrm{O}_{3}$ was added and it was then stirred at $0{ }^{\circ} \mathrm{C}$ for $2 \mathrm{hr}$. The mixture was extracted with EtOAc $30 \mathrm{~mL}$ X 2. The organic layer was washed with $4 \mathrm{~mL}$ saturated aqueous $\mathrm{NaHCO}_{3}, 4 \mathrm{~mL}$ saturated aqueous $\mathrm{NaCl}$, dried over $\mathrm{Na}_{2} \mathrm{SO}_{4}$, and concentrated under reduced pressure to give a residue which was subjected to chromatography on silica gel column. Elution with hexaneEtOAc (1:1) afforded phosphate II-41 (42 mg, 70\%).

II-41: Colorless oil; $R_{f}=0.36\left(1: 2(\mathrm{v} / \mathrm{v})\right.$ hexane/EtOAc); $[\alpha]_{\mathrm{D}}{ }^{20}-3$ (c 2.2, MeOH); IR (thin film, $\left.\mathrm{cm}^{-1}\right) v 3035,2934,1712,1497,1455,1270,990 ;{ }^{1} \mathrm{H} \mathrm{NMR}\left(\mathrm{CD}_{3} \mathrm{OD}, 600 \mathrm{MHz}\right) \delta 7.38(\mathrm{~m}, 10 \mathrm{H})$, $5.13(\mathrm{dd}, J=12.0,9.6 \mathrm{~Hz}, 2 \mathrm{H}), 5.09$ (dd, $J=12.0,10.2 \mathrm{~Hz}, 2 \mathrm{H}), 4.73(\mathrm{~m}, 1 \mathrm{H}), 2.58$ (ddd, $J=$ 19.8, 12.6, 6.6 Hz, 1H), 2.52 (ddd, $J=14.4,14.4,5.4 \mathrm{~Hz}, 1 \mathrm{H}), 2.19$ (m, 1H), 2.13 (ddd, $J=14.4$, 4.8, $2.4 \mathrm{~Hz}, 1 \mathrm{H}$ ), 1.87 (ddddd, $J=11.8,11.8,4.8,2.4,2.4 \mathrm{~Hz}, 1 \mathrm{H}), 1.58$ (dddd, $J=11.8,11.8$, 2.4, $2.4 \mathrm{~Hz}, 1 \mathrm{H}), 0.89$ (d, $J=6.6 \mathrm{~Hz}, 1 \mathrm{H}) ;{ }^{13} \mathrm{C} \mathrm{NMR}\left(\mathrm{CD}_{3} \mathrm{OD}, 150 \mathrm{MHz}\right) \delta 213.6,137.5(\mathrm{~d}, J=$ $5.7 \mathrm{~Hz}), 137.4$ (d, $J=5.7 \mathrm{~Hz}), 130.0,129.9,129.4$ (d, $J=2.2 \mathrm{~Hz}), 75.6$ (d, $J=5.7 \mathrm{~Hz}), 71.1$ (d, $J$ $=5.7 \mathrm{~Hz}), 41.4(\mathrm{~d}, J=1.6 \mathrm{~Hz}), 40.3,37.0,33.5(\mathrm{~d}, J=4.6 \mathrm{~Hz}), 14.4$; HRMS (ESI) calcd. for $\left[\mathrm{C}_{21} \mathrm{H}_{25} \mathrm{O}_{5} \mathrm{P}+\mathrm{H}\right]^{+}:$389.15124, Found: 389.15117 .

\section{(1R,3S)-3-methyl-4-oxocyclohexyl dihydrogen phosphate (II-31)}

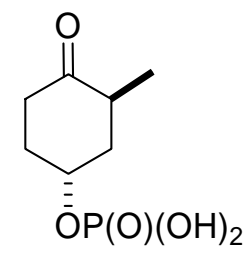

$38 \mathrm{mg} \mathrm{10 \%} \mathrm{Pd/C} \mathrm{was} \mathrm{first} \mathrm{washed} \mathrm{with} \mathrm{HPLC} \mathrm{grade} \mathrm{MeOH} 2 \mathrm{~mL} \mathrm{X} \mathrm{2,} \mathrm{then} \mathrm{it} \mathrm{was} \mathrm{transferred}$ with another $2 \mathrm{~mL}$ HPLC grade $\mathrm{MeOH}$ to $25 \mathrm{~mL}$ flask which contained $22 \mathrm{mg}$ dibenzyl phosphate II-41 (0.06 mmol). The mixture was degassed using vacuum and refilled with $\mathrm{H}_{2}$ at - 
$30{ }^{\circ} \mathrm{C}$ three times. The reaction was stirred under balloon pressure of $\mathrm{H}_{2}$ at room temperature for $3 \mathrm{hr}$. The reaction mixture was filtered and rinsed with HPLC grade $\mathrm{MeOH}$ and concentrated under reduced pressure to give a residue, which was then treated under vacuum at RT overnight to afford phosphate II-31 (8.5 mg, 73\%). ${ }^{1} \mathrm{H}$ and ${ }^{31} \mathrm{P}$ NMR show it is a mixture of two epimers on the $\alpha$-methyl group (dr $\boldsymbol{\alpha}: \boldsymbol{\beta} 2: 1$ ).

II-31 (dr $\boldsymbol{\alpha}: \boldsymbol{\beta}$ 2:1): Colorless oil; $[\alpha]_{D}^{20}-4\left(c\right.$ 0.74, MeOH); IR (thin film, $\left.\mathrm{cm}^{-1}\right) v$ 3500-2500 (broad), 2936, 1702, 1456, 1378, 1150, 1013, 990; HRMS (ESI) calcd. for $\left[\mathrm{C}_{7} \mathrm{H}_{13} \mathrm{O} 5 \mathrm{P}-\mathrm{H}\right]$ : 207.04278, Found: 207.04273.

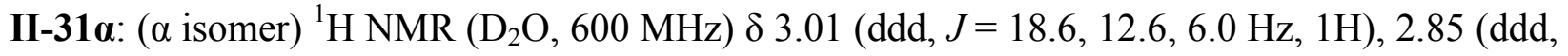
$J=13.8,13.8,6.0 \mathrm{~Hz}, 1 \mathrm{H}), 2.36(\mathrm{~m}, 3 \mathrm{H}), 2.02(\mathrm{dd}, J=14.4,13.8 \mathrm{~Hz}, 1 \mathrm{H}), 1.77$ (dd, $J=13.8$, $13.2 \mathrm{~Hz}, 1 \mathrm{H}), 1.03(\mathrm{~d}, J=6.6 \mathrm{~Hz}, 1 \mathrm{H}) ;{ }^{13} \mathrm{C} \mathrm{NMR}\left(\mathrm{D}_{2} \mathrm{O}, 150 \mathrm{MHz}\right) \delta 220.0,71.1(\mathrm{~d}, J=5.1 \mathrm{~Hz})$, $40.6(\mathrm{~d}, J=3.8 \mathrm{~Hz}), 39.6,36.2,32.7$ (d, $J=4.5 \mathrm{~Hz}), 13.6 ;{ }^{31} \mathrm{P}$ NMR $\left(\mathrm{D}_{2} \mathrm{O}, 242 \mathrm{MHz}\right) \delta 0.66$;

II-31ß: ( $\beta$ epimer) ${ }^{1} \mathrm{H}$ NMR $\left(\mathrm{D}_{2} \mathrm{O}, 600 \mathrm{MHz}\right) \delta 2.75(\mathrm{ddd}, J=19.8,13.2,6.6 \mathrm{~Hz}, 1 \mathrm{H}), 2.63$ (ddd, $J=14.4,14.4,7.8 \mathrm{~Hz}, 1 \mathrm{H}), 2.36(\mathrm{~m}, 3 \mathrm{H}), 1.89$ (dd, $J=15.0,15.0,4.8 \mathrm{~Hz}, 1 \mathrm{H}), 1.68(\mathrm{dd}, J=$ $13.8,13.2 \mathrm{~Hz}, 1 \mathrm{H}), 1.05(\mathrm{~d}, J=6.6 \mathrm{~Hz}, 1 \mathrm{H}) ;{ }^{13} \mathrm{C} \mathrm{NMR}\left(\mathrm{D}_{2} \mathrm{O}, 150 \mathrm{MHz}\right) \delta 218.4,72.8(\mathrm{~d}, J=5.7$ $\mathrm{Hz}), 41.7,40.8(\mathrm{~d}, J=4.5 \mathrm{~Hz}), 37.8,33.0(\mathrm{~d}, J=3.9 \mathrm{~Hz}), 13.9 ;{ }^{31} \mathrm{P} \mathrm{NMR}\left(\mathrm{D}_{2} \mathrm{O}, 242 \mathrm{MHz}\right) \delta 0.46$;

\section{Dibenzyl (1S,4R,5S)-4-hydroxy-5-methylcyclohex-2-enyl phosphate (II-42)}

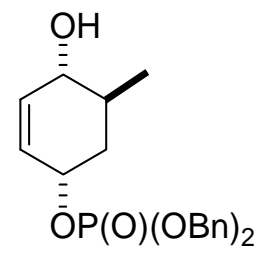

To a solution of $89 \mathrm{mg}$ phosphate $\mathbf{I I - 3 9}(0.23 \mathrm{mmol})$ in $3.5 \mathrm{~mL}$ THF at $-78{ }^{\circ} \mathrm{C}$ was added $20 \mathrm{mg}$ $\mathrm{LiAlH}_{4}(0.50 \mathrm{mmol})$. The reaction mixture was stirred at $-78{ }^{\circ} \mathrm{C}$ for $30 \mathrm{~min}$, then $1 \mathrm{~mL}$ acetone was added at $-78{ }^{\circ} \mathrm{C}$ to quench the reaction. Then $6 \mathrm{~mL}$ saturated aqueous $\mathrm{NH}_{4} \mathrm{Cl}$ was added and the reaction was stirred at $0{ }^{\circ} \mathrm{C}$ for $15 \mathrm{~min}$. The mixture was passed through a pad of sand-silica 
gel-celite, eluting with EtOAc-MeOH (10:1) and the effluent was dried over $\mathrm{Na}_{2} \mathrm{SO}_{4}$. The solution was concentrated under reduced pressure to give a residue. Crude ${ }^{1} \mathrm{H}$ NMR showed that the diastereoselectivity is $11: 1$. The residue was subjected to silica gel chromatography and elution with hexane-EtOAc (2:3) afforded allylic alcohol II-42 (67 mg, 87\%).

II-42: colorless oil; $R_{f}=0.71(10: 1 \mathrm{v} / \mathrm{v}$ EtOAc/MeOH$) ;[\alpha]_{\mathrm{D}}{ }^{20}-6\left(c 2.50, \mathrm{CHCl}_{3}\right)$; IR (thin film, $\left.\mathrm{cm}^{-1}\right)$ v 3412, 3034, 2954, 1498, 1456, 1380, 1252, 1215, 1034, 982; ${ }^{1} \mathrm{H}\left(\mathrm{CDCl}_{3}, 600 \mathrm{MHz}\right) \delta$ $7.34(\mathrm{~m}, 10 \mathrm{H}), 5.90$ (dd, $J=10.2,1.8 \mathrm{~Hz}, 1 \mathrm{H}), 5.76$ (dddd, $J=10.2,4.2,1.8,1.8 \mathrm{~Hz}, 1 \mathrm{H}), 5.04$ (ddd, $J=12.0,8.4,3.0 \mathrm{~Hz}, 2 \mathrm{H}$ ), 5.01 (ddd, $J=11.4,8.4,3.0 \mathrm{~Hz}, 2 \mathrm{H}$ ), 4.78 (dddd, $J=7.2,4.2$, 3.6, $3.0 \mathrm{~Hz}, 1 \mathrm{H}$ ), 3.66 (ddd, $J=9.0,3.0,1.8 \mathrm{~Hz}, 1 \mathrm{H}$ ), 1.90 (dddd, $J=14.4,2.4,2.4,1.2 \mathrm{~Hz}, 1 \mathrm{H}$ ), 1.77 (ddqd, $J=12.6,12.6,6.6,3.0 \mathrm{~Hz}, 1 \mathrm{H}), 1.48$ (dddd, $J=14.4,12.0,3.6,1.8 \mathrm{~Hz}, 1 \mathrm{H}$ ), 1.02 (d, $J=7.2 \mathrm{~Hz}, 3 \mathrm{H}) ;{ }^{13} \mathrm{C} \mathrm{NMR}\left(\mathrm{CDCl}_{3}, 150 \mathrm{MHz}\right) \delta 136.9,135.9(\mathrm{~d}, J=7.0 \mathrm{~Hz}), 128.5,128.4(\mathrm{~d}, J=$ $1.2 \mathrm{~Hz}), 127.9$ (d, $J=4.4 \mathrm{~Hz}), 125.8$ (d, $J=4.4 \mathrm{~Hz}), 73.0,70.9$ (d, $J=6.0 \mathrm{~Hz}), 69.1$ (d, $J=5.4$ $\mathrm{Hz}), 35.6(\mathrm{~d}, J=4.8 \mathrm{~Hz}), 32.2$, 17.9; HRMS (ESI) calcd. for $\left[\mathrm{C}_{21} \mathrm{H}_{25} \mathrm{O}_{5} \mathrm{P}+\mathrm{Na}\right]^{+}: 411.13318$, Found: 411.13294 .

\section{Dibenzyl (1R,3S,4S)-4-hydroxy-3-methylcyclohexyl phosphate (II-43)}

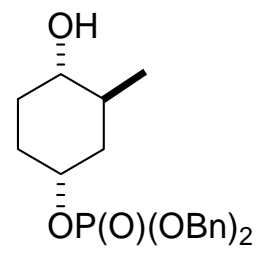

To a solution of $30 \mathrm{mg}$ allylic alcohol $\mathbf{I I}-\mathbf{4 2}(0.077 \mathrm{mmol})$ in $0.35 \mathrm{~mL} \mathrm{~N}$-methylmorpholine was added $167 \mathrm{mg}(0.77 \mathrm{mmol}) o$-nitrobenzenesulfonylhydrazide and $128 \mu 1 \mathrm{Et}_{3} \mathrm{~N}$. It was stirred at RT for $18 \mathrm{hr}$, then the reaction mixture was diluted with $30 \mathrm{~mL}$ EtOAc and washed at $0{ }^{\circ} \mathrm{C}$ by 8 $\mathrm{mL} 0.5 \mathrm{M} \mathrm{NaHSO}_{4}$. The aqueous layer was extracted with EtOAc $30 \mathrm{~mL} \mathrm{X} \mathrm{2.} \mathrm{The} \mathrm{organic} \mathrm{layer}$ was washed with $5 \mathrm{~mL}$ saturated aqueous $\mathrm{NaHCO}_{3}, 5 \mathrm{~mL}$ saturated brine, and dried over $\mathrm{Na}_{2} \mathrm{SO}_{4}$. The organic layer was concentrated under reduced pressure to give crude product, which was then subjected to chromatography on silica gel column. Elution with hexane-EtOAc (1:1.5-1:2) gave saturated alcohol II-43 (18 mg, 60\%) and recovered starting material II-42 (6 mg, 20\% recovery). 
II-43: Colorless oil; $R_{f}=0.19\left(1: 3 \mathrm{v} / \mathrm{v}\right.$ hexane/EtOAc); $[\alpha]_{\mathrm{D}}{ }^{20}+0.3\left(c 0.76, \mathrm{CHCl}_{3}\right)$; IR (thin film, $\left.\mathrm{cm}^{-1}\right)$ v 3420, 3064, 2977, 2934, 1498, 1456, 1256, 1213, 997; ${ }^{1} \mathrm{H} \mathrm{NMR}\left(\mathrm{CDCl}_{3}, 600 \mathrm{MHz}\right) \delta$ $7.35(\mathrm{~m}, 10 \mathrm{H}), 5.06(\mathrm{dd}, J=12.0,8.4 \mathrm{~Hz}, 2 \mathrm{H}), 5.02$ (ddd, $J=12.0,8.4,1.2 \mathrm{~Hz}, 2 \mathrm{H}), 4.61$ (ddddd, $J=6.0,3.6,3.0,3.0,2.4 \mathrm{~Hz}, 1 \mathrm{H}), 3.13(\mathrm{ddd}, J=10.2,10.2,4.2 \mathrm{~Hz}, 1 \mathrm{H}), 1.97$ (dddd, $J=13.8,6.6$, 3.6, 3.0 Hz, 1H), 1.91 (ddd, $J=14.4,7.2,3,6 \mathrm{~Hz}, 1 \mathrm{H}), 1.74(\mathrm{ddd}, J=12.0,7.2,3.6 \mathrm{~Hz}, 1 \mathrm{H}), 1.67$ (ddqd, $J=13.2,12.6,6.6,3.0 \mathrm{~Hz}, 1 \mathrm{H}), 1.53(\mathrm{~m}, 2 \mathrm{H}), 1.18(\mathrm{dddd}, J=14.4,12.0,2.4,1.8 \mathrm{~Hz}, 1 \mathrm{H})$, $0.95(\mathrm{~d}, J=6.6 \mathrm{~Hz}, 3 \mathrm{H}) ;{ }^{13} \mathrm{C} \mathrm{NMR}\left(\mathrm{CDCl}_{3}, 150 \mathrm{MHz}\right) \delta 136.0(\mathrm{~d}, J=5.4 \mathrm{~Hz}), 128.5,128.4$, 127.8, 75.1, 74.2 (d, $J=6.0 \mathrm{~Hz}), 69.1$ (d, $J=5.4 \mathrm{~Hz}), 38.1(\mathrm{~d}, J=4.4 \mathrm{~Hz}), 34.0,30.2(\mathrm{~d}, J=4.4$ $\mathrm{Hz}), 20.1,17.8 ;{ }^{31} \mathrm{P}$ NMR $\left(\mathrm{CD}_{3} \mathrm{OD}, 242 \mathrm{MHz}\right) \delta-0.419$; HRMS (ESI) calcd. for $\left[\mathrm{C}_{21} \mathrm{H}_{27} \mathrm{O}_{5} \mathrm{P}+\mathrm{H}\right]^{+}$: 391.16687, Found: 391.16685.

(1R,3S,4S)-4-hydroxy-3-methylcyclohexyl dihydrogen phosphate (II-32)

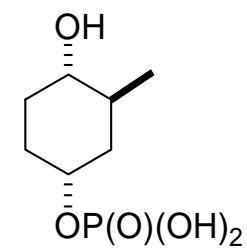

$38 \mathrm{mg} \mathrm{10 \%} \mathrm{Pd/C} \mathrm{was} \mathrm{first} \mathrm{washed} \mathrm{with} \mathrm{HPLC} \mathrm{grade} \mathrm{MeOH} 2 \mathrm{~mL} \mathrm{X} \mathrm{2,} \mathrm{then} \mathrm{it} \mathrm{was} \mathrm{transferred}$ with another $2 \mathrm{~mL}$ HPLC grade $\mathrm{MeOH}$ to $25 \mathrm{~mL}$ flask which contained $21 \mathrm{mg}$ dibenzyl phosphate II-43 (0.05 mmol). The mixture was degassed using vacuum and refilled with $\mathrm{H}_{2}$ at $30{ }^{\circ} \mathrm{C}$ three times. The reaction was stirred under balloon pressure of $\mathrm{H}_{2}$ at room temperature for $3 \mathrm{hr}$. The reaction mixture was filtered and rinsed with HPLC grade $\mathrm{MeOH}$ and concentrated under reduced pressure to give a residue, which was then treated under vacuum at RT overnight to afford phosphate II-32 (9.5 mg, 85\%). 
II-32: Colorless oil; $[\alpha]_{\mathrm{D}}^{20}+14(c 0.95, \mathrm{MeOH})$; IR (thin film, $\left.\mathrm{cm}^{-1}\right)$ v 3500-2500 (broad), 3254, 2935, 2872, 1654, 1446, 1377, 1162, 1001, 980; ${ }^{1} \mathrm{H}$ NMR ( $\left.\mathrm{D}_{2} \mathrm{O}, 600 \mathrm{MHz}\right) \delta 4.50$ (m, 1H), 3.29 (ddd, $J=10.2,10.2,4.2 \mathrm{~Hz}, 1 \mathrm{H}), 2.00(\mathrm{~m}, 1 \mathrm{H}), 1.97(\mathrm{~m}, 2 \mathrm{H}), 1.79(\mathrm{~m}, 2 \mathrm{H}), 1.65(\mathrm{~m}, 2 \mathrm{H}), 1.35$ (dddd, $J=13.8,12.6,1.2,1.2 \mathrm{~Hz}, 1 \mathrm{H}), 1.00(\mathrm{~d}, J=6.6 \mathrm{~Hz}, 3 \mathrm{H}) ;{ }^{13} \mathrm{C} \mathrm{NMR}\left(\mathrm{D}_{2} \mathrm{O}, 150 \mathrm{MHz}\right) \delta$ 75.2, $72.8(\mathrm{~d}, J=5.6 \mathrm{~Hz}), 38.0(\mathrm{~d}, J=4.4 \mathrm{~Hz}), 33.4,29.9(\mathrm{~d}, J=4.4 \mathrm{~Hz}), 28.3,17.6 ;{ }^{31} \mathrm{P}$ NMR $\left(\mathrm{D}_{2} \mathrm{O}, 242 \mathrm{MHz}\right) \delta$-0.492; HRMS (ESI) calcd. for $\left[\mathrm{C}_{7} \mathrm{H}_{15} \mathrm{O}_{5} \mathrm{P}-\mathrm{H}\right]^{-}: 209.05843$, Found: 209.05843.

\section{Dibenzyl (1S,2S,3S,4R,5S)-2,3,4-trihydroxy-5-methylcyclohexyl phosphate (II-44)}

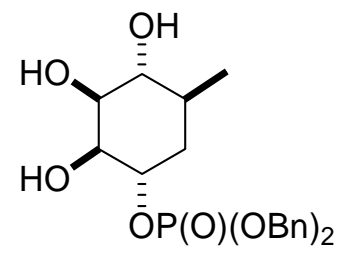

To a solution of $60 \mathrm{mg}$ allylic alcohol II-42 $(0.154 \mathrm{mmol})$ in $1.5 \mathrm{~mL}$ t-BuOH-Actone-50\% NMO (aq.) $(1: 1: 1 \mathrm{v} / \mathrm{v} / \mathrm{v})$ was added a small piece of crystalline $\mathrm{OsO}_{4}$ at $0{ }^{\circ} \mathrm{C}$. The reaction mixture was stirred at $0{ }^{\circ} \mathrm{C}$ for overnight, then $1.5 \mathrm{~mL}$ saturated aqueous $\mathrm{Na}_{2} \mathrm{~S}_{2} \mathrm{O}_{3}$ was added and it was stirred at $0{ }^{\circ} \mathrm{C}$ for another $2 \mathrm{hr}$. The reaction mixture was passed through a pad of sand-silica gel-celite, washing with EtOAc-MeOH (1:1, v/v). The solution was dried over $\mathrm{Na}_{2} \mathrm{SO}_{4}$ and concentrated under reduced pressure to give a residue. Crude ${ }^{1} \mathrm{H}-\mathrm{NMR}$ showed only one single diastereomer was formed. The residue was subjected to chromatography on silica gel column, eluting with EtOAc-MeOH (20:1) gave triol II-44 (50 mg, 77\%).

II-44: Colorless oil: $R_{f}=0.77\left(10: 1(\mathrm{v} / \mathrm{v})\right.$ EtOAc/MeOH); $[\alpha]_{\mathrm{D}}{ }^{20}+6(c$ 1.84, MeOH); IR (thin film, $\left.\mathrm{cm}^{-1}\right)$ 3378, 3066, 3035, 2952, 2926, 1498, 1456, 1378, 1247, 1215, 995, 970; ${ }^{1} \mathrm{H}$ NMR $\left(\mathrm{CD}_{3} \mathrm{OD}, 600 \mathrm{MHz}\right) \delta 7.37(\mathrm{~m}, 10 \mathrm{H}), 5.06(\mathrm{ddd}, J=11.4,8.4,1.2 \mathrm{~Hz}, 2 \mathrm{H}), 5.03$ (ddd, $J=11.4$, 9.0, 4.2 Hz, 2H), 4.46 (dddd, $J=6.6,4.2,3.0,2.4 \mathrm{~Hz}, 1 \mathrm{H}), 3.95$ (dd, $J=3.6,3.6 \mathrm{~Hz}, 1 \mathrm{H}), 3.49$ $(\mathrm{dd}, J=9.6,3.6 \mathrm{~Hz}, 1 \mathrm{H}), 3.25(\mathrm{dd}, J=9.6,9.6 \mathrm{~Hz}, 1 \mathrm{H}), 1.64(\mathrm{~m}, 3 \mathrm{H}), 0.96(\mathrm{~d}, J=6.6 \mathrm{~Hz}, 3 \mathrm{H})$; ${ }^{13} \mathrm{C}$ NMR $\left(\mathrm{CD}_{3} \mathrm{OD}, 150 \mathrm{MHz}\right) \delta 137.3(\mathrm{~d}, J=5.8 \mathrm{~Hz}), 129.9(\mathrm{~d}, J=2.8 \mathrm{~Hz}), 129.8(\mathrm{~d}, J=1.2$ Hz), $129.3(\mathrm{~d}, J=7.5 \mathrm{~Hz}), 78.9(\mathrm{~d}, J=6.3 \mathrm{~Hz}), 76.2,74.0,72.8(\mathrm{~d}, J=5.8 \mathrm{~Hz}), 71.1(\mathrm{~d}, J=6.4$ 
$\mathrm{Hz}), 71.0(\mathrm{~d}, J=6.4 \mathrm{~Hz}), 34.2(\mathrm{~d}, J=4.0 \mathrm{~Hz}), 33.2,18.2 ;{ }^{31} \mathrm{P} \mathrm{NMR}\left(\mathrm{CD}_{3} \mathrm{OD}, 242 \mathrm{MHz}\right) \delta$-1.003; HRMS (ESI) calcd. for $\left[\mathrm{C}_{21} \mathrm{H}_{27} \mathrm{O}_{7} \mathrm{P}+\mathrm{H}\right]^{+}: 423.15672$, Found: 423.15630.

$(1 S, 2 S, 3 S, 4 R, 5 S)-2,3,4-$ trihydroxy-5-methylcyclohexyl dihydrogen phosphate (II-33)

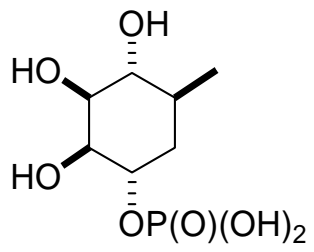

$39 \mathrm{mg} \mathrm{10 \%} \mathrm{Pd} / \mathrm{C}$ was first washed with HPLC grade $\mathrm{MeOH} 2 \mathrm{~mL} \mathrm{X} \mathrm{2,} \mathrm{then} \mathrm{it} \mathrm{was} \mathrm{transferred}$ with another $2 \mathrm{~mL}$ HPLC grade $\mathrm{MeOH}$ to $25 \mathrm{~mL}$ flask which contained $24 \mathrm{mg}$ dibenzyl phosphate II-44 (0.06 mmol). The mixture was degassed using vacuum and refilled with $\mathrm{H}_{2}$ at $\sim-$ $30{ }^{\circ} \mathrm{C}$ three times. The reaction was stirred under balloon pressure of $\mathrm{H}_{2}$ at room temperature for $4 \mathrm{hr}$. The reaction mixture was filtered and rinsed with HPLC grade $\mathrm{MeOH}$ and concentrated under reduced pressure to give a residue, which was then treated with vacuum at RT overnight to afford phosphate II-33 (12 mg, 87\%).

II-33: Colorless oil; $[\alpha]_{\mathrm{D}}^{20}+4\left(c\right.$ 1.13, MeOH); IR (thin film, $\left.\mathrm{cm}^{-1}\right) v$ 3283, 2918, 1376, 1011, 972; ${ }^{1} \mathrm{H}$ NMR $\left(\mathrm{D}_{2} \mathrm{O}, 600 \mathrm{MHz}\right) \delta 4.37$ (ddd, $\left.J=9.0,6.0,3.0,1 \mathrm{H}\right), 4.12(\mathrm{ddd}, J=3.6,3.6,1.2 \mathrm{~Hz}$, 1H), 3.73 (dd, $J=9.6,3.0 \mathrm{~Hz}, 1 \mathrm{H}$ ), 3.35 (dd, $J=10.2,10.2 \mathrm{~Hz}, 1 \mathrm{H}$ ), 1.89 (dddd, $J=14.4,3.0$, 2.4, $1.8 \mathrm{~Hz}, 1 \mathrm{H}$ ), 1.83 (ddqd, $J=13.2,10.8,6.6,4.2 \mathrm{~Hz}, 1 \mathrm{H}$ ), 1.63 (dddd, $J=13.2,13.2,2.4,1.2$ $\mathrm{Hz}, 1 \mathrm{H}), 1.05(\mathrm{~d}, J=6.0 \mathrm{~Hz}, 3 \mathrm{H}) ;{ }^{13} \mathrm{C} \mathrm{NMR}\left(\mathrm{D}_{2} \mathrm{O}, 150 \mathrm{MHz}\right) \delta 75.1,74.5(\mathrm{~d}, J=5.6 \mathrm{~Hz}), 72.2$, $71.9(\mathrm{~d}, J=5.4 \mathrm{~Hz}), 32.8(\mathrm{~d}, J=3.4 \mathrm{~Hz}), 31.6,17.1 ;{ }^{31} \mathrm{P}$ NMR $\left(\mathrm{D}_{2} \mathrm{O}, 242 \mathrm{MHz}\right) \delta-0.612$; HRMS (ESI) calcd. for $\left.\left[\mathrm{C}_{7} \mathrm{H}_{15} \mathrm{O}_{7} \mathrm{P}-\mathrm{H}\right]\right]^{-}: 241.04826$, Found: 241.04805.

(3aS,5R,7S,7aR)-hexahydro-2,2,7-trimethylbenzo[d][1,3]dioxol-5-ol (II-45) 


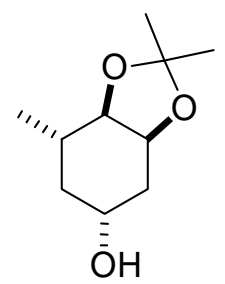

To a solution of $130 \mathrm{mg}$ carbonate II-22 $(0.45 \mathrm{mmol})$ in $5 \mathrm{~mL}$ THF at $0{ }^{\circ} \mathrm{C}$ was added $48 \mathrm{mg}$ $\mathrm{LiAlH}_{4}(1.26 \mathrm{mmol})$. The reaction mixture was warmed up and stirred at RT for $2 \mathrm{hr}$, then it was diluted with $30 \mathrm{~mL}$ EtOAc and cooled down to $0{ }^{\circ} \mathrm{C}$, and $4 \mathrm{~mL}$ saturated aqueous $\mathrm{NaHCO}_{3}$ was added and it was stirred for another 20 min to quench the reaction. The mixture was passed through a pad of sand-silica gel-celite, rinsing with EtOAc. The effluent was dried over $\mathrm{Na}_{2} \mathrm{SO}_{4}$. The solution was concentrated under reduced pressure to give a residue, which was subjected to chromatography on silica gel column, elution with hexane-EtOAc (5:1) afforded alcohol II-45 (78 mg, 93\%).

II-45: White solid; $\mathrm{mp}: 61.5-62.0^{\circ} \mathrm{C} ; R_{f}=0.28(1: 2(\mathrm{v} / \mathrm{v})$ hexane-EtOAc $) ;[\alpha]_{\mathrm{D}}{ }^{20}+54(c 0.66$, $\mathrm{CHCl}_{3}$ ); IR (thin film, $\mathrm{cm}^{-1}$ ) v 3257, 2990, 2940, 2923, 2884, 1458, 1433, 1378, 1364, 1242, 1214, 1197, 1151, 1130, 1064, 1042, 1024, 960; ${ }^{1} \mathrm{H} \mathrm{NMR}\left(\mathrm{CDCl}_{3}, 600 \mathrm{MHz}\right) \delta 4.28$ (ddd, $J=$ 4.8, 4.8, $2.4 \mathrm{~Hz}, 1 \mathrm{H}), 3.96$ (ddd, $J=10.8,10.8,4.2,4.2 \mathrm{~Hz}, 1 \mathrm{H}), 3.51$ (dd, $J=9.6,5.4 \mathrm{~Hz}, 1 \mathrm{H}$ ), 2.39 (dddd, $J=14.4,4.8,2.4,2.4 \mathrm{~Hz}, 1 \mathrm{H}), 1.85$ (s, OH), 1.77 (ddd, $J=12.6,6.6,3.6 \mathrm{~Hz}, 1 \mathrm{H}$ ), 1.65 (ddqd, $J=10.2,10.2,6.6,3.6 \mathrm{~Hz}, 1 \mathrm{H}), 1.59$ (ddd, $J=15.0,10.8,6.4 \mathrm{~Hz}, 1 \mathrm{H}), 1.43$ (s, 3H), $1.32(\mathrm{~s}, 3 \mathrm{H}), 1.01(\mathrm{~d}, J=6.6 \mathrm{~Hz}, 3 \mathrm{H}), 1.00(\mathrm{ddd}, J=13.2,13.2,10.8 \mathrm{~Hz}, 1 \mathrm{H}) ;{ }^{13} \mathrm{C} \mathrm{NMR}\left(\mathrm{CDCl}_{3}\right.$, $150 \mathrm{MHz}) \delta 108.2,80.7,74.5,66.0,40.5,36.2,33.7,28.5,26.1,18.7$; HRMS (ESI) calcd. for $\left[\mathrm{C}_{10} \mathrm{H}_{18} \mathrm{O}_{3}+\mathrm{H}\right]^{+}:$187.13287, Found: 187.13288 .

Dibenzyl (3aR,4S,6R,7aS)-hexahydro-2,2,4-trimethylbenzo[d][1,3]dioxol-6-yl phosphate (II46) 


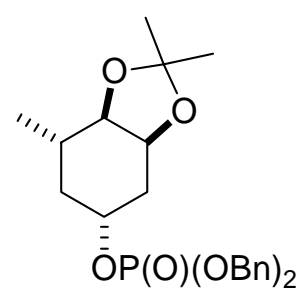

Into a solution of $74 \mathrm{mg}$ alcohol II-45 $(0.40 \mathrm{mmol})$ in $1.8 \mathrm{~mL} \mathrm{CH}_{2} \mathrm{Cl}_{2}$ was added a solution of $0.39 \mathrm{~mL} 85 \% \mathrm{~N}$,N-diethyl dibenzylphosphoramidite $(1.2 \mathrm{mmol})$ in $1.8 \mathrm{~mL} \mathrm{CH}_{2} \mathrm{Cl}_{2}$ at room temperature followed by addition of $2.6 \mathrm{~mL} 0.45 \mathrm{M} 1 H$-tetrazole in acetonitrile $(1.2 \mathrm{mmol})$. The mixture was stirred at RT for $1 \mathrm{hr}$. Then it was cooled down to $0{ }^{\circ} \mathrm{C}$, and added $1.8 \mathrm{~mL} 30 \%$ $\mathrm{H}_{2} \mathrm{O}_{2}$. It was stirred at to $0{ }^{\circ} \mathrm{C}$ for $30 \mathrm{~min}$. To the mixture was added $3 \mathrm{~mL}$ saturated aqueous $\mathrm{Na}_{2} \mathrm{~S}_{2} \mathrm{O}_{3}$ and it was then stirred $0{ }^{\circ} \mathrm{C}$ for $2 \mathrm{hr}$. The mixture was extracted with EtOAc $30 \mathrm{~mL}$ X 3 . The organic layer was washed with $5 \mathrm{~mL}$ saturated aqueous $\mathrm{NaHCO}_{3}, 5 \mathrm{~mL}$ saturated aqueous $\mathrm{NaCl}$, dried over $\mathrm{Na}_{2} \mathrm{SO}_{4}$, and concentrated under reduced pressure to give a residue which was subjected to chromatography on silica gel column. Elution with hexane-EtOAc (3:1) afforded phosphate II-46 (131 mg, 74\%).

II-46: Colorless oil: $R_{f}=0.38\left(1: 1(\mathrm{v} / \mathrm{v})\right.$ hexane/EtOAc); $[\alpha]_{\mathrm{D}}{ }^{20}+14\left(c 2.95, \mathrm{CHCl}_{3}\right)$; IR (thin film, $\mathrm{cm}^{-1}$ ) $v$ 3034, 2955, 2934, 1497, 1456, 1370, 1264, 1243, 1216, 1065, 993; ${ }^{1} \mathrm{H}$ NMR $\left(\mathrm{CDCl}_{3}, 600 \mathrm{MHz}\right) \delta 7.35(\mathrm{~m}, 10 \mathrm{H}), 5.03(\mathrm{~m}, 4 \mathrm{H}), 4.60$ (ddddd, $J=10.8,10.8,7.2,4.2,4.2 \mathrm{~Hz}$, 1H), 4.24 (ddd, $J=4.8,4.8,2.4 \mathrm{~Hz}, 1 \mathrm{H}), 3.49$ (dd, $J=9.6,5.4 \mathrm{~Hz}, 1 \mathrm{H}), 2.45$ (dddd, $J=14.4$, 4.8, 2.4, $2.4 \mathrm{~Hz}, 1 \mathrm{H}), 1.90(\mathrm{ddd}, J=13.2,6.6,3.61 \mathrm{H}) ; 1.76(\mathrm{ddd}, J=14.4,10.2,4.2 \mathrm{~Hz}, 1 \mathrm{H})$, $1.60(\mathrm{ddqd}, J=10.2,10.2,6.6,3.6 \mathrm{~Hz}, 1 \mathrm{H}), 1.43$ (s, 3H), 1.31 (s, 3H), 1.12 (ddd, $J=12.6,12.6$, $10.8 \mathrm{~Hz}, 1 \mathrm{H}) ; 0.98(\mathrm{~d}, J=6.6 \mathrm{~Hz}, 3 \mathrm{H}) ;{ }^{13} \mathrm{C} \mathrm{NMR}\left(\mathrm{CDCl}_{3}, 150 \mathrm{MHz}\right) \delta 135.9(\mathrm{~d}, J=7.0 \mathrm{~Hz})$, $128.5(\mathrm{~d}, J=2.7 \mathrm{~Hz}), 128.4(\mathrm{~d}, J=1.6 \mathrm{~Hz}), 127.9,80.1,73.9,73.7(\mathrm{~d}, J=6.0 \mathrm{~Hz}), 69.2(\mathrm{~d}, J=$ $5.6 \mathrm{~Hz}), 37.9(\mathrm{~d}, J=3.9 \mathrm{~Hz}), 34.1(\mathrm{~d}, J=6.0 \mathrm{~Hz}), 33.3,28.4,26.1,18.5$; HRMS (ESI) calcd. for $\left[\mathrm{C}_{24} \mathrm{H}_{31} \mathrm{O}_{6} \mathrm{P}+\mathrm{H}\right]^{+}:$447.19310, Found: 447.19283 .

Dibenzyl (1R,3S,4R,5S)-3,4-dihydroxy-5-methylcyclohexyl phosphate (II-47) 


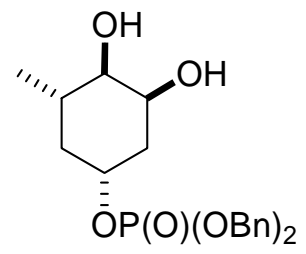

A solution of $100 \mathrm{mg}(0.22 \mathrm{mmol})$ of acetonide II-46 was dissolved in $1.4 \mathrm{~mL}$ THF-10\% $\mathrm{HCl}$ $(\mathrm{v} / \mathrm{v}, 1: 1)$ and stirred at room temperature for $30 \mathrm{~min}$. The mixture was cooled down to $0{ }^{\circ} \mathrm{C}$, and diluted with $50 \mathrm{~mL}$ EtOAc, then $2 \mathrm{~mL}$ saturated aqueous $\mathrm{NaHCO}_{3}$ and $400 \mathrm{mg} \mathrm{NaHCO}_{3}(\mathrm{~s})$ was slowly added to quench the reaction. The mixture was filtered through a pad of sand-silica gelcelite, rinsing with EtOAc-MeOH $(10: 1 \mathrm{v} / \mathrm{v})$. The effluent was dried over $\mathrm{Na}_{2} \mathrm{SO}_{4}$ and concentrated to a residue, which was loaded to a silica gel column. Elution with hexane-EtOAc $(1: 7 \mathrm{v} / \mathrm{v})$ gave diol II-47 (93mg, 95\%).

II-47: White solid; mp: $122-123{ }^{\circ} \mathrm{C} ; R_{f}=0.13\left(1: 2(\mathrm{v} / \mathrm{v})\right.$ hexane/EtOAc); $[\alpha]_{\mathrm{D}}{ }^{20}+4(c 0.91$, $\mathrm{MeOH}$ ); IR (thin film, $\mathrm{cm}^{-1}$ ) v 3454, 3394, 3038, 2956, 2926, 2869, 1605, 1497, 1456, 1377 , 1251, 1215, 1057, 1007; ${ }^{1} \mathrm{H}$ NMR $\left(\mathrm{CD}_{3} \mathrm{OD}, 600 \mathrm{MHz}\right) \delta 7.36(\mathrm{~m}, 10 \mathrm{H}), 5.03(\mathrm{dd}, J=12.6,8.0$ $\mathrm{Hz}, 1 \mathrm{H}), 5.01$ (ddd, $J=12.0,8.4,1.8 \mathrm{~Hz}, 1 \mathrm{H}$ ), 4.60 (ddddd, $J=11.4,11.4,7.2,4.8,4.8 \mathrm{~Hz}, 1 \mathrm{H}$ ), $3.93(\mathrm{ddd}, J=3.6,3.0,3.0 \mathrm{~Hz}, 1 \mathrm{H}), 3.06(\mathrm{dd}, J=10.8,3.0 \mathrm{~Hz}, 1 \mathrm{H}), 2.24$ (dddd, $J=13.2,4.2$, 3.0, $3.0 \mathrm{~Hz}, 1 \mathrm{H}), 1.92$ (dddd, $J=12.6,4.2,3.6,3.6 \mathrm{~Hz}, 1 \mathrm{H}) ; 1.82$ (ddqd, $J=13.2,10.2,6.6,3.6$ $\mathrm{Hz}, 1 \mathrm{H}), 1.59$ (ddd, $J=13.8,11.4,3.0 \mathrm{~Hz}, 1 \mathrm{H}), 1.17$ (ddd, $J=12.6,12.6,12.6 \mathrm{~Hz}, 1 \mathrm{H}) ; 0.97$ (d, $J=6.6 \mathrm{~Hz}, 3 \mathrm{H}) ;{ }^{13} \mathrm{C} \mathrm{NMR}\left(\mathrm{CD}_{3} \mathrm{OD}, 150 \mathrm{MHz}\right) \delta 137.4(\mathrm{~d}, J=6.0 \mathrm{~Hz}), 129.8,129.7,129.3,77.5$, $75.9(\mathrm{~d}, J=6.6 \mathrm{~Hz}), 71.1,70.8(\mathrm{~d}, J=5.4 \mathrm{~Hz}), 41.0$ (d, $J=4.5 \mathrm{~Hz}), 39.9$ (d, $J=4.8 \mathrm{~Hz}), 33.2$, 18.9; ${ }^{31} \mathrm{P}$ NMR $\left(\mathrm{CD}_{3} \mathrm{OD}, 242 \mathrm{MHz}\right) \delta$-1.003; HRMS (ESI) calcd. for $\left[\mathrm{C}_{21} \mathrm{H}_{27} \mathrm{O}_{6} \mathrm{P}+\mathrm{H}\right]^{+}: 407.1618$, Found: 407.1617.

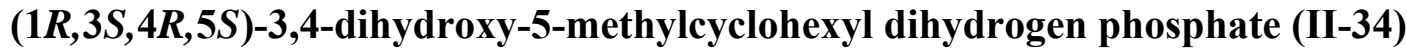

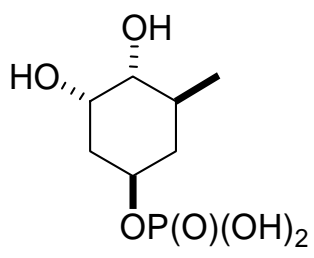


A $37 \mathrm{mg}$ sample of $10 \% \mathrm{Pd} / \mathrm{C}$ was first washed with HPLC grade $\mathrm{MeOH} 2 \mathrm{~mL} \mathrm{X} \mathrm{2,} \mathrm{then} \mathrm{it} \mathrm{was}$ transferred with another $2 \mathrm{~mL}$ HPLC grade $\mathrm{MeOH}$ to $25 \mathrm{~mL}$ flask which contained $21 \mathrm{mg}$ dibenzyl phosphate II-47 (0.05 mmol). The mixture was degassed using vacuum and refilled with $\mathrm{H}_{2}$ at $\sim-30{ }^{\circ} \mathrm{C}$ three times. The reaction was stirred under balloon pressure of $\mathrm{H}_{2}$ at room temperature for $3 \mathrm{hr}$. The reaction mixture was filtered and rinsed with HPLC grade $\mathrm{MeOH}$ and concentrated under reduced pressure to give a residue, which was then treated with vacuum at RT overnight to afford phosphate II-34 (9.0 mg, 78\%).

II-34: Colorless oil; $[\alpha]_{\mathrm{D}}^{20}+14(c 0.85, \mathrm{MeOH})$; IR (thin film, $\left.\mathrm{cm}^{-1}\right) v$ 3311, 2933, 1653, 1459, $1379,1158,1124,1088,1005,960,917 ;{ }^{1} \mathrm{H}$ NMR $\left(\mathrm{D}_{2} \mathrm{O}, 600 \mathrm{MHz}\right) \delta 4.49$ (ddddd, $J=11.4,11.4$, 9.0, 4.8, $4.8 \mathrm{~Hz}, 1 \mathrm{H}), 4.12$ (ddd, $J=3.0,3.0,3.0 \mathrm{~Hz}, 1 \mathrm{H}), 3.32$ (dd, $J=10.8,3.0 \mathrm{~Hz}, 1 \mathrm{H}), 2.29$ (dddd, $J=13.2,4.2,3.6,3.6 \mathrm{~Hz}, 1 \mathrm{H}), 2.12$ (dddd, $J=12.6,4.2,3.6,3.6 \mathrm{~Hz}, 1 \mathrm{H}$ ); 1.90 (ddqd, $J=$ 13.2, 10.2, 6.6, $3.6 \mathrm{~Hz}, 1 \mathrm{H}), 1.71$ (ddd, $J=13.8,12.0,2.4 \mathrm{~Hz}, 1 \mathrm{H}$ ), 1.30 (ddd, $J=12.0,12.0$, $12.0 \mathrm{~Hz}, 1 \mathrm{H}) ; 1.05(\mathrm{~d}, J=6.6 \mathrm{~Hz}, 3 \mathrm{H}) ;{ }^{13} \mathrm{C}$ NMR $\left(\mathrm{D}_{2} \mathrm{O}, 150 \mathrm{MHz}\right) \delta 76.2,71.7(\mathrm{~d}, J=5.4 \mathrm{~Hz})$, 70.0, $39.4(\mathrm{~d}, J=4.1 \mathrm{~Hz}), 38.2(\mathrm{~d}, J=4.4 \mathrm{~Hz}), 30.6,17.8 ;{ }^{31} \mathrm{P}$ NMR $\left(\mathrm{D}_{2} \mathrm{O}, 242 \mathrm{MHz}\right) \delta-0.413$; HRMS (ESI) calcd. for $\left[\mathrm{C}_{7} \mathrm{H}_{15} \mathrm{O}_{6} \mathrm{P}-\mathrm{H}\right]^{-}: 225.05335$, Found: 225.05335.

$(1 R, 4 S, 5 R)-4-t$-butyldimethylsilyloxy-5-methylcyclohex-2-enyl 4-nitrobenzoate (II-27a)

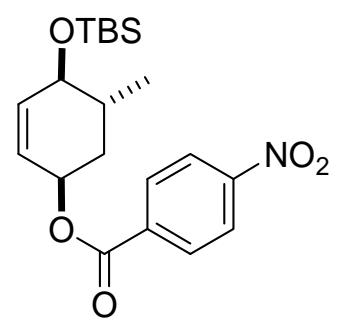

A solution of $1.15 \mathrm{~g}$ allylic alcohol II-27 $(4.18 \mathrm{mmol})$ in $42 \mathrm{~mL} \mathrm{CH}_{2} \mathrm{Cl}_{2}$ was added $1.14 \mathrm{~g}$ (16.7 $\mathrm{mmol})$ imidazole when stirring, followed by addition of $1.30 \mathrm{~g}$ TBSCl $(16.7 \mathrm{mmol})$ in portions. It was stirred at $\mathrm{RT}$ overnight, then it was diluted with $200 \mathrm{~mL}$ hexane- $\mathrm{Et}_{2} \mathrm{O}(1: 3 \mathrm{v} / \mathrm{v})$ and cooled down to $0{ }^{\circ} \mathrm{C}$, and $25 \mathrm{~mL}$ saturated aqueous $\mathrm{NaHCO}_{3}$ was added. It was stirred at $0{ }^{\circ} \mathrm{C}$ for 30 $\mathrm{min}$, and then the aqueous layer was extracted with $\mathrm{Et}_{2} \mathrm{O} 60 \mathrm{~mL} \times 2$. The pooled organic layer was washed with $50 \mathrm{~mL}$ saturated aqueous $\mathrm{NaCl}$, dried over $\mathrm{Na}_{2} \mathrm{SO}_{4}$. Concentration of the 113 
solution under reduced pressure gave crude product which will be used directly for next step reaction. For characterization, small amount of the crude product was subjected to chromatography on silica gel column, eluting with hexane-EtOAc $(20: 1, \mathrm{v} / \mathrm{v})$ gave product silylether II-27a.

II-27a: White solid; mp: 77.3-78.3 ${ }^{\circ} \mathrm{C} ; R_{f}=0.51\left(6: 1(\mathrm{v} / \mathrm{v})\right.$ hexane/EtOAc); $[\alpha]_{\mathrm{D}}{ }^{20}+97(c$ 4.12, $\mathrm{CHCl}_{3}$ ); IR (thin film, $\mathrm{cm}^{-1}$ ) v 3039, 2953, 2929, 2898, 2857, 1713, 1609, 1600, 1522, 1462, 1390, 1338, 1271, 1249, 1101, 1081, 1008, 932; ${ }^{1} \mathrm{H}$ NMR $\left(\mathrm{CDCl}_{3}, 600 \mathrm{MHz}\right) \delta 8.27$ (d, $J=8.4$ $\mathrm{Hz}, 1 \mathrm{H}), 8.21(\mathrm{~d}, J=9.0 \mathrm{~Hz}, 1 \mathrm{H}), 5.95(\mathrm{dd}, J=10.2,1.8 \mathrm{~Hz}, 1 \mathrm{H}), 5.84$ (dddd, $J=10.2,3.6,1.8$, $1.2 \mathrm{~Hz}, 1 \mathrm{H}$ ), 5.45 (ddd, $J=6.0,3.0,1.2 \mathrm{~Hz}, 1 \mathrm{H}$ ), 3.80 (ddd, $J=8.4,3.6,1.8 \mathrm{~Hz}, 1 \mathrm{H}$ ), 1.98 (ddd, $J=14.4,4.2,3.2 \mathrm{~Hz} 1 \mathrm{H}), 1.94(\mathrm{ddqd}, J=12.0,9.6,6.6,3.0 \mathrm{~Hz} 1 \mathrm{H}), 1.68(\mathrm{ddd}, J=16.2,12.0,4.8$ $\mathrm{Hz} 1 \mathrm{H}), 1.05$ (d, $J=6.6 \mathrm{~Hz}, 3 \mathrm{H}), 0.93(\mathrm{~s}, 9 \mathrm{H}), 0.12(\mathrm{~s}, 3 \mathrm{H}), 0.11(\mathrm{~s}, 3 \mathrm{H}) ;{ }^{13} \mathrm{C} \mathrm{NMR}\left(\mathrm{CDCl}_{3}, 150\right.$ MHz) $\delta 164.2,150.5,138.7,136.0,130.8,124.0,123.4,73.8,68.4,34.5,32.8,25.8,18.5,18.1$, 4.2, -4.6; HRMS (ESI) calcd. for $\left[\mathrm{C}_{20} \mathrm{H}_{29} \mathrm{NO}_{5} \mathrm{Si}+\mathrm{Na}\right]^{+}: 414.17072$, Found: 414.17035.

\section{t-Butyldimethylsilyl (1S,4R,6R)-4-hydroxy-6-methylcyclohex-2-enyl ether (II-48)}<smiles>C[C@H]1CC(O)C=CC1[OH+]</smiles>

The crude product of II-27a obtained from last step was redissolved in $45 \mathrm{~mL} \mathrm{MeOH}$ at $0{ }^{\circ} \mathrm{C}$. Then $115.4 \mathrm{mg} \mathrm{LiOH}(4.81 \mathrm{mmol})$ was slowly added into the solution. The reaction mixture was stirred at $0{ }^{\circ} \mathrm{C}$ for $2 \mathrm{hr}$. It was then diluted with $450 \mathrm{~mL}$ hexane-EtOAc $(1: 2 \mathrm{v} / \mathrm{v})$ and $35 \mathrm{~mL}$ saturated aqueous $\mathrm{NH}_{4} \mathrm{Cl}$ was added in. The aqueous layer was extracted with hexane-EtOAc $(1: 2 \mathrm{v} / \mathrm{v}) 100 \mathrm{~mL}$ X 2 . The pooled organic layer was washed with $50 \mathrm{~mL}$ saturated aqueous $\mathrm{NaCl}$, dried over $\mathrm{Na}_{2} \mathrm{SO}_{4}$. Concentration of the solution under reduced pressure gave a residue, then washed with $30 \mathrm{~mL}$ saturated brine, dried over $\mathrm{Na}_{2} \mathrm{SO}_{4}$. The solution was concentrated under reduced pressure to give $\sim 5 \mathrm{~mL}$ residue, which was subjected to chromatography on silica gel column. Elution with hexane-EtOAc (8:1) afforded allylic alcohol II-48 (873 mg, 85\% for two 
steps). II-48: Colorless oil; $R_{f}=0.49$ (2:1 (v/v) hexane/EtOAc); $[\alpha]_{\mathrm{D}}{ }^{20}-21\left(c 1.74, \mathrm{CHCl}_{3}\right)$; IR (thin film, $\mathrm{cm}^{-1}$ ) $v 3335,3032,2953,2929,2896,2858,1472,1462,1388,1251,1098,1078$, 1061, 989; ${ }^{1} \mathrm{H}$ NMR $\left(\mathrm{CDCl}_{3}, 600 \mathrm{MHz}\right) \delta 5.75(\mathrm{dddd}, J=10.2,3.0,1.2,1.2 \mathrm{~Hz}, 1 \mathrm{H}), 5.71(\mathrm{dd}, J$ $=10.2,1.8 \mathrm{~Hz}, 1 \mathrm{H}), 4.10(\mathrm{ddd}, J=6.6,3.0 \mathrm{~Hz}, 1 \mathrm{H}), 3.70(\mathrm{ddd}, J=7.8,3.0,1.8 \mathrm{~Hz}, 1 \mathrm{H}), 1.80(\mathrm{~m}$, 2H), 1.50 (ddd, $J=16.8,12.6,4.8 \mathrm{~Hz} 1 \mathrm{H}), 0.99$ (d, $J=6.6 \mathrm{~Hz}, 3 \mathrm{H}), 0.90$ (s, 9H), 0.08 (s, 3H), 0.07 (s, 3H); ${ }^{13} \mathrm{C}$ NMR $\left(\mathrm{CDCl}_{3}, 150 \mathrm{MHz}\right) \delta$ 135.5, 128.7, 74.0, 64.0, 37.4, 32.0, 25.8, 18.5, 18.1, $-4.2,-4.7$; HRMS (ESI) calcd. for $\left[\mathrm{C}_{13} \mathrm{H}_{26} \mathrm{O}_{2} \mathrm{Si}+\mathrm{H}\right]^{+}: 243.17748$, Found: 243.17744.

\section{Dibenzyl (1S,4R,5S)-4-t-butyldimethylsiloxy-5-methylcyclohex-2-enyl phosphate (II-49)}

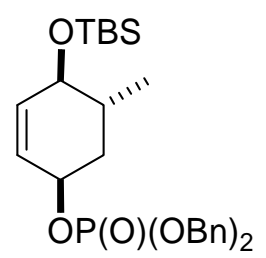

Into a solution of $36.7 \mathrm{mg}$ allylic alcohol $\mathbf{I I - 4 8}(0.15 \mathrm{mmol})$ in $0.5 \mathrm{~mL} \mathrm{CH}_{2} \mathrm{Cl}_{2}$ was added a

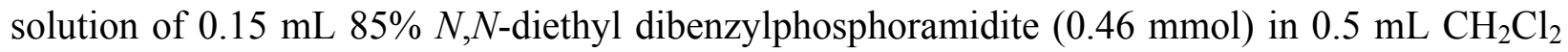
at room temperature, followed by addition of $1.0 \mathrm{~mL} 0.45 \mathrm{M} 1 H$-tetrazole in acetonitrile $(0.45$ mmol). The mixture was stirred at RT for $1 \mathrm{hr}$. Then it was cooled down to $0{ }^{\circ} \mathrm{C}$, and added 0.75 $\mathrm{mL} 30 \% \mathrm{H}_{2} \mathrm{O}_{2}$. It was stirred at to $0{ }^{\circ} \mathrm{C}$ for $30 \mathrm{~min}$. Into the mixture $1 \mathrm{~mL}$ saturated aqueous $\mathrm{Na}_{2} \mathrm{~S}_{2} \mathrm{O}_{3}$ was added and it was then stirred $0{ }^{\circ} \mathrm{C}$ for $20 \mathrm{~min}$. The mixture was extracted with EtOAc $15 \mathrm{~mL}$ X 3. The organic layer was washed with $4 \mathrm{~mL}$ saturated aqueous $\mathrm{NaHCO}_{3}, 4 \mathrm{~mL}$ saturated aqueous $\mathrm{NaCl}$, dried over $\mathrm{Na}_{2} \mathrm{SO}_{4}$, and concentrated under reduced pressure to give a residue which was subjected to chromatography on silica gel column. Elution with hexaneEtOAc (4:1) afforded phosphate II-49 (49 mg, 66\%).

II-49: Colorless oil; $R_{f}=0.30\left(2: 1 \mathrm{v} / \mathrm{v}\right.$ EtOAc/MeOH); $[\alpha]_{\mathrm{D}}{ }^{20}+18\left(c 2.0, \mathrm{CHCl}_{3}\right)$; IR (thin film, $\left.\mathrm{cm}^{-1}\right) v 3035,2955,2929,2857,1498,1456,1394,1252,1214,1082,985 ;{ }^{1} \mathrm{H}\left(\mathrm{CDCl}_{3}, 600 \mathrm{MHz}\right)$ $\delta 7.34(\mathrm{~m}, 10 \mathrm{H}), 5.82(\mathrm{dd}, J=10.2,1.8 \mathrm{~Hz}, 1 \mathrm{H}), 5.76$ (dddd, $J=10.2,4.2,1.8,1.8 \mathrm{~Hz}, 1 \mathrm{H}), 5.04$ (ddd, $J=11.4,7.8,2.4 \mathrm{~Hz}, 2 \mathrm{H}$ ), 5.02 (ddd, $J=11.4,7.8,3.0 \mathrm{~Hz}, 2 \mathrm{H}$ ), 4.79 (m, 1H), 3.66 (ddd, $J$ $=8.4,2.4,1.8 \mathrm{~Hz}, 1 \mathrm{H}), 1.91$ (dddd, $J=14.4,4.2,2.4 \mathrm{~Hz}, 1 \mathrm{H}), 1.85$ (ddqd, $J=13.2,9.0,6.6,2.4$ 
$\mathrm{Hz}, 1 \mathrm{H}), 1.48$ (dddd, $J=14.4,12.6,4.2,1.8 \mathrm{~Hz}, 1 \mathrm{H}), 0.95(\mathrm{~d}, J=6.6 \mathrm{~Hz}, 3 \mathrm{H}) ;{ }^{13} \mathrm{C} \mathrm{NMR}\left(\mathrm{CDCl}_{3}\right.$, $150 \mathrm{MHz}) \delta 138.0,136.0(\mathrm{~d}, J=6.9 \mathrm{~Hz}), 128.5,128.4,127.8(\mathrm{~d}, J=8.7 \mathrm{~Hz}), 124.9(\mathrm{~d}, J=4.0$ Hz), 73.7, $71.1(\mathrm{~d}, J=5.8 \mathrm{~Hz}), 69.1(\mathrm{~d}, J=5.8 \mathrm{~Hz}), 35.7$ (d, $J=5.2 \mathrm{~Hz}), 32.0,25.8,18.3,18.1$, 4.3, -4.7; HRMS (ESI) calcd. for $\left[\mathrm{C}_{27} \mathrm{H}_{39} \mathrm{O}_{5} \mathrm{PSi}+\mathrm{H}\right]^{+}$: 503.23771, Found: 503.23769.

Dibenzyl (1S,2S,3S,4R,5S)-4-t-butyldimethylsilyloxy-2,3-dihydroxy-5-methylcyclohexyl phosphate (II-49a)

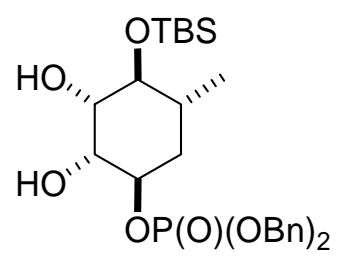

To a solution of $72 \mathrm{mg}$ olefin II-49 (0.146 mmol) in $1.5 \mathrm{~mL} t$-BuOH-Actone-50\% NMO (aq.) $(1: 1: 1 \mathrm{v} / \mathrm{v} / \mathrm{v})$ was added a small piece of crystalline $\mathrm{OsO}_{4}$ at $0{ }^{\circ} \mathrm{C}$. The reaction mixture was stirred at $0{ }^{\circ} \mathrm{C}$ for overnight, then $1 \mathrm{~mL}$ saturated aqueous $\mathrm{Na}_{2} \mathrm{~S}_{2} \mathrm{O}_{3}$ was added and it was stirred at $0{ }^{\circ} \mathrm{C}$ for another $2 \mathrm{hr}$. The reaction mixture was passed through a pad of sand-silica gel-celite, washing with EtOAc-MeOH $(1: 1, \mathrm{v} / \mathrm{v})$. The solution was dried over $\mathrm{Na}_{2} \mathrm{SO}_{4}$ and concentrated under reduced pressure to give a residue. Crude ${ }^{1} \mathrm{H}-\mathrm{NMR}$ showed only one single diastereomer was formed. The residue was subjected to chromatography on silica gel column, eluting with hexane-EtOAc (1:1) gave diol II-49a (62 mg, 81\%).

II-49a: Colorless oil: $R_{f}=0.36\left(1: 2(\mathrm{v} / \mathrm{v})\right.$ hexane-EtOAc); $[\alpha]_{\mathrm{D}}{ }^{20}-8\left(c\right.$ 2.43, $\left.\mathrm{CHCl}_{3}\right)$; IR (thin film, $\left.\mathrm{cm}^{-1}\right) v$ 3380, 3066, 3036, 2953, 2928, 2893, 2856, 1498, 1457, 1386, 1361, 1247, 1215, 1096, 997; ${ }^{1} \mathrm{H}$ NMR $\left(\mathrm{CDCl}_{3}, 600 \mathrm{MHz}\right) \delta 7.34$ (m, 10H), 5.04 (m, 2H), 5.01 (m, 2H), 4.52 (dddd, $J=8.4,5.4,5.4,3.0 \mathrm{~Hz}, 1 \mathrm{H}), 3.95(\mathrm{dd}, J=4.2,4.2 \mathrm{~Hz}, 1 \mathrm{H}), 3.60$ (dd, $J=7.2,5.0 \mathrm{~Hz}, 1 \mathrm{H}), 3.45$ (dd, $J=7.8,7.2 \mathrm{~Hz}, 1 \mathrm{H}), 3.2$ (brs, 1H), 2.34 (brs, 1H), 1.72 (m, 2H), 1.58 (d, $J=10.8,10.8,3.6$, $3.0 \mathrm{~Hz}, 1 \mathrm{H}), 0.93(\mathrm{~d}, J=7.2 \mathrm{~Hz}, 3 \mathrm{H}), 0.89$ (s, 9H), $0.08(\mathrm{~s}, 3 \mathrm{H}), 0.06(\mathrm{~s}, 3 \mathrm{H}) ;{ }^{13} \mathrm{C} \mathrm{NMR}\left(\mathrm{CDCl}_{3}\right.$, $150 \mathrm{MHz}) \delta 135.7(\mathrm{~d}, J=6.3 \mathrm{~Hz}), 128.6,128.0,127.9,76.3(\mathrm{~d}, J=5.8 \mathrm{~Hz}), 76.0,73.7,71.9(\mathrm{~d}, J$ $=5.1 \mathrm{~Hz}), 69.5(\mathrm{~d}, J=4.6 \mathrm{~Hz}), 71.0(\mathrm{~d}, J=6.4 \mathrm{~Hz}), 33.0(\mathrm{~d}, J=4.6 \mathrm{~Hz}), 32.9,25.9,18.2,18.0$, - 
4.1, -4.4; ${ }^{31} \mathrm{P}$ NMR $\left(\mathrm{CD}_{3} \mathrm{OD}, 242 \mathrm{MHz}\right) \delta$-1.003; HRMS (ESI) calcd. for $\left[\mathrm{C}_{27} \mathrm{H}_{41} \mathrm{O}_{7} \mathrm{PSi}+\mathrm{H}\right]^{+}$: 537.24319, Found: 537.24241.

Dibenzyl (1R,2R,3R,4S,5R)-2,3,4-trihydroxy-5-methylcyclohexyl phosphate (II-50)<smiles>CCOCO[C@H]1C[C@H](C)C(O)[C@H](O)[C@H]1O</smiles>

A $60 \mathrm{mg}$ sample of diol II-49a $(0.114 \mathrm{mmol})$ was dissolved in $1.2 \mathrm{~mL} \mathrm{MeOH}$ at $0{ }^{\circ} \mathrm{C}$ and $4.4 \mathrm{mg}$ $(0.023 \mathrm{mmol}) p-\mathrm{TsOH} \cdot \mathrm{H}_{2} \mathrm{O}$ was added. It was stirred at $0{ }^{\circ} \mathrm{C}$ for $24 \mathrm{hr}$, then was diluted with 10 $\mathrm{mL}$ EtOAc and quenched with $0.4 \mathrm{~mL}$ saturated aqueous $\mathrm{NaHCO}_{3}$. The mixture was concentrated under reduced pressure to give a residue, which was subjected to chromatography on silica gel column. Elution with EtOAc-MeOH (20:1) afforded triol II-50 (46 mg, 98\%).

II-50: Colorless oil: $R_{f}=0.31\left(10: 1(\mathrm{v} / \mathrm{v})\right.$ EtOAc-MeOH); $[\alpha]_{\mathrm{D}}{ }^{20}-6(c$ 1.57, MeOH); IR (thin film, $\left.\mathrm{cm}^{-1}\right) v 3376,3065,3036,2953,2926,1498,1456,1379,1247,1215,995,970 ;{ }^{1} \mathrm{H}$ NMR $\left(\mathrm{CD}_{3} \mathrm{OD}, 600 \mathrm{MHz}\right) \delta 7.37(\mathrm{~m}, 10 \mathrm{H}), 5.06(\mathrm{ddd}, J=12.0,9.0,1.2 \mathrm{~Hz}, 2 \mathrm{H}), 5.03$ (ddd, $J=11.4$, 9.0, $4.2 \mathrm{~Hz}, 1 \mathrm{H}$ ), 4.46 (dddd, $J=6.6,4.2,3.6,3.0 \mathrm{~Hz}, 1 \mathrm{H}), 3.96$ (dd, $J=3.0,3.0 \mathrm{~Hz}, 1 \mathrm{H}), 3.50$ (dd, $J=9.6,3.0 \mathrm{~Hz}, 1 \mathrm{H}), 3.26(\mathrm{dd}, J=9.6,9.0 \mathrm{~Hz}, 1 \mathrm{H}), 1.64(\mathrm{~m}, 3 \mathrm{H}), 0.96(\mathrm{~d}, J=6.6 \mathrm{~Hz}, 3 \mathrm{H})$; ${ }^{13} \mathrm{C}$ NMR $\left(\mathrm{CD}_{3} \mathrm{OD}, 150 \mathrm{MHz}\right) \delta 137.3(\mathrm{~d}, J=6.3 \mathrm{~Hz}), 129.9(\mathrm{~d}, J=2.2 \mathrm{~Hz}), 129.8(\mathrm{~d}, J=1.2$ $\mathrm{Hz}), 129.3(\mathrm{~d}, J=6.9 \mathrm{~Hz}), 78.9(\mathrm{~d}, J=6.3 \mathrm{~Hz}), 76.2,73.9,72.8(\mathrm{~d}, J=5.8 \mathrm{~Hz}), 71.1$ (d, $J=5.8$ $\mathrm{Hz}), 71.0(\mathrm{~d}, J=5.8 \mathrm{~Hz}), 34.1(\mathrm{~d}, J=4.0 \mathrm{~Hz}), 33.2,18.2$; HRMS (ESI) calcd. for $\left[\mathrm{C}_{21} \mathrm{H}_{27} \mathrm{O}_{7} \mathrm{P}+\mathrm{H}\right]^{+}: 423.15672$, Found: 423.15655 .

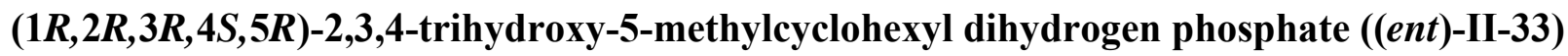




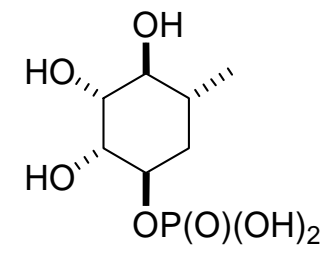

A $37 \mathrm{mg}$ sample of $10 \% \mathrm{Pd} / \mathrm{C}$ was first washed with HPLC grade $\mathrm{MeOH} 2 \mathrm{~mL} \times 2$, then it was transferred with another $2 \mathrm{~mL}$ HPLC grade $\mathrm{MeOH}$ to $25 \mathrm{~mL}$ flask which contained $22 \mathrm{mg}$ dibenzyl phosphate II-50 $(0.05 \mathrm{mmol})$. The mixture was degassed using vacuum and refilled with $\mathrm{H}_{2}$ at $\sim-30{ }^{\circ} \mathrm{C}$ three times. The reaction was stirred under balloon pressure of $\mathrm{H}_{2}$ at room temperature for $4 \mathrm{hr}$. The reaction mixture was filtered and rinsed with HPLC grade $\mathrm{MeOH}$ and concentrated under reduced pressure to give a residue, which was then treated with vacuum at RT overnight to afford phosphate (ent)-II-33 (10 mg, 81\%).

(ent)-II-33: Colorless oil; $[\alpha]_{\mathrm{D}}^{20}-6\left(c\right.$ 0.73, MeOH); IR (thin film, $\left.\mathrm{cm}^{-1}\right) v$ 3278, 2933, 1440, 1376, 1137, 1010, 972; ${ }^{1} \mathrm{H}$ NMR $\left(\mathrm{D}_{2} \mathrm{O}, 600 \mathrm{MHz}\right) \delta 4.37$ (ddd, $\left.J=9.0,6.6,3.0,1 \mathrm{H}\right), 4.12$ (ddd, $J=3.0,3.0,1.2 \mathrm{~Hz}, 1 \mathrm{H}), 3.74(\mathrm{dd}, J=9.6,3.0 \mathrm{~Hz}, 1 \mathrm{H}), 3.35$ (dd, $J=10.2,9.6 \mathrm{~Hz}, 1 \mathrm{H}), 1.89$ (dddd, $J=14.4,3.0,2.4,1.8 \mathrm{~Hz}, 1 \mathrm{H}$ ), 1.83 (ddqd, $J=13.2,10.2,6.6,3.0 \mathrm{~Hz}, 1 \mathrm{H}$ ), 1.63 (dddd, $J$ $=13.2,13.2,2.4,1.2 \mathrm{~Hz}, 1 \mathrm{H}), 1.05(\mathrm{~d}, J=6.6 \mathrm{~Hz}, 3 \mathrm{H}) ;{ }^{13} \mathrm{C} \mathrm{NMR}\left(\mathrm{D}_{2} \mathrm{O}, 150 \mathrm{MHz}\right) \delta 75.1,74.4$ $(\mathrm{d}, J=5.6 \mathrm{~Hz}), 72.2,71.9(\mathrm{~d}, J=5.4 \mathrm{~Hz}), 32.8(\mathrm{~d}, J=3.9 \mathrm{~Hz}), 31.6,17.1 ;{ }^{31} \mathrm{P}$ NMR $\left(\mathrm{D}_{2} \mathrm{O}, 242\right.$ MHz) $\delta$-0.594; HRMS (ESI) calcd. for $\left.\left[\mathrm{C}_{7} \mathrm{H}_{15} \mathrm{O} \mathrm{O}_{7} \mathrm{P}-\mathrm{H}\right]\right]^{-}: 241.04826$, Found: 241.04805.

\section{V.4. Chapter III Experimental}

(4S,6S)-4-(benzyloxy)-6-methylcyclohex-2-enone (III-7) 


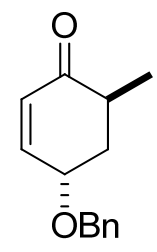

A solution of $832 \mathrm{mg}(3.68 \mathrm{mmol}) \alpha$-D-Boc-enone (ent)-III-4 and $0.70 \mathrm{~mL}(6.77 \mathrm{mmol}) \mathrm{BnOH}$ in $3 \mathrm{~mL} \mathrm{CH} \mathrm{Cl}_{2}$ at $0{ }^{\circ} \mathrm{C}$ was added a solution of $95 \mathrm{mg}(0.092 \mathrm{mmol}) \mathrm{Pd}_{2}(\mathrm{dba})_{3} \cdot \mathrm{CHCl}_{3}$ and 96

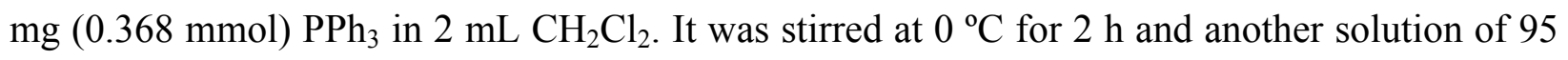
mg (0.092 mmol) $\mathrm{Pd}_{2}\left(\mathrm{dba}_{3} \cdot \mathrm{CHCl}_{3}\right.$ and $96 \mathrm{mg}(0.368 \mathrm{mmol}) \mathrm{PPh}_{3}$ in $2 \mathrm{~mL} \mathrm{CH}_{2} \mathrm{Cl}_{2}$ was added. The reaction mixture was stirred at $0{ }^{\circ} \mathrm{C}$ for another $3 \mathrm{~h}$. The mixture was directly loaded onto silica gel column. Elution with hexane-EtOAc (15:1, v/v) afforded glycosylated enone III-7 (475 mg 60\%) as colorless oil. $R_{f}=0.35\left(4: 1(\mathrm{v} / \mathrm{v})\right.$ hexane/EtOAc); $[\alpha]_{\mathrm{D}}{ }^{20}-146\left(\right.$ c $\left.2.56, \mathrm{CHCl}_{3}\right)$; IR (thin film, $\mathrm{cm}^{-1}$ ) $v$ 3033, 2964, 2931, 2872, 1678, 1497, 1454, 1377, 1331, 1250, 1205, 1220 , 1087, 1068, 1027, 946; ${ }^{1} \mathrm{H}$ NMR $\left(\mathrm{CDCl}_{3}, 600 \mathrm{MHz}\right) \delta$ 7.38-7.35 (m, 4H), $7.31(\mathrm{~m}, 1 \mathrm{H}), 6.89(\mathrm{dd}$, $J=10.2,3.6 \mathrm{~Hz}, 1 \mathrm{H}), 5.99$ (d, $J=9.6 \mathrm{~Hz}, 1 \mathrm{H}), 4.67$ (d, $J=12.0 \mathrm{~Hz}, 1 \mathrm{H}), 4.62(\mathrm{~d}, J=12.0 \mathrm{~Hz}$, 1H), 4.20 (ddd, $J=4.8,4.2,4.2 \mathrm{~Hz}, 1 \mathrm{H}$ ), 2.80 (dqd, $J=9.0,7.2,4.8 \mathrm{~Hz}, 1 \mathrm{H}$ ), 2.55 (dddd, $J=$ 13.8, 6.0, 4.8, $1.2 \mathrm{~Hz}, 1 \mathrm{H}), 1.98(\mathrm{ddd}, J=13.8,9.0,4.2 \mathrm{~Hz}, 1 \mathrm{H}), 1.15(\mathrm{~d}, J=7.2 \mathrm{~Hz}, 3 \mathrm{H}) ;{ }^{13} \mathrm{C}$ $\mathrm{NMR}\left(\mathrm{CDCl}_{3}, 150 \mathrm{MHz}\right) \delta 201.9,146.2,138.0,129.9,128.5,127.9,127.7,71.3,69.8,37.8,35.6$, 15.2; HRMS (ESI): calcd. for $\left[\mathrm{C}_{14} \mathrm{H}_{16} \mathrm{O}_{2}+\mathrm{H}\right]^{+}: 217.1223$, Found: 217.1223 .

\section{General procedure of palladium(0)-catalyzed cyclitolization for phenols}

A solution of $0.1 \mathrm{mmol}$ phenol and $27 \mathrm{mg}(0.12 \mathrm{mmol}) \alpha$-D-Boc-enone (ent)-III-4 in $1.5 \mathrm{~mL}$ $\mathrm{CH}_{2} \mathrm{Cl}_{2}$ at $0{ }^{\circ} \mathrm{C}$ was added a solution of $2.6 \mathrm{mg}\left(0.0025 \mathrm{mmol} \mathrm{Pd}_{2}(\mathrm{dba})_{3} \cdot \mathrm{CHCl}_{3}\right.$ and $2.6 \mathrm{mg}$ (0.010 mmol) $\mathrm{PPh}_{3}$ in $0.5 \mathrm{~mL} \mathrm{CH}_{2} \mathrm{Cl}_{2}$. The reaction mixture was stirred at $0{ }^{\circ} \mathrm{C}$ for $0.5-2 \mathrm{~h}$, then the mixture was directly subjected to silica gel column chromatography to afford the corresponding glycosylated product.

(4S,6S)-6-methyl-4-phenoxycyclohex-2-enone (III-21) 


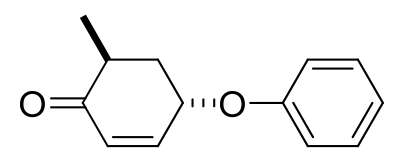

Colorles oil, $R_{f}=0.43\left(4: 1(\mathrm{v} / \mathrm{v})\right.$ hexane/EtOAc); $[\alpha]_{\mathrm{D}}{ }^{20}-150\left(c 1.2, \mathrm{CHCl}_{3}\right) ; \mathrm{IR}$ (thin film, $\mathrm{cm}^{-1}$ ) $v$ 3039, 2932, 1683, 1596, 1492, 1455, 1384, 1290, 1227, 1054, 980; ${ }^{1} \mathrm{H}$ NMR $\left(\mathrm{CDCl}_{3}, 600 \mathrm{MHz}\right)$ $\delta 7.32(\mathrm{dd}, J=7.2,7.2 \mathrm{~Hz}, 2 \mathrm{H}), 7.01(\mathrm{t}, J=7.2 \mathrm{~Hz}, 1 \mathrm{H}), 6.96(\mathrm{~m}, 3 \mathrm{H}), 6.08(\mathrm{~d}, J=10.2 \mathrm{~Hz}, 1 \mathrm{H})$, 5.04 (ddd, $J=4.8,4.2,4.2 \mathrm{~Hz}, 1 \mathrm{H}$ ), 2.87 (dqd, $J=9.6,7.2,4.8 \mathrm{~Hz}, 1 \mathrm{H}$ ), 2.39 (ddd, $J=13.8,4.8$,

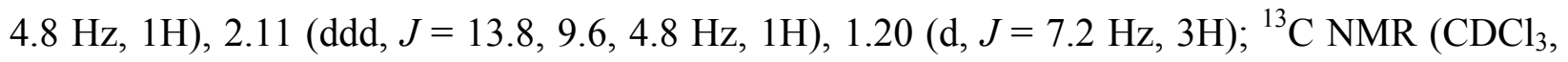
$150 \mathrm{MHz}) \delta 201.5,157.3,144.6,130.7,129.8,121.7,115.9,68.6,37.7,35.5,15.1$; HRMS (ESI): calcd. for $\left[\mathrm{C}_{13} \mathrm{H}_{14} \mathrm{O}_{2}+\mathrm{H}\right]^{+}:$203.1066, Found: 203.1066 .

(4S,6S)-4-(4-methoxyphenoxy)-6-methylcyclohex-2-enone (III-22)

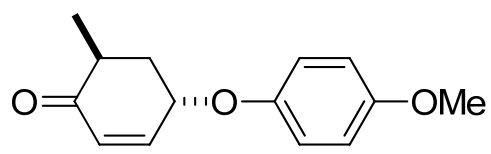

Colorles oil, $R_{f}=0.31$ (4:1 (v/v) hexane/EtOAc); $[\alpha]_{\mathrm{D}}{ }^{20}-233\left(c 0.07, \mathrm{CHCl}_{3}\right)$; IR (thin film, $\mathrm{cm}^{-1}$ ) v 3042, 2933, 1680, 1591, 1504, 1463, 1441, 1383, 1289, 1212, 1033, 980; ${ }^{1} \mathrm{H} \mathrm{NMR}\left(\mathrm{CDCl}_{3}\right.$, $600 \mathrm{MHz}) \delta 6.95(\mathrm{dd}, J=10.2,3.6 \mathrm{~Hz}, 1 \mathrm{H}), 6.91(\mathrm{~d}, J=9.0 \mathrm{~Hz}, 2 \mathrm{H}), 6.86(\mathrm{~d}, J=9.0 \mathrm{~Hz}, 2 \mathrm{H})$, $6.06(\mathrm{~d}, J=10.2 \mathrm{~Hz}, 1 \mathrm{H}), 4.90(\mathrm{ddd}, J=4.8,4.8,4.2 \mathrm{~Hz}, 1 \mathrm{H}), 2.86(\mathrm{dqd}, J=9.0,7.2,4.8 \mathrm{~Hz}$, 1H), 2.36 (ddd, $J=13.8,5.4,4.8 \mathrm{~Hz}, 1 \mathrm{H}$ ), 2.07 (ddd, $J=13.8,9.0,4.2 \mathrm{~Hz}, 1 \mathrm{H}$ ), 1.19 (d, $J=7.2$ $\mathrm{Hz}, 3 \mathrm{H}) ;{ }^{13} \mathrm{C} \mathrm{NMR}\left(\mathrm{CDCl}_{3}, 150 \mathrm{MHz}\right) \delta 201.6,154.7,151.3,144.9,130.5,117.6,114.9,70.0$, 55.7, 37.7, 35.6, 15.1; HRMS (ESI): calcd. for $\left[\mathrm{C}_{14} \mathrm{H}_{16} \mathrm{O}_{3}+\mathrm{H}\right]^{+}: 233.1172$, Found: 233.1173 .

(4S,6S)-4-(7-bromo-5,8-dimethoxynaphthalen-1-yloxy)-6-methylcyclohex-2-enone (III-23) 


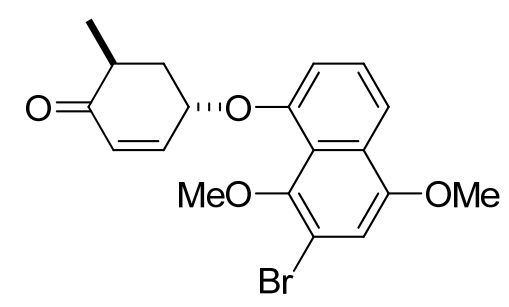

Brown solid, mp: 85.0-86.5 ${ }^{\circ} \mathrm{C} ; R_{f}=0.36\left(4: 1(\mathrm{v} / \mathrm{v})\right.$ hexane/EtOAc); $[\alpha]_{\mathrm{D}}{ }^{20}-138\left(c 1.51, \mathrm{CHCl}_{3}\right)$; IR (thin film, $\mathrm{cm}^{-1}$ ) $v$ 3074, 2963, 2931, 2834, 1680, 1576, 1505, 1453, 1441, 1430, 1408, 1329 , 1259, 1209, 1178, 1057, 1041, 975; ${ }^{1} \mathrm{H}$ NMR $\left(\mathrm{CDCl}_{3}, 600 \mathrm{MHz}\right) \delta 7.94(\mathrm{dd}, J=8.4,1.2 \mathrm{~Hz}, 1 \mathrm{H})$, 7.40 (dd, $J=8.4,7.2 \mathrm{~Hz}, 1 \mathrm{H}), 7.06$ (ddd, $J=9.6,4.2,0.6 \mathrm{~Hz}, 1 \mathrm{H}), 7.06(\mathrm{~d}, J=7.2 \mathrm{~Hz}, 1 \mathrm{H}), 6.96$ (s, 1H), 6.12 (dd, $J=9.6,0.6 \mathrm{~Hz}, 1 \mathrm{H}), 5.09$ (ddd, $J=4.8,4.2,4.2 \mathrm{~Hz}, 1 \mathrm{H}), 3.97$ (s, 3H), 3.82 (s, 3H), 3.01 (dqd, $J=9.6,7.2,5.4 \mathrm{~Hz}, 1 \mathrm{H}$ ), 2.50 (dddd, $J=14.4,5.4,4.8,0.6 \mathrm{~Hz}, 1 \mathrm{H}$ ), 2.11 (ddd, $J$ $=13.8,9.6,4.2 \mathrm{~Hz}, 1 \mathrm{H}), 1.21(\mathrm{~d}, J=7.2 \mathrm{~Hz}, 3 \mathrm{H}) ;{ }^{13} \mathrm{C} \mathrm{NMR}\left(\mathrm{CDCl}_{3}, 150 \mathrm{MHz}\right) \delta 201.7,152.5$, 152.0, 146.3, 144.6, 130.8, 128.6, 125.8, 122.3, 117.1, 114.7, 114.0, 109.1, 71.1, 61.8, 56.0, 37.8, 35.4, 15.1; HRMS (ESI): calcd. for $\left[\mathrm{C}_{19} \mathrm{H}_{19} \mathrm{BrO}_{4}+\mathrm{Na}\right]^{+}$: 413.0359, Found: 413.0361.

(4S,6S)-6-methyl-4-(o-tolyloxy)cyclohex-2-enone (III-24)

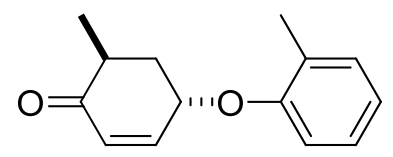

Colorles oil, $R_{f}=0.42\left(4: 1(\mathrm{v} / \mathrm{v})\right.$ hexane/EtOAc); $[\alpha]_{\mathrm{D}}{ }^{20}-190\left(c 1.0, \mathrm{CHCl}_{3}\right)$; IR (thin film, $\left.\mathrm{cm}^{-1}\right)$ v 3024, 2966, 2931, 2874, 1681, 1601, 1589, 1490, 1460, 1438, 1380, 1232, 1188, 1117, 1048, 980; ${ }^{1} \mathrm{H}$ NMR $\left(\mathrm{CDCl}_{3}, 600 \mathrm{MHz}\right) \delta 7.17(\mathrm{~m}, 2 \mathrm{H}), 6.97(\mathrm{dd}, J=10.2,3.6 \mathrm{~Hz}, 1 \mathrm{H}), 6.92(\mathrm{~m}, 2 \mathrm{H})$, $6.08(\mathrm{~d}, J=10.2 \mathrm{~Hz}, 1 \mathrm{H}), 5.03$ (ddd, $J=4.8,4.2,4.2 \mathrm{~Hz}, 1 \mathrm{H}), 2.89$ (dqd, $J=9.0,7.2,4.8 \mathrm{~Hz}$, 1H), 2.41 (ddd, $J=13.8,5.4,4.8 \mathrm{~Hz}, 1 \mathrm{H}), 2.23$ (s, 3H), 2.12 (ddd, $J=13.8,9.0,4.2 \mathrm{~Hz}, 1 \mathrm{H}$ ), $1.21(\mathrm{~d}, J=7.2 \mathrm{~Hz}, 3 \mathrm{H}) ;{ }^{13} \mathrm{C} \mathrm{NMR}\left(\mathrm{CDCl}_{3}, 150 \mathrm{MHz}\right) \delta 201.5,155.5,144.9,131.3,130.5,128.1$, 126.8, 121.4, 112.9, 68.9, 37.8, 35.8, 16.3, 15.1; HRMS (ESI): calcd. for $\left[\mathrm{C}_{14} \mathrm{H}_{16} \mathrm{O}_{2}+\mathrm{H}\right]^{+}$: 217.1223, Found: 217.1223.

(4S,6S)-4-(2-t-butylphenoxy)-6-methylcyclohex-2-enone (III-25) 


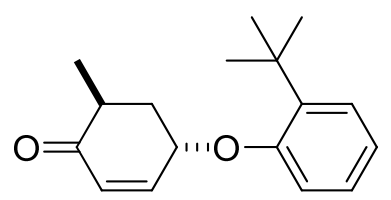

Colorless oil; $R_{f}=0.42\left(4: 1(\mathrm{v} / \mathrm{v})\right.$ hexane/EtOAc); $[\alpha]_{\mathrm{D}}{ }^{20}-218\left(c 1.85, \mathrm{CHCl}_{3}\right)$; IR (thin film, $\mathrm{cm}^{-}$ 1) $v 3005,2985,2960,2950,2940,2895,1689,1593,1484,1441,1378,1290,1249,1222,1200$, 1125, 1091, 1051, 985; ${ }^{1} \mathrm{H}$ NMR $\left(\mathrm{CDCl}_{3}, 600 \mathrm{MHz}\right) \delta 7.34$ (dd, $\left.J=7.8,1.8 \mathrm{~Hz}, 1 \mathrm{H}\right), 7.20$ (ddd, $J=9.0,7.2,1.8 \mathrm{~Hz}, 1 \mathrm{H}), 7.01(\mathrm{dd}, J=10.2,4.2 \mathrm{~Hz}, 1 \mathrm{H}), 6.93(\mathrm{ddd}, J=7.2,7.2,1.2 \mathrm{~Hz}, 1 \mathrm{H})$, $6.91(\mathrm{ddd}, J=7.8,1.2,0.6 \mathrm{~Hz}, 1 \mathrm{H}), 6.10$ (dd, $J=10.2,1.2 \mathrm{~Hz}, 1 \mathrm{H}), 5.18(\mathrm{ddd}, J=4.8,4.8,4.2$ Hz, 1H), 2.87 (dqd, $J=9.0,7.2,4.8 \mathrm{~Hz}, 1 \mathrm{H}), 2.48$ (dddd, $J=13.8,5.4,4.8,1.2 \mathrm{~Hz}, 1 \mathrm{H}), 2.19$ (ddd, $J=13.8,9.0,4.2 \mathrm{~Hz}, 1 \mathrm{H}), 1.24(\mathrm{~d}, J=7.2 \mathrm{~Hz}, 3 \mathrm{H}) ;{ }^{13} \mathrm{C} \mathrm{NMR}\left(\mathrm{CDCl}_{3}, 150 \mathrm{MHz}\right) \delta 201.4$, $155.7,144.6,138.6,130.6,127.3,127.0,120.8,111.7,67.5,38.1$, 35.6, 34.9, 29.9, 15.2; HRMS (ESI): calcd. for $\left[\mathrm{C}_{17} \mathrm{H}_{22} \mathrm{O}_{2}+\mathrm{H}\right]^{+}: 259.1692$, Found: 259.1693 .

\section{(4S,6S)-4-(4-bromophenoxy)-6-methylcyclohex-2-enone (III-26)}

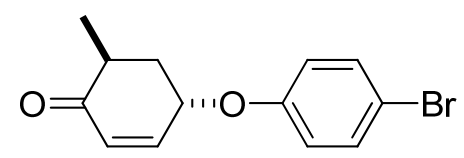

Colorles oil, $R_{f}=0.54\left(2: 1(\mathrm{v} / \mathrm{v})\right.$ hexane/EtOAc); $[\alpha]_{\mathrm{D}}{ }^{20}-148\left(c 1.78, \mathrm{CHCl}_{3}\right)$; IR (thin film, $\left.\mathrm{cm}^{-1}\right)$ v 3040, 2970, 2931, 2875, 1680, 1578, 1384, 1280, 1230, 1173, 1148, 978; ${ }^{1} \mathrm{H} \mathrm{NMR}\left(\mathrm{CDCl}_{3}\right.$, $600 \mathrm{MHz}) \delta 7.41(\mathrm{~d}, J=9.0 \mathrm{~Hz}, 2 \mathrm{H}), 6.92(\mathrm{dd}, J=10.2,4.2 \mathrm{~Hz}, 1 \mathrm{H}), 6.84(\mathrm{~d}, J=9.0 \mathrm{~Hz}, 2 \mathrm{H})$, $6.08(\mathrm{dd}, J=10.2,1.2 \mathrm{~Hz}, 1 \mathrm{H}), 4.98(\mathrm{ddd}, J=4.8,4.2,4.2 \mathrm{~Hz}, 1 \mathrm{H}), 2.85(\mathrm{dqd}, J=9.0,7.2,4.8$ Hz, 1H), 2.36 (dddd, $J=14.4,5.4,4.8,1.2 \mathrm{~Hz}, 1 \mathrm{H}), 2.09$ (ddd, $J=13.2,9.0,4.2 \mathrm{~Hz}, 1 \mathrm{H}), 1.20$ (d, $J=7.2 \mathrm{~Hz}, 3 \mathrm{H}) ;{ }^{13} \mathrm{C} \mathrm{NMR}\left(\mathrm{CDCl}_{3}, 150 \mathrm{MHz}\right) \delta 201.2,156.4,143.9,132.6,131.0,117.7,113.9$, $69.0,37.6,35.4,15.1$.

Methyl 2-((1S,5S)-5-methyl-4-oxocyclohex-2-enyloxy)benzoate (III-27) 


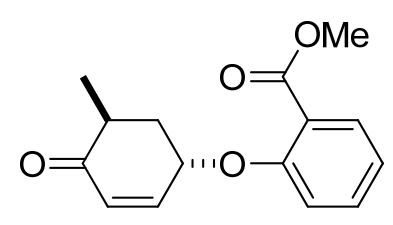

Colorles oil, $R_{f}=0.33\left(2: 1(\mathrm{v} / \mathrm{v})\right.$ hexane/EtOAc); $[\alpha]_{\mathrm{D}}{ }^{20}-194\left(c 1.42, \mathrm{CHCl}_{3}\right)$; IR (thin film, $\left.\mathrm{cm}^{-1}\right)$ v 3040, 2951, 2875, 1725, 1680, 1599, 1485, 1450, 1299, 1240, 1126, 1081, 1045; ${ }^{1} \mathrm{H}$ NMR $\left(\mathrm{CDCl}_{3}, 600 \mathrm{MHz}\right) \delta 7.82(\mathrm{ddd}, J=8.4,2.4,0.6 \mathrm{~Hz}, 1 \mathrm{H}), 7.48(\mathrm{ddd}, J=7.8,7.8,1.8,0.6 \mathrm{~Hz}, 1 \mathrm{H})$, 7.07 (ddd, $J=7.2,7.2,1.2 \mathrm{~Hz}, 1 \mathrm{H}), 7.06$ (d, $J=7.8 \mathrm{~Hz}, 1 \mathrm{H}$ ), 6.98 (dd, $J=10.2,4.2 \mathrm{~Hz}, 1 \mathrm{H}$ ), 6.09 (d, $J=10.2 \mathrm{~Hz}, 1 \mathrm{H}$ ), 5.04 (ddd, $J=4.8,4.2,4.2 \mathrm{~Hz}, 1 \mathrm{H}$ ), 3.86 (s, 3H), 2.97 (dqd, $J=9.6$, 7.2, $4.8 \mathrm{~Hz}, 1 \mathrm{H}), 2.44$ (ddd, $J=13.8,4.8,4.8 \mathrm{~Hz}, 1 \mathrm{H}), 2.10$ (ddd, $J=13.8,9.6,4.2 \mathrm{~Hz}, 1 \mathrm{H}), 1.20$ $(\mathrm{d}, J=7.2 \mathrm{~Hz}, 3 \mathrm{H}) ;{ }^{13} \mathrm{C} \mathrm{NMR}\left(\mathrm{CDCl}_{3}, 150 \mathrm{MHz}\right) \delta 201.5,166.4,156.9,144.0,133.3,132.0$, 131.0, 122.5, 121.9, 116.6, 70.9, 52.0, 37.6, 35.8, 15.0; HRMS (ESI): calcd. for $\left[\mathrm{C}_{15} \mathrm{H}_{16} \mathrm{O}_{4}+\mathrm{Na}\right]^{+}$: 283.0941, Found: 283.0942.

(4S,6S)- 4-(4-acetylphenoxy)-6-methylcyclohex-2-enone (III-28)

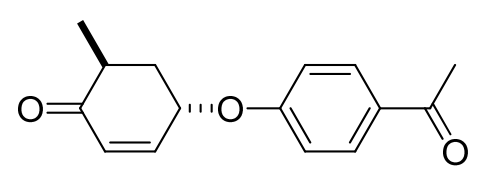

Colorless oil; $R_{f}=0.27\left(2: 1(\mathrm{v} / \mathrm{v})\right.$ hexane/EtOAc); $[\alpha]_{\mathrm{D}}{ }^{20}-194\left(c 1.79, \mathrm{CHCl}_{3}\right)$; IR (thin film, $\mathrm{cm}^{-}$ 1) $v$ 3075, 3040, 2975, 2965, 2935, 2870, 1677, 1597, 1576, 1506, 1359, 1271, 1242, 1174, 954; ${ }^{1} \mathrm{H}$ NMR $\left(\mathrm{CDCl}_{3}, 600 \mathrm{MHz}\right) \delta 7.96(\mathrm{~d}, J=9.0 \mathrm{~Hz}, 2 \mathrm{H}), 6.98(\mathrm{dd}, J=9.0 \mathrm{~Hz}, 2 \mathrm{H}), 6.94(\mathrm{dd}, J=$ 10.2, $4.2 \mathrm{~Hz}, 1 \mathrm{H}), 6.11(\mathrm{dd}, J=10.2,0.6 \mathrm{~Hz}, 1 \mathrm{H}), 5.14$ (ddd, $J=4.8,4.2,4.2 \mathrm{~Hz}, 1 \mathrm{H}), 2.85$ (dqd, $J=9.6,7.2,4.8 \mathrm{~Hz}, 1 \mathrm{H}), 2.56$ (s, 3H), 2.40 (dddd, $J=13.8,4.8,4.8,0.6 \mathrm{~Hz}, 1 \mathrm{H}), 2.14$ (ddd, $J=$ 14.4, 9.6, $4.8 \mathrm{~Hz}, 1 \mathrm{H}), 1.21(\mathrm{~d}, J=7.2 \mathrm{~Hz}, 3 \mathrm{H}) ;{ }^{13} \mathrm{C} \mathrm{NMR}\left(\mathrm{CDCl}_{3}, 150 \mathrm{MHz}\right) \delta 200.9,196.5$, 161.1, 143.3, 131.3, 131.0, 130.8, 115.1, 68.6, 37.6, 35.4, 26.3, 15.0; HRMS (ESI): calcd. for $\left[\mathrm{C}_{15} \mathrm{H}_{16} \mathrm{O}_{3}+\mathrm{H}\right]^{+}: 245.1172$, Found: 245.1173 . 


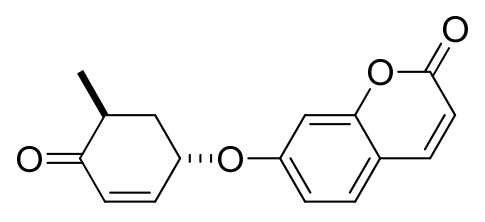

White solid, mp: $137.2-137.8{ }^{\circ} \mathrm{C} ; R_{f}=0.36\left(1: 1(\mathrm{v} / \mathrm{v})\right.$ hexane/EtOAc); $[\alpha]_{\mathrm{D}}{ }^{20}-104$ (c 1.97 , $\mathrm{CHCl}_{3}$ ); IR (thin film, cm ${ }^{-1}$ ) v 3075, 3055, 2980, 2970, 2920, 2890, 1703, 1683, 1608, 1501, $1403,1352,1403,1276,1232,1211,1196,1124,1079,1031,1005,985 ;{ }^{1} \mathrm{H} \mathrm{NMR}\left(\mathrm{CDCl}_{3}, 600\right.$ MHz) $\delta 7.64(\mathrm{~d}, J=9.6 \mathrm{~Hz}, 1 \mathrm{H}), 7.41(\mathrm{~d}, J=9.0 \mathrm{~Hz}, 1 \mathrm{H}), 6.94(\mathrm{dd}, J=10.2,4.2 \mathrm{~Hz}, 1 \mathrm{H}), 6.88$ $(\mathrm{m}, 2 \mathrm{H}), 6.28(\mathrm{~d}, J=9.6 \mathrm{~Hz}, 1 \mathrm{H}), 6.12(\mathrm{~d}, J=10.2 \mathrm{~Hz}, 1 \mathrm{H}), 5.11(\mathrm{ddd}, J=4.8,4.8,4.2 \mathrm{~Hz}, 1 \mathrm{H})$, $2.86(\mathrm{dqd}, J=9.6,7.2,5.4 \mathrm{~Hz}, 1 \mathrm{H}), 2.40(\mathrm{ddd}, J=13.8,4.8,4.8 \mathrm{~Hz} 1 \mathrm{H}), 2.16(\mathrm{ddd}, J=13.8,9.6$, $4.8 \mathrm{~Hz}, 1 \mathrm{H}), 1.21(\mathrm{~d}, J=6.6 \mathrm{~Hz}, 3 \mathrm{H}) ;{ }^{13} \mathrm{C} \mathrm{NMR}\left(\mathrm{CDCl}_{3}, 150 \mathrm{MHz}\right) \delta 200.8,160.8,160.4,155.8$, 143.1, 142.9, 131.4, 129.1, 113.7, 113.5, 113.1, 102.4, 69.1, 37.6, 35.2, 15.0; HRMS (ESI): calcd. for $\left[\mathrm{C}_{16} \mathrm{H}_{14} \mathrm{O}_{4}+\mathrm{H}\right]^{+}:$271.0965, Found: 271.0966.

\section{2-((1S,5S)-5-methyl-4-oxocyclohex-2-enyloxy)naphthalene-1,4-dione (III-30)}

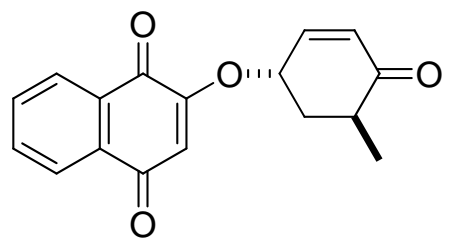

Red brown solid, mp: $60-62{ }^{\circ} \mathrm{C} ; R_{f}=0.58\left(10: 1(\mathrm{v} / \mathrm{v}) \mathrm{CH}_{2} \mathrm{Cl}_{2} / \mathrm{MeOH}\right) ;[\alpha]_{\mathrm{D}}{ }^{20}-97\left(c\right.$ 0.55, $\left.\mathrm{CHCl}_{3}\right)$; IR (thin film, $\mathrm{cm}^{-1}$ ) v 3280, 2975, 2925, 2870, 1740, 1720, 1664, 1642, 1595, 1460, 1368, 1340, $1273,1246,1214,1153 ;{ }^{1} \mathrm{H} \mathrm{NMR}\left(\mathrm{CDCl}_{3}, 600 \mathrm{MHz}\right) \delta 8.15(\mathrm{~d}, J=7.8 \mathrm{~Hz}, 1 \mathrm{H}), 8.12(\mathrm{~d}, J=7.8$ $\mathrm{Hz}, 1 \mathrm{H}), 7.80$ (ddd, $J=7.8,7.2,1.2 \mathrm{~Hz}, 1 \mathrm{H}), 7.73$ (ddd, $J=7.8,7.2,1.2 \mathrm{~Hz}, 1 \mathrm{H}), 7.54$ (s, 1H), $6.87(\mathrm{ddd}, J=10.2,1.8,1.2 \mathrm{~Hz}, 1 \mathrm{H}), 6.07$ (dd, $J=10.2,2.4 \mathrm{~Hz}, 1 \mathrm{H}), 4.32$ (dddd, $J=10.2,5.4$, 2.4, $2.4 \mathrm{~Hz}, 1 \mathrm{H}), 2.68(\mathrm{dqd}, J=12.0,7.2,4.8 \mathrm{~Hz}, 1 \mathrm{H}), 2.45$ (ddd, $J=13.8,9.6,4.8 \mathrm{~Hz} 1 \mathrm{H}), 1.94$ (dddd, $J=13.2,4.8,4.8,1.2 \mathrm{~Hz}, 1 \mathrm{H}), 1.29(\mathrm{~d}, J=7.2 \mathrm{~Hz}, 3 \mathrm{H}) ;{ }^{13} \mathrm{C} \mathrm{NMR}\left(\mathrm{CDCl}_{3}, 150 \mathrm{MHz}\right) \delta$ 202.2, 183.7, 181.2, 153.7, 151.0, 135.4, 133.3, 132.7, 129.2, 127.7, 127.1, 126.4, 123.4, 40.2, 34.2, 29.4, 15.6; HRMS (ESI): calcd. for $\left[\mathrm{C}_{17} \mathrm{H}_{14} \mathrm{O}_{4}+\mathrm{H}\right]^{+}:$283.0965, Found: 283.0966. 
(1R,4S,6S)-4-(benzyloxy)-6-methylcyclohex-2-enol (III-31)

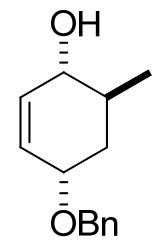

A solution of $528 \mathrm{mg}(2.44 \mathrm{mmol})$ enone III-7 in $10 \mathrm{~mL}$ THF at $-78{ }^{\circ} \mathrm{C}$ was added $93 \mathrm{mg}(2.44$ mmol) $\mathrm{LiAlH}_{4}$ in one portion. It was stirred at $-78{ }^{\circ} \mathrm{C}$ for $1 \mathrm{~h}$ and then diluted with $50 \mathrm{~mL}$ EtOAc and quenched with $16 \mathrm{~mL}$ saturated aqueous $\mathrm{NaHCO}_{3}$. The mixture was stirred at $0{ }^{\circ} \mathrm{C}$ for 15 min. The organic layer was separated and the aqueous layer was extracted with EtOAc. The pooled organic layer was washed with saturated brine and dried over $\mathrm{Na}_{2} \mathrm{SO}_{4}$. After removal of the solvent under reduced pressure, the residue was subjected to silica gel column chromatography. Elution with hexane-EtOAc (4:1, v/v) afforded allylic alcohol III-31 (454 mg $85 \%) . R_{f}=0.48\left(1: 1(\mathrm{v} / \mathrm{v})\right.$ hexane/EtOAc); $[\alpha]_{\mathrm{D}}{ }^{20}-90\left(c 1.33, \mathrm{CHCl}_{3}\right) ;$ IR (thin film, $\left.\mathrm{cm}^{-1}\right) v$ 3357 (broad), 3064, 3029, 2952, 2928, 2908, 2869, 1497, 1454, 1390, 1375, 1326, 1305, 1196, 1067, 1037, 1026, 971; ${ }^{1} \mathrm{H} \mathrm{NMR}\left(\mathrm{CDCl}_{3}, 600 \mathrm{MHz}\right) \delta 7.37-7.32(\mathrm{~m}, 4 \mathrm{H}), 7.30-7.26(\mathrm{~m}, 1 \mathrm{H})$, $5.88(\mathrm{~m}, 2 \mathrm{H}), 4.60(\mathrm{~d}, J=12.0 \mathrm{~Hz}, 1 \mathrm{H}), 4.54(\mathrm{~d}, J=12.0 \mathrm{~Hz}, 1 \mathrm{H}), 3.87(\mathrm{~m}, 1 \mathrm{H}), 3.68(\mathrm{~d}, J=9.0$ $\mathrm{Hz}, 1 \mathrm{H}), 1.94$ (dddd, $J=14.4,3.6,3.0,1.2 \mathrm{~Hz}, 1 \mathrm{H}), 1.68(\mathrm{ddqd}, J=11.4,8.4,6.6,3.0 \mathrm{~Hz}, 1 \mathrm{H})$, 1.43 (dddd, $J=13.8,11.4,4.2 \mathrm{~Hz}, 1 \mathrm{H}), 1.08(\mathrm{~d}, J=7.2 \mathrm{~Hz}, 3 \mathrm{H}) ;{ }^{13} \mathrm{C} \mathrm{NMR}\left(\mathrm{CDCl}_{3}, 150 \mathrm{MHz}\right) \delta$ 138.8, 134.9, 128.4, 127.7, 127.6, 127.5, 73.6, 70.8, 70.3, 38.9, 32.8, 18.2; HRMS (ESI): calcd. for $\left[\mathrm{C}_{14} \mathrm{H}_{18} \mathrm{O}_{2}+\mathrm{Na}\right]^{+}$: 241.1199, Found: 241.1199.

\section{(1R,2S,3S,4S,6S)-4-(benzyloxy)-6-methylcyclohexane-1,2,3-triol (III-33)}




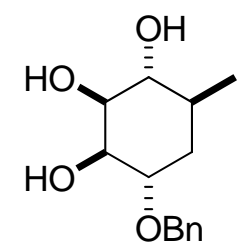

To a solution of $22 \mathrm{mg}$ olefin III-31 $(0.10 \mathrm{mmol})$ in $0.9 \mathrm{~mL} t$-BuOH-actone- $50 \% \mathrm{NMO}_{\text {(aq.) }}(1: 1: 1$ $\mathrm{v} / \mathrm{v} / \mathrm{v}$ ) was added a small piece of crystalline $\mathrm{OsO}_{4}$ at $0{ }^{\circ} \mathrm{C}$. The reaction mixture was stirred at $0{ }^{\circ} \mathrm{C}$ for $2 \mathrm{~h}$, and then it was diluted with EtOAc $20 \mathrm{~mL}$ and $1.5 \mathrm{~mL}$ saturated aqueous $\mathrm{Na}_{2} \mathrm{~S}_{2} \mathrm{O}_{3}$ was added at $0{ }^{\circ} \mathrm{C}$. It was stirred at $0{ }^{\circ} \mathrm{C}$ for $30 \mathrm{~min}$. The mixture was passed through a pad of sand-silica gel-celite, eluting with EtOAc-MeOH (3:1, v/v). The effluent was dried over $\mathrm{Na}_{2} \mathrm{SO}_{4}$ and concentrated under reduced pressure to give a residue, which was purified by silica gel column. Elution with EtOAc-MeOH (20:1) gave triol III-33 (25 mg, 98\%) as viscous colorless oil: $R_{f}=0.46(10: 1(\mathrm{v} / \mathrm{v}) \mathrm{EtOAc} / \mathrm{MeOH}) ;[\alpha]_{\mathrm{D}}^{20}+14(c 2.58, \mathrm{MeOH}) ;$ IR (thin film, $\left.\mathrm{cm}^{-1}\right) v 3357$ (broad), 3060, 3025, 2920, 2870, 1497, 1453, 1055, 1027, 976; ${ }^{1} \mathrm{H}$ NMR $\left(\mathrm{CD}_{3} \mathrm{OD}, 600 \mathrm{MHz}\right) \delta$ 7.35-7.30 (m, 4H), 7.28-7.23 (m, 1H), 4.57 (d, $J=12.0 \mathrm{~Hz}, 1 \mathrm{H}), 4.51(\mathrm{~d}, J=12.0 \mathrm{~Hz}, 1 \mathrm{H}), 4.01$ $(\mathrm{dd}, J=3.0,3.0 \mathrm{~Hz}, 1 \mathrm{H}), 3.61(\mathrm{~m}, 1 \mathrm{H}), 3.60(\mathrm{dd}, J=9.6,3.6 \mathrm{~Hz}, 1 \mathrm{H}), 3.27(\mathrm{dd}, J=9.6,9.6 \mathrm{~Hz}$, 1H), 1.77 (m, 1H), 1.73 (ddqd, $J=13.2,10.2,6.6,3.6 \mathrm{~Hz}, 1 \mathrm{H}), 1.52$ (ddd, $J=14.4,12.6,1.8 \mathrm{~Hz}$ $1 \mathrm{H}), 1.01(\mathrm{~d}, J=6.6 \mathrm{~Hz}, 3 \mathrm{H}) ;{ }^{13} \mathrm{C} \mathrm{NMR}\left(\mathrm{CD}_{3} \mathrm{OD}, 150 \mathrm{MHz}\right) \delta 140.3,129.5,128.8,128.7,78.6$, 76.8, 74.7, 72.9, 71.9, 33.4, 33.0, 18.6; HRMS (ESI): calcd. for $\left[\mathrm{C}_{14} \mathrm{H}_{20} \mathrm{O}_{4}+\mathrm{Na}\right]^{+}$: 275.1254, Found: 275.1255 .

5,7-bis(benzyloxy)-2-(4-(benzyloxy)phenyl)-3-((1R,5R)-5-methyl-4-oxocyclohex-2-enyloxy)4H-chromen-4-one (III-9) 


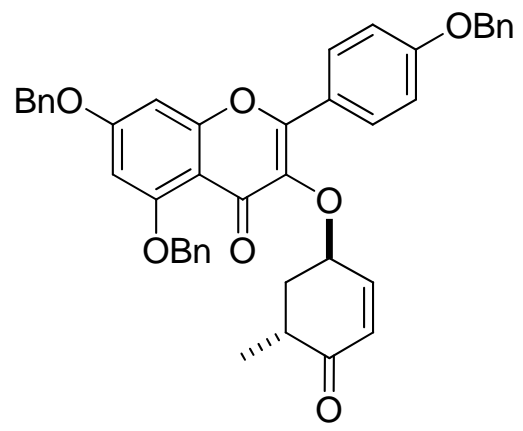

$133 \mathrm{mg}(0.24 \mathrm{mmol})$ perbenzylated flavonol III-8 and $65 \mathrm{mg}(0.29 \mathrm{mmol}) \alpha$-L-Boc-enone (III-4) was suspended in $2.5 \mathrm{~mL} \mathrm{CH} \mathrm{Cl}_{2}$ at $0{ }^{\circ} \mathrm{C}$. A solution of $6.2 \mathrm{mg}(0.006 \mathrm{mmol}) \mathrm{Pd}_{2}\left(\mathrm{dba}_{3} \cdot{ }_{3} \cdot \mathrm{CHCl}_{3}\right.$ and $6.3 \mathrm{mg} \mathrm{PPh}(0.024 \mathrm{mmol})$ in $1 \mathrm{~mL} \mathrm{CH}_{2} \mathrm{Cl}_{2}$ wad added via a syringe at $0{ }^{\circ} \mathrm{C}$. The reaction mixture was stirred at $0{ }^{\circ} \mathrm{C}$ for $30 \mathrm{~min}$ and then directly loaded onto a silica gel column, elution with hexane-EtOAc (3:1) gave glycosylated enone III-9 (133 mg, 84\%): yellow solid, mp: 59-61 ${ }^{\circ} \mathrm{C} ; R_{f}=0.60\left(1: 1(\mathrm{v} / \mathrm{v})\right.$ hexane/EtOAc); $[\alpha]_{\mathrm{D}}{ }^{20}+57\left(c 3.1, \mathrm{CHCl}_{3}\right) ;$ IR (thin film, $\left.\mathrm{cm}^{-1}\right) v 3063$, 3034, 2931, 2872, 1734, 1681, 1626, 1602, 1572, 1508, 1452, 1436, 1375, 1355, 1296, 1248, 1195, 1173, 1100, 1043, 1003, 972. ${ }^{1} \mathrm{H}$ NMR $\left(\mathrm{CDCl}_{3}, 600 \mathrm{MHz}\right) \delta 7.96(\mathrm{~d}, J=9.0 \mathrm{~Hz}, 2 \mathrm{H}), 7.58$ (d, $J=7.8 \mathrm{~Hz}, 2 \mathrm{H}), 7.46-7.29(\mathrm{~m}, 13 \mathrm{H}), 7.04(\mathrm{~d}, J=9.0 \mathrm{~Hz}, 2 \mathrm{H}), 7.04$ (dd, $J=10.2,4.2 \mathrm{~Hz}, 1 \mathrm{H})$, $6.59(\mathrm{~d}, J=1.8 \mathrm{~Hz}, 1 \mathrm{H}), 6.48(\mathrm{~d}, J=2.4 \mathrm{~Hz}, 1 \mathrm{H}), 5.92(\mathrm{dd}, J=9.6,1.2 \mathrm{~Hz}, 1 \mathrm{H}), 5.29(\mathrm{~s}, 2 \mathrm{H})$, 5.14 (s, 2H), 5.09 (s, 2H), 5.07 (ddd, $J=4.8,4.2,4.2 \mathrm{~Hz}, 1 \mathrm{H}), 2.60$ (dqd, $J=10.2,6.6,4.8 \mathrm{~Hz}$, 1H), 2.11 (ddd, $J=13.8,4.8,4.2 \mathrm{~Hz}, 1 \mathrm{H}), 1.86$ (ddd, $J=14.4,10.2,4.8 \mathrm{~Hz}, 1 \mathrm{H}), 0.94$ (d, $J=7.2$ $\mathrm{Hz}, 3 \mathrm{H}) ;{ }^{13} \mathrm{C} \mathrm{NMR}\left(\mathrm{CDCl}_{3}, 150 \mathrm{MHz}\right) \delta 201.9,173.7,162.9,160.4,159.8,158.8,154.1,145.6$, 138.7, 136.3, 135.6, 130.4, 130.0, 128.7, 128.6, 128.6, 128.4, 128.2, 127.7, 127.5, 127.4, 126.6, 123.3, 114.6, 109.9, 98.3, 94.0, 72.8, 70.8, 70.5, 70.1, 37.8, 35.8, 14.7; HRMS (ESI): calcd. for $\left[\mathrm{C}_{43} \mathrm{H}_{36} \mathrm{O}_{7}+\mathrm{H}\right]^{+}:$665.2534, Found: 665.2530 .

5,7-bis(benzyloxy)-2-(4-(benzyloxy)phenyl)-3-((1R,4S,5R)-4-hydroxy-5-methylcyclohex-2enyloxy)-4H-chromen-4-one (III-34) 


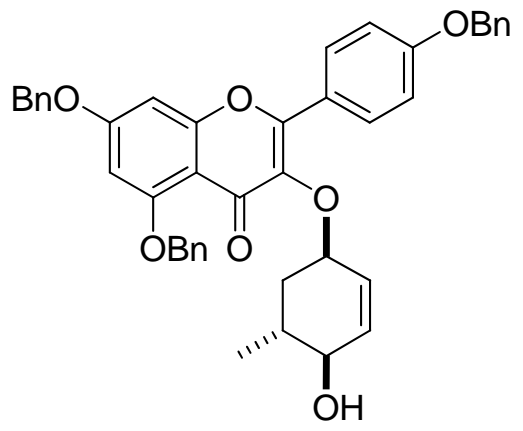

A solution of $115 \mathrm{mg}(0.17 \mathrm{mmol})$ enone III-9 in $14 \mathrm{~mL}$ THF at $-78{ }^{\circ} \mathrm{C}$ was added $13 \mathrm{mg}(0.35$ mmol) $\mathrm{LiAlH}_{4}$ in two portions. It was stirred at $-78^{\circ} \mathrm{C}$ for $1 \mathrm{~h}$ and then $3 \mathrm{~mL}$ acetone was slowly added via syringe into the reaction mixture at $-78^{\circ} \mathrm{C}$. The mixture was stirred at $-78{ }^{\circ} \mathrm{C}$ for 20 min and diluted with $20 \mathrm{~mL}$ hexane-EtOAc $(1: 1, \mathrm{v} / \mathrm{v})$ and quenched with $6 \mathrm{~mL}$ saturated aqueous $\mathrm{NaHCO}_{3}$. The mixture was stirred at $0{ }^{\circ} \mathrm{C}$ for $20 \mathrm{~min}$. The organic layer was separated and the aqueous layer was extracted with EtOAc. The pooled organic layer was washed with saturated brine and dried over $\mathrm{Na}_{2} \mathrm{SO}_{4}$. After removal of the solvent under reduced pressure, the residue was subjected to silica gel column chromatography. Elution with hexane-EtOAc (1.5:1, $\mathrm{v} / \mathrm{v})$ afforded allylic alcohol III-34 (97 mg, 89\%). $R_{f}=0.38\left(1: 1(\mathrm{v} / \mathrm{v})\right.$ hexane/EtOAc); $[\alpha]_{\mathrm{D}}{ }^{20}+$ 10 (c 3.7, $\mathrm{CHCl}_{3}$ ); IR (thin film, $\mathrm{cm}^{-1}$ ) v 3507, 3035, 2949, 2900, 2862, 1626, 1597, 1507, 1447, 1379, 1361, 1303, 1245, 1179, 1103, 1038, 947; ${ }^{1} \mathrm{H}$ NMR $\left(\mathrm{CDCl}_{3}, 600 \mathrm{MHz}\right) \delta 7.99$ (d, $J=9.0$ $\mathrm{Hz}, 2 \mathrm{H}), 7.58(\mathrm{~d}, J=7.2 \mathrm{~Hz}, 2 \mathrm{H}), 7.46-7.27(\mathrm{~m}, 13 \mathrm{H}), 7.05(\mathrm{~d}, J=9.0 \mathrm{~Hz}, 2 \mathrm{H}), 6.57$ (d, $J=2.4$ $\mathrm{Hz}, 1 \mathrm{H}), 6.45$ (d, $J=1.8 \mathrm{~Hz}, 1 \mathrm{H}), 6.03$ (dddd, $J=9.6,4.2,1.8,1.8 \mathrm{~Hz}, 1 \mathrm{H}), 5.80$ (dd, $J=9.6,1.8$ Hz, 1H), 5.28 (s, 2H), 5.15 (s, 2H), 5.08 (s, 2H), 4.73 (m, 1H), 3.59 (d, $J=8.4 \mathrm{~Hz}, 1 \mathrm{H}), 1.73$ (ddd, $J=14.4,4.2,2.4 \mathrm{~Hz}, 1 \mathrm{H}$ ), 1.63 (ddqd, $J=13.2,9.6,6.6,3.0 \mathrm{~Hz}, 1 \mathrm{H}$ ), 1.29 (ddd, $J=15.0$, 12.6, $4.8 \mathrm{~Hz}, 1 \mathrm{H}), 0.79(\mathrm{~d}, J=6.6 \mathrm{~Hz}, 3 \mathrm{H}) ;{ }^{13} \mathrm{C} \mathrm{NMR}\left(\mathrm{CDCl}_{3}, 150 \mathrm{MHz}\right) \delta 174.0,162.6,160.2$, $159.8,158.8,154.0,139.3,136.5,136.4,135.7,135.4,130.6,128.7,128.7,128.6,128.4,128.1$, $127.7,127.6,127.5,127.4,126.7,123.8,114.4,110.1,98.2,94.0,73.7,73.5,70.8,70.4,70.1$, 34.5, 33.0, 17.8; HRMS (ESI): calcd. for $\left[\mathrm{C}_{43} \mathrm{H}_{38} \mathrm{O}_{7}+\mathrm{H}\right]^{+}:$667.2696, Found: 667.2678.

(1S,4R,6R)-4-(5,7-bis(benzyloxy)-2-(4-(benzyloxy)phenyl)-4-oxo-4H-chromen-3-yloxy)-6methylcyclohex-2-enyl acetate (III-35) 


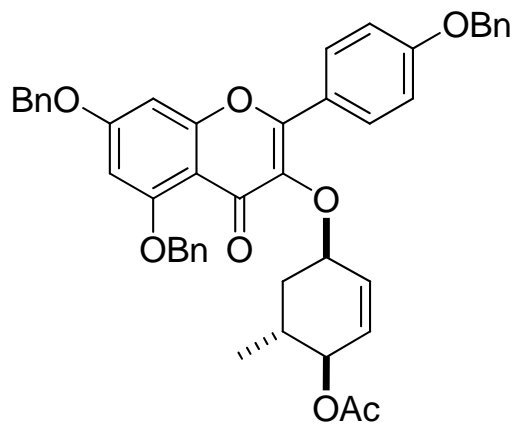

A solution of $95 \mathrm{mg}(0.14 \mathrm{mmol})$ enone III-34 and $1.7 \mathrm{mg}(0.014 \mathrm{mmol})$ DMAP in $2.5 \mathrm{~mL}$ $\mathrm{CH}_{2} \mathrm{Cl}_{2}$ at $0{ }^{\circ} \mathrm{C}$ was added $250 \mu \mathrm{L}(3.09 \mathrm{mmol})$ pyridine and $150 \mu \mathrm{L}(1.59 \mathrm{mmol}) \mathrm{Ac}_{2} \mathrm{O}$ via syringe. It was stirred at $0{ }^{\circ} \mathrm{C}$ for $2 \mathrm{~h}$ and diluted with $20 \mathrm{~mL}$ hexane-EtOAc $(1: 1, \mathrm{v} / \mathrm{v})$ and washed with $6 \mathrm{~mL} 0.5 \mathrm{M}$ aqueous $\mathrm{NaHSO}_{4}$. The organic layer was separated and the aqueous layer was extracted with EtOAc. The pooled organic layer was washed with saturated aqueous $\mathrm{NaHCO}_{3}$ and saturated brine subsequently and then dried over $\mathrm{Na}_{2} \mathrm{SO}_{4}$. After removal of the solvent under reduced pressure, the residue was subjected to silica gel column chromatography. Elution with hexane-EtOAc (3:1, v/v) afforded allylic acetate III-35 (94 mg, 93\%). Pale yellow solid, mp: $68-70^{\circ} \mathrm{C} ; R_{f}=0.30$ (hexane/EtOAc 2:1, v/v); $[\alpha]_{\mathrm{D}}{ }^{20}-33\left(c 3.15, \mathrm{CHCl}_{3}\right)$; IR (thin film, $\mathrm{cm}^{-1}$ ) v 3063, 3033, 2930, 1729, 1625, 1602, 1508, 1453, 1434, 1372, 1356, 1295, 1243, 1172, 1100, 1021, 920; ${ }^{1} \mathrm{H}$ NMR $\left(\mathrm{CDCl}_{3}, 600 \mathrm{MHz}\right) \delta 8.02(\mathrm{~d}, J=9.0 \mathrm{~Hz}, 2 \mathrm{H}), 7.58(\mathrm{~d}, J=7.2 \mathrm{~Hz}$, 2H), 7.47-7.27 (m, 13H), $7.06(\mathrm{~d}, J=9.6 \mathrm{~Hz}, 2 \mathrm{H}), 6.58(\mathrm{~d}, J=1.8 \mathrm{~Hz}, 1 \mathrm{H}), 6.45(\mathrm{~d}, J=2.4 \mathrm{~Hz}$, 1H), 6.13 (dddd, $J=10.2,4.2,1.8,1.2 \mathrm{~Hz}, 1 \mathrm{H}), 5.71$ (dd, $J=10.2,1.8 \mathrm{~Hz}, 1 \mathrm{H}), 5.28(\mathrm{~s}, 2 \mathrm{H})$, 5.15 (s, 2H), 5.08 (s, 2H), 4.88 (dddd, $J=9.0,2.4,1.8,1.2 \mathrm{~Hz}, 1 \mathrm{H}), 4.74(\mathrm{~m}, 1 \mathrm{H}), 2.09$ (s, 3H), 1.94 (ddqd, $J=13.2,9.6,6.6,3.0 \mathrm{~Hz}, 1 \mathrm{H}), 1.77(\mathrm{~m}, 1 \mathrm{H}), 1.40$ (ddd, $J=14.4,12.6,4.8 \mathrm{~Hz}, 1 \mathrm{H})$, $0.70(\mathrm{~d}, J=6.6 \mathrm{~Hz}, 3 \mathrm{H}) ;{ }^{13} \mathrm{C} \mathrm{NMR}\left(\mathrm{CDCl}_{3}, 150 \mathrm{MHz}\right) \delta 173.9,171.0,162.7,160.2,159.8,158.8$, 153.8, 139.4, 136.5, 136.4, 135.7, 131.1, 130.5, 129.2, 128.7, 128.7, 128.6, 128.4, 128.1, 127.6, 127.5, 127.4, 126.6, 123.8, 114.4, 110.1, 98.2, 94.0, 75.2, 73.5, 70.8, 70.4, 70.1, 34.3, 29.5, 21.2, 17.6 ; HRMS (ESI): calcd. for $\left[\mathrm{C}_{45} \mathrm{H}_{40} \mathrm{O}_{8}+\mathrm{H}\right]^{+}:$709.2796, Found: 709.2782.

(1S,2S,3R,4R,6R)-4-(5,7-bis(benzyloxy)-2-(4-(benzyloxy)phenyl)-4-oxo-4H-chromen-3yloxy)-2,3-dihydroxy-6-methylcyclohexyl acetate (III-36) 


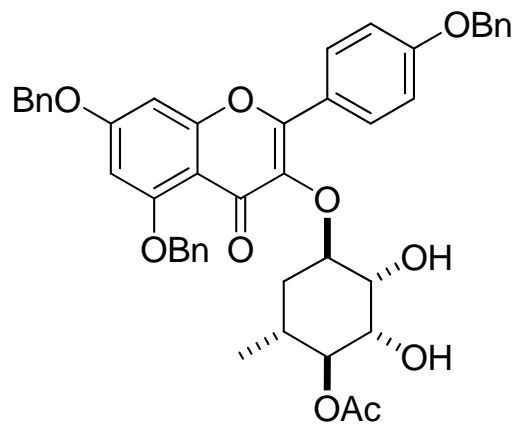

A solution of $92 \mathrm{mg}(0.13 \mathrm{mmol})$ allylic acetate (III-35) in $1 \mathrm{~mL}$ acetone- $t$-BuOH (1:1) was cooled down to $0{ }^{\circ} \mathrm{C}$. To the solution was added $0.5 \mathrm{~mL} \mathrm{NMO}-\mathrm{H}_{2} \mathrm{O}(\mathrm{w} / \mathrm{w} 1: 1)$, and a small piece of crystalline $\mathrm{OsO}_{4}$ was added into the mixture. The reaction mixture was stirred at $0{ }^{\circ} \mathrm{C}$ for 20 hours. The reaction was diluted with $20 \mathrm{~mL}$ EtOAc and then quenched with $1 \mathrm{~mL}$ saturated $\mathrm{Na}_{2} \mathrm{~S}_{2} \mathrm{O}_{3}$ (aq.) and stirred for $1 \mathrm{~h}$ at $0{ }^{\circ} \mathrm{C}$. Then the mixture was passed through a small pad of celite/silica gel/sand and washed with EtOAc-MeOH (3:1). The effluent was dried over $\mathrm{Na}_{2} \mathrm{SO}_{4}$, and concentrated under reduced pressure to give a residue. Chromatography on silica gel eluting with hexane-EtOAc (1.5:1) gave diol III-36 (70 mg, 73\%): White solid, mp: 97-99 ${ }^{\circ} \mathrm{C} ; R_{f}=0.24$ (1:2 (v/v), hexane/EtOAc); $[\alpha]_{D}^{20}-47\left(c 2.7, \mathrm{CHCl}_{3}\right)$; IR (thin film, $\left.\mathrm{cm}^{-1}\right) v 3404,3064,2932$, 1727, 1601, 1508, 1453, 1433, 1373, 1297, 1247, 1196, 1172, 1099, 1036, 959; ${ }^{1} \mathrm{H}$ NMR $\left(\mathrm{CDCl}_{3}\right.$, $600 \mathrm{MHz}) \delta 7.90(\mathrm{~d}, J=9.0 \mathrm{~Hz}, 2 \mathrm{H}), 7.55(\mathrm{~d}, J=7.2 \mathrm{~Hz}, 2 \mathrm{H}), 7.47-7.27(\mathrm{~m}, 13 \mathrm{H}), 7.08(\mathrm{~d}, J=$ $9.0 \mathrm{~Hz}, 2 \mathrm{H}), 6.56(\mathrm{~d}, J=2.4 \mathrm{~Hz}, 1 \mathrm{H}), 6.46(\mathrm{~d}, J=2.4 \mathrm{~Hz}, 1 \mathrm{H}), 5.26(\mathrm{~s}, 2 \mathrm{H}), 5.15(\mathrm{~s}, 2 \mathrm{H}), 5.07$ (s, 2H), 4.79 (dd, $J=7.8,7.2 \mathrm{~Hz}, 1 \mathrm{H}), 4.28$ (ddd, $J=5.4,5.4,3.0 \mathrm{~Hz}, 1 \mathrm{H}), 4.17$ (dd, $J=3.6,4.8 \mathrm{~Hz}$, 1H), 3.85 (dd, $J=7.2,3.0 \mathrm{~Hz}, 1 \mathrm{H}), 2.85$ (brs, 1H), 2.08 (s, 3H), 1.80 (m, 1H), 1.69 (ddd, $J=$ 14.4, 5.4, $4.8 \mathrm{~Hz}, 1 \mathrm{H}$ ), 1.41 (ddd, $J=13.8,9.6,3.6 \mathrm{~Hz}, 1 \mathrm{H}), 0.81$ (d, $J=6.6 \mathrm{~Hz}, 3 \mathrm{H}) ;{ }^{13} \mathrm{C} \mathrm{NMR}$ $\left(\mathrm{CDCl}_{3}, 150 \mathrm{MHz}\right) \delta 174.2,171.3,163.0,160.5,159.8,158.8,154.4,139.3,136.4,136.3,135.6$, 130.6, 128.7, 128.7, 128.6, 128.4, 128.2, 127.7, 127.5, 127.4, 126.7, 123.4, 114.6, 109.8, 98.4, 94.0, 80.6, 76.9, 72.0, 71.9, 70.9, 70.5, 70.1, 32.7, 31.1, 21.1, 17.3; HRMS (ESI) calcd. for $\left[\mathrm{C}_{45} \mathrm{H}_{42} \mathrm{O}_{10}+\mathrm{H}\right]^{+}:$743.2851, Found: 743.2835 .

(1S,2S,3R,4R,6R)-4-(5,7-dihydroxy-2-(4-hydroxyphenyl)-4-oxo-4H-chromen-3-yloxy)-2,3dihydroxy-6-methylcyclohexyl acetate (III-1) 


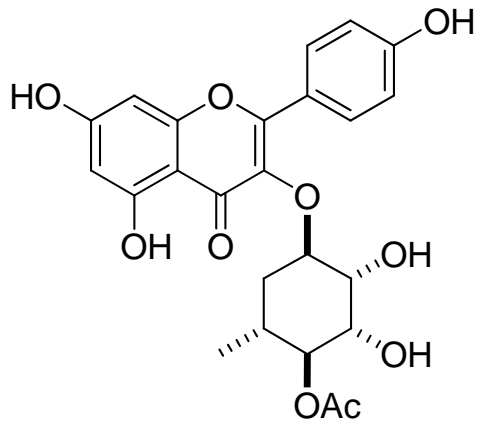

To a solution of $17 \mathrm{mg}(0.023 \mathrm{mmol})$ diol III-36 in $2.5 \mathrm{~mL}$ THF-EtOH (1:1) was added $21 \mathrm{mg}$ $\mathrm{Pd} / \mathrm{C}(10 \%)$. The reaction mixture was degassed at -50 to $-40{ }^{\circ} \mathrm{C}$ using vacuum and refilled with $\mathrm{H}_{2}$. This procedure was repeated three times, then the bath was removed and the reaction was warmed up to room temperature. The reaction mixture was stirred under a $\mathrm{H}_{2}$ atmosphere for 12 hours. The reaction mixture was loaded onto silica gel and elution with hexane-EtOAc (1.5:1) to give product (III-1) $(7.3 \mathrm{mg}, 68 \%)$ : Yellow solid, mp: $161-163{ }^{\circ} \mathrm{C} ; R_{f}=0.24(1: 4(\mathrm{v} / \mathrm{v})$ hexane/EtOAc); $[\alpha]_{\mathrm{D}}{ }^{20}-31$ (c 0.49, MeOH); IR (thin film, $\mathrm{cm}^{-1}$ ) v 3283, 2923, 1710, 1649, 1607, 1499, 1436, 1360, 1259, 1208, 1170, 1085, 1039, 971; ${ }^{1} \mathrm{H}$ NMR $\left(\mathrm{CD}_{3} \mathrm{OD}, 600 \mathrm{MHz}\right) \delta 7.78(\mathrm{~d}, J$ $=9.0 \mathrm{~Hz}, 2 \mathrm{H}), 6.94(\mathrm{~d}, J=8.4 \mathrm{~Hz}, 2 \mathrm{H}), 6.38(\mathrm{~d}, J=1.8 \mathrm{~Hz}, 1 \mathrm{H}), 6.21(\mathrm{~d}, J=1.8 \mathrm{~Hz}, 1 \mathrm{H}), 4.76$ (dd, $J=10.2,10.2 \mathrm{~Hz}, 1 \mathrm{H}), 4.31$ (ddd, $J=3.0,3.0,3.0 \mathrm{~Hz}, 1 \mathrm{H}), 4.08(\mathrm{dd}, J=3.0,3.0 \mathrm{~Hz}, 1 \mathrm{H}$ ), $3.72(\mathrm{dd}, J=9.6,3.0 \mathrm{~Hz}, 1 \mathrm{H}), 2.07$ (s, 3H), 1.70 (ddqd, $J=12.6,10.2,6.0,3.0 \mathrm{~Hz}, 1 \mathrm{H}), 1.64$ (dddd, $J=15.0,4.2,3.0,1.2 \mathrm{~Hz}, 1 \mathrm{H}$ ), 1.50 (ddd, $J=15.0,12.6,2.4 \mathrm{~Hz}, 1 \mathrm{H}$ ), 0.70 (d, $J=5.4 \mathrm{~Hz}$, $3 \mathrm{H}) ;{ }^{13} \mathrm{C}$ NMR $\left(\mathrm{CD}_{3} \mathrm{OD}, 150 \mathrm{MHz}\right) \delta 180.2,173.5,166.0,163.4,161.8,159.4,158.7,137.7$, 132.2, 123.0, 116.6, 106.2, 99.9, 94.9, 82.1, 79.0, 72.9, 72.3, 33.3, 32.1, 21.2, 18.0; HRMS (ESI) calcd. for $\left[\mathrm{C}_{24} \mathrm{H}_{24} \mathrm{O}_{10}+\mathrm{H}\right]^{+}: 473.1442$, Found: 473.1441 .

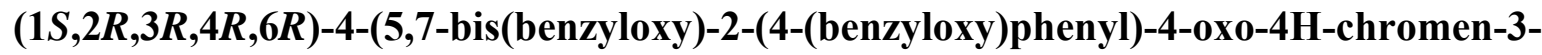
yloxy)-2-hydroxy-6-methylcyclohexane-1,3-diyl diacetate (III-37) 
<smiles>CC(=O)OC1c2cc(OCc3ccccc3)cc(OCc3ccccc3)c2C(=O)C(OCc2ccccc2)[C@H]1OC1C[C@H](C)[C@@H](OC(C)=O)[C@H](O)C1Cc1ccccc1</smiles>

(1S,2S,3S,4R,6R)-4-(5,7-bis(benzyloxy)-2-(4-(benzyloxy)phenyl)-4-oxo-4H-chromen-3yloxy)-3-hydroxy-6-methylcyclohexane-1,2-diyl diacetate (III-38)<smiles>CC(=O)OC1CCC[C@H]1OC1C[C@H](C)[C@@H](OC(C)=O)[C@H](O)C1Oc1c(-c2ccc(OCc3ccccc3)cc2)oc2cc(OCc3ccccc3)cc(OCc3ccccc3)c2c1=O</smiles>

To a solution of $46 \mathrm{mg}(0.062 \mathrm{mmol})$ diol III-36 in $1.3 \mathrm{~mL} \mathrm{CH}_{2} \mathrm{Cl}_{2}$ at $0{ }^{\circ} \mathrm{C}$ was added $1.0 \mathrm{mg}$ $(5.5 \mu \mathrm{mol}) p$ - $\mathrm{TsOH} \cdot \mathrm{H}_{2} \mathrm{O}, 48 \mu \mathrm{L}$ trimethylorthoacetate $(0.36 \mathrm{mmol})$. After stirring at $0{ }^{\circ} \mathrm{C}$ for 20 min, $0.5 \mathrm{~mL} 90 \%$ HOAc (aq.) was added at $0{ }^{\circ} \mathrm{C}$, and the reaction mixture was stirred for another $15 \mathrm{~min}$ at $0{ }^{\circ} \mathrm{C}$. The reaction mixture was diluted with $30 \mathrm{~mL}$ EtOAc and washed at $0{ }^{\circ} \mathrm{C}$ with 15 $\mathrm{mL}$ saturated aqueous $\mathrm{NaHCO}_{3}$. It was then washed by $10 \mathrm{~mL}$ saturated brine, and dried over $\mathrm{Na}_{2} \mathrm{SO}_{4}$. The organic layer was concentrated under reduced pressure to give crude product, which was then subjected to chromatography on silica gel. Elution with hexane-EtOAc $(1: 1)$ gave diacetate III-37 (26 mg, 54\%) and III-38 (16.5 mg, 34\%):

III-37: White solid, mp: $99-101{ }^{\circ} \mathrm{C} ; R_{f}=0.36\left(1: 1.5(\mathrm{v} / \mathrm{v})\right.$ hexane/EtOAc); $[\alpha]_{\mathrm{D}}{ }^{20}+26(c 1.23$, $\mathrm{CHCl}_{3}$ ); IR (thin film, $\mathrm{cm}^{-1}$ ) v 3471, 3030, 2932, 1731, 1626, 1603, 1574, 1509, 1453, 1432 , 1370, 1298, 1228, 1172, 1101, 1039, 963; ${ }^{1} \mathrm{H} \mathrm{NMR}\left(\mathrm{CDCl}_{3}, 600 \mathrm{MHz}\right) \delta 7.85$ (d, $J=9.0 \mathrm{~Hz}$, 2H), $7.56(\mathrm{~d}, J=7.2 \mathrm{~Hz}, 2 \mathrm{H}), 7.47-7.27(\mathrm{~m}, 13 \mathrm{H}), 7.11(\mathrm{~d}, J=9.0 \mathrm{~Hz}, 2 \mathrm{H}), 6.56(\mathrm{~d}, J=2.4 \mathrm{~Hz}$, $1 \mathrm{H}), 6.44(\mathrm{~d}, J=2.4 \mathrm{~Hz}, 1 \mathrm{H}), 5.28(\mathrm{~s}, 2 \mathrm{H}), 5.15(\mathrm{~s}, 2 \mathrm{H}), 5.07$ (s, 2H), 5.06 (ddd, $J=3.6,3.6,1.2$ $\mathrm{Hz}, 1 \mathrm{H}), 4.70(\mathrm{dd}, J=10.8,10.2 \mathrm{~Hz}, 1 \mathrm{H}), 4.39$ (dddd, $J=3.0,3.0,2.4 \mathrm{~Hz}, 1 \mathrm{H}), 3.76(\mathrm{ddd}, J=$ 
9.6, 6.0, 3.6 Hz, 1H), 2.18 (dddd, $J=14.4,4.2,3.0,1.8 \mathrm{~Hz}, 1 \mathrm{H}), 2.11$ (s, 3H), 2.01 (s, 3H), 1.97 (ddqd, $J=13.2,10.2,6.0,3.6 \mathrm{~Hz}, 1 \mathrm{H}$ ), 1.85 (d, $J=5.4 \mathrm{~Hz}, 1 \mathrm{H}$ ), 1.49 (ddd, $J=15.0,13.2,3.0$ $\mathrm{Hz}, 1 \mathrm{H}), 0.88(\mathrm{~d}, J=6.6 \mathrm{~Hz}, 3 \mathrm{H}) ;{ }^{13} \mathrm{C} \mathrm{NMR}\left(\mathrm{CDCl}_{3}, 150 \mathrm{MHz}\right) \delta 173.5,171.9,170.1,162.8$, 160.6, 159.8, 158.8, 154.1, 139.1, 136.4, 135.7, 130.5, 128.8, 128.7, 128.6, 128.4, 128.2, 127.7, 127.5, 127.4, 126.7, 123.3, 114.8, 110.1, 98.4, 94.0, 77.7, 73.1, 70.8, 70.5, 70.3, 70.2, 33.5, 30.4, 21.1, 21.0, 17.4; HRMS (ESI) calcd. for $\left[\mathrm{C}_{47} \mathrm{H}_{44} \mathrm{O}_{11}+\mathrm{H}\right]^{+}:$785.2956, Found: 785.2940.

III-38: White solid, mp: $89-91{ }^{\circ} \mathrm{C} ; R_{f}=0.47\left(1: 1.5(\mathrm{v} / \mathrm{v})\right.$ hexane/EtOAc); $[\alpha]_{\mathrm{D}}{ }^{20}-63$ (c 1.32, $\mathrm{CHCl}_{3}$ ); IR (thin film, $\mathrm{cm}^{-1}$ ) $v$ 3426, 3031, 2930, 1735, 1623, 1603, 1573, 1508, 1453, 1432, 1367, 1297, 1249, 1224, 1173, 1101, 1040, 962; ${ }^{1} \mathrm{H}$ NMR $\left(\mathrm{CDCl}_{3}, 600 \mathrm{MHz}\right) \delta 7.90(\mathrm{~d}, J=9.0$ $\mathrm{Hz}, 2 \mathrm{H}), 7.56(\mathrm{~d}, J=7.8 \mathrm{~Hz}, 2 \mathrm{H}), 7.47-7.27(\mathrm{~m}, 13 \mathrm{H}), 7.11(\mathrm{~d}, J=9.0 \mathrm{~Hz}, 2 \mathrm{H}), 6.57$ (d, $J=2.4$ $\mathrm{Hz}, 1 \mathrm{H}), 6.45(\mathrm{~d}, J=2.4 \mathrm{~Hz}, 1 \mathrm{H}), 5.26(\mathrm{~s}, 2 \mathrm{H}), 5.22(\mathrm{dd}, J=9.0,3.0 \mathrm{~Hz}, 1 \mathrm{H}), 5.16(\mathrm{~d}, J=11.4 \mathrm{~Hz}$, 1H), 5.15 (d, $J=12.0 \mathrm{~Hz}, 1 \mathrm{H}), 5.08$ (s, 2H), 4.92 (dd, $J=9.6,9.0 \mathrm{~Hz}, 1 \mathrm{H}$ ), 4.41 (ddd, $J=4.2$, 3.6, 3.6 Hz, 1H), 4.31 (dd, $J=3.6,3.6 \mathrm{~Hz}, 1 \mathrm{H}), 2.93$ (brs, 1H), 2.02 (s, 3H), 2.02 (s, 3H), 1.81 (ddqd, $J=13.2,10.8,6.6,3.6 \mathrm{~Hz}, 1 \mathrm{H}), 1.73$ (ddd, $J=14.4,4.2,4.2 \mathrm{~Hz}, 1 \mathrm{H}), 1.52$ (ddd, $J=14.4$, $10.8,3.0 \mathrm{~Hz}, 1 \mathrm{H}), 0.74(\mathrm{~d}, J=6.6 \mathrm{~Hz}, 3 \mathrm{H}) ;{ }^{13} \mathrm{C} \mathrm{NMR}\left(\mathrm{CDCl}_{3}, 150 \mathrm{MHz}\right) \delta 173.8,170.5,169.5$, $162.8,160.5,159.8,158.8,154.1,139.3,136.5,136.3,135.6,130.5,128.8,128.7,128.6,128.4$, $128.1,127.7,127.6,127.4,126.7,123.3,114.8,110.0,98.3,94.0,79.7,74.5,73.4,70.9,70.8$, 70.5, 70.1, 32.3, 31.0, 20.9, 20.9, 17.2; HRMS (ESI) calcd. for $\left[\mathrm{C}_{47} \mathrm{H}_{44} \mathrm{O}_{11}+\mathrm{H}\right]^{+}:$785.2956, Found: 785.2942 .

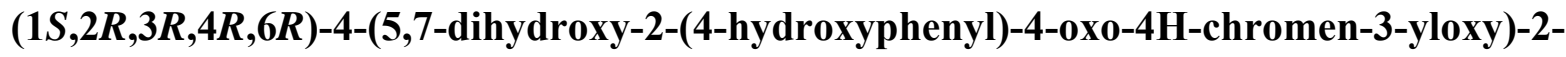
hydroxy-6-methylcyclohexane-1,3-diyl diacetate (III-2) 


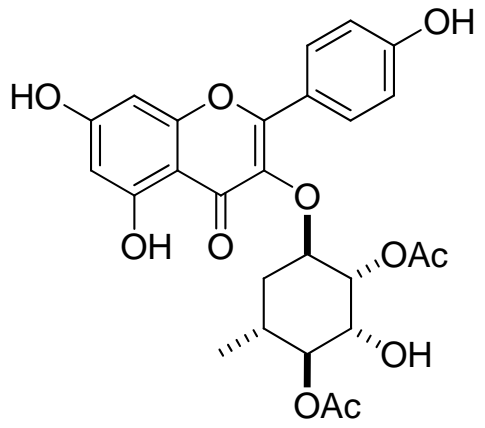

To a solution of $19 \mathrm{mg}(0.024 \mathrm{mmol})$ diol III-37 in $2.5 \mathrm{~mL}$ THF-EtOH (1:1) was added $23 \mathrm{mg}$ $\mathrm{Pd} / \mathrm{C}(10 \%)$. The reaction mixture was degassed at -50 to $-40{ }^{\circ} \mathrm{C}$ using vacuum and refilled with $\mathrm{H}_{2}$. This procedure was repeated three times, then the bath was removed and the reaction was warmed up to room temperature. The reaction mixture was stirred under a $\mathrm{H}_{2}$ atmosphere for 12 hours. The reaction mixture was loaded onto silica gel and elution with hexane-EtOAc (1:1) to give product (III-2) $(7.7 \mathrm{mg}, 63 \%)$ : Yellow solid, mp: $165-167{ }^{\circ} \mathrm{C} ; R_{f}=0.31(1: 3(\mathrm{v} / \mathrm{v})$ hexane/EtOAc); $[\alpha]_{\mathrm{D}}^{20}+4\left(c\right.$ 0.51, MeOH); IR (thin film, $\left.\mathrm{cm}^{-1}\right) v 3220,2961,1717,1650,1607$, $1505,1441,1366,1248,1210,1174,1108,1084,1040,1008,972 ;{ }^{1} \mathrm{H}$ NMR $\left(\mathrm{CD}_{3} \mathrm{OD}, 600 \mathrm{MHz}\right)$ $\delta 7.78(\mathrm{~d}, J=8.4 \mathrm{~Hz}, 2 \mathrm{H}), 6.94(\mathrm{~d}, J=9.0 \mathrm{~Hz}, 2 \mathrm{H}), 6.38(\mathrm{~d}, J=2.4 \mathrm{~Hz}, 1 \mathrm{H}), 6.21(\mathrm{~d}, J=1.8 \mathrm{~Hz}$, 1H), 5.22 (ddd, $J=3.6,3.6,1.2 \mathrm{~Hz}, 1 \mathrm{H}), 4.73$ (dd, $J=10.2,10.2 \mathrm{~Hz}, 1 \mathrm{H}), 4.28$ (ddd, $J=3.0,3.0$, $3.0 \mathrm{~Hz}, 1 \mathrm{H}), 3.91$ (dd, $J=9.6,3.0 \mathrm{~Hz}, 1 \mathrm{H}), 2.08$ (s, 3H), 2.02 (s, 3H), 1.83 (ddqd, $J=13.2,10.2$, 6.6, 3.6 Hz, 1H), 1.82 (ddd, $J=15.6,3.6,3.0,1.2 \mathrm{~Hz}, 1 \mathrm{H}), 1.44$ (ddd, $J=16.2,13.8,3.0 \mathrm{~Hz}, 1 \mathrm{H}$ ), $0.78(\mathrm{~d}, J=6.6 \mathrm{~Hz}, 3 \mathrm{H}) ;{ }^{13} \mathrm{C} \mathrm{NMR}\left(\mathrm{CD}_{3} \mathrm{OD}, 150 \mathrm{MHz}\right) \delta 179.9,173.2,172.0,166.0,163.4$, 161.9, 159.4, 158.7, 137.4, 132.1, 122.8, 116.7, 106.1, 100.0, 94.9, 78.8, 78.8, 74.5, 70.4, 34.1, 32.0, 21.2, 21.0, 18.0; HRMS (ESI) calcd. for $\left[\mathrm{C}_{26} \mathrm{H}_{26} \mathrm{O}_{11}+\mathrm{H}\right]^{+}: 515.1548$, Found: 515.1547.

(1S,2S,3S,4R,6R)-4-(5,7-dihydroxy-2-(4-hydroxyphenyl)-4-oxo-4H-chromen-3-yloxy)-3hydroxy-6-methylcyclohexane-1,2-diyl diacetate (III-3) 


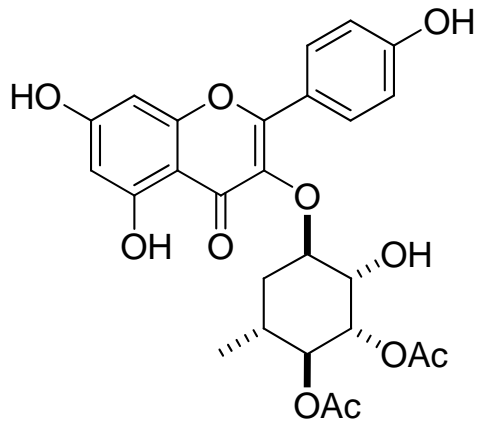

To a solution of $20 \mathrm{mg}(0.0255 \mathrm{mmol})$ diol III-38 in $2.5 \mathrm{~mL}$ THF-EtOH (1:1) was added 21.6 $\mathrm{mg} \mathrm{Pd} / \mathrm{C}(10 \%)$. The reaction mixture was degassed at -50 to $-40{ }^{\circ} \mathrm{C}$ using vacuum and refilled with $\mathrm{H}_{2}$. This procedure was repeated three times, then the bath was removed and the reaction was warmed up to room temperature. The reaction mixture was stirred under a $\mathrm{H}_{2}$ atmosphere for 7 hours. The reaction mixture was loaded onto silica gel and elution with hexane-EtOAc (1.5:1) to give product (III-3) $(9.8 \mathrm{mg}, 75 \%)$ : Yellow solid, $\mathrm{mp}: 127-129{ }^{\circ} \mathrm{C} ; R_{f}=0.36(1: 3(\mathrm{v} / \mathrm{v})$ hexane/EtOAc); $[\alpha]_{\mathrm{D}}{ }^{20}-93$ (c 0.75, MeOH); IR (thin film, $\mathrm{cm}^{-1}$ ) v 3342, 2927, 1716, 1650, 1607, 1505, 1437, 1364, 1208, 1171, 1084, 1042, 1008, 972; ${ }^{1} \mathrm{H}$ NMR $\left(\mathrm{CD}_{3} \mathrm{OD}, 600 \mathrm{MHz}\right) \delta 7.79$ (d, $J$ $=9.0 \mathrm{~Hz}, 2 \mathrm{H}), 6.94(\mathrm{~d}, J=8.4 \mathrm{~Hz}, 2 \mathrm{H}), 6.38(\mathrm{~d}, J=1.8 \mathrm{~Hz}, 1 \mathrm{H}), 6.20(\mathrm{~d}, J=2.4 \mathrm{~Hz}, 1 \mathrm{H}), 5.06$ (dd, $J=10.2,3.0 \mathrm{~Hz}, 1 \mathrm{H}), 4.94$ (dd, $J=10.8,10.2 \mathrm{~Hz}, 1 \mathrm{H}$ ), 4.47 (ddd, $J=3.0,3.0,3.0 \mathrm{~Hz}, 1 \mathrm{H}$ ), 4.26 (ddd, $J=4.2,4.2,1.2 \mathrm{~Hz}, 1 \mathrm{H}), 2.02$ (s, 3H), 1.99 (s, 3H), 1.65 (ddqd, $J=12.6,10.2,6.0,3.6$ $\mathrm{Hz}, 1 \mathrm{H}), 1.62$ (ddd, $J=15.6,4.2,3.0,1.2 \mathrm{~Hz}, 1 \mathrm{H}), 1.50$ (ddd, $J=15.0,13.2,2.4 \mathrm{~Hz}, 1 \mathrm{H}), 0.67$ (d, $J=6.6 \mathrm{~Hz}, 3 \mathrm{H}) ;{ }^{13} \mathrm{C} \mathrm{NMR}\left(\mathrm{CD}_{3} \mathrm{OD}, 150 \mathrm{MHz}\right) \delta 180.1,172.7,172.2,166.0,163.4,161.7,159.5$, 158.7, 137.2, 132.2, 122.9, 116.6, 106.2, 99.9, 94.9, 81.3, 75.9, 75.1, 70.8, 32.7, 32.2, 21.0, 20.9, 17.9; HRMS (ESI) calcd. for $\left[\mathrm{C}_{26} \mathrm{H}_{26} \mathrm{O}_{11}+\mathrm{H}\right]^{+}: 515.1548$, Found: 515.1547.

5,7-bis(benzyloxy)-2-(4-(benzyloxy)phenyl)-3-((1S,5S)-5-methyl-4-oxocyclohex-2-enyloxy)4H-chromen-4-one ((ent)-III-9) 


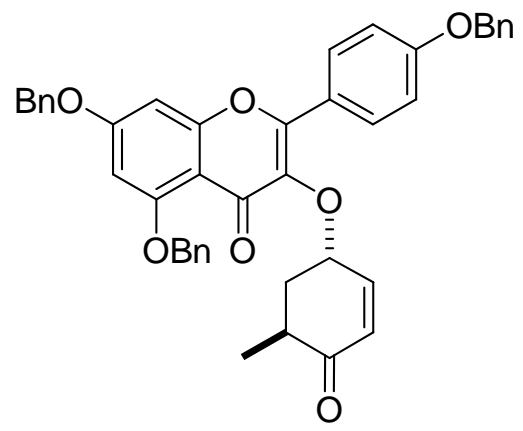

$85 \mathrm{mg}(0.15 \mathrm{mmol})$ perbenzylated flavonol III-8 and $50 \mathrm{mg}(0.22 \mathrm{mmol}) \alpha$-D-Boc-enone (ent)III-4 was suspended in $1.5 \mathrm{~mL} \mathrm{CH}_{2} \mathrm{Cl}_{2}$ at $0{ }^{\circ} \mathrm{C}$. A solution of $4.0 \mathrm{mg}(0.004 \mathrm{mmol})$ $\mathrm{Pd}_{2}(\mathrm{DBA})_{3} \cdot \mathrm{CHCl}_{3}$ and $4.0 \mathrm{mg} \mathrm{PPh} 3(0.015 \mathrm{mmol})$ in $0.5 \mathrm{~mL} \mathrm{CH}_{2} \mathrm{Cl}_{2}$ wad added via a syringe at $0{ }^{\circ} \mathrm{C}$. The reaction mixture was stirred at $0{ }^{\circ} \mathrm{C}$ for $30 \mathrm{~min}$ and then directly loaded onto a silica gel column, elution with hexane-EtOAc (3:1) gave glycosylated enone (ent)-III-9 (86 mg, 84\%). Off white powder, mp: $63-65^{\circ} \mathrm{C} ; R_{f}=0.60\left(1: 1(\mathrm{v} / \mathrm{v})\right.$ hexane/EtOAc); $[\alpha]_{\mathrm{D}}{ }^{20}-63\left(c 2.2, \mathrm{CHCl}_{3}\right)$; IR (thin film, $\mathrm{cm}^{-1}$ ) $v$ 3033, 2931, 1731, 1681, 1625, 1602, 1572, 1508, 1452, 1433, 1375, 1354, 1297, 1250, 1171, 1100, 1002, 972. ${ }^{1} \mathrm{H}$ NMR $\left(\mathrm{CDCl}_{3}, 600 \mathrm{MHz}\right) \delta 7.96(\mathrm{~d}, J=8.4 \mathrm{~Hz}, 2 \mathrm{H}), 7.58$ (d, $J=7.2 \mathrm{~Hz}, 2 \mathrm{H}), 7.46-7.29$ (m, 13H), 7.04 (d, $J=9.0 \mathrm{~Hz}, 2 \mathrm{H}), 7.04$ (dd, $J=10.2,4.2 \mathrm{~Hz}, 1 \mathrm{H})$, $6.59(\mathrm{~d}, J=2.4 \mathrm{~Hz}, 1 \mathrm{H}), 6.47(\mathrm{~d}, J=2.4 \mathrm{~Hz}, 1 \mathrm{H}), 5.92(\mathrm{~d}, J=9.6 \mathrm{~Hz}, 1 \mathrm{H}), 5.28(\mathrm{~s}, 2 \mathrm{H}), 5.14$ (s, 2H), 5.09 (s, 2H), 5.07 (ddd, $J=4.8,4.2,4.2 \mathrm{~Hz}, 1 \mathrm{H}), 2.60$ (dqd, $J=10.2,7.2,4.8 \mathrm{~Hz}, 1 \mathrm{H}), 2.11$ (ddd, $J=13.8,4.2,4.2 \mathrm{~Hz}, 1 \mathrm{H}), 1.86$ (ddd, $J=14.4,10.2,4.8 \mathrm{~Hz}, 1 \mathrm{H}), 0.95$ (d, $J=6.6 \mathrm{~Hz}, 3 \mathrm{H}$ ); ${ }^{13} \mathrm{C}$ NMR $\left(\mathrm{CDCl}_{3}, 150 \mathrm{MHz}\right) \delta 201.9,173.7,162.9,160.5,159.8,158.8,154.1,145.6,138.7$, 136.3, 135.6, 130.4, 130.0, 128.7, 128.7, 128.6, 128.4, 128.2, 127.7, 127.5, 127.4, 126.6, 123.3, $114.6,110.0,98.3,94.0,72.8,70.9,70.5,70.1,37.8,35.8,14.7$.

\section{5,7-bis(benzyloxy)-2-(4-(benzyloxy)phenyl)-3-((1S,4R,5S)-4-hydroxy-5-methylcyclohex-2- enyloxy)-4H-chromen-4-one ((ent)-III-34)}




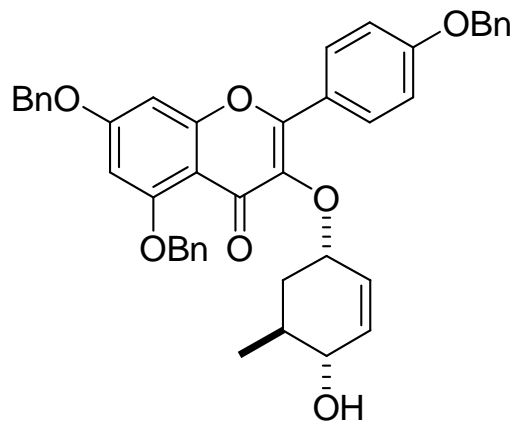

A solution of $135 \mathrm{mg}(0.20 \mathrm{mmol})$ enone (ent)-III-9 in $15 \mathrm{~mL}$ THF at $-78^{\circ} \mathrm{C}$ was added $15 \mathrm{mg}$ $(0.4 \mathrm{mmol}) \mathrm{LiAlH}_{4}$ in two portions. It was stirred at $-78{ }^{\circ} \mathrm{C}$ for $1 \mathrm{~h}$ and then $3 \mathrm{~mL}$ acetone was slowly added via syringe into the reaction mixture at $-78{ }^{\circ} \mathrm{C}$. The mixture was stirred at $-78{ }^{\circ} \mathrm{C}$ for $20 \mathrm{~min}$ and diluted with $20 \mathrm{~mL}$ hexane-EtOAc $(1: 1, \mathrm{v} / \mathrm{v})$ and quenched with $6 \mathrm{~mL}$ saturated aqueous $\mathrm{NaHCO}_{3}$. The mixture was stirred at $0{ }^{\circ} \mathrm{C}$ for $20 \mathrm{~min}$. The organic layer was separated and the aqueous layer was extracted with EtOAc. The pooled organic layer was washed with saturated brine and dried over $\mathrm{Na}_{2} \mathrm{SO}_{4}$. After removal of the solvent under reduced pressure, the residue was subjected to silica gel column chromatography. Elution with hexane-EtOAc (1.5:1, v/v) afforded allylic alcohol (ent)-III-34 (118 mg, 89\%). Pale yellow solid, mp: $72-74{ }^{\circ} \mathrm{C} ; R_{f}=$ $0.38\left(1: 1(\mathrm{v} / \mathrm{v})\right.$ hexane/EtOAc); $[\alpha]_{\mathrm{D}}{ }^{20}-12\left(c 2.1, \mathrm{CHCl}_{3}\right)$; IR (thin film, $\left.\mathrm{cm}^{-1}\right) v 3422,3064,3032$, 2927, 1735, 1620, 1601, 1508, 1452, 1373, 1355, 1296, 1249, 1172, 1100, 1041, 1013, 966; ${ }^{1} \mathrm{H}$ NMR $\left(\mathrm{CDCl}_{3}, 600 \mathrm{MHz}\right) \delta 7.99(\mathrm{~d}, J=8.4 \mathrm{~Hz}, 2 \mathrm{H}), 7.58(\mathrm{~d}, J=7.2 \mathrm{~Hz}, 2 \mathrm{H}), 7.46-7.27$ (m, $13 \mathrm{H}), 7.05(\mathrm{~d}, J=9.0 \mathrm{~Hz}, 2 \mathrm{H}), 6.57(\mathrm{~d}, J=2.4 \mathrm{~Hz}, 1 \mathrm{H}), 6.45$ (d, $J=2.4 \mathrm{~Hz}, 1 \mathrm{H}), 6.03$ (dddd, $J=$ 9.6, 3.6, 1.8, 1.2 Hz, 1H), $5.80(\mathrm{dd}, J=9.6,1.8 \mathrm{~Hz}, 1 \mathrm{H}), 5.28(\mathrm{~s}, 2 \mathrm{H}), 5.15(\mathrm{~s}, 2 \mathrm{H}), 5.08(\mathrm{~s}, 2 \mathrm{H})$, $4.73(\mathrm{~m}, 1 \mathrm{H}), 3.59(\mathrm{dd}, J=7.8,7.2 \mathrm{~Hz}, 1 \mathrm{H}), 1.73(\mathrm{ddd}, J=14.4,4.2,3.0 \mathrm{~Hz}, 1 \mathrm{H}), 1.63$ (ddqd, $J$ $=13.2,9.6,6.6,3.0 \mathrm{~Hz}, 1 \mathrm{H}), 1.29(\mathrm{ddd}, J=14.4,12.0,4.2 \mathrm{~Hz}, 1 \mathrm{H}), 0.79(\mathrm{~d}, J=6.6 \mathrm{~Hz}, 3 \mathrm{H}) ;{ }^{13} \mathrm{C}$ NMR $\left(\mathrm{CDCl}_{3}, 150 \mathrm{MHz}\right) \delta 174.0,162.7,160.2,159.8,158.8,154.0,139.3,136.5,136.4,135.7$, $135.4,130.6,128.7,128.7,128.6,128.4,128.1,127.7,127.6,127.6,127.4,126.7,123.8,114.4$, $110.1,98.2,94.0,73.6,73.5,70.8,70.4,70.1,34.5,33.0,17.8$.

(1R,4S,6S)-4-(5,7-bis(benzyloxy)-2-(4-(benzyloxy)phenyl)-4-oxo-4H-chromen-3-yloxy)-6methylcyclohex-2-enyl acetate ((ent)-III-35) 


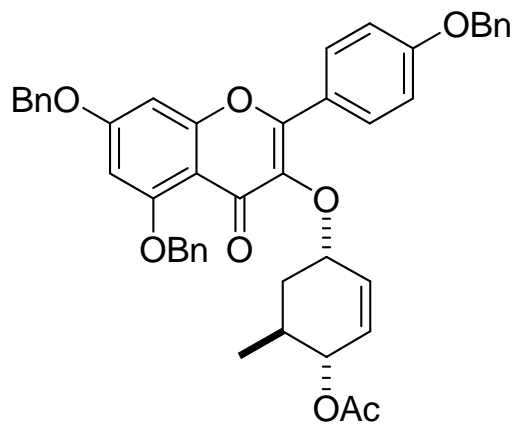

A solution of $99 \mathrm{mg}(0.15 \mathrm{mmol})$ enone (ent)-III-34 and $1.8 \mathrm{mg}(0.015 \mathrm{mmol})$ DMAP in $2.0 \mathrm{~mL}$ $\mathrm{CH}_{2} \mathrm{Cl}_{2}$ at $0{ }^{\circ} \mathrm{C}$ was added $200 \mu \mathrm{L}(2.0 \mathrm{mmol})$ pyridine and $94 \mu \mathrm{L}(1.0 \mathrm{mmol}) \mathrm{Ac}_{2} \mathrm{O}$ via syringe. It was stirred at $0{ }^{\circ} \mathrm{C}$ for $2 \mathrm{~h}$ and diluted with $20 \mathrm{~mL}$ hexane-EtOAc $(1: 1, \mathrm{v} / \mathrm{v})$ and washed with 5 $\mathrm{mL} 0.5 \mathrm{M}$ aqueous $\mathrm{NaHSO}_{4}$. The organic layer was separated and the aqueous layer was extracted with EtOAc. The pooled organic layer was washed with saturated aqueous $\mathrm{NaHCO}_{3}$ and saturated brine subsequently and then dried over $\mathrm{Na}_{2} \mathrm{SO}_{4}$. After removal of the solvent under reduced pressure, the residue was subjected to silica gel column chromatography. Elution with hexane-EtOAc (3:1, v/v) afforded allylic acetate (ent)-III-35 (103 mg, 98\%). Pale yellow solid, mp: 61-63 ${ }^{\circ} \mathrm{C}, R_{f}=0.30\left(\right.$ hexane/EtOAc 2:1, v/v); $[\alpha]_{\mathrm{D}}{ }^{20}+31\left(\right.$ c 3.25, $\left.\mathrm{CHCl}_{3}\right)$; IR (thin film, $\mathrm{cm}^{-1}$ ) $v$ 3065, 3034, 2958, 2931, 2873, 1732, 1626, 1602, 1508, 1452, 1434, 1372, 1356, 1295, 1242, 1173, 1101, 1022, 920; ${ }^{1} \mathrm{H} \mathrm{NMR}\left(\mathrm{CDCl}_{3}, 600 \mathrm{MHz}\right) \delta 8.02(\mathrm{~d}, J=9.0 \mathrm{~Hz}, 2 \mathrm{H}), 7.58(\mathrm{~d}, J=7.2$ $\mathrm{Hz}, 2 \mathrm{H}), 7.47-7.27(\mathrm{~m}, 13 \mathrm{H}), 7.06(\mathrm{~d}, J=9.0 \mathrm{~Hz}, 2 \mathrm{H}), 6.58(\mathrm{~d}, J=1.8 \mathrm{~Hz}, 1 \mathrm{H}), 6.45$ (d, $J=2.4$ $\mathrm{Hz}, 1 \mathrm{H}), 6.13$ (dddd, $J=10.2,4.8,1.8,1.8 \mathrm{~Hz}, 1 \mathrm{H}), 5.71$ (dd, $J=10.2,1.8 \mathrm{~Hz}, 1 \mathrm{H}), 5.28$ (s, 2H), 5.15 (s, 2H), 5.08 (s, 2H), 4.88 (dddd, $J=9.0,1.8,1.8,1.8 \mathrm{~Hz}, 1 \mathrm{H}), 4.74(\mathrm{~m}, 1 \mathrm{H}), 2.09$ (s, 3H), 1.94 (ddqd, $J=13.2,9.6,6.6,3.0 \mathrm{~Hz}, 1 \mathrm{H}), 1.77$ (m, $1 \mathrm{H}), 1.40$ (ddd, $J=14.4,12.6,4.8 \mathrm{~Hz}, 1 \mathrm{H})$, $0.70(\mathrm{~d}, J=6.6 \mathrm{~Hz}, 3 \mathrm{H}) ;{ }^{13} \mathrm{C} \mathrm{NMR}\left(\mathrm{CDCl}_{3}, 150 \mathrm{MHz}\right) \delta 173.9,171.0,162.7,160.2,159.8,158.8$, $153.8,139.4,136.5,136.4,135.7,131.1,130.5,129.2,128.7,128.7,128.6,128.4,128.1,127.7$, $127.6,127.4,126.7,123.8,114.4,110.1,98.2,94.0,75.2,73.5,70.8,70.4,70.1,34.3,29.5,21.2$, 17.6 .

(1R,2R,3S,4S,6S)-4-(5,7-bis(benzyloxy)-2-(4-(benzyloxy)phenyl)-4-oxo-4H-chromen-3yloxy)-2,3-dihydroxy-6-methylcyclohexyl acetate ((ent)-III-36) 


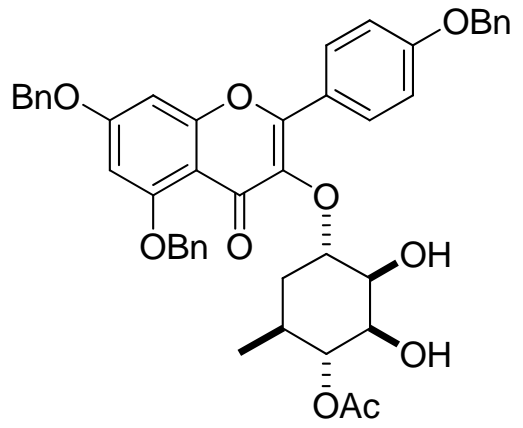

A solution of $103 \mathrm{mg}(0.145 \mathrm{mmol})$ allylic acetate $((e n t)$-III-35) in $2 \mathrm{~mL}$ acetone-t-BuOH $(1: 1)$ was cooled down to $0{ }^{\circ} \mathrm{C}$. To the solution was added $1 \mathrm{~mL} \mathrm{NMO}-\mathrm{H}_{2} \mathrm{O}(\mathrm{w} / \mathrm{w} 1: 1)$, and a small piece of crystalline $\mathrm{OsO}_{4}$ was added into the mixture. The reaction mixture was stirred at $0{ }^{\circ} \mathrm{C}$ for 12 hours. The reaction was diluted with $20 \mathrm{~mL}$ EtOAc and then quenched with $2 \mathrm{~mL}$ saturated $\mathrm{Na}_{2} \mathrm{~S}_{2} \mathrm{O}_{3}$ (aq.) and stirred for $2 \mathrm{~h}$ at $0{ }^{\circ} \mathrm{C}$. Then the mixture was passed through a small pad of celite/silica gel/sand and washed with EtOAc-MeOH (3:1). The effluent was dried over $\mathrm{Na}_{2} \mathrm{SO}_{4}$, and concentrated under reduced pressure to give a residue. Chromatography on silica gel eluting with hexane-EtOAc (1:1.5) gave diol (ent)-III-36 (89 mg, 82\%): White solid, mp: 91$93{ }^{\circ} \mathrm{C} ; R_{f}=0.12\left(1: 1(\mathrm{v} / \mathrm{v})\right.$, hexane/EtOAc); $[\alpha]_{\mathrm{D}}{ }^{20}+47\left(c 2.95, \mathrm{CHCl}_{3}\right)$; IR (thin film, $\left.\mathrm{cm}^{-1}\right) v$ 3418,3032 , 2929, 1727, 1602, 1508, 1452, 1432, 1372, 1357, 1298, 1247, 1197, 1172, 1099, 1036, 959; ${ }^{1} \mathrm{H} \mathrm{NMR}\left(\mathrm{CDCl}_{3}, 600 \mathrm{MHz}\right) \delta 7.90(\mathrm{~d}, J=8.4 \mathrm{~Hz}, 2 \mathrm{H}), 7.55(\mathrm{~d}, J=7.8 \mathrm{~Hz}, 2 \mathrm{H})$, 7.47-7.27 (m, 13H), $7.08(\mathrm{~d}, J=8.4 \mathrm{~Hz}, 2 \mathrm{H}), 6.56(\mathrm{~d}, J=2.4 \mathrm{~Hz}, 1 \mathrm{H}), 6.45(\mathrm{~d}, J=1.8 \mathrm{~Hz}, 1 \mathrm{H})$, $5.26(\mathrm{~s}, 2 \mathrm{H}), 5.15(\mathrm{~s}, 2 \mathrm{H}), 5.08(\mathrm{~s}, 2 \mathrm{H}), 4.80(\mathrm{dd}, J=7.8,7.2 \mathrm{~Hz}, 1 \mathrm{H}), 4.28(\mathrm{ddd}, J=5.4,5.4,3.0$ $\mathrm{Hz}, 1 \mathrm{H}), 4.17$ (ddd, $J=5.4,3.0,3.0 \mathrm{~Hz}, 1 \mathrm{H}), 3.85$ (ddd, $J=7.2,4.2,4.2 \mathrm{~Hz}, 1 \mathrm{H}), 2.86(\mathrm{~d}, J=4.2$ Hz, 1H), 2.08 (s, 3H), 1.80 (m, 1H), 1.69 (ddd, $J=13.8,5.4,4.8$ Hz, 1H ), 1.41 (ddd, $J=13.8$, 9.6, 3.6 Hz, $1 \mathrm{H}), 0.81(\mathrm{~d}, J=7.2 \mathrm{~Hz}, 3 \mathrm{H}) ;{ }^{13} \mathrm{C} \mathrm{NMR}\left(\mathrm{CDCl}_{3}, 150 \mathrm{MHz}\right) \delta 174.1,171.3,162.9$, $160.5,159.8,158.8,154.4,139.2$, 136.3, 136.2, 135.6, 130.6, 128.7, 128.7, 128.6, 128.4, 128.2, $127.7,127.5,127.4,126.7,123.4,114.6,109.8,98.3,94.0,80.6,76.9,72.0,71.9,70.8,70.5,70.1$, $32.7,31.0,21.1,17.3$.

(1R,2R,3S,4S,6S)-4-(5,7-dihydroxy-2-(4-hydroxyphenyl)-4-ox0-4H-chromen-3-yloxy)-2,3dihydroxy-6-methylcyclohexyl acetate ((ent)-III-1) 


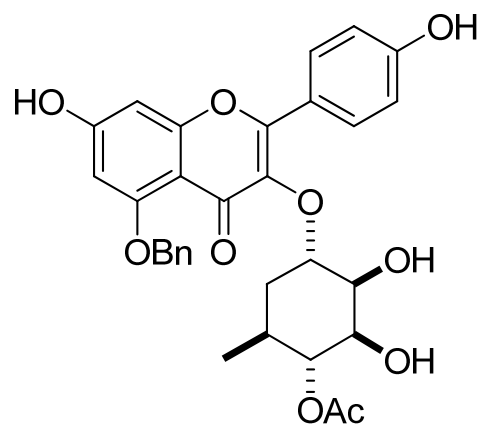

To a solution of $26 \mathrm{mg}(0.035 \mathrm{mmol})$ diol (ent)-III-36 in $2 \mathrm{~mL}$ THF-EtOH (1:1) was added 26 $\mathrm{mg} \mathrm{Pd} / \mathrm{C}(10 \%)$. The reaction mixture was degassed at -50 to $-40{ }^{\circ} \mathrm{C}$ using vacuum and refilled with $\mathrm{H}_{2}$. This procedure was repeated three times, then the bath was removed and the reaction was warmed up to room temperature. The reaction mixture was stirred under a $\mathrm{H}_{2}$ atmosphere for 7 hours. The reaction mixture was loaded onto silica gel and elution with hexane-EtOAc $(1: 1.5)$ to give product (ent)-(III-1) (12 mg, 73\%): Yellow solid, mp: $148-150{ }^{\circ} \mathrm{C} ; R_{f}=0.24(1: 4(\mathrm{v} / \mathrm{v})$ hexane/EtOAc); $[\alpha]_{\mathrm{D}}^{20}+34\left(c\right.$ 0.78, MeOH); IR (thin film, $\left.\mathrm{cm}^{-1}\right) v 3217,2927,1709,1650,1607$, $1497,1440,1361,1258,1207,1171,1085,1037,970 ;{ }^{1} \mathrm{H}$ NMR $\left(\mathrm{CD}_{3} \mathrm{OD}, 600 \mathrm{MHz}\right) \delta 7.78(\mathrm{~d}, J$ $=9.0 \mathrm{~Hz}, 2 \mathrm{H}), 6.94(\mathrm{~d}, J=9.0 \mathrm{~Hz}, 2 \mathrm{H}), 6.38(\mathrm{~d}, J=2.4 \mathrm{~Hz}, 1 \mathrm{H}), 6.20(\mathrm{~d}, J=1.8 \mathrm{~Hz}, 1 \mathrm{H}), 4.76$ (dd, $J=10.8,9.6 \mathrm{~Hz}, 1 \mathrm{H}), 4.31$ (ddd, $J=3.0,3.0,3.0 \mathrm{~Hz}, 1 \mathrm{H}$ ), 4.08 (ddd, $J=3.6,3.0,1.2 \mathrm{~Hz}$, 1H), $3.72(\mathrm{dd}, J=9.6,3.0 \mathrm{~Hz}, 1 \mathrm{H}), 2.07$ (s, 3H), $1.70(\mathrm{ddqd}, J=13.2,10.8,7.2,3.6 \mathrm{~Hz}, 1 \mathrm{H})$, 1.64 (dddd, $J=15.0,4.2,3.0,1.8 \mathrm{~Hz}, 1 \mathrm{H}$ ), 1.50 (ddd, $J=15.0,12.6,2.4 \mathrm{~Hz}, 1 \mathrm{H}), 0.69$ (d, $J=$ $6.6 \mathrm{~Hz}, 3 \mathrm{H}) ;{ }^{13} \mathrm{C}$ NMR $\left(\mathrm{CD}_{3} \mathrm{OD}, 150 \mathrm{MHz}\right) \delta 180.1,173.5,166.0,163.4,161.8,159.4,158.7$, $137.7,132.2,123.0,116.6,106.2,99.9,94.9,82.1,79.0,72.9,72.3,33.3,32.1,21.2,18.0$.

(1R,2S,3S,4S,6S)-4-(5,7-bis(benzyloxy)-2-(4-(benzyloxy)phenyl)-4-oxo-4H-chromen-3yloxy)-2-hydroxy-6-methylcyclohexane-1,3-diyl diacetate ((ent)-III-37) 
<smiles>CC(=O)OC1C(O)[C@H](OC(C)=O)C(C)C[C@H]1Oc1c(-c2ccc(OCc3ccccc3)cc2)oc2cc(OCc3ccccc3)cc(OCc3ccccc3)c2c1=O</smiles>

(1R,2R,3R,4S,6S)-4-(5,7-bis(benzyloxy)-2-(4-(benzyloxy)phenyl)-4-oxo-4H-chromen-3yloxy)-3-hydroxy-6-methylcyclohexane-1,2-diyl diacetate ((ent)-III-38)<smiles>CC(=O)OC1[C@H](O)[C@@H](Oc2c(-c3ccc(OCc4ccccc4)cc3)oc3cc(OCc4ccccc4)cc(OCc4ccccc4)c3c2=O)C[C@H](C)[C@H]1OC(C)=O</smiles>

To a solution of $59 \mathrm{mg}(0.080 \mathrm{mmol})$ diol (ent)-III-36 in $1 \mathrm{~mL} \mathrm{CH}_{2} \mathrm{Cl}_{2}$ at $0{ }^{\circ} \mathrm{C}$ was added 0.6 mg $(3.2 \mu \mathrm{mol}) p$ - $\mathrm{TsOH} \cdot \mathrm{H}_{2} \mathrm{O}, 35 \mu \mathrm{L}(0.27 \mathrm{mmol})$ trimethylorthoacetate. After stirring at $0{ }^{\circ} \mathrm{C}$ for $20 \mathrm{~min}, 0.5 \mathrm{~mL} \mathrm{90 \%} \mathrm{HOAc} \mathrm{(aq.)} \mathrm{was} \mathrm{added} \mathrm{at} 0{ }^{\circ} \mathrm{C}$, and the reaction mixture was stirred for another $20 \mathrm{~min}$ at $0{ }^{\circ} \mathrm{C}$. The reaction mixture was diluted with $20 \mathrm{~mL}$ EtOAc and washed at $0{ }^{\circ} \mathrm{C}$ with $15 \mathrm{~mL}$ saturated aqueous $\mathrm{NaHCO}_{3}$. It was then washed with $15 \mathrm{~mL}$ saturated brine, and dried over $\mathrm{Na}_{2} \mathrm{SO}_{4}$. The organic layer was concentrated under reduced pressure to give crude product, which was then subjected to chromatography on silica gel. Elution with hexane-EtOAc (1:1) gave diacetate (ent)-III-37 (28 mg, 45\%) and elution with hexane-EtOAc (1:1.25) gave (ent)-III-38 (27.5 mg, 44\%):

(ent)-III-37: White solid, mp: $99-101{ }^{\circ} \mathrm{C} ; R_{f}=0.27\left(1: 1.5(\mathrm{v} / \mathrm{v})\right.$ hexane/EtOAc); $[\alpha]_{\mathrm{D}}{ }^{20}-28(c$ 1.46, $\mathrm{CHCl}_{3}$ ); IR (thin film, $\mathrm{cm}^{-1}$ ) v 3474, 3066, 2930, 1732, 1626, 1603, 1574, 1509, 1453, 1433, 1370, 1298, 1225, 1172, 1100, 1039, 963; ${ }^{1} \mathrm{H} \mathrm{NMR}\left(\mathrm{CDCl}_{3}, 600 \mathrm{MHz}\right) \delta 7.85$ (d, $J=9.0 \mathrm{~Hz}$, 
2H), $7.56(\mathrm{~d}, J=7.2 \mathrm{~Hz}, 2 \mathrm{H}), 7.47-7.27(\mathrm{~m}, 13 \mathrm{H}), 7.11(\mathrm{~d}, J=9.0 \mathrm{~Hz}, 2 \mathrm{H}), 6.56(\mathrm{~d}, J=2.4 \mathrm{~Hz}$, $1 \mathrm{H}), 6.44(\mathrm{~d}, J=2.4 \mathrm{~Hz}, 1 \mathrm{H}), 5.28(\mathrm{~s}, 2 \mathrm{H}), 5.15(\mathrm{~s}, 2 \mathrm{H}), 5.07(\mathrm{~s}, 2 \mathrm{H}), 5.06$ (ddd, $J=3.0,3.0,1.8$ $\mathrm{Hz}, 1 \mathrm{H}), 4.70$ (dd, $J=10.8,10.2 \mathrm{~Hz}, 1 \mathrm{H}), 4.39$ (ddd, $J=3.0,3.0,3.0 \mathrm{~Hz}, 1 \mathrm{H}), 3.75(\mathrm{~m}, 1 \mathrm{H})$, 2.18 (ddd, $J=15.0,3.6,2.4,1.2 \mathrm{~Hz}, 1 \mathrm{H}), 2.10$ (s, 3H), 2.01 (s, 3H), 1.97 (ddqd, $J=13.2,10.8$, 6.6, $4.2 \mathrm{~Hz}, 1 \mathrm{H}$ ), 1.85 (d, $J=5.4 \mathrm{~Hz}, 1 \mathrm{H}$ ), 1.49 (ddd, $J=15.0,12.6,3.0 \mathrm{~Hz}, 1 \mathrm{H}$ ), 0.88 (d, $J=6.0$ $\mathrm{Hz}, 3 \mathrm{H}) ;{ }^{13} \mathrm{C} \mathrm{NMR}\left(\mathrm{CDCl}_{3}, 150 \mathrm{MHz}\right) \delta 173.5,171.9,170.1,162.8,160.6,159.8,158.8,154.1$, 139.1, 136.4, 135.6, 130.5, 128.7, 128.7, 128.6, 128.4, 128.2, 127.7, 127.5, 127.4, 126.6, 123.3, $114.8,110.1,98.4,94.0,77.7,73.1,70.8,70.5,70.3,70.2,33.5,30.3,21.1,21.0,17.4$.

(ent)-III-38: White solid, mp: $98-100{ }^{\circ} \mathrm{C} ; R_{f}=0.38(1: 1.5(\mathrm{v} / \mathrm{v})$ hexane/EtOAc $) ;[\alpha]_{\mathrm{D}}{ }^{20}+66(c$ 1.95, $\mathrm{CHCl}_{3}$ ); IR (thin film, $\mathrm{cm}^{-1}$ ) v 3436, 3033, 2932, 1735, 1603, 1573, 1508, 1453, 1432, 1366 , 1298, 1248, 1223, 1172, 1100, 1039, 962; ${ }^{1} \mathrm{H} \mathrm{NMR}\left(\mathrm{CDCl}_{3}, 600 \mathrm{MHz}\right) \delta 7.90(\mathrm{~d}, J=8.4 \mathrm{~Hz}$, 2H), $7.56(\mathrm{~d}, J=7.2 \mathrm{~Hz}, 2 \mathrm{H}), 7.47-7.27(\mathrm{~m}, 13 \mathrm{H}), 7.11(\mathrm{~d}, J=9.0 \mathrm{~Hz}, 2 \mathrm{H}), 6.56(\mathrm{~d}, J=2.4 \mathrm{~Hz}$, $1 \mathrm{H}), 6.45(\mathrm{~d}, J=2.4 \mathrm{~Hz}, 1 \mathrm{H}), 5.26(\mathrm{~s}, 2 \mathrm{H}), 5.22(\mathrm{dd}, J=9.0,3.6 \mathrm{~Hz}, 1 \mathrm{H}), 5.16(\mathrm{~d}, J=11.4 \mathrm{~Hz}$, 1H), 5.15 (d, $J=12.0 \mathrm{~Hz}, 1 \mathrm{H}), 5.08$ (s, 2H), 4.93 (dd, $J=9.6,9.0 \mathrm{~Hz}, 1 \mathrm{H}), 4.42$ (ddd, $J=4.2$, 3.6, $3.6 \mathrm{~Hz}, 1 \mathrm{H}), 4.32$ (dd, $J=4.2,4.2 \mathrm{~Hz}, 1 \mathrm{H}), 2.98$ (brs, 1H), 2.02 (s, 3H), 2.02 (s, 3H), 1.80 (ddqd, $J=13.8,10.8,6.6,4.2 \mathrm{~Hz}, 1 \mathrm{H}$ ), 1.73 (ddd, $J=14.4,4.2,3.6 \mathrm{~Hz}, 1 \mathrm{H}$ ), 1.52 (ddd, $J=14.4$, 11.4, $3.0 \mathrm{~Hz}, 1 \mathrm{H}), 0.73(\mathrm{~d}, J=6.6 \mathrm{~Hz}, 3 \mathrm{H}) ;{ }^{13} \mathrm{C} \mathrm{NMR}\left(\mathrm{CDCl}_{3}, 150 \mathrm{MHz}\right) \delta 173.7,170.4,169.5$, $162.8,160.4,159.8,158.8,154.1,139.2,136.5,136.3,135.6,130.5,128.7,128.6,128.6,128.4$, $128.1,127.7,127.5,127.4,126.7,123.3,114.7,110.0,98.3,93.9,79.7,74.5,73.4,70.8,70.8$, $70.5,70.1,32.3,31.0,20.9,20.9,17.2$.

(1R,2S,3S,4S,6S)-4-(5,7-dihydroxy-2-(4-hydroxyphenyl)-4-0x0-4H-chromen-3-yloxy)-2hydroxy-6-methylcyclohexane-1,3-diyl diacetate ((ent)-III-2) 


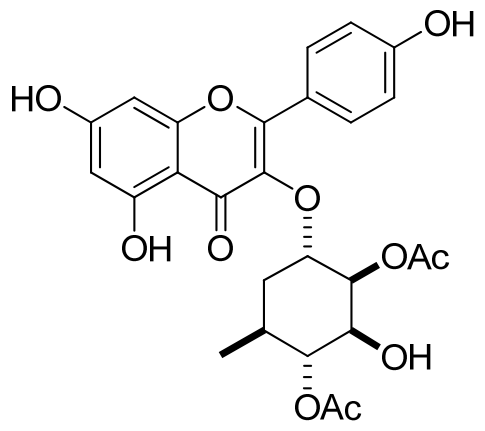

To a solution of $26 \mathrm{mg}(0.033 \mathrm{mmol})$ diol (ent)-III-37 in $1.5 \mathrm{~mL}$ THF-EtOH (1:1) was added 26 $\mathrm{mg} \mathrm{Pd} / \mathrm{C}(10 \%)$. The reaction mixture was degassed at -50 to $-40{ }^{\circ} \mathrm{C}$ using vacuum and refilled with $\mathrm{H}_{2}$. This procedure was repeated three times, then the bath was removed and the reaction was warmed up to room temperature. The reaction mixture was stirred under a $\mathrm{H}_{2}$ atmosphere for 5 hours. The reaction mixture was loaded onto silica gel and elution with hexane-EtOAc (1:1.5) to give product (ent)-III-2 (11 mg, 65\%): Yellow solid, mp: $167-169{ }^{\circ} \mathrm{C} ; R_{f}=0.11(1: 1.5(\mathrm{v} / \mathrm{v})$ hexane/EtOAc); $[\alpha]_{\mathrm{D}}^{20}-5$ (c 0.76, MeOH); IR (thin film, $\mathrm{cm}^{-1}$ ) v 3200, 2926, 1717, 1650, 1607, 1503, 1441, 1361, 1248, 1208, 1171, 1109, 1083, 1038, 1008, 972; ${ }^{1} \mathrm{H}$ NMR ( $\left.\mathrm{CD}_{3} \mathrm{OD}, 600 \mathrm{MHz}\right)$ $\delta 7.78(\mathrm{~d}, J=8.4 \mathrm{~Hz}, 2 \mathrm{H}), 6.94(\mathrm{~d}, J=9.0 \mathrm{~Hz}, 2 \mathrm{H}), 6.38(\mathrm{~d}, J=1.8 \mathrm{~Hz}, 1 \mathrm{H}), 6.21(\mathrm{~d}, J=1.8 \mathrm{~Hz}$, 1H), 5.22 (ddd, $J=3.6,3.6,1.2 \mathrm{~Hz}, 1 \mathrm{H}$ ), 4.73 (dd, $J=10.2,10.2 \mathrm{~Hz}, 1 \mathrm{H}$ ), 4.28 (ddd, $J=3.0,3.0$, $3.0 \mathrm{~Hz}, 1 \mathrm{H}), 3.91(\mathrm{dd}, J=10.2,3.6 \mathrm{~Hz}, 1 \mathrm{H}), 2.08(\mathrm{~s}, 3 \mathrm{H}), 2.02$ (s, $3 \mathrm{H}), 1.83(\mathrm{ddqd}, J=13.2,10.2$, 6.6, $3.0 \mathrm{~Hz}, 1 \mathrm{H}$ ), 1.82 (ddd, $J=15.6,4.2,3.0,1.8 \mathrm{~Hz}, 1 \mathrm{H}$ ), 1.44 (ddd, $J=15.6,13.8,3.0 \mathrm{~Hz}, 1 \mathrm{H}$ ), $0.78(\mathrm{~d}, J=6.6 \mathrm{~Hz}, 3 \mathrm{H}) ;{ }^{13} \mathrm{C} \mathrm{NMR}\left(\mathrm{CD}_{3} \mathrm{OD}, 150 \mathrm{MHz}\right) \delta 179.9,173.2,172.0,166.0,163.4$, 161.9, 159.4, 158.7, 137.4, 132.1, 122.8, 116.7, 106.1, 100.0, 94.9, 78.8, 78.8, 74.5, 70.4, 34.1, $32.0,21.2,21.0,18.0$.

(1R,2R,3R,4S,6S)-4-(5,7-dihydroxy-2-(4-hydroxyphenyl)-4-oxo-4H-chromen-3-yloxy)-3hydroxy-6-methylcyclohexane-1,2-diyl diacetate ((ent)-III-3) 


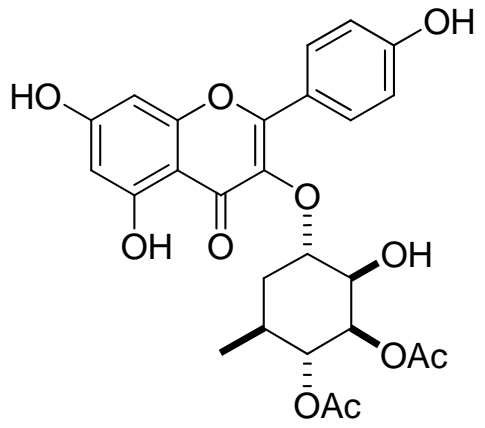

To a solution of $26 \mathrm{mg}(0.033 \mathrm{mmol})$ diol (ent)-III-38 in $3 \mathrm{~mL}$ THF-EtOH (1:1) was added 23 $\mathrm{mg} \mathrm{Pd} / \mathrm{C}(10 \%)$. The reaction mixture was degassed at -50 to $-40{ }^{\circ} \mathrm{C}$ using vacuum and refilled with $\mathrm{H}_{2}$. This procedure was repeated three times, then the bath was removed and the reaction was warmed up to room temperature. The reaction mixture was stirred under a $\mathrm{H}_{2}$ atmosphere for 7 hours. The reaction mixture was loaded onto silica gel and elution with hexane-EtOAc (1:1) to give product (ent)-III-3 (12 mg, 70\%): Yellow solid, mp: 147-149 ${ }^{\circ} \mathrm{C} ; R_{f}=0.47(1: 3(\mathrm{v} / \mathrm{v})$ hexane/EtOAc); $[\alpha]_{\mathrm{D}}{ }^{20}+114(c 0.74, \mathrm{MeOH})$; IR (thin film, $\left.\mathrm{cm}^{-1}\right) v 3335,2931,1716,1653$, $1608,1499,1440,1363,1207,1170,1085,1041,972 ;{ }^{1} \mathrm{H}$ NMR $\left(\mathrm{CD}_{3} \mathrm{OD}, 600 \mathrm{MHz}\right) \delta 7.79$ (d, $J$ $=9.0 \mathrm{~Hz}, 2 \mathrm{H}), 6.94(\mathrm{~d}, J=9.0 \mathrm{~Hz}, 2 \mathrm{H}), 6.38(\mathrm{~d}, J=1.8 \mathrm{~Hz}, 1 \mathrm{H}), 6.20(\mathrm{~d}, J=1.8 \mathrm{~Hz}, 1 \mathrm{H}), 5.06$ (dd, $J=9.6,3.0 \mathrm{~Hz}, 1 \mathrm{H}), 4.94$ (dd, $J=10.8,10.2 \mathrm{~Hz}, 1 \mathrm{H}), 4.47$ (ddd, $J=3.0,3.0,2.4 \mathrm{~Hz}, 1 \mathrm{H}$ ), 4.26 (ddd, $J=3.6,3.6,1.2 \mathrm{~Hz}, 1 \mathrm{H}), 2.02$ (s, 3H), 1.99 (s, 3H), 1.66 (ddqd, $J=12.6,10.2,6.0,3.6$ $\mathrm{Hz}, 1 \mathrm{H}), 1.62$ (ddd, $J=15.0,3.6,2.4,1.2 \mathrm{~Hz}, 1 \mathrm{H}), 1.50$ (ddd, $J=15.0,12.6,2.4 \mathrm{~Hz}, 1 \mathrm{H}), 0.67$ (d, $J=6.6 \mathrm{~Hz}, 3 \mathrm{H}) ;{ }^{13} \mathrm{C} \mathrm{NMR}\left(\mathrm{CD}_{3} \mathrm{OD}, 150 \mathrm{MHz}\right) \delta 180.1,172.7,172.2,165.9,163.4,161.7,159.4$, 158.7, 137.2, 132.2, 122.9, 116.6, 106.2, 99.9, 94.9, 81.3, 75.9, 75.1, 70.8, 32.7, 32.1, 21.0, 21.0, 17.9.

\section{V.5. Chapter IV Experimental}


<smiles>CC1O[C@H](OCc2ccccc2)C=CC1OC(=O)OC(C)(C)C</smiles>

A solution of $519 \mathrm{mg}(2.38 \mathrm{mmol})$ allylic alcohol IV-24 and $14.5 \mathrm{mg}(0.119 \mathrm{mmol})$ DMAP in 8 $\mathrm{mL} \mathrm{CH}_{2} \mathrm{Cl}_{2}$ at $0{ }^{\circ} \mathrm{C}$ was added a solution of $778 \mathrm{mg}(3.57 \mathrm{mmol}) \mathrm{Boc}_{2} \mathrm{O}$ in $2.5 \mathrm{~mL} \mathrm{CH}_{2} \mathrm{Cl}_{2}$. Then the reaction mixture was stirred at RT for $1 \mathrm{~h}$. It was diluted with $20 \mathrm{~mL}$ EtOAc and quenched with saturated aqueous $\mathrm{NaHCO}_{3}$ at $0{ }^{\circ} \mathrm{C}$. The mixture was stirred at $0{ }^{\circ} \mathrm{C}$ for $20 \mathrm{~min}$, then was extracted with $100 \mathrm{~mL} \mathrm{Et}_{2} \mathrm{O}$ and the aqueous layer was extracted again with $50 \mathrm{~mL} \mathrm{Et} \mathrm{O}_{2} \mathrm{O}$. The pooled organic layer was subsequently washed with saturated aqueous $\mathrm{NH}_{4} \mathrm{Cl}, \mathrm{NaHCO}_{3}$, brine and dried over $\mathrm{Na}_{2} \mathrm{SO}_{4}$. After removal of the solvent under reduced pressure, the residue was subjected to silica gel column chromatography, elution with hexane-EtOAc $(20: 1, \mathrm{v} / \mathrm{v})$ afforded the carbonate IV-25 $663 \mathrm{mg}(87 \%)$ as white solid: mp 60-62 ${ }^{\circ} \mathrm{C} ; R_{f}$ (hexanes/EtOAc, 3:1 v/v) = 0.60; $[\alpha]^{20}{ }_{\mathrm{D}}+89\left(c\right.$ 2.99, $\mathrm{CHCl}_{3}$ ); IR (thin film, $\mathrm{cm}^{-1}$ ) v 2982, 2935, 1732, 1455, 1370, 1336, 1317, 1286, 1269, 1254, 1154, 1094, 1049, 1028, 969; ${ }^{1} \mathrm{H}$ NMR $\left(\mathrm{CDCl}_{3}, 600 \mathrm{MHz}\right): \delta 7.38-7.32$ (m, 4H), $7.29(\mathrm{~m}, 1 \mathrm{H}), 5.93(\mathrm{~d}, J=10.2 \mathrm{~Hz}, 1 \mathrm{H}), 5.82$ (ddd, $J=10.8,2.4,1.8 \mathrm{~Hz}, 1 \mathrm{H}), 5.06$ (brs, $1 \mathrm{H}), 4.86$ (ddd, $J=9.0,3.0,1.8 \mathrm{~Hz}, 1 \mathrm{H}), 4.77$ (d, $J=12.0 \mathrm{~Hz}, 1 \mathrm{H}), 4.60$ (d, $J=12.0 \mathrm{~Hz}$,

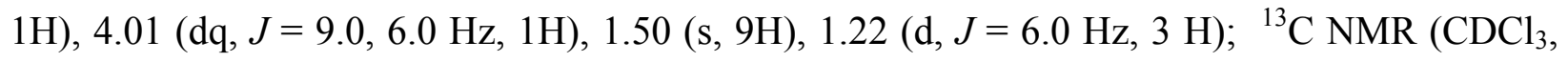
$150 \mathrm{MHz}): \delta$ 153.1, 138.1, 129.7, 128.4, 127.9, 127.8, 127.6, 93.7, 82.6, 73.5, 70.1, 64.9, 27.7, 17.8; HRESIMS: Calcd. for $\left[\mathrm{C}_{18} \mathrm{H}_{24} \mathrm{O}_{5}+\mathrm{Na}\right]^{+}: 343.1516$, Found: 343.1517.

Benzyl (2R,3S,6S)-6-(benzyloxy)-2-methyl-3,6-dihydro-2H-pyran-3-yl carbonate (IV-26) 


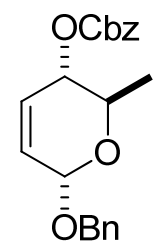

A solution of $224 \mathrm{mg}(1.03 \mathrm{mmol})$ allylic alcohol IV-24 in $4 \mathrm{~mL} \mathrm{CH}_{2} \mathrm{Cl}_{2}$ at RT was added 220

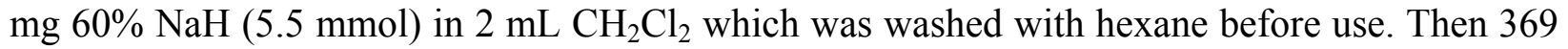
$\mathrm{mg} n-\mathrm{Bu}_{4} \mathrm{NI}(1 \mathrm{mmol})$ was added to the mixture followed by the addition of $61 \mathrm{mg}$ DMAP $(0.5$ mmol). The reaction mixture was stirred at $\mathrm{RT}$ for $2 \mathrm{~h}$. It was then diluted with $\mathrm{Et}_{2} \mathrm{O}$ and washed with saturated aqueous $\mathrm{NH}_{4} \mathrm{Cl}$ and the aqueous layer was extracted with $\mathrm{Et}_{2} \mathrm{O}$ twice and the pooled organic layer was then washed with saturated aqueous $\mathrm{NaHCO}_{3}$, brine and dried over $\mathrm{Na}_{2} \mathrm{SO}_{4}$. After removal of the solvent under reduced pressure, the residue was subjected silica gel column chromatography, elution with hexane-EtOAc (15:1, v/v) afforded the carbonate IV-26 $270 \mathrm{mg}(74 \%)$ as colorless viscous oil: $R_{f}($ hexanes/EtOAc, $3: 1 \mathrm{v} / \mathrm{v})=0.44 ;[\alpha]^{20}{ }_{\mathrm{D}}+96(c 4.48$, $\mathrm{CHCl}_{3}$ ); IR (thin film, $\mathrm{cm}^{-1}$ ) v 3032, 2979, 2936, 1747, 1497, 1456, 1382, 1250, 1049, 1020, 962; ${ }^{1} \mathrm{H} \mathrm{NMR}\left(\mathrm{CDCl}_{3}, 600 \mathrm{MHz}\right): \delta 7.41-7.34(\mathrm{~m}, 9 \mathrm{H}), 7.31(\mathrm{~m}, 1 \mathrm{H}), 5.96(\mathrm{~d}, J=10.2 \mathrm{~Hz}, 1 \mathrm{H}), 5.85$ (ddd, $J=9.6,12.4,1.8 \mathrm{~Hz}, 1 \mathrm{H}), 5.21(\mathrm{~d}, J=12.6 \mathrm{~Hz}, 1 \mathrm{H}), 5.18$ (d, $J=12.0 \mathrm{~Hz}, 1 \mathrm{H}), 5.08$ (brs, 1H), 4.94 (ddd, $J=9.0,3.0,1.8 \mathrm{~Hz}, 1 \mathrm{H}), 4.79$ (d, $J=12.0 \mathrm{~Hz}, 1 \mathrm{H}), 4.62$ (d, $J=12.0 \mathrm{~Hz}, 1 \mathrm{H})$, $4.05(\mathrm{dq}, J=9.6,6.6 \mathrm{~Hz}, 1 \mathrm{H}), 1.24(\mathrm{~d}, J=6.0 \mathrm{~Hz}, 3 \mathrm{H}) ;{ }^{13} \mathrm{C} \mathrm{NMR}\left(\mathrm{CDCl}_{3}, 150 \mathrm{MHz}\right): \delta 154.7$, 137.9, 135.0, 129.1, 128.6, 128.5, 128.4, 128.2, 128.1, 127.9, 127.6, 93.6, 74.7, 70.1, 69.8, 64.7, 17.8; HRESIMS: Calculated for $\left[\mathrm{C}_{21} \mathrm{H}_{22} \mathrm{O}_{5}+\mathrm{Na}\right]^{+}: 377.1359$, Found: 377.1362 .

(3aR,4R,6S,7S,7aR)-6-(benzyloxy)-7-iodo-4-methyltetrahydro-3aH-[1,3]dioxolo[4,5clpyran-2-one (IV-27)

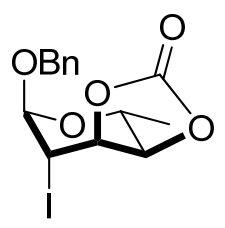

A solution of $285 \mathrm{mg}(0.90 \mathrm{mmol})$ allyl carbonate IV-25 in $3 \mathrm{~mL}$ HOAc was added $320 \mathrm{mg}$ NIS 
(1.42 mmol) at RT. It was stirred at RT $12 \mathrm{~h}$, then another $230 \mathrm{mg}(1.0 \mathrm{mmol})$ NIS was added and continued stirring at RT for another $12 \mathrm{~h}$. The reaction mixture was diluted with EtOAc and cooled down to $0{ }^{\circ} \mathrm{C}$ and quenched by addition of saturated aqueous $\mathrm{NaHCO}_{3}$. The mixture was extracted with EtOAc and the organic layer was washed subsequently with saturated aqueous $\mathrm{Na}_{2} \mathrm{~S}_{2} \mathrm{O}_{3}$, brine and dried over $\mathrm{Na}_{2} \mathrm{SO}_{4}$. After removal of the solvent under reduced pressure, the residue was subjected to silica gel column chromatography, elution with hexane-EtOAc $(5: 1, \mathrm{v} / \mathrm{v})$ afforded the iodo-carbonate IV-27 $335 \mathrm{mg}(95 \%)$ as white solid: $\mathrm{mp}$ 80.5-82.5 ${ }^{\circ} \mathrm{C}$; $R_{f}$ (hexanes/EtOAc, 3:1 v/v) $=0.38 ;[\alpha]^{20}{ }_{\mathrm{D}}+52\left(c\right.$ 2.65, $\left.\mathrm{CHCl}_{3}\right)$; IR (thin film, $\left.\mathrm{cm}^{-1}\right) v 3030,2980$, 2935, 1839, 1806, 1497, 1454, 1347, 1331, 1147, 1087, 1053, 1021, 970; ${ }^{1} \mathrm{H}$ NMR $\left(\mathrm{CDCl}_{3}, 600\right.$ MHz): $\delta 7.39-7.34(\mathrm{~m}, 4 \mathrm{H}), 7.32(\mathrm{~m}, 1 \mathrm{H}), 5.16(\mathrm{~d}, J=5.4 \mathrm{~Hz}, 1 \mathrm{H}), 4.96(\mathrm{dd}, J=8.4,7.8 \mathrm{~Hz}, 1$ H), $4.76(\mathrm{~d}, J=12.0 \mathrm{~Hz}, 1 \mathrm{H}), 4.59$ (d, $J=12.0 \mathrm{~Hz}, 1 \mathrm{H}), 4.40(\mathrm{dd}, J=9.0,7.8 \mathrm{~Hz}, 1 \mathrm{H}), 4.21(\mathrm{dd}$, $J=8.4,6.0 \mathrm{~Hz}, 1 \mathrm{H}), 4.05(\mathrm{dq}, J=9.0,6.6 \mathrm{~Hz}, 1 \mathrm{H}), 1.35(\mathrm{~d}, J=6.0 \mathrm{~Hz}, 3 \mathrm{H}) ;{ }^{13} \mathrm{C} \mathrm{NMR}\left(\mathrm{CDCl}_{3}\right.$, $150 \mathrm{MHz}): \delta \quad 152.9,136.6,128.5,128.1,127.9,101.0,78.7,77.1,70.2,64.0,21.0,18.6$; HRESIMS: Calculated for $\left[\mathrm{C}_{14} \mathrm{H}_{15} \mathrm{IO}_{5}+\mathrm{Na}\right]^{+}: 412.9856$, Found: 412.9859.

$\mathrm{MgBr}_{2} \bullet \mathrm{Et}_{2} \mathrm{O}$ catalyzed iodo-carbonation of Boc-carbonate IV-25

A solution of $31.8 \mathrm{mg}(0.1 \mathrm{mmol})$ allyl carbonate $\mathbf{I V - 2 5}$ and $33.8 \mathrm{mg}(0.15 \mathrm{mmol})$ NIS in $0.8 \mathrm{~mL}$ $\mathrm{CH}_{2} \mathrm{Cl}_{2}$ was added $25.8 \mathrm{mg}(0.1 \mathrm{mmol}) \mathrm{MgBr}_{2} \cdot \mathrm{Et}_{2} \mathrm{O}$ at $\mathrm{RT}$. It was stirred at $\mathrm{RT} 12 \mathrm{~h}$, then another $22.5 \mathrm{mg}(0.1 \mathrm{mmol})$ NIS was added and continued stirring at RT for another $24 \mathrm{~h}$. The reaction mixture was diluted with EtOAc and cooled down to $0{ }^{\circ} \mathrm{C}$ and quenched by addition of saturated aqueous $\mathrm{NaHCO}_{3}$. The mixture was extracted with EtOAc and the organic layer was washed subsequently with saturated aqueous $\mathrm{Na}_{2} \mathrm{~S}_{2} \mathrm{O}_{3}$, brine and dried over $\mathrm{Na}_{2} \mathrm{SO}_{4}$. After removal of the solvent under reduced pressure, a crude ${ }^{1} \mathrm{H}$ NMR experiment showed that the ratio of product and remaining starting material is $2: 1$. Then the residue was subjected to silica gel column chromatography, elution with hexane-EtOAc $(7: 1, \mathrm{v} / \mathrm{v})$ afforded $10.1 \mathrm{mg}$ iodocarbonate IV-27 (26\%). 
Iodo-carbonation of Cbz-carbonate IV-26

A solution of $35.4 \mathrm{mg}(0.10 \mathrm{mmol})$ allyl carbonate $\mathbf{I V - 2 6}$ in $3 \mathrm{~mL}$ HOAc was added $33.8 \mathrm{mg}$ NIS $(0.15 \mathrm{mmol})$ at RT. It was stirred at RT $12 \mathrm{~h}$, then another $22.5 \mathrm{mg}(0.1 \mathrm{mmol})$ NIS was added and continued stirring at RT for another $24 \mathrm{~h}$. Once again $22.5 \mathrm{mg}(0.1 \mathrm{mmol})$ NIS was added and continued stirring at RT for another $24 \mathrm{~h}$. The reaction mixture was diluted with EtOAc and cooled down to $0{ }^{\circ} \mathrm{C}$ and quenched by addition of saturated aqueous $\mathrm{NaHCO}_{3}$. The mixture was extracted with EtOAc and the organic layer was washed subsequently with saturated aqueous $\mathrm{Na}_{2} \mathrm{~S}_{2} \mathrm{O}_{3}$, brine and dried over $\mathrm{Na}_{2} \mathrm{SO}_{4}$. After removal of the solvent under reduced pressure, the residue was subjected to silica gel column chromatography. Elution with hexaneEtOAc (7:1, v/v) afforded the iodo-carbonate IV-27 $20 \mathrm{mg}$ (51\%)

(3aR,4R,6S,7aS)-6-(benzyloxy)-4-methyltetrahydro-3aH-[1,3]dioxolo[4,5-c]pyran-2-one (IV-28)

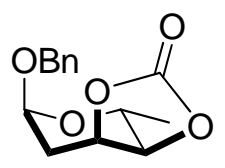

A solution of $59 \mathrm{mg}(0.15 \mathrm{mmol})$ iodo-carbonate $\mathbf{I V - 2 7}$ in $2 \mathrm{~mL}$ toluene was added $70 \mu \mathrm{L}(0.22$ mmol) TMSS and then quickly $5 \mathrm{mg}(0.03 \mathrm{mmol})$ solid AIBN was added in one portion at $0{ }^{\circ} \mathrm{C}$. The system was then cooled down to $\sim-50{ }^{\circ} \mathrm{C}$ and degassed with vacuum and refilled with Ar. This procedure was repeated three times and then the mixture was heated up to $75^{\circ} \mathrm{C}$ for $40 \mathrm{~min}$. The reaction mixture was cooled down to RT, and then directly loaded onto silica gel column. Elution with hexane-EtOAc (3:1, v/v) afforded carbonate IV-28 $17.8 \mathrm{mg}$ (45\%) as colorless viscous oil; $R_{f}($ hexanes/EtOAc, $2: 1 \mathrm{v} / \mathrm{v})=0.29 ;[\alpha]_{\mathrm{D}}^{20}+129\left(c 0.93, \mathrm{CHCl}_{3}\right)$; IR (thin film, $\left.\mathrm{cm}^{-1}\right)$ v 3035, 2975, 2940, 1798, 1454, 1356, 1175, 1152, 1066, 1036, 1023, 919; ${ }^{1} \mathrm{H} \mathrm{NMR}\left(\mathrm{CDCl}_{3}\right.$, $600 \mathrm{MHz}): \delta 7.36-7.27(\mathrm{~m}, 5 \mathrm{H}), 4.92(\mathrm{dd}, J=5.4,4.8 \mathrm{~Hz}, 1 \mathrm{H}), 4.78(\mathrm{ddd}, J=7.2,7.2,6.0 \mathrm{~Hz}, 1$ H), $4.71(\mathrm{~d}, J=12.6 \mathrm{~Hz}, 1 \mathrm{H}), 4.52(\mathrm{~d}, J=12.0 \mathrm{~Hz}, 1 \mathrm{H}), 4.26(\mathrm{dd}, J=9.0,7.8 \mathrm{~Hz}, 1 \mathrm{H}), 4.01$ (dq, $J=9.0,6.0 \mathrm{~Hz}, 1 \mathrm{H}), 2.31(\mathrm{ddd}, J=15.0,5.4,5.4 \mathrm{~Hz}, 1 \mathrm{H}), 2.19(\mathrm{ddd}, J=15.0,7.2,4.8 \mathrm{~Hz}, 1 \mathrm{H})$, $1.31(\mathrm{~d}, J=6.6 \mathrm{~Hz}, 3 \mathrm{H}) ;{ }^{13} \mathrm{C} \mathrm{NMR}\left(\mathrm{CDCl}_{3}, 150 \mathrm{MHz}\right): \delta 154.3,137.2,128.5,127.8,127.7,94.2$, 
77.0, 72.5, 69.3, 63.3, 30.7, 18.5; HRESIMS: Calculated for $\left[\mathrm{C}_{14} \mathrm{H}_{16} \mathrm{O}_{5}+\mathrm{Na}\right]^{+}: 287.0890$, Found: 287.0890 .

\section{(2R,3S,4S,6S)-6-(benzyloxy)-2-methyltetrahydro-2H-pyran-3,4-diol (IV-29) ${ }^{65}$}

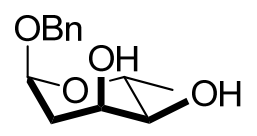

A solution of $16 \mathrm{mg}(0.06 \mathrm{mmol})$ carbonate IV-28 in $2 \mathrm{~mL}$ THF was added $1 \mathrm{~mL} 2 \mathrm{M} \mathrm{NaOH}$. The mixture was stirred at RT for $1 \mathrm{~h}$. It was then diluted with EtOAc and passed through a pad of celite-silica gel-sand, eluting with EtOAc-MeOH $(3: 1, \mathrm{v} / \mathrm{v})$. The effluent was dried over $\mathrm{Na}_{2} \mathrm{SO}_{4}$. After removal of the solvent under reduced pressure, the residue was subjected to silica gel column chromatography, elution with hexane-EtOAc (1.5:1, v/v) afforded the $\alpha$-digitoxoside IV-29 $12 \mathrm{mg}(84 \%)$ as colorless oil; $R_{f}($ hexanes/EtOAc, $1: 1 \mathrm{v} / \mathrm{v})=0.25 ;[\alpha]^{20}{ }_{\mathrm{D}}+107(c 1.2$, $\mathrm{CHCl}_{3}$ ); IR (thin film, $\mathrm{cm}^{-1}$ ) v 3461 (broad), 3090, 3065, 3033, 2975, 2918, 1497, 1454, 1407, 1235, 1148, 1120, 1101, 1054, 1010, 970; ${ }^{1} \mathrm{H}$ NMR $\left(\mathrm{CDCl}_{3}, 600 \mathrm{MHz}\right): \delta 7.39-7.35$ (m, 2H), 7.34-7.30 (m, 3H), 4.97 (d, $J=3.6 \mathrm{~Hz}, 1 \mathrm{H}), 4.72(\mathrm{~d}, J=12.0 \mathrm{~Hz}, 1 \mathrm{H}), 4.50(\mathrm{~d}, J=11.4 \mathrm{~Hz}, 1 \mathrm{H})$, 3.96 (brs, $1 \mathrm{H}$ ), 3.77 (dq, $J=9.6,6.0 \mathrm{~Hz}, 1 \mathrm{H}), 3.45$ (brs, OH), 3.16 (dd, $J=10.2,3.6 \mathrm{~Hz}, 1 \mathrm{H}$ ), 2.48 (brs, OH), 2.22 (ddd, $J=15.0,3.0,1.2 \mathrm{~Hz}, 1 \mathrm{H}$ ), 1.94 (ddd, $J=15.0,3.6,3.6 \mathrm{~Hz}, 1 \mathrm{H}), 1.33$ $(\mathrm{d}, J=6.6 \mathrm{~Hz}, 3 \mathrm{H}) ;{ }^{13} \mathrm{C} \mathrm{NMR}\left(\mathrm{CDCl}_{3}, 150 \mathrm{MHz}\right): \delta 136.9,128.6,128.1,127.9,96.4,72.6,69.5$, $67.3,64.7,35.1,17.8$.

\section{Ethyl 3-acetyl-4-hydroxy-5-oxo-2-(2-oxopropyl)-2,5-dihydrofuran-2-carboxylate (IV-36) ${ }^{54}$}

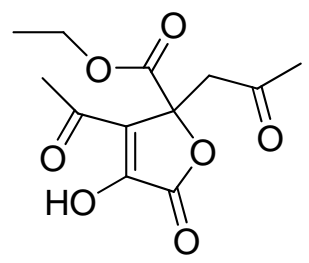

A sample of $49.5 \mathrm{~g}(0.313 \mathrm{~mol})$ in a $500 \mathrm{~mL}$ flask was added a solution of $25.7 \mathrm{~g} \mathrm{NaOAc}(0.312$ 149 
mol) in $54 \mathrm{~mL} \mathrm{HOAc}$ and $71 \mathrm{~mL} \mathrm{H}_{2} \mathrm{O}$ at $0{ }^{\circ} \mathrm{C}$. The bath was removed after complete addition, and the mixture was stirred at RT $24 \mathrm{~h}$. Then it was poured into a mixture of $165 \mathrm{~g}$ ice and $26 \mathrm{~mL}$ concentrated $\mathrm{H}_{2} \mathrm{SO}_{4}$. Gentle shaking of the mixture resulted in formation of precipitate. Then it was cooled down at $0{ }^{\circ} \mathrm{C}$ for $15 \mathrm{~min}$. The mixture was filtered under reduced pressure and the solid was washed with ice cold water $(100 \mathrm{~mL} \mathrm{X} \mathrm{3).} \mathrm{The} \mathrm{crude} \mathrm{product} \mathrm{was} \mathrm{directly} \mathrm{used} \mathrm{for}$ next step except that small amount of solid was dried and used for NMR test. White solid: ${ }^{1} \mathrm{H}$ NMR ( $\left.\mathrm{CDCl}_{3}, 270 \mathrm{MHz}\right): \delta 4.54$ (brs, $\left.1 \mathrm{H}\right), 4.21$ (q, $\left.J=6.9 \mathrm{~Hz}, 2 \mathrm{H}\right), 3.66$ (d, $\left.J=17.8 \mathrm{~Hz}, 1 \mathrm{H}\right)$, $3.29(\mathrm{~d}, J=17.8 \mathrm{~Hz}, 1 \mathrm{H}), 2.50(\mathrm{~s}, 1 \mathrm{H}), 2.15(\mathrm{~s}, 3 \mathrm{H}), 1.24(\mathrm{t}, J=7.2 \mathrm{~Hz}, 3 \mathrm{H}) ;{ }^{13} \mathrm{C} \mathrm{NMR}\left(\mathrm{CDCl}_{3}\right.$, 67.5 MHz): $\delta 203.7,194.0,167.7,166.8,149.5,123.5,82.9,63.1,44.1,31.1,30.0,13.8$.

\section{Methyl 3-hydroxy-5-methylbenzoate $(\mathrm{IV}-37)^{54}$}

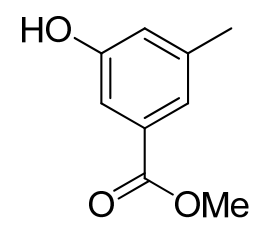

The crude product IV-36 from last reaction was suspended in $500 \mathrm{~mL} \mathrm{H}_{2} \mathrm{O}$ and $45 \mathrm{~g}(0.77 \mathrm{~mol})$ $\mathrm{Mg}(\mathrm{OH})_{2}$ was slowly added at RT when stirring. The mixture was stirred at reflux for $1.5 \mathrm{~h}$. The hot solution was filtered under reduced pressure and the precipitate was rinsed with warm water. The mother liquor was concentrated under reduced pressure to remove the water to afforded crude solid product, which was then suspended in $270 \mathrm{~mL}$ methanol at RT. $13 \mathrm{~mL}$ concentrated $\mathrm{H}_{2} \mathrm{SO}_{4}$ was added into the mixture when stirring. After addition, the mixture was heated up and stirred under reflux for $12 \mathrm{~h}$. Then it was cooled down to $0{ }^{\circ} \mathrm{C}$ and slowly $400 \mathrm{~mL}$ saturated aqueous $\mathrm{NaHCO}_{3}$ was added followed by addition of another $20 \mathrm{~g}$ solid $\mathrm{NaHCO}_{3}$. The mixture was extracted with EtOAc (400 mL X 3). The organic layer was washed with saturated brine and dried over $\mathrm{Na}_{2} \mathrm{SO}_{4}$. After removal of the solvent, the residue was subjected to silica gel column chromatography, eluting with hexane-EtOAc (8:1,v/v) afforded methyl ester IV-37 $14.5 \mathrm{~g}(56 \%$ over three steps) as white solid: ${ }^{1} \mathrm{H}$ NMR $\left(\mathrm{CDCl}_{3}, 270 \mathrm{MHz}\right): \delta 7.41(\mathrm{~s}, 2 \mathrm{H}), 6.9(\mathrm{~s}, 1 \mathrm{H}), 6.20$ (brs, 1H), 3.90 (s, 3H), 2.33 (s, 3H); ${ }^{13} \mathrm{C} \mathrm{NMR}\left(\mathrm{CDCl}_{3}, 67.5 \mathrm{MHz}\right): \delta 167.7,155.8,140.0,130.9$, 
122.6, 121.1, 113.5, 52.4, 21.2.

\section{Methyl 3-(tert-butyldimethylsilyloxy)-5-methylbenzoate (IV-37a)}<smiles>COC(=O)c1cc(C)cc(OC(C)(C)C)c1</smiles>

A solution of $4 \mathrm{~g}$ (24.1 mmol) methyl ester IV-37 in $24 \mathrm{ml} \mathrm{CH} \mathrm{Cl}_{2}$ was added $2.46 \mathrm{~g}$ (36.2 mmol) imidazole at $0{ }^{\circ} \mathrm{C}$ followed by a addition of $3.99 \mathrm{~g}(26.5 \mathrm{mmol}) \mathrm{TBSCl}$. The ice bath was removed and the mixture was then stirred at RT for $2 \mathrm{~h}$. It was diluted with $150 \mathrm{~mL}$ hexaneEtOAc $(2: 1, \mathrm{v} / \mathrm{v})$ and cooled down to $0{ }^{\circ} \mathrm{C}$, and then $70 \mathrm{~mL} \mathrm{H}_{2} \mathrm{O}$ was added. After separation, the aqueous layer was extracted with hexane-EtOAc $(2: 1, \mathrm{v} / \mathrm{v})(100 \mathrm{~mL} \mathrm{X} \mathrm{2).} \mathrm{The} \mathrm{pooled} \mathrm{organic}$ layer was subsequently washed with saturated aqueous $\mathrm{NaHCO}_{3}$, saturated brine and dried over $\mathrm{Na}_{2} \mathrm{SO}_{4}$. After removal of the solvent under reduced pressure, the residue was subjected to silica gel flash column chromatography. Elution with hexane-EtOAc $(50: 1, \mathrm{v} / \mathrm{v})$ afforded TBS ether IV-37a $6.7 \mathrm{~g}(100 \%)$ as clear oil: $R_{f}($ hexanes/EtOAc, $3.5: 1 \mathrm{v} / \mathrm{v})=0.62 ;$ IR (thin film, $\left.\mathrm{cm}^{-1}\right) v$ 2954, 2931, 2898, 2859, 1724, 1594, 1454, 1435, 1322, 1252, 1226, 1160, 1105, 1035, 982; ${ }^{1} \mathrm{H}$ NMR (CDCl $3,270 \mathrm{MHz}): \delta 7.46(\mathrm{~s}, 1 \mathrm{H}), 7.29(\mathrm{~s}, 1 \mathrm{H}), 6.85(\mathrm{~s}, 1 \mathrm{H}), 3.89(\mathrm{~s}, 3 \mathrm{H}), 2.33$ (s, 3H), $0.99(\mathrm{~s}, 9 \mathrm{H}), 0.20(\mathrm{~s}, 6 \mathrm{H}) ;{ }^{13} \mathrm{C} \mathrm{NMR}\left(\mathrm{CDCl}_{3}, 67.5 \mathrm{MHz}\right): \delta 167.1,155.5,139.5,131.1,125.5$, 123.3, 118.0, 52.0, 25.6, 21.2, 18.1, -4.5; HRMS (ESI): Cacld. for $\left[\mathrm{C}_{15} \mathrm{H}_{24} \mathrm{O}_{3} \mathrm{Si}+\mathrm{H}\right]^{+}: 281.1568$, Found: 281.1569.

\section{(3-(tert-butyldimethylsilyloxy)-5-methylphenyl)methanol (IV-38)}<smiles>Cc1cc(CO)cc(O[AsH3])c1</smiles> 
A solution of $6.7 \mathrm{~g}(24.1 \mathrm{mmol})$ ester IV-37a in $48 \mathrm{~mL}$ THF was slowly added $1.83 \mathrm{~g}$ (48.2 mmol) $\mathrm{LiAlH}_{4}$ in several portions at $0{ }^{\circ} \mathrm{C}$. After addition, the ice bath was removed and the mixture was then stirred at RT for $3 \mathrm{~h}$. It was cooled down to $0{ }^{\circ} \mathrm{C}$ and diluted with $400 \mathrm{~mL} \mathrm{Et}_{2} \mathrm{O}$, then carefully $3.6 \mathrm{~mL} 2 \mathrm{M} \mathrm{NaOH}$ was added dropwise, followed by the addition of $5.4 \mathrm{~mL}_{2} \mathrm{O}$. The mixture was stirred at $0{ }^{\circ} \mathrm{C}$ for $15 \mathrm{~min}$, and then the precipitate was rinsed with ether. The mother liquor was subsequently washed with saturated aqueous $\mathrm{NaHCO}_{3}$, saturated brine and dried over $\mathrm{Na}_{2} \mathrm{SO}_{4}$. After removal of the solvent under reduced pressure, the residue was subjected to silica gel flash column chromatography. Elution with hexane-EtOAc $(10: 1, \mathrm{v} / \mathrm{v})$ afforded benzyl alcohol IV-38 $5.4 \mathrm{~g}(89 \%)$ as clear oil: $R_{f}($ hexanes/EtOAc, 3:1 v/v) =0.40; IR (thin film, $\mathrm{cm}^{-1}$ ) v 3306 (broad), 2956, 2930, 2886, 2859, 1595, 1462, 1315, 1253, 1161, 1042, 1019, 947; ${ }^{1} \mathrm{H} \mathrm{NMR}\left(\mathrm{CDCl}_{3}, 270 \mathrm{MHz}\right): \delta 6.77$ (s, 1H), 6.65 (s, 1H), 6.59 (s, 1H), 4.59 (s, 2H), $2.30(\mathrm{~s}, 3 \mathrm{H}), 1.72(\mathrm{~s}, 1 \mathrm{H}), 0.99(\mathrm{~s}, 9 \mathrm{H}), 0.20(\mathrm{~s}, 6 \mathrm{H}) ;{ }^{13} \mathrm{C} \mathrm{NMR}\left(\mathrm{CDCl}_{3}, 67.5 \mathrm{MHz}\right): \delta 155.7$, 142.1, 139.6, 120.6, 120.0, 115.5, 65.2, 25.6, 21.4, 18.1, -4.4; HRMS (ESI): Cacld. for $\left[\mathrm{C}_{14} \mathrm{H}_{24} \mathrm{O}_{2} \mathrm{Si}+\mathrm{H}\right]^{+}:$253.1618, Found: 253.1620.

\section{3-(tert-butyldimethylsilyloxy)-5-methylbenzaldehyde (IV-39) ${ }^{66}$}

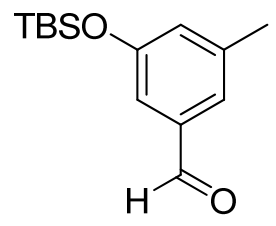

A solution of $5.4 \mathrm{~g}(21.4 \mathrm{mmol})$ benzyl alcohol IV-38 in $33 \mathrm{~mL}$ hexane was added $18.6 \mathrm{~g}$ (214 mmol) activated $\mathrm{MnO}_{2}$ at RT. It was stirred at RT for $10 \mathrm{~h}$. The mixture was filtered and the precipitate was rinsed with ether. After removal of the solvent under reduced pressure, the residue was subjected to silica gel flash column chromatography. Elution with hexane-EtOAc $(25: 1, \mathrm{v} / \mathrm{v})$ afforded aldehyde IV-39 $4.5 \mathrm{~g}(84 \%)$ as clear oil: $R_{f}($ hexanes/EtOAc, 3:1 v/v) $=0.62$; ${ }^{1} \mathrm{H}$ NMR $\left(\mathrm{CDCl}_{3}, 270 \mathrm{MHz}\right): \delta 9.90$ (s, 1H), 7.29 (s, 1H), 7.12 (s, 1H), 6.92 (s, 1H), 2.37 (s, 3H), 0.99 (s, 9H), $0.21(\mathrm{~s}, 6 \mathrm{H}) ;{ }^{13} \mathrm{C} \mathrm{NMR}\left(\mathrm{CDCl}_{3}, 67.5 \mathrm{MHz}\right): \delta$ 192.4, 156.2, 140.4, 137.7, 127.3, $124.2,117.2,25.6,21.2,18.2,-4.4$. 
3-hydroxy-5-methylbenzaldehyde (IV-34) ${ }^{67}$

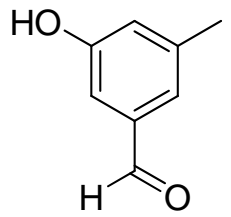

A solution of $3.94 \mathrm{~g}(15.8 \mathrm{mmol})$ TBS ether IV-39 in $20 \mathrm{~mL} \mathrm{MeOH}$ was added $898 \mathrm{mg}$ (4.73 mmol) solid $p-\mathrm{TsOH} \cdot \mathrm{H} 2 \mathrm{O}$ when stirring at $\mathrm{RT}$. The mixture was stirred at $\mathrm{RT}$ for $24 \mathrm{~h}$. Then it was diluted with $200 \mathrm{~mL}$ EtOAc and cooled down to $0{ }^{\circ} \mathrm{C}$. Into the solution, $20 \mathrm{~mL}$ saturated aqueous $\mathrm{NaHCO}_{3}$ was slowly added. The mixture was stirred at $0{ }^{\circ} \mathrm{C}$ for $10 \mathrm{~min}$. After separation, the aqueous layer was extracted with EtOAc and the combined organic layer was washed with saturated brine and dried over $\mathrm{Na}_{2} \mathrm{SO}_{4}$. After removal of the solvent, the residue was subjected to silica gel column chromatography, eluting with hexane-EtOAc $(5: 1, v / v)$ afforded phenol IV-34 $2.0 \mathrm{~g}(93 \%)$ as white solid: $R_{f}$ (hexanes/EtOAc, $\left.2: 1 \mathrm{v} / \mathrm{v}\right)=0.42 ;{ }^{1} \mathrm{H} \mathrm{NMR}\left(\mathrm{CDCl}_{3}, 270 \mathrm{MHz}\right)$ : $\delta 9.90(\mathrm{~s}, 1 \mathrm{H}), 7.26(\mathrm{~s}, 1 \mathrm{H}), 7.19(\mathrm{~s}, 1 \mathrm{H}), 6.98(\mathrm{~s}, 1 \mathrm{H}), 2.38(\mathrm{~s}, 3 \mathrm{H}) ;{ }^{13} \mathrm{C} \mathrm{NMR}\left(\mathrm{CDCl}_{3}, 67.5\right.$ $\mathrm{MHz}): \delta 192.9,156.4,140.8,137.6,124.2,122.8,112.2,21.1$.

\section{(2S,3S)-tert-butyl 2-amino-3-methylpentanoate (IV-40)}

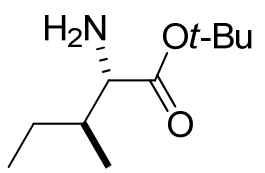

A sample of $2.62 \mathrm{~g}(20.0 \mathrm{mmol})$ isoleucine IV-6 was placed in a $40 \mathrm{~mL}$ pressure vessel, into which a premixed $20 \mathrm{~mL}$ THF and $5 \mathrm{~mL}$ concentrated $\mathrm{H}_{2} \mathrm{SO}_{4}$ was added when stirring. The suspension was cooled down to $-78^{\circ} \mathrm{C}$ under Ar. Then the inlet of Ar was closed, and isobutene was allowed to bubble through the solution via a needle. The bubbling was stopped until about 3 $\mathrm{mL}$ liquid of isobutene was seen floating on the solution. Quickly switch the septum to a pressure gauge and the pressure vessel was sealed. The mixture was stirred at RT for $15 \mathrm{~h}$. The solution was cooled down to $0{ }^{\circ} \mathrm{C}$, and then the pressure was released and quickly the mixture was poured into a suspension of $100 \mathrm{~g}$ ice in $200 \mathrm{~mL} 1.25 \mathrm{M}$ aqueous $\mathrm{NaOH}$ when stirring. The 
mixture was stirred at $0{ }^{\circ} \mathrm{C}$ for $15 \mathrm{~min}$ and extracted with EtOAc twice. The organic layer was subsequently washed with saturated aqueous $\mathrm{NaHCO}_{3}$ and brine, then was dried over $\mathrm{Na}_{2} \mathrm{SO}_{4}$. Concentration of the solution resulted in isoleucine $t$-butyl ester IV-40 $2.0 \mathrm{~g}(54 \%)$ as clear oil: ${ }^{1} \mathrm{H} \mathrm{NMR}\left(\mathrm{CDCl}_{3}, 270 \mathrm{MHz}\right): \delta 3.25(\mathrm{~d}, J=4.70 \mathrm{~Hz}, 1 \mathrm{H}), 2.03$ (brs, 2H), $1.72(\mathrm{~m}, 1 \mathrm{H}), 1.45$ (s, 9H), $1.21(\mathrm{~m}, 2 \mathrm{H}), 0.93(\mathrm{~d}, J=6.9 \mathrm{~Hz}, 3 \mathrm{H}), 0.90(\mathrm{t}, J=7.4 \mathrm{~Hz}, 3 \mathrm{H}) ;{ }^{13} \mathrm{C} \mathrm{NMR}\left(\mathrm{CDCl}_{3}, 67.5\right.$ MHz): $\delta 174.5,80.9,59.3,39.2,28.1,24.8,15.6,11.8$.

\section{2-Bromo-8-hydroxynaphthalene-1,4-dione (IV-42)}<smiles>O=C1C=C(Br)C(=O)c2c(O)cccc21</smiles>

A suspension of $3.0 \mathrm{~g}(17.2 \mathrm{mmol})$ juglone (IV-41) in $45 \mathrm{~mL}$ HOAc was added $0.9 \mathrm{~mL}(17.2$ mmol) $\mathrm{Br}_{2}$ when stirring at RT. The mixture was stirred at RT for $15 \mathrm{~min}$. Then it was poured into $50 \mathrm{~g}$ ice followed by addition of $150 \mathrm{~mL} \mathrm{H}_{2} \mathrm{O}$. It was stirred at RT for $10 \mathrm{~min}$. The mixture was filtered and the solid was washed with cold water, which was then transferred to flask followed by addition of $20 \mathrm{~mL}$ ethanol. The mixture was stirred at a preheated oil bath (100-110 ${ }^{\circ} \mathrm{C}$ ) for $10 \mathrm{~min}$ and then cooled down to RT. The solid was collected by filtration. Chromatography of the crude product on silica gel column, eluting with hexane- $\mathrm{CH}_{2} \mathrm{Cl}_{2}(1: 1$, $\mathrm{v} / \mathrm{v})$, afforded a $3.5 \mathrm{~g}$ orange solid mixture ( $80 \%$ total yield), in which contained $81 \% 3$ bromojuglone (IV-42) and 19\% 2-bromojuglone.

3-bromojuglone (IV-42): $R_{f}$ (hexanes/EtOAc, 3:1 v/v) $=0.54 ;{ }^{1} \mathrm{H}$ NMR $\left(\mathrm{CDCl}_{3}, 270 \mathrm{MHz}\right)$ : $\delta 11.73(\mathrm{~s}, 1 \mathrm{H}), 7.68$ (dd, $J=7.7,7.4 \mathrm{~Hz}, 1 \mathrm{H}), 7.64$ (dd, $J=6.5,1.0 \mathrm{~Hz}, 1 \mathrm{H}), 7.49$ (s, 1H), 7.31 $(\mathrm{dd}, J=7.4,2.2 \mathrm{~Hz}, 1 \mathrm{H}) ;{ }^{13} \mathrm{C} \mathrm{NMR}\left(\mathrm{CDCl}_{3}, 150 \mathrm{MHz}\right): \delta 182.9,181.6,162.1,141.2,139.3$, 137.2, 131.7, 124.7, 119.9, 114.0. 
(2S,3S)-tert-butyl

methylpentanoate (IV-43) 2-(8-hydroxy-1,4-dioxo-1,4-dihydronaphthalen-2-ylamino)-3-

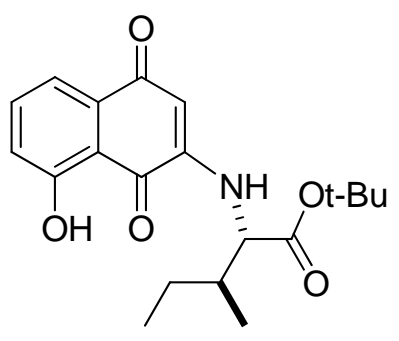

A solution of $253 \mathrm{mg}$ (1.0 mmol) juglone mixture which contain 81\% 2-bromojuglone (IV-42) in $1 \mathrm{~mL} \mathrm{CH}_{2} \mathrm{Cl}_{2}$ at $0{ }^{\circ} \mathrm{C}$ was added a solution of $234 \mathrm{mg}(1.25 \mathrm{mmol})$ isoleucine $t$-butyl ester in 1 $\mathrm{mL} \mathrm{CH} \mathrm{Cl}_{2}$ when stirring, followed by addition of $139 \mu \mathrm{L} \mathrm{Et}_{3} \mathrm{~N}(1.0 \mathrm{mmol})$ in $1 \mathrm{~mL} \mathrm{CH}_{2} \mathrm{Cl}_{2}$. The mixture was stirred at $0{ }^{\circ} \mathrm{C}$ for $15 \mathrm{~min}$ and then diluted with $30 \mathrm{~mL}$ hexane-EtOAc $(1: 1, \mathrm{v} / \mathrm{v})$ and washed with $6 \mathrm{~mL}$ water. The aqueous layer was extracted with hexane-EtOAc $(1: 1, \mathrm{v} / \mathrm{v}) 20 \mathrm{~mL}$. The pooled organic layer was subsequently washed with saturated aqueous $\mathrm{NaHCO}_{3}$, brine and dried over $\mathrm{Na}_{2} \mathrm{SO}_{4}$. After removal of the solvent, the residue was subjected to silica gel column chromatography. Elution with hexane-EtOAc $(10: 1, \mathrm{v} / \mathrm{v})$ afforded aminojuglone IV-43 $240 \mathrm{mg}$ $(67 \%)$ as dark red oil: $R_{f}($ hexanes/EtOAc, $3: 1 \mathrm{v} / \mathrm{v})=0.60 ;[\alpha]^{20}{ }_{\mathrm{D}}-60\left(c 0.59, \mathrm{CHCl}_{3}\right)$; IR (thin film, $\mathrm{cm}^{-1}$ ) v 3361, 2967, 2934, 1731, 1680, 1624, 1604, 1509, 1469, 1385, 1368, 1293, 1247 , 1227, 1156, 1041, 918; ${ }^{1} \mathrm{H}$ NMR $\left(\mathrm{CDCl}_{3}, 600 \mathrm{MHz}\right): \delta 12.95$ (s, 1H), $7.61(\mathrm{dd}, J=7.2,1.2 \mathrm{~Hz}, 1$ H), $7.47(\mathrm{dd}, J=8.4,7.8 \mathrm{~Hz}, 1 \mathrm{H}), 7.24(\mathrm{dd}, J=8.4,1.2 \mathrm{~Hz}, 1 \mathrm{H}), 6.57$ (d, $J=7.8 \mathrm{~Hz}, 1 \mathrm{H}), 5.59$ (s, 1H), 3.87 (dd, $J=8.4,5.4 \mathrm{~Hz}, 1 \mathrm{H}), 1.97$ (dqdd, $J=9.0,7.2,5.4,5.4 \mathrm{~Hz}, 1 \mathrm{H}), 1.58$ (dqd, $J=$ 13.8, 7.2, $5.4 \mathrm{~Hz}, 1 \mathrm{H}), 1.50$ (s, 9H), 1.35 (ddq, $J=14.4,9.0,7.2 \mathrm{~Hz}, 1 \mathrm{H}) ; 1.00$ (dd, $J=7.8,7.8$ $\mathrm{Hz}, 3 \mathrm{H}) ; 0.98(\mathrm{~d}, J=6.6 \mathrm{~Hz}, 3 \mathrm{H}) ;{ }^{13} \mathrm{C} \mathrm{NMR}\left(\mathrm{CDCl}_{3}, 150 \mathrm{MHz}\right): \delta 189.2,180.7,169.1,161.1$, $147.7,134.0,130.5,125.8,119.1,114.8,100.5,83.1,59.7,37.5,28.1,25.9,15.2,11.6$; HRESIMS: Calcd. for $\left[\mathrm{C}_{20} \mathrm{H}_{25} \mathrm{NO}_{5}+\mathrm{H}\right]^{+}: 360.1806$, Found: 360.1807. 


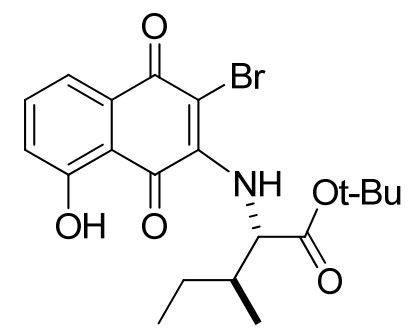

A solution of $152 \mathrm{mg}(0.42 \mathrm{mmol})$ aminojuglone $\mathbf{I V - 4 3}$ in $4 \mathrm{~mL} \mathrm{CH}_{2} \mathrm{Cl}_{2}$ at $0{ }^{\circ} \mathrm{C}$ was added a solution of $24 \mu \mathrm{L}(0.46 \mathrm{mmol}) \mathrm{Br}_{2}$ and $38 \mu \mathrm{L}(0.46 \mathrm{mmol})$ pyridine in $4 \mathrm{~mL} \mathrm{CH}_{2} \mathrm{Cl}_{2}$. It was stirred at $0{ }^{\circ} \mathrm{C}$ for $10 \mathrm{~min}$, then it was diluted with hexane-EtOAc $(1: 1, \mathrm{v} / \mathrm{v}) 80 \mathrm{~mL}$ followed by addition of $15 \mathrm{~mL} \mathrm{H}_{2} \mathrm{O}$. The organic layer was separated and subsequently washed with saturated aqueous $\mathrm{NaHCO}_{3}$, saturated brine and dried over $\mathrm{Na}_{2} \mathrm{SO}_{4}$. After removal of the solvent, the residue was subjected to silica gel column chromatography. Elution with hexane-EtOAc (20:1,v/v) afforded 2-bromo-3-aminojuglone IV-44 $140 \mathrm{mg}(76 \%)$ as dark red oil: $R_{f}$ $($ hexanes/EtOAc, $1: 1 \mathrm{v} / \mathrm{v})=0.78 ;[\alpha]^{20}{ }_{\mathrm{D}}+20\left(c\right.$ 2.9, $\left.\mathrm{CHCl}_{3}\right)$; IR (thin film, $\left.\mathrm{cm}^{-1}\right) v 3300,2965$, 2930, 2875, 1725, 1680, 1620, 1605, 1586, 1458, 1237, 1214, 1151, 1131, 1108, ; ${ }^{1} \mathrm{H}$ NMR $\left(\mathrm{CDCl}_{3}, 600 \mathrm{MHz}\right): \delta 12.56(\mathrm{~s}, 1 \mathrm{H}), 7.56$ (brs, $\left.1 \mathrm{H}\right), 7.46(\mathrm{dd}, J=8.4,7.8 \mathrm{~Hz}, 1 \mathrm{H}), 7.22(\mathrm{dd}, J=$ $8.4 \mathrm{~Hz}, 1 \mathrm{H}), 5.18$ (brs, 1H), 1.98 (brs, 1H), 1.62 (dqd, $J=12.6,7.2,4.8 \mathrm{~Hz}, 1 \mathrm{H}), 1.49$ (s, 9H), 1.37 (ddq, $J=14.4,9.6,7.2 \mathrm{~Hz}, 1 \mathrm{H}) ; 1.01(\mathrm{dd}, J=7.8,7.2 \mathrm{~Hz}, 3 \mathrm{H}) ; 0.96(\mathrm{~d}, J=7.2 \mathrm{~Hz}, 3 \mathrm{H})$; HRESIMS: Calculated for $\left[\mathrm{C}_{20} \mathrm{H}_{24} \mathrm{BrNO}_{5}+\mathrm{H}\right]^{+}: 438.0906$, Found: 438.0913.

\section{3-(1,3-dioxan-2-yl)-5-methylphenol (IV-34a) ${ }^{58}$}

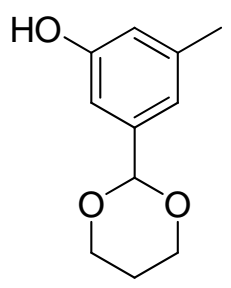

\footnotetext{
${ }^{\S}$ An average optical rotation was given due to the unstable data that was obtained. The ${ }^{13} \mathrm{C}$ NMR data was not given due to the broadening of most signals of carbons on the quinone part, a copy of the spectra was enclosed in the spetra section. 
A solution of $1.77 \mathrm{~g}(13.0 \mathrm{mmol})$ benzaldehyde $\mathbf{I V - 3 4}$ in $70 \mathrm{~mL}$ benzene was placed in a $100 \mathrm{~mL}$ flask which was equipped with a distillation assembly. Into the solution was added $1.88 \mathrm{~mL}$ (26.0 mmol) 1,3-propanediol and $123 \mathrm{mg}(0.65 \mathrm{mmol})$ solid $p-\mathrm{TsOH} \bullet \mathrm{H}_{2} \mathrm{O}$. The mixture was then gradually heated up under Ar. The distillation was allowed to proceed until the total volume of solution was about 5-10 mL. Then it was cooled down to $0{ }^{\circ} \mathrm{C}$ and diluted with $\mathrm{Et}_{2} \mathrm{O} 130 \mathrm{~mL} .50$ $\mathrm{mL}$ saturated aqueous $\mathrm{NaHCO}_{3}$ was added to the mixture. The aqueous layer was extracted with $\mathrm{Et}_{2} \mathrm{O}$ and the pooled organic layer was washed with saturated brine and dried over $\mathrm{Na}_{2} \mathrm{SO}_{4}$. After removal of the solvent, the residue was subjected to silica gel column chromatography, eluting with hexane-EtOAc $(5: 1, \mathrm{v} / \mathrm{v})$ afforded acetal IV-34a $2.11 \mathrm{~g}(84 \%)$ as white solid: $R_{f}$ (hexanes/EtOAc, 2:1 v/v) $=0.31 ;{ }^{1} \mathrm{H}$ NMR $\left(\mathrm{CDCl}_{3}, 270 \mathrm{MHz}\right): \delta 6.86(\mathrm{~s}, 1 \mathrm{H}), 6.75(\mathrm{~s}, 1 \mathrm{H}), 6.59$ (s, 1H), 6.06 (brs, 1H), $5.42(\mathrm{~s}, 1 \mathrm{H}), 4.25(\mathrm{dd}, J=11.6,4.9 \mathrm{~Hz}, 2 \mathrm{H}), 3.96$ (ddd, $J=12.2,10.2$, $2.0 \mathrm{~Hz}, 2 \mathrm{H}), 2.23(\mathrm{~s}, 3 \mathrm{H}), 2.17(\mathrm{~m}, 1 \mathrm{H}), 1.43(\mathrm{~d}, 13.6 \mathrm{~Hz}, 1 \mathrm{H}) ;{ }^{13} \mathrm{C} \mathrm{NMR}\left(\mathrm{CDCl}_{3}, 67.5 \mathrm{MHz}\right): \delta$ $155.6,139.7,139.3,118.7,116.7,110.3,101.6,67.3,25.5,21.2$.

\section{2-(3-(methoxymethoxy)-5-methylphenyl)-1,3-dioxane (IV-45)}

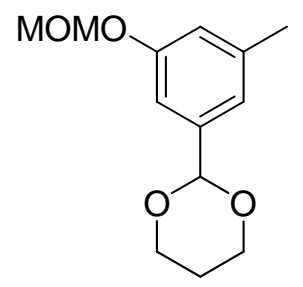

A solution of $172 \mathrm{mg}(0.89 \mathrm{mmol})$ phenol $\mathbf{I V - 3 4 a}$ in $1.5 \mathrm{~mL} \mathrm{CH}_{2} \mathrm{Cl}_{2}$ at ${ }^{\circ} \mathrm{C}$ was added $0.55 \mathrm{~mL}$ (3.33 mmol) DIPEA followed by addition of $150 \mu \mathrm{L}(1.98 \mathrm{mmol}) \mathrm{MOMCl}$. It was stirred at RT for $8 \mathrm{~h}$. The mixture was cooled down to $0{ }^{\circ} \mathrm{C}$ and diluted with hexane-Et $2 \mathrm{O}(1: 1, \mathrm{v} / \mathrm{v}) 50 \mathrm{~mL}$. Then $10 \mathrm{~mL}$ water was added and it was then stirred at $0{ }^{\circ} \mathrm{C}$ for $20 \mathrm{~min}$. The aqueous layer was extracted with hexan-Et ${ }_{2} \mathrm{O}(1: 1, \mathrm{v} / \mathrm{v})$ and the pooled organic layer was subsequently washed with saturated aqueous $\mathrm{NaHCO}_{3}$, saturated brine and dried over $\mathrm{Na}_{2} \mathrm{SO}_{4}$. After removal of the solvent, the residue was subjected to silica gel column chromatography, eluting with hexane-EtOAc (6:1, v/v) afforded MOM ether IV-45 $150 \mathrm{mg}(68 \%)$ and elution with hexane-EtOAc (3:1,v/v) recovered $42 \mathrm{mg}$ starting material (20\%). MOM ether IV-45: White solid; $R_{f}$ (hexanes/EtOAc, 
$2: 1 \mathrm{v} / \mathrm{v})=0.49 ;{ }^{1} \mathrm{H} \mathrm{NMR}\left(\mathrm{CDCl}_{3}, 600 \mathrm{MHz}\right): \delta 6.93(\mathrm{~s}, 1 \mathrm{H}), 6.90(\mathrm{~s}, 1 \mathrm{H}), 6.82(\mathrm{~s}, 1 \mathrm{H}), 5.45(\mathrm{~s}$, 1H), 5.14 (s, 2H), 4.19 (dddd, $J=10.2,4.8,1.2,1.2 \mathrm{~Hz}, 2 \mathrm{H}) ; 4.00$ (dddd, $J=12.0,10.2,2.4,1.2$ $\mathrm{Hz}, 2 \mathrm{H}), 3.43(\mathrm{~s}, 3 \mathrm{H}), 2.30(\mathrm{~s}, 3 \mathrm{H}), 2.13(\mathrm{dtt}, J=13.8,12.6,5.4 \mathrm{~Hz}, 1 \mathrm{H}), 1.46(\mathrm{dtt}, J=13.8,2.4$, $1.2 \mathrm{~Hz}, 1 \mathrm{H}) ;{ }^{13} \mathrm{C} \mathrm{NMR}\left(\mathrm{CDCl}_{3}, 150 \mathrm{MHz}\right): \delta 158.7,141.7,140.5,121.7,118.5,112.4,102.9$, $95.7,68.5,56.3,27.1,21.6$.

\section{(2-(1,3-dioxan-2-yl)-6-(methoxymethoxy)-4-methylphenyl)tributylstannane (IV-46)}

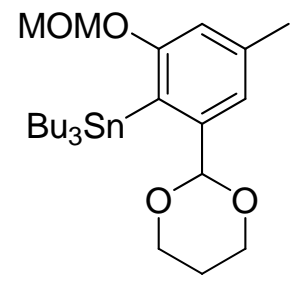

A solution of $1.82 \mathrm{~g}(7.65 \mathrm{mmol}) \mathrm{MOM}$ ether $\mathbf{I V}-45$ in $23 \mathrm{~mL}$ hexane at ${ }^{\circ} \mathrm{C}$ was added $4.9 \mathrm{~mL}$ 2.34 M (11.5 mmol) n-BuLi. The mixture was stirred at ${ }^{\circ} \mathrm{C}$ for $1 \mathrm{~h}$. Then $3.3 \mathrm{~mL} \mathrm{Bu} \mathrm{SnCl}$ was slowly added in to the reaction mixture at ${ }^{\circ} \mathrm{C}$. It was stirred at ${ }^{\circ} \mathrm{C}$ for another $1 \mathrm{~h}$. It was then diluted with hexane- $\mathrm{Et}_{2} \mathrm{O}(1: 1, \mathrm{v} / \mathrm{v}) 200 \mathrm{~mL}$ followed by addition of $60 \mathrm{~mL}$ saturated aqueous $\mathrm{NaHCO}_{3}$. The mixture was stirred at ${ }^{\circ} \mathrm{C}$ for $1 \mathrm{~h}$. The organic layer was separated and the aqueous layer was extracted with hexane- $\mathrm{Et}_{2} \mathrm{O}(1: 1, \mathrm{v} / \mathrm{v})$. The pooled organic layer was subsequently washed with saturated brine and dried over $\mathrm{Na}_{2} \mathrm{SO}_{4}$. After removal of the solvent, the residue was subjected to silica gel column chromatography, eluting with hexane-EtOAc $(25: 1, \mathrm{v} / \mathrm{v})$ afforded phenylstannane IV-46 $1.94 \mathrm{~g}$ (49\%). Elution with hexane-EtOAc $(5: 1, \mathrm{v} / \mathrm{v})$ recovered $650 \mathrm{mg}$ starting material (36\%). Phenylstannane IV-46: clear oil; $R_{f}($ hexanes/EtOAc, 2:1 v/v) = 0.78; ${ }^{1} \mathrm{H}$ NMR (benzene-d $\left.6,600 \mathrm{MHz}\right): \delta 7.68(\mathrm{~s}, 1 \mathrm{H}), 7.06(\mathrm{~s}, 1 \mathrm{H}), 5.55(\mathrm{~s}, 1 \mathrm{H}), 4.93(\mathrm{~s}, 2 \mathrm{H})$, $3.96(\mathrm{dd}, J=12.0,5.4 \mathrm{~Hz}, 2 \mathrm{H}), 3.69$ (ddd, $J=12.6,12.0,2.4 \mathrm{~Hz}, 2 \mathrm{H}), 3.24$ (s, 3H), 2.14 (s, 3H), $1.94(\mathrm{dtt}, J=12.6,12.6,4.8 \mathrm{~Hz}, 1 \mathrm{H}) ; 1.74(\mathrm{~m}, 6 \mathrm{H}) ; 1.48(\mathrm{~m}, 6 \mathrm{H}) ; 1.29(\mathrm{~m}, 6 \mathrm{H}), 0.98(\mathrm{t}, J=7.2$ $\mathrm{Hz}, 9 \mathrm{H}) ; 0.75(\mathrm{~d}, J=13.2 \mathrm{~Hz}, 1 \mathrm{H}) ;{ }^{13} \mathrm{C}$ NMR (benzene-d $\left.6,150 \mathrm{MHz}\right): \delta 162.9,148.1,140.4$, $125.9,122.6,114.4,103.0,95.2,67.5,56.0,30.1,28.3,26.4,21.9,14.4,13.0$. 


\section{Naphthalene-1,5-diyl diacetate (IV-48) ${ }^{59}$}<smiles>CC(=O)Oc1cccc2c(OC(C)=O)cccc12</smiles>

A solution of $5 \mathrm{~g}(31.2 \mathrm{mmol})$ naphthalenediol $\mathbf{I V}-\mathbf{- 4 7}$ in $40 \mathrm{~mL}$ pyridine at $0{ }^{\circ} \mathrm{C}$ was slowly added $8.9 \mathrm{~mL}(125 \mathrm{mmol}) \mathrm{AcCl}$ dropwise. It was stirred at RT for $10 \mathrm{~min}$, then it was poured into a $500 \mathrm{~mL}$ water when stirring. The solid was collected by filtratin, washing with water several times. After being dried by using vacuum, $7.4 \mathrm{~g}$ ( 97\%) diacetate IV-48 was afforded as brown solid: $R_{f}($ hexanes/EtOAc, $1: 1 \mathrm{v} / \mathrm{v})=0.55 ;{ }^{1} \mathrm{H} \mathrm{NMR}\left(\mathrm{CDCl}_{3}, 270 \mathrm{MHz}\right): \delta 7.80(\mathrm{~d}, J=8.4$ $\mathrm{Hz}, 2 \mathrm{H}), 7.52(\mathrm{dd}, J=8.2,7.7 \mathrm{~Hz}, 2 \mathrm{H}), 7.31$ (d, $J=7.4 \mathrm{~Hz}, 2 \mathrm{H}), 2.46(\mathrm{~s}, 6 \mathrm{H}) ;{ }^{13} \mathrm{C}$ NMR $\left(\mathrm{CDCl}_{3}, 67.5 \mathrm{MHz}\right): \delta$ 169.2, 146.6, 128.0, 126.0, 119.2, 118.7, 20.9.

\section{6-Bromo-5,8-dioxo-5,8-dihydronaphthalen-1-yl acetate $\left(\right.$ IV-49) ${ }^{59}$}<smiles>CC(=O)Oc1cccc2c1C(=O)C=C(Br)C2=O</smiles>

$2.44 \mathrm{~g}(10.0 \mathrm{mmol})$ diacetate IV-48 was suspended in $100 \mathrm{~mL}$ HOAc at $50-60{ }^{\circ} \mathrm{C}$, a solution of $7.12 \mathrm{~g} \mathrm{(40} \mathrm{mmol)} \mathrm{NBS} \mathrm{in} 100 \mathrm{~mL} H O A c$ and $200 \mathrm{~mL} \mathrm{H} \mathrm{H}_{2} \mathrm{O}$ was added into the mixture dropwise over $1 \mathrm{~h}$. After addition, the reaction mixture was stirred at $60{ }^{\circ} \mathrm{C}$ for $2 \mathrm{~h}$. It was cooled down to RT. Yellow precipitate started to form. Filtration and washing with water. After drying, $1.9 \mathrm{~g}$ 2bromojuglone acetate (IV-49) was obtained (66\%). Yellow crystal: $R_{f}$ (hexanes/EtOAc, 2:1 v/v) $=0.46 ;{ }^{1} \mathrm{H} \mathrm{NMR}\left(\mathrm{CDCl}_{3}, 600 \mathrm{MHz}\right): \delta 8.14(\mathrm{dd}, J=7.8,1.2 \mathrm{~Hz}, 1 \mathrm{H}), 7.77(\mathrm{dd}, J=7.8,7.8 \mathrm{~Hz}$, $1 \mathrm{H}), 7.42(\mathrm{dd}, J=7.8,1.2 \mathrm{~Hz}, 1 \mathrm{H}), 7.39(\mathrm{~s}, 1 \mathrm{H}), 2.44(\mathrm{~s}, 3 \mathrm{H}) ;{ }^{13} \mathrm{C} \mathrm{NMR}\left(\mathrm{CDCl}_{3}, 150 \mathrm{MHz}\right): \delta$ $180.9,177.4,169.2,149.9,141.4,138.5,134.9,132.6,130.3,126.3,123.1,21.0$. 


\section{2-Bromo-5-hydroxynaphthalene-1,4-dione (IV-50) ${ }^{59}$}<smiles>O=C1C=C(Br)C(=O)c2c(O)cccc21</smiles>

A solution of $765 \mathrm{mg}$ (2.59 mmol) acetate IV-49 in $26 \mathrm{~mL} \mathrm{95 \%} \mathrm{ethanol} \mathrm{was} \mathrm{added} 8.5 \mathrm{~mL} 1.5 \mathrm{M}$ aqueous $\mathrm{H}_{2} \mathrm{SO}_{4}$. The solution was stirred under reflux condition for $3 \mathrm{~h}$. It was then cooled down to RT and diluted with EtOAc $100 \mathrm{~mL}$ and washed with water $150 \mathrm{~mL}$ twice. The organic layer was subsequently washed with saturated aqueous $\mathrm{NaHCO}_{3}$, saturated brine and then dried over $\mathrm{Na}_{2} \mathrm{SO}_{4}$. After removal of the solvent, the residue was subjected to silica gel column chromatography, eluting with hexane-EtOAc (15:1,v/v) afforded 2-bromojuglone (IV-50) 459 mg (70\%). Orange solid: $R_{f}$ (hexanes/EtOAc, $\left.2: 1 \mathrm{v} / \mathrm{v}\right)=0.51 ;{ }^{1} \mathrm{H} \mathrm{NMR}\left(\mathrm{CDCl}_{3}, 270 \mathrm{MHz}\right)$ : $\delta 11.75(\mathrm{~s}, 1 \mathrm{H}), 7.72(\mathrm{dd}, J=7.2,1.2 \mathrm{~Hz}, 1 \mathrm{H}), 7.63(\mathrm{ddd}, J=7.8,7.2,0.6 \mathrm{~Hz}, 1 \mathrm{H}), 7.45(\mathrm{~s}, 1 \mathrm{H})$, $7.30(\mathrm{dd}, J=8.4,1.2 \mathrm{~Hz}, 1 \mathrm{H}) ;{ }^{13} \mathrm{C} \mathrm{NMR}\left(\mathrm{CDCl}_{3}, 67.5 \mathrm{MHz}\right): \delta 187.5,177.2,161.7,140.9$, $140.3,136.4,130.7,125.1,121.0,114.7$.

\section{2-(2-(1,3-dioxan-2-yl)-6-(methoxymethoxy)-4-methylphenyl)-5-hydroxynaphthalene-1,4-} dione (IV-51)<smiles>COc1cc(C)cc(C2OCCCO2)c1C1=CC(=O)c2c(O)cccc2C1=O</smiles>

A suspension of $253 \mathrm{mg}(1.0 \mathrm{mmol})$ juglone IV-50, $526 \mathrm{mg}(1.0 \mathrm{mmol})$ stannane $\mathbf{I V - 4 6}$, and $19.0 \mathrm{mg}(0.10 \mathrm{mmol}) \mathrm{CuI}$ in $8 \mathrm{~mL}$ THF at RT was added a solution of $25.9 \mathrm{mg}(0.025 \mathrm{mmol})$ $\mathrm{Pd}_{2}(\mathrm{dba})_{3} \cdot \mathrm{CHCl}_{3}$ and $26.2 \mathrm{mg}(0.10 \mathrm{mmol}) \mathrm{PPh}_{3}$ in $2 \mathrm{~mL}$ THF. The mixture was stirred at $75^{\circ} \mathrm{C}$ for $5 \mathrm{~h}$. It was then cooled down to $0{ }^{\circ} \mathrm{C}$ and diluted with $100 \mathrm{~mL}$ EtOAc and washed with saturated aqueous $\mathrm{NaHCO}_{3}$, saturated brine, then dried over $\mathrm{Na}_{2} \mathrm{SO}_{4}$. After removal of the 
solvent, the residue was subjected to silica gel column chromatography, eluting with hexaneEtOAc (5:1,v/v) afforded coupled product IV-51 $310 \mathrm{mg}$ (76\%). Orange solid, mp: 44-46 ${ }^{\circ} \mathrm{C} ; R_{f}$ (hexanes/EtOAc, 2:1 v/v) $=0.47$; IR (thin film, $\mathrm{cm}^{-1}$ ) $v$ 2955, 2925, 2850, 1639, 1455, 1247 ,

1148, 1050, 999; ${ }^{1} \mathrm{H}$ NMR $\left(\mathrm{CD}_{3} \mathrm{OD}, 600 \mathrm{MHz}\right): \delta 7.70(\mathrm{dd}, J=8.4,7.8 \mathrm{~Hz}, 1 \mathrm{H}), 7.61(\mathrm{dd}, J=$ 7.2, $1.2 \mathrm{~Hz}, 1 \mathrm{H}), 7.31$ (dd, $J=8.4,1.2 \mathrm{~Hz}, 1 \mathrm{H}), 7.12$ (brs, 1H), 7.04 (brs, 1H), 6.86 (s, 1H), 5.38 (s, 1H), $5.08(\mathrm{~d}, J=6.6 \mathrm{~Hz}, 1 \mathrm{H}), 5.07$ (d, $J=7.2 \mathrm{~Hz}, 1 \mathrm{H}), 3.99$ (m, 2H); 3.80 (ddd, $J=12.0,12.0$, $3.0 \mathrm{~Hz}, 1 \mathrm{H}), 3.76$ (ddd, $J=12.0,12.0,2.4 \mathrm{~Hz}, 1 \mathrm{H}), 3.33$ (s, 3H), 2.38 (s, 3H), 1.94 (ddddd, $J=$ 13.2, 12.0, 12.0, 4.8, $4.8 \mathrm{~Hz}, 1 \mathrm{H}), 1.30$ (ddddd, $J=13.2,2.4,2.4,1.2,1.2 \mathrm{~Hz}, 1 \mathrm{H}$ ); ${ }^{13} \mathrm{C}$ NMR $\left(\mathrm{CD}_{3} \mathrm{OD}, 150 \mathrm{MHz}\right): \delta 191.7,184.5,162.5,155.9,150.4,141.7,139.2,138.4,137.8,134.0$, 125.0, 121.8, 121.2, 120.4, 116.5, 101.8, 95.9, 68.4, 68.3, 56.6, 26.8, 21.9; HRESIMS: Calculated for $\left[\mathrm{C}_{23} \mathrm{H}_{22} \mathrm{O}_{7}+\mathrm{H}\right]^{+}: 411.1438$, Found: 411.1440 .

3-hydroxy-2-(5-hydroxy-1,4-dioxo-1,4-dihydronaphthalen-2-yl)-5-methylbenzaldehyde (IV53)<smiles>Cc1cc(O)c(C2=CC(=O)c3c(O)cccc3C2=O)c(C=O)c1</smiles>

A sample of $80 \mathrm{mg}(0.20 \mathrm{mmol})$ MOM ether $\mathbf{I V - 5 1}$ was placed in a $50 \mathrm{~mL}$ flask. A premixed solution of $5 \mathrm{~mL} 4.5 \mathrm{M} \mathrm{HCl}_{(\mathrm{aq} .)} / \mathrm{THF}(1: 1, \mathrm{v} / \mathrm{v})$ was added at RT. The solution was stirred on preheated oil bath $\left(65^{\circ} \mathrm{C}\right)$ for $10 \mathrm{~min}$ under Ar. The reaction mixture was then quickly cooled down to $0{ }^{\circ} \mathrm{C}$, and diluted with $20 \mathrm{~mL}$ EtOAc, followed by addition of $20 \mathrm{~mL}$ saturated aqueous $\mathrm{NaHCO}_{3}$. After separation, the aqueous layer was extracted with EtOAc and the pooled organic layer was washed with saturated brine and dried over $\mathrm{Na}_{2} \mathrm{SO}_{4}$. After removal of the solvent, the residue was subjected to silica gel column chromatography, eluting with hexane-EtOAc $(2: 1, \mathrm{v} / \mathrm{v})$ afforded deprotected product IV-53 $14 \mathrm{mg}$ (23\%). Orange solid, mp: 144-150 ${ }^{\circ} \mathrm{C} ; R_{f}$ $\left(\right.$ hexanes/EtOAc, 2:1 v/v) = 0.28; IR (thin film, $\mathrm{cm}^{-1}$ ) v 3250 (brs), 2925, 2860, 1670, 1637, 1604, 1453, 1363, 1310, 1244, 1162, 1047, 900; ${ }^{1} \mathrm{H}$ NMR (CD $\left.{ }_{3} \mathrm{OD}, 600 \mathrm{MHz}\right): \delta 9.8$ (s, 1H), 7.71 (dd, 
$J=8.4,7.8 \mathrm{~Hz}, 1 \mathrm{H}), 7.60(\mathrm{ddd}, J=7.2,1.2 \mathrm{~Hz}, 1 \mathrm{H}), 7.33(\mathrm{q}, J=1.2 \mathrm{~Hz}, 1 \mathrm{H}), 7.32(\mathrm{dd}, J=8.4$, $1.2 \mathrm{~Hz}, 1 \mathrm{H}), 7.09$ (d, $J=1.2 \mathrm{~Hz}, 1 \mathrm{H}), 6.87(\mathrm{~s}, 1 \mathrm{H}), 2.44$ (s, 3H); ${ }^{13} \mathrm{C}$ NMR $\left(\mathrm{CD}_{3} \mathrm{OD}, 150 \mathrm{MHz}\right): \delta$ 194.1, 191.6, 184.8, 162.5, 156.8, 149.9, 142.6, 137.9, 137.8, 137.6, 134.4, 127.2, 124.9, 123.6, 120.3, 118.7, 116.7, 21.3; HRESIMS: Calculated for $\left[\mathrm{C}_{18} \mathrm{H}_{12} \mathrm{O}_{5}+\mathrm{H}\right]^{+}$: 309.0758, Found: 309.0759 .

Jadomycin A (IV-1) $)^{44 a}$

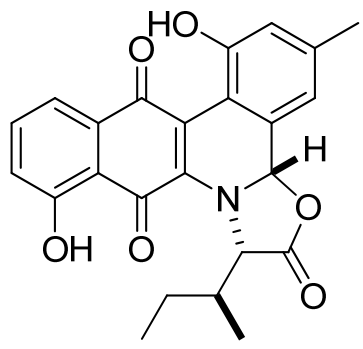

A suspension of $14 \mathrm{mg}(0.045 \mathrm{mmol})$ aldehyde IV-53 in $3 \mathrm{~mL}$ toluene was added $51 \mu \mathrm{L}(0.14$ mmol) isoleucine $t$-butyl ester. The mixture was stirred for 2 days at RT with The flask only loosely capped in order that air could get in. The mixture was directly subjected to silica gel column chromatography. Elution with hexane-EtOAc $(8: 1, \mathrm{v} / \mathrm{v})$ afforded a crude product, which was then treated with $0.15 \mathrm{~mL}$ TFA at RT for $1 \mathrm{~h}$. Then it was diluted with $20 \mathrm{~mL}$ EtOAc and subsequently washed with saturated aqueous $\mathrm{NaHCO}_{3}$ and saturated brine, then dried over $\mathrm{Na}_{2} \mathrm{SO}_{4}$. After removal the solvent, a NMR experiment showed it's a mixture of two isomers with 6:1 ratio with the cis product as major. The two isomers can't be separted with silica gel column chromatography. Jadomycin A (IV-1): $R_{f}$ (hexanes/EtOAc, $\left.2: 1 \mathrm{v} / \mathrm{v}\right)=0.65 ;{ }^{1} \mathrm{H}$ NMR $\left(\mathrm{CDCl}_{3}, 600 \mathrm{MHz}\right): \delta 11.72(\mathrm{~s}, 1 \mathrm{H}), 10.18(\mathrm{~s}, 1 \mathrm{H}), 7.81$ (d, $\left.J=7.8 \mathrm{~Hz}, 1 \mathrm{H}\right), 7.67$ (dd, $J=8.4$, $7.2 \mathrm{~Hz}, 1 \mathrm{H}), 7.25$ (d, J=7.2 Hz, 1H), 6.91 (s, 1H), 6.82 (s, 1H), 6.16 (s, 1H), 5.30 (d, $J=3.0 \mathrm{~Hz}$, 1H), $2.37(\mathrm{~s}, 3 \mathrm{H}), 1.83(\mathrm{~m}, 2 \mathrm{H}), 1.56(\mathrm{~m}, 1 \mathrm{H}), 1.04(\mathrm{t}, J=7.2 \mathrm{~Hz}, 1 \mathrm{H}), 0.45(\mathrm{~d}, J=6.6 \mathrm{~Hz}, 1 \mathrm{H})$; ${ }^{13} \mathrm{C}$ NMR $\left(\mathrm{CDCl}_{3}, 150 \mathrm{MHz}\right): \delta 185.7,183.5,170.0,162.0,154.1,143.3,142.1,137.7,133.1$, 129.9, 124.4, 122.1, 120.9, 120.9, 114.5, 113.3, 111.1, 87.2, 62.6, 40.0, 25.2, 21.3, 13.8, 11.9; HRESIMS: Calculated for $\left[\mathrm{C}_{24} \mathrm{H}_{21} \mathrm{NO}_{6}+\mathrm{H}\right]^{+}: 420.1442$, Found: 420.1443. 


\section{References and Notes}

${ }^{1}$ Shan, M.; O'Doherty, G. A. Org. Lett., 2006, 8, 5149-5152.

${ }^{2}$ (a) Smith, J. A.; Poteet-Smith, C. E.; Xu, Y.; Errington, T. M.; Hecht, S. M.; Lannigan, D. A. Cancer Res. 2005, 65, 1027-1034. (b) Xu, Y.; Smith, J. A.; Lannigan, D. A.; Hecht, S. M. Bioorg. Med. Chem. 2006, 14, 3974-3977.

${ }^{3}$ For the isolation of I-1a to I-1e see ref. $2 b$ and: (a) Matthes, H. W. D.; Luu, B.; Ourisson, G. Phytochem. 1980, 19, 2643-2650. For I-1b, see: (b) Kaouadji, M. Phytochem. 1990, 29, 22952297. For I-1b and I-1d, see: (c) Masuda, T.; Jitoe, A.; Kato, S.; Nakatani, N. Phytochem. 1991, 30, 2391-2392. For I-1a and I-1e, see: (d) Nakatani, N.; Jitoe, A.; Masuda, T. Agric. Biol. Chem. 1991, 55, 455-460. For I-1c see: (e) Usia, T.; Iwata, H.; Hiratsuka, A.; Watabe, T.; Kadota, S.; Tezuka, Y. J. Nat. Prod. 2004, 67, 1079-1083. (f) For I-1b, see: Deng, J.-Z.; Marshall, R.; Jones, S. H.; Johnson, R. K.; Hecht, S. M. J. Nat. Prod. 2002, 65, 1930-1932.

${ }^{4}$ (a) Muir, S. R.; Collins, G. J.; Robinson, S.; Hughes, S.; Bovy, A.; Ric De Vos, C. H.; van Tunen, A. J.; Verhoeyen, M. E. Nat. Biotechnol. 2001, 19, 470-474. (b) Hertog, M. G. L.; Feskens E. J. M.; Hollman P. C. H.; Katan, M. B. Kromhout D. Lancet 1993, 342, 1007-1011.

${ }^{5}$ Maloney, D. J.; Hecht, S. M. Org. Lett. 2005, 7, 1097-1099.

${ }^{6}$ (a) Babu, R. S.; Zhou, M.; O’Doherty, G. A. J. Am. Chem. Soc. 2004, 126, 3428-3429. (b) Li, M.; Scott, J. G.; O’Doherty, G. A. Tetrahedron Lett. 2004, 45, 1005-1009. (c) Guo, H.; O’Doherty, G. A. Org. Lett. 2005, 7, 3921-3924. (d) Shan, M.; O'Doherty, G. A. Org. Lett., 2006, $8,5149-5152$.

7 (a) Harris, J. M.; Keranen, M. D.; O’Doherty, G. A. J. Org. Chem. 1999, 64, 2982-2983. (b) Harris, J. M.; Keranen, M. D.; Nguyen, H.; Young, V. G.; O’Doherty, G. A. Carbohydr. Res. 2000, 328, 17-36. 
${ }^{8}$ (a) Guo, H.; O’Doherty, G. A. Org. Lett. 2005, 7, 3921-3924. (b) Guppi, S. R.; Zhou, M.; O’Doherty, G. A. Org. Lett. 2006, 8, 293-296. (c) Babu, R. S.; Guppi, S. R.; O’Doherty, G. A. Org. Lett. 2006, 8, 1605-1608.

${ }^{9}$ VanRheenen, V.; Kelly, R. C.; Cha, D. Y. Tetrahedron Lett. 1976, 17, 1973-1976.

${ }^{10}$ According to carbohydrate nomenclature, rhamnose is 6-deoxy-mannose.

${ }^{11}$ For the selective acylation of an axial alcohol see: ref. 7 and (a) King, J. F.; Allbutt, A. D. Can. J. Chem. 1970, 48, 1754-1769. (b) Lowary, T. L.; Hindsgaul, O. Carbohydr. Res. 1994, 251, 3367.

12 (a) Doerschuk, A. P. J. Am. Chem. Soc. 1952, 74, 4202-4203. (b) Pettit, G. R.; Cragg, G. M.; Suffness, M. J. Org. Chem. 1985, 50, 5060-5063.

${ }^{13}$ Shan, M.; O’Doherty, George A. Synthesis 2008, 3171-3179.

${ }^{14}$ Postema, M, H, D C-Glycoside Synthesis, 1st ed.; CRC Press: Boca Raton, 1995.

${ }^{15}$ Carbohydrate Mimics. Concepts and Methods; Chapleur, Y., Ed.; Wiley-VCH: Weinheim, New York, 1998.

${ }^{16}$ For a review on cyclitol chemistry, see: Arjona, O.; Gomez, A. M.; Lopez, C.; Plumet, J. Chem. Rev., 2007, 107, 1919-2036.

${ }^{17}$ For synthetic approach to cyclitol published after ref. 16, see: a) Ortiz, J. C.; Ozores, L.; Cagide-Fagin, F.; Alonso, R. Chem. Comm. 2006, 4239-4241. b) Chiara, J. L.; Garcia, A.; Sesmilo, E.; Vacas, T. Org. Lett. 2006, 8, 3935-3938. c) Chakraborty, C.; Vyavahare, V. P.; Dhavale, D. D. Tetrahedron, 2007, 63, 11984-11990. d) Lysek, R.; Schutz, C.; Favre, S.; O’Sullivan, A. C.; Pillonel, C.; Krulle, T.; Jung, P. M. J.; Clotet-Codina, I.; Este, J.; Vogel, R. Bioorg. Med. Chem. 2006, 14, 6355-6282. e) Corsaro, A.; Pistara, V.; Catelani, G.; D’Andrea, F.; Adamo, R.; Chiacchio, M. A. Tetrahedron Lett. 2006, 47, 6591-6594. f) Frigell, J.; Cumpstey, I. Tetrahedron Lett. 2007, 48, 9073-9076. g) Sardinha, J. Guieu, S.; Deleuze, A.; FernandezAlonso, M. C.; Rauter, A. P.; Sinay, P.; Marrot, J.; Jimenez-Babero, J.; Sollogoub, M. Carbohyd. 
Res. 2007, 342, 1689-1703. h) Plumet, J.; Gomez, A. M.; Lopez, J. C. Mini-Rev. in Org. Chem. 2007, 4, 201-216.

${ }^{18}$ Zhou, M.; O'Doherty, G. A. Current Topics in Medicinal Chemistry, 2008, 8, 114-125.

${ }^{19}$ An Achmatowicz reaction is the oxidative rearrangement of furyl alcohols to 2-substituted 6hydroxy-2H-pyran-3(6H)-ones. a) Achmatowicz, O. ; Bielski, R. Carbohydr. Res. 1977, 55, 165-176. b) Grapsas, I., K.; Couladouros, E. A.; Georgiadis, M. P. Pol. J. Chem. 1990, 64, 823826. For its use in carbohydrate synthesis see: c) Harris, J. M.; Keranen, M. D.; O’Doherty, G. A. J. Org. Chem. 1999, 64, 2982-2983. d) Harris, J. M.; Keranen, M. D.; Nguyen, H.; Young, V. G.; O'Doherty, G. A. Carbohydrate Res. 2000, 328, 17-36. For examples of oligosaccharides, see: e) Guo, H.; O'Doherty, G. A. Angew. Chem., Int. Ed. 2007, 46, 5206-5208. f) Babu, R. S.; Zhou, M.; O'Doherty, G. A. J. Am. Chem. Soc. 2004, 126, 3428-3429.

${ }^{20}$ a) Federspiel, M.; Fischer, R.; Hennig, M.; Mair, H.-J.; Oberhauser, T.; Rimmler, G.; Albiez, T.; Bruhin, J.; Estermann, H.; Gandert, C.; Gockel, V.; Gotzo, S.; Hoffmann, U.; Huber, G.; Janatsch, G.; Lauper, S.; Rockel-Stabler, O.; Trussardi, R.; Zwahlen, A. G. Org. Process Res. Dev. 1999, 3, 266-274. b) Trost, B. M.; Romero A. G. J. Org. Chem. 1986, 51, 2332-2342. c) Audia, J. E.; Boisvert, L.; Patten, A. D.; Villalobos, A.; Danishefsky, S. J. J. Org. Chem. 1989, 54, 3738-3740. d) Barros, M. T.; Maycock, C. D.; Ventura, M. R. J. Org. Chem. 1997, 62, 3984-3988.

${ }^{21}$ Tsuda, T.; Satomi, H.; Hayashi, T.; Saegusa T. J. Org. Chem. 1987, 52, 439-443.

22 a) Lipshutz, B. H.; Chrisman, W.; Noson, K.; Papa, P.; Sclafani, J. A.; Vivian, R. W.; Keith, J. M. Tetrahedron 2000, 56, 2779-2788. b) Mahoney, W. S; Stryker, J. M. J. Am. Chem. Soc. 1989, $111,8818-8823$.

${ }^{23}$ For recent review, see: Kulinkovich, O. G. Chem. Rev. 2003, 103, 2597-2632.

${ }^{24}$ a) Ojima, I.; Kogure, T. Organometallics, 1982, 1, 1390-1399. b) Tojo, Shingo; Isobe, M. Synthesis 2005, 8, 1237-1244. 
${ }^{25}$ For a review on Simmons-Smith cyclopropanation, see: a) Hoveyda, A. H.; Evans, D. A.; Fu, G. C. Chem. Rev. 1993, 93, 1307-1370. b) Lautens, M.; Klute, W.; Tam, W. Chem. Rev. 1996, 96, 49. c) Lebel, H.; Marcoux, J.-F.; Molinaro, C.; Charette, A. B. Chem. Rev. 2003, 103, $977-$ 1050.

${ }^{26}$ Acid and base opening see ref. 23; for examples of acid catalyzed opening, see: a) Schreiber, S. L.; Smith, D. B.; Schulte, G. J. Org. Chem. 1989, 54; 5994-5996. b) Paquette, L. A.; Ross, R. J.; Shi, Y. J. J. Org. Chem. 1990, 55, 1589-1598; for examples of base catalyzed opening, see: c) Barnier, J. P.; Blanco, L.; Rousseau, G.; Guibe-Jampel, E.; Fresse, I. J. Org. Chem. 1993, 58, 1570-1574.

${ }^{27}$ Park, S.-B.; Cha, J. K. Org. Lett. 2000, 2, 147-149.

${ }^{28}$ Tarros, M. T.; Maycock, C. D.; Ventura, M. R. J. Org. Chem. 1997, 62, 3984-3988.

${ }^{29}$ For examples of the trapping of organometallic intermediates with hydrogen see: (a) Ngai, M.-Y.; Kong, J.-R.; Krische, M. J. J. Org. Chem. 2007, 72, 1063-1072. (b) Iida, H.; Krische, M. J. Top. Curr. Chem. 2007, 279, 77-104. (c) Skucas, E.; Ngai, M.-Y.; Komanduri, V.; Krische, M. J. Acc. Chem. Res. 2007, 40, 1394-1401.

${ }^{30}$ Montchamp, J.-L.; Tian, F.; Hart, M. E.; Frost, J. W. J. Org. Chem. 1996, 61, 3897-3899. b) Barros, M. T.; Maycock, C. D.; Ventura, M. R. J. C. S., Perkin Trans. I, 2001, 166-173.

${ }^{31}$ Myers, A. G.; Zheng, B. J. Am. Chem. Soc. 1996, 118, 4492-4493. b) Myers, A. G.; Zheng, B. Tetrahedron Lett. 1996, 37, 4841-4844. c) Myers, A. G.; Zheng, B.; Movassaghi, M. J. Org. Chem. 1997, 62, 7507. d) Haukaas, M. H.; O'Doherty, G. A. Org. Lett. 2002, 4, 1771-1774. e) Zhou, M.; O'Doherty, G. A. Org. Lett. 2006, 8, 4339-4342.

${ }^{32}$ Mitsunobu, O.; Yamada, Y. Bull. Chem. Soc. Japan 1967, 40, 2380-2382. b) Mitsunobu, O. Synthesis 1981, 1-28.

${ }^{33}$ The optical rotations of our II-7 and (ent)-II-7 material were significantly higher than reported; however, the remaining spectral data for compound II-7 and (ent)-II-7 matched that for the 166 
reported data for (ent)-II-7 prepared by Redlich, see: Redlich, H.; Sudau, W.; Szadenings, A. K.; Vollerthun, R. Carbohydr. Res. 1992, 226, 57-78.

${ }^{34}$ Shan, M.; O’Doherty, George A. Org. Lett. 2008, 10, 3381-3384.

35 Johnson, D. A.; Liu, H.-w. Comprehensive Natural Products Chemistry; Elsevier: Amsterdam, New York, 1999; Vol. 3, pp 311-365.

${ }^{36}$ Thibodeaux, C.; Melancüon, C. E., III; Liu, H.-w. Nature 2007, 446, 1008-1016.

${ }^{37}$ Kren, V.; Martinkova, L. Curr. Med. Chem. 2001, 8, 1303-1328.

${ }^{38}$ Zhou, M.; O’Doherty, G. A. Current Topics in Medicinal Chemistry, 2008, 8, 114-125.

${ }^{39}$ Hong, L.; Zhao, Z.; Melancüon, C. E. III; Zhang, H.; Liu H.-w. J. Am. Chem. Soc. 2008, 130, 4954-4967.

${ }^{40}$ (a) Moffatt, J. G.; Khorana, H. G. J. Am. Chem. Soc. 1961, 83, 649-658. (b) Melancüon, C. E., III; Hong, L.; White, J. A.; Liu, Y. N.; Liu, H.-w. Biochemistry 2007, 46, 577-590.

${ }^{41}$ Ko, K.-S.; Zea, C. J.; Pohl, N. L. J. Am. Chem. Soc. 2004, 126, 13188-13189.

${ }^{42}$ VanRheenen, V.; Kelly, R. C.; Cha, D. Y. Tetrahedron Lett. 1976, 17, 1973-1976.

43 (a) Babu, R. S.; O’Doherty, G. A. J. Am. Chem. Soc. 2003, 125, 12406-12407. (b) Babu, R. S.; Zhou, M.; O’Doherty, G. A. J. Am. Chem. Soc. 2004, 126, 3428-3429. (c) Harris, J. M.; Keranen, M. D.; O’Doherty, G. A. J. Org. Chem. 1999, 64, 2982-2983. (d) Harris, J. M.; Keranen, M. D.; Nguyen, H.; Young, V. G.; O’Doherty, G. A. Carbohydr. Res. 2000, 328, 17-36. (e) Guo, H.; O’Doherty, G. A. Org. Lett. 2005, 7, 3921-3924. (f) Guppi, S. R.; Zhou, M.; O’Doherty, G. A. Org. Lett. 2006, 8, 293-296. (g) Babu, R. S.; Guppi, S. R.; O’Doherty, G. A. Org. Lett. 2006, 8, 1605-1608. (h) Shan, M.; O’Doherty, G. A. Org. Lett. 2006, 8, 5149-5152.

${ }^{44}$ (a) Ayer, S. W.; McInnes, A. G.; Thibault, P.; Walter, J. A.; Doull, J. L.; Parnell, T.; Vining, L. C. Tetrahedron Lett. 1991, 32, 6301-6304. (b) Doull, J. L.; Ayer, S. W.; Singh, A. K.; Thibault, 
P. J. Antibiot. 1993, 46, 869-871. (c) Doull, J. L.; Singh, A. K.; Hoare, M.; Ayer, S. W. J. Ind. Microbiol. 1994, 13, 120-125.

${ }^{45}$ Rix, U.; Zheng, J.; Remsing Rix, L. L.; Greenwell, L.; Yang, K. ; Rohr, J. J. Am. Chem. Soc. 2004, 126, 4496-4497.

${ }^{46}$ (a) Borissow, C. N.; Graham, C. L.; Syvitski, R. T.; Reid, T. R.; Blay, J.; Jakeman, D. L. Chembiochem 2007, 8, 198-1203. (b) J.-T. Zheng, Rix, U.; Zhao, L.; Mattingly, C.; Adams, V.; Chen, Q.; Rohr, J.; Yang, K.-Q. J. Antibiot. 2005, 58, 405-408. (c) Jakeman, D. L.; Bandi, S.; Graham, C. L.; Reid, T. R.; Wentzell, J. R.; Douglas, S. E. Antimicrob. Agents Chemother. ASAP.

${ }^{47}$ Wang, L.; White, R. L.; Vining, L. C. Microbiology 2002, 148, 1091-1103.

${ }^{48}$ (a) Jakeman, D. L.; Farrell, S.; Young, W.; Doucet, R. J.; Timmons, S. C. Bioorg. Med. Chem. Lett. 2005, 15, 1447-1449. (b) Jakeman, D. L., Graham, C. L.; Reid, T. R. Bioorg. Med. Chem. Lett. 2005, 15, 5280-5283. (c) Burdock, T. J.; Giffin, A. H.; Brooks, M.S.; Ghaly, A.E. Am. J. Biochem. Biotech. 2008, 4, 7-18.

49 (a) Syvitski, R. T.; Borissow, C. N.; Graham, C. L.; Jakeman, D. L. Org. Lett. 2006, 8, 697700. (b) Jakeman, D. L.; Borissow, C. N.; Graham, C. L.; Timmons, S. C.; Reid, T. R.; Syvitski, R. T. Chem. Commun. 2006, 3738-3740. (c) Kharel, M. K.; Zhu, L.; Liu, T.; Rohr, J. J. Am. Chem. Soc. 2007, 129, 3780-3781.

${ }^{50}$ Zhou, M.; O'Doherty, G. A. Org. Lett., 2006, 8, 4339-4342.

${ }^{51}$ (a) Pauls, H. W.; Fraser-Reid, B. J. Carbohydr. Chem. 1985, 4, 1-14. (b) Martin, S. F.; Zinke, P. W. J. Org. Chem. 1991, 56, 6600-6606. (c) Cardillo, G.; Orena, M. Tetrahedron 1990, 46, 3321-3408.

52 (a) Babu, R. S.; Zhou, M.; O’Doherty, G. A. J. Am. Chem. Soc. 2004, 126, 3428-3429. (b) Li, M.; Scott, J. G.; O’Doherty, G. A. Tetrahedron Lett. 2004, 45, 1005-1009. (c) Guo, H.; 
O’Doherty, G. A. Org. Lett. 2005, 7, 3921-3924. (d) Shan, M.; O'Doherty, G. A. Org. Lett., 2006, $8,5149-5152$.

${ }^{53}$ Chatgilialoglu, C.; Griller, D.; Lesage, M. J. Org. Chem. 1988, 53, 3641-3642.

54 Turner, F. A.; Gearien, J. E. J. Org. Chem. 1959, 24, 1952-1955.

${ }^{55}$ Tietze, L. F.; Singidi, R. R.; Gericke, K. M. Chem. Eur. J. 2007, 13, 9939-9947.

${ }^{56}$ Couladouros, E. A.; Plyta, Z. F.; Papageorgiou, V. P. J. Org. Chem. 1996, 61, 3031-3033.

${ }^{57}$ Dampier, M. F.; Chen, C. W.; Whitlock, H. W. Jr.; J. Am. Chem. Soc. 1976, 98 , 7064-7069.

${ }^{58}$ De Frutos, $\mathrm{O}^{\prime}$.; Atienza, C.; Echavarren, A. M. Eur. J. Org. Chem. 2001, 163-171

${ }^{59}$ Jung, M. E.; Hagenah, J. A. J. Org. Chem. 1987, 52, 1889-1902.

${ }^{60}$ These known compounds were characterized based on the comparison with authentic sample that our group prepared before: (a) Guo, H.; O’Doherty, G. A. Org. Lett. 2005, 7, 3921-3924. (b) Li, M.; Scott, J. G.; O’Doherty, G. A. Tetrahedron Lett. 2004, 45, 1005-1009.

${ }^{61}$ Ohkuma, T.; Koizumi, M.; Yoshida, M.; Noyori R. Org. Lett., 2000, 2, 1749-1751.

62 (a) Matthes, H. W. D.; Luu, B.; Ourisson, G. Phytochemistry 1980, 19, 2643-2650. (b) Kaouadji, M. Phytochemistry 1990, 29, 2295-2297. (c) Masuda, T.; Jitoe, A.; Kato, S.; Nakatani, N. Phytochemistry 1991, 30, 2391-2392. (d) Deng, J.-Z.; Marshall, R.; Jones, S. H.; Johnson, R. K.; Hecht, S. M. J. Nat. Prod. 2002, 65, 1930-1932.

${ }^{63}$ Usia, T.; Iwata, H.; Hiratsuka, A.; Watabe, T.; Kadota, S.; Tezuka, Y. J. Nat. Prod. 2004, 67, 1079-1083.

${ }^{64}$ Nakatani, N.; Jitoe, A.; Masuda, T. Agric. Biol. Chem. 1991, 55(2), 455-460.

${ }^{65}$ Binkley, R. W.; Schneider, J. S. J. Carbohydr. Chem. 1988, 7, 157-167. 
${ }^{66}$ Roush, W. R. and Neitz, R. J. J. Org. Chem., 2004, 69, 4906-4912.

${ }^{67}$ Brown, P. M. and Thomson, R. H. J. Chem. Soc., Perkin Trans. 1, 1976, 997-1000.

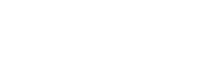

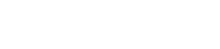

Hage

$\mathrm{n}$ 


\section{Supporting Spectra}

For

\section{Asymmetric Synthesis of SL0101, Cyclitol and Jadomycin \\ Mingde Shan}

Dissertation submitted to the

Eberly College of Arts and Sciences

at West Virginia University

in partial fulfillment of the requirements

for the degree of

Doctor of Philosophy

In

Organic Chemistry

George A. O'Doherty, Ph.D., Chair

Patrick S. Callery, Ph.D.

Jeffrey L. Petersen, Ph.D.

X. Michael Shi, Ph.D.

Björn C. G. Söderberg, Ph.D.

C. Eugene Bennett Department of Chemistry

Morgantown, West Virginia

2009

Keywords: SL0101, Cyclitol and Jadomycin

Copyright 2009 Mingde Shan 


\section{Table of Contents for Spectra}

Compound No.

Page No. for NMR Spectra

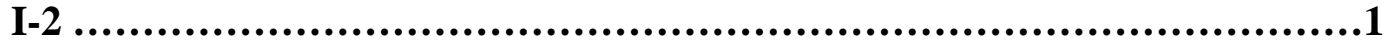

I-11

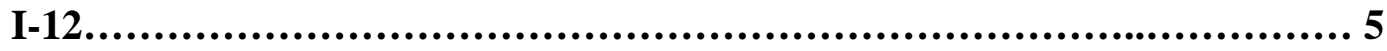

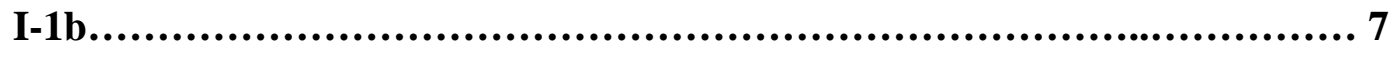

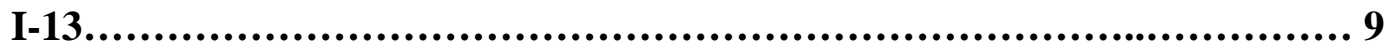

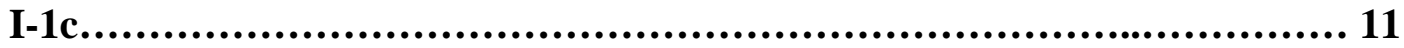

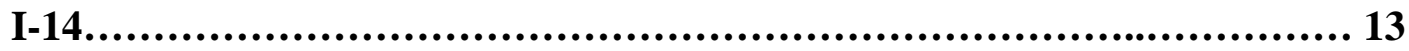

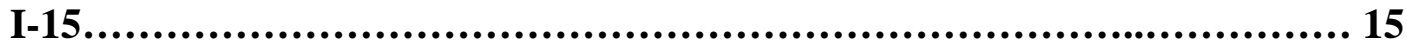

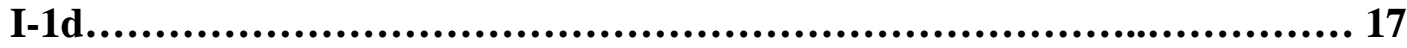

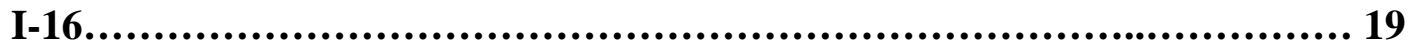

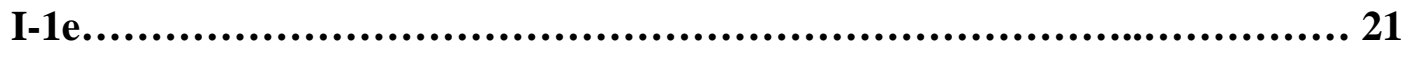

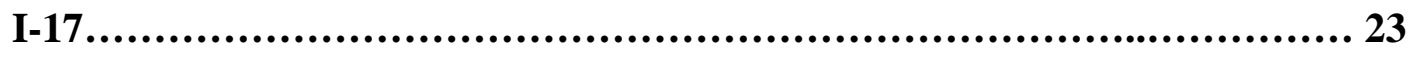

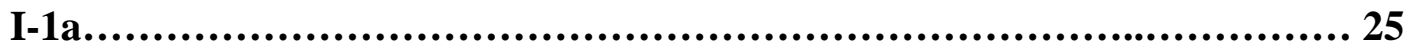

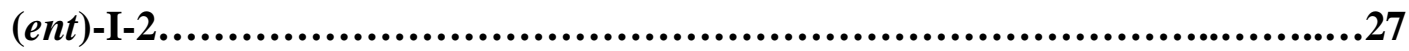

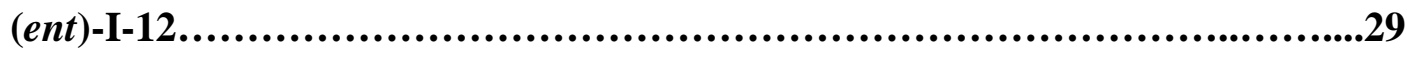

(ent)-I-1b......................................................................31

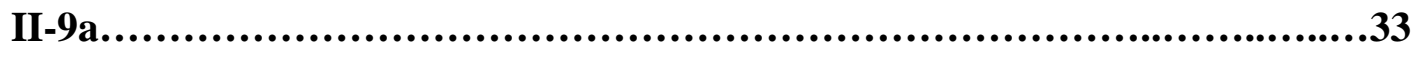

II-9b...........................................................................35

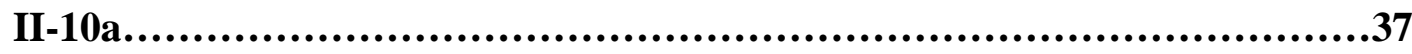

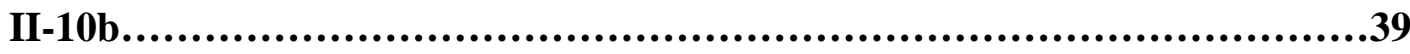

II-11a.........................................................................41 
II-13......................................................................43

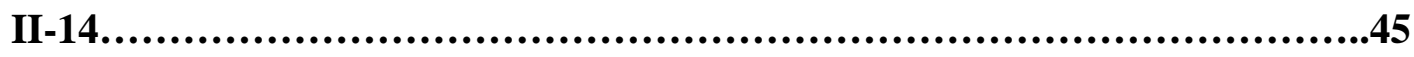

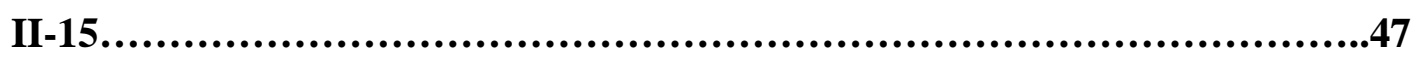

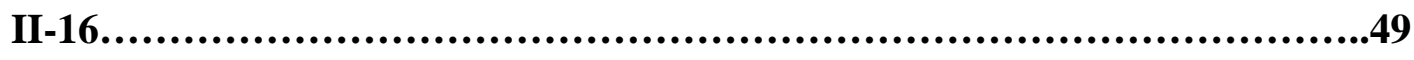

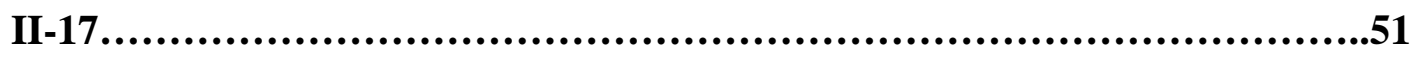

II-7..........................................................................53

II-18b...........................................................................55

II-19a.....................................................................57

II-19b....................................................................59

II-20.........................................................................61

II-20a...........................................................................63

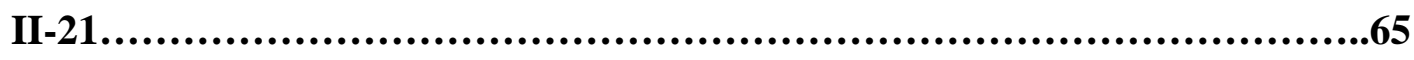

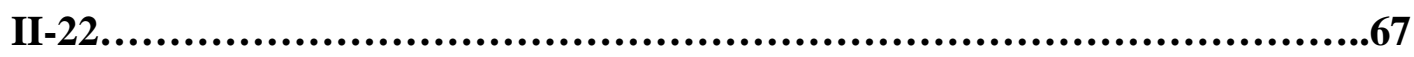

II-8.......................................................................69

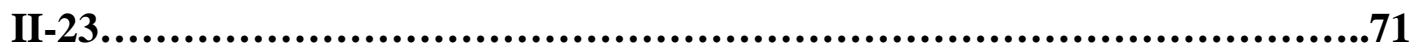

(ent)-II-15..............................................................73

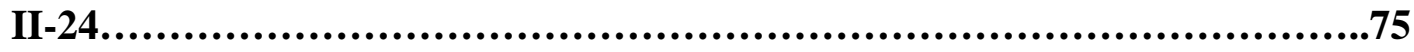

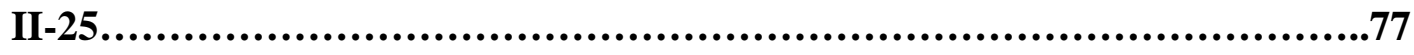

II-12b..........................................................................79

II-26.......................................................................81

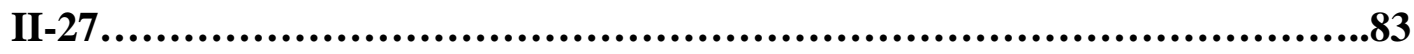

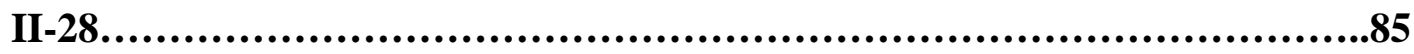

(ent)-II-7..............................................................87

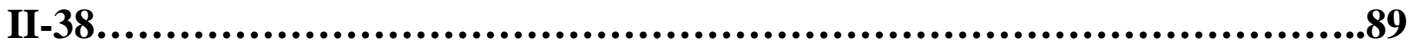


II-39...................................................................91

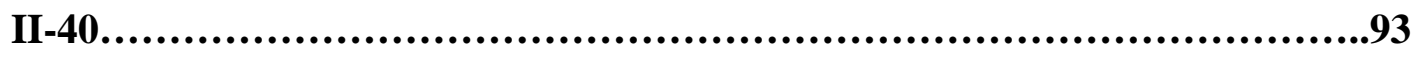

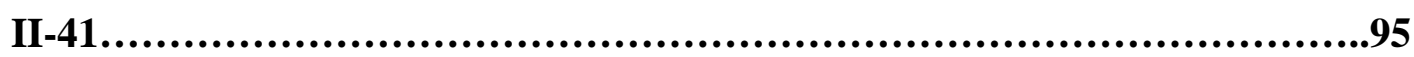

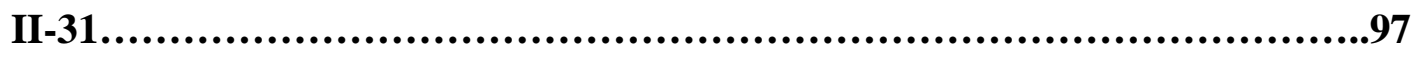

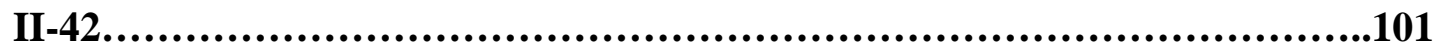

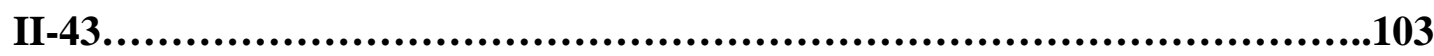

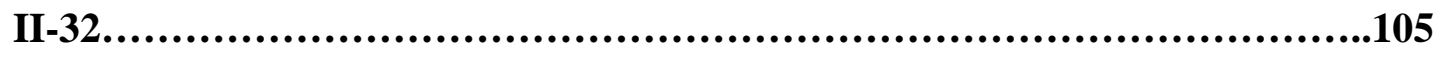

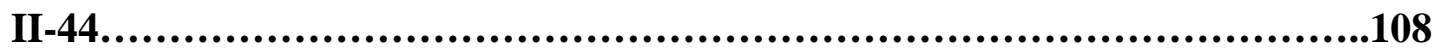

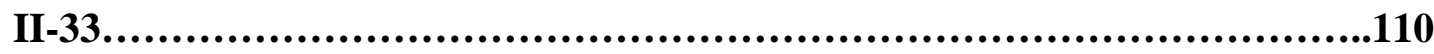

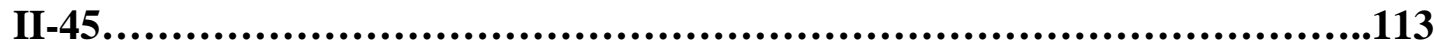

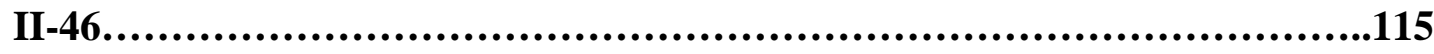

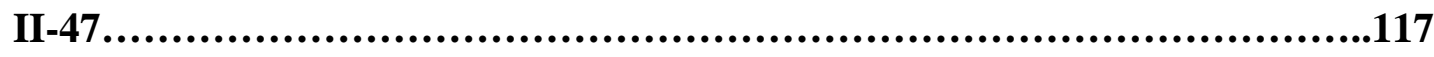

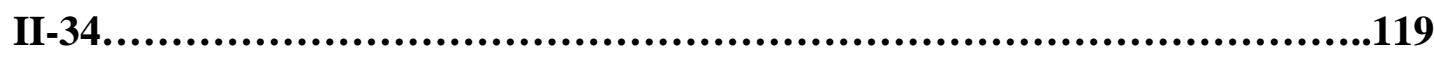

II-27a......................................................................122

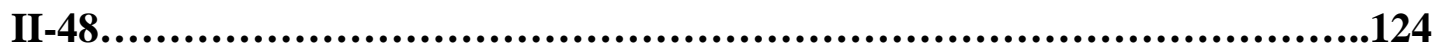

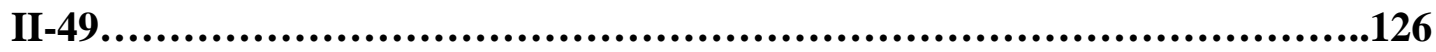

II-49a........................................................................128

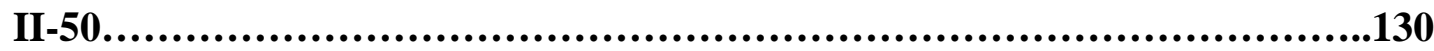

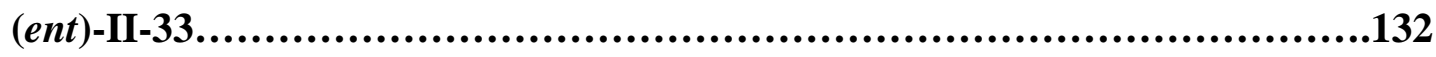

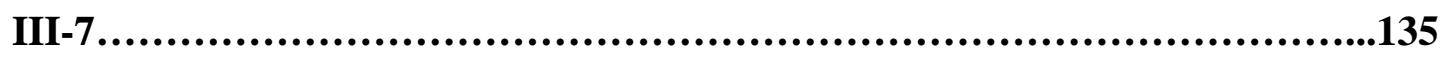

III-21.......................................................................137

III-22..................................................................139

III-23......................................................................141

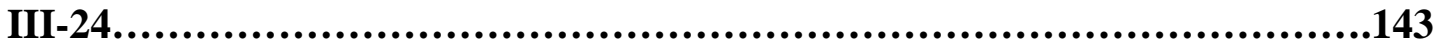




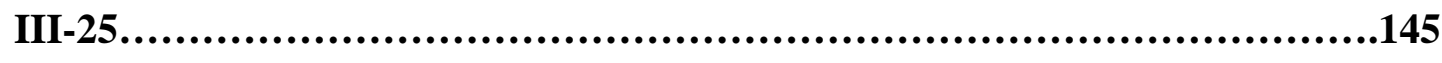

III-26...................................................................147

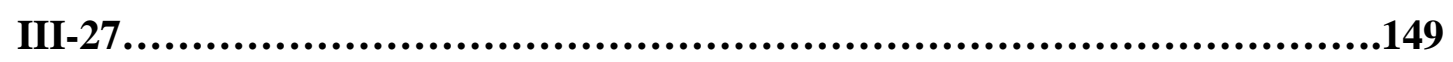

III-28..........................................................................151

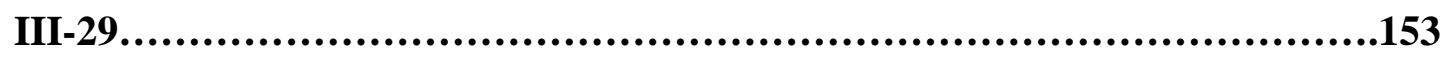

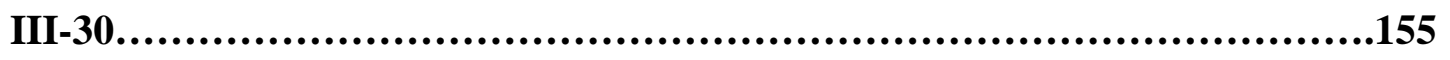

III-31........................................................................157

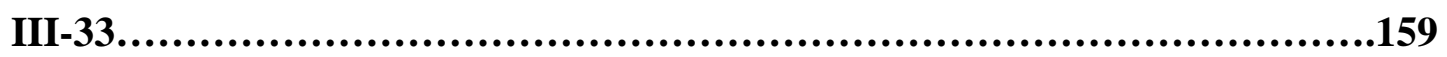

III-9......................................................................161

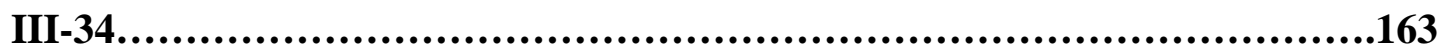

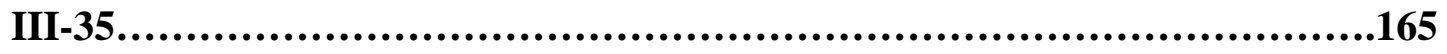

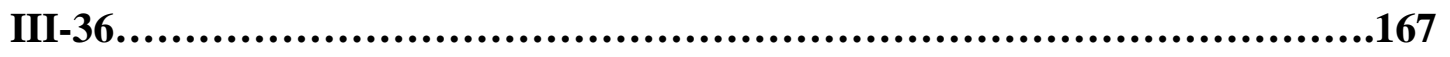

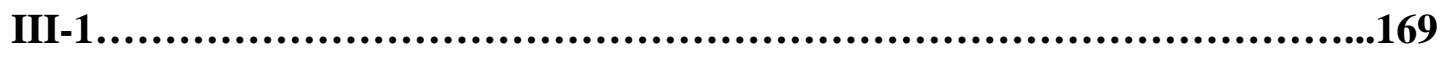

III-37......................................................................171

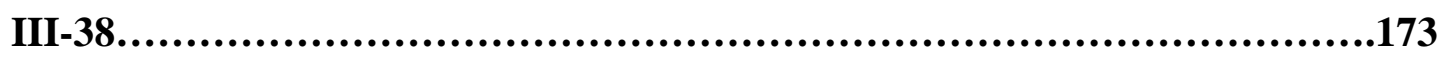

III-2...................................................................175

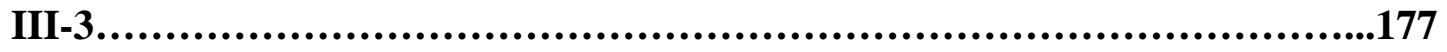

(ent)-III-9......................................................................179

(ent)-III-34................................................................181

(ent)-III-35.............................................................183

(ent)-III-36.............................................................185

(ent)-III-1.......................................................................187

(ent)-III-37.................................................................189

(ent)-III-38...................................................................191 


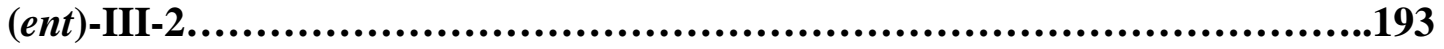

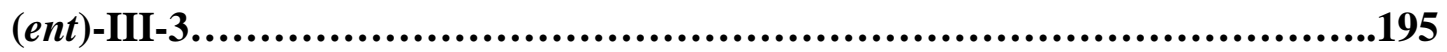

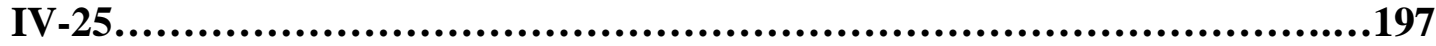

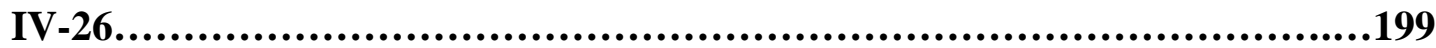

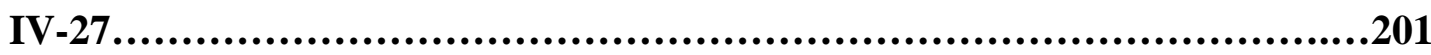

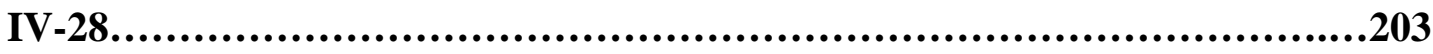

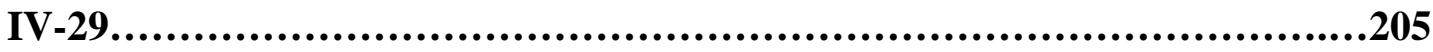

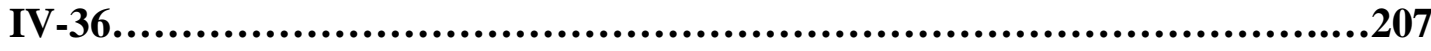

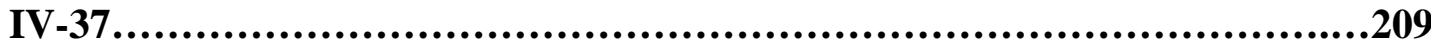

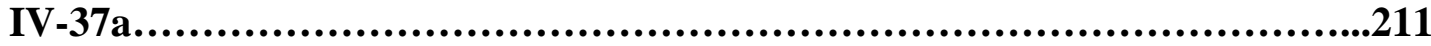

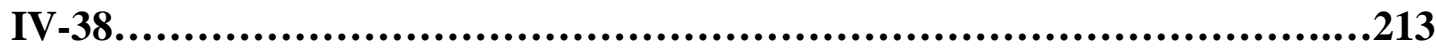

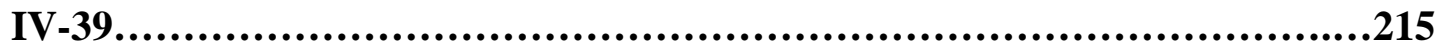

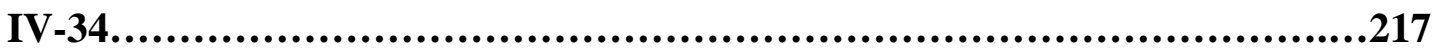

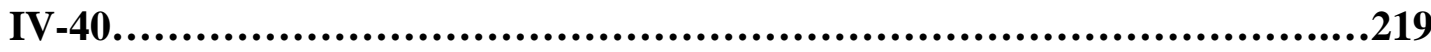

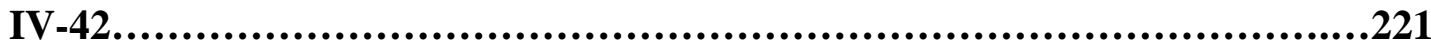

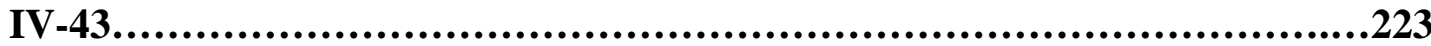

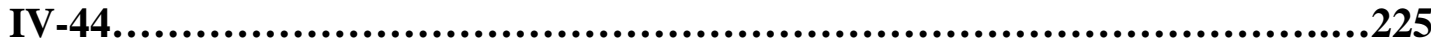

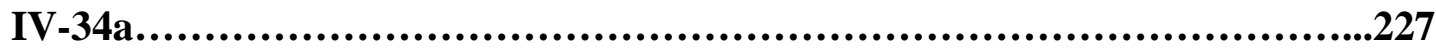

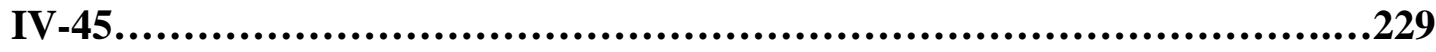

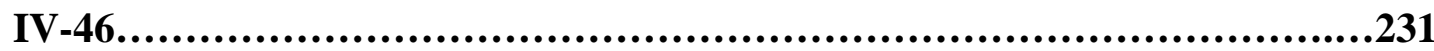

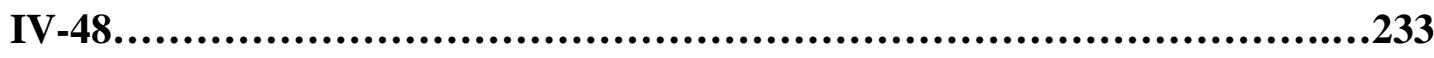

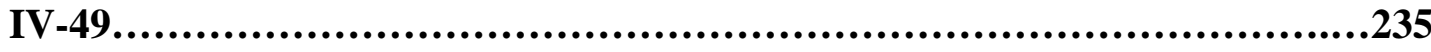

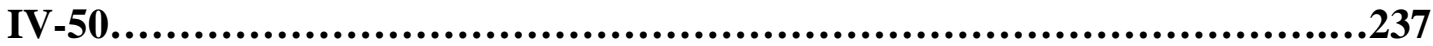

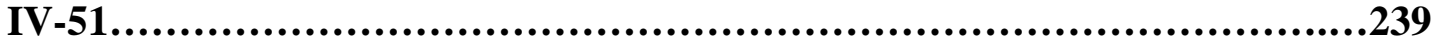




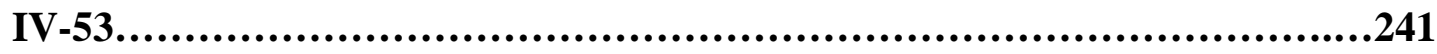

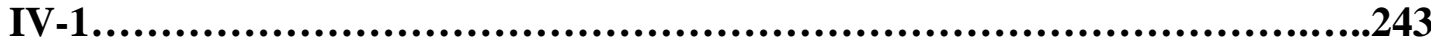




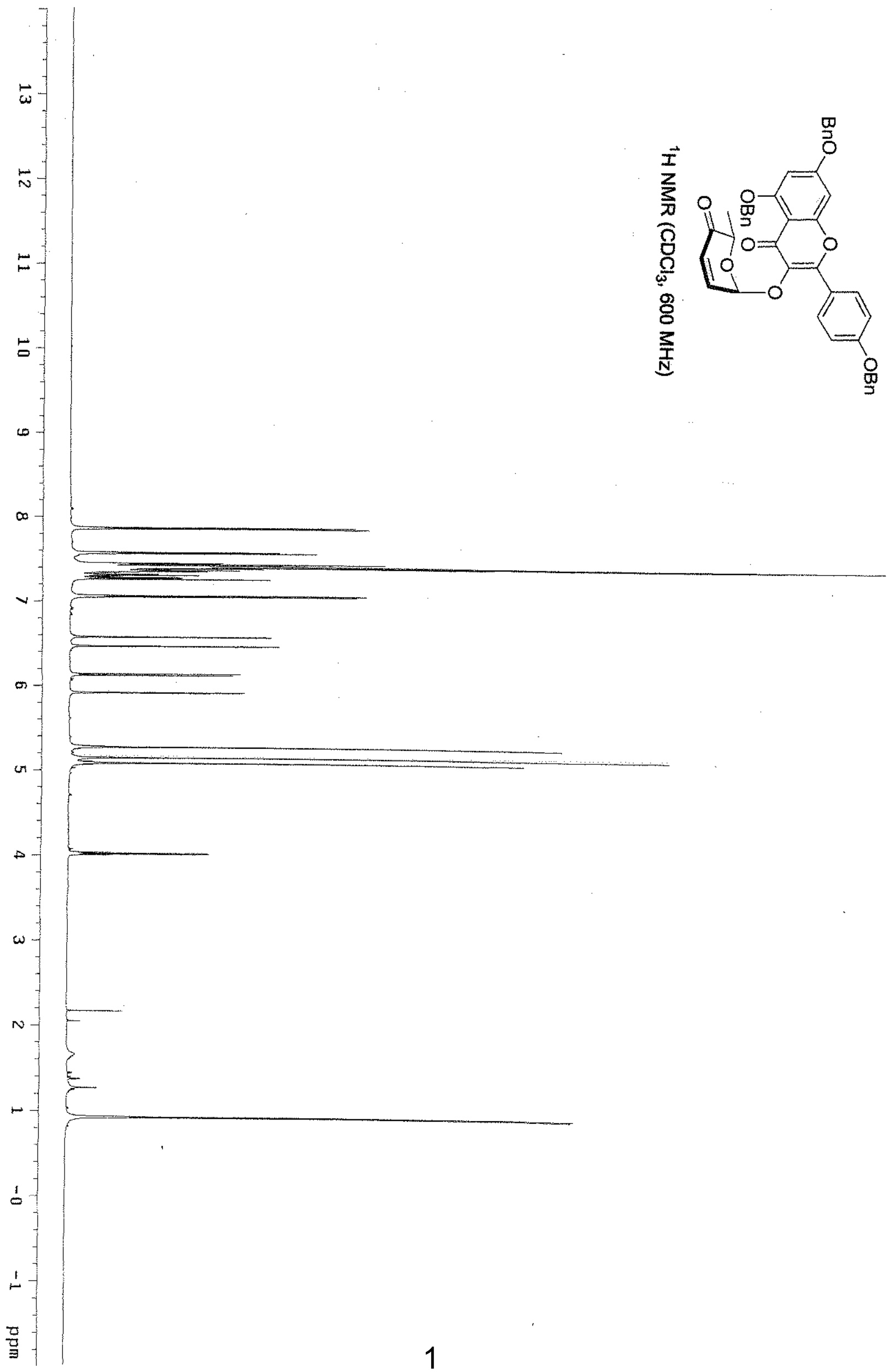




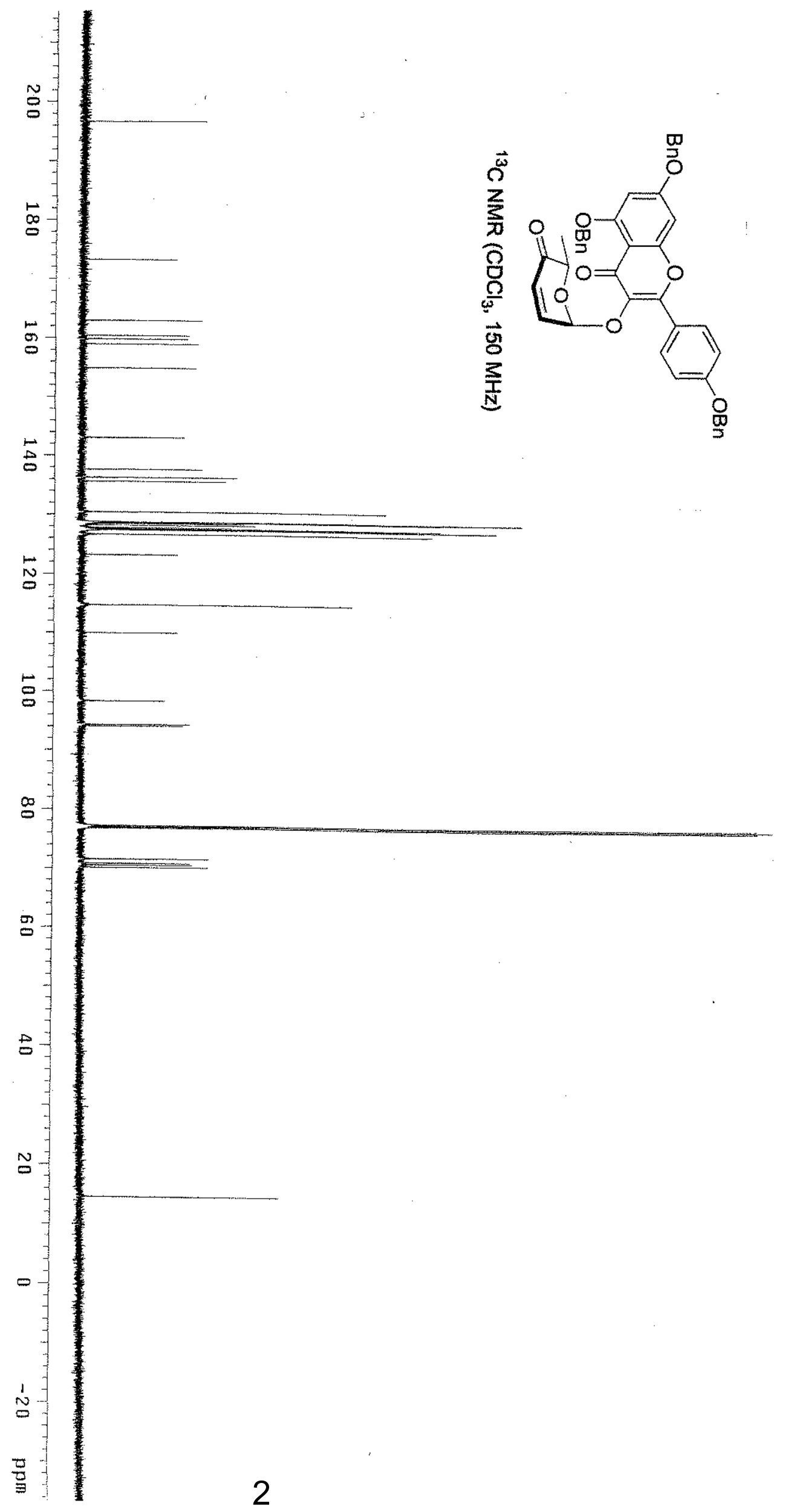




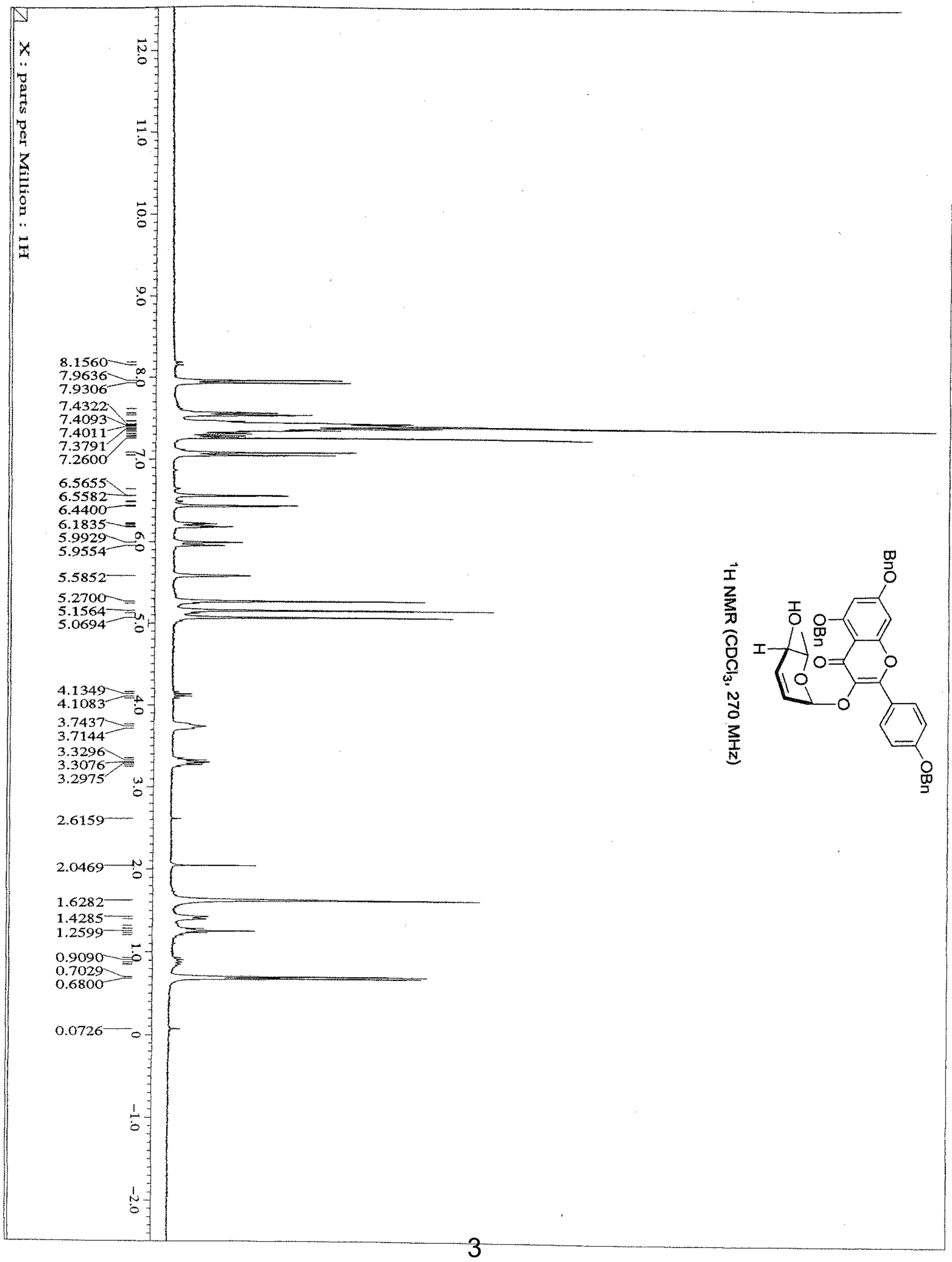




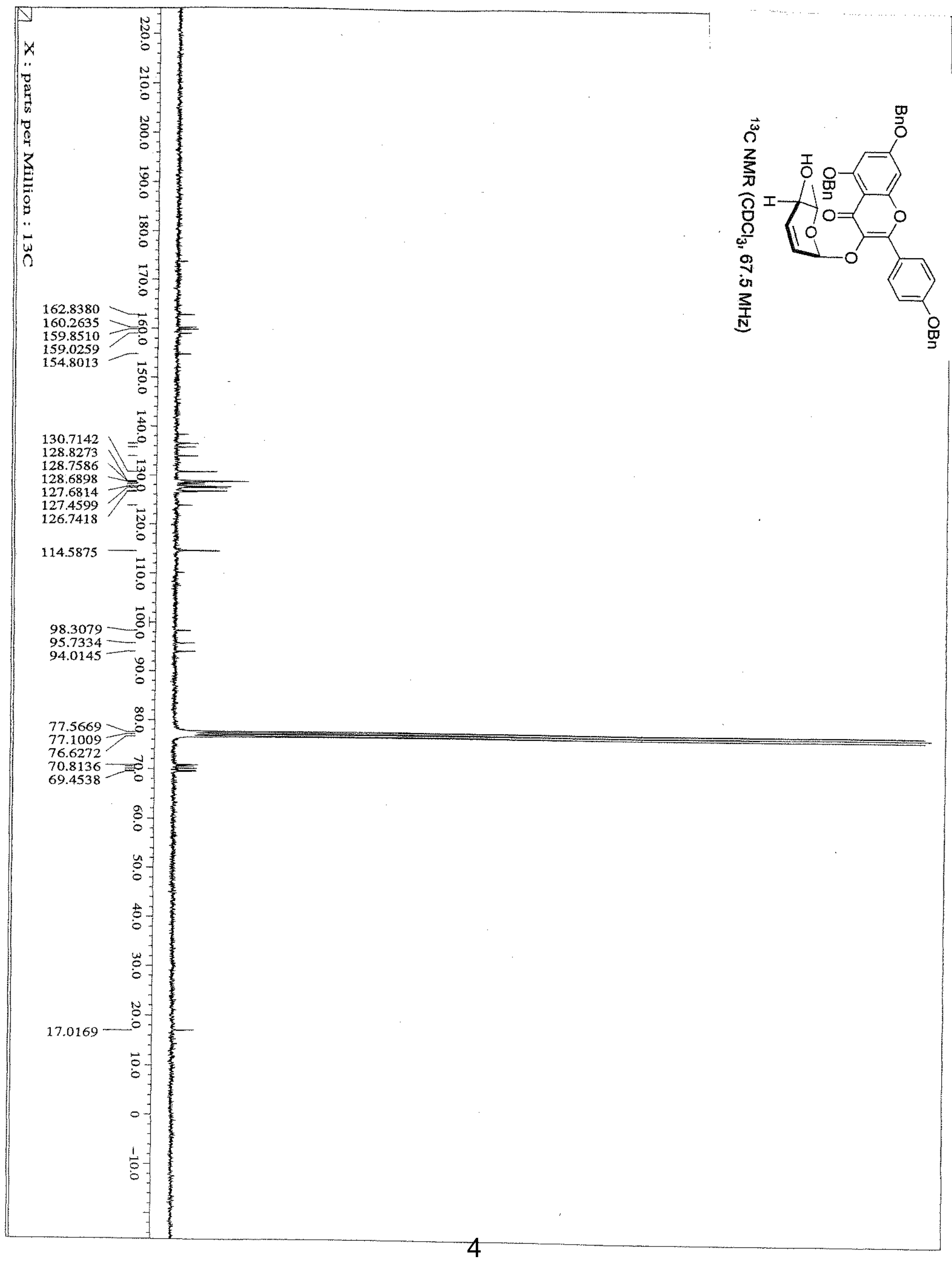




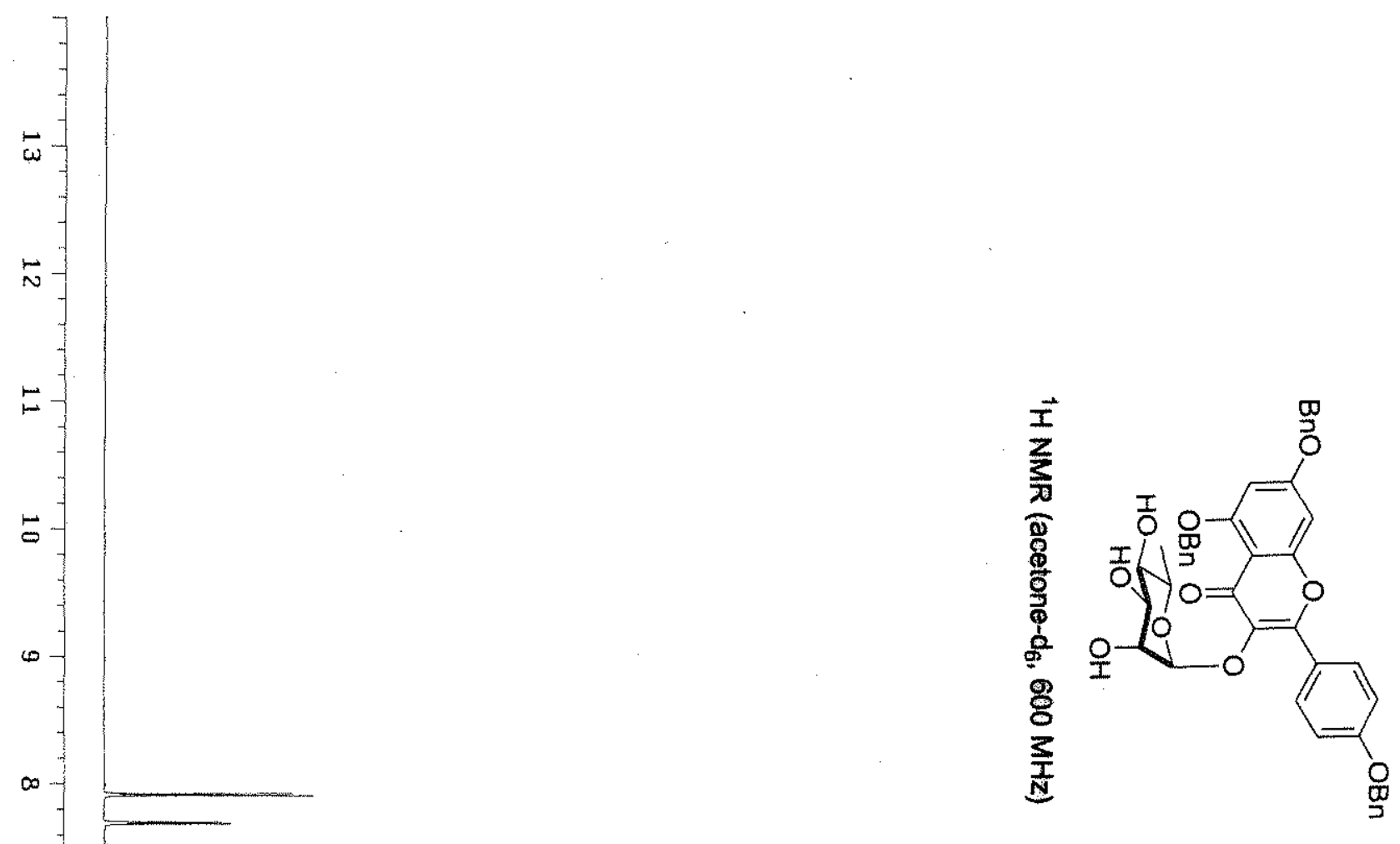




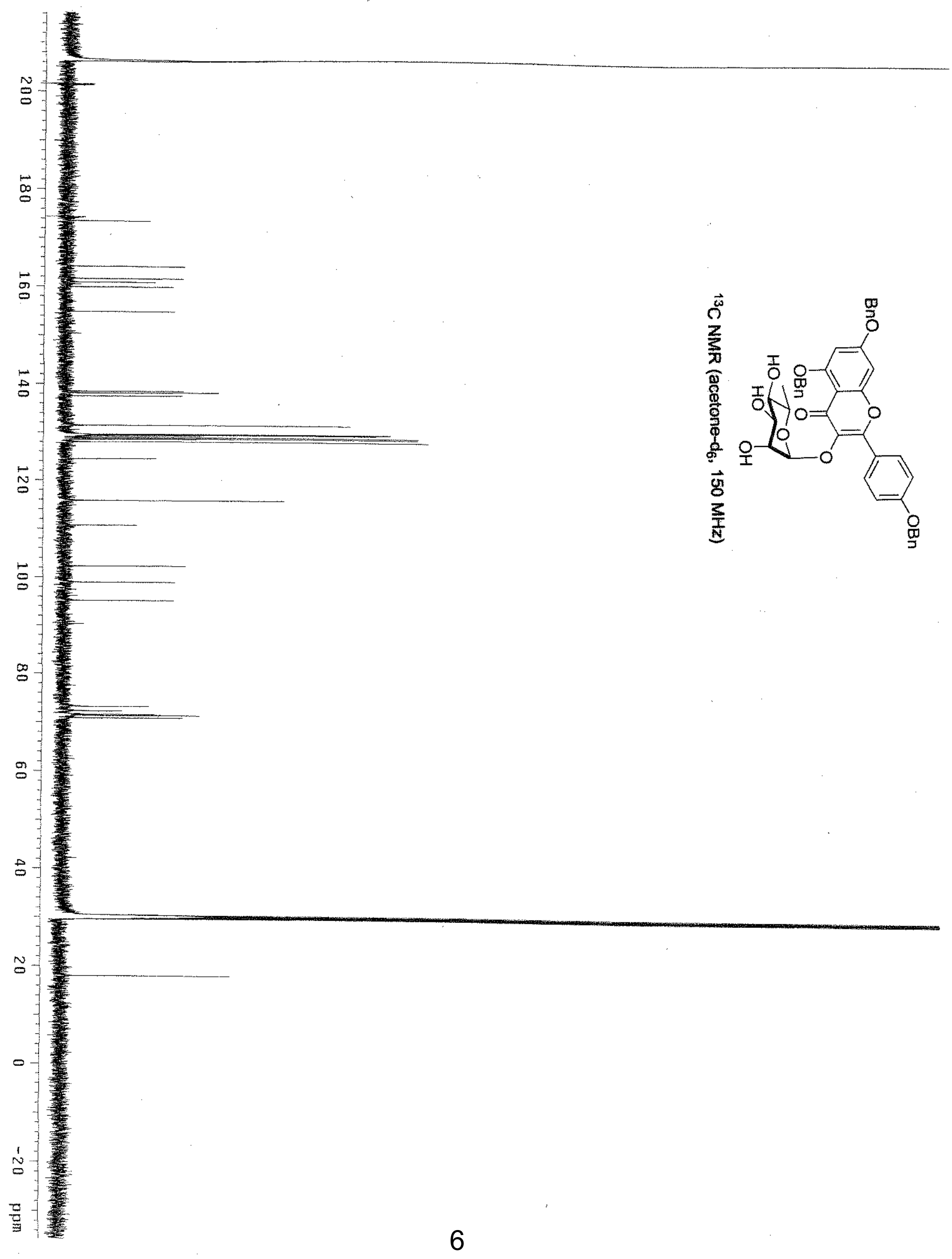




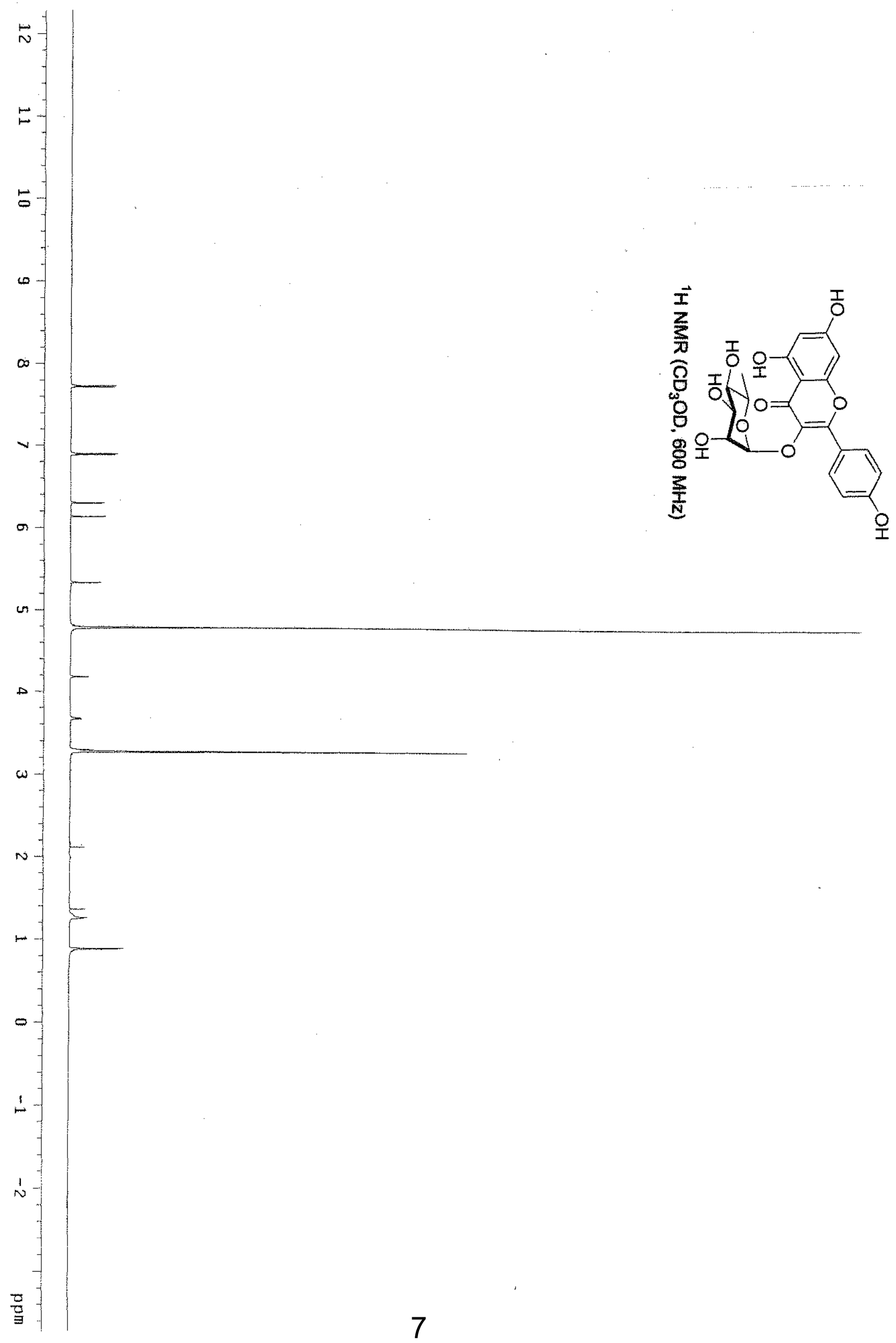




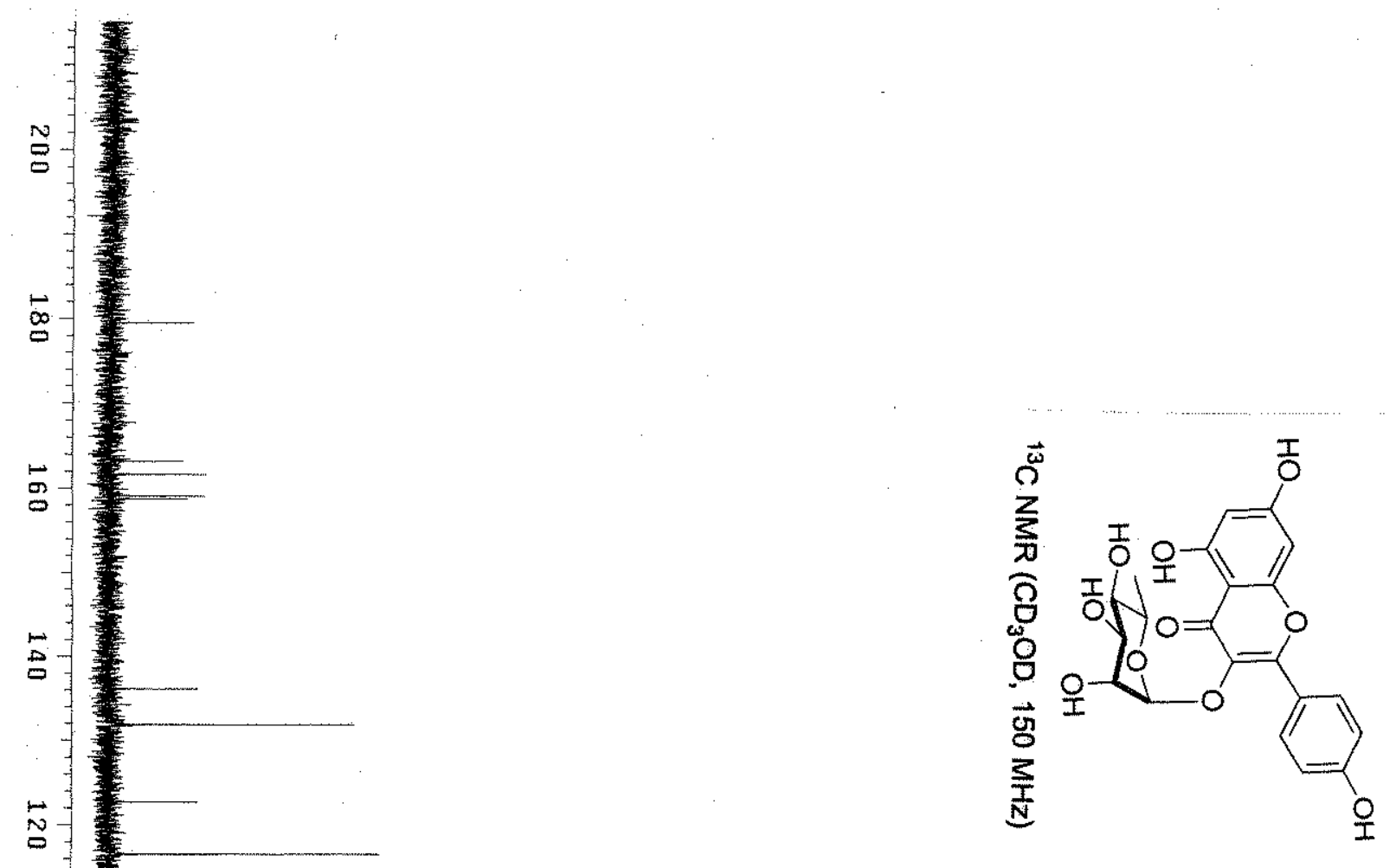




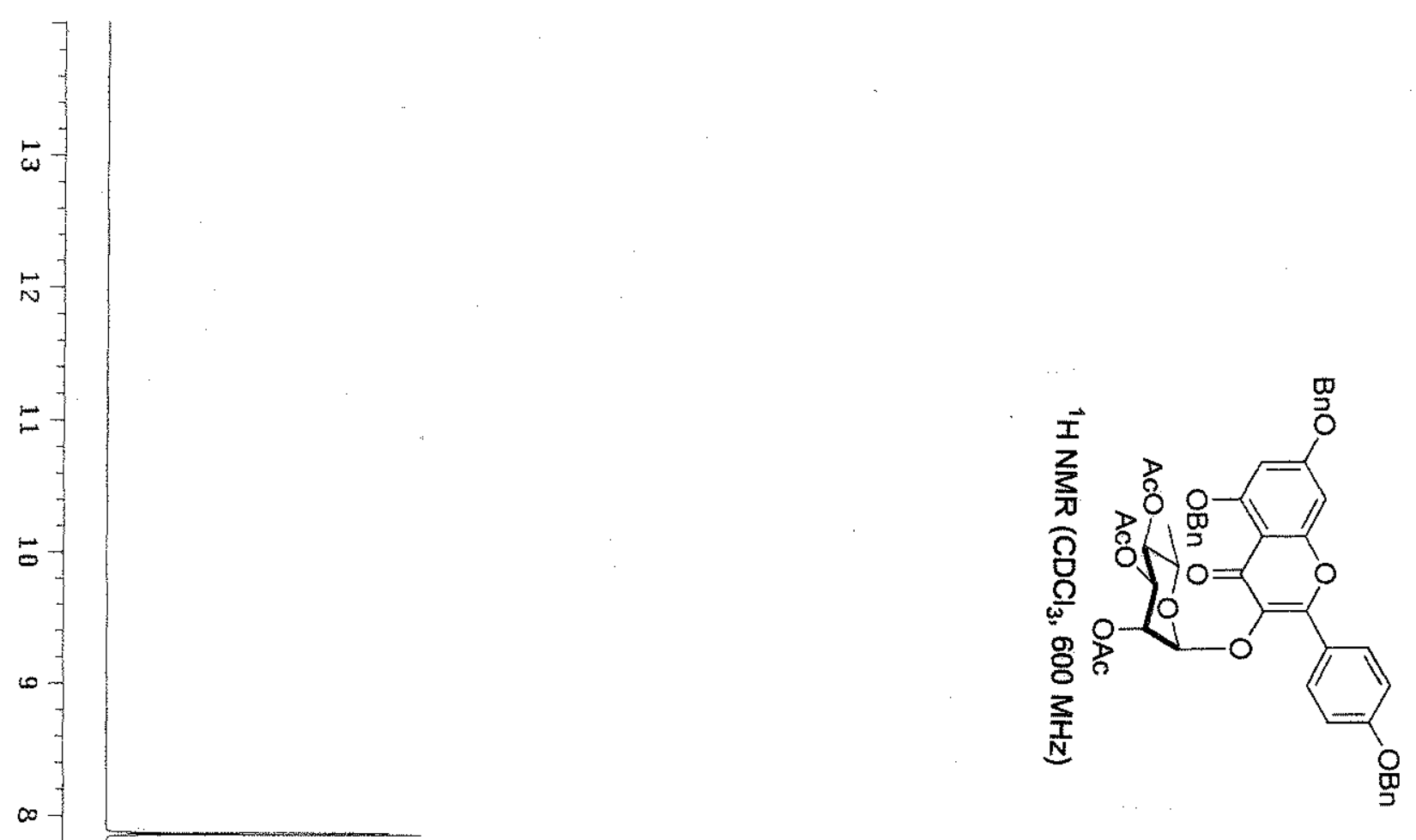




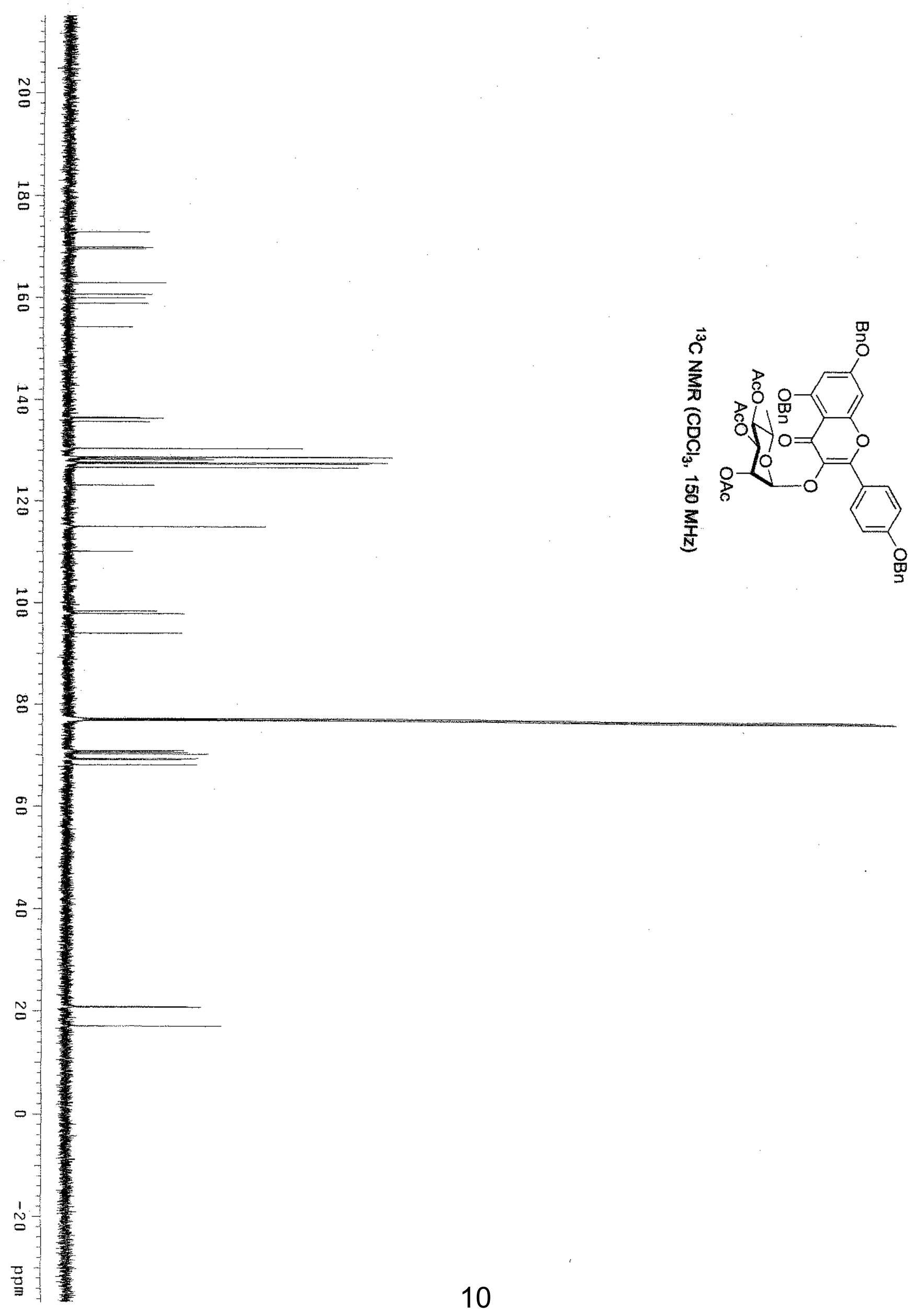




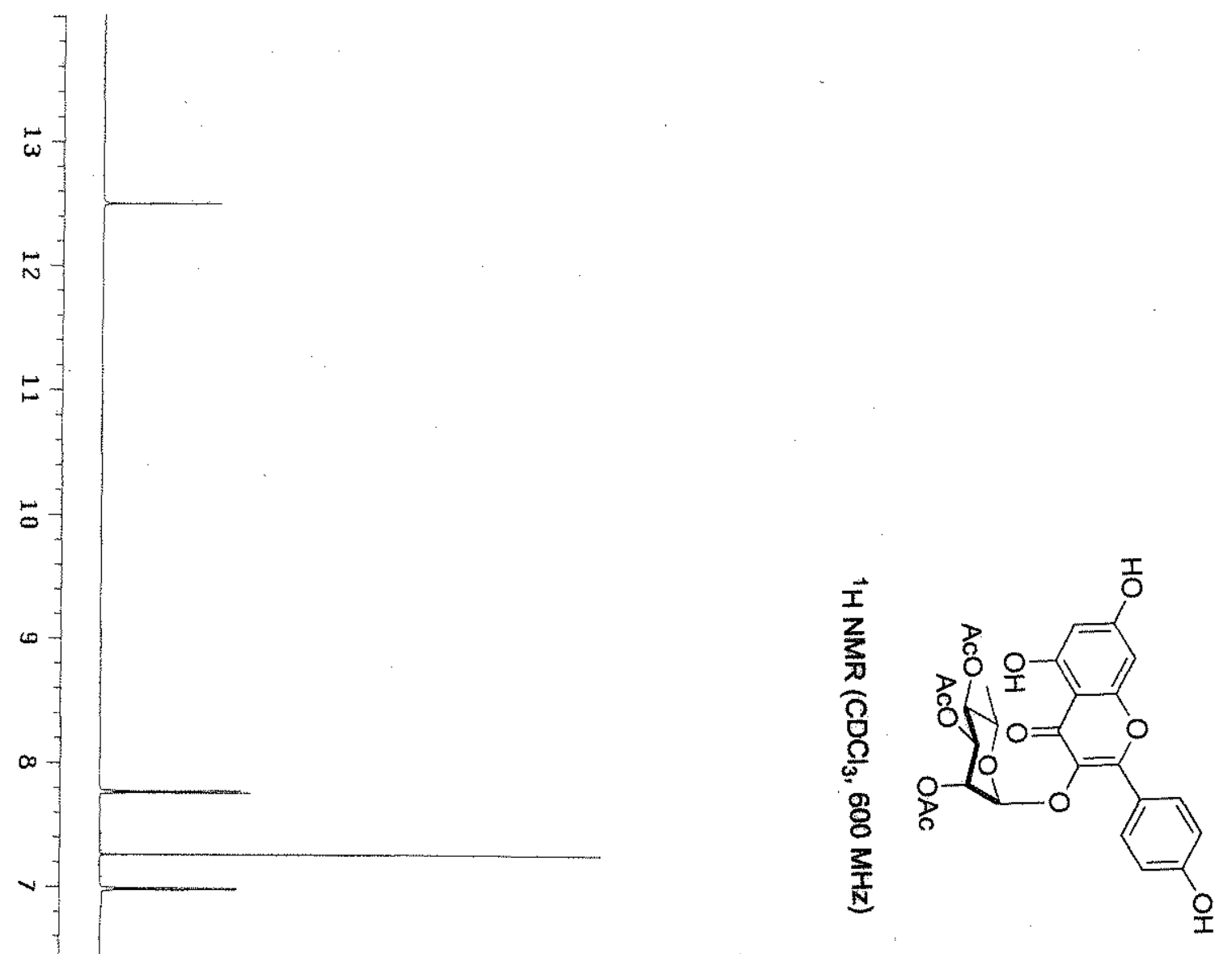




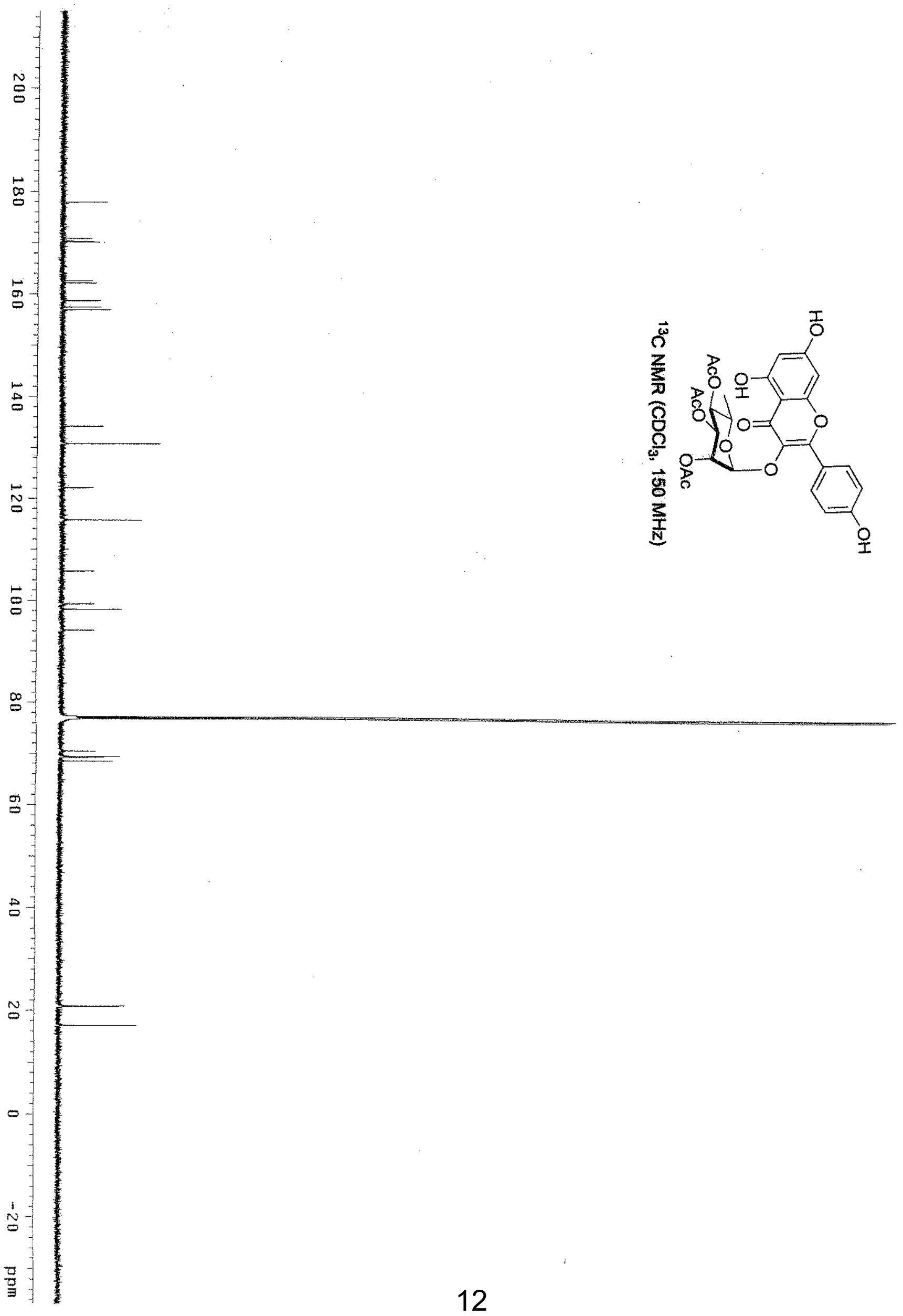




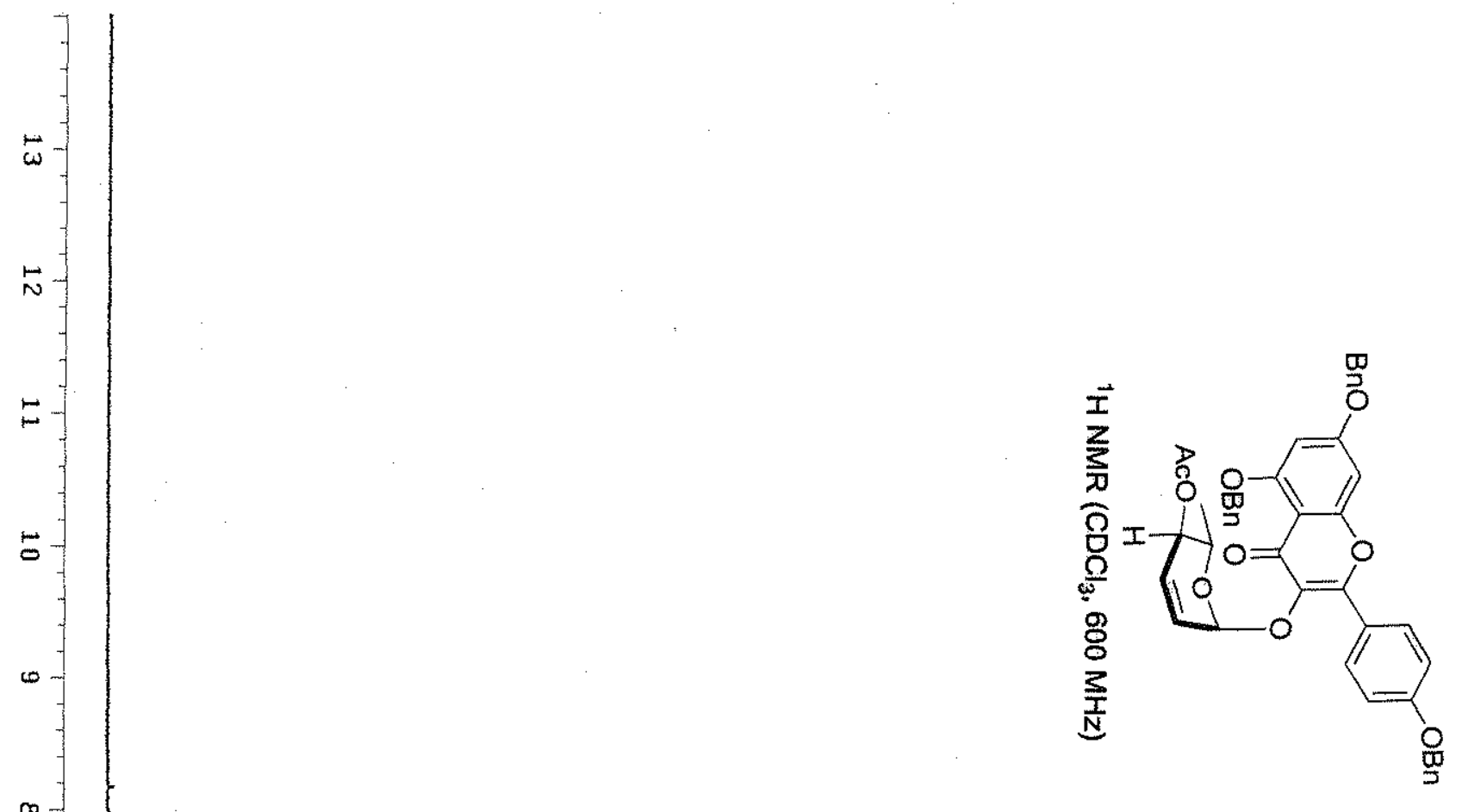



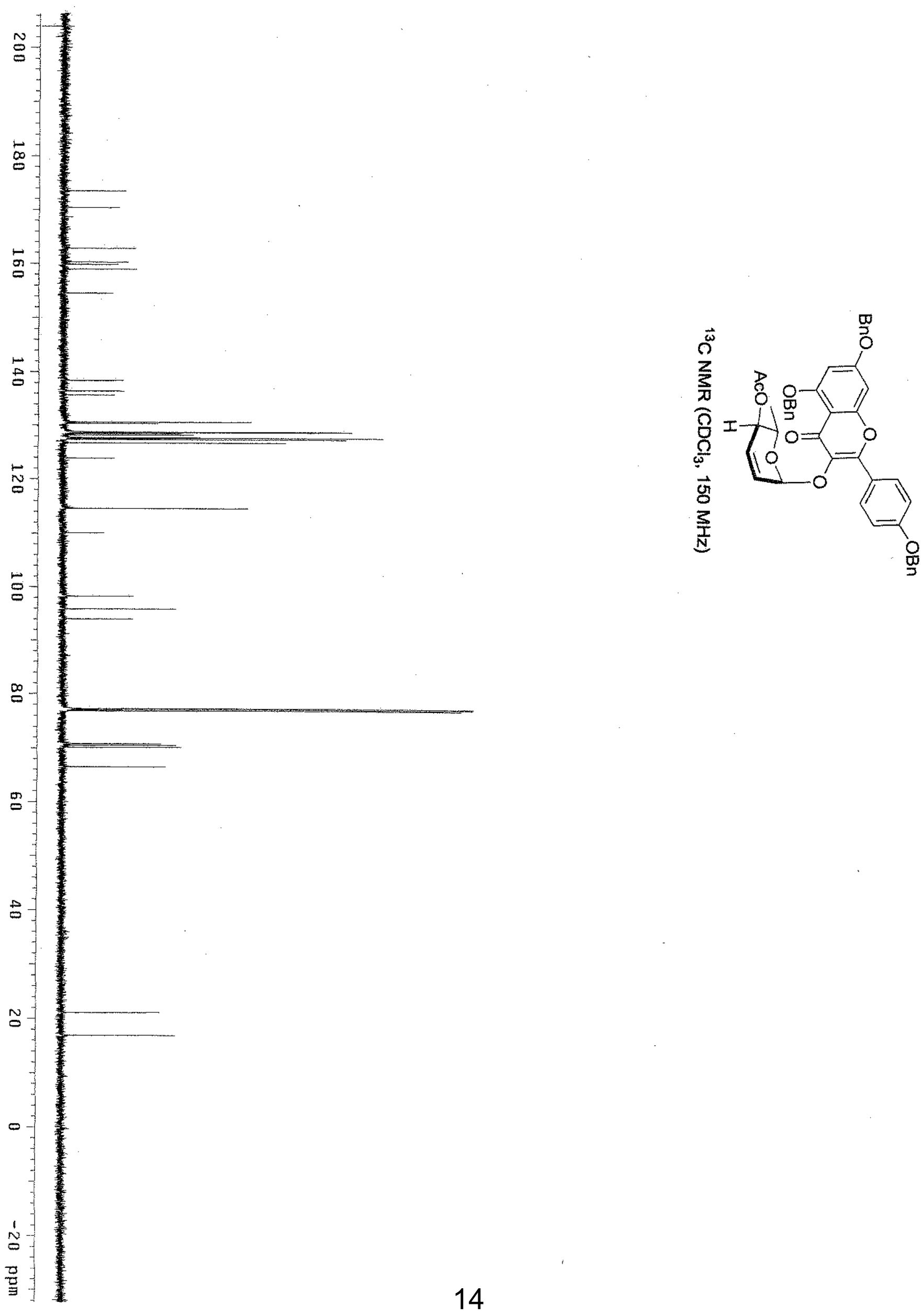


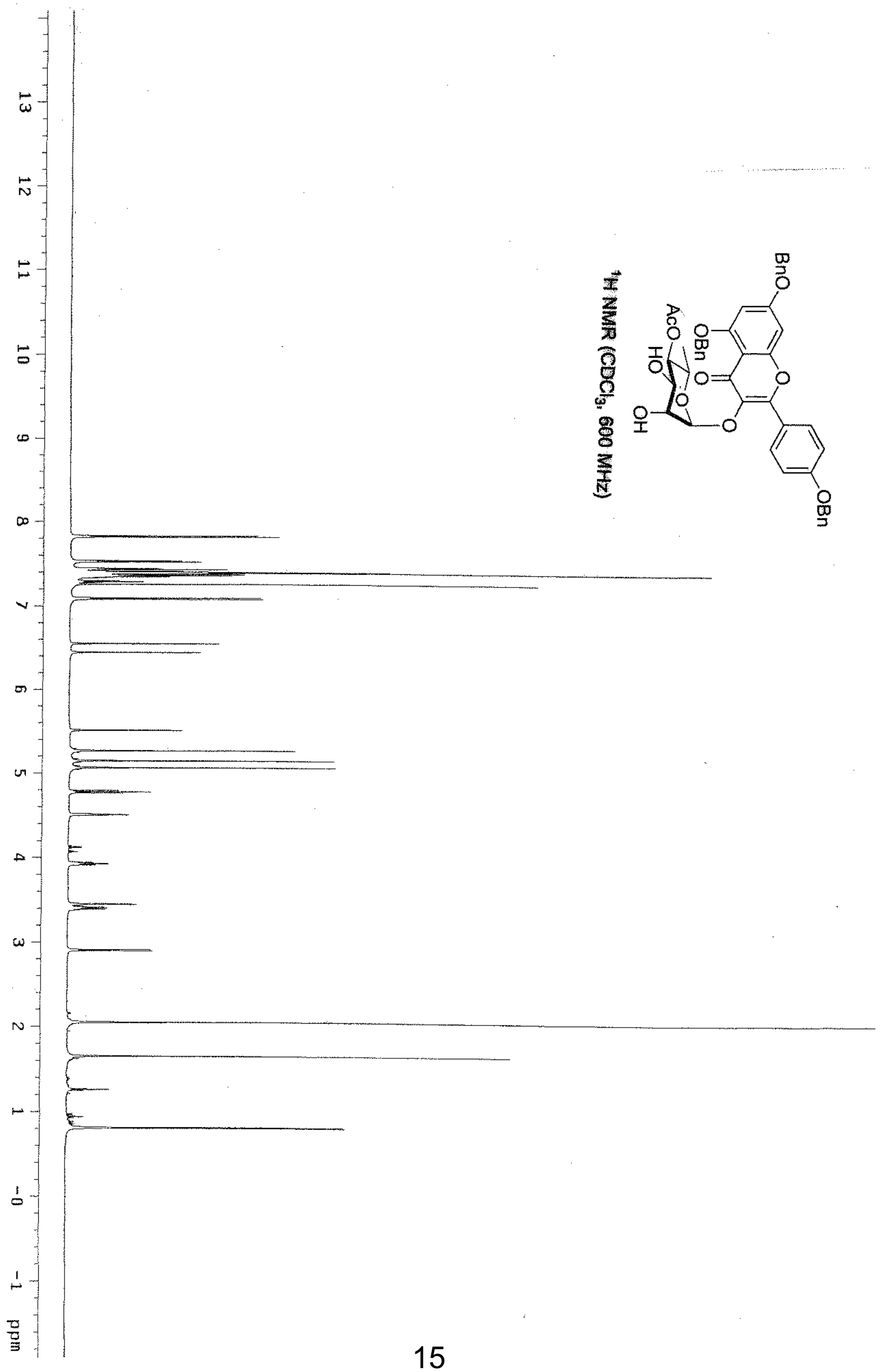




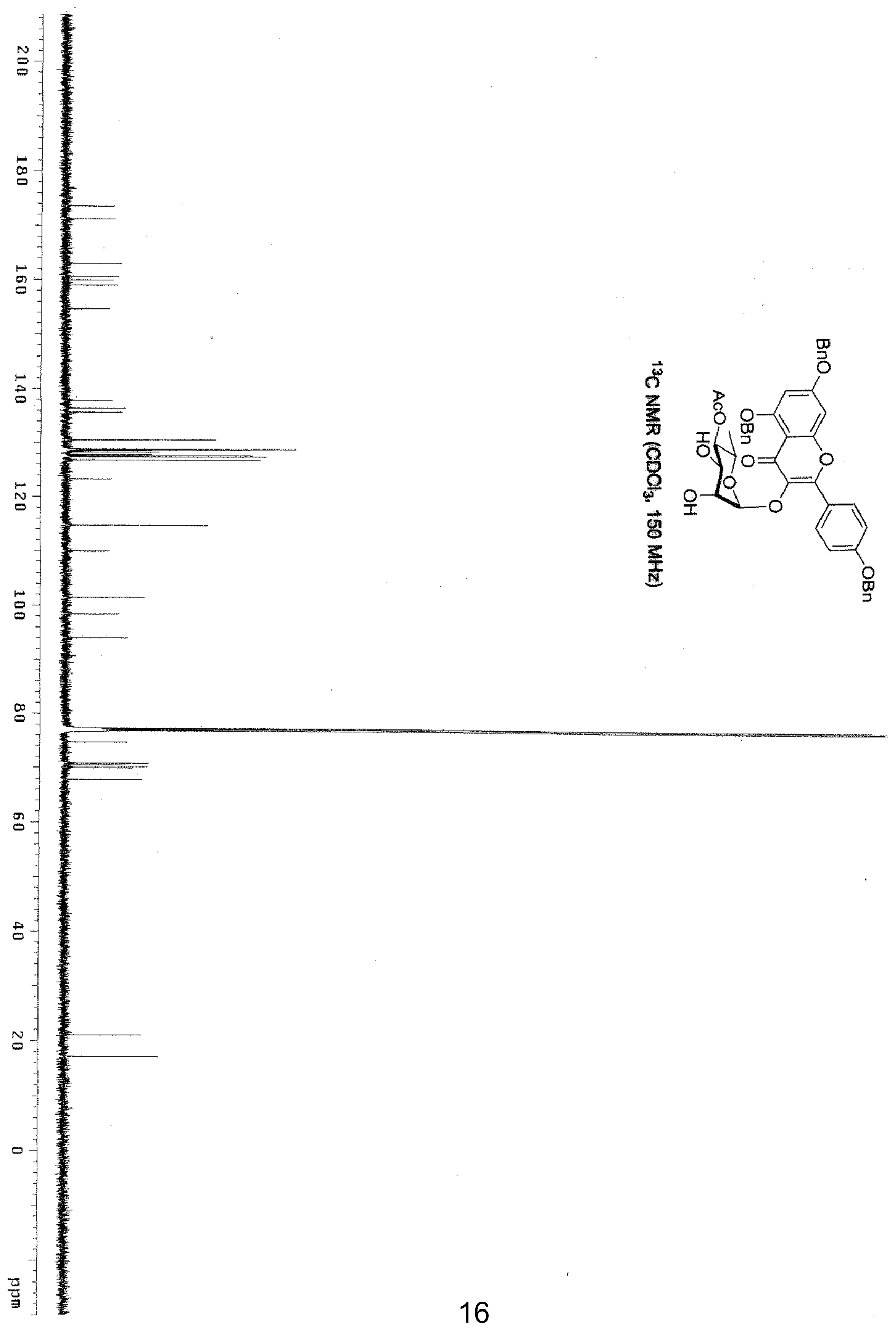




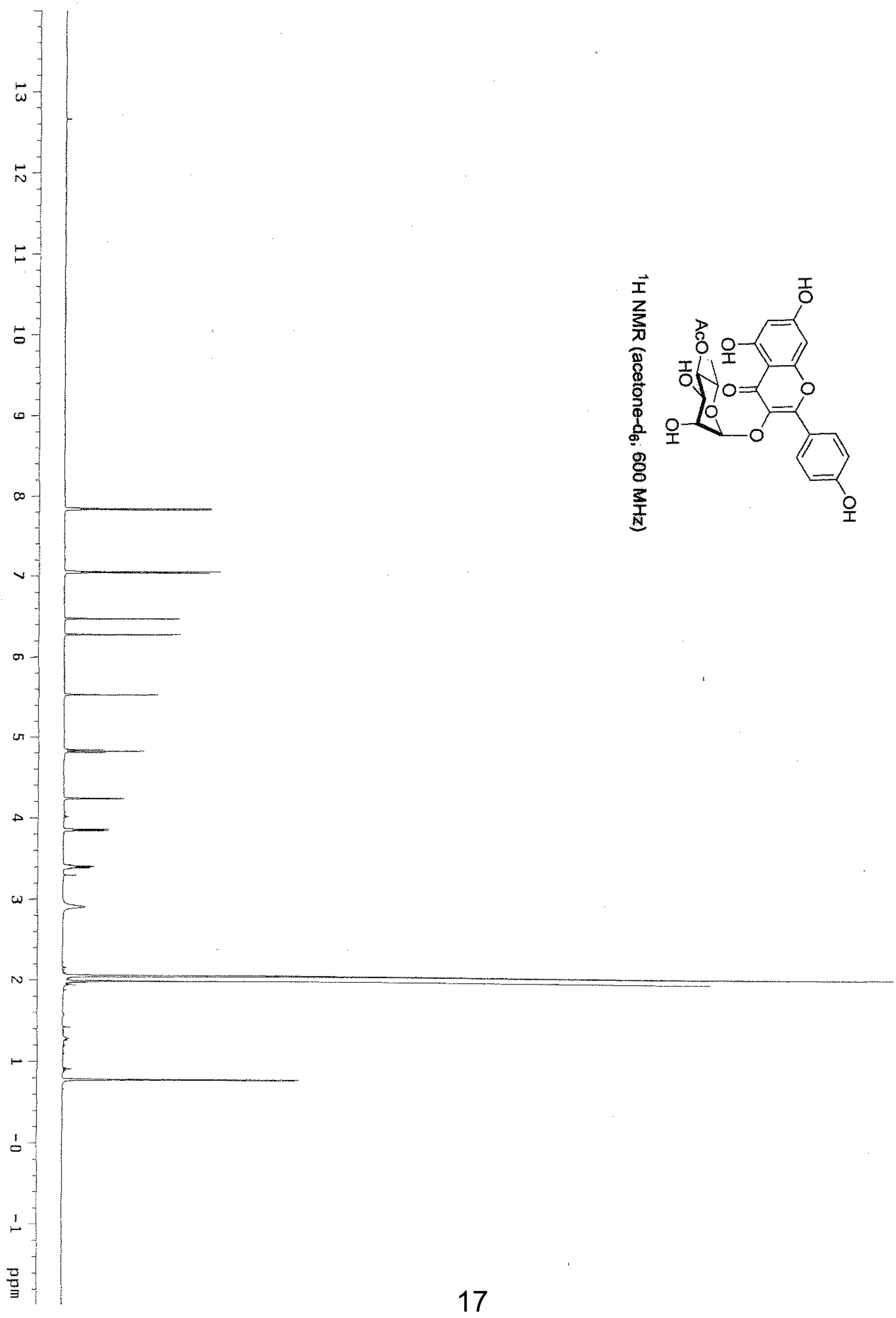




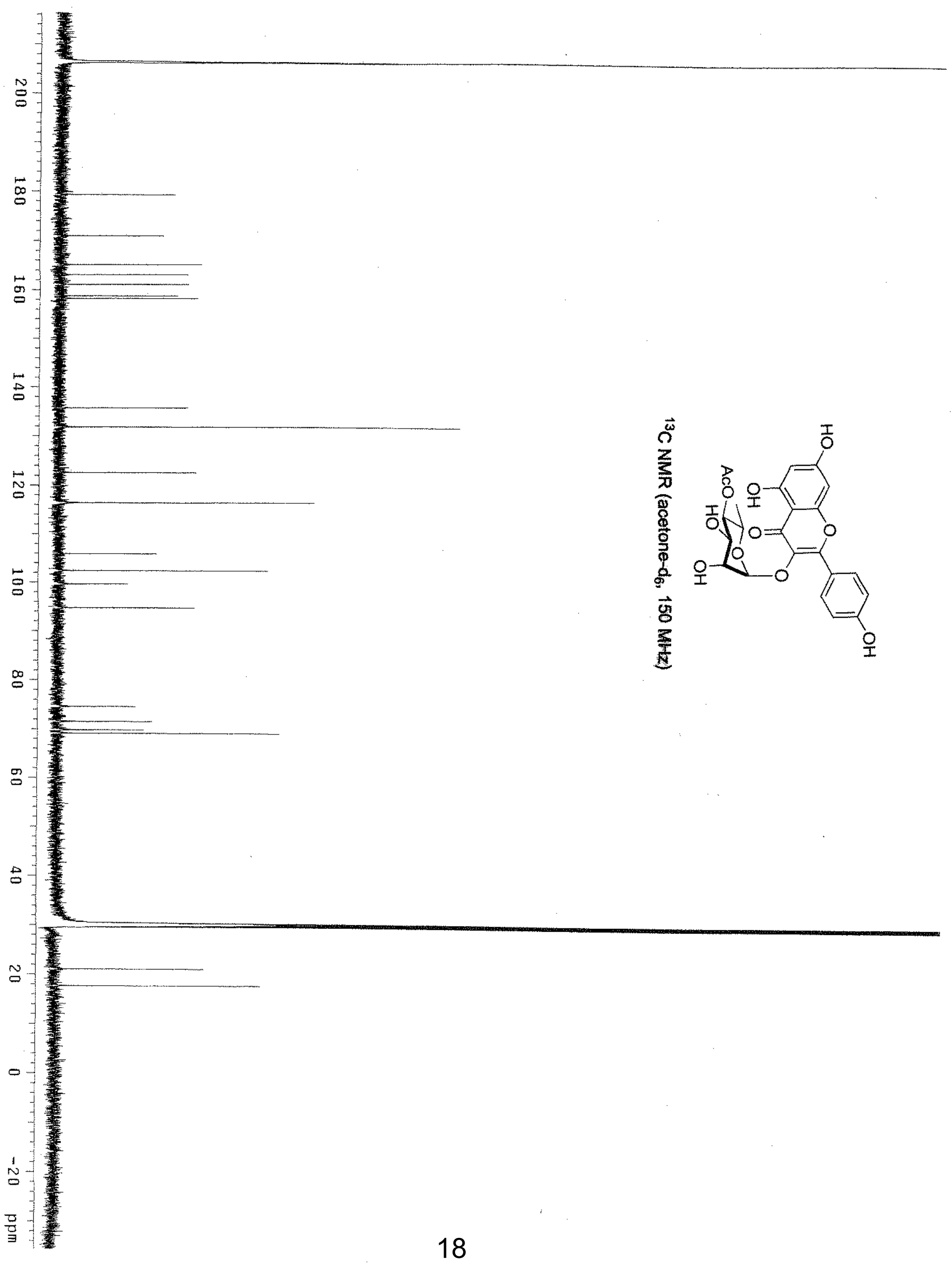




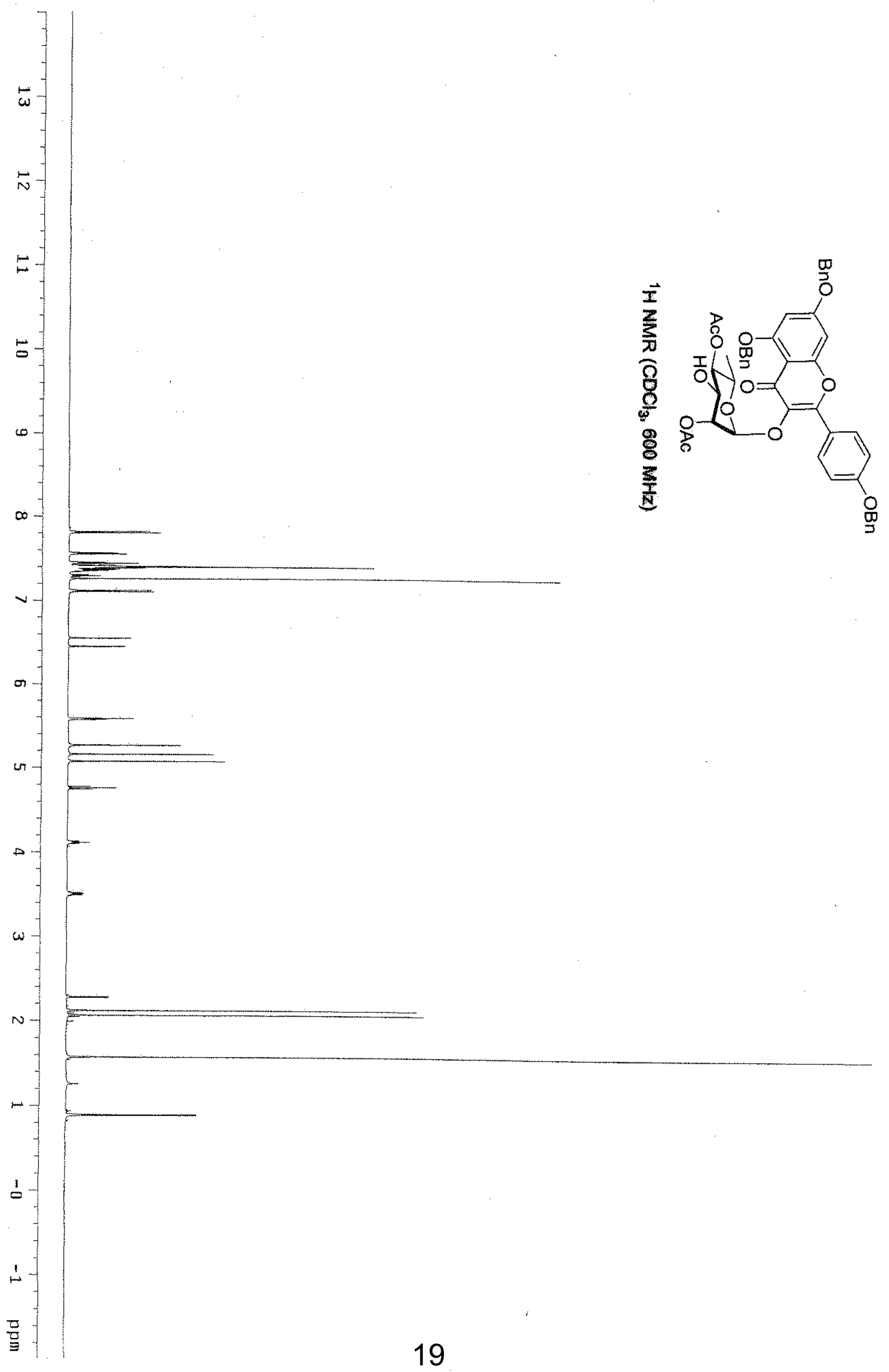




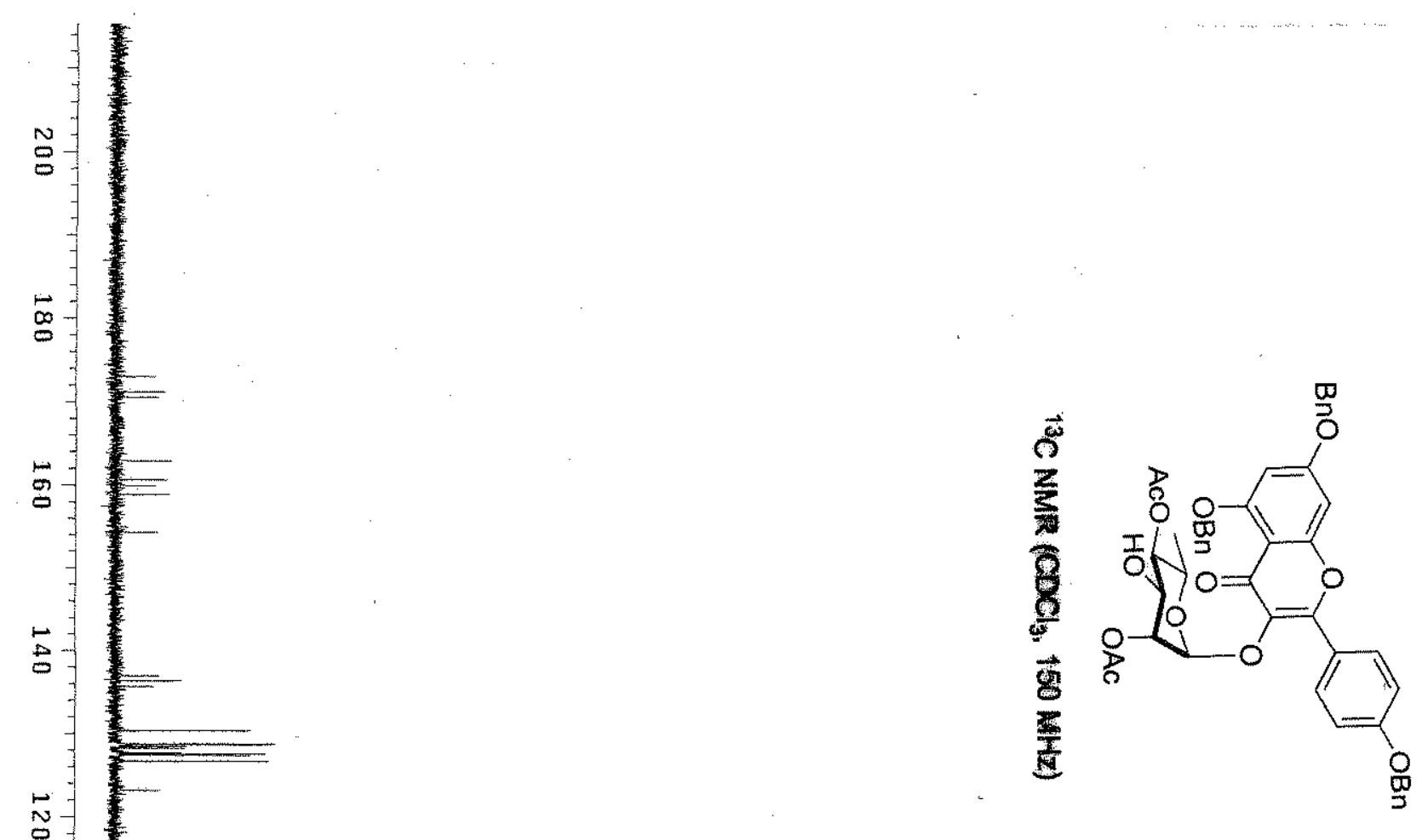

$\stackrel{\infty}{\circ}$

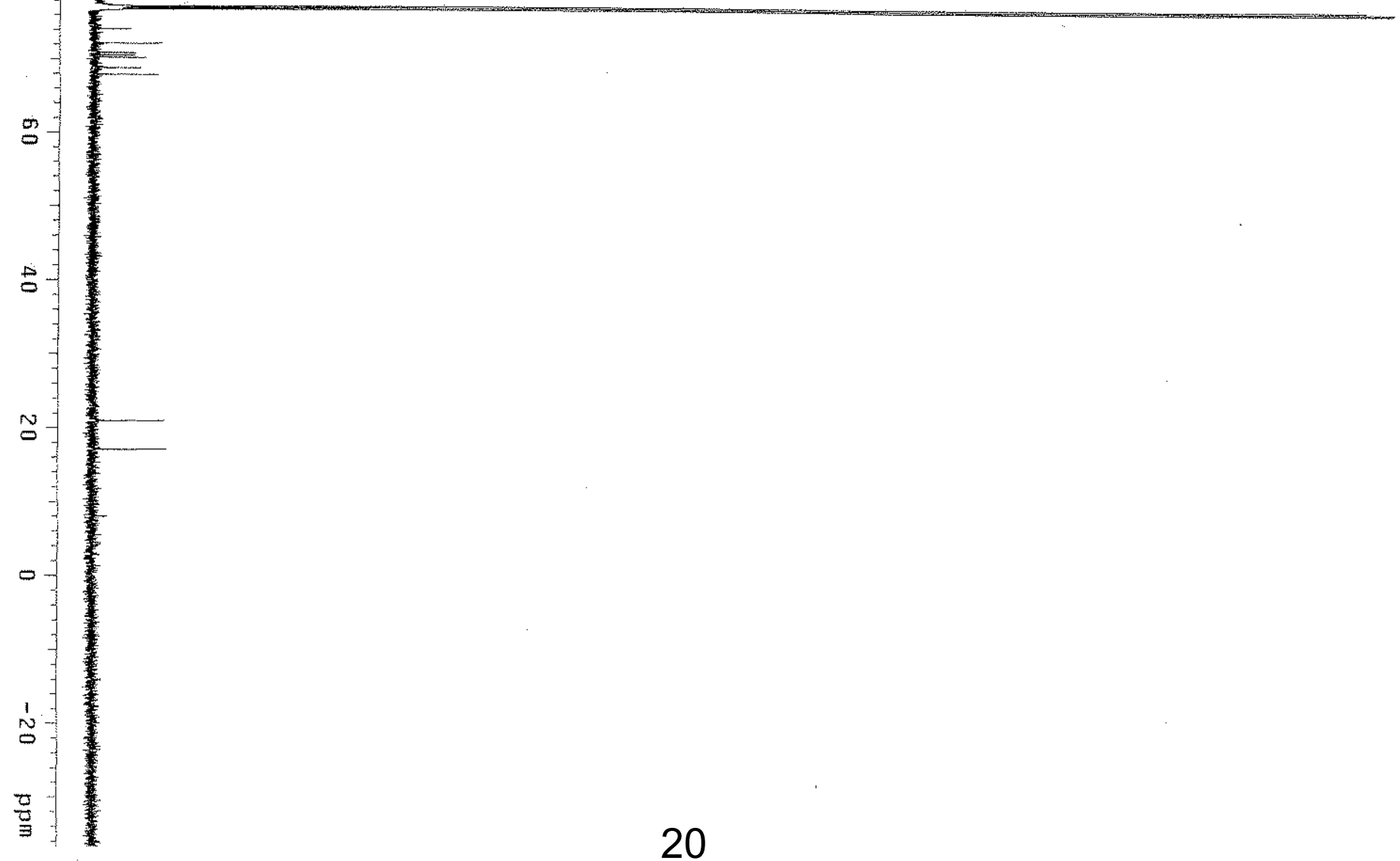




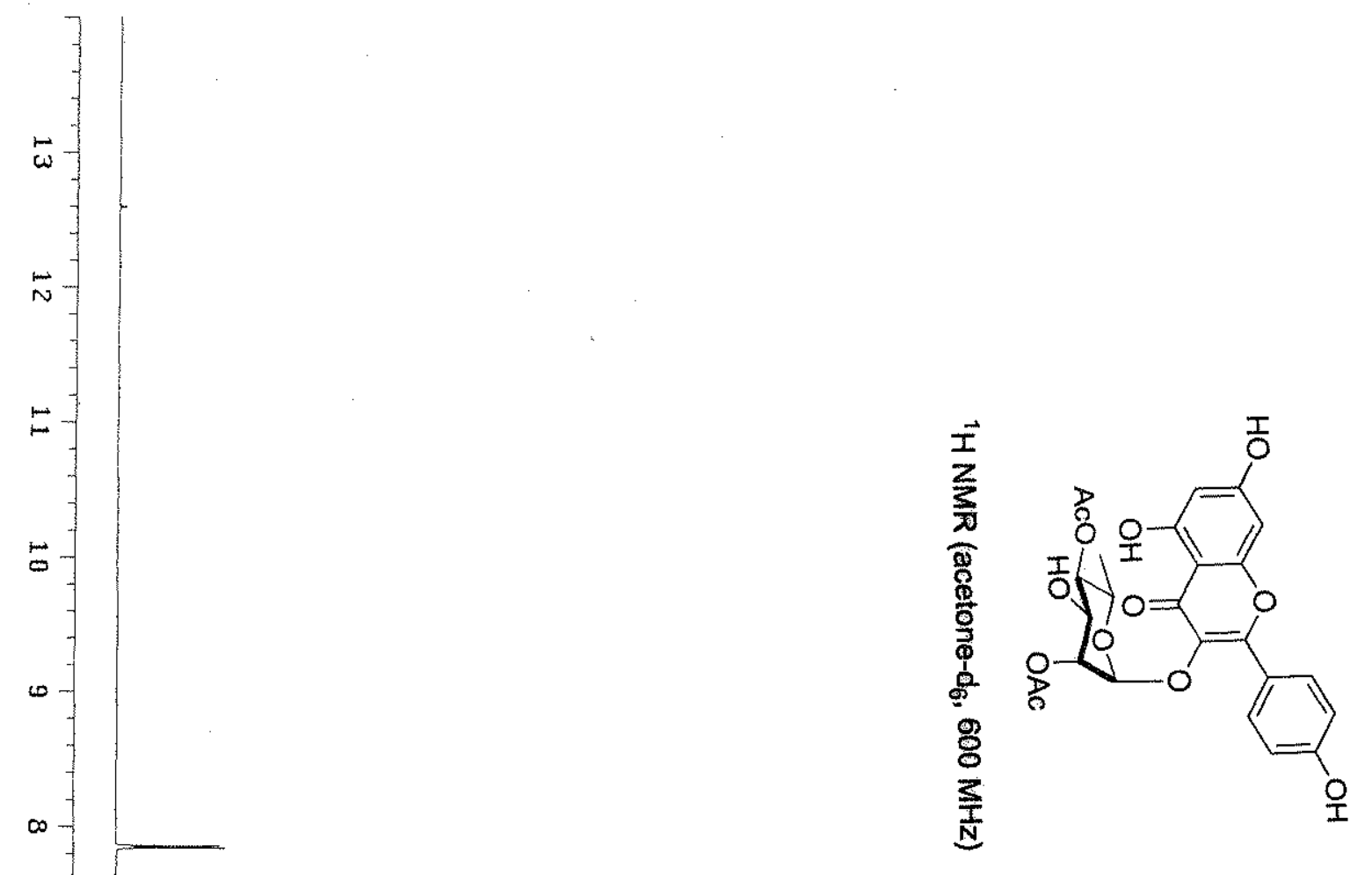




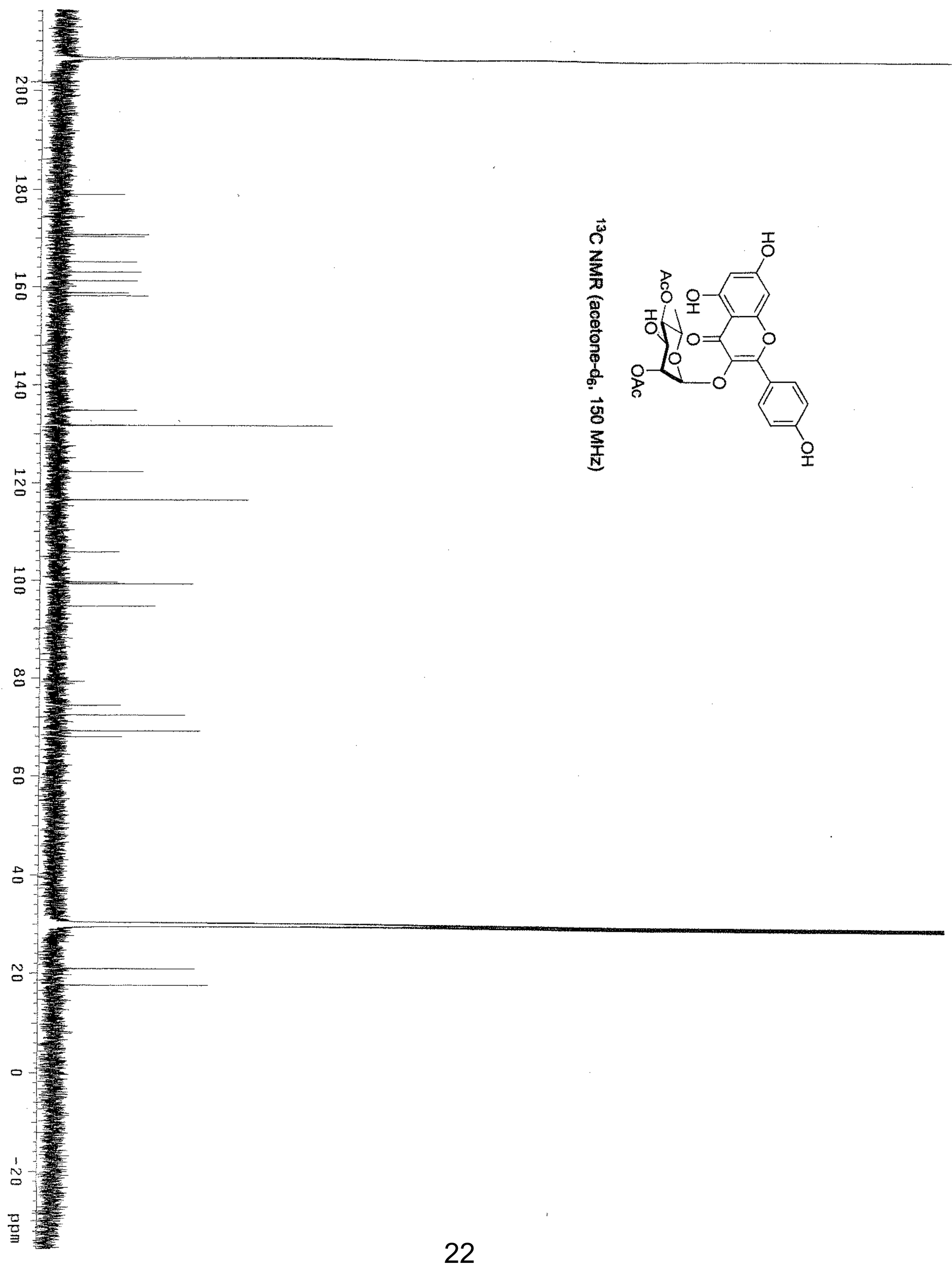




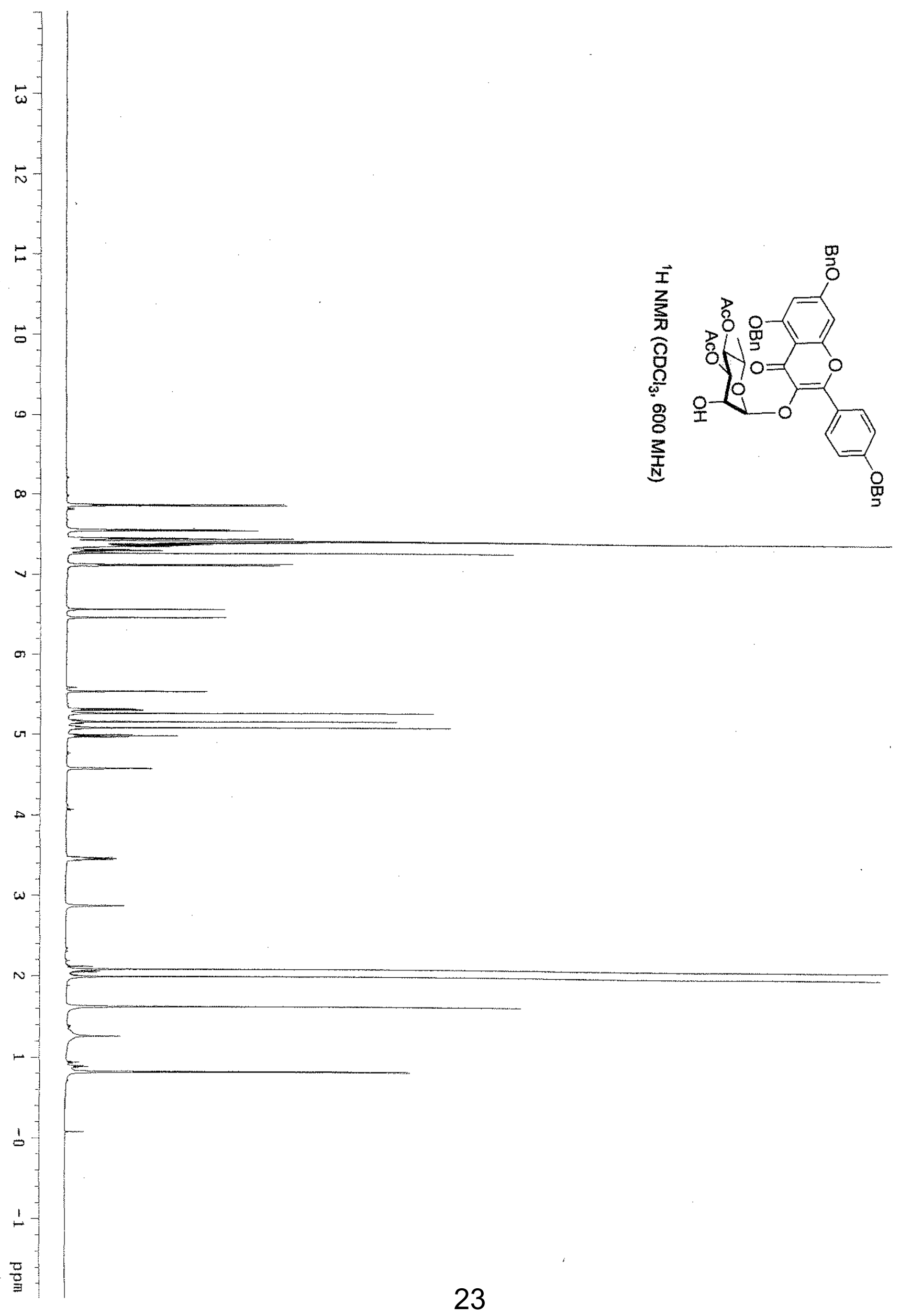



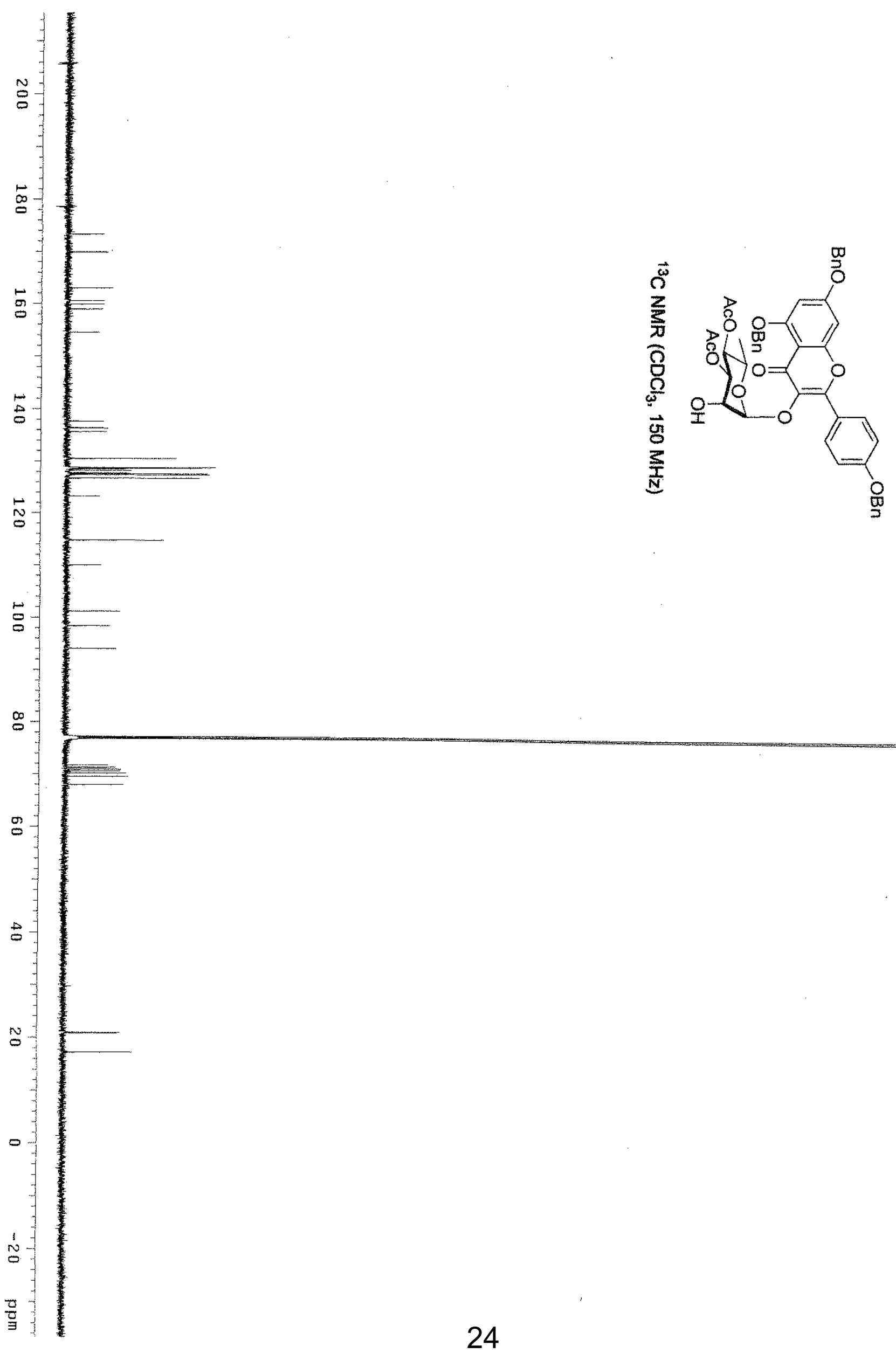


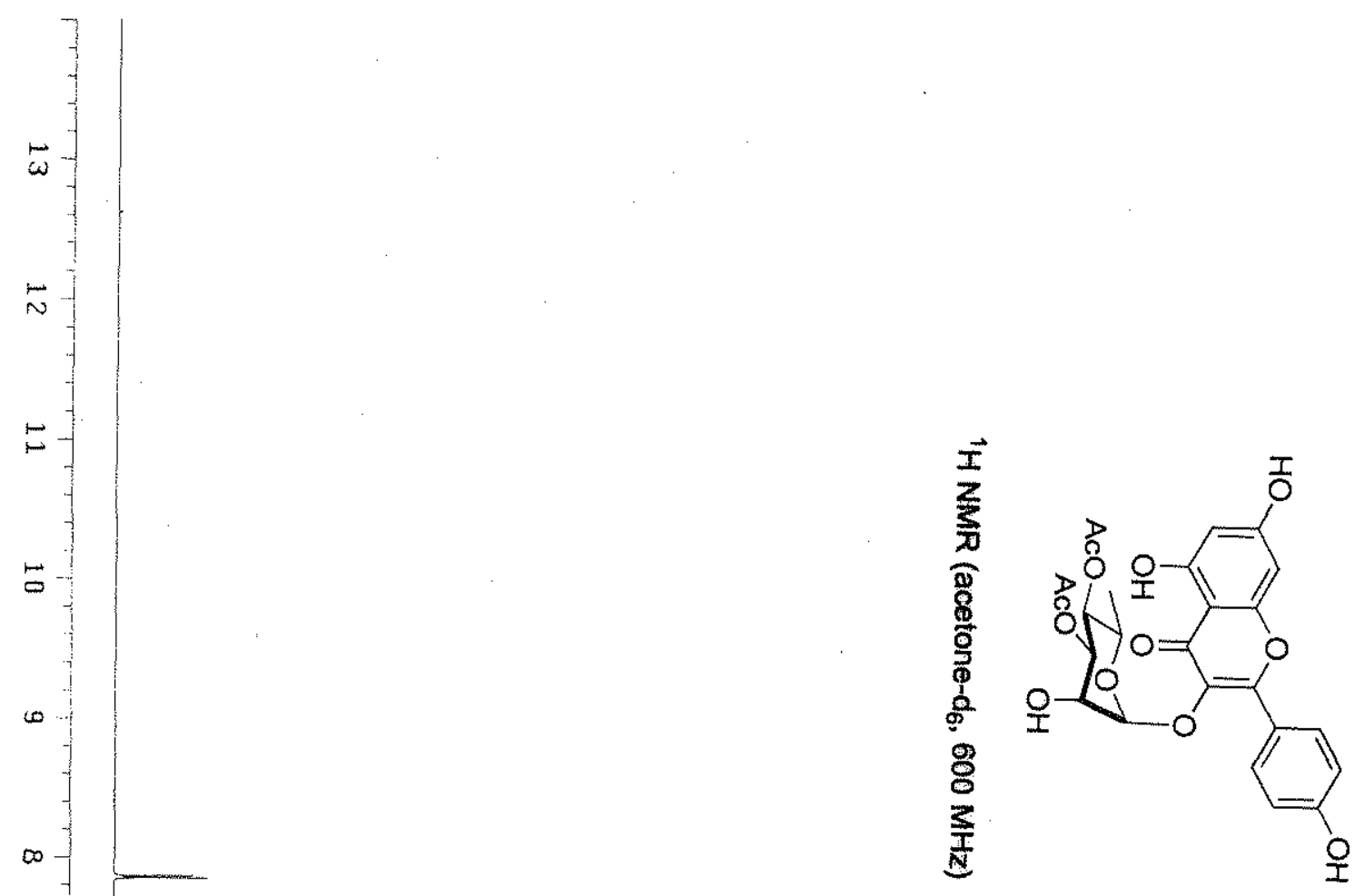




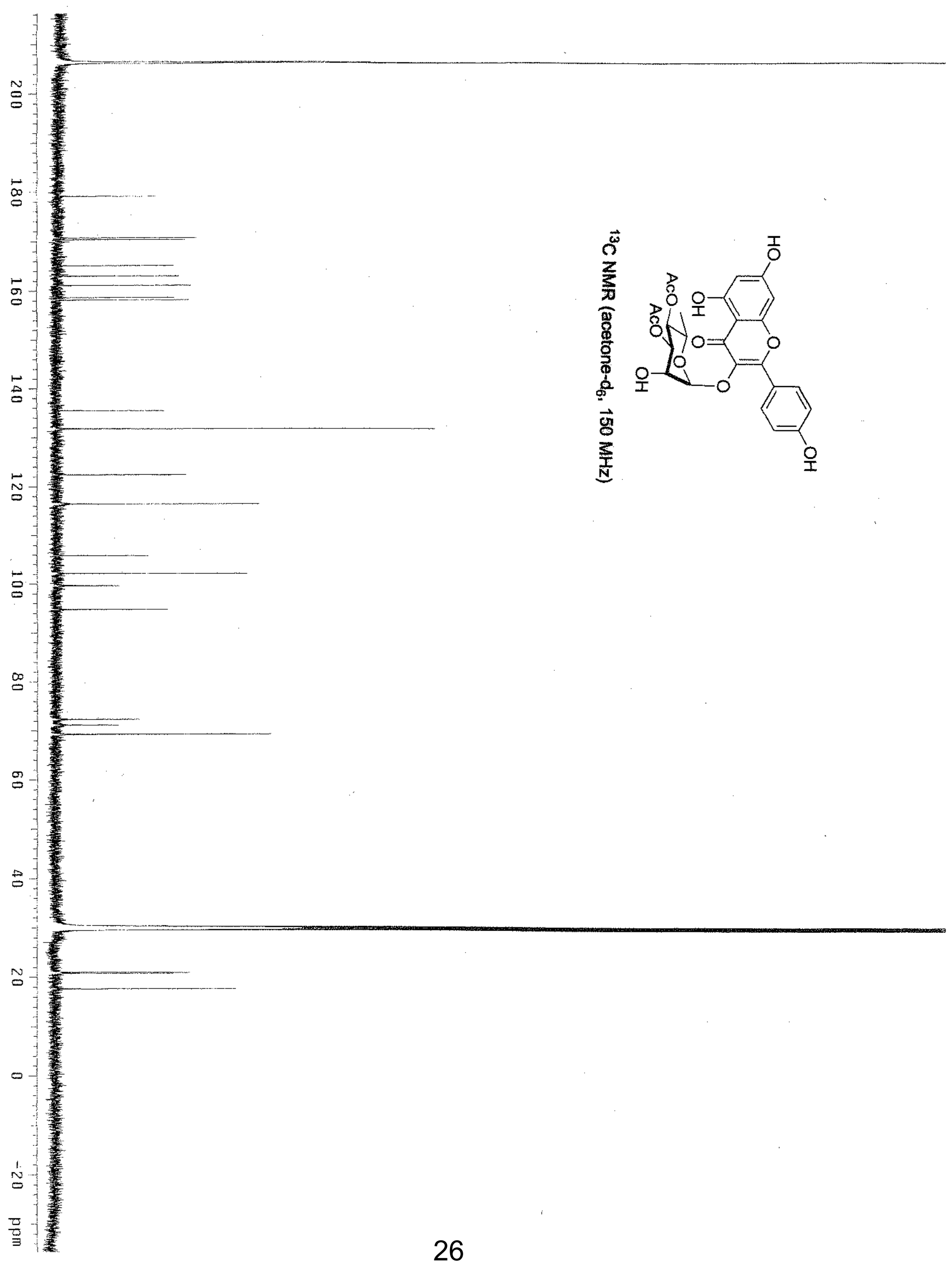




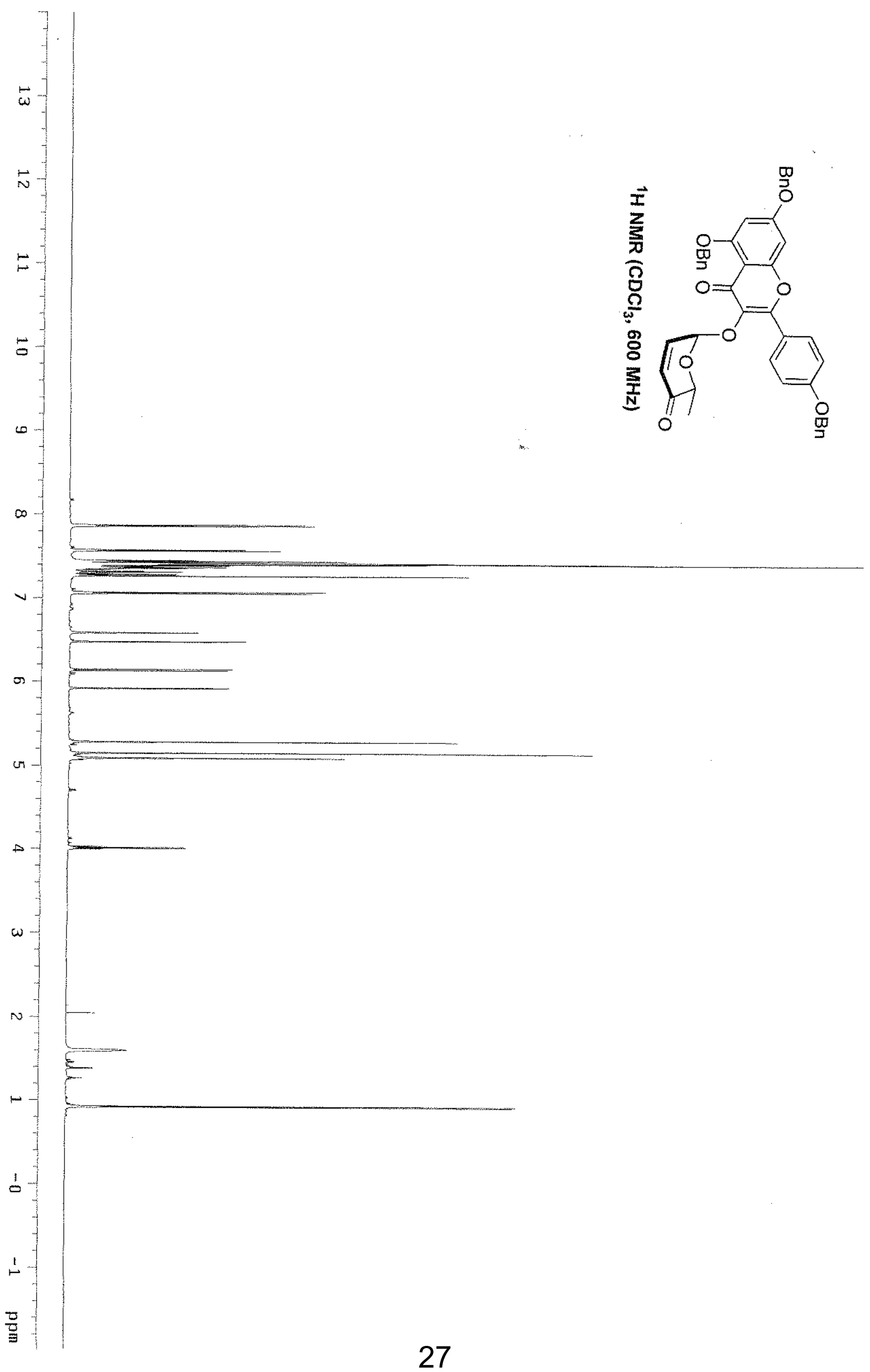




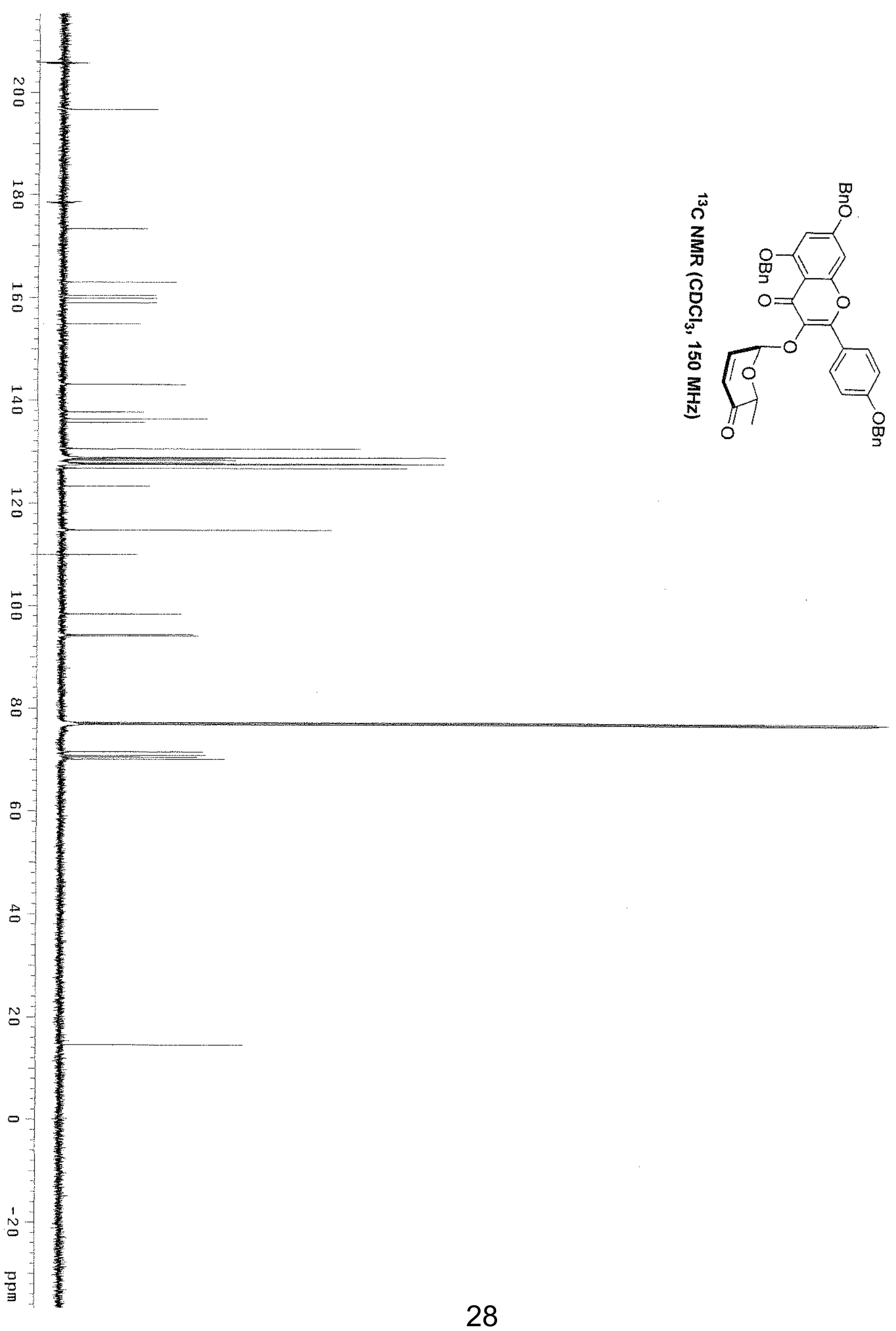



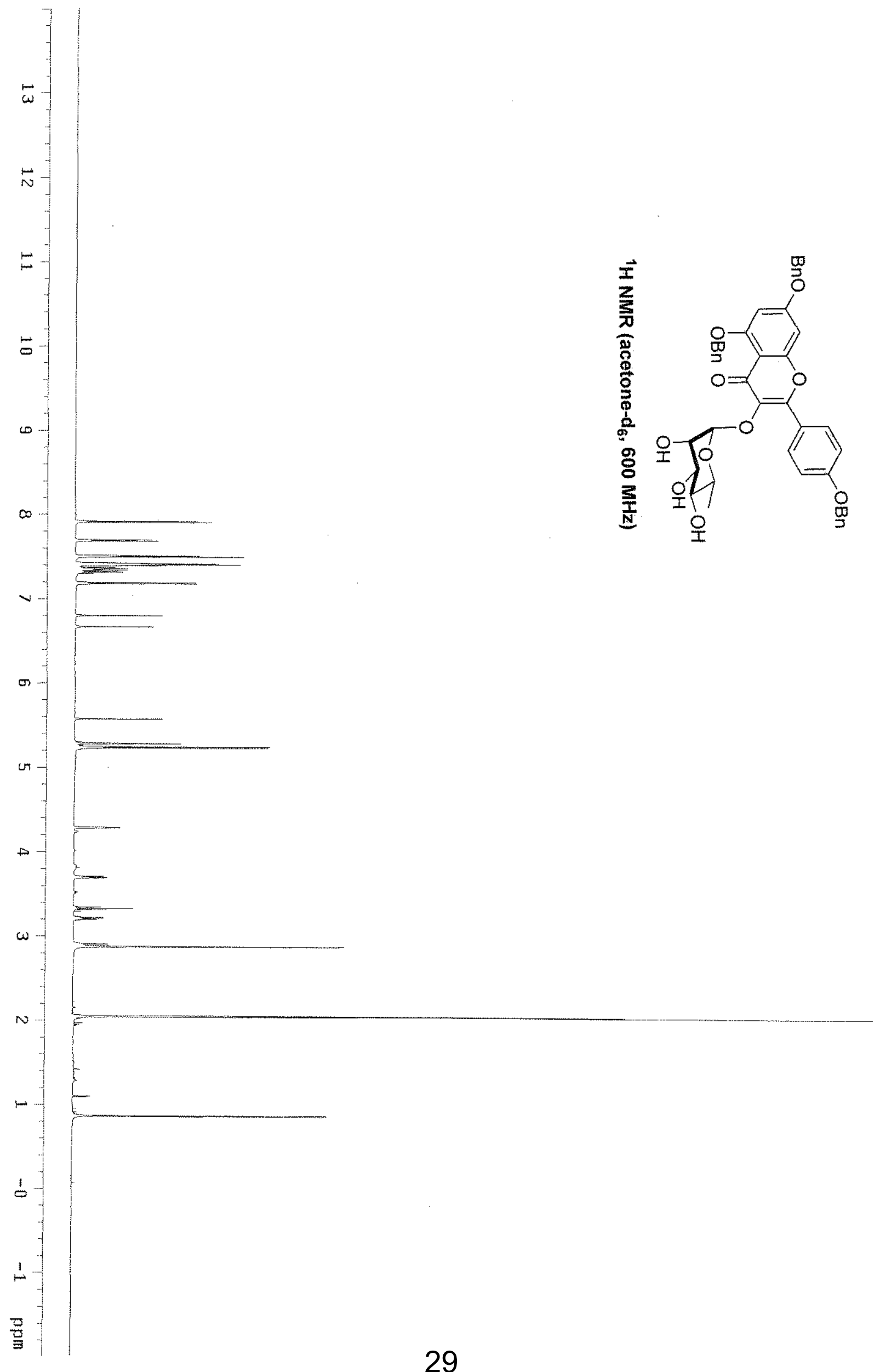


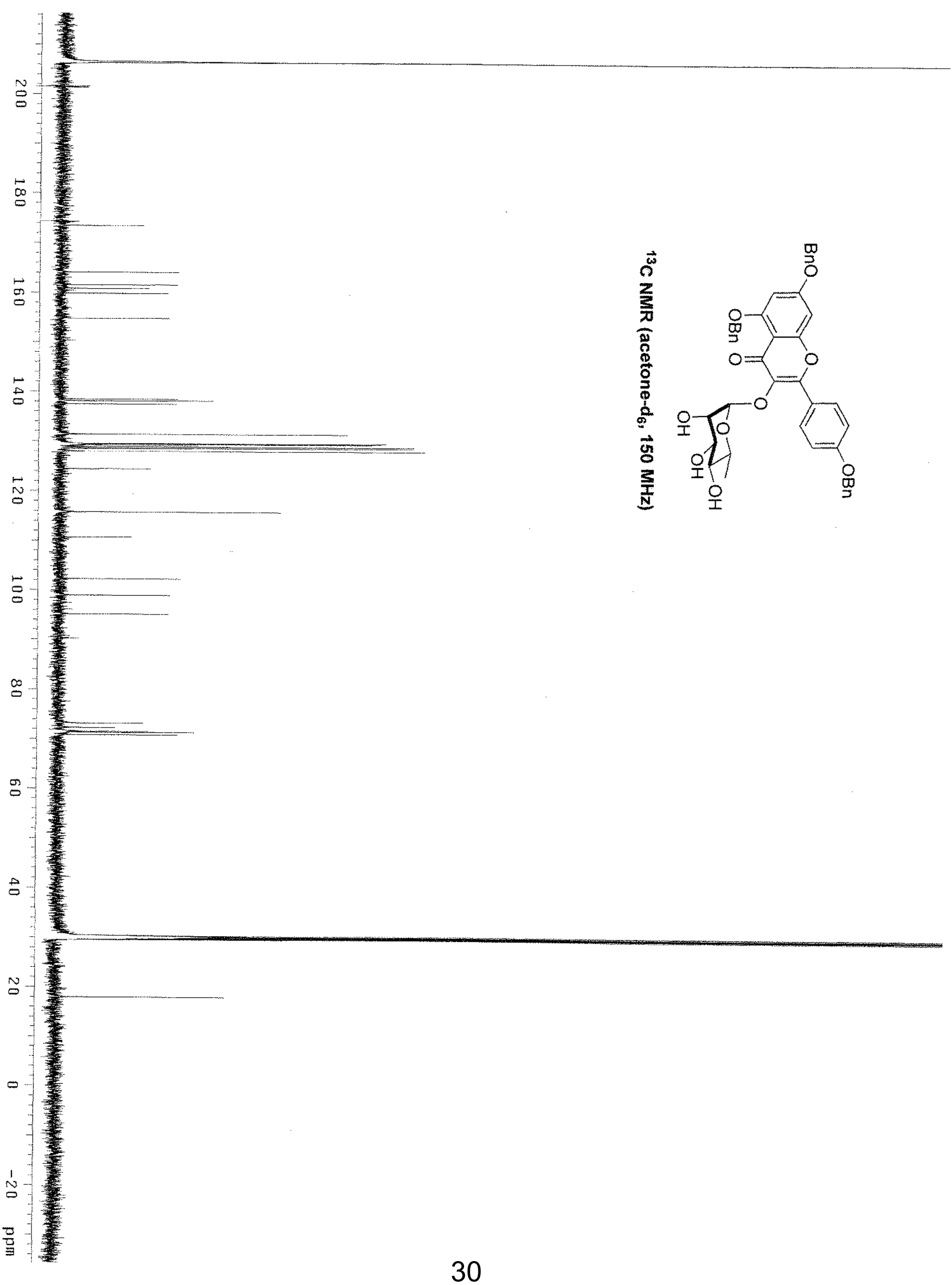



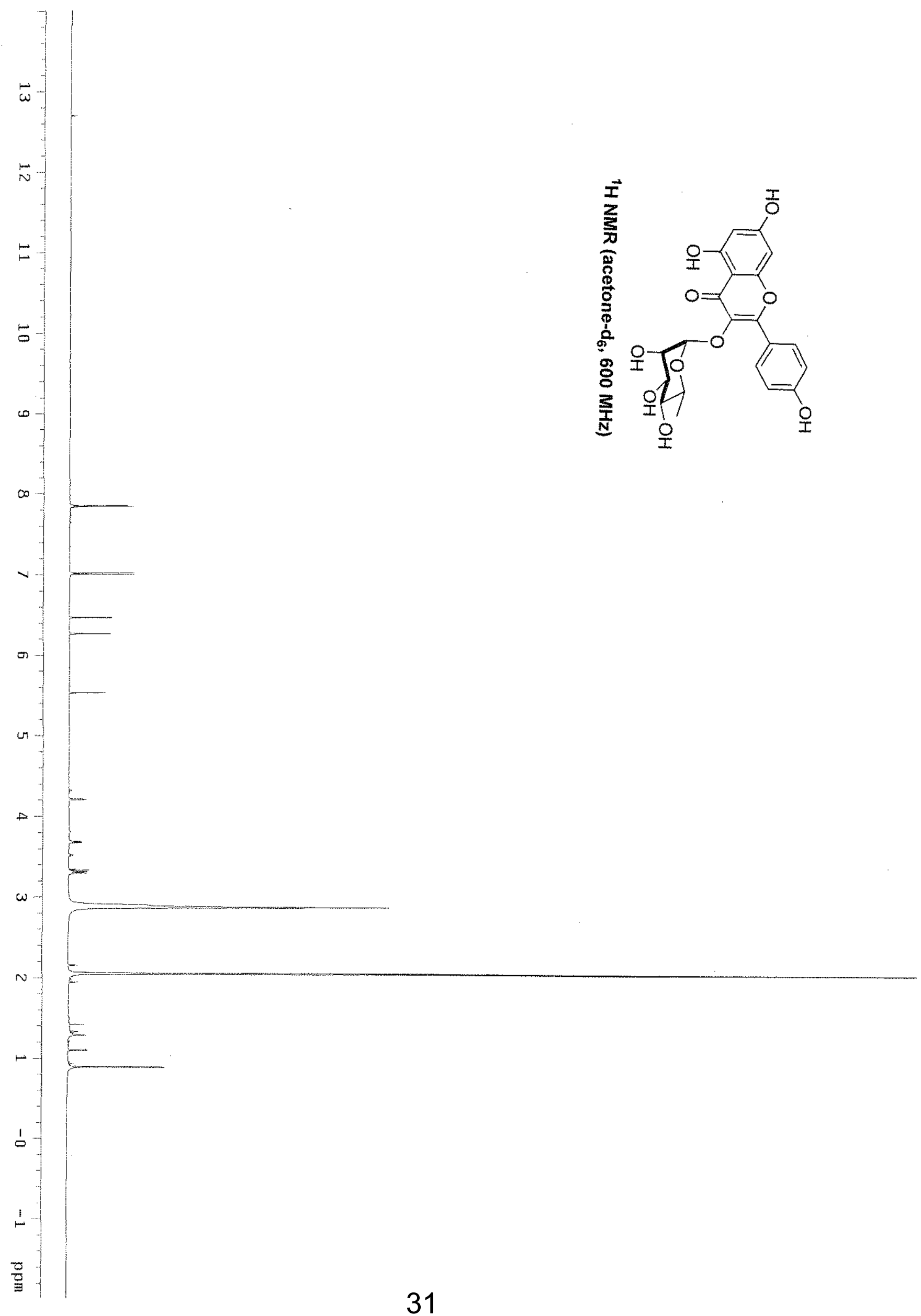


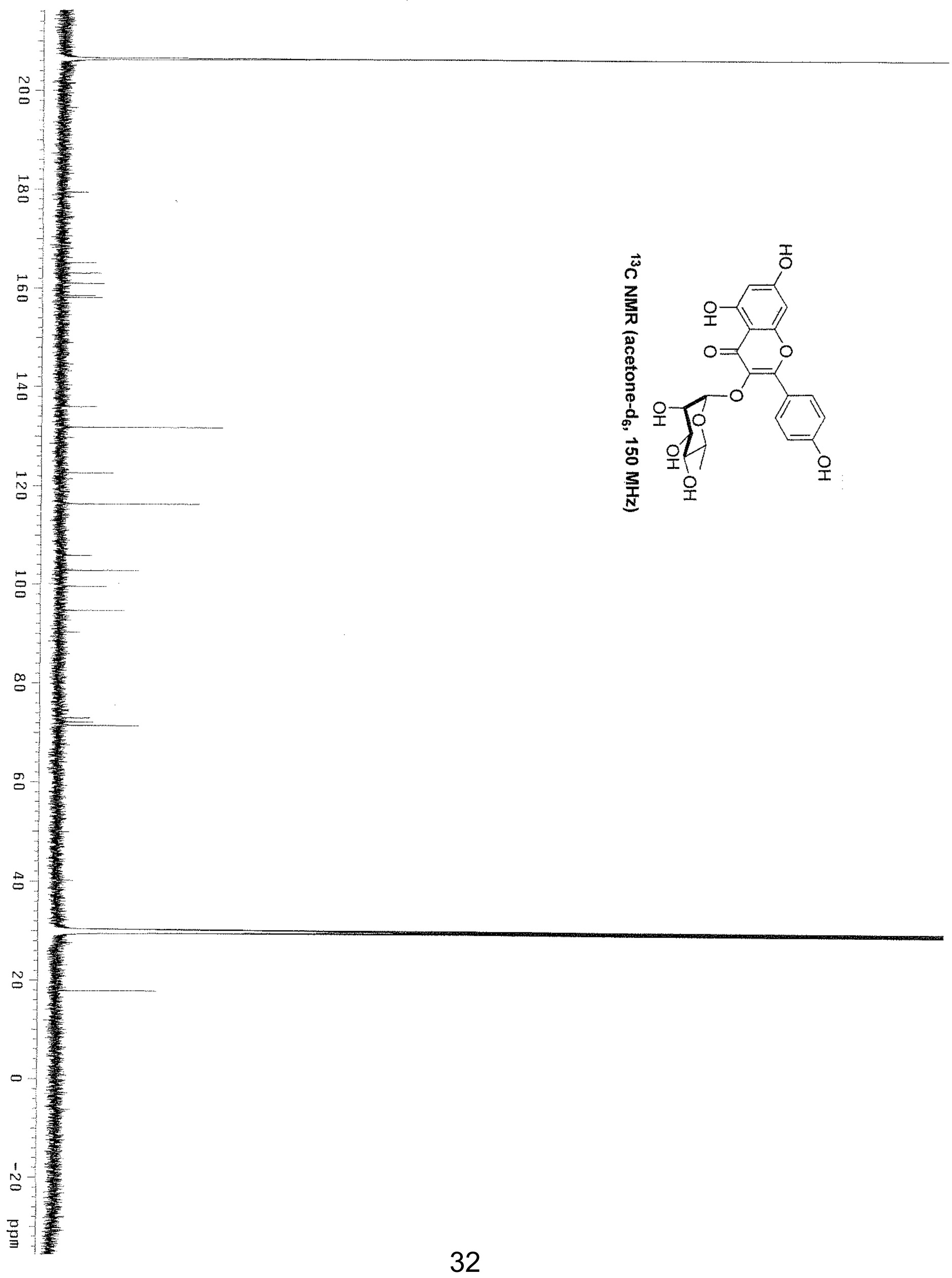




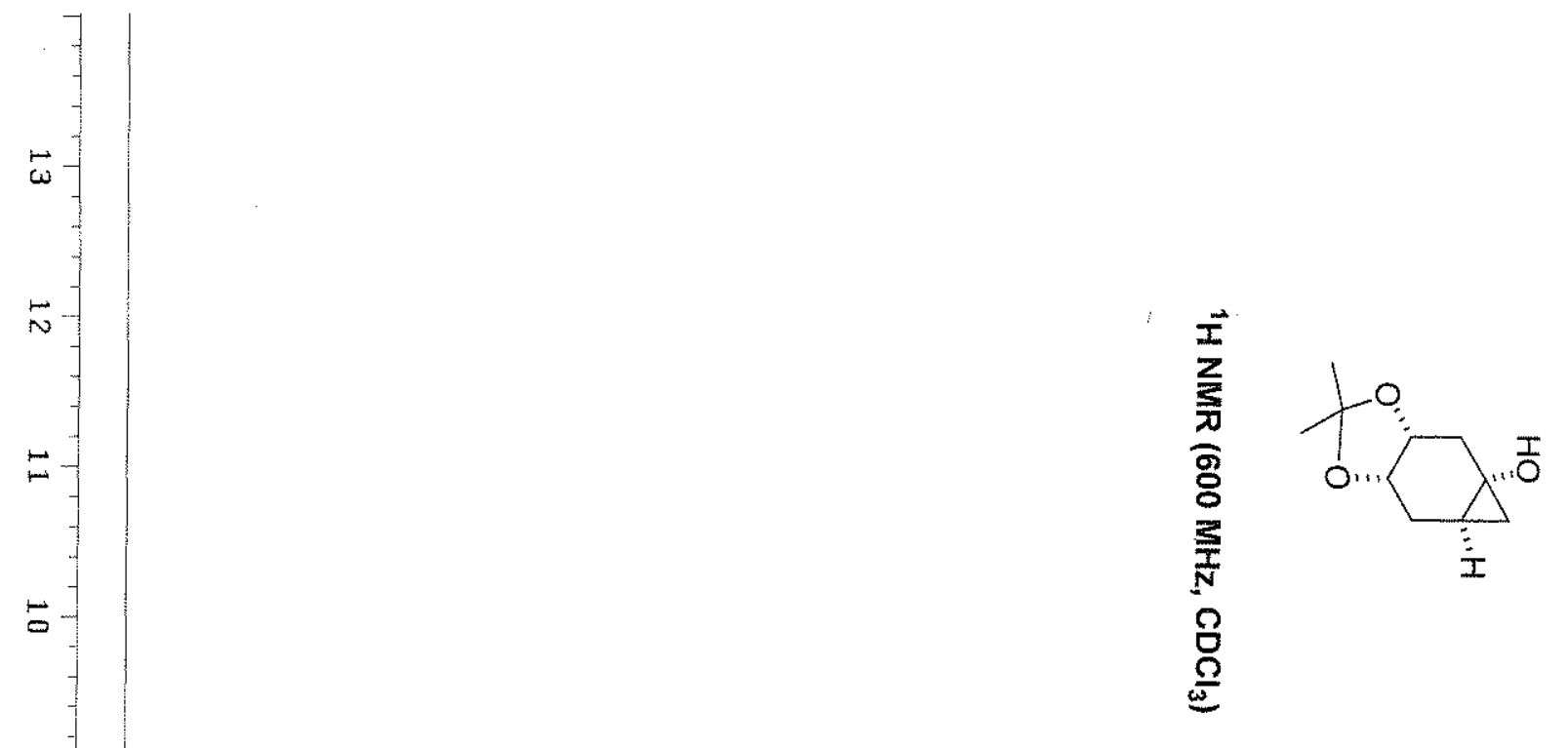

$\infty$

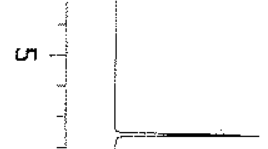

$\omega$
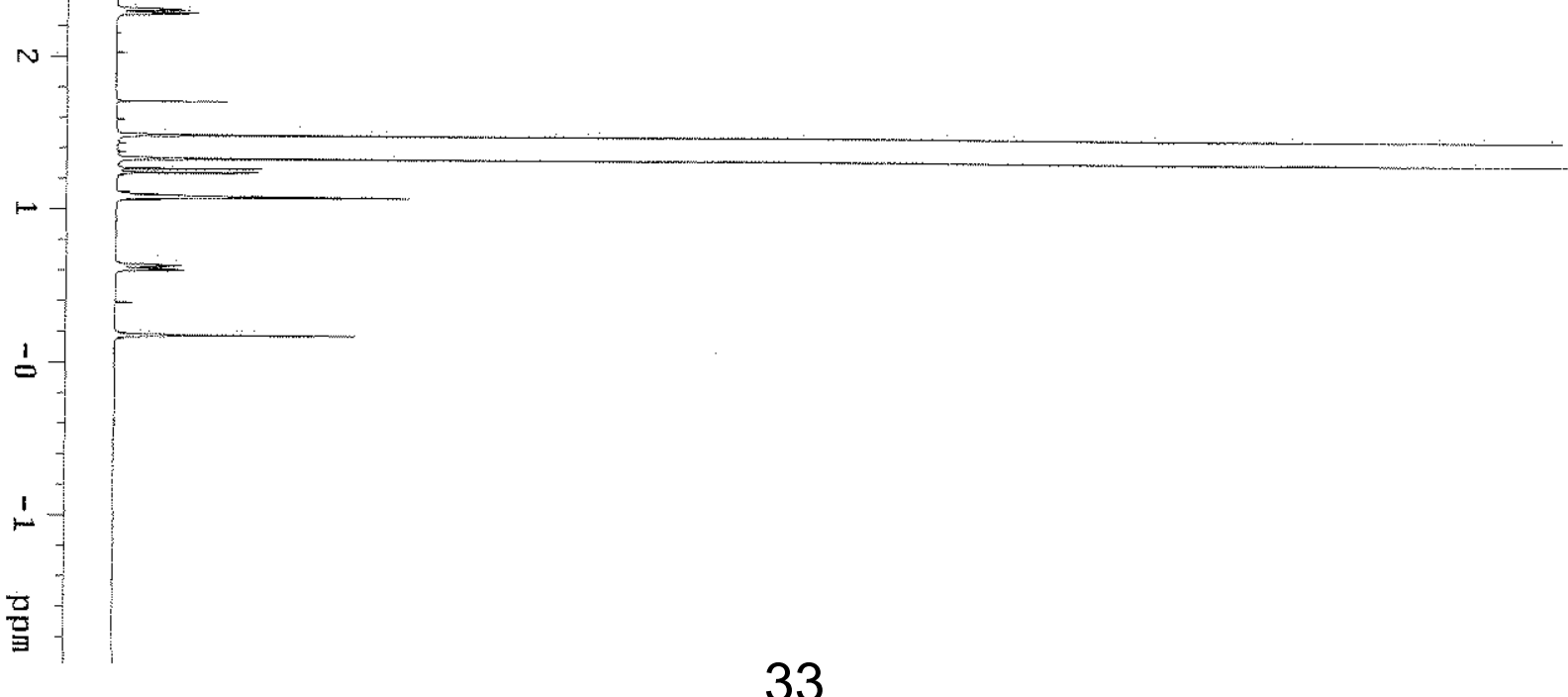


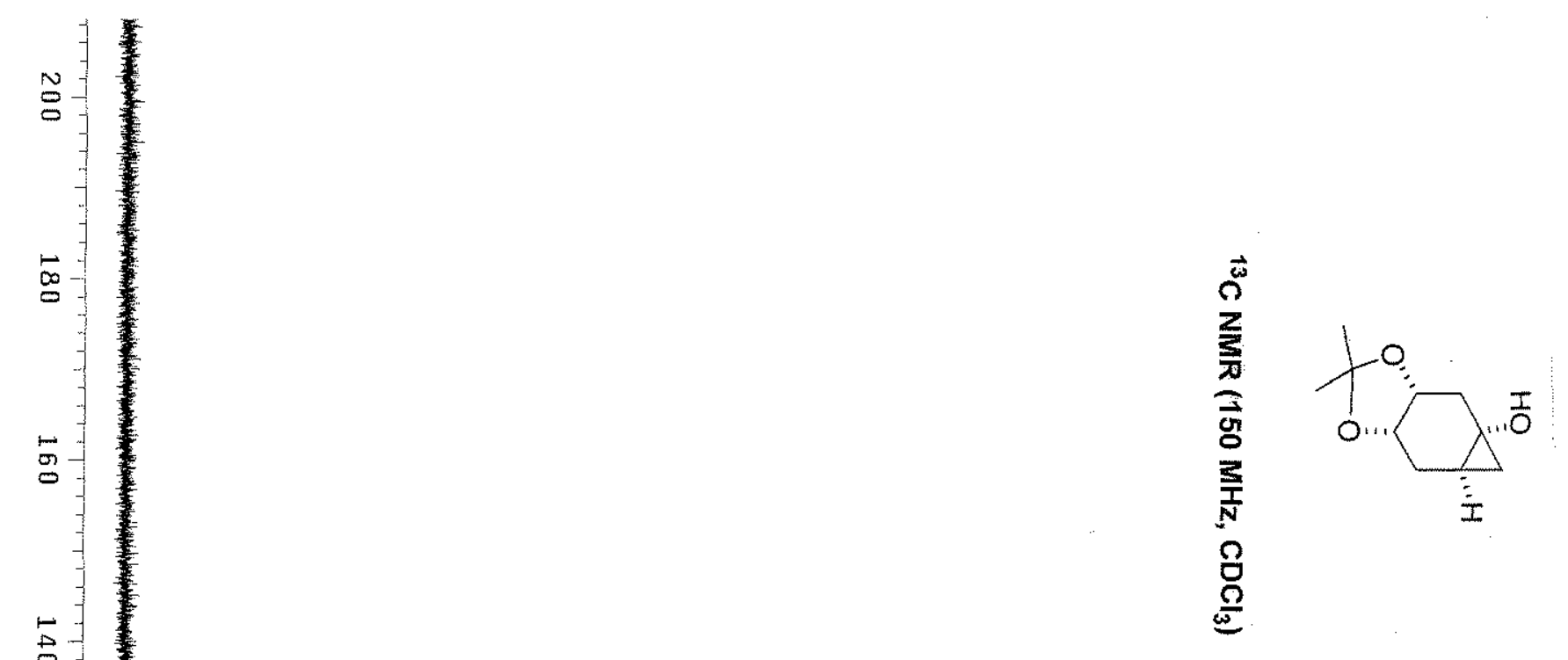




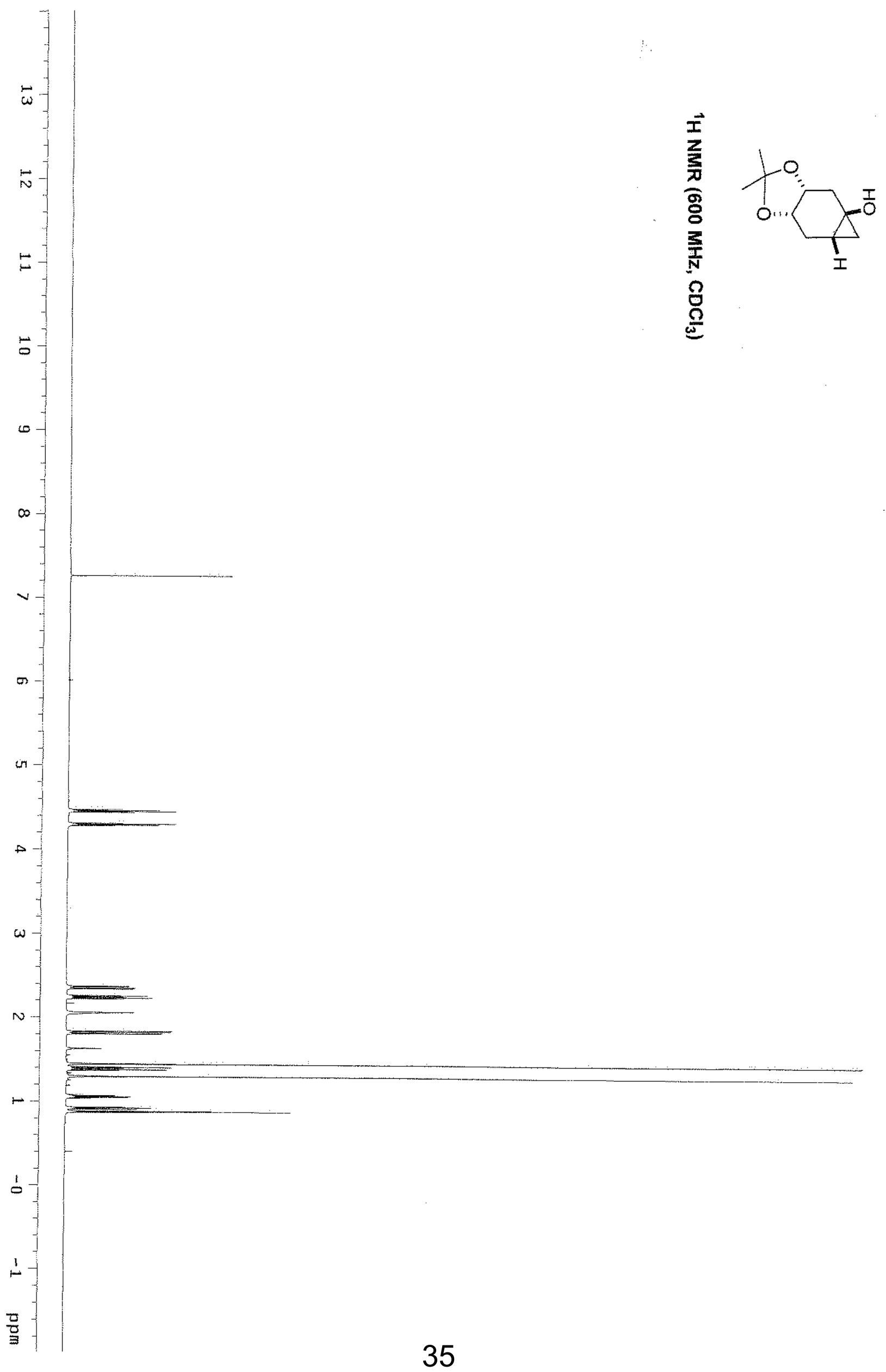




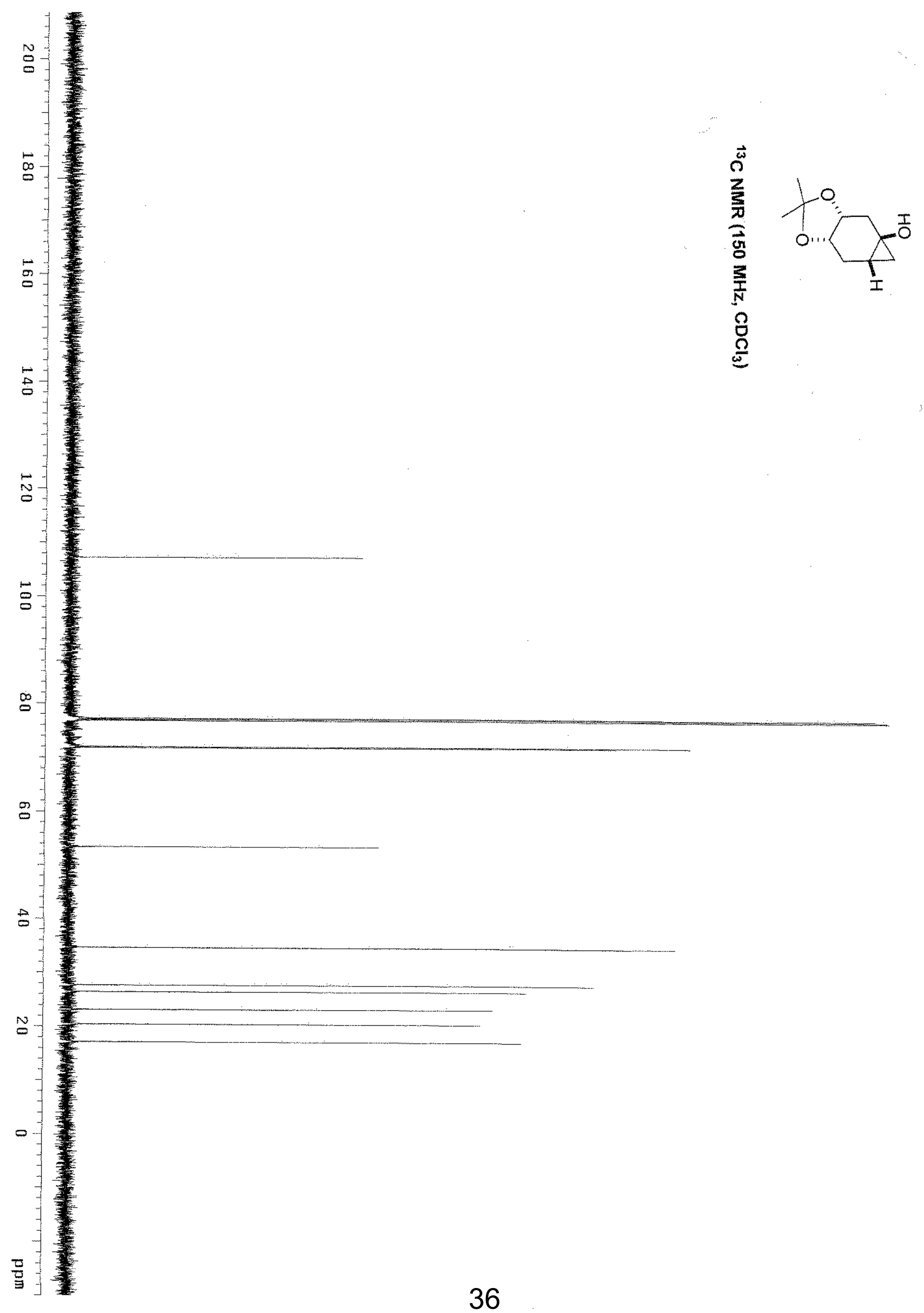




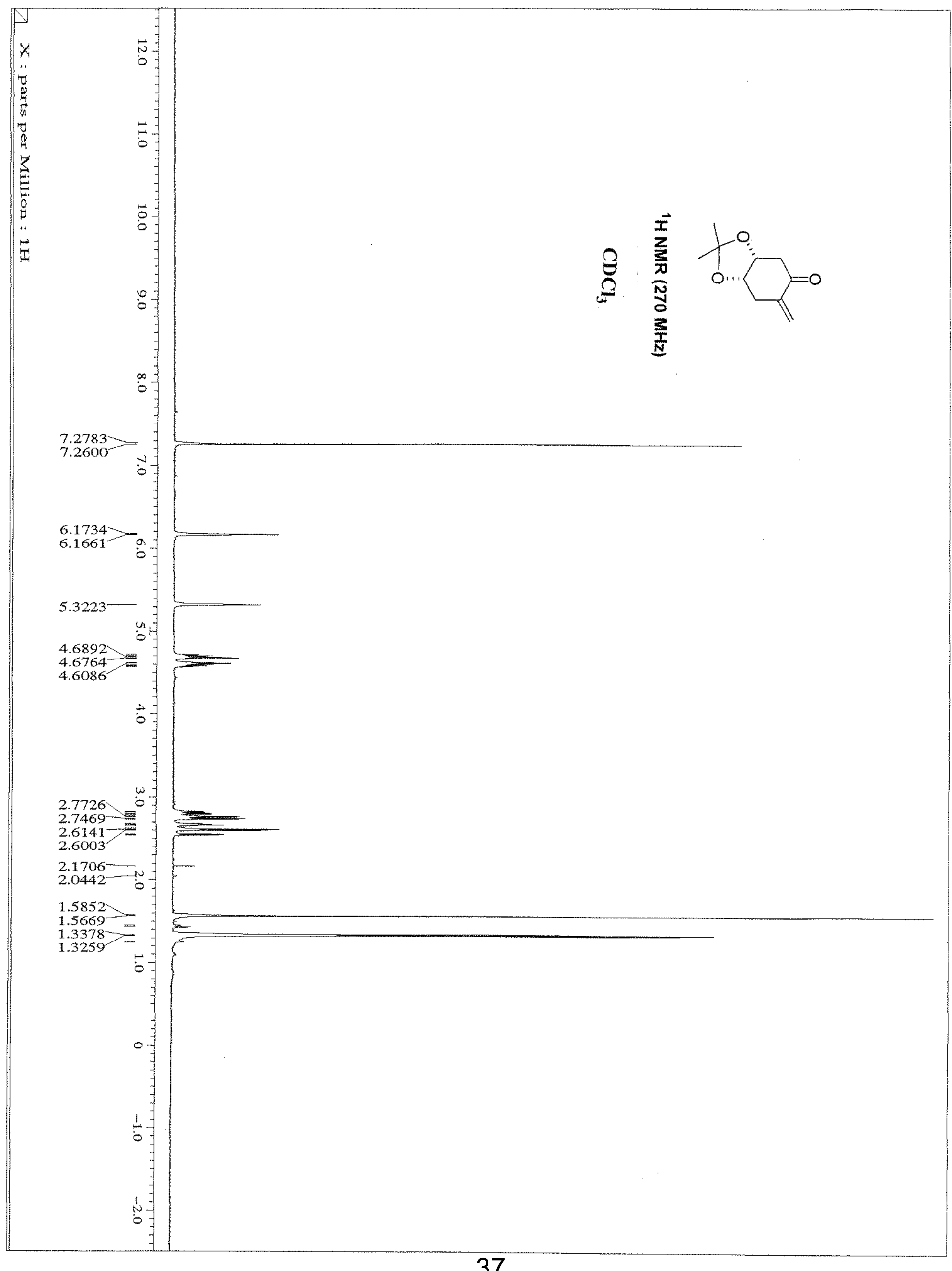




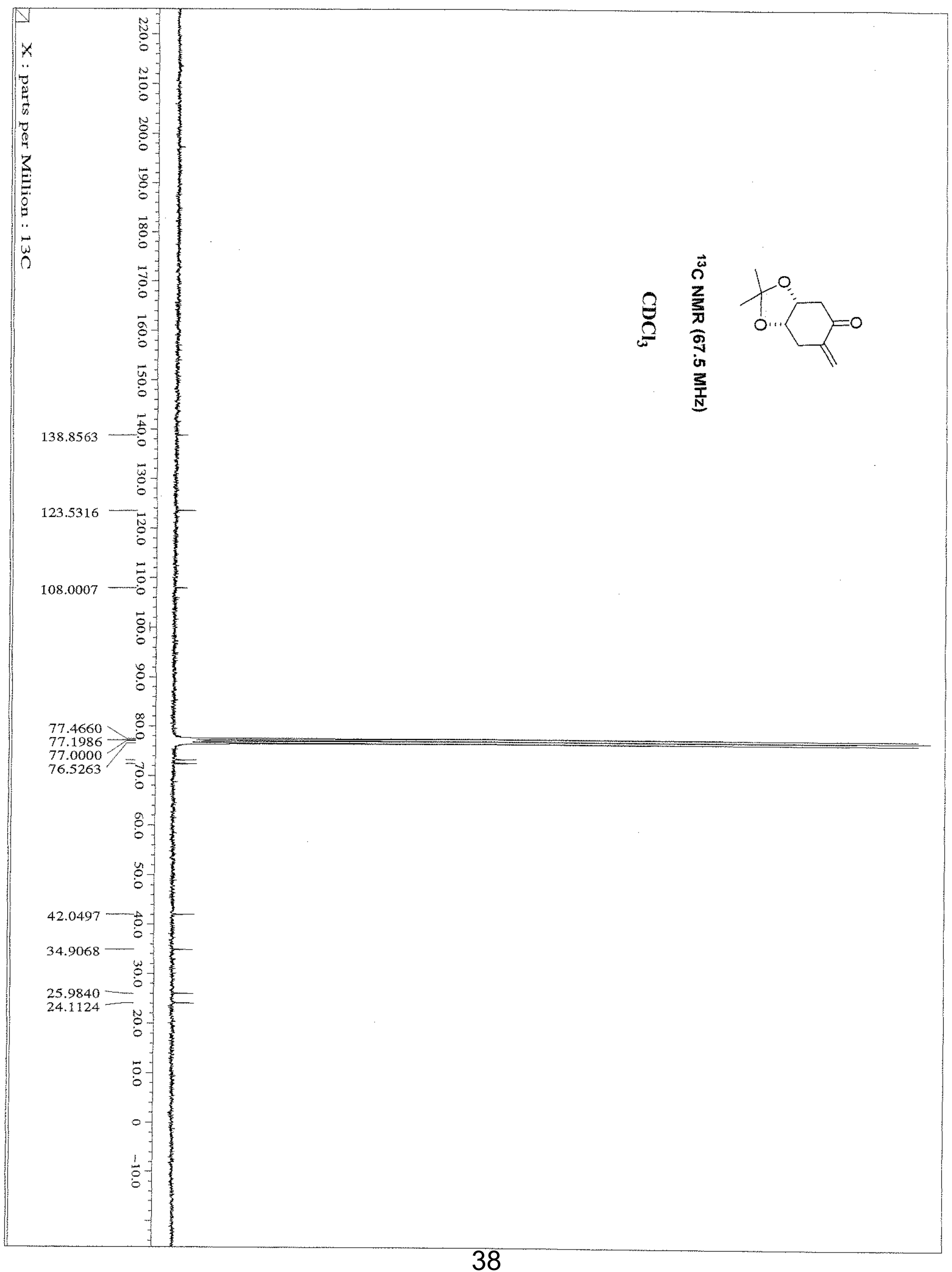




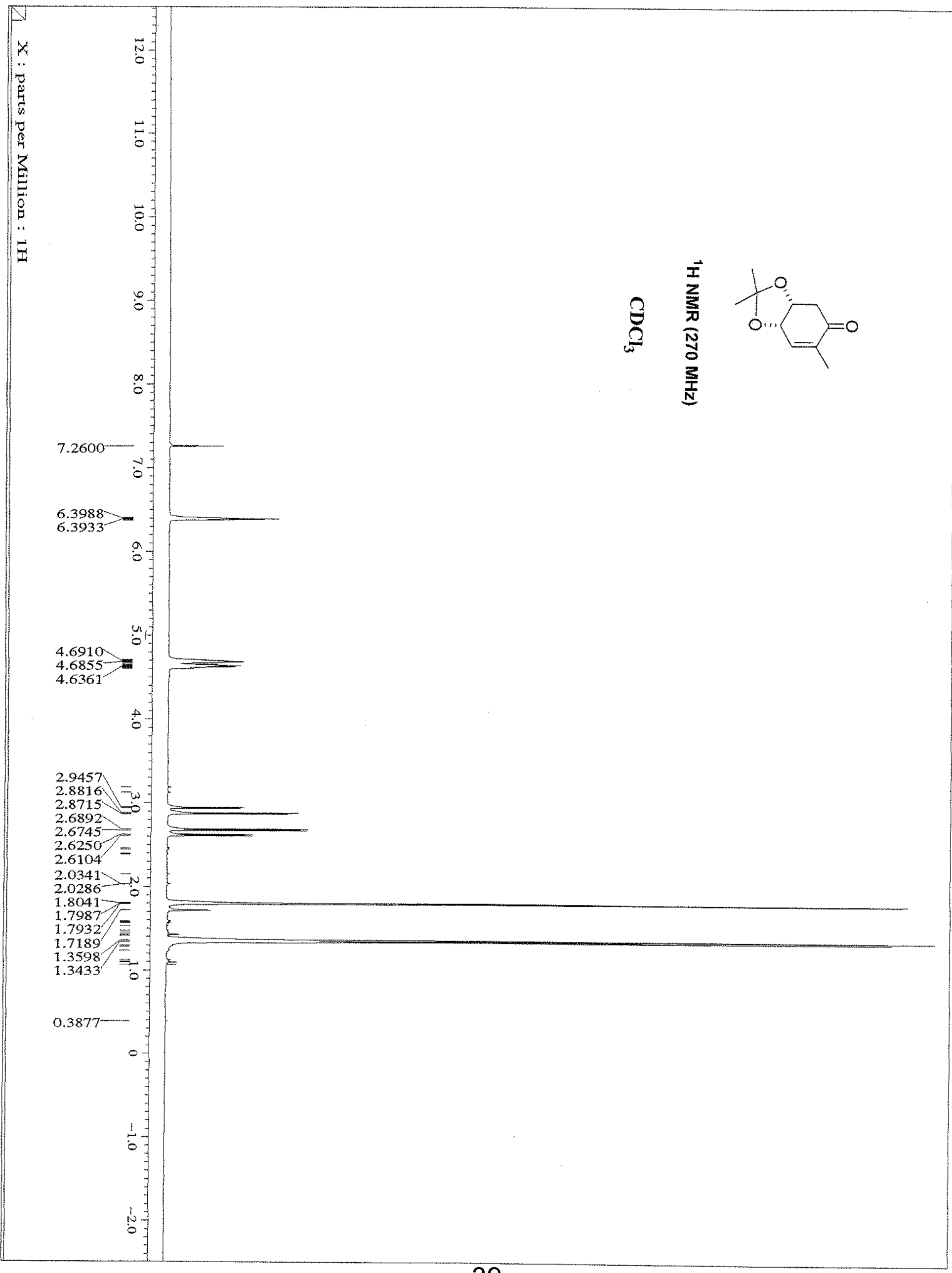




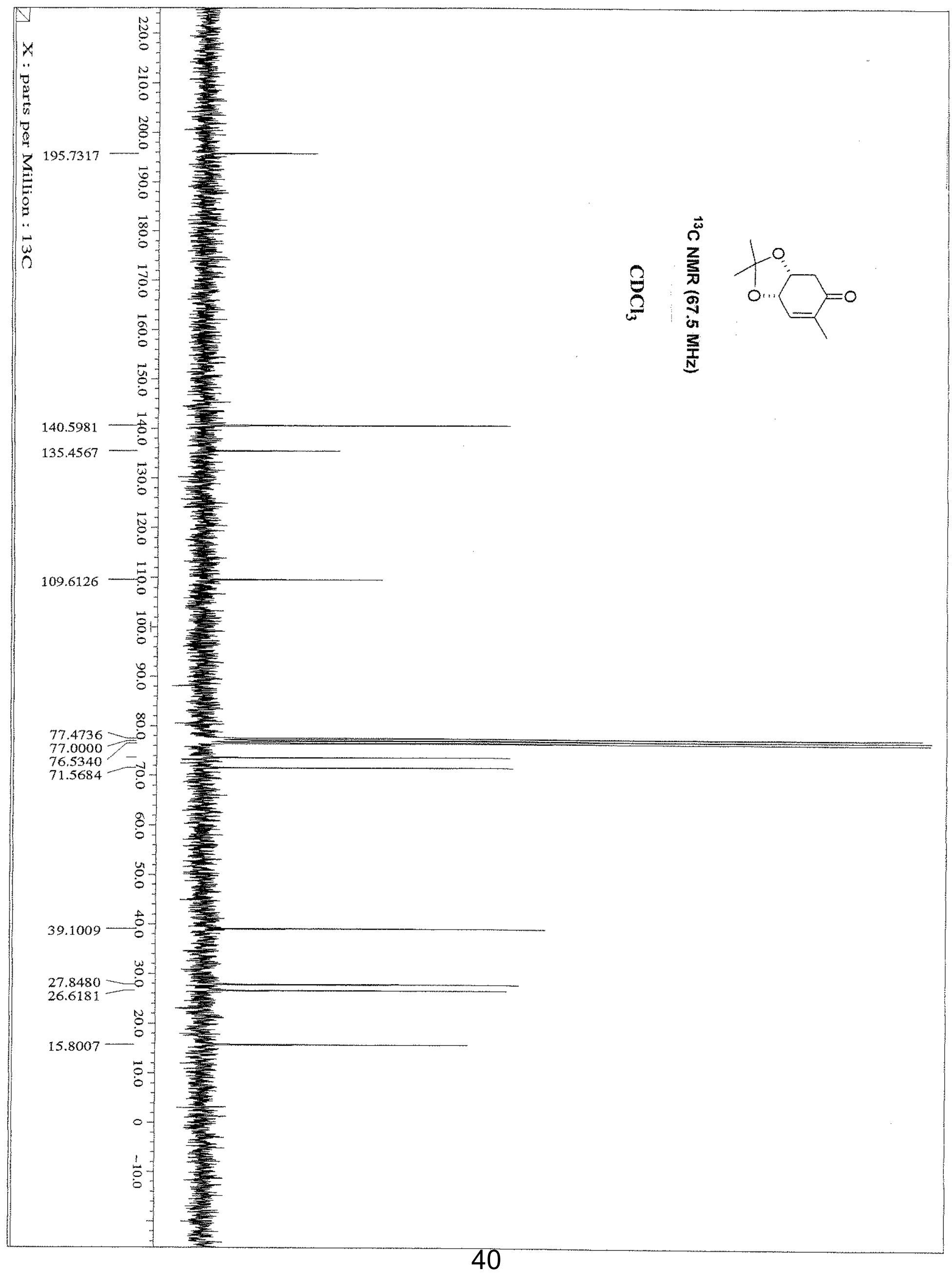




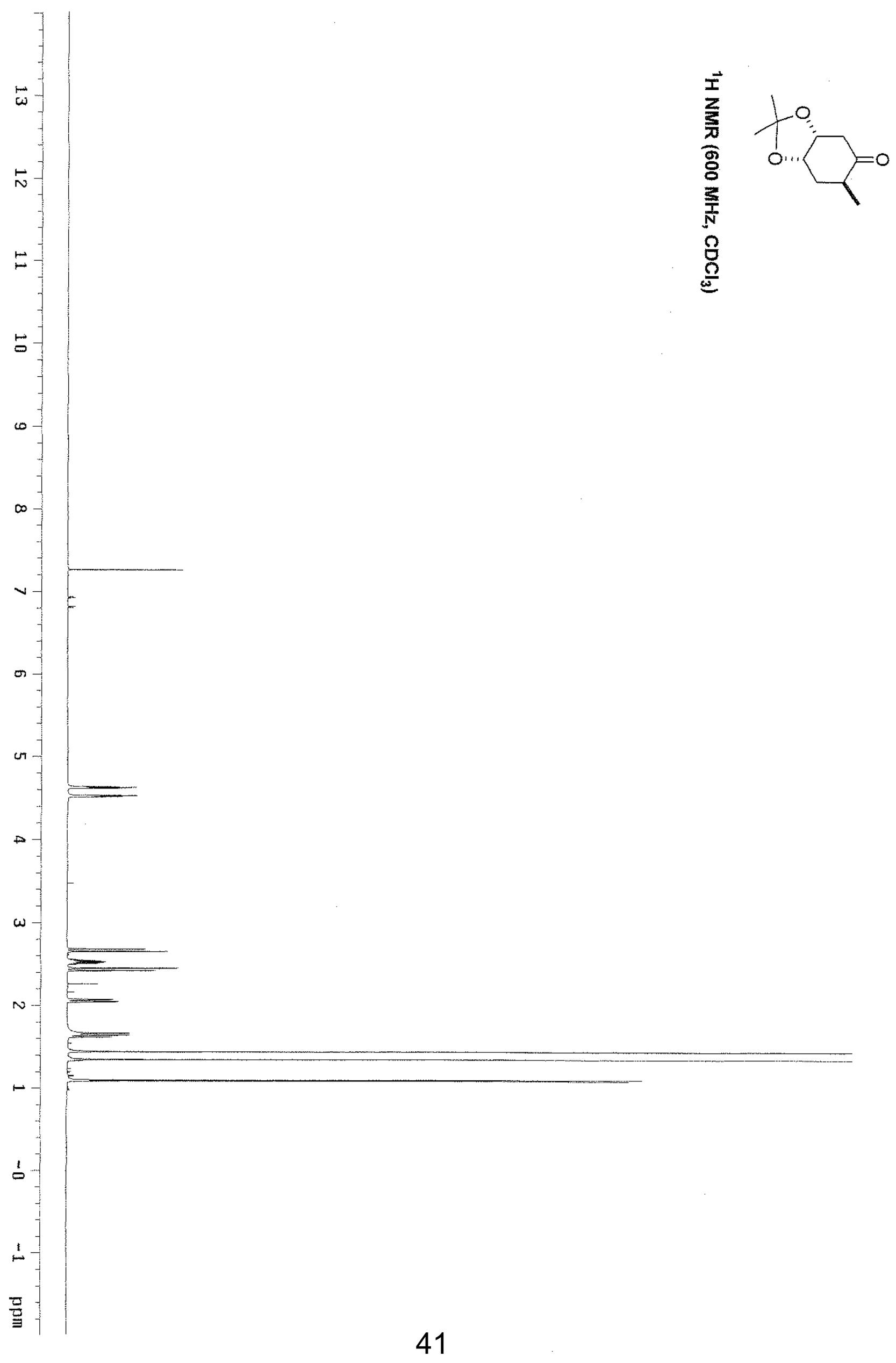




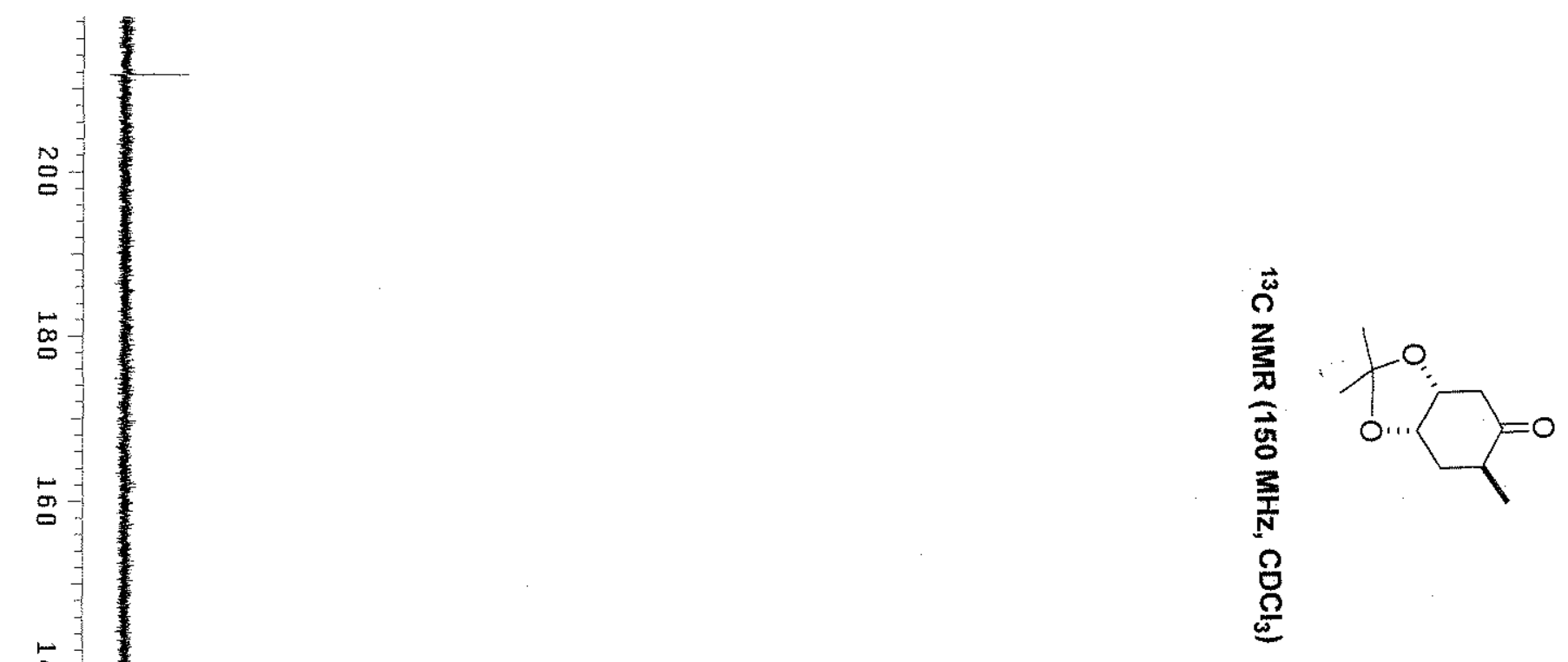

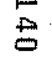

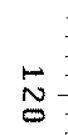

吕

$\stackrel{\infty}{=}$

g

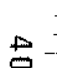
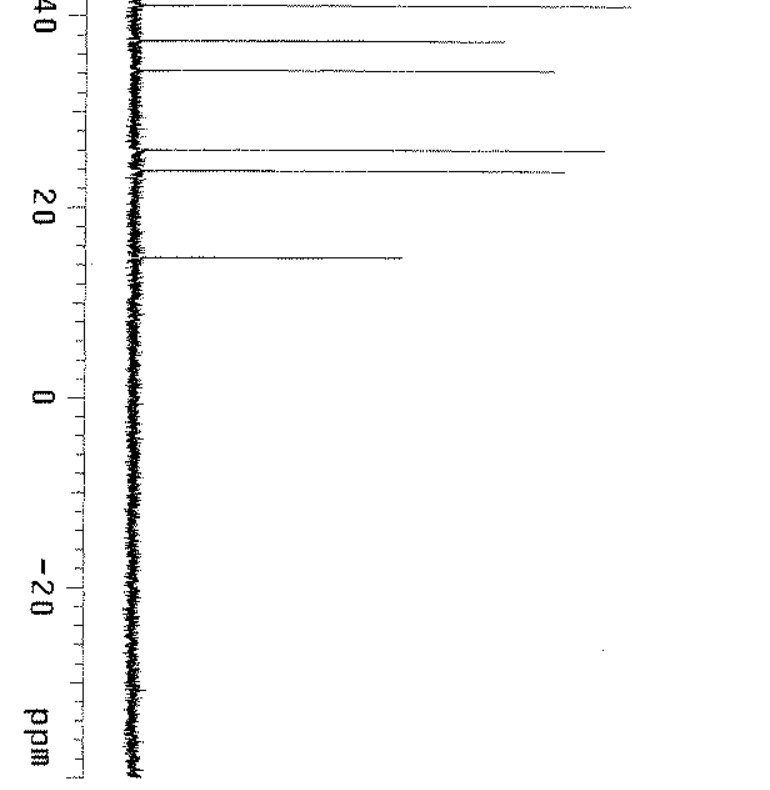


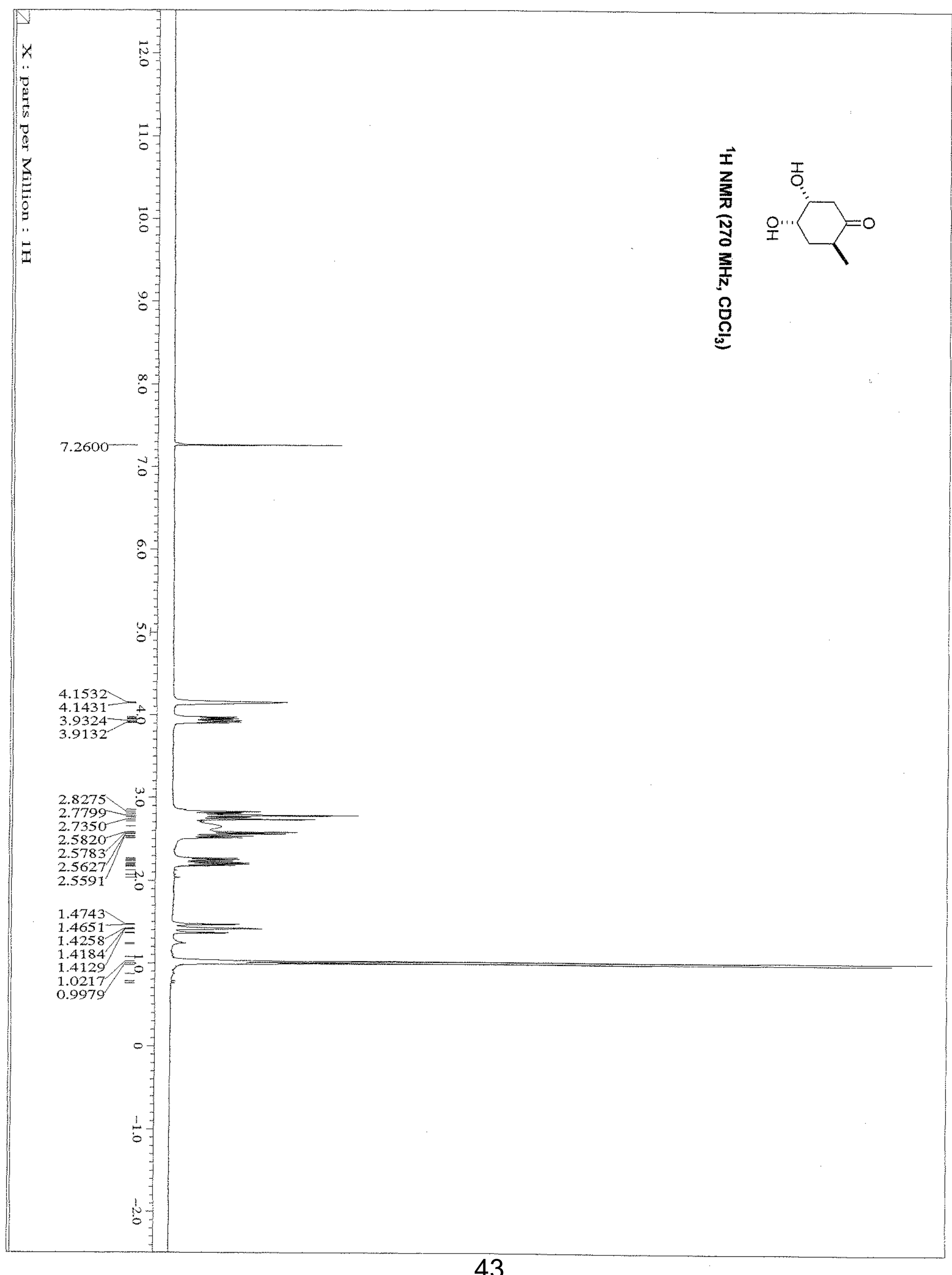




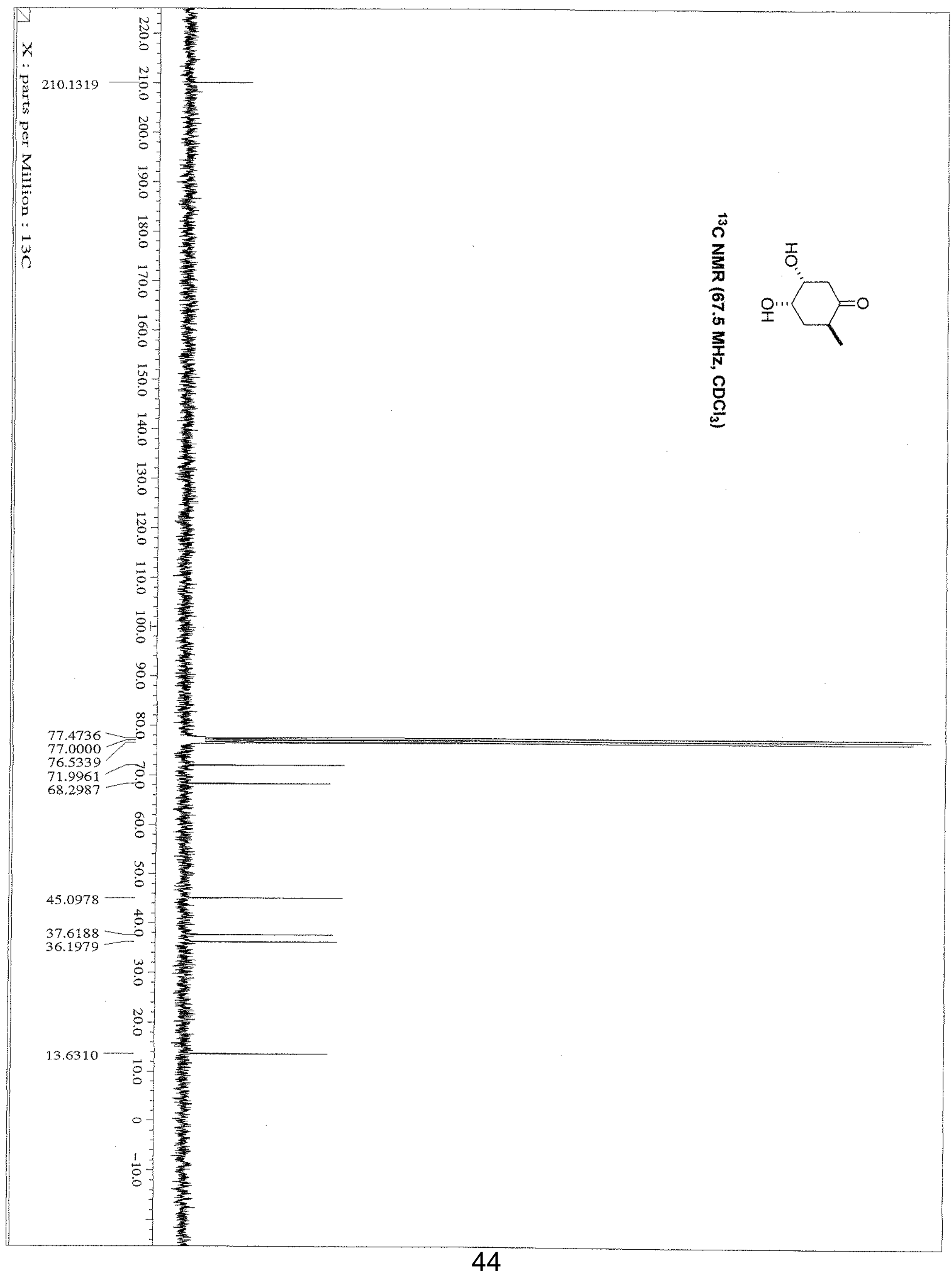




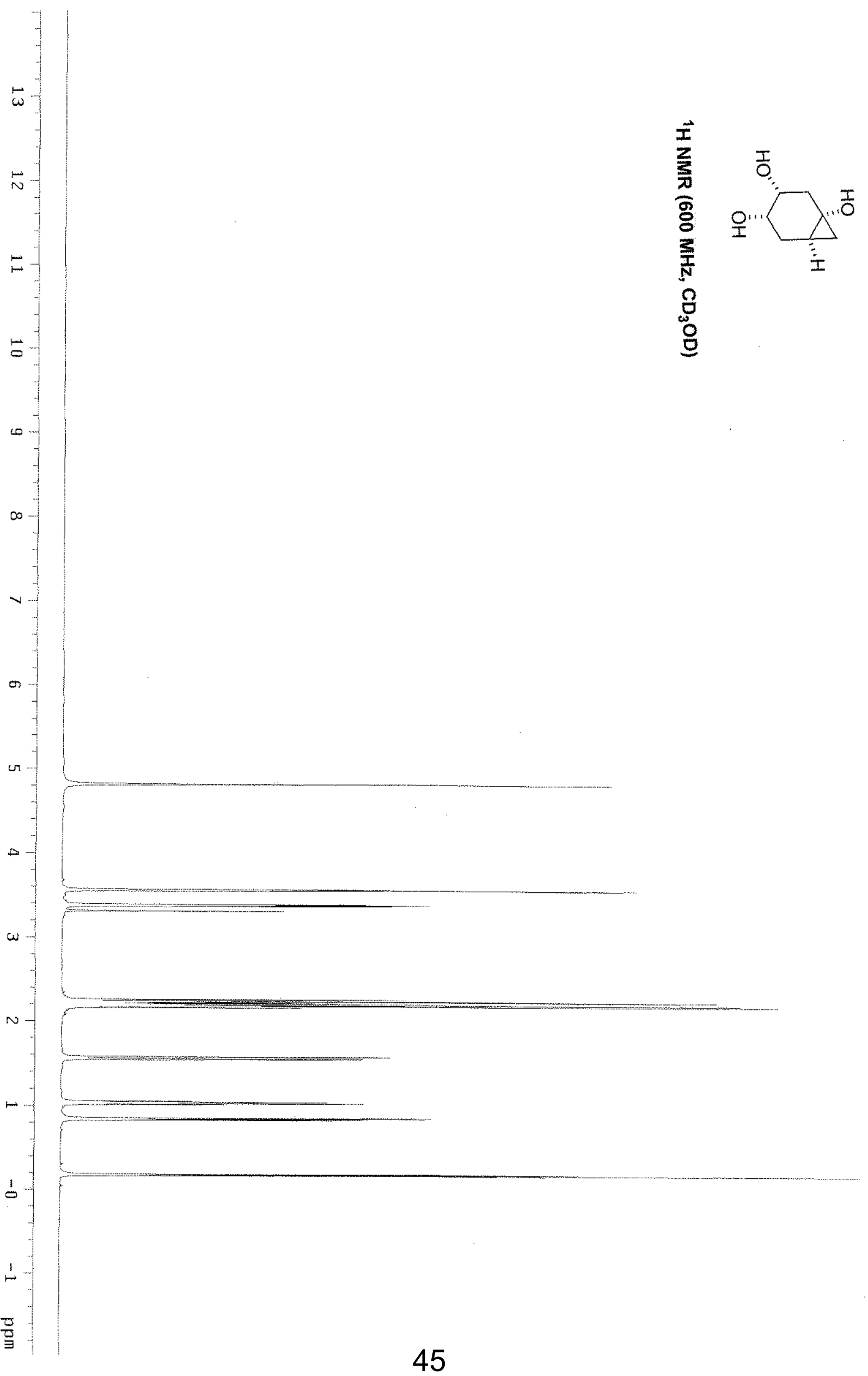




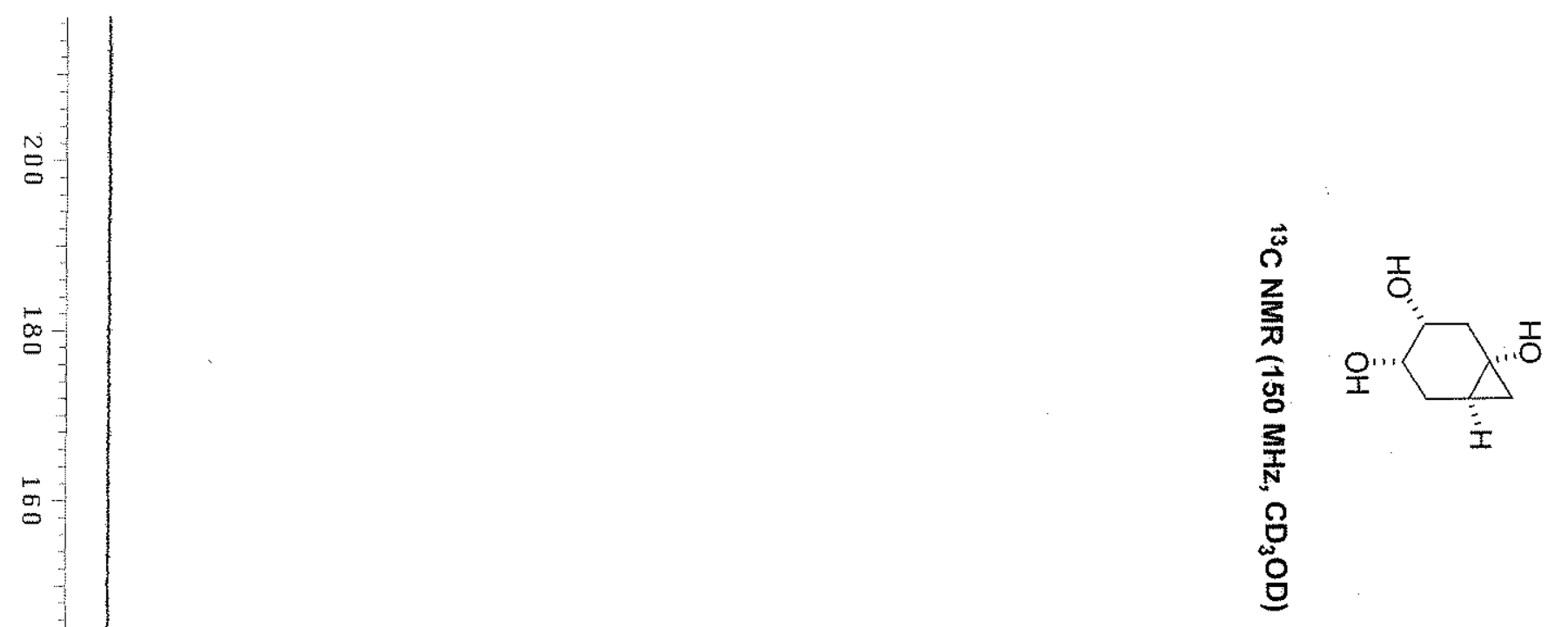

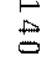

$\stackrel{\sim}{\circ}$

8

$\infty$

9

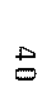

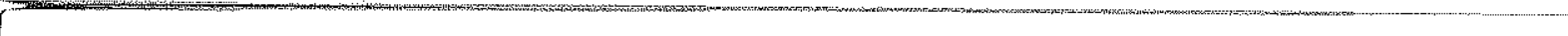

$\stackrel{\circ}{\circ}$

$\circ$

N

뫀 


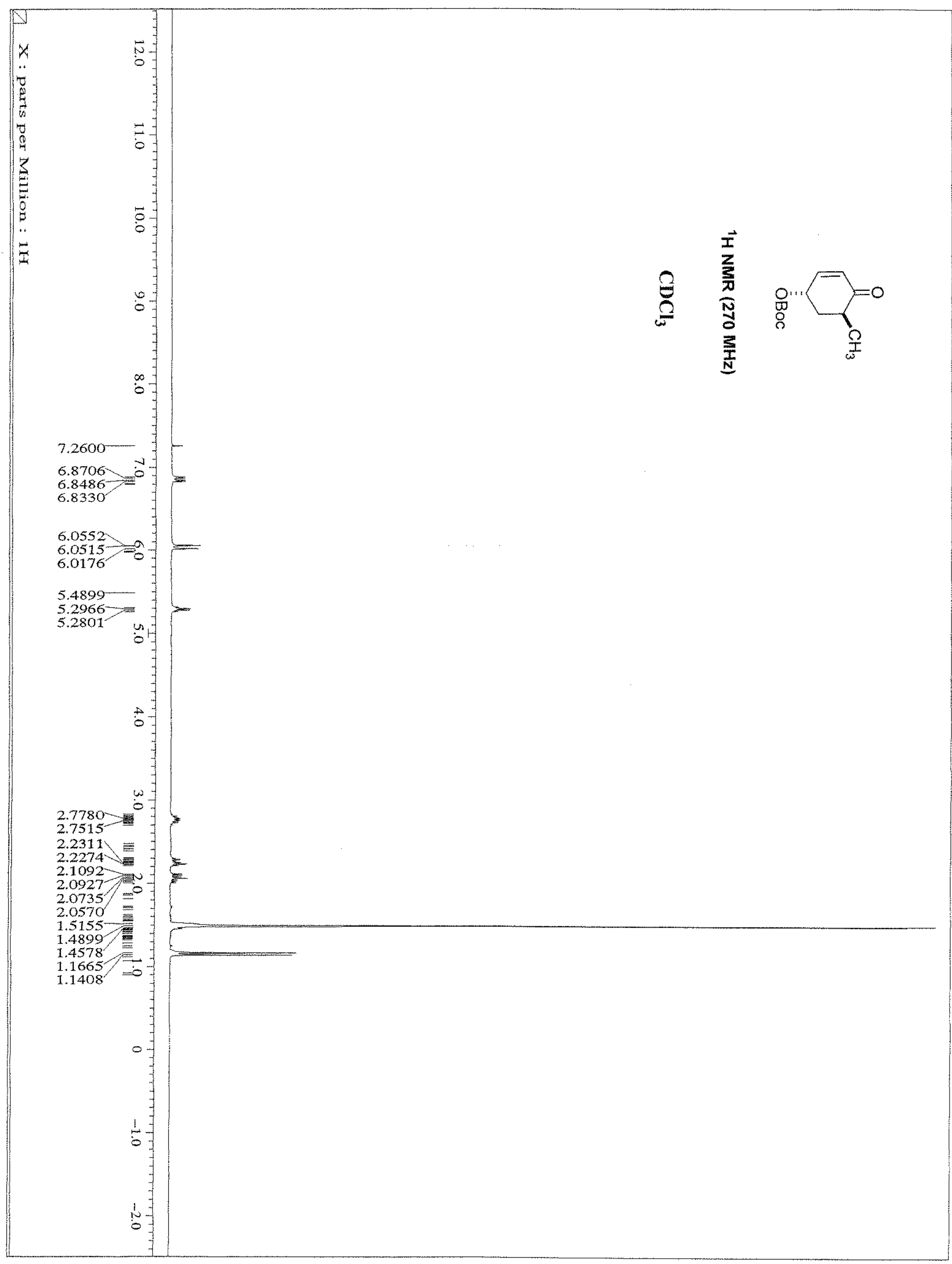




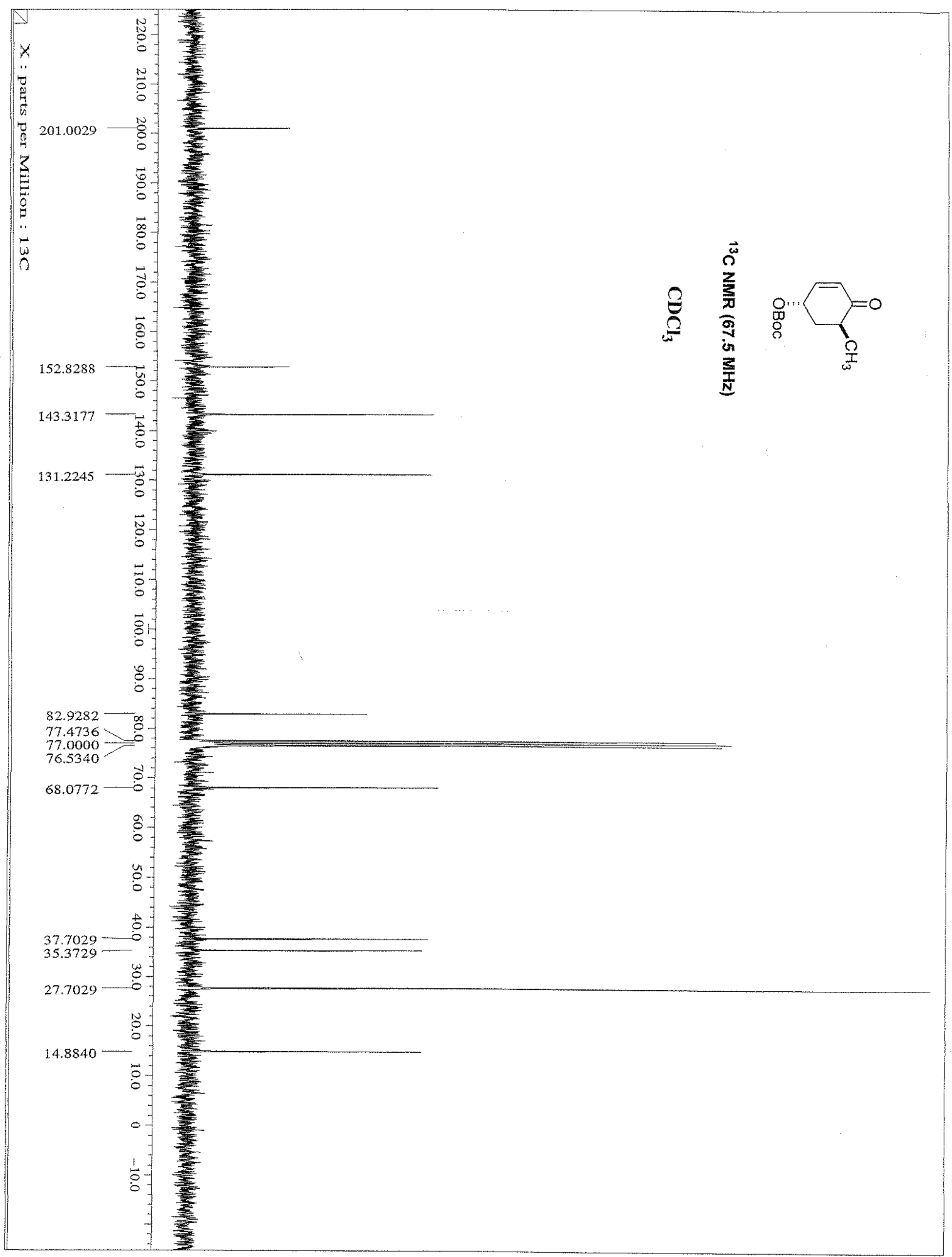




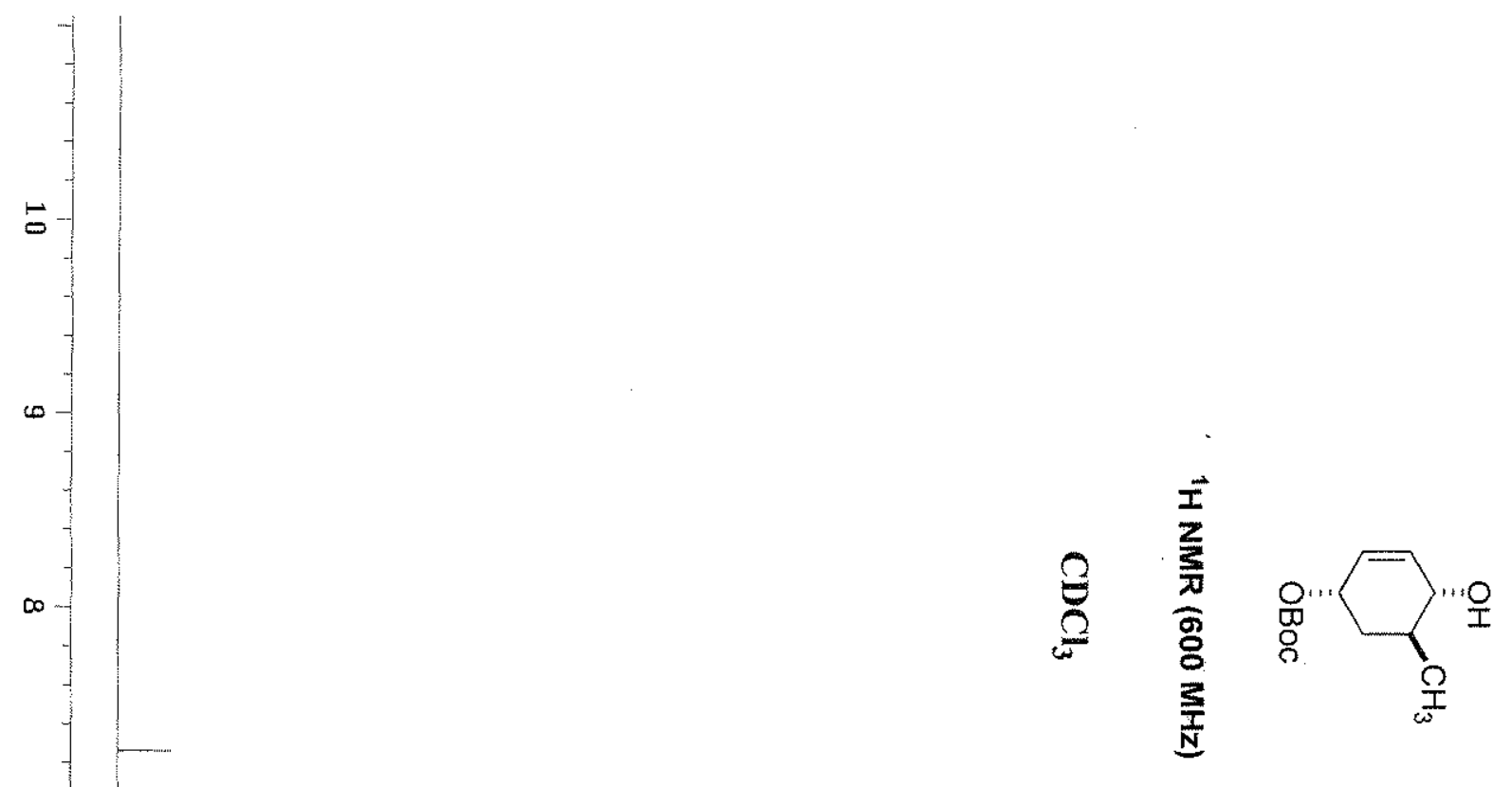




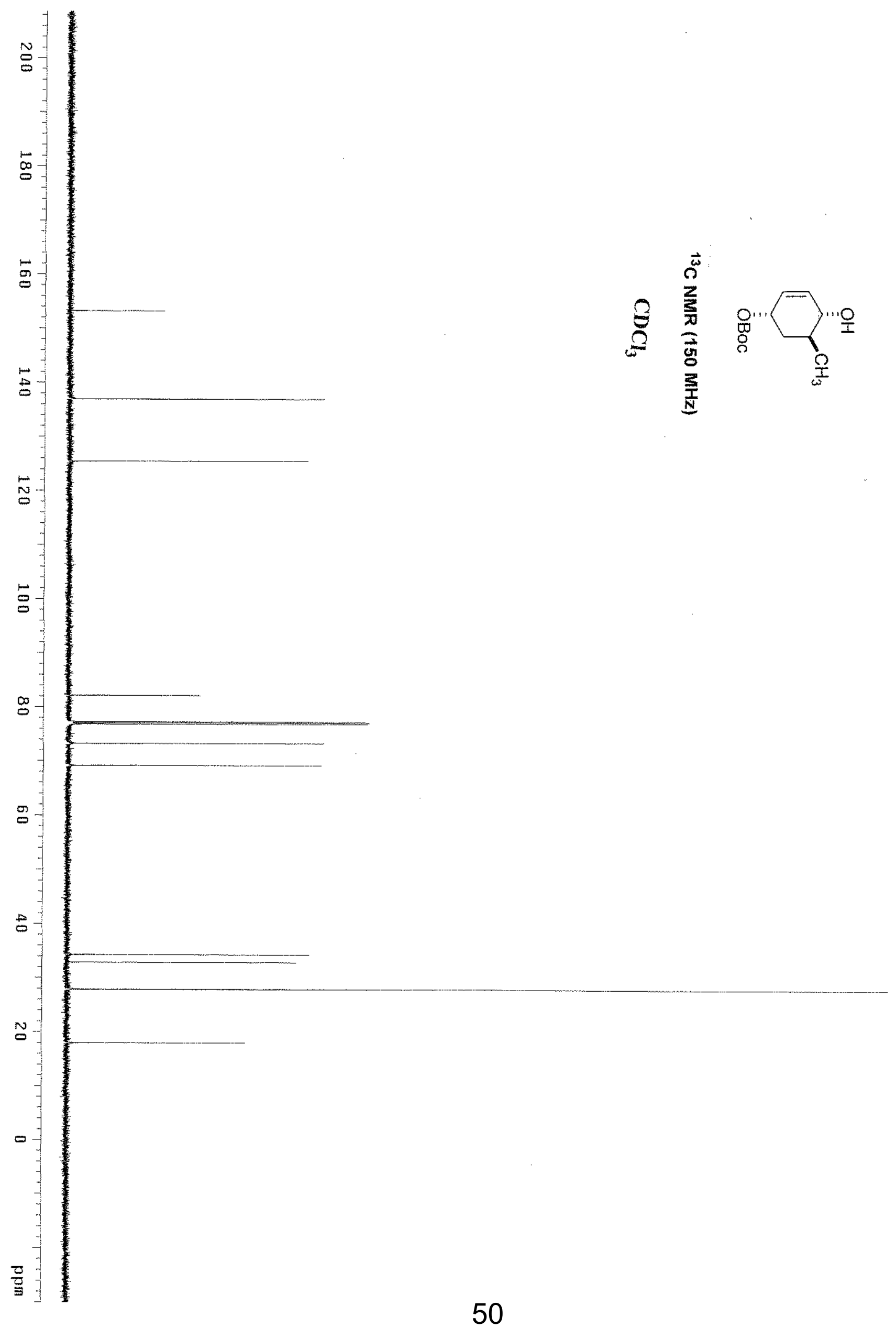




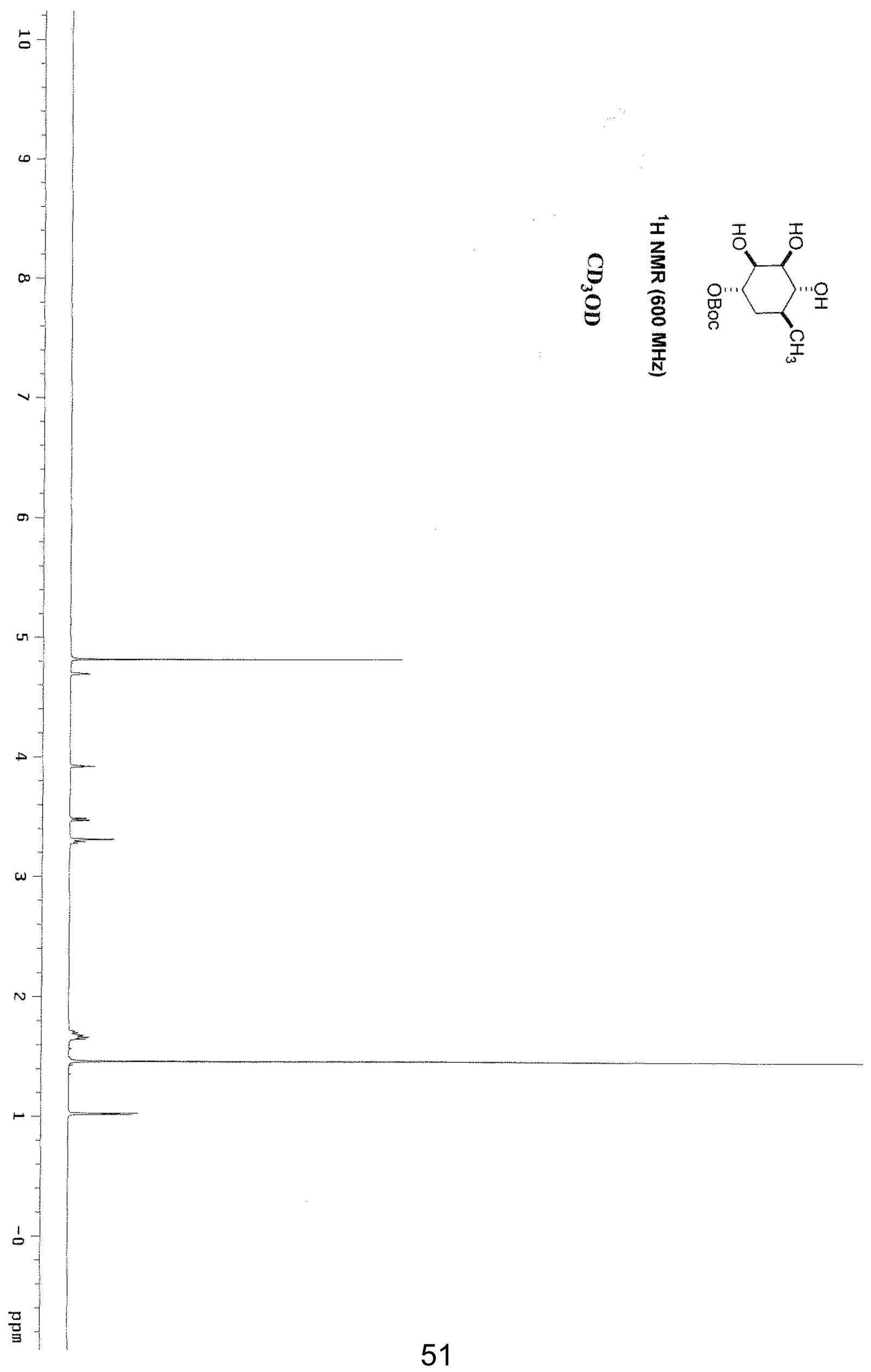




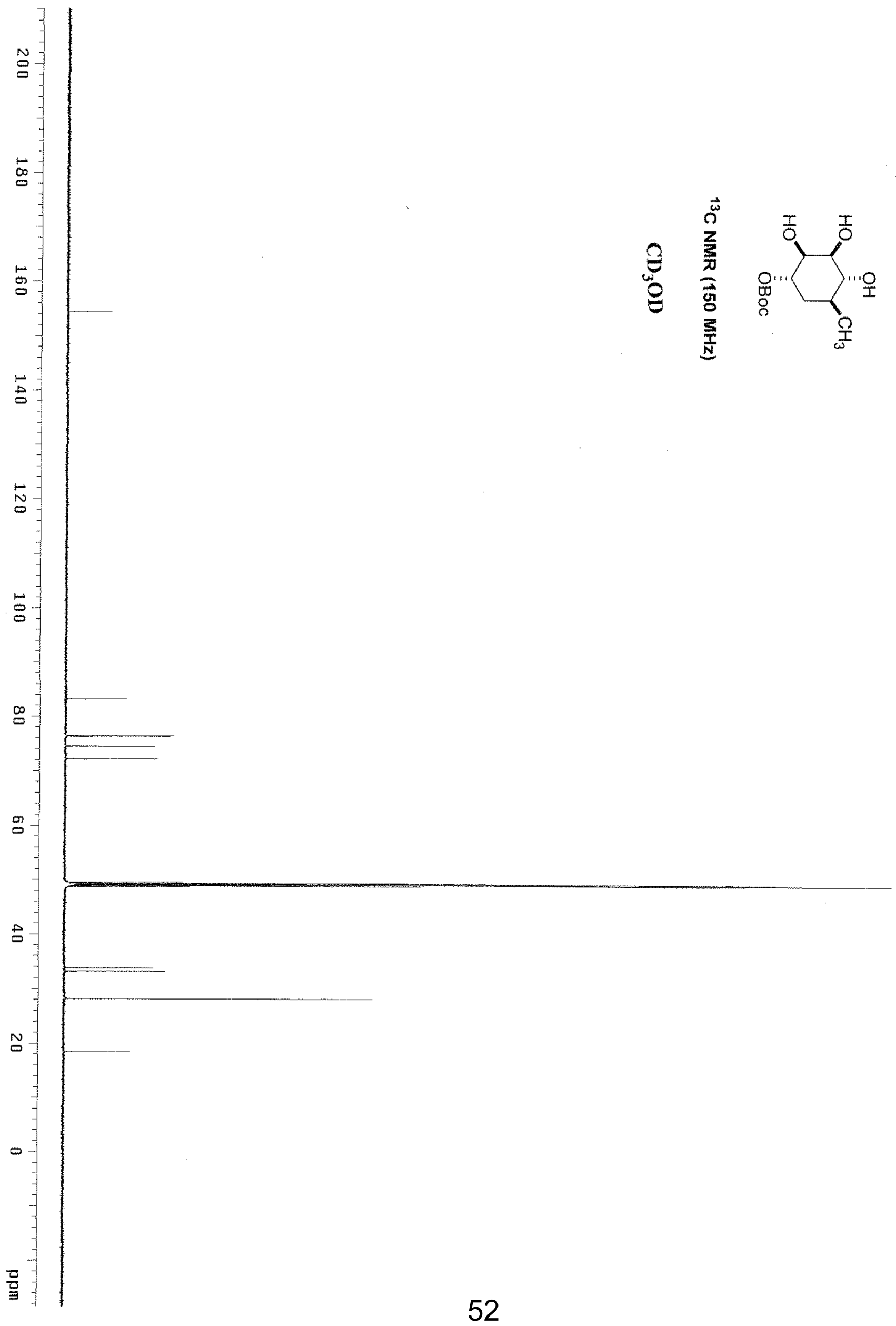




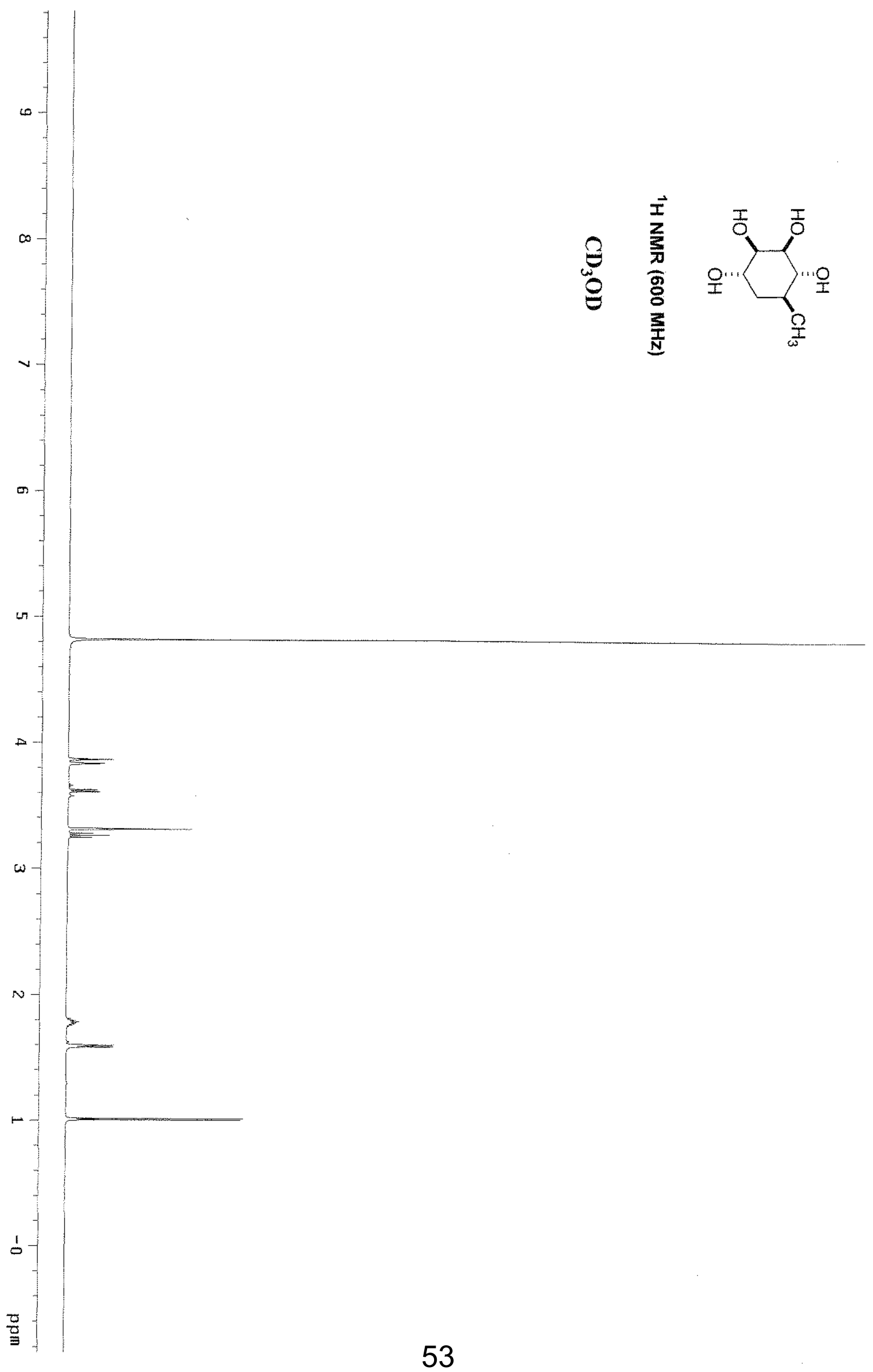




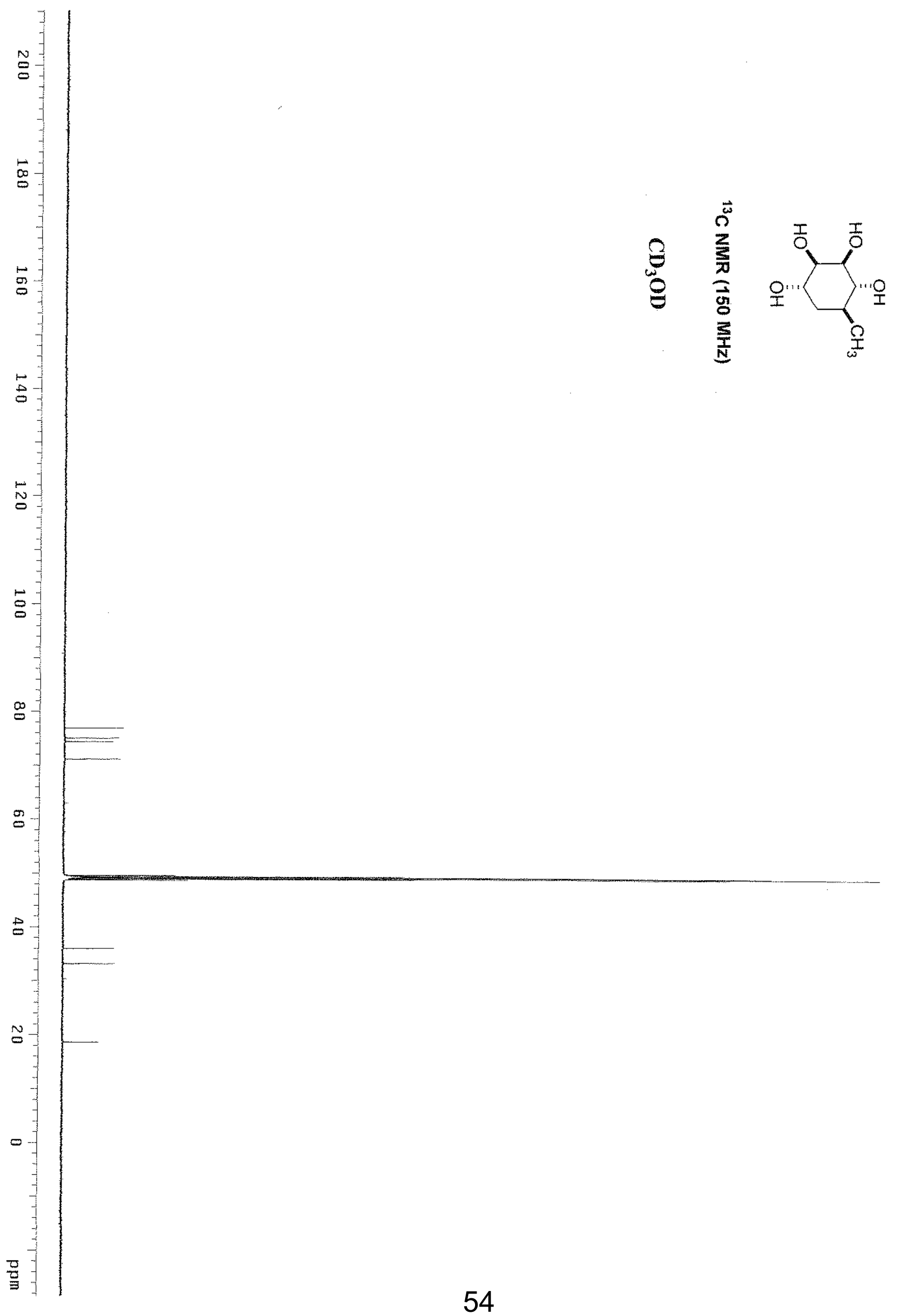




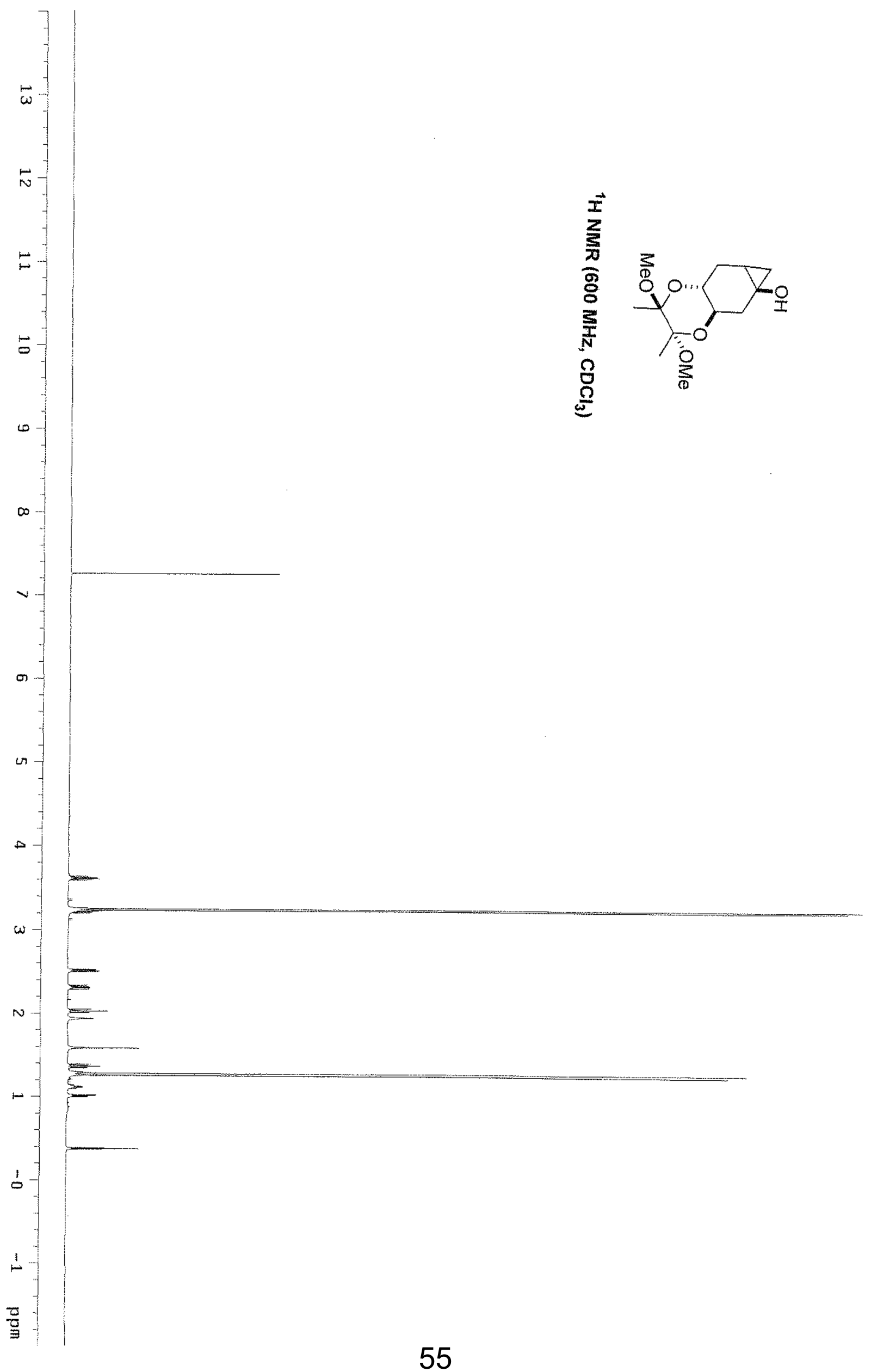




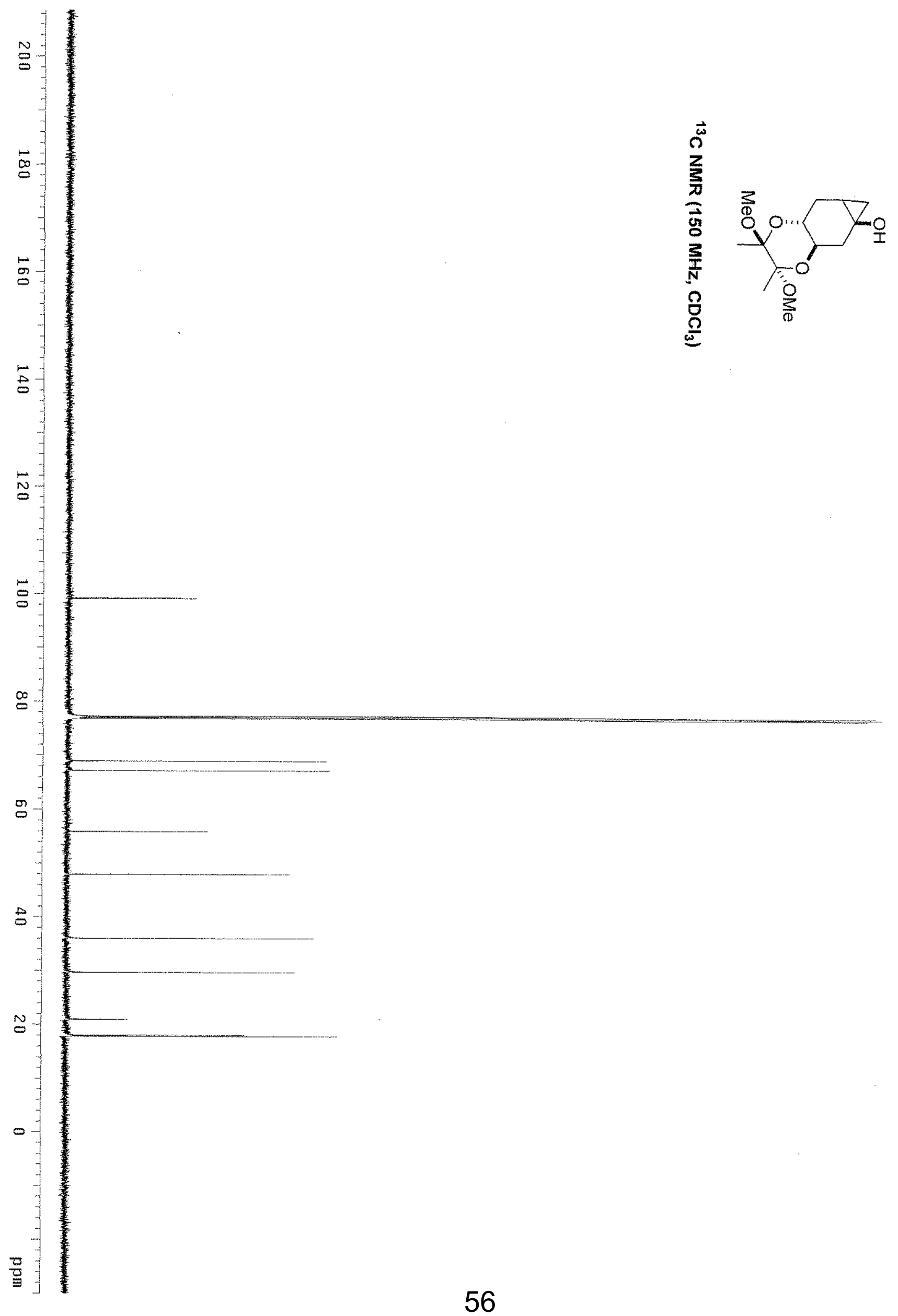




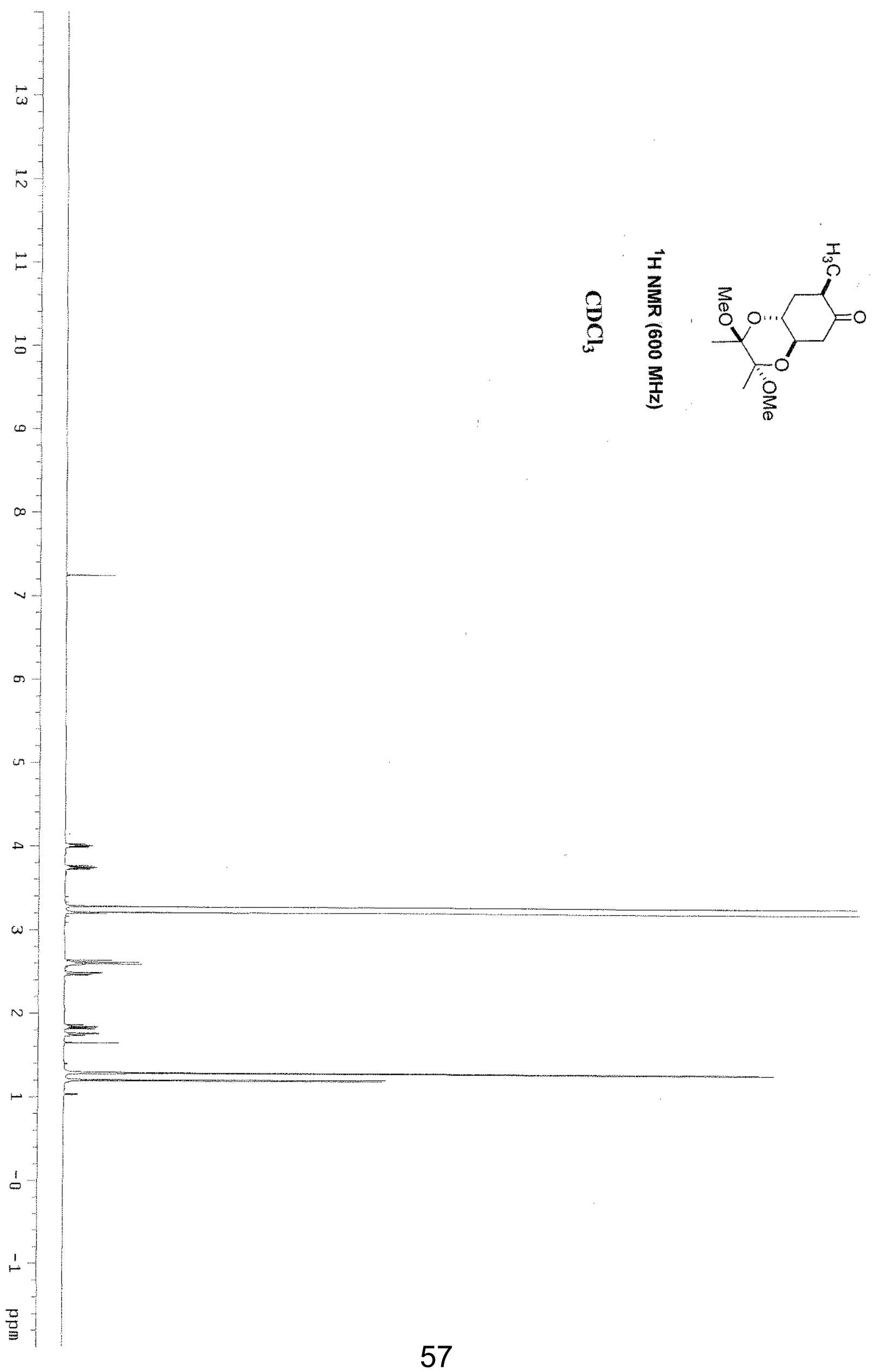




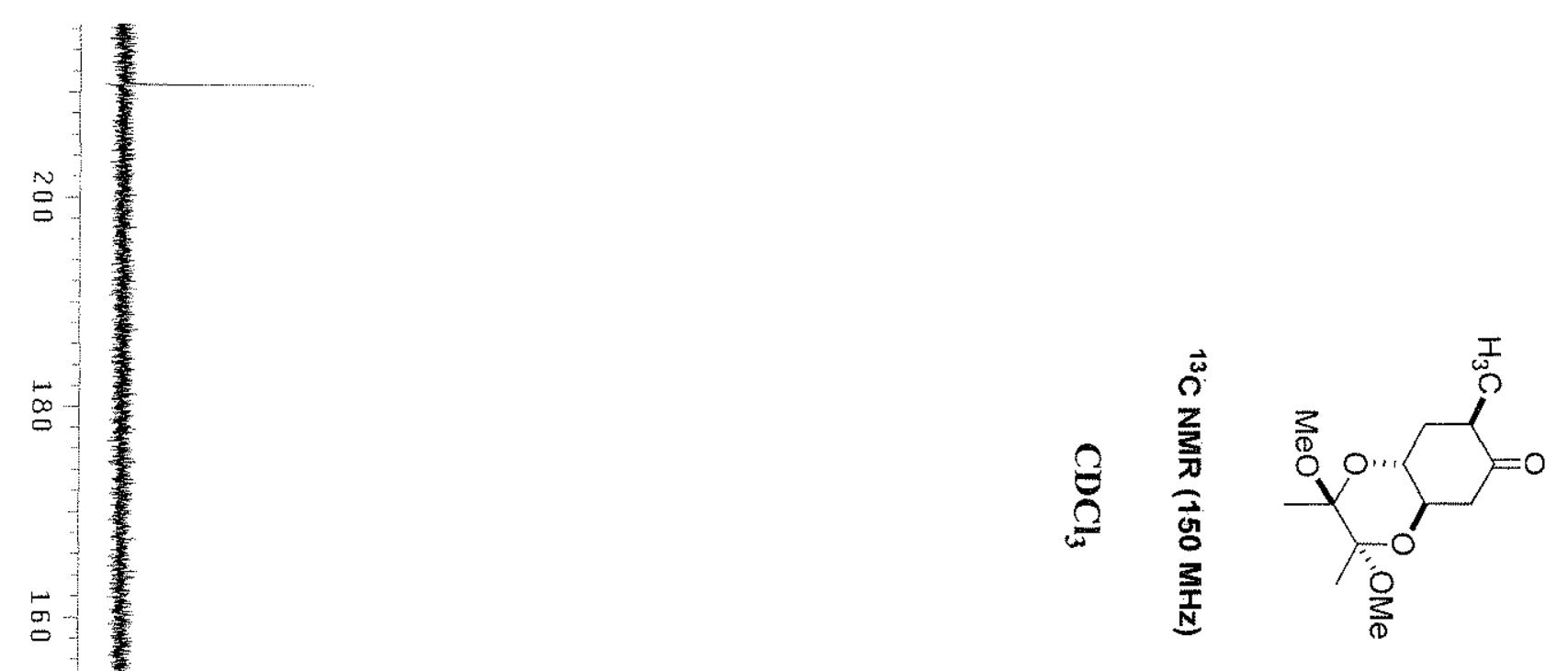

s.

s

5

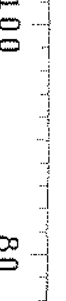

$\stackrel{\infty}{\circ}$

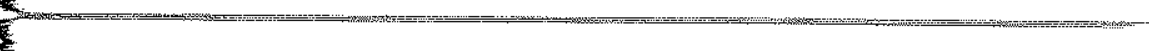

g.

妾

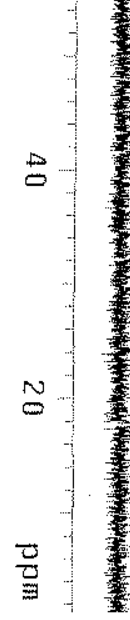

$\frac{4}{4}$ 


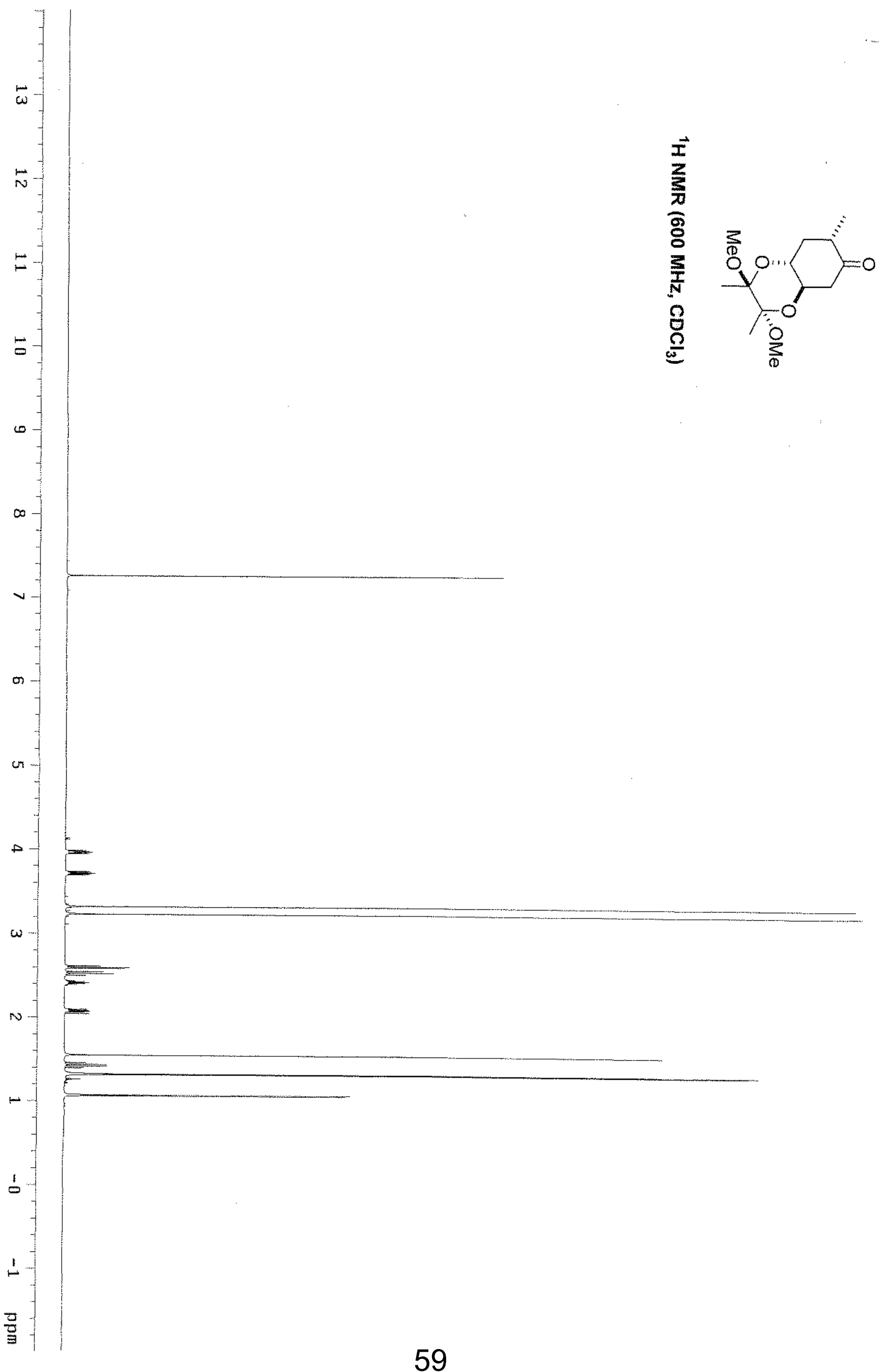




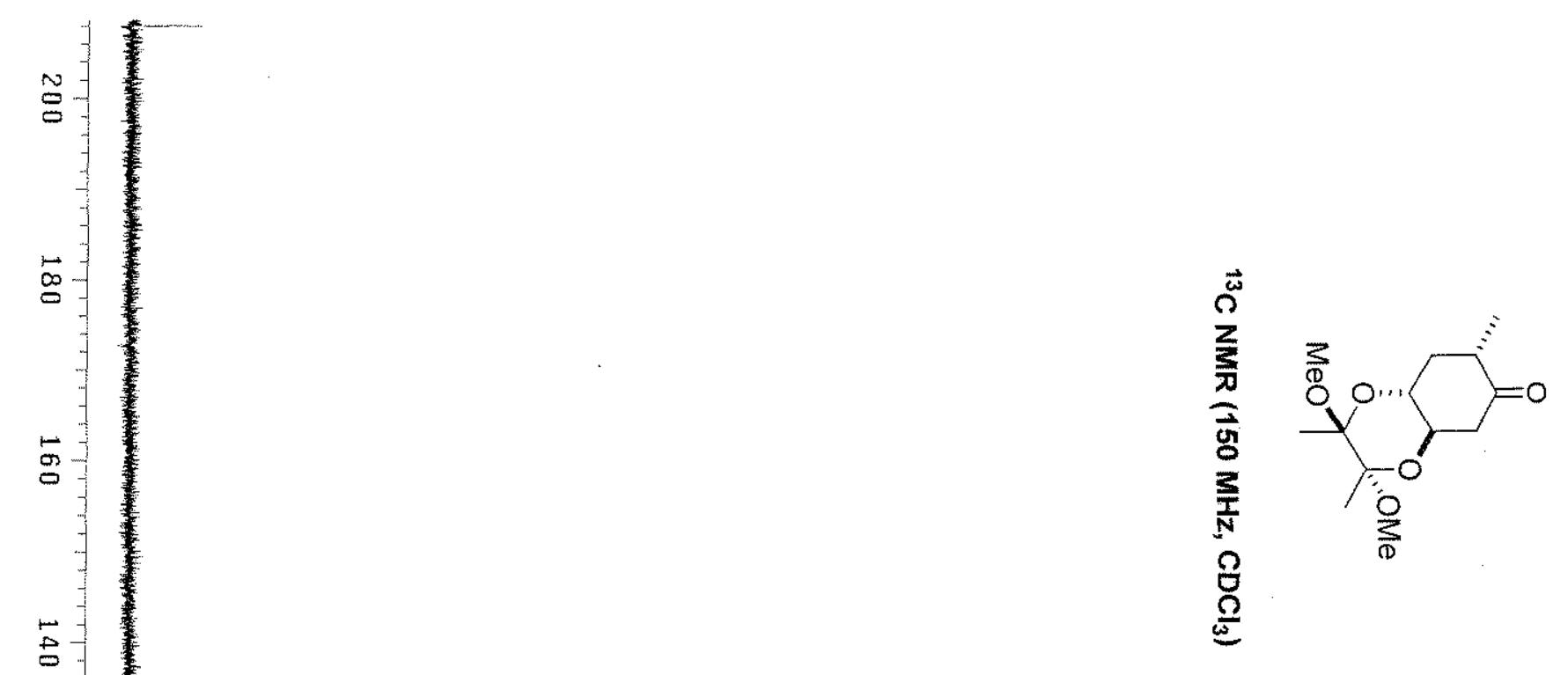

$\stackrel{\infty}{\circ}$

艺

5

8

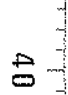

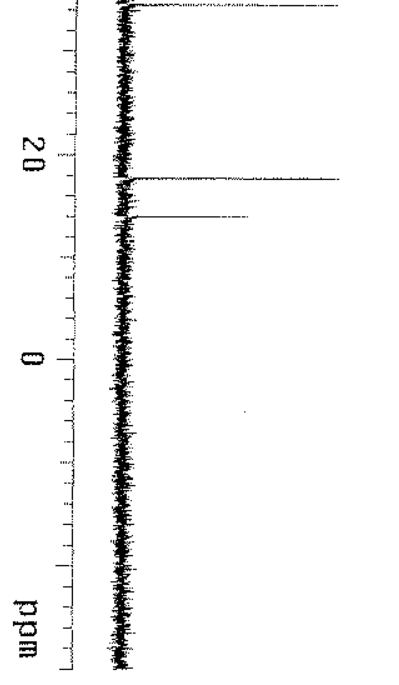




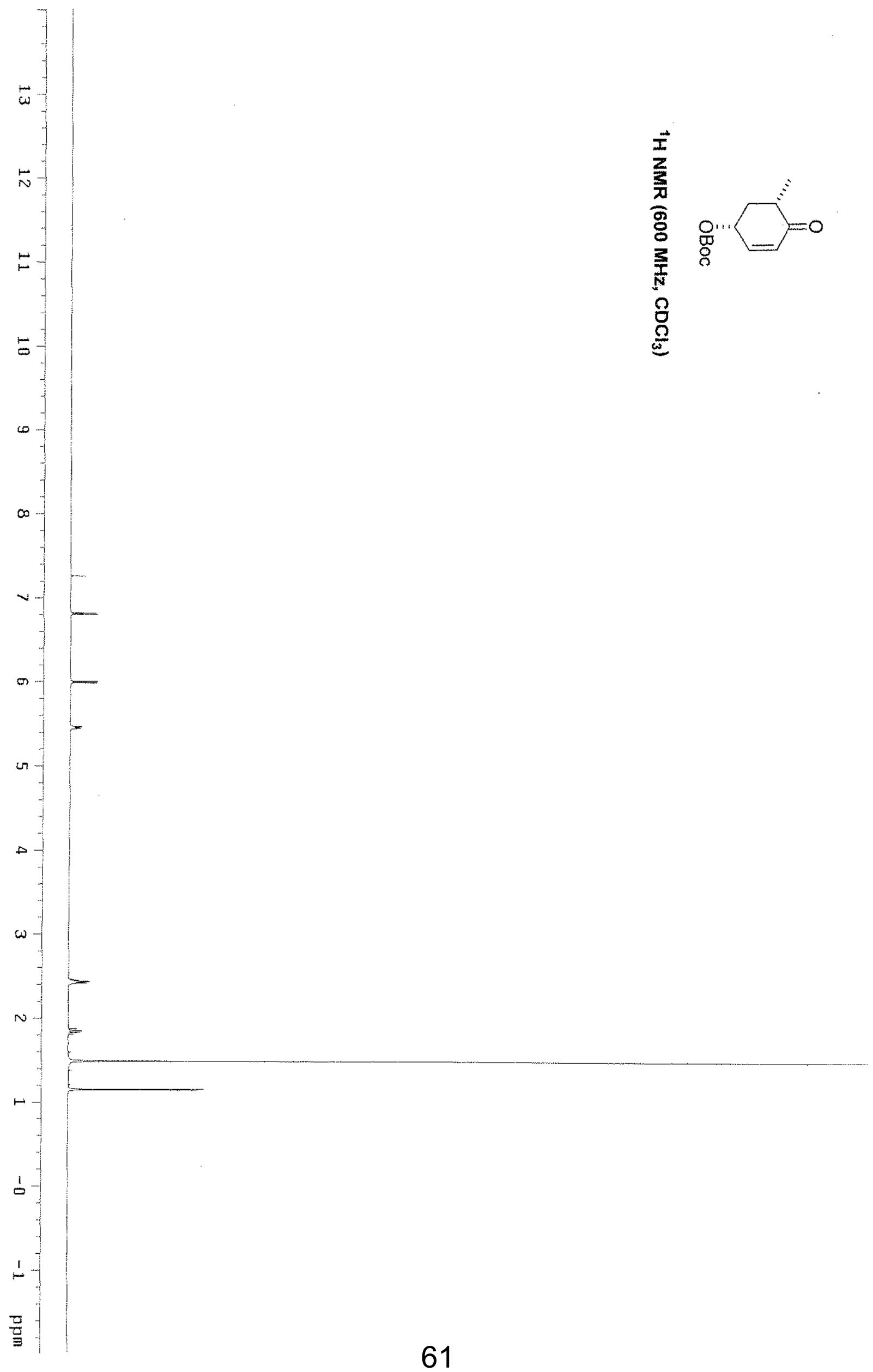




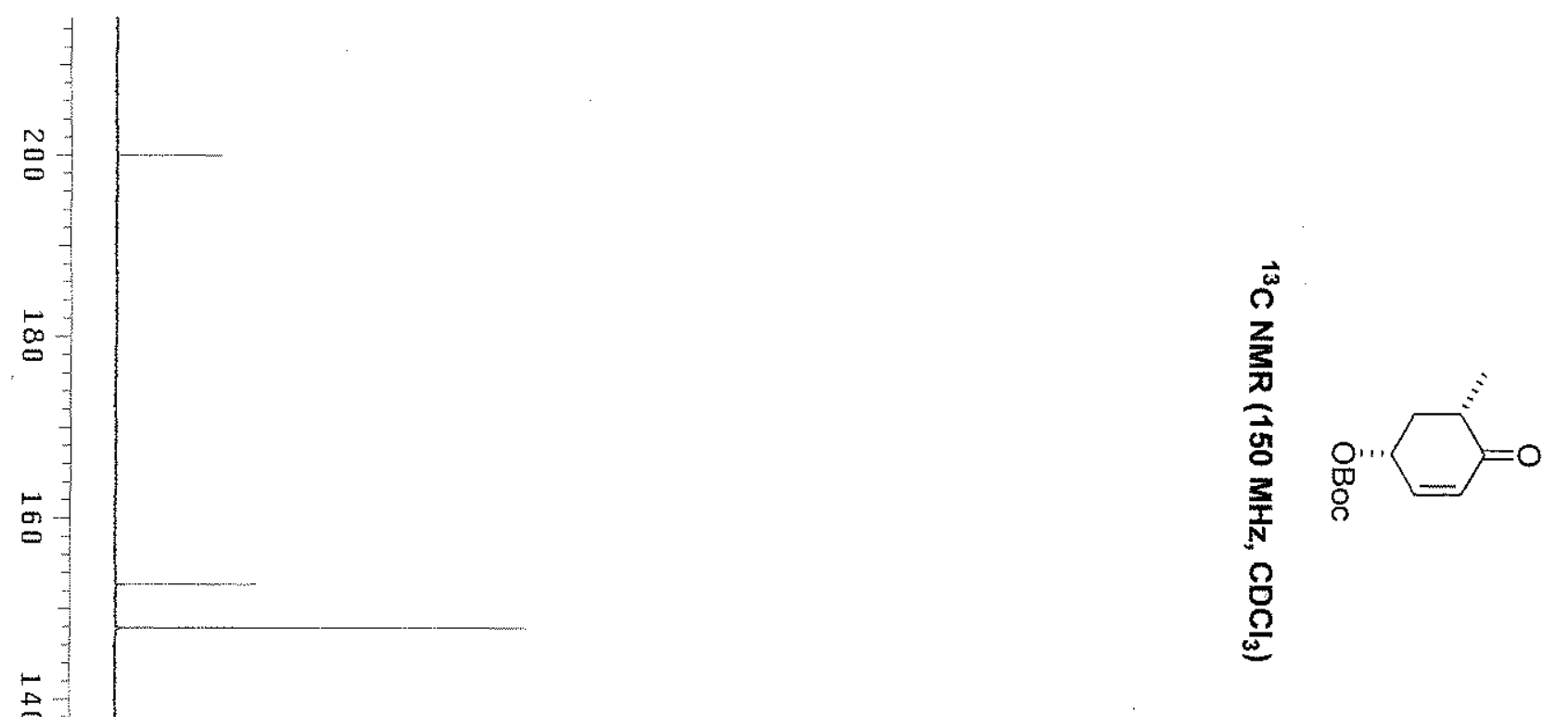

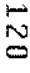

$\stackrel{\bullet}{0}$

$\infty$

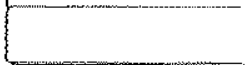

$\sigma$

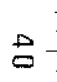

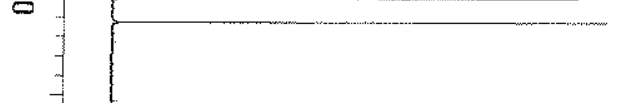

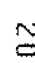

$-$

$\stackrel{1}{0}$

믐 


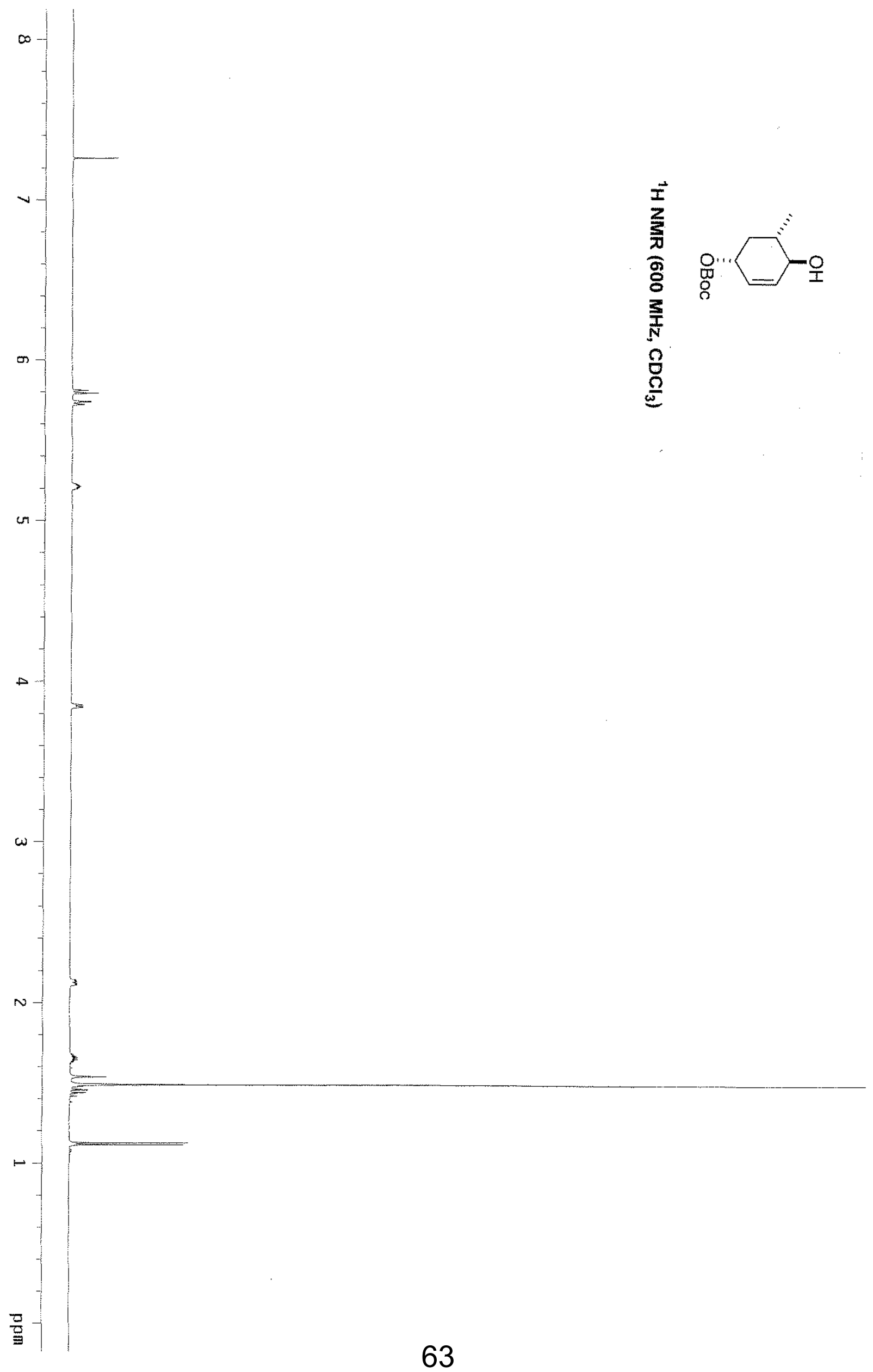




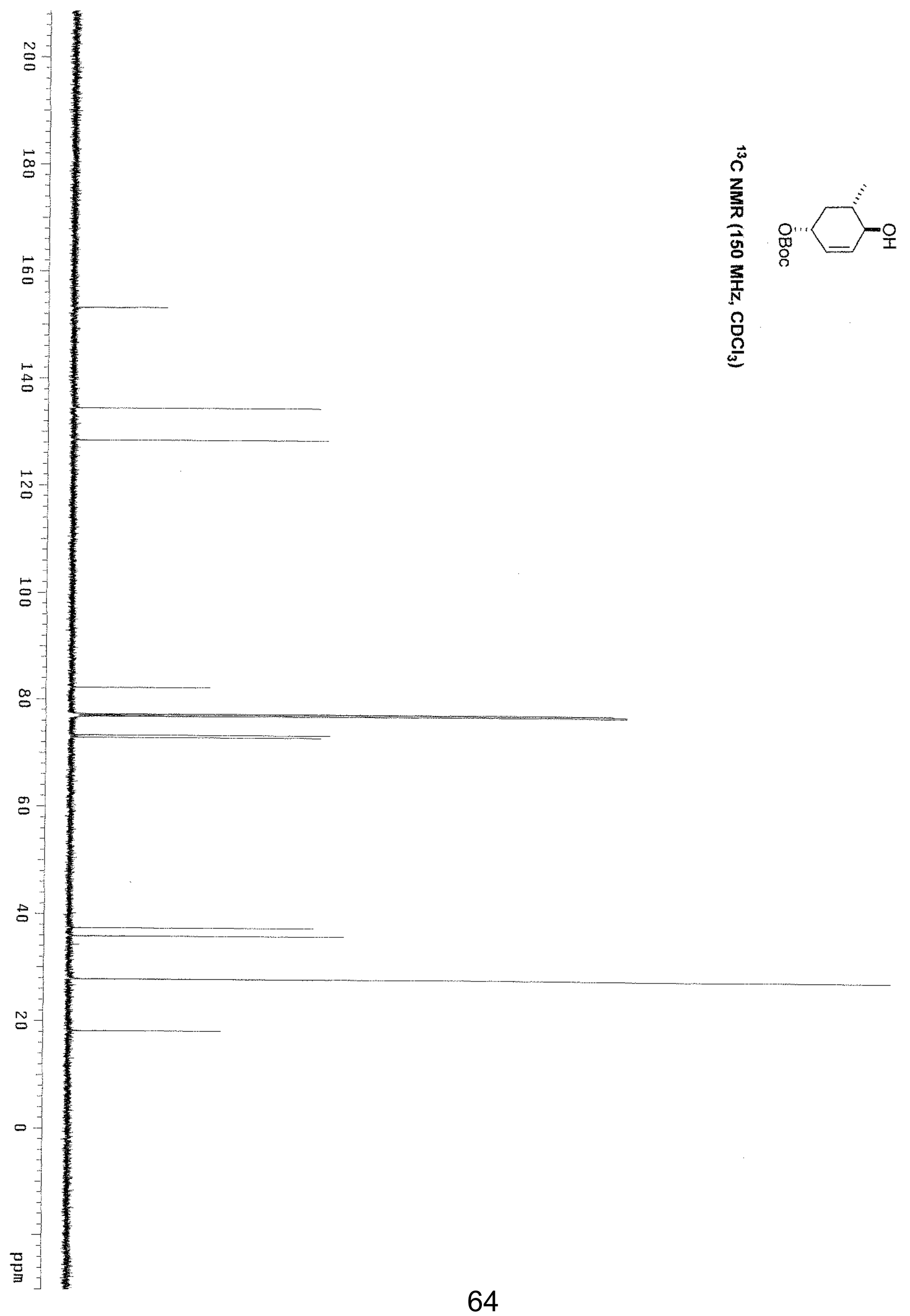




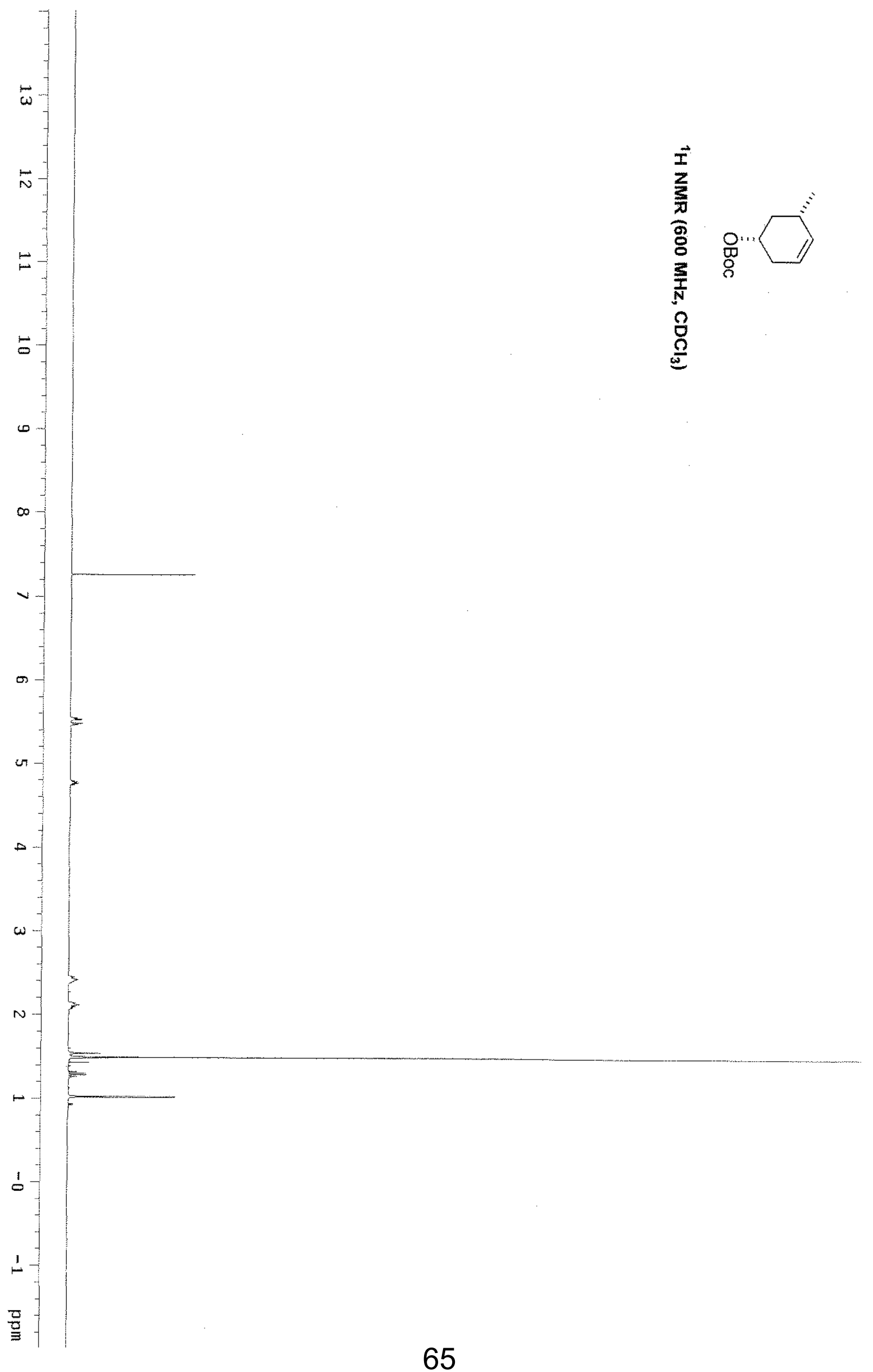




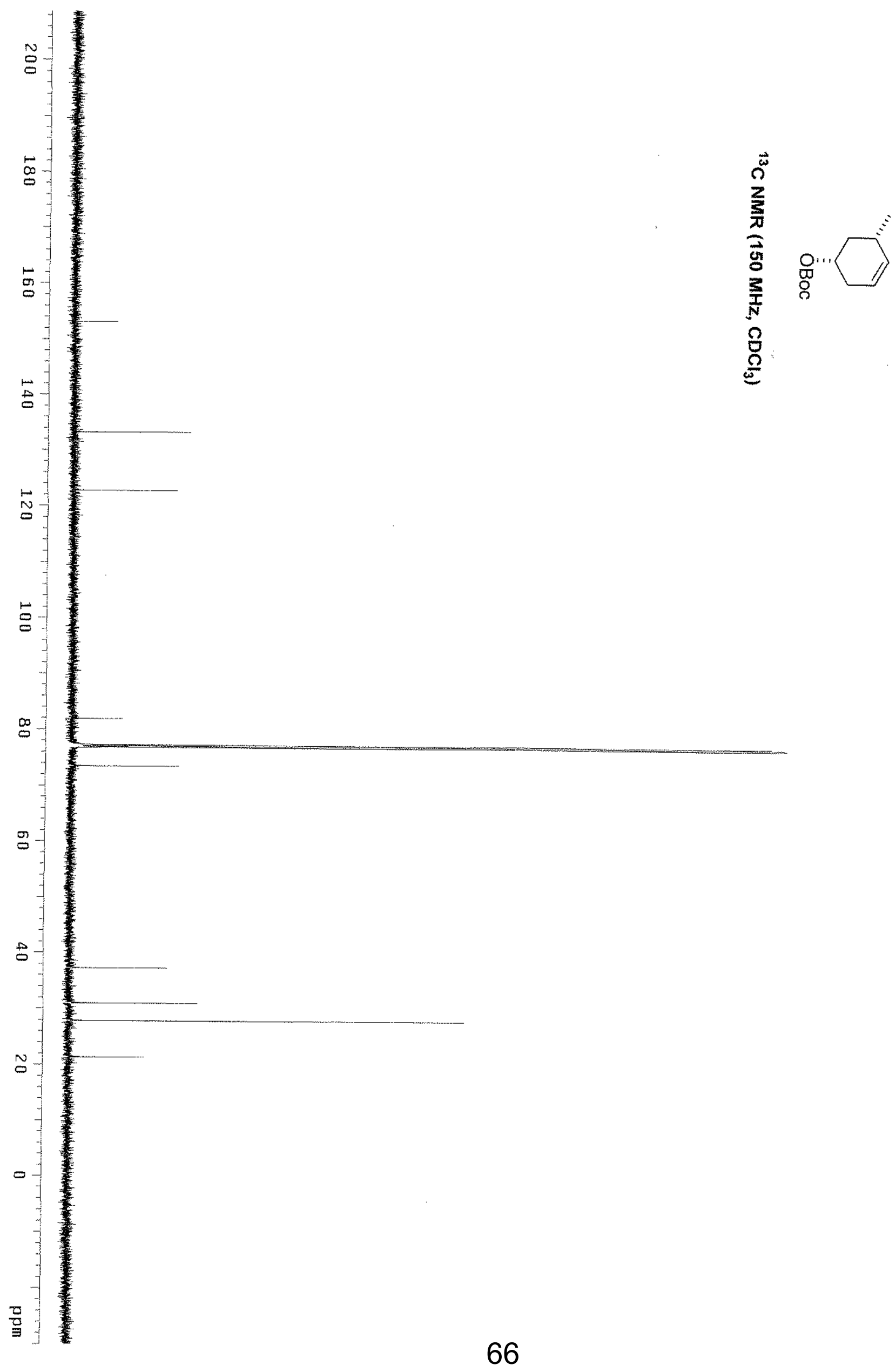




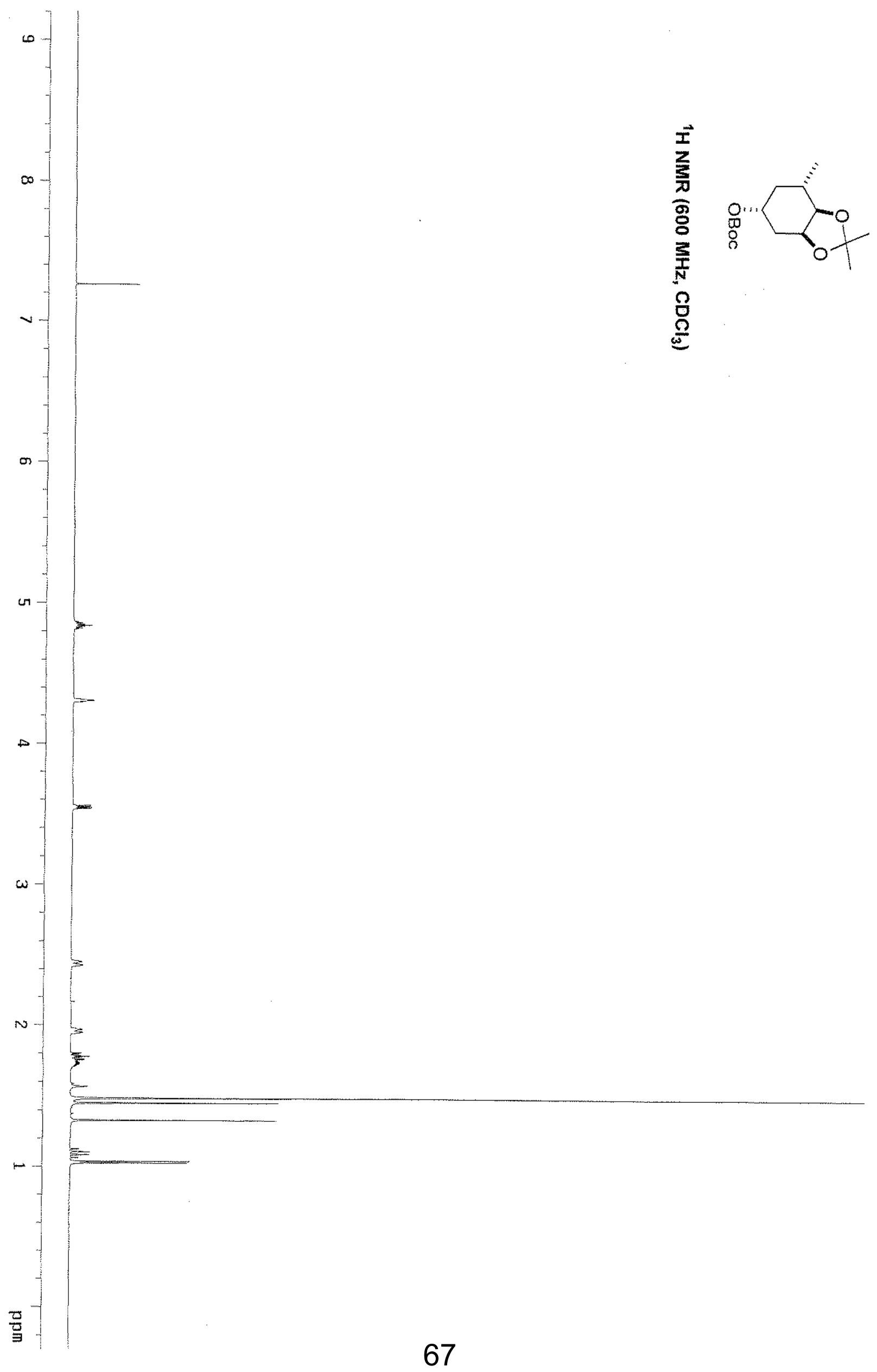




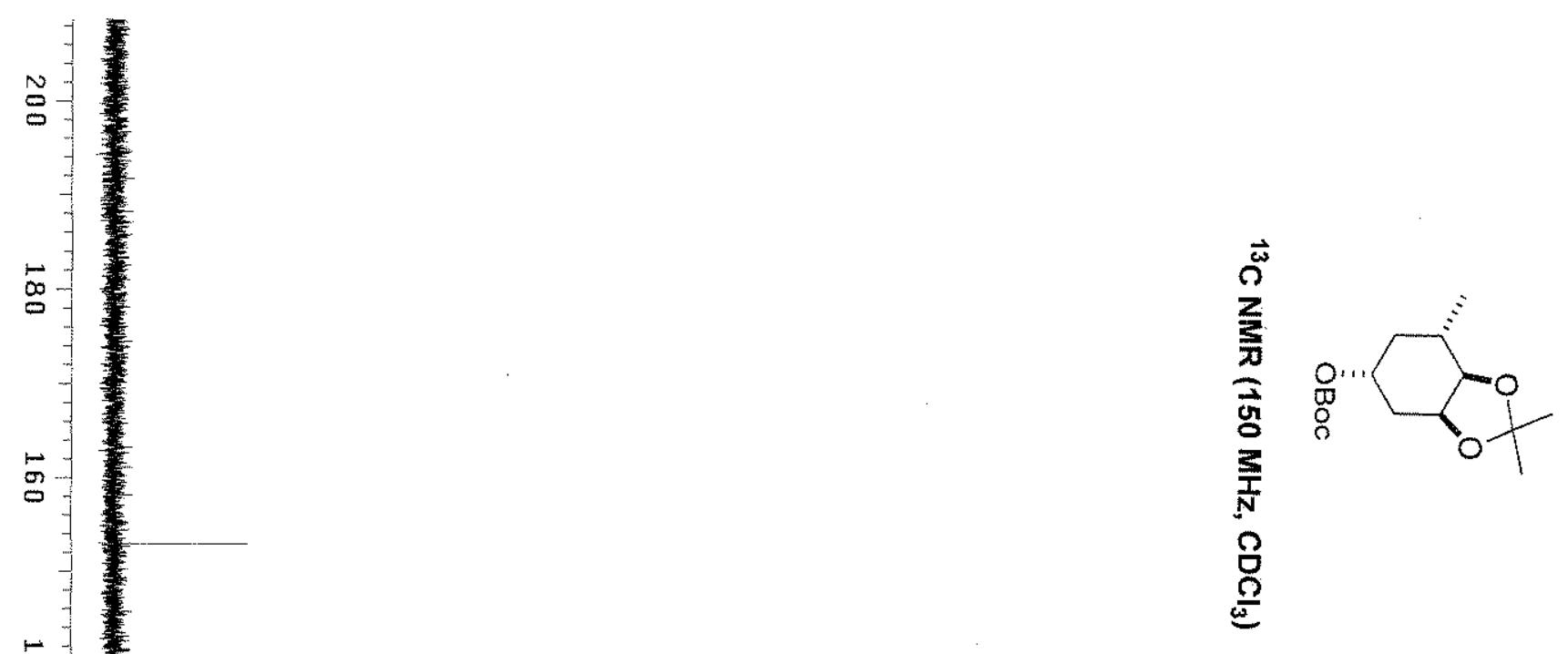

$\infty$

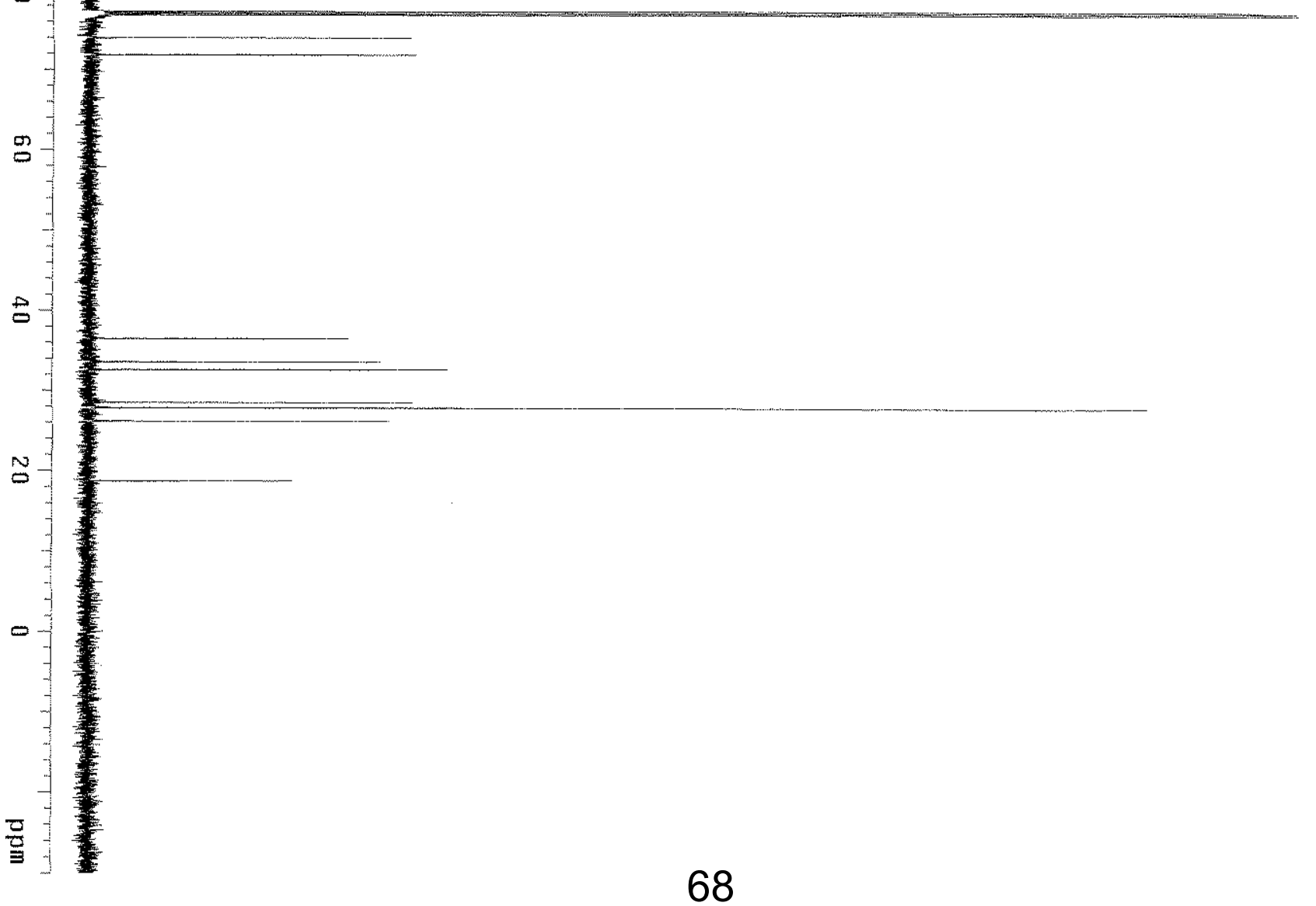




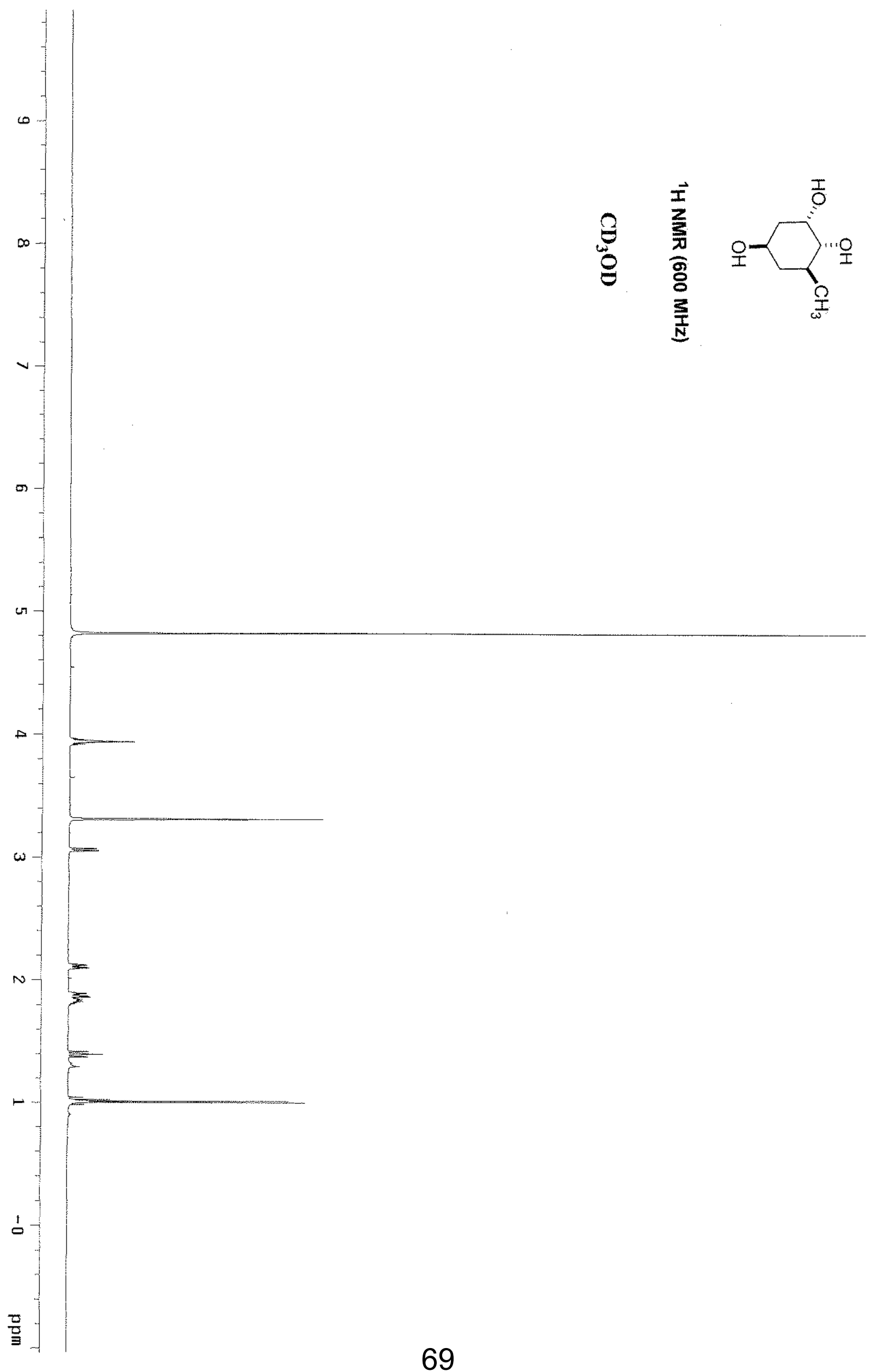




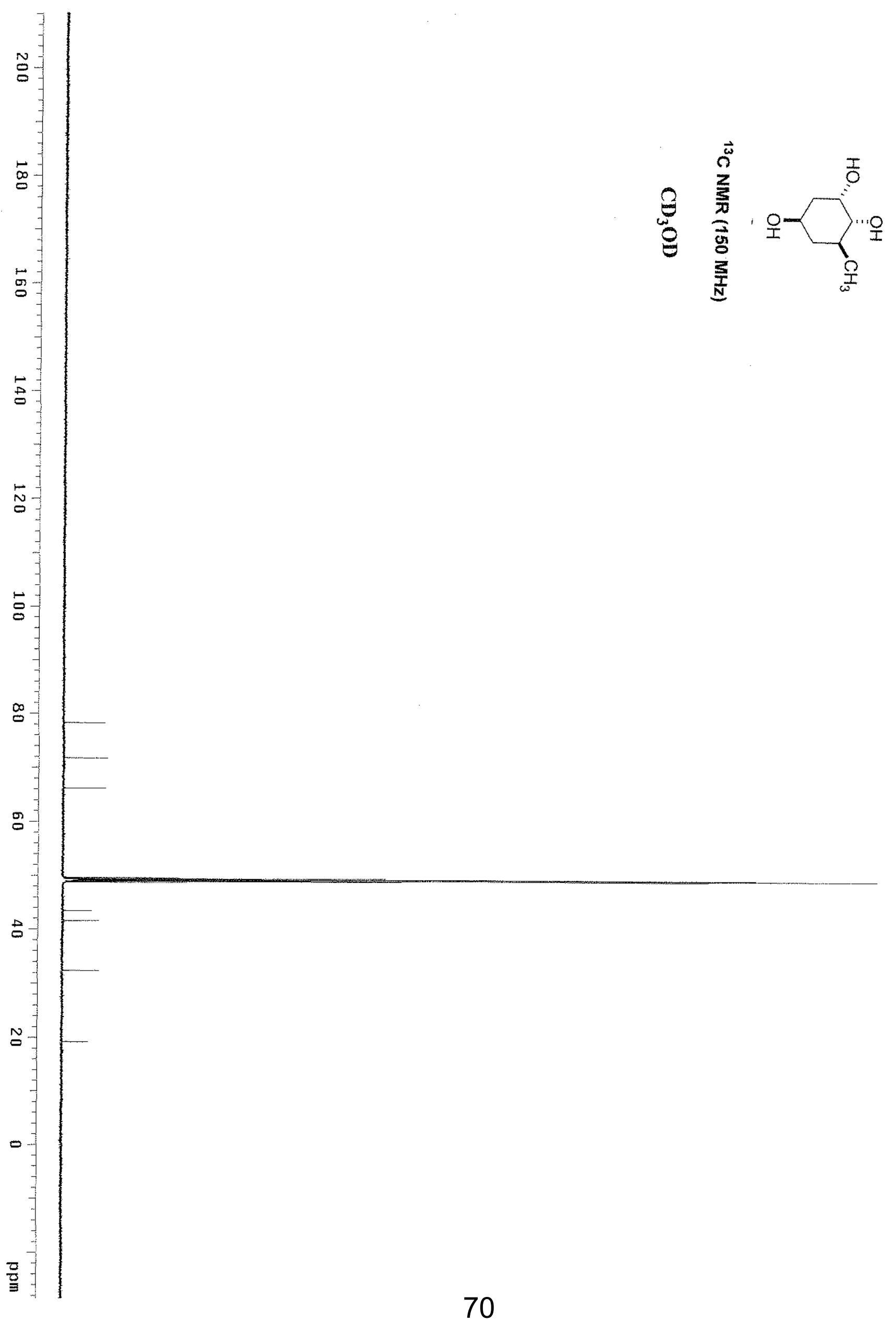




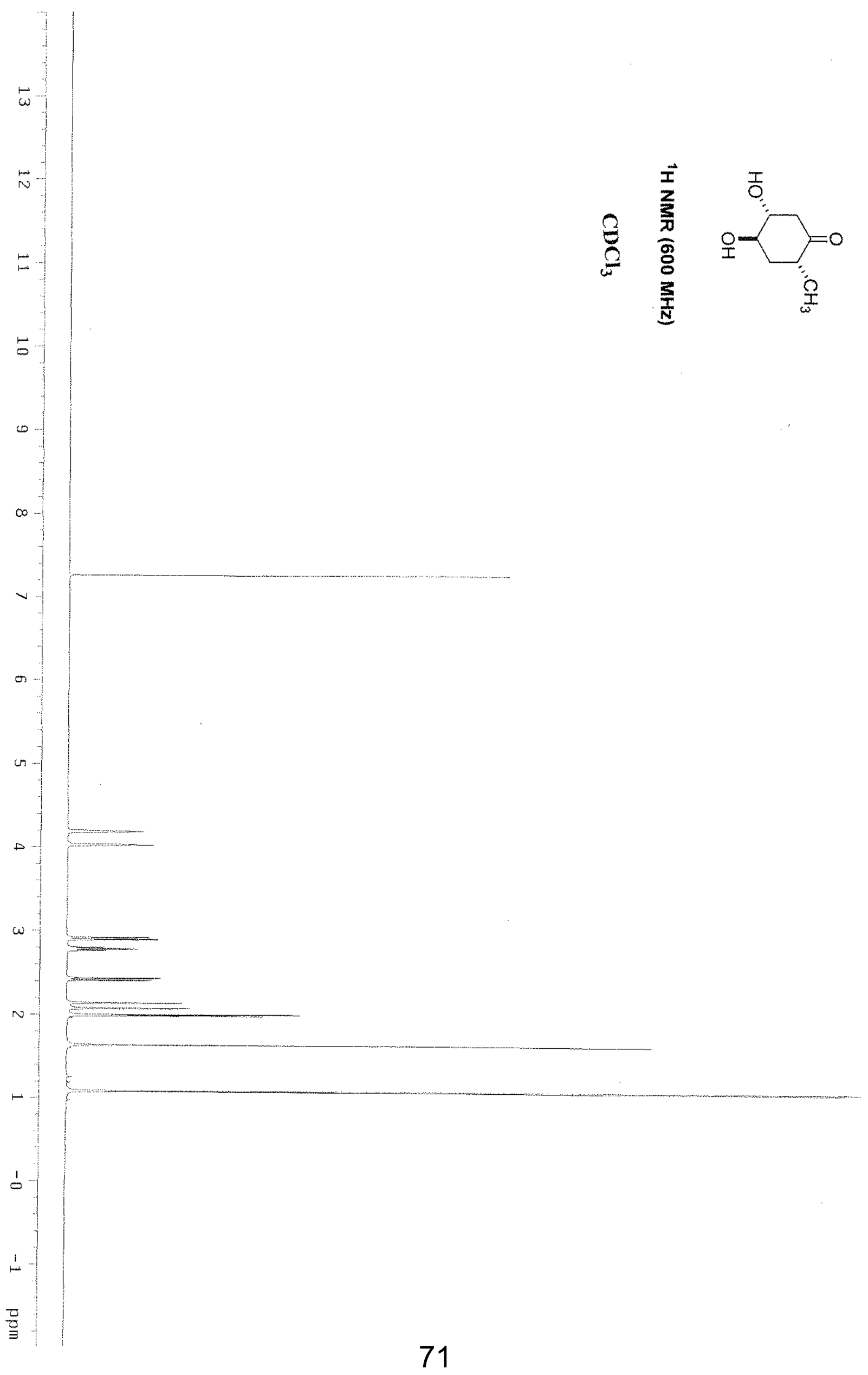




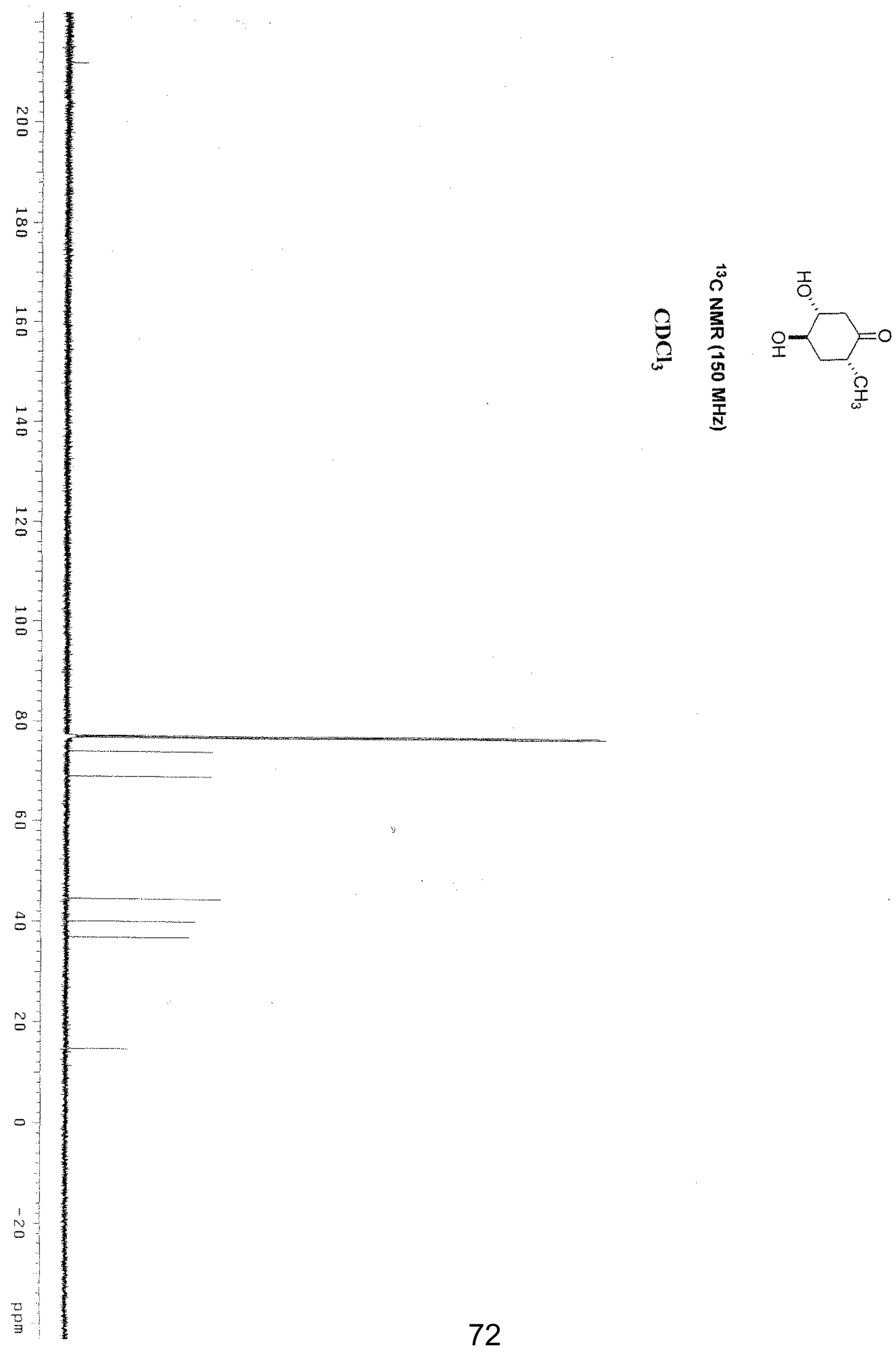




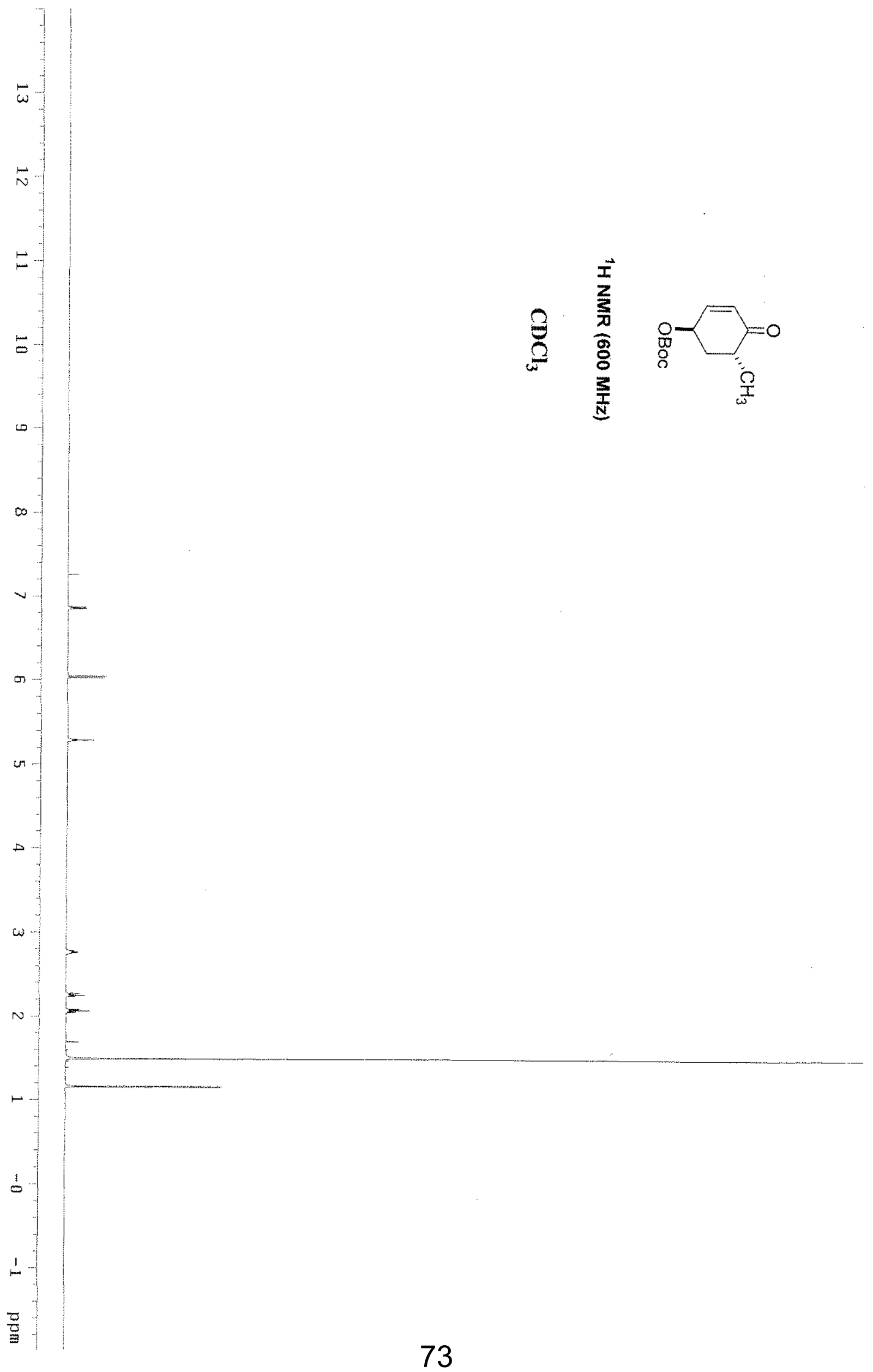



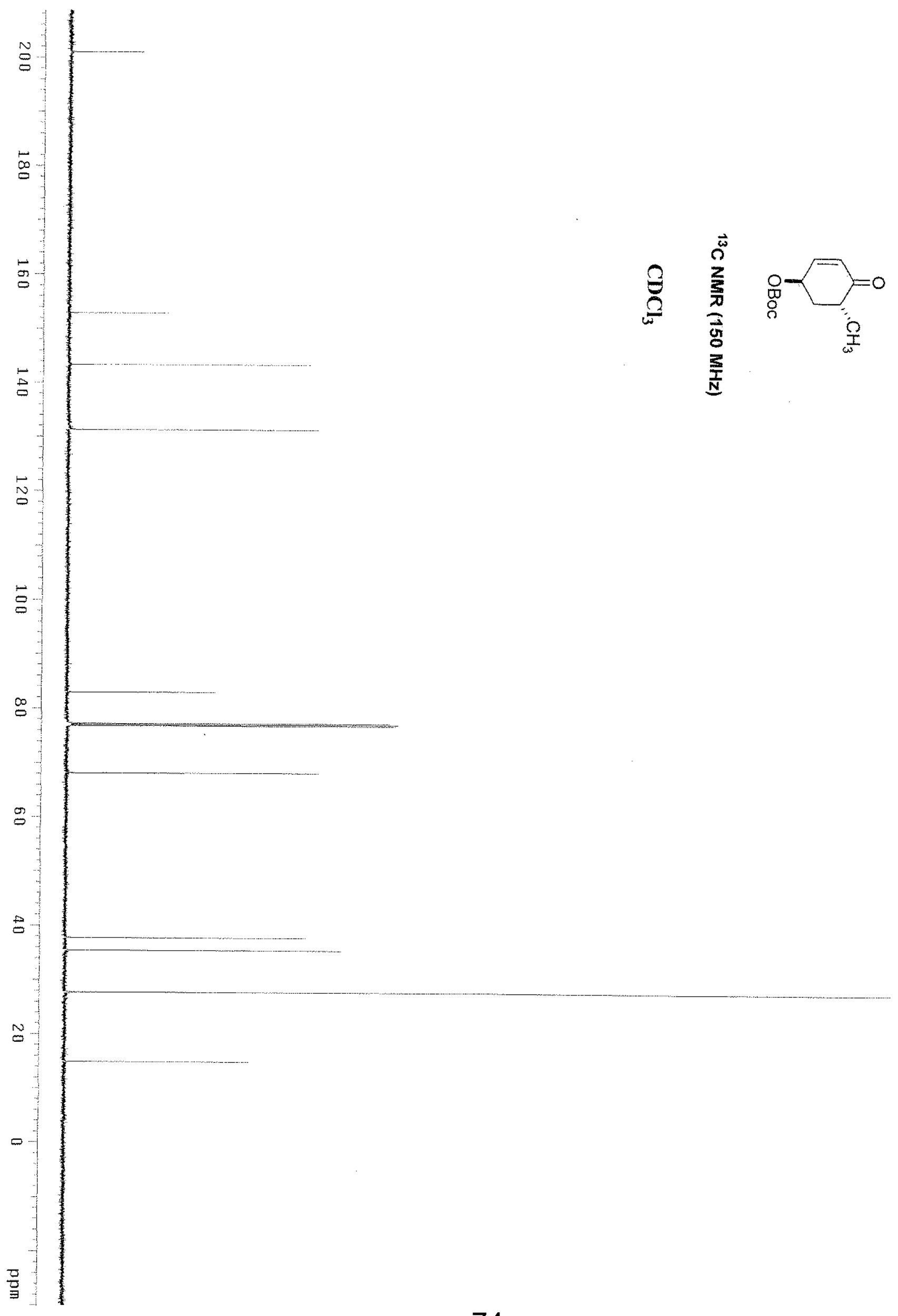


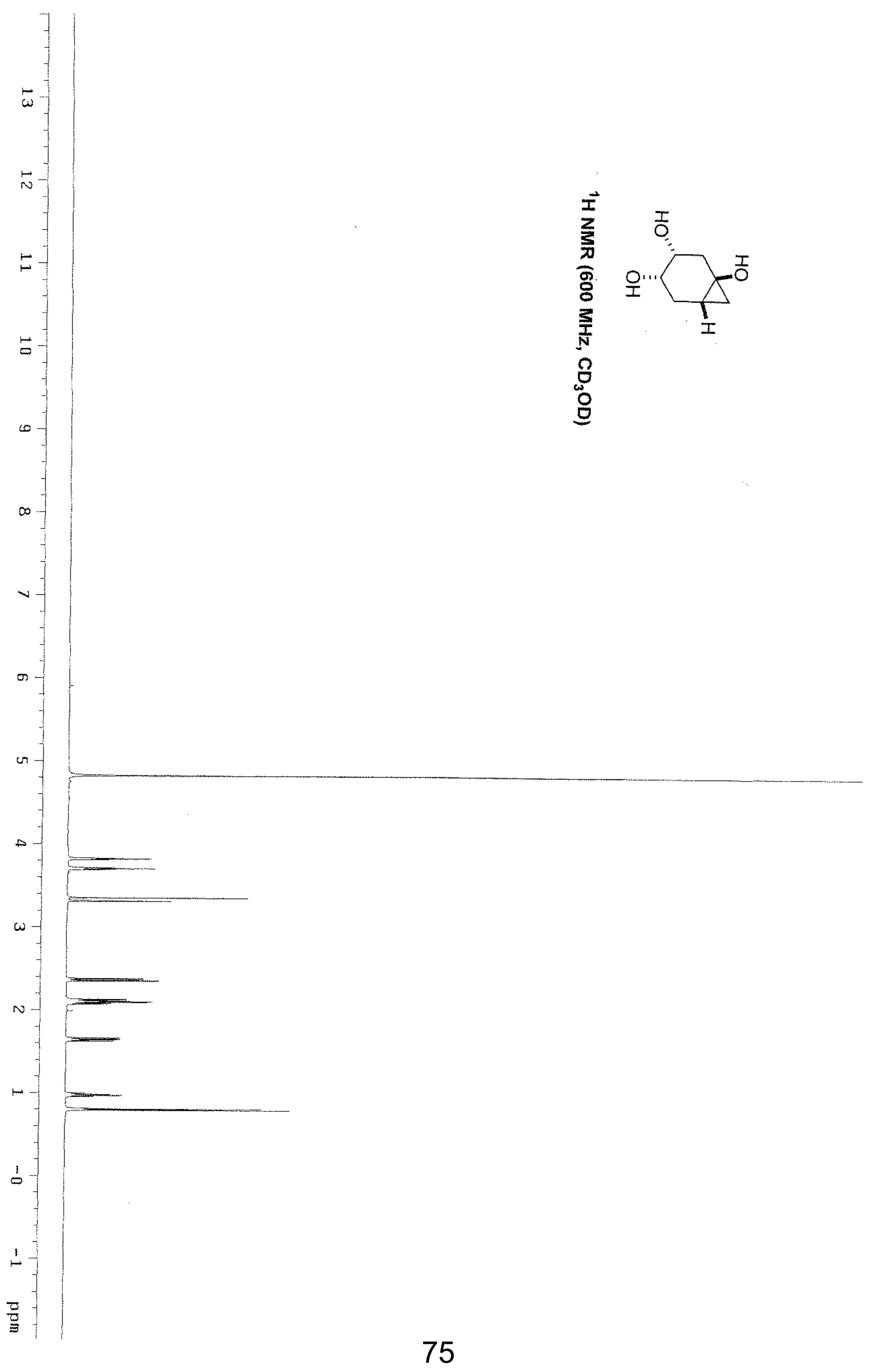




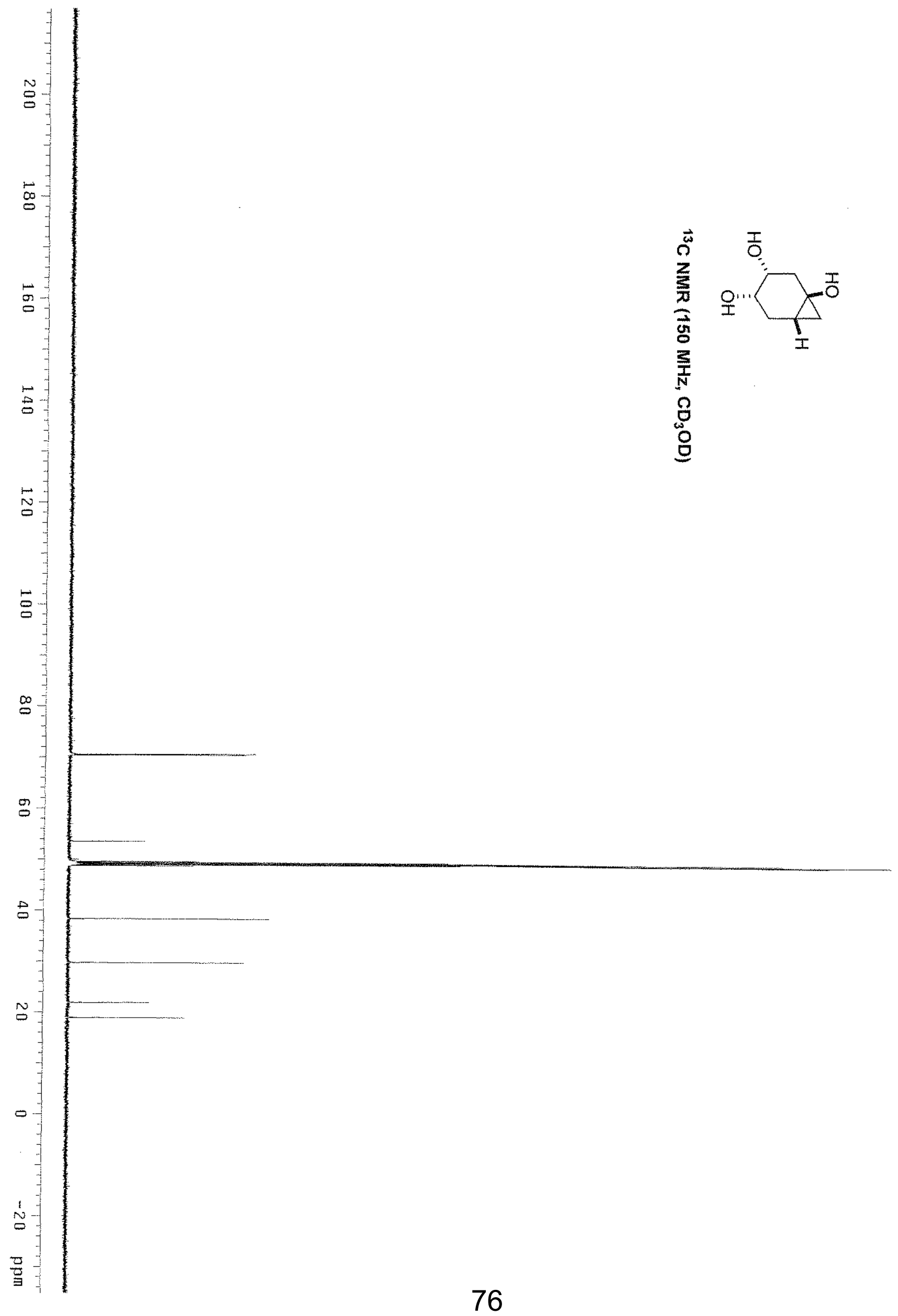




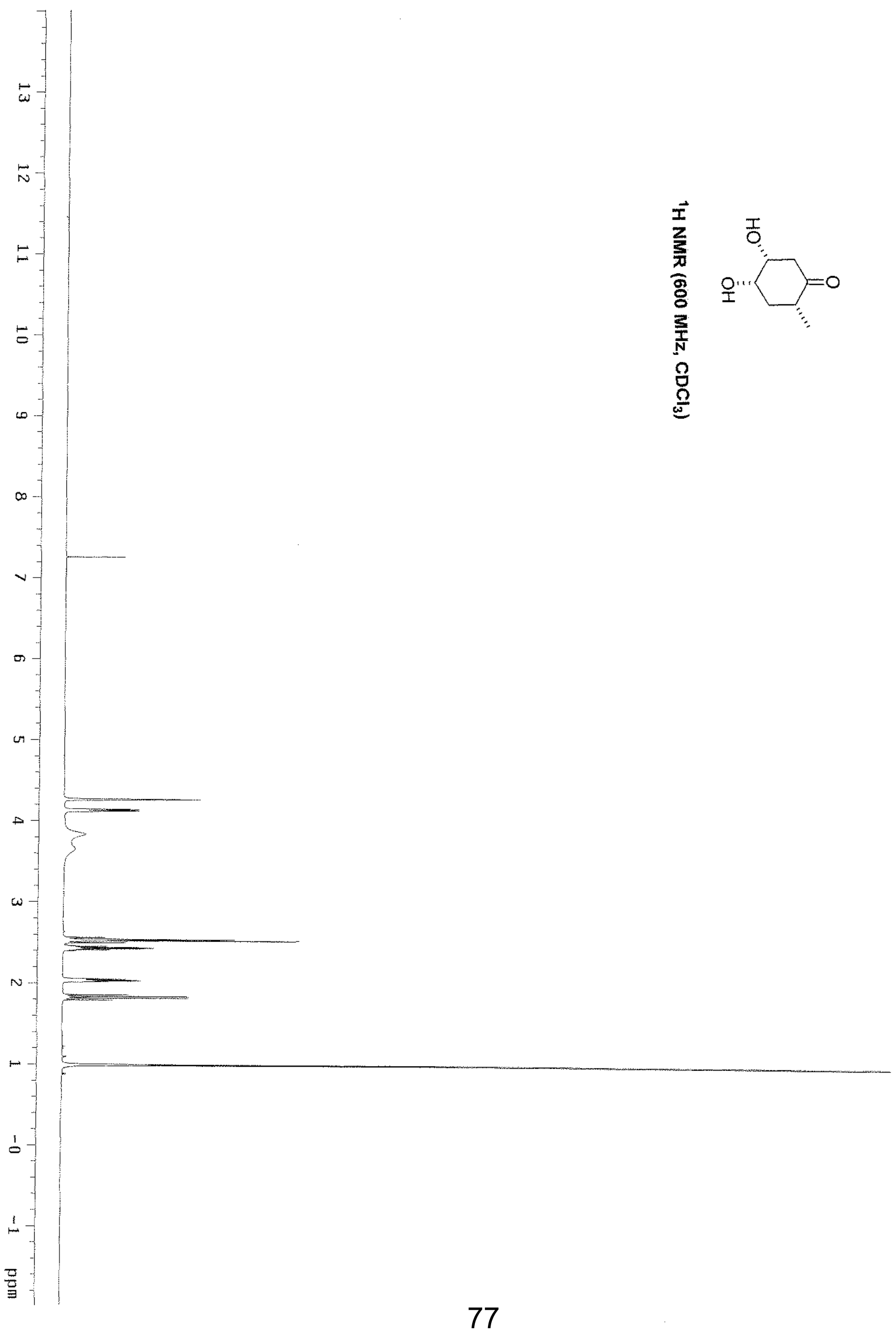




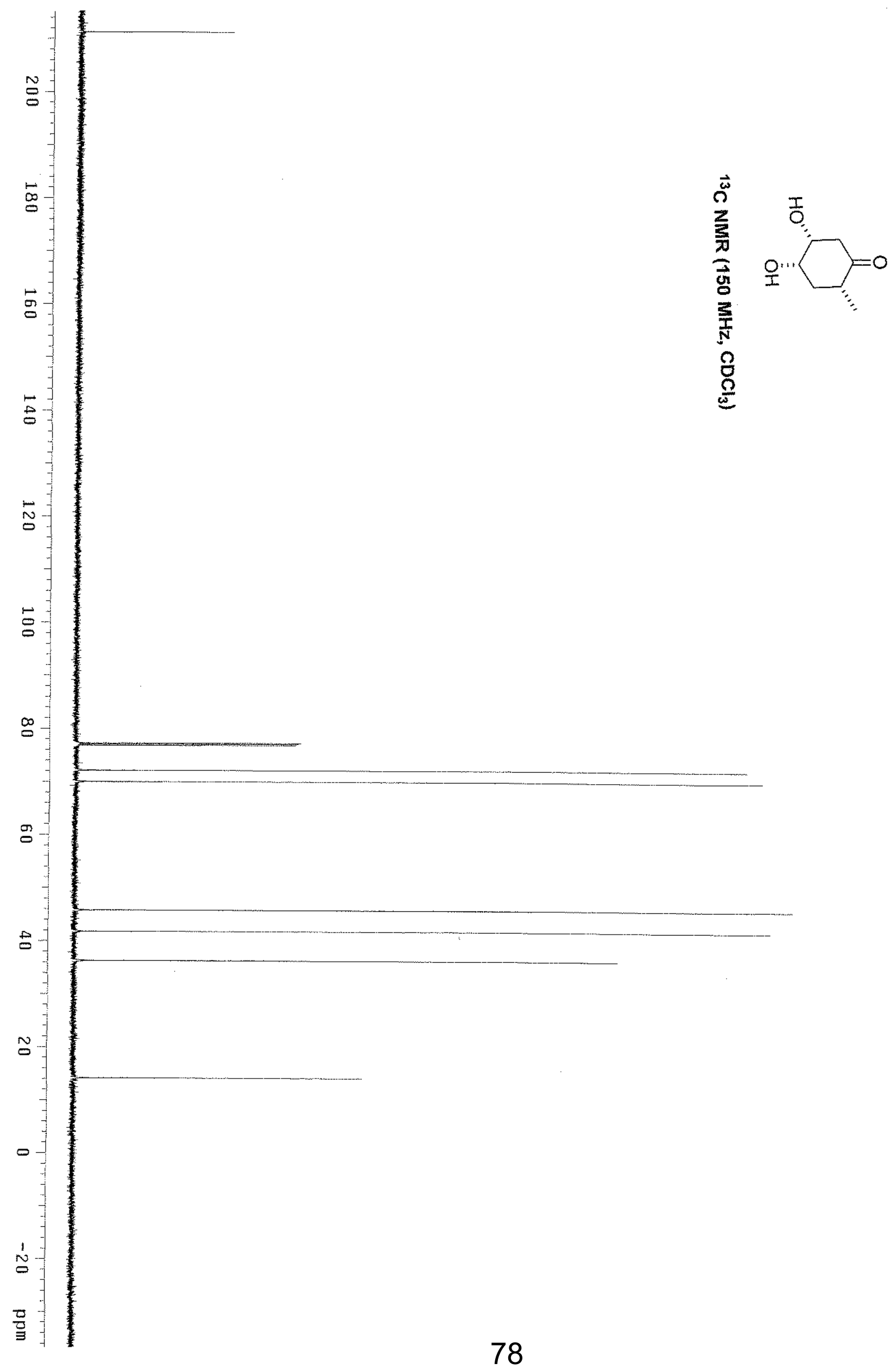




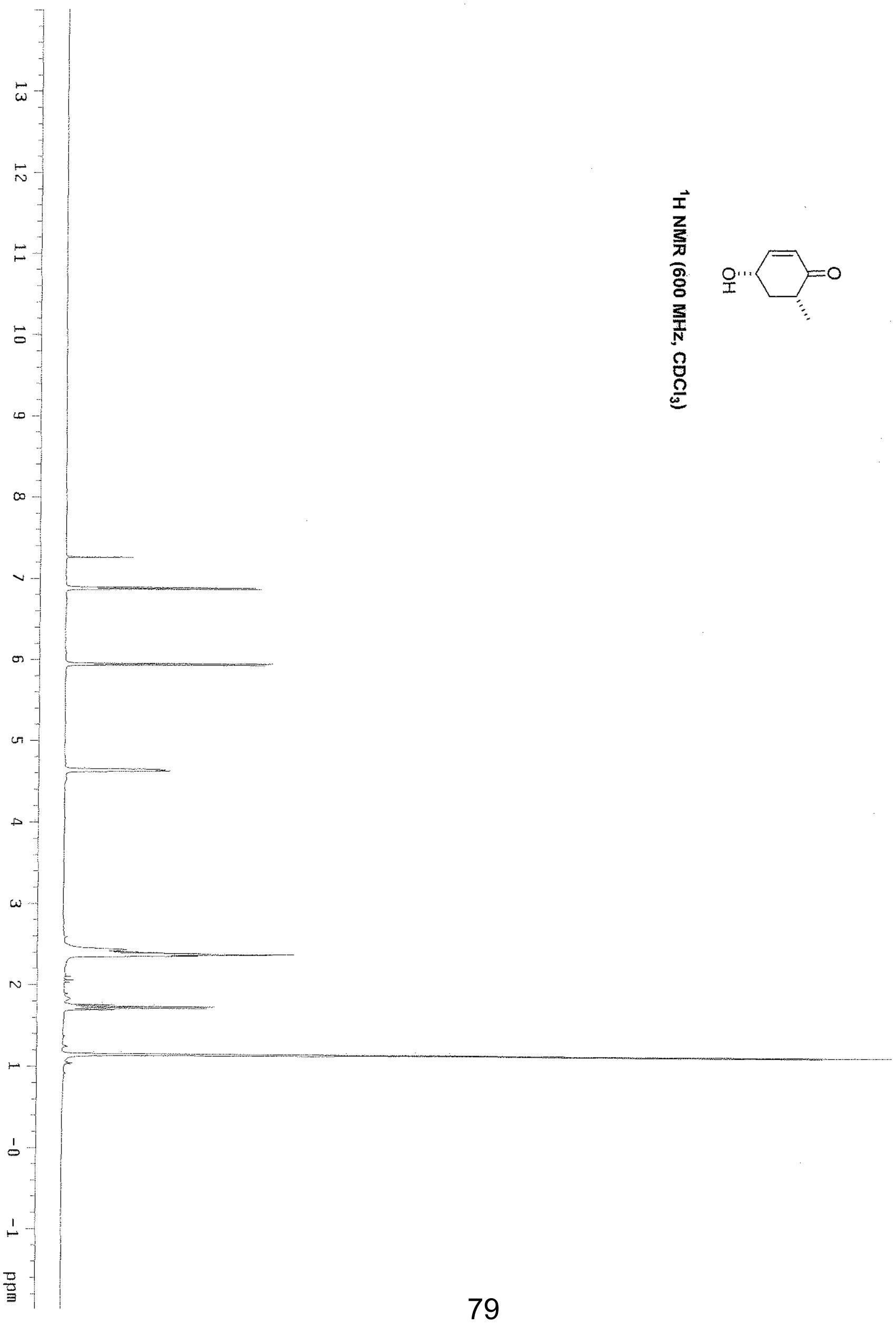




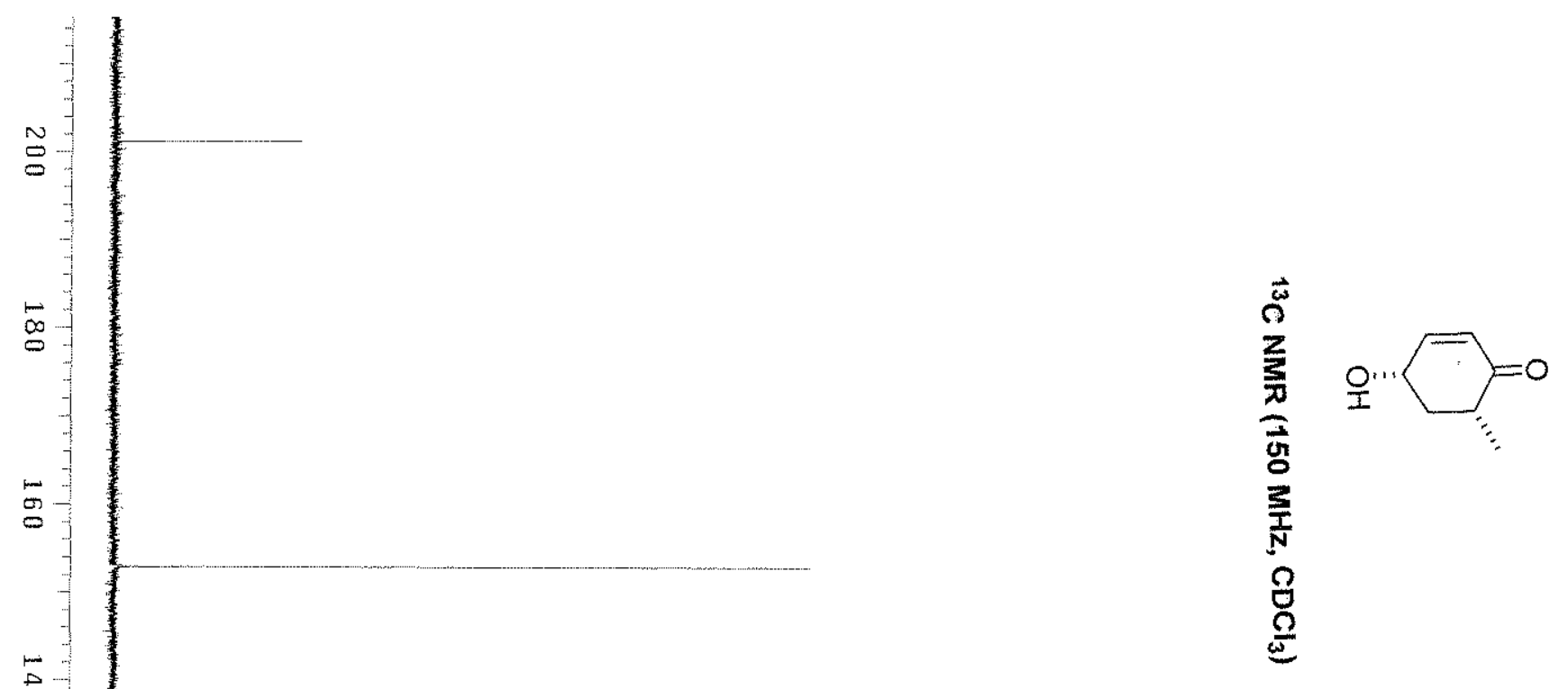

$B$

5

:

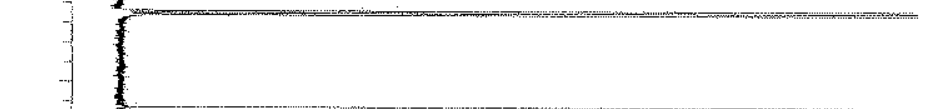

ㅇ․ㄱ.

s

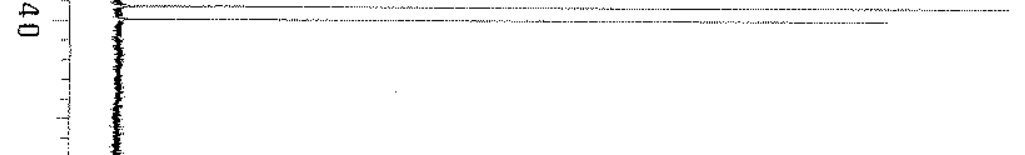

N

$\circ$$$
\text { 。 }
$$

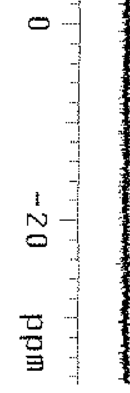




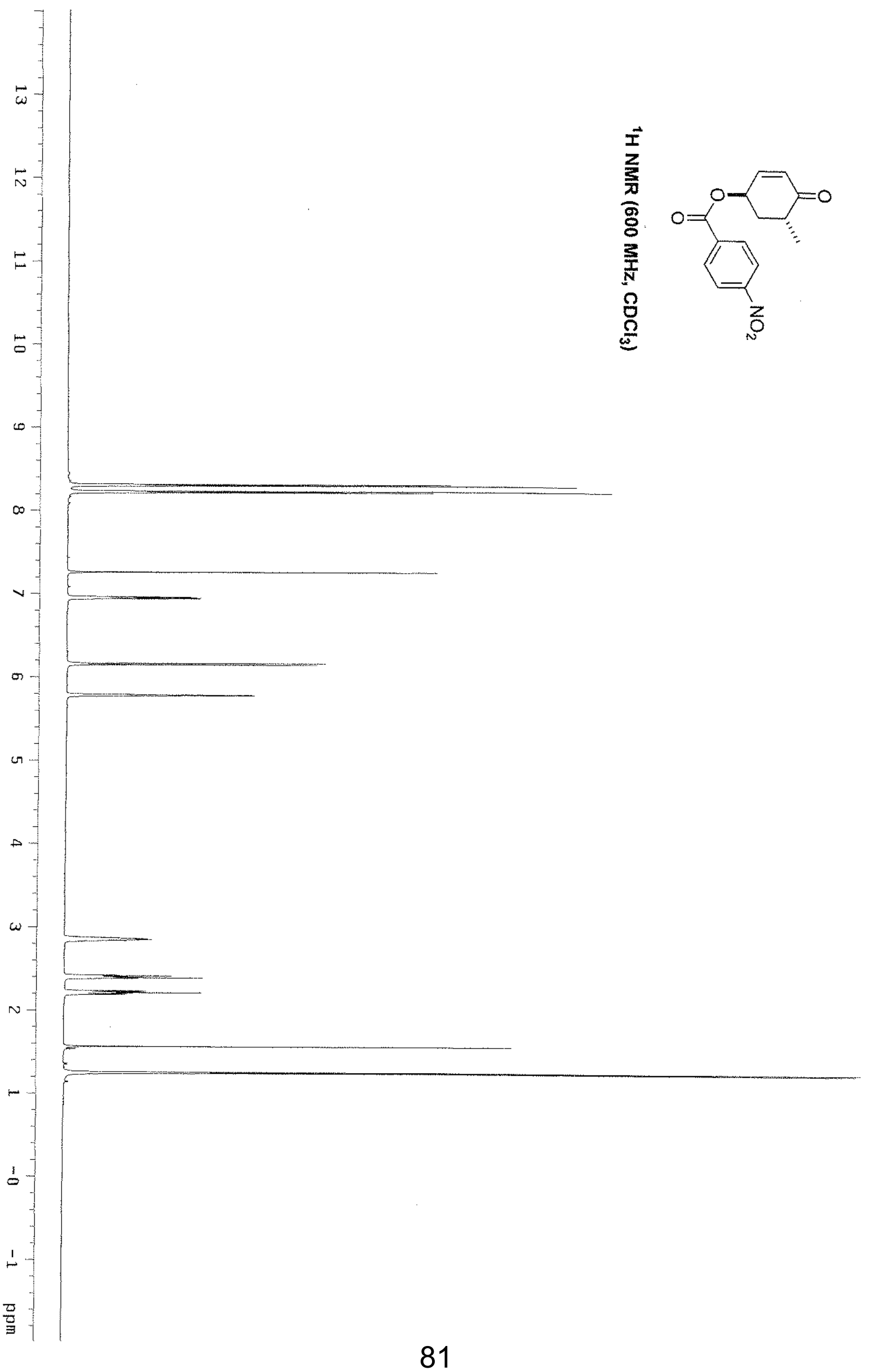




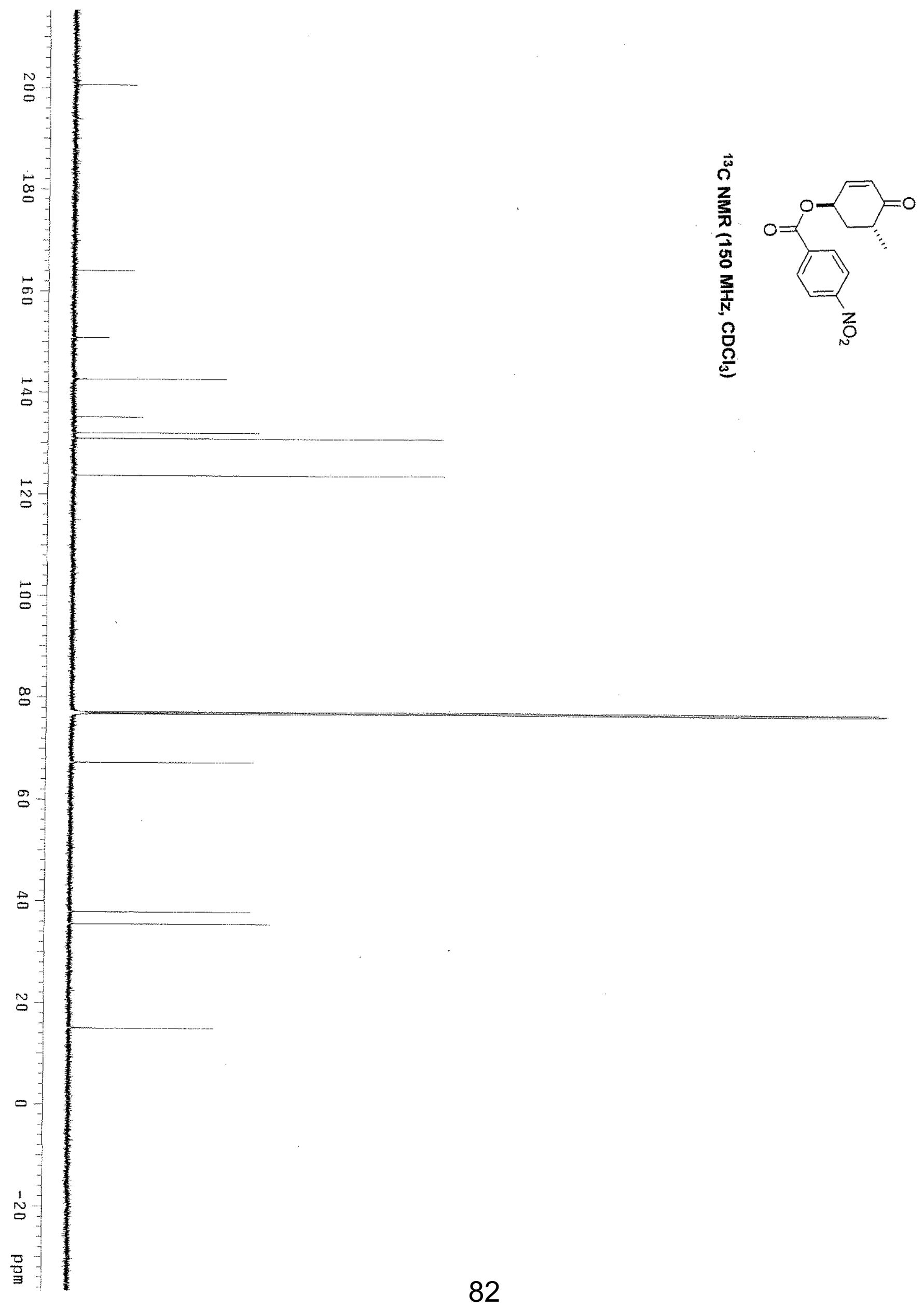




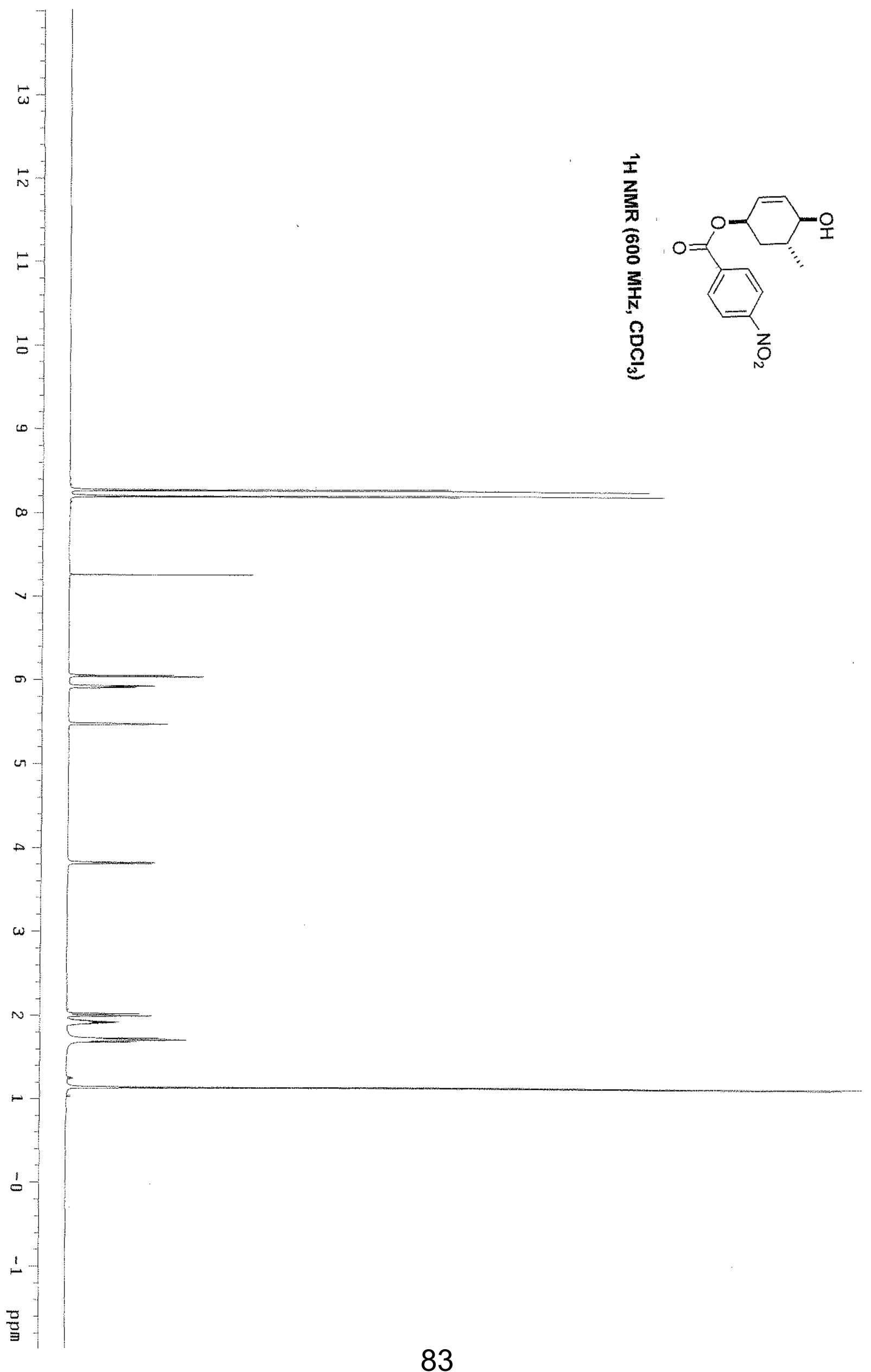



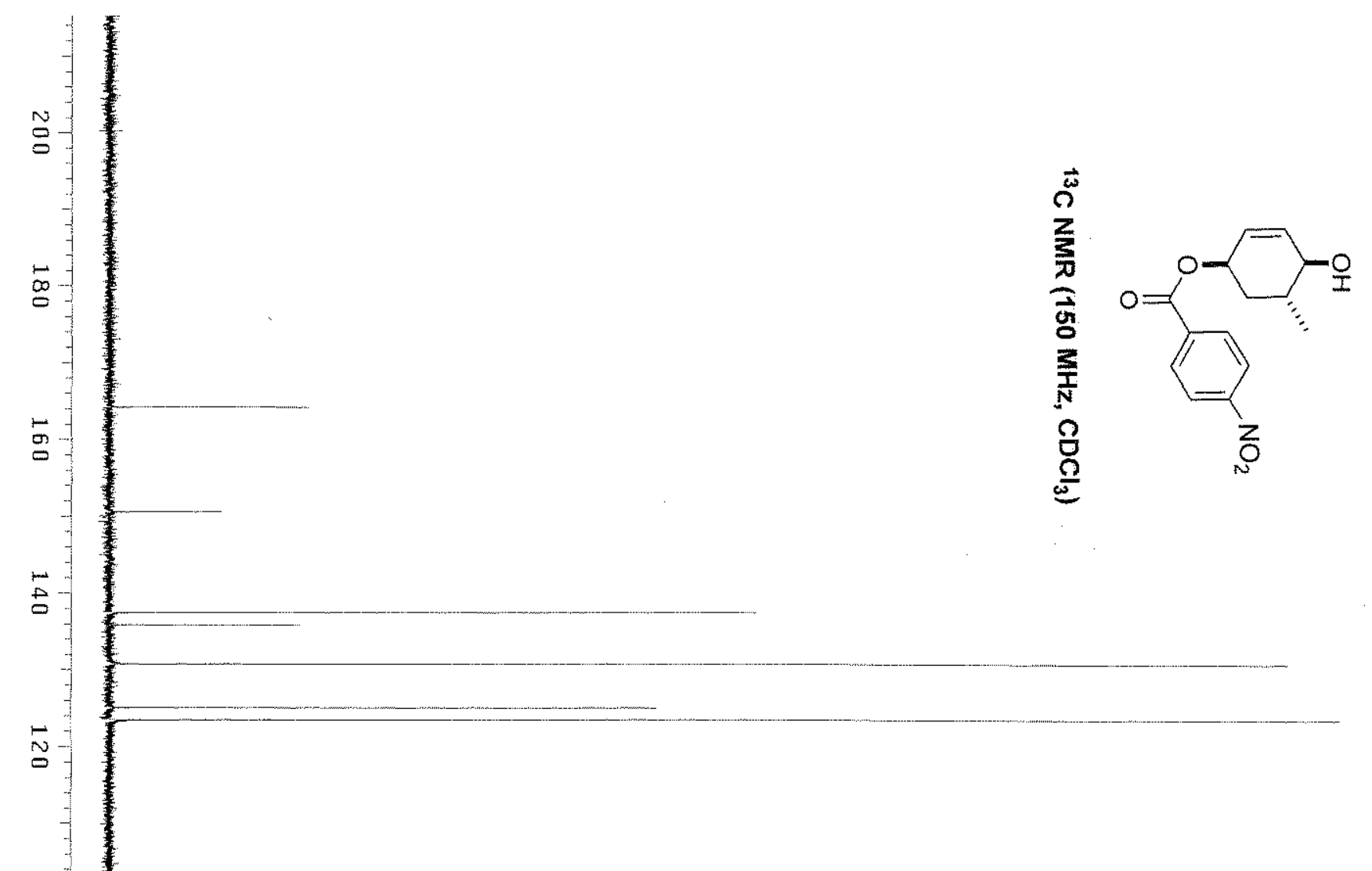

亭

$\infty$

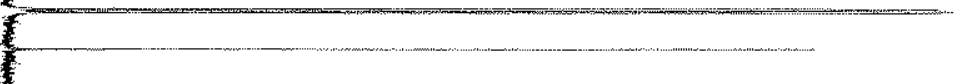

0

8

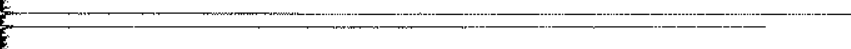

N

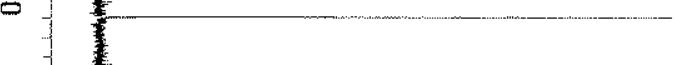

2
0
3
0
0
0
0 


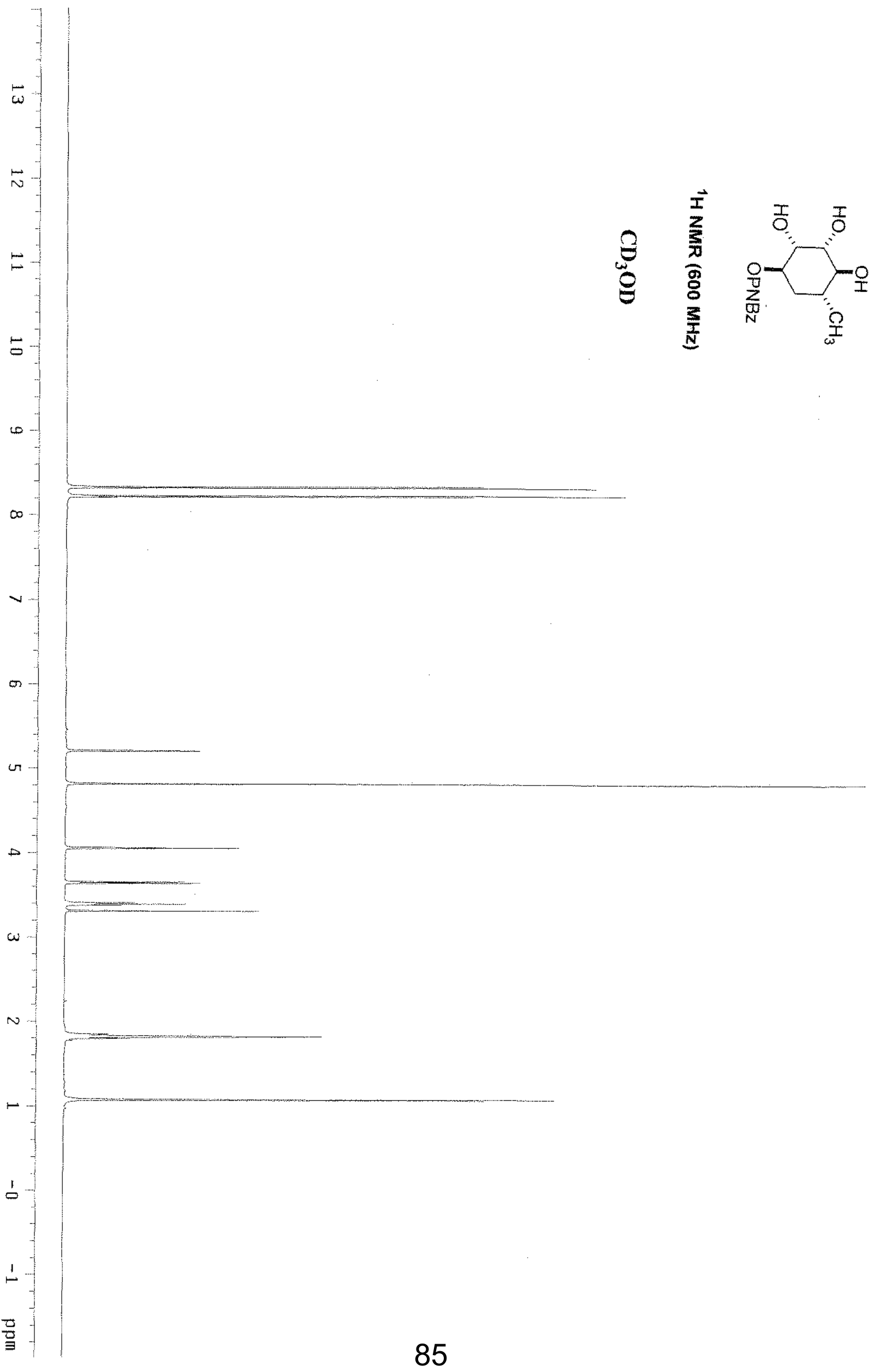




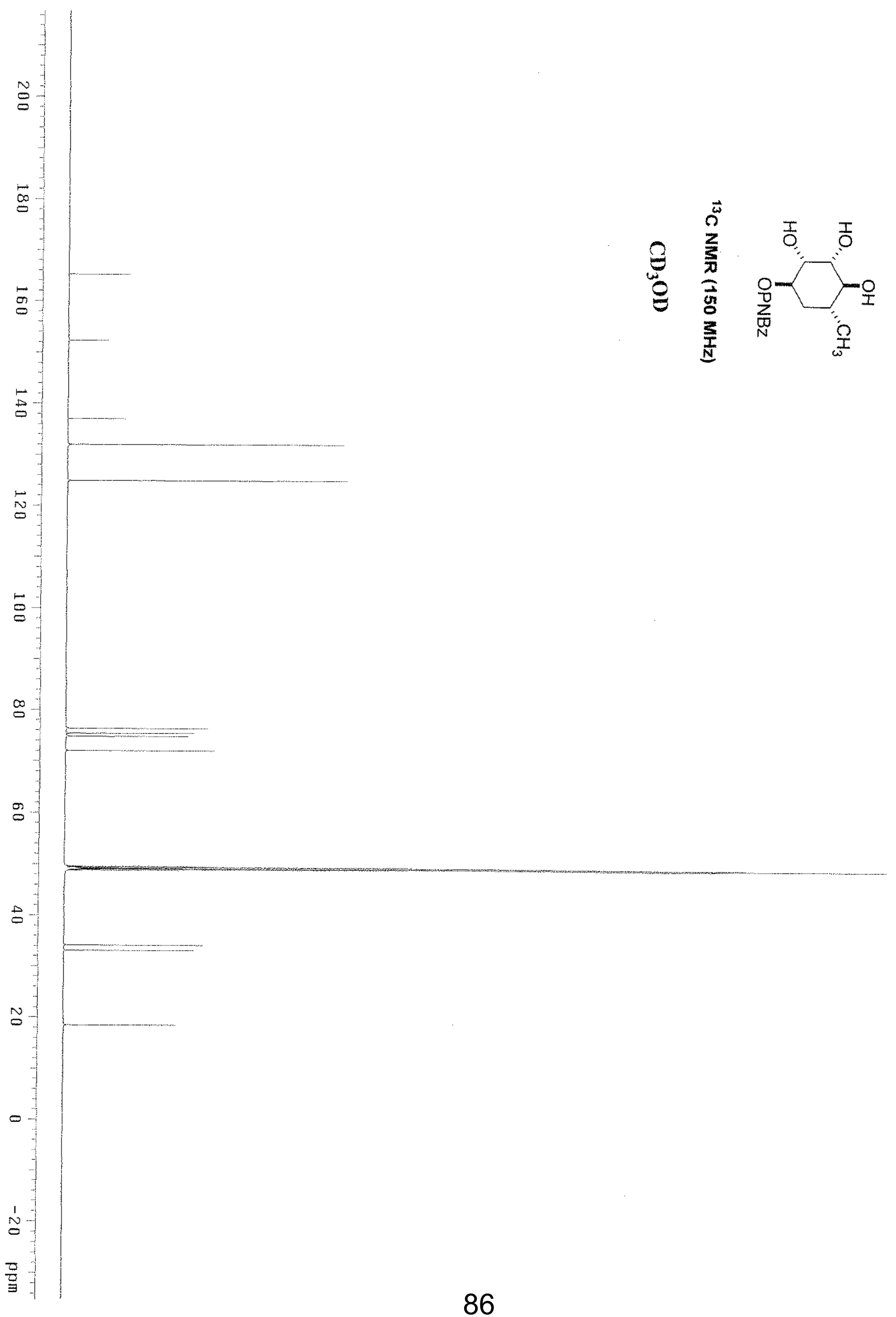




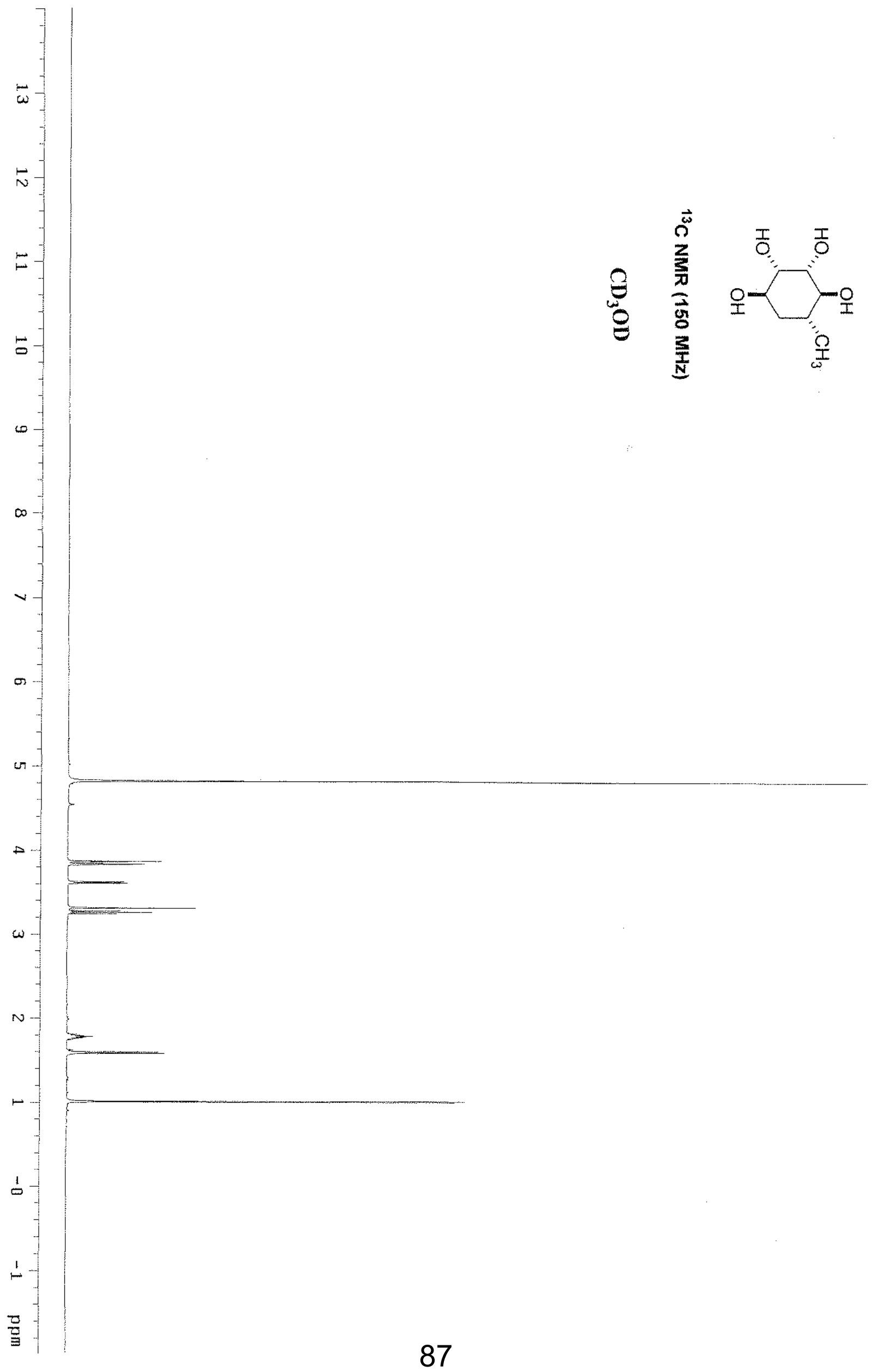




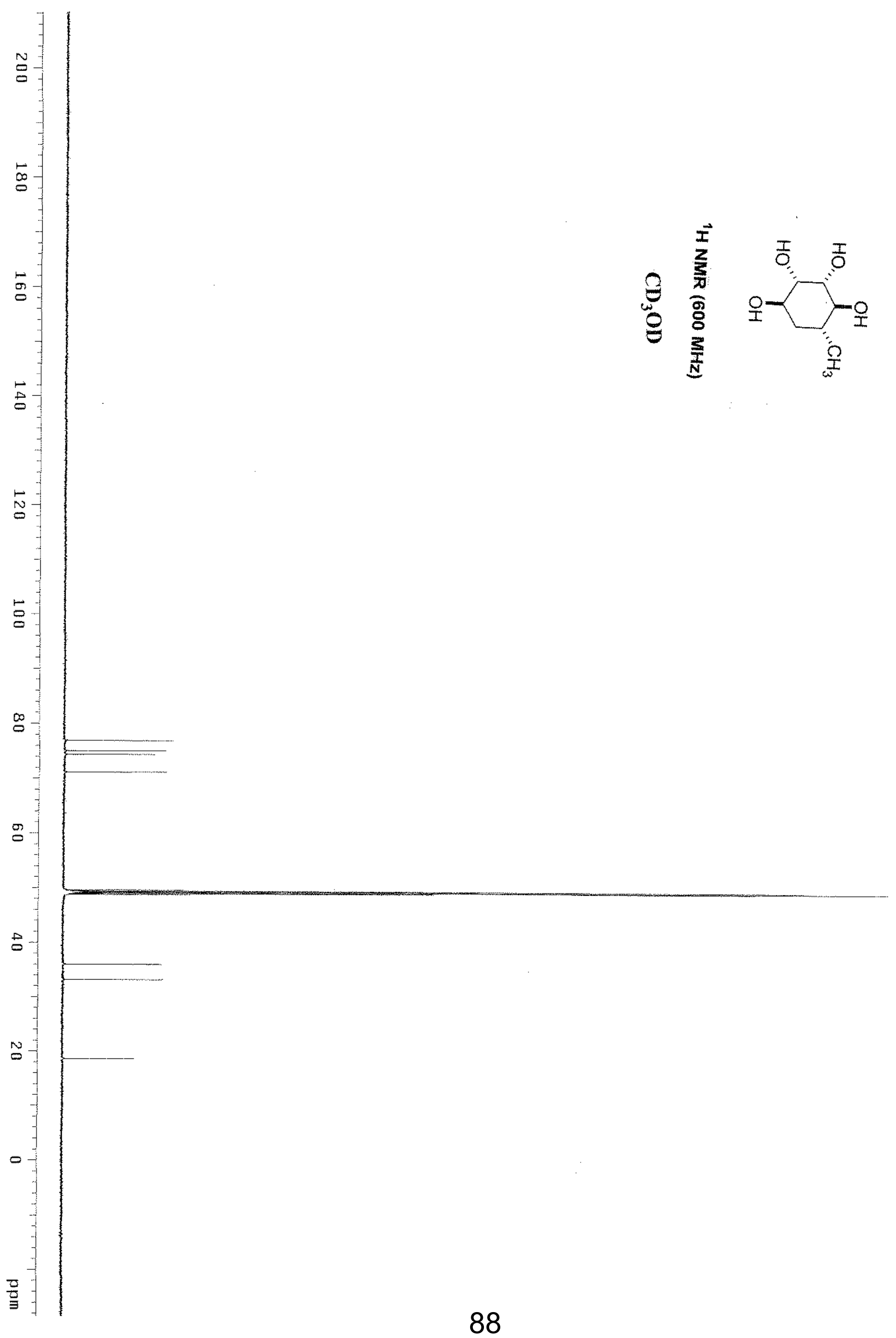




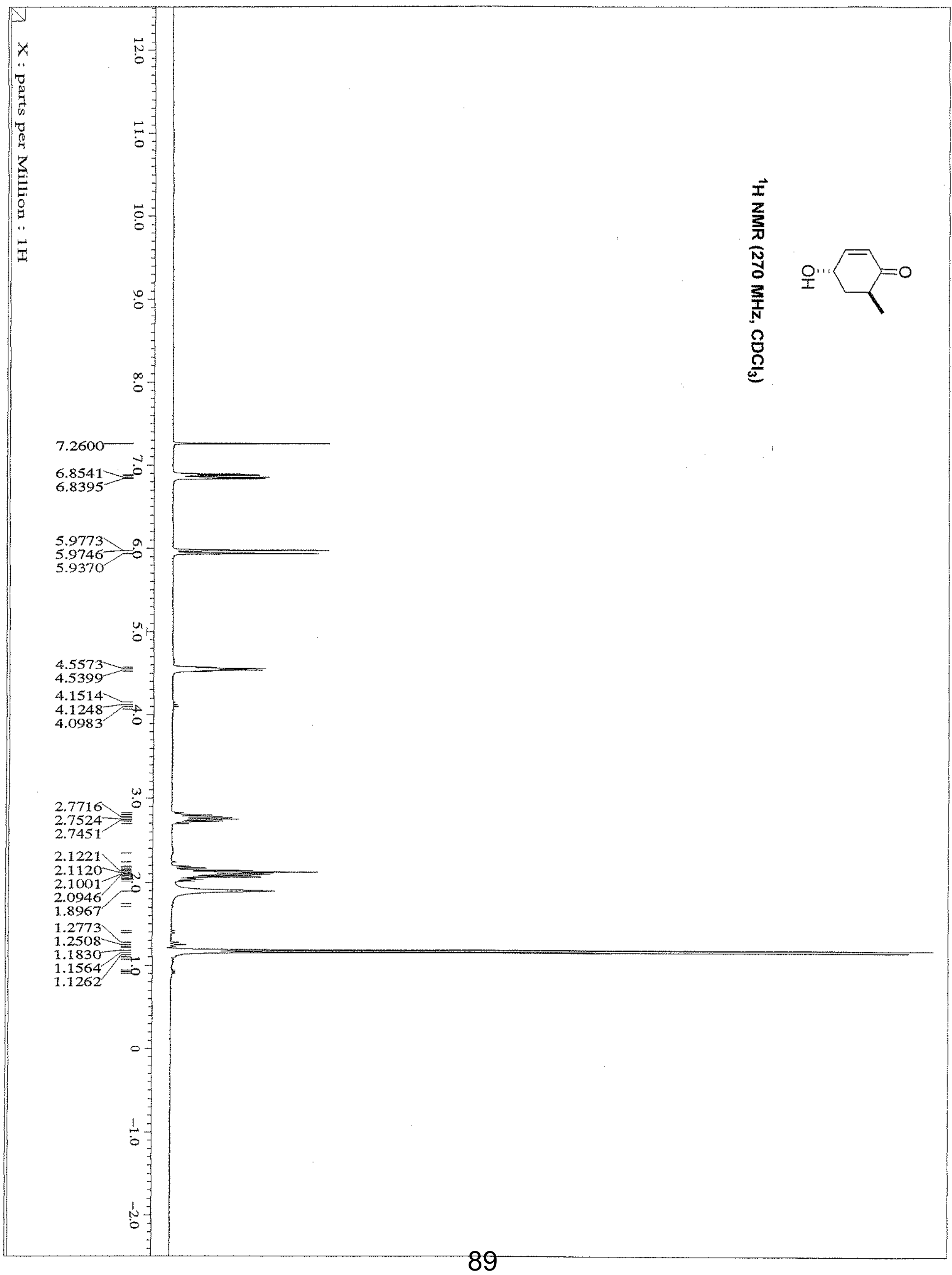




$$
11
$$




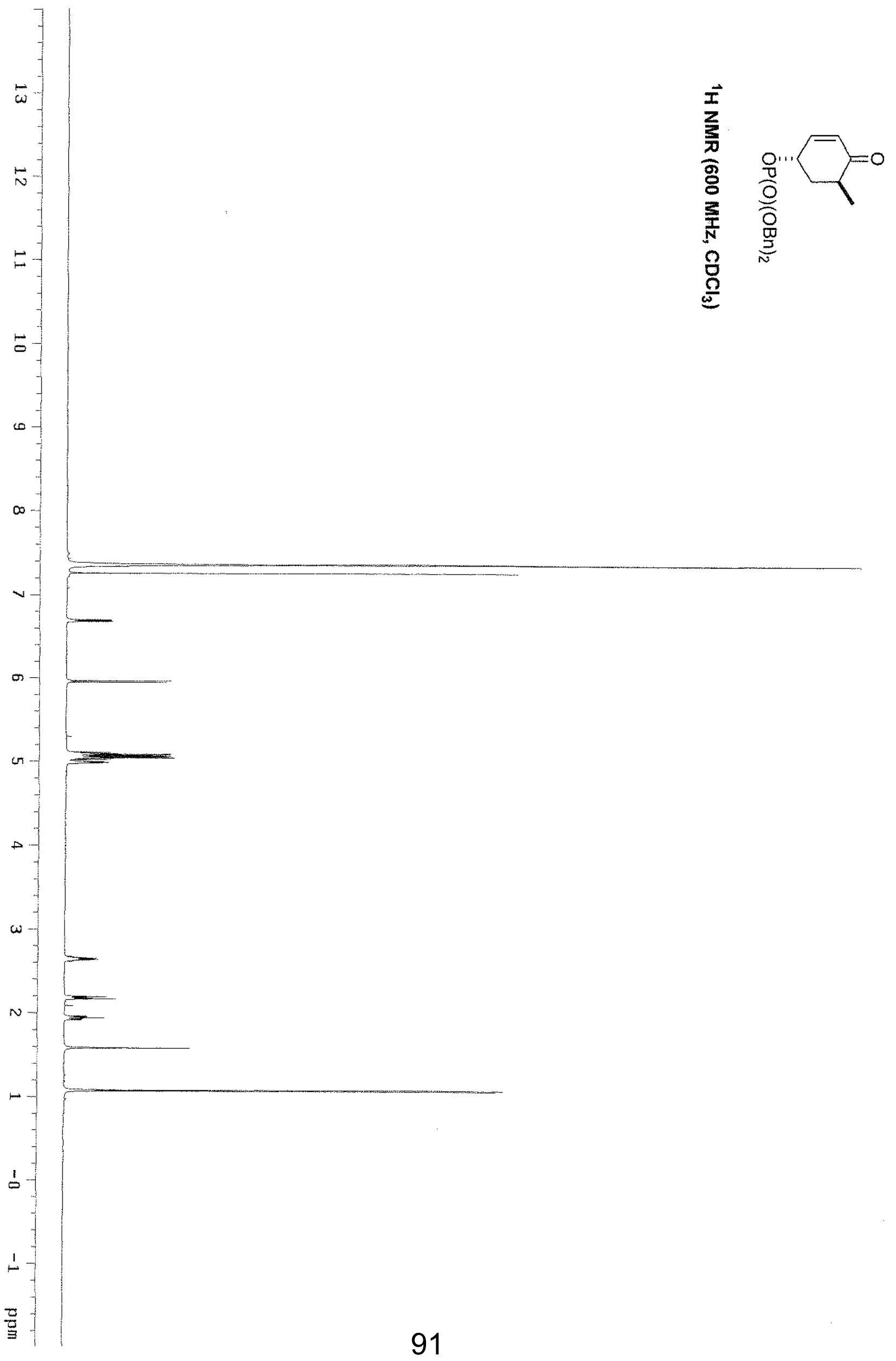




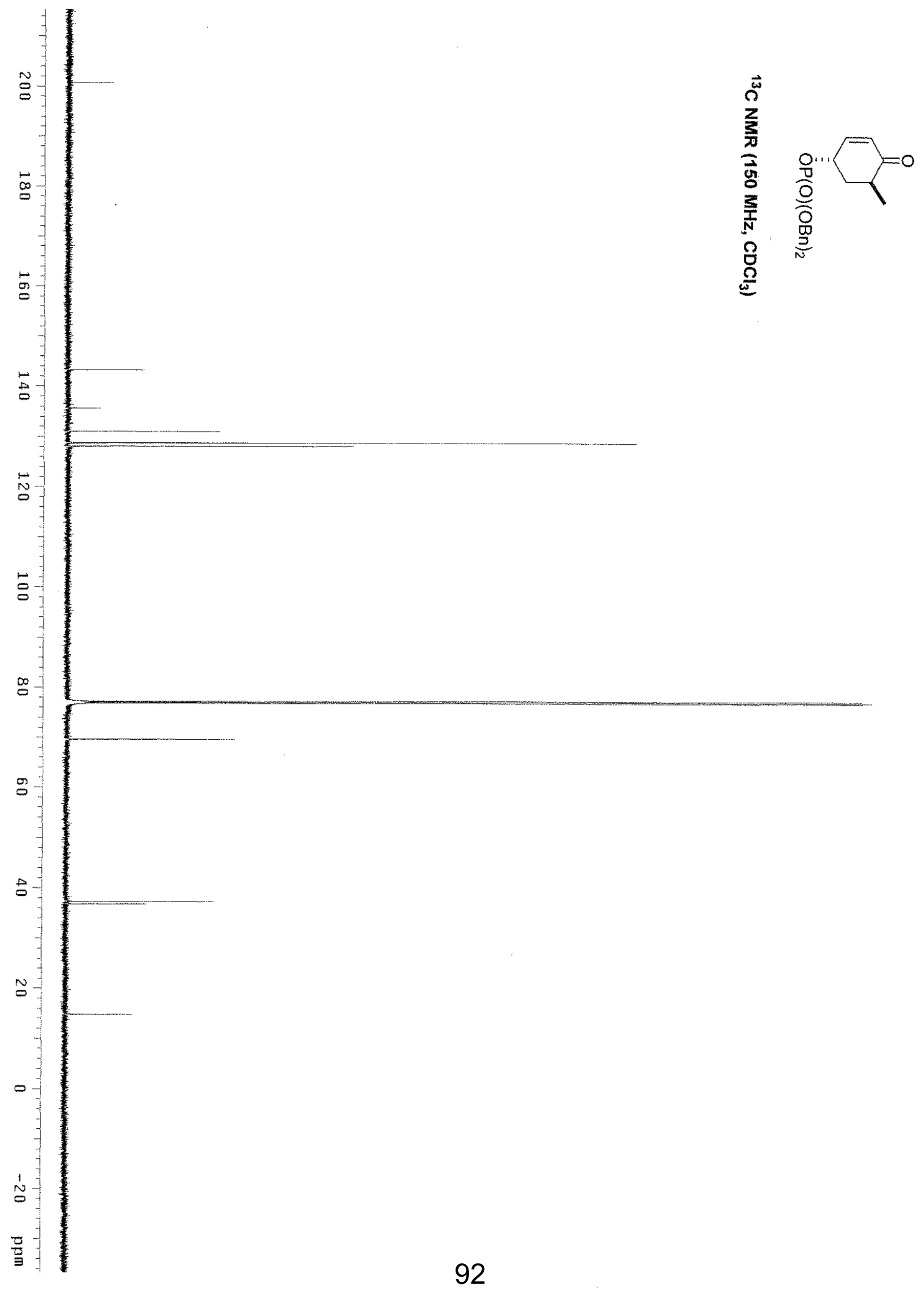




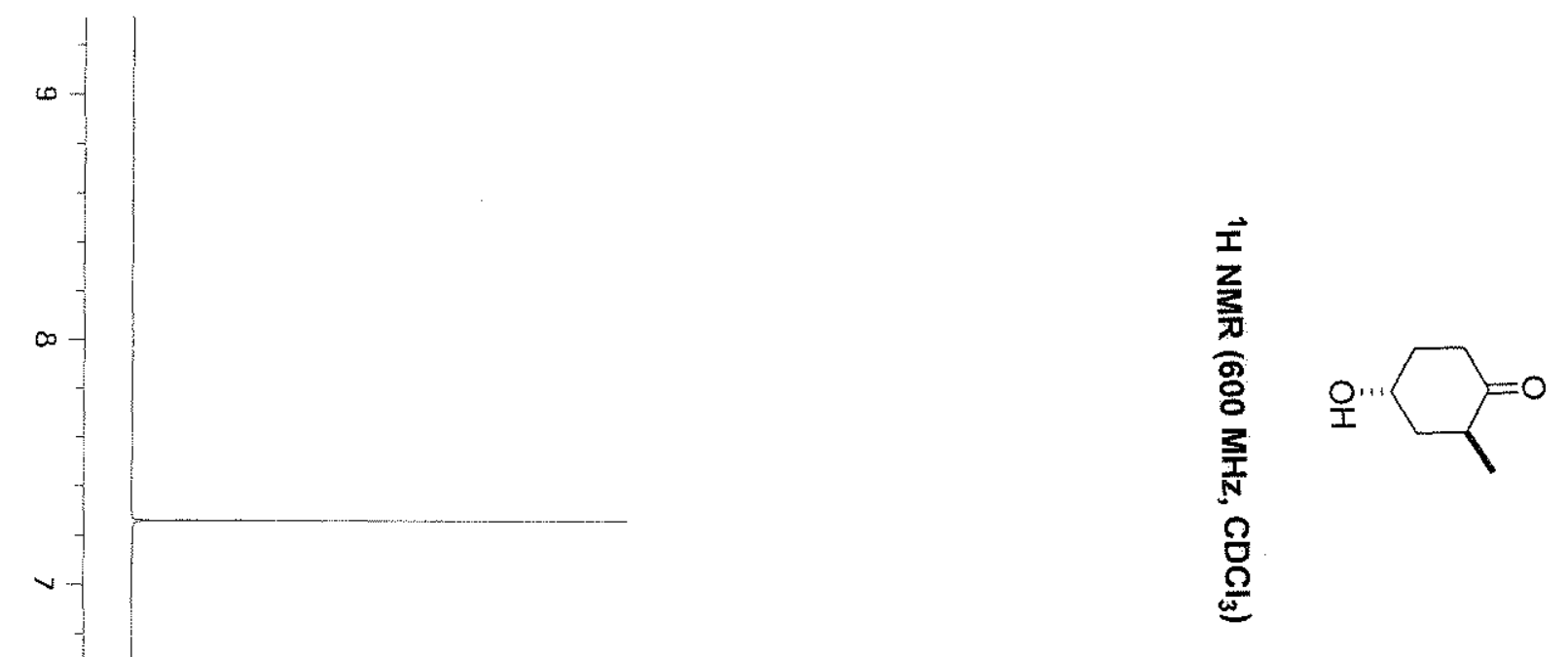

ar

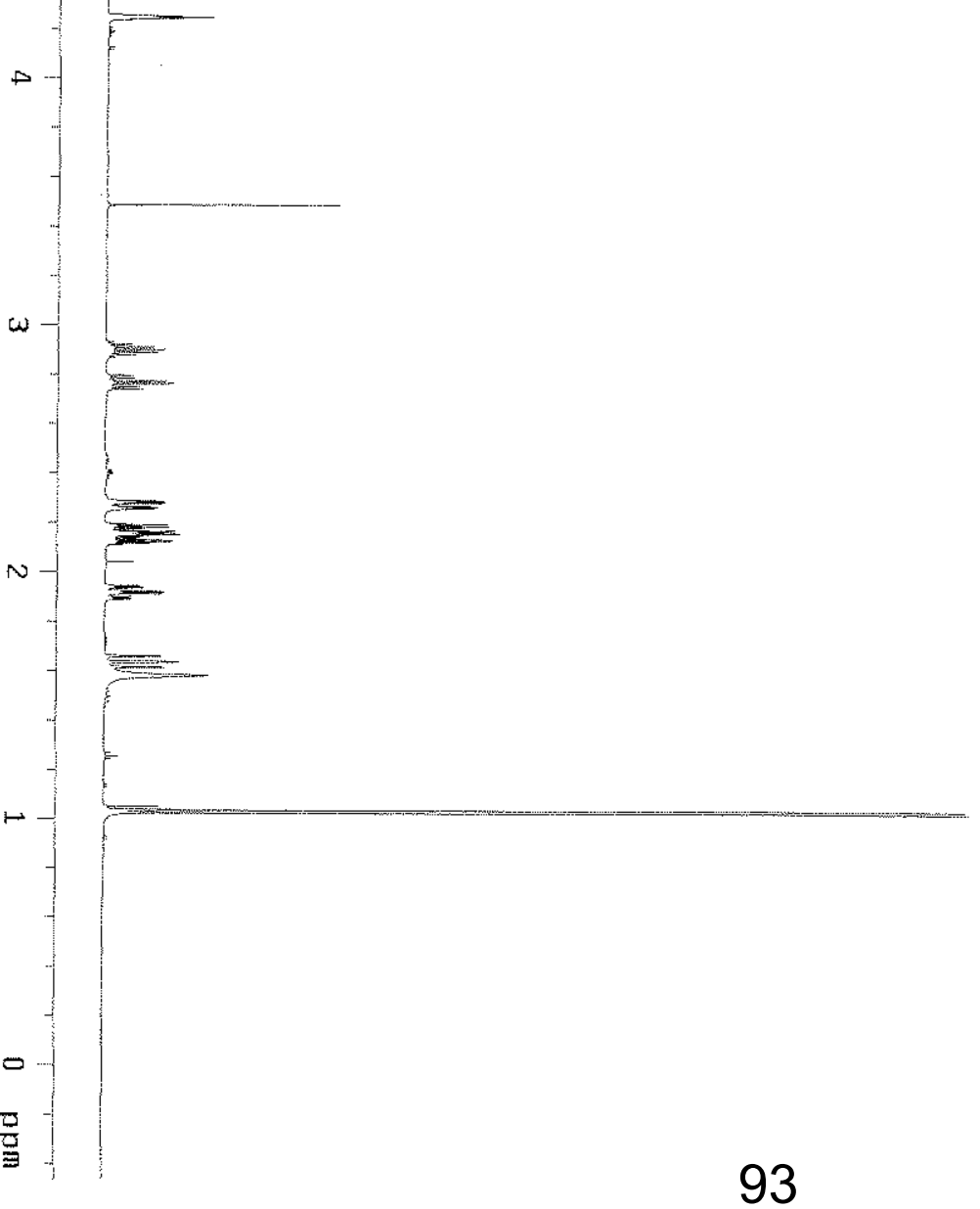




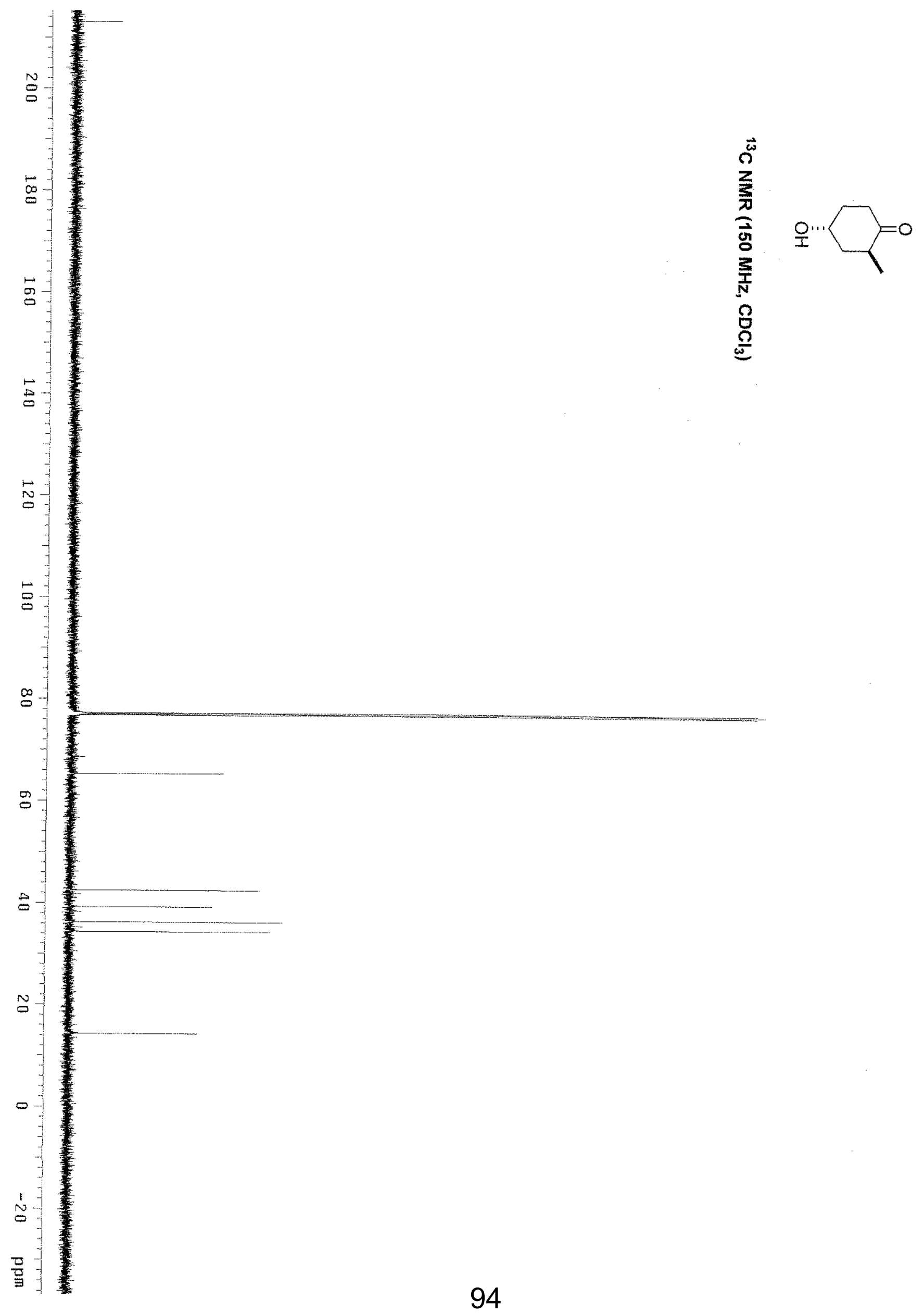




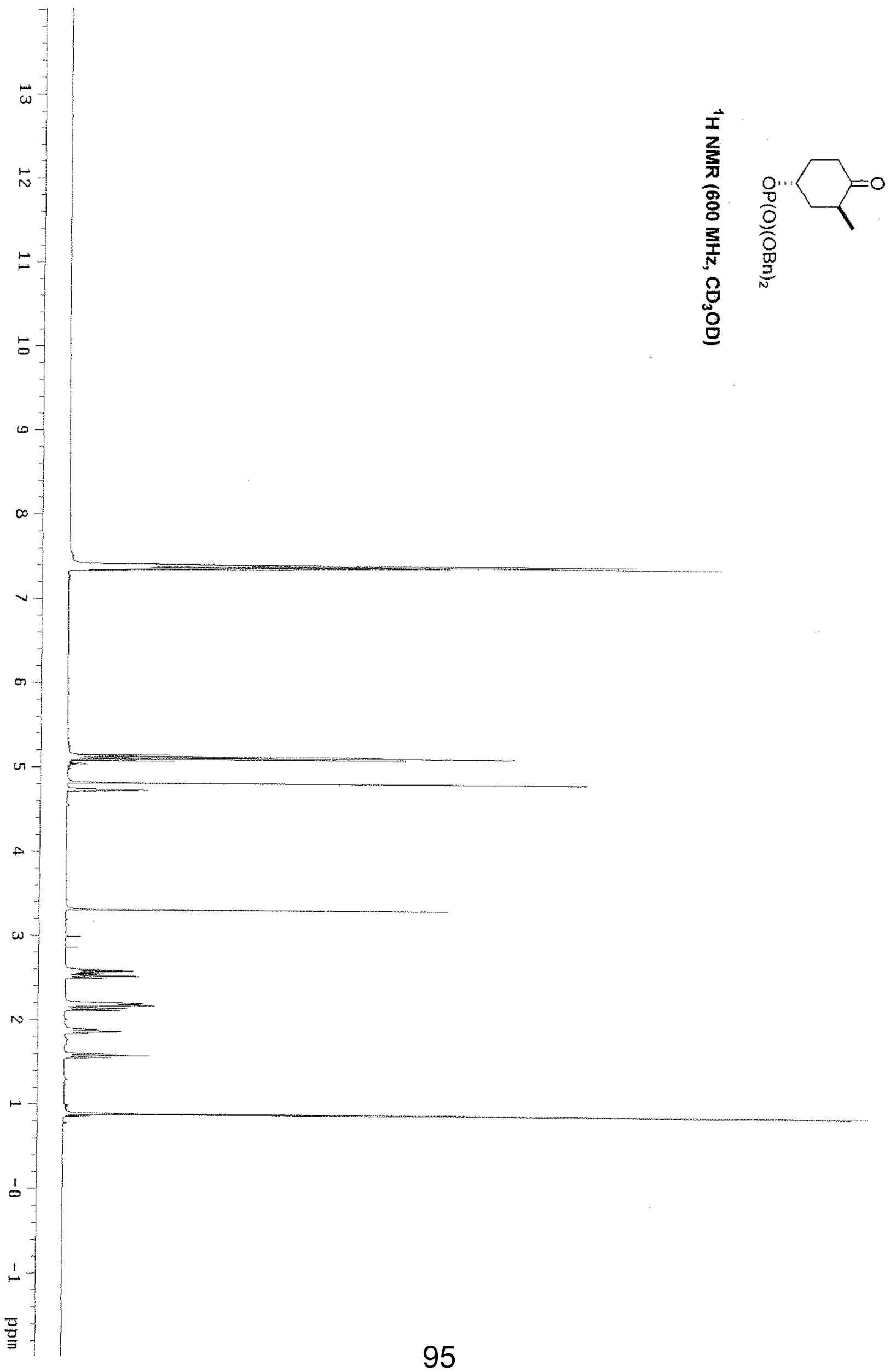




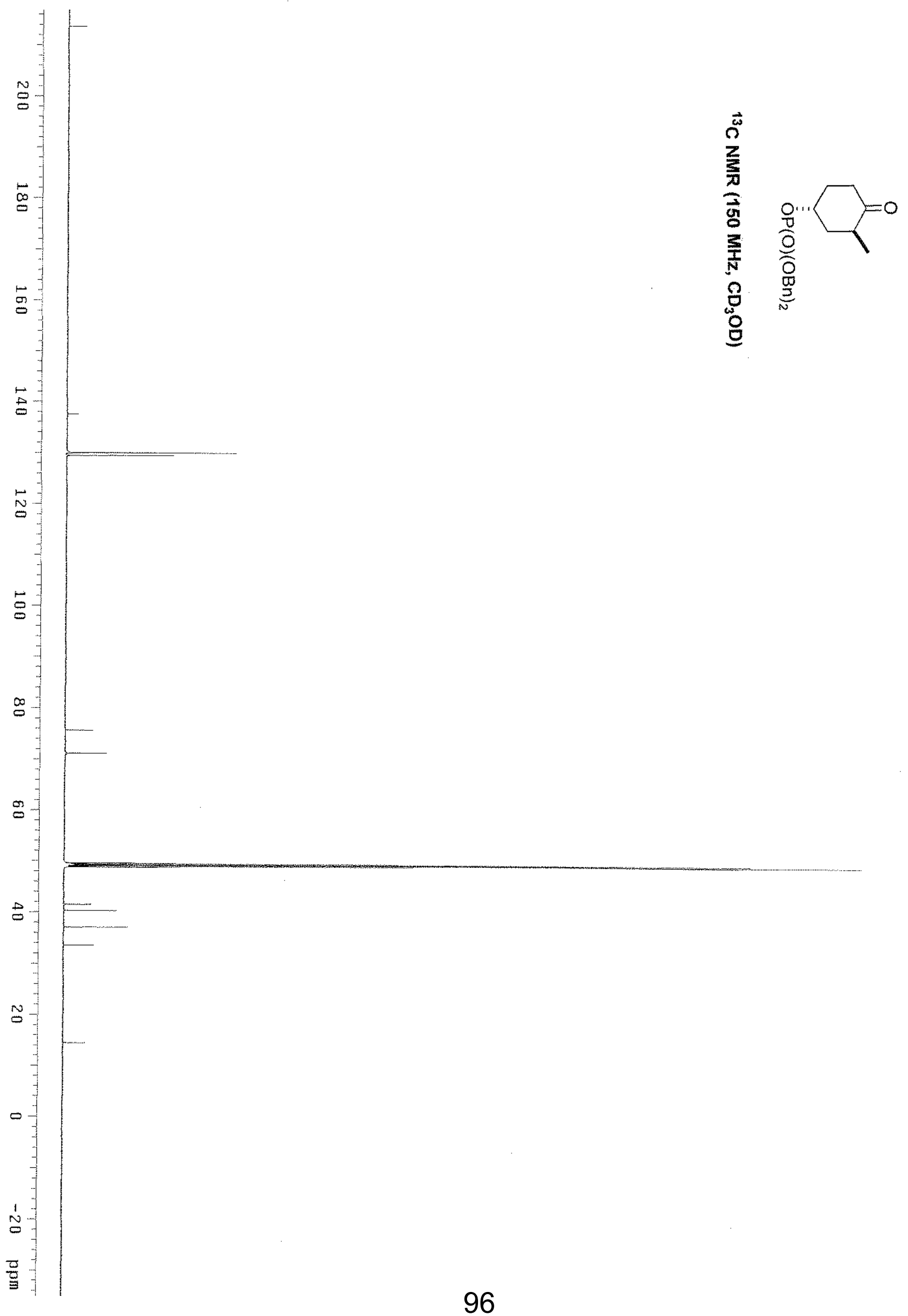




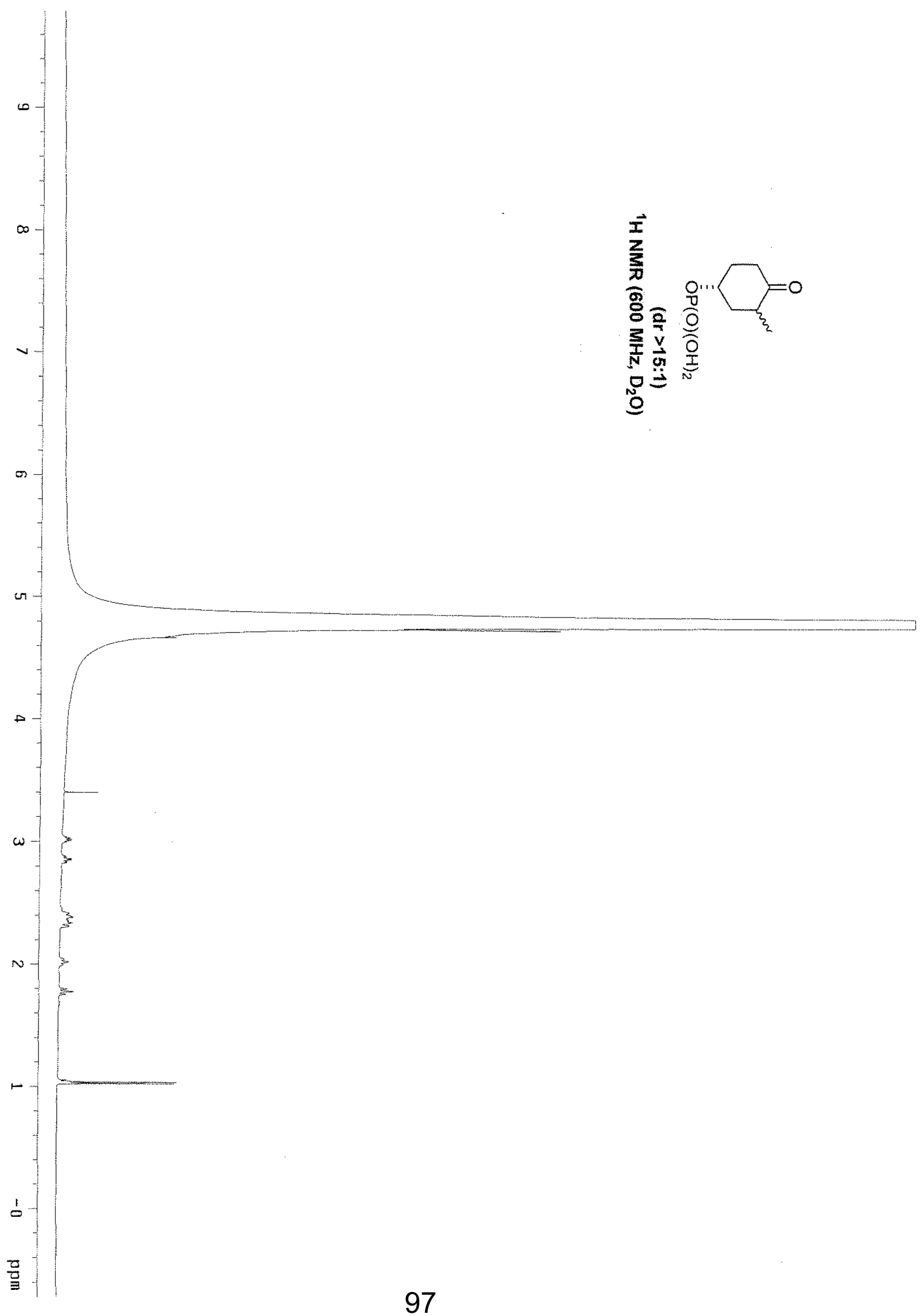




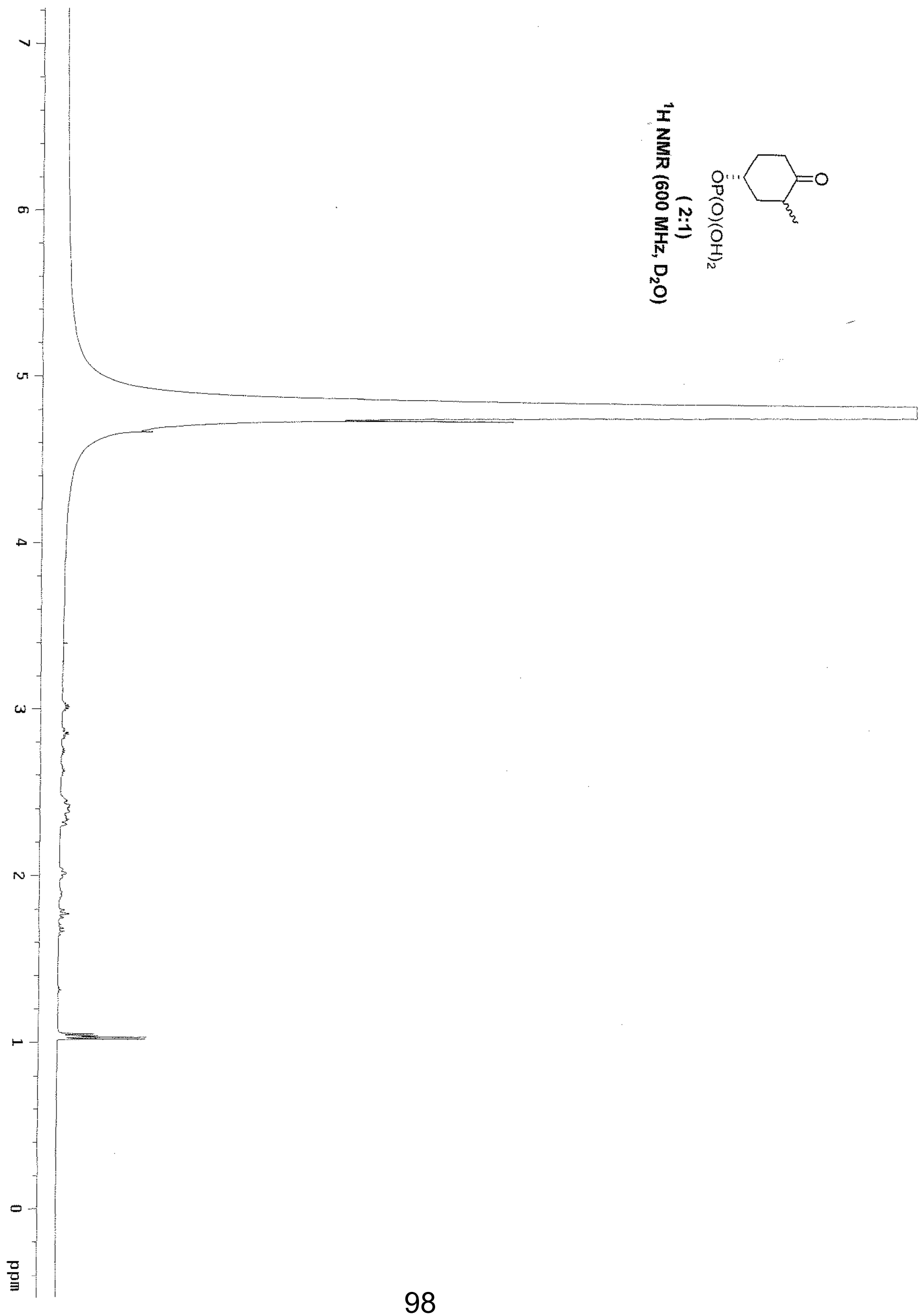




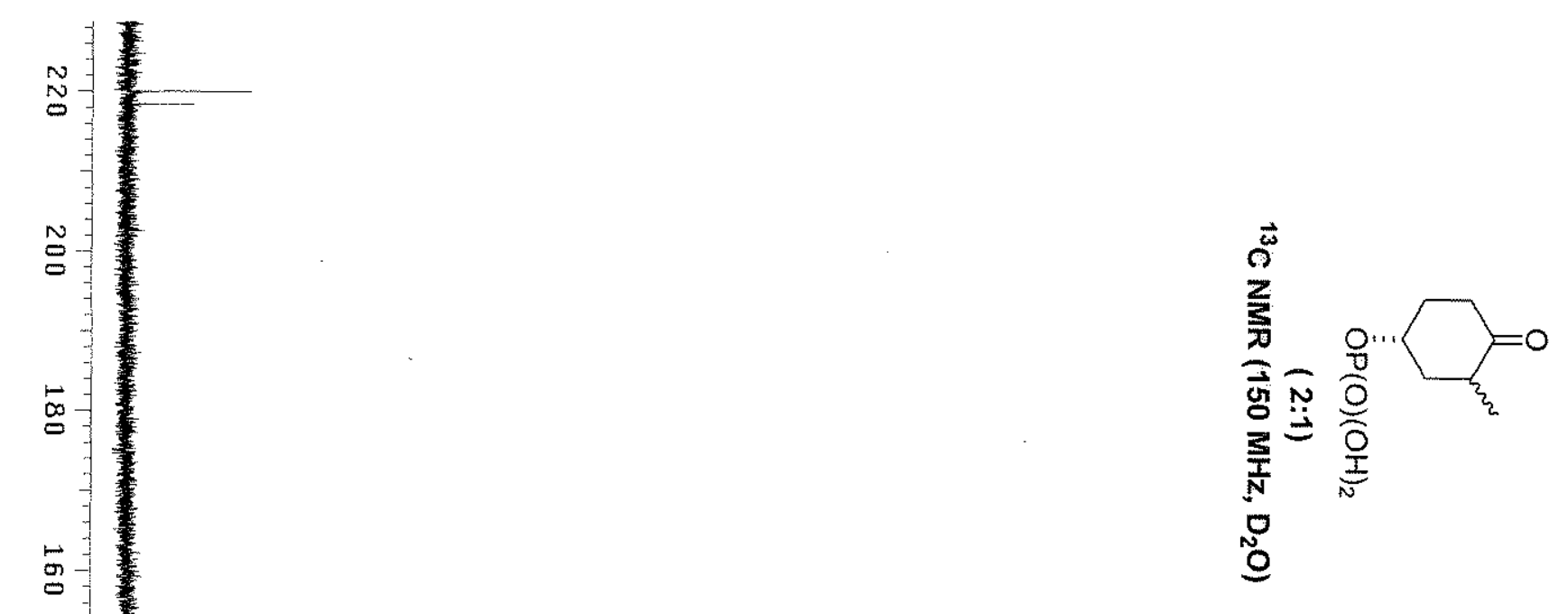




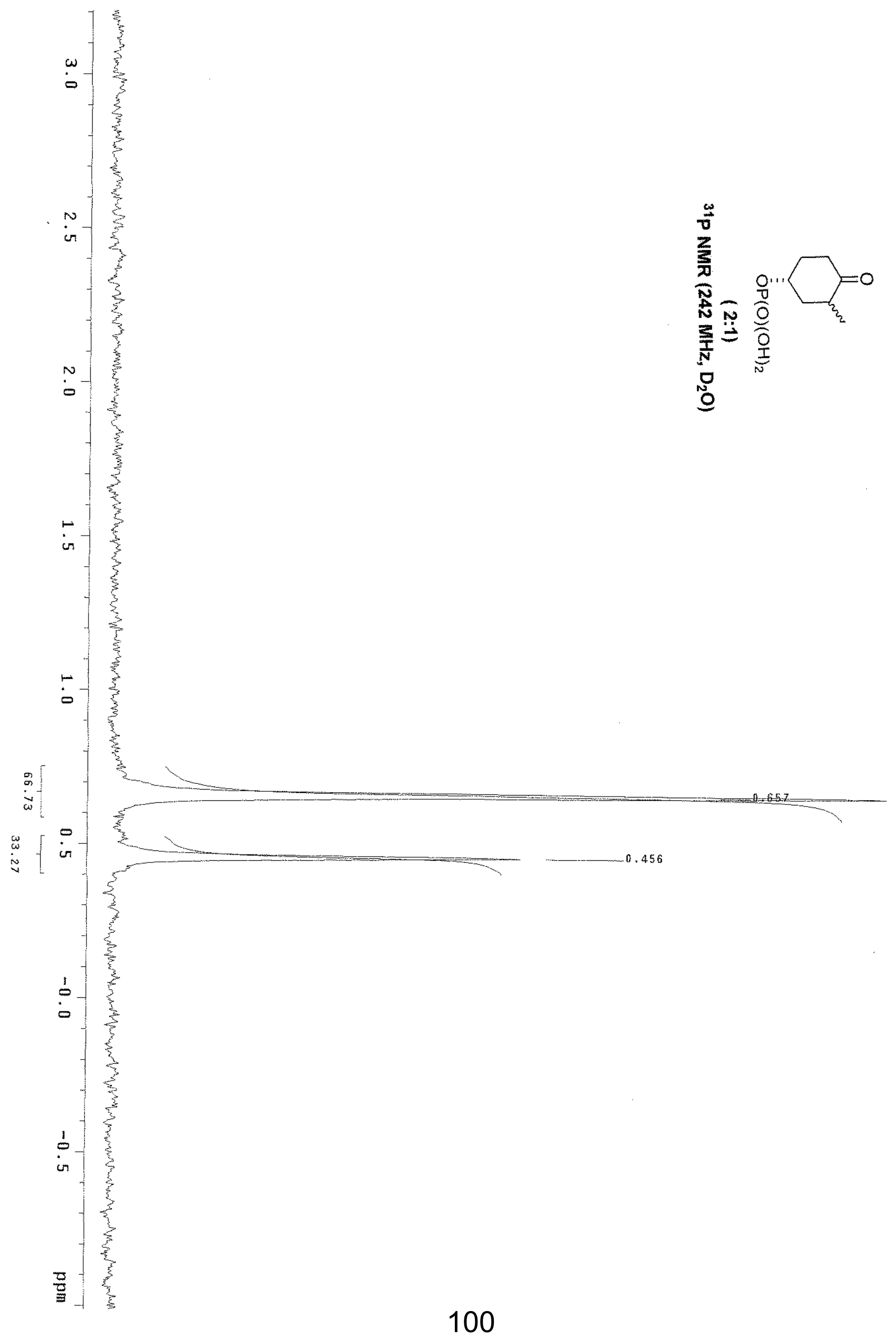




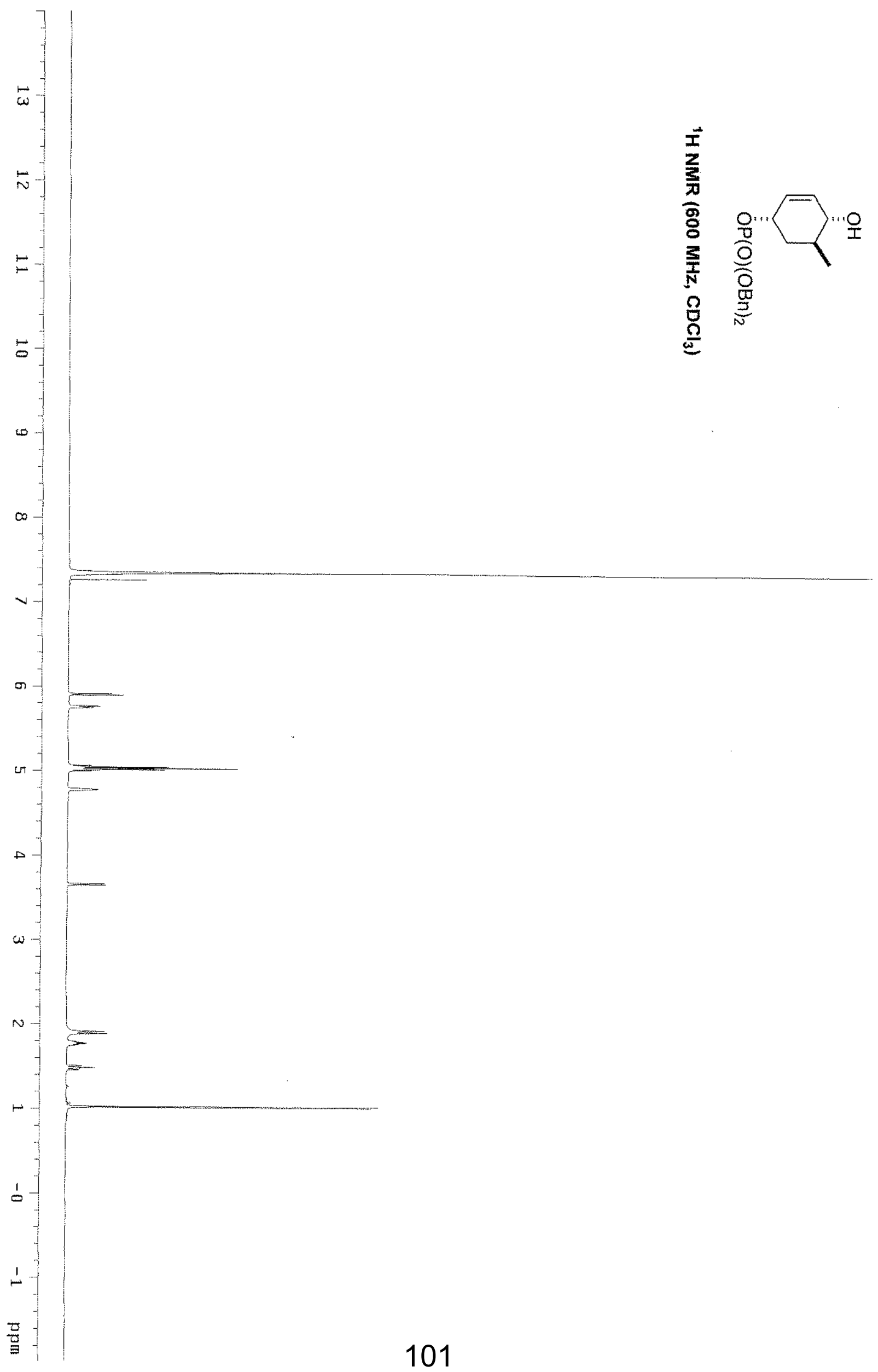




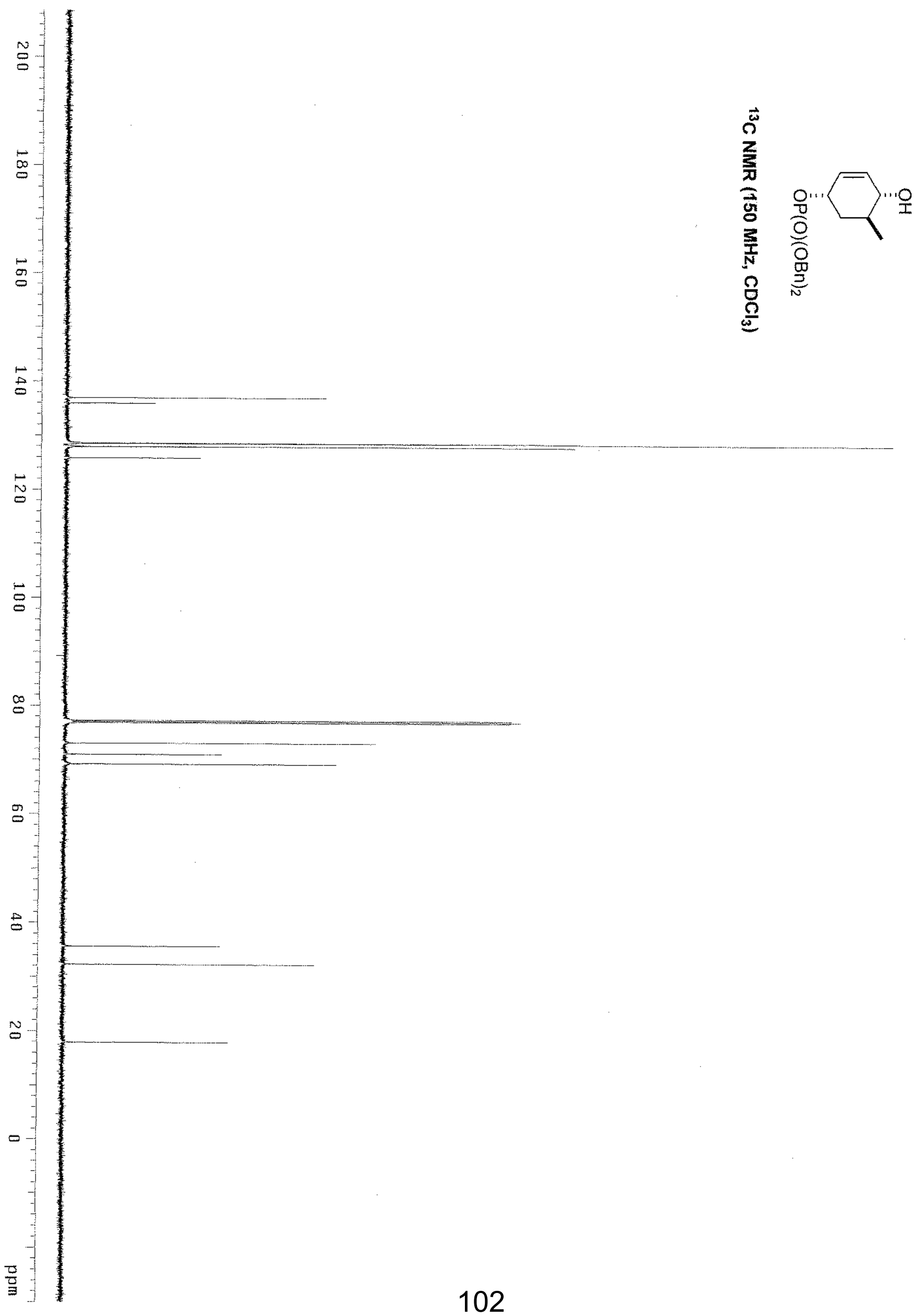




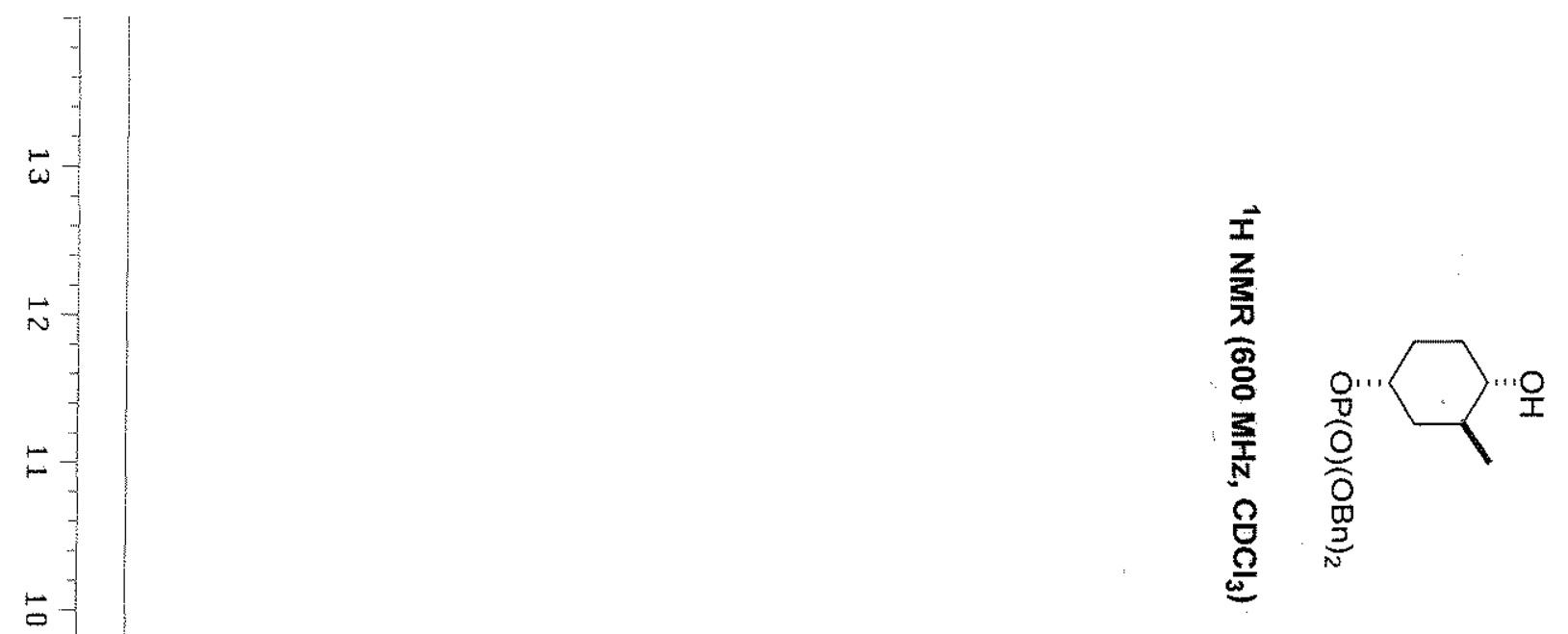

0

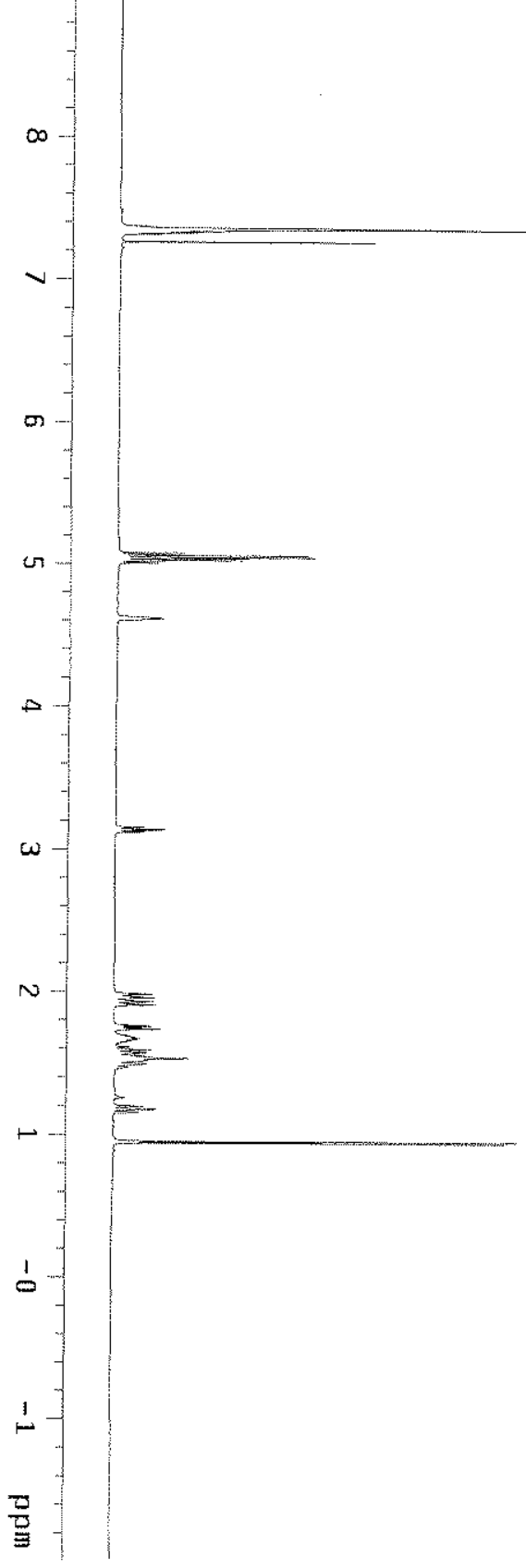




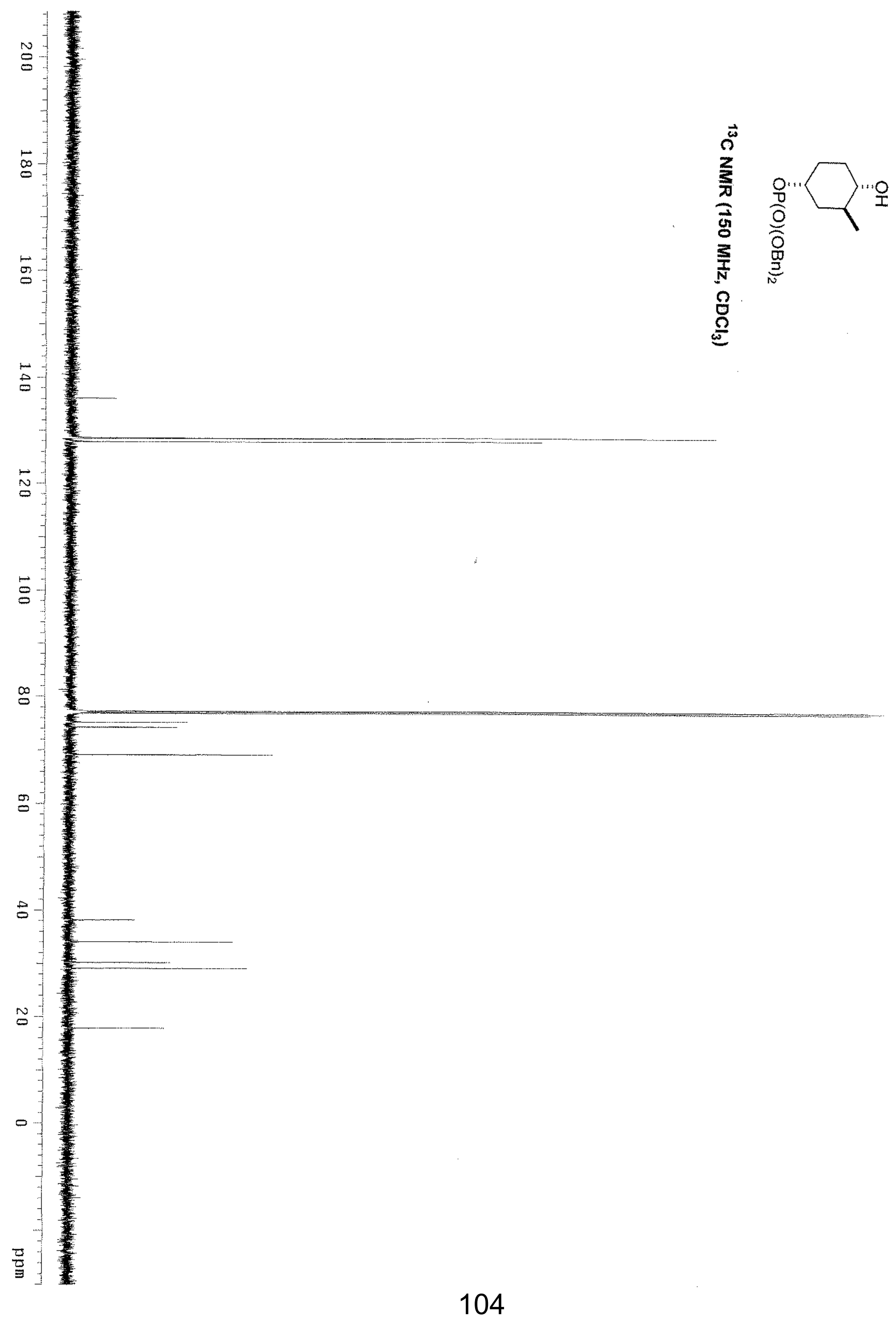




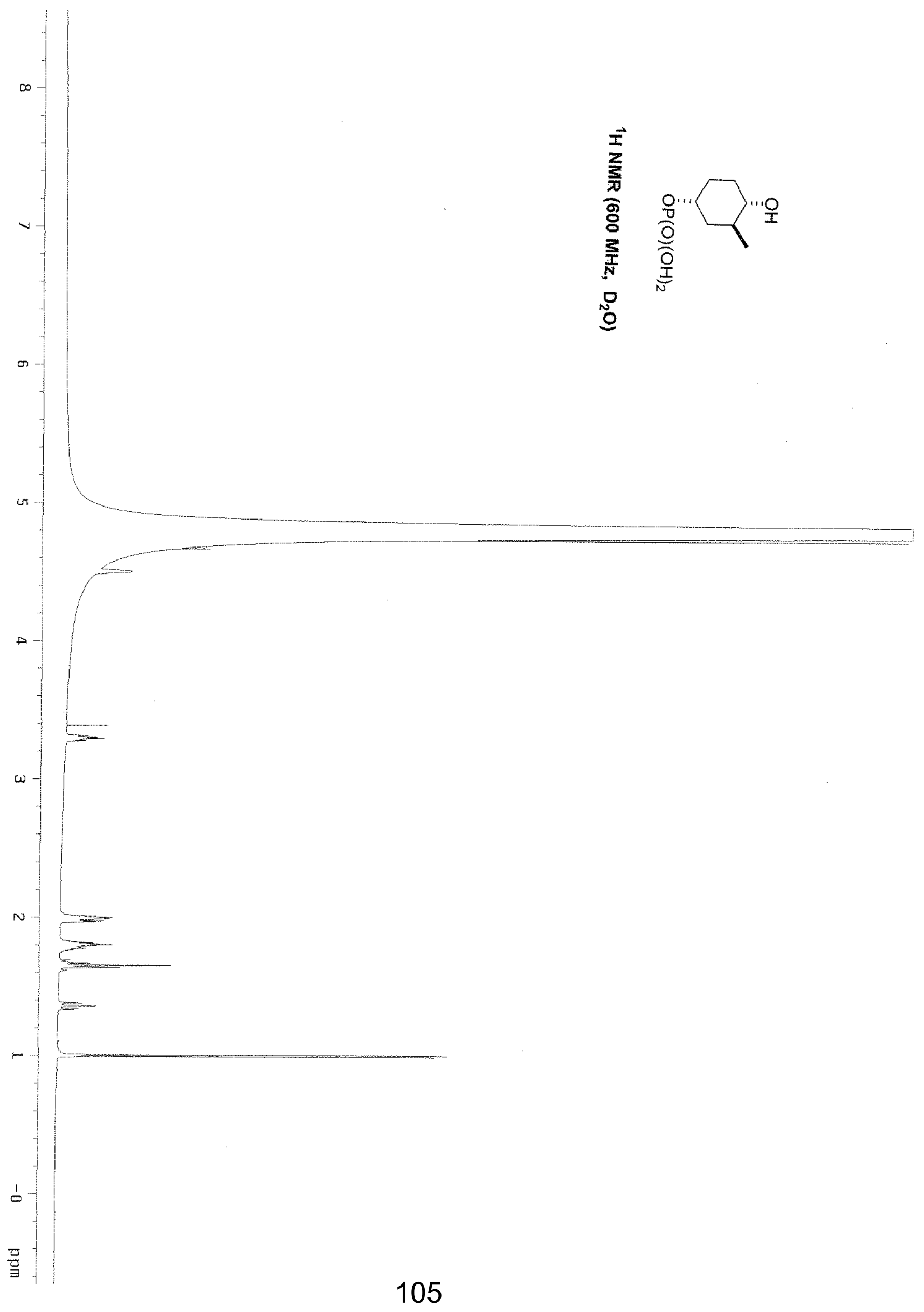




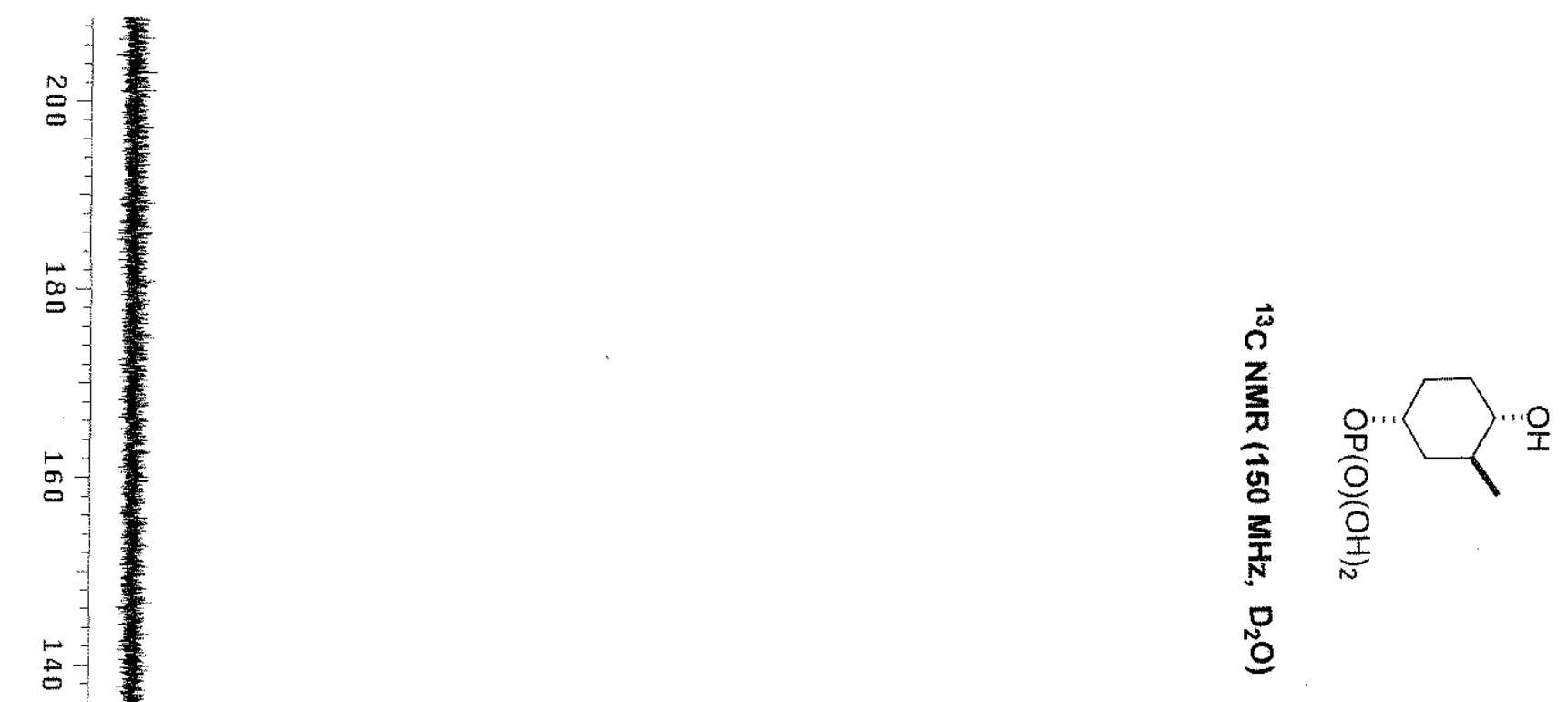




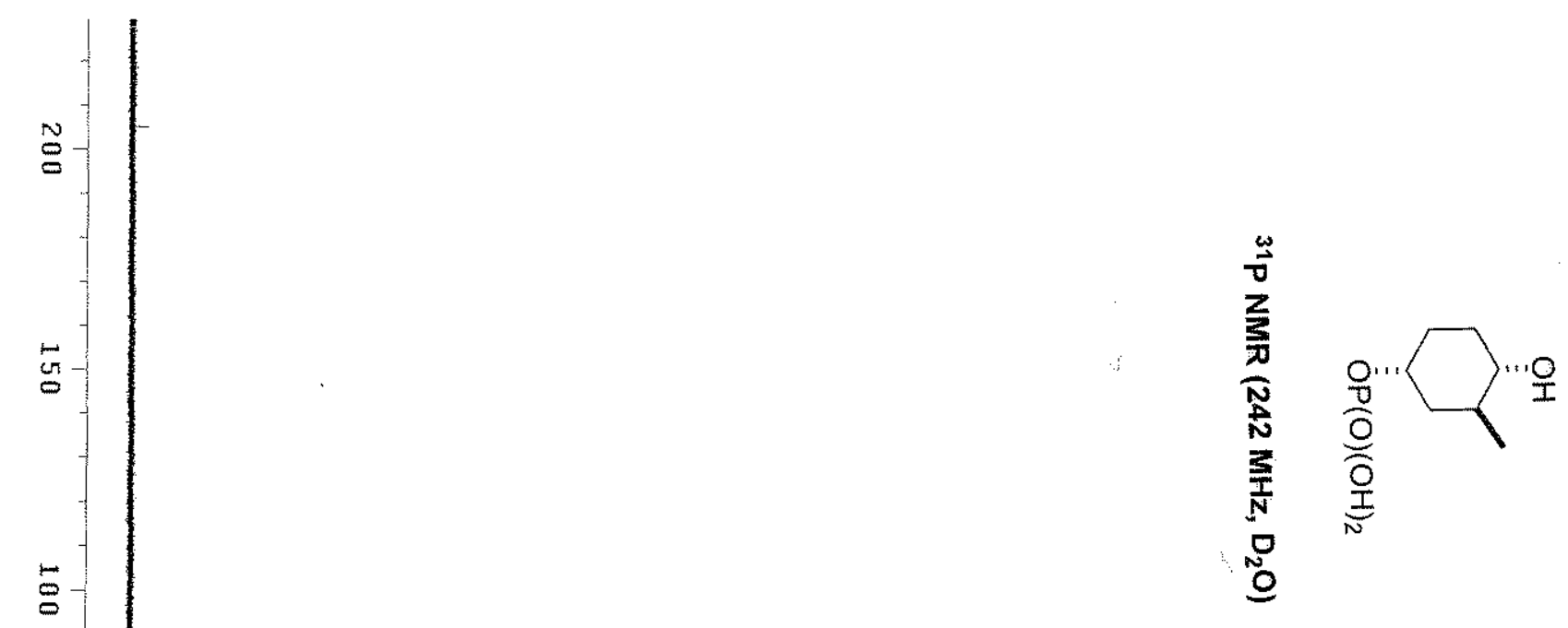

b

:

点

:

n

0

G

.

1
$N$
$u$
0

윰] 


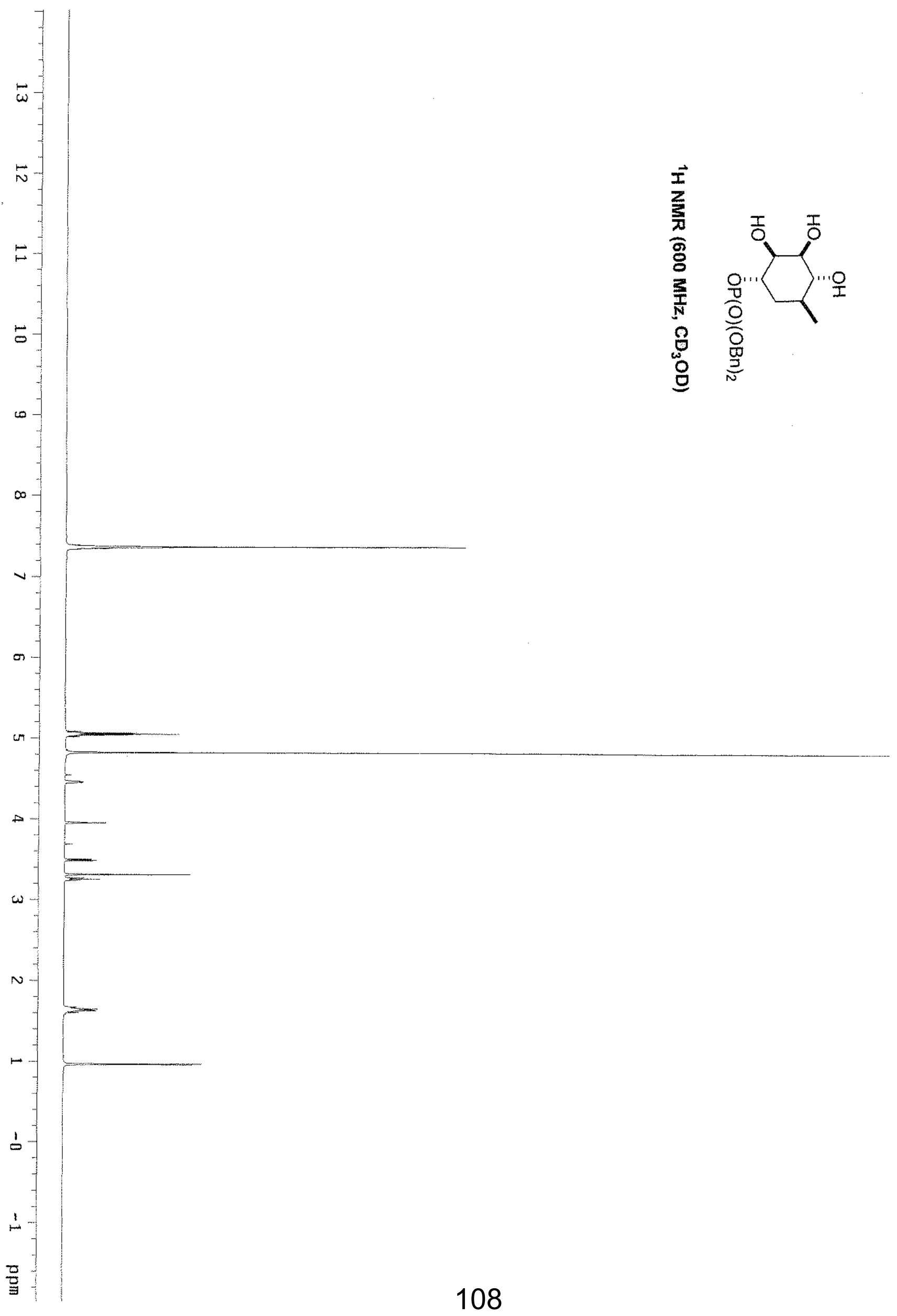




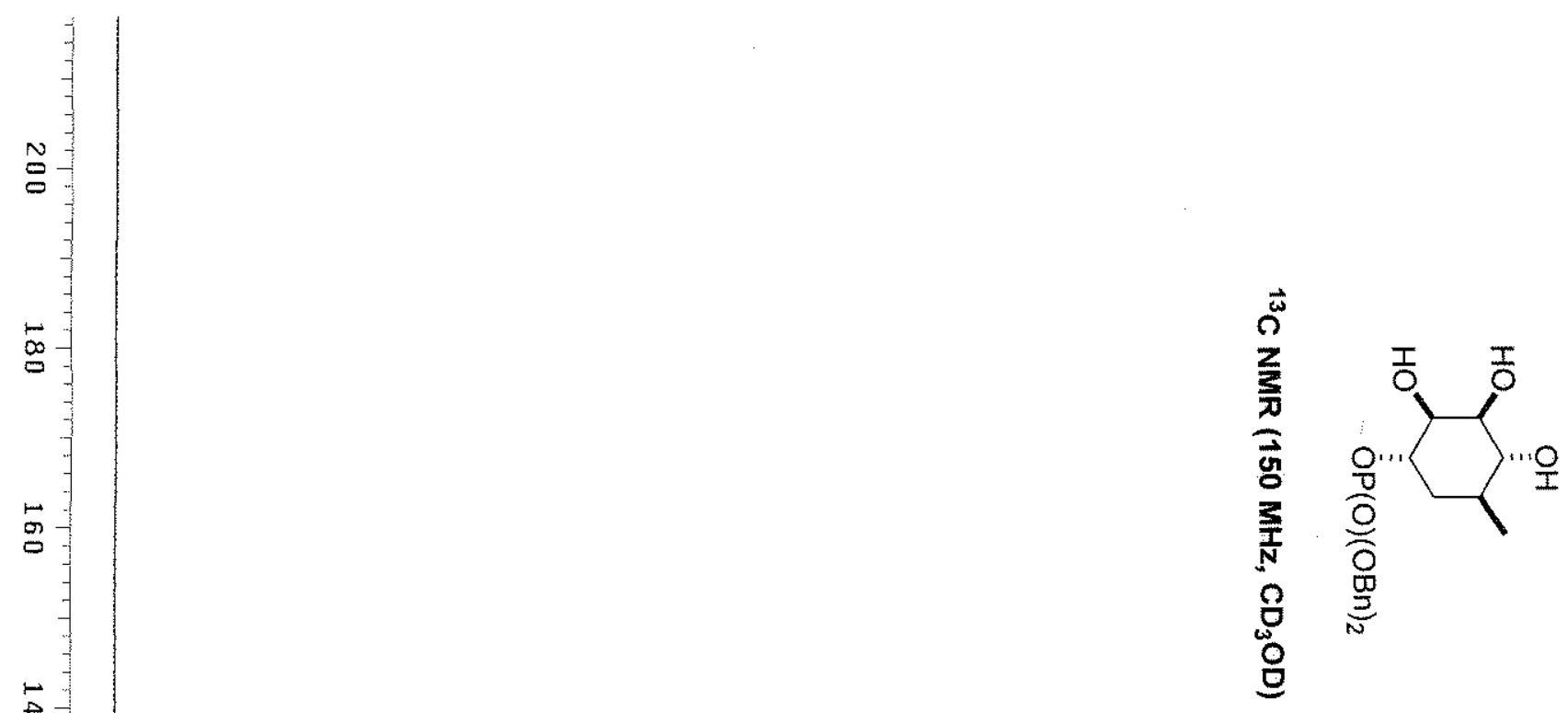

8

N

$\stackrel{5}{\circ}$

$\stackrel{\infty}{\circ}$

g

号

$\stackrel{0}{0}$

$\square$

$\stackrel{1}{0}$

$\stackrel{\circ}{\circ}$

믐 


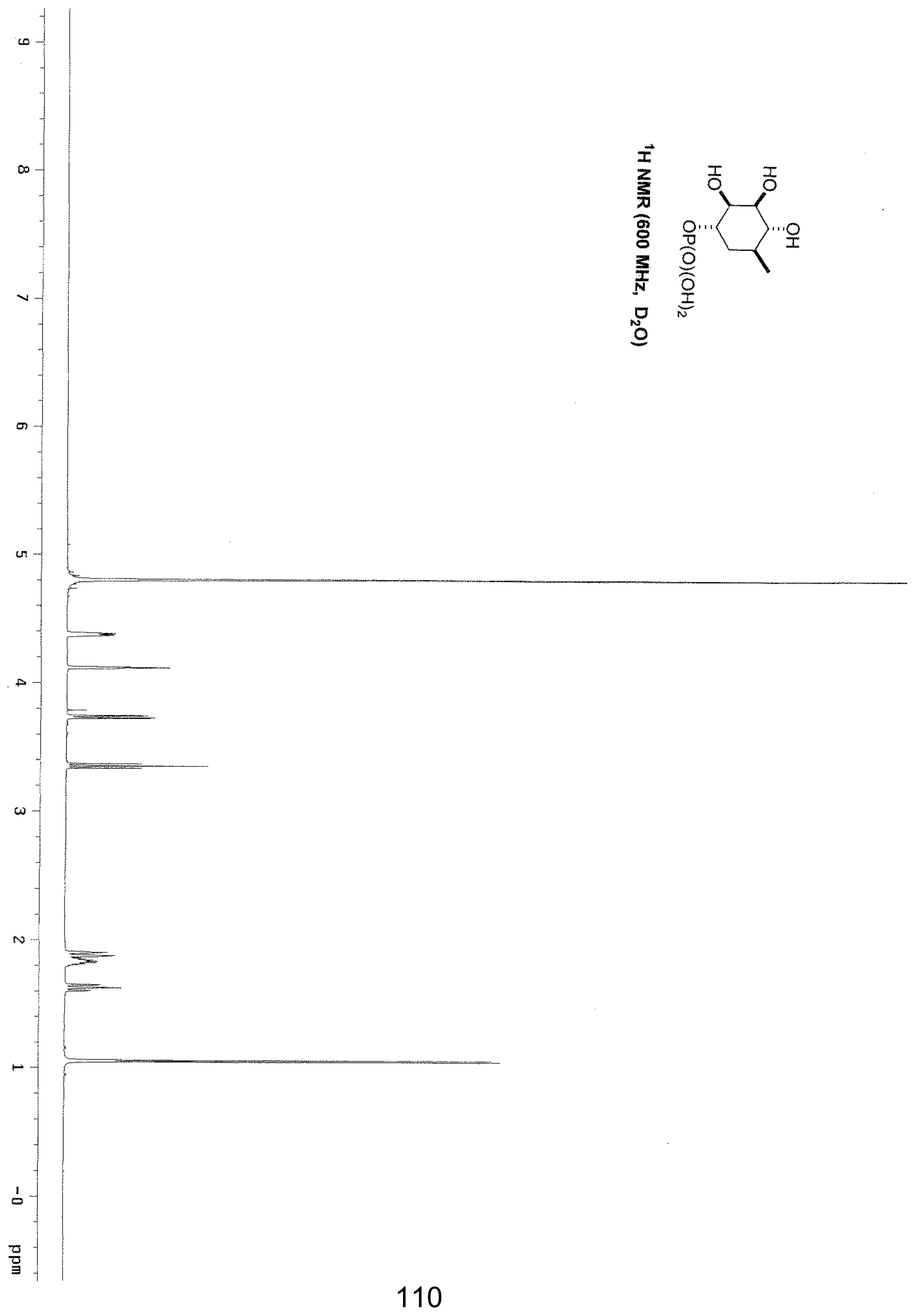




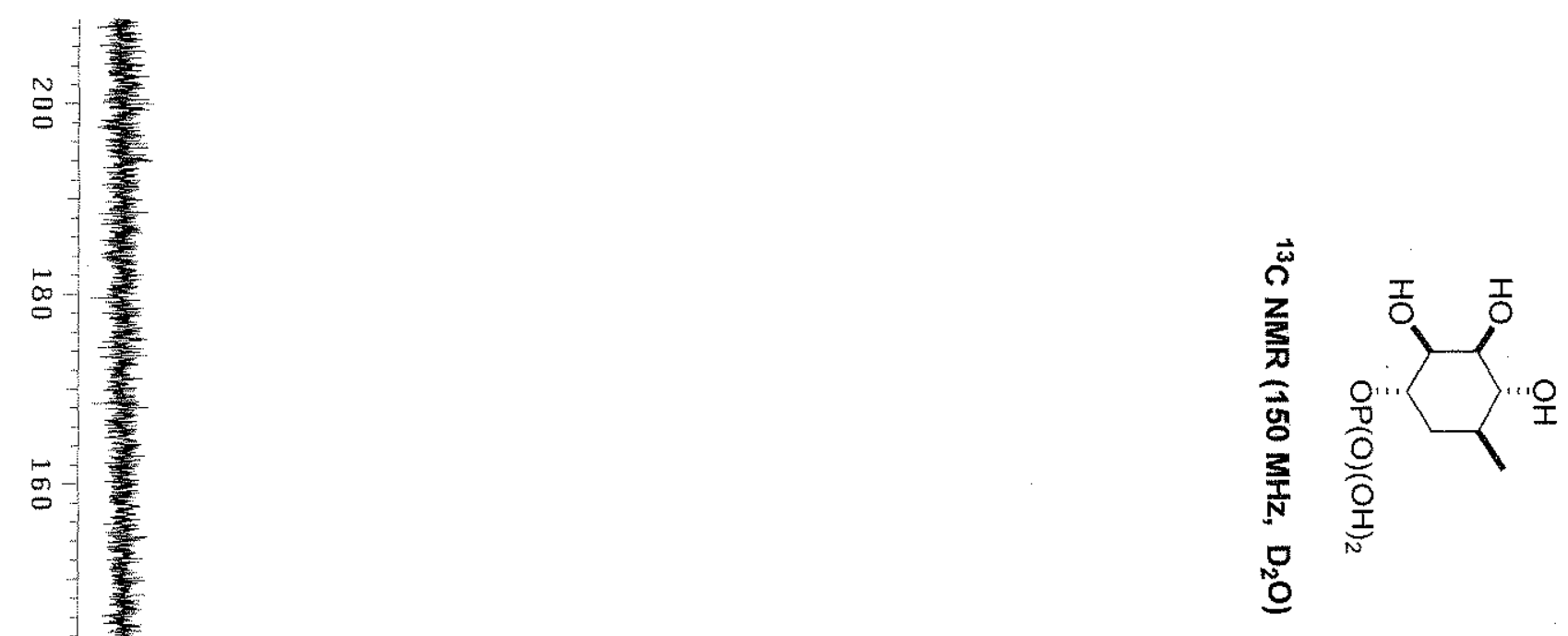

E

N

$\varnothing$

:

䲞

$\infty$

g

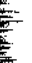




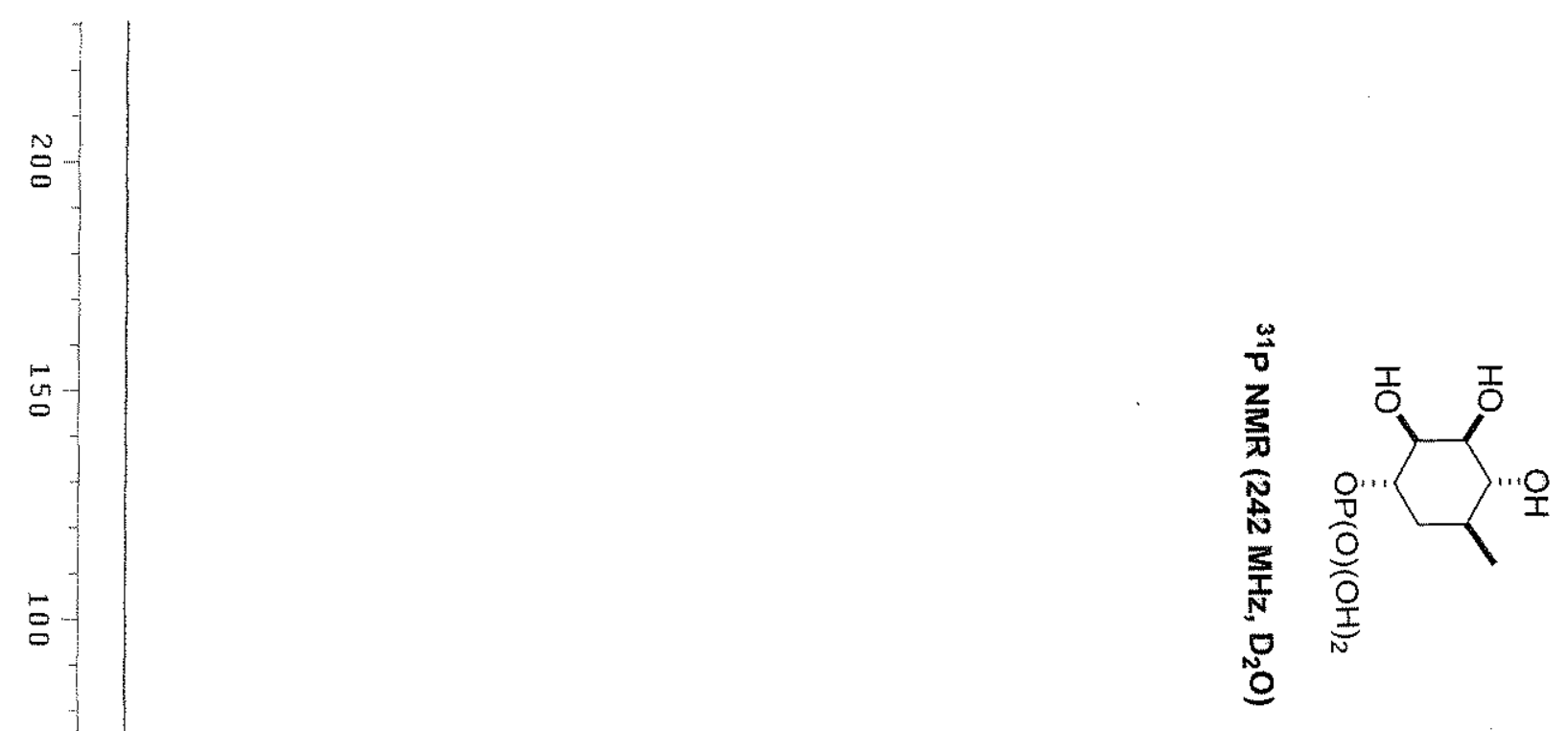

I

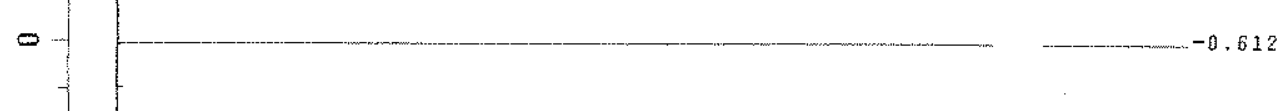

1
5
5

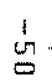

$\frac{1}{8}$

$\stackrel{1}{N}$

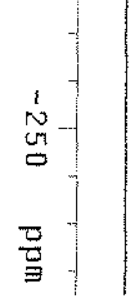




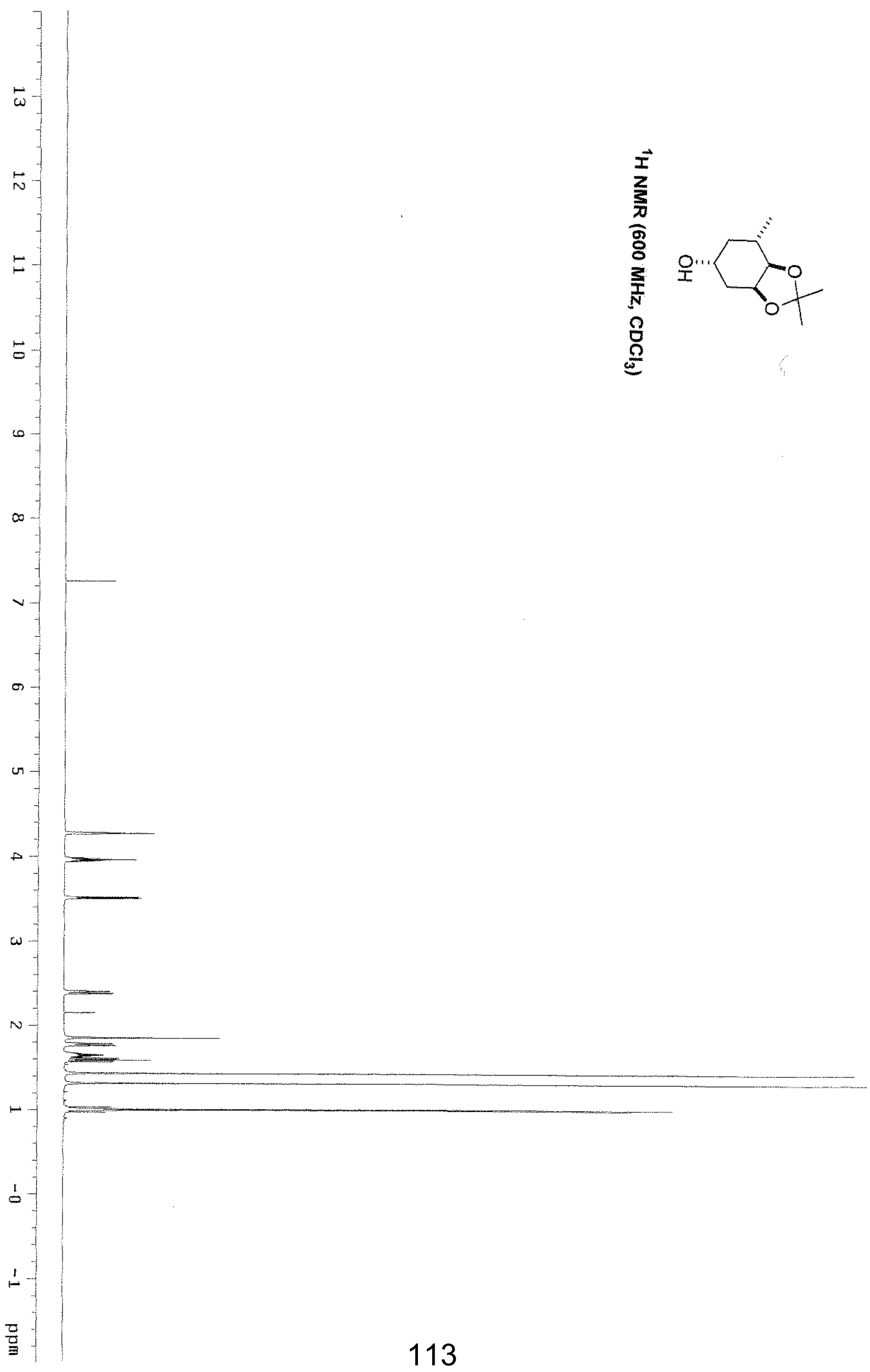




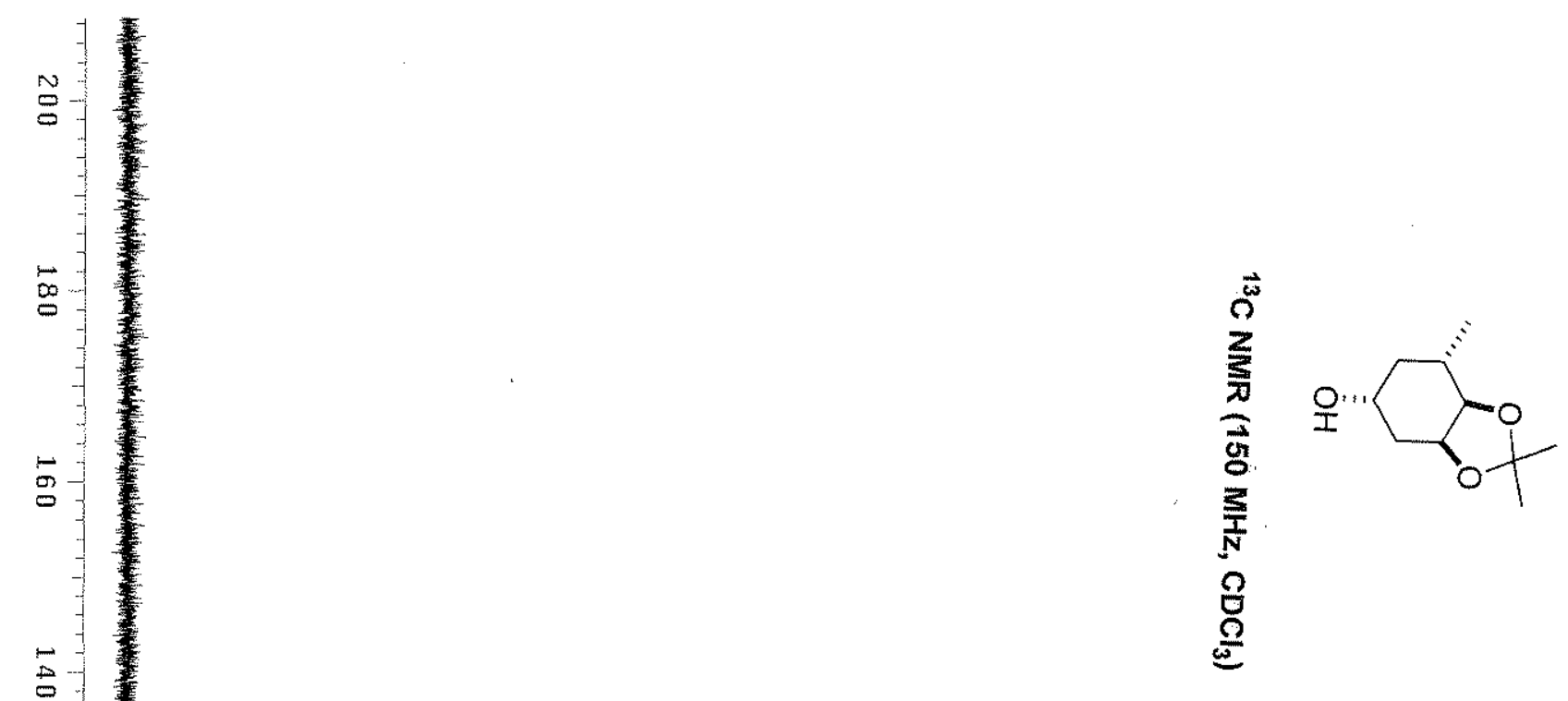

$\stackrel{\infty}{\circ}$

$g$

:

$B$

t.

t?

-

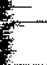

s.
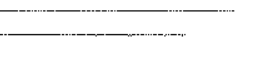


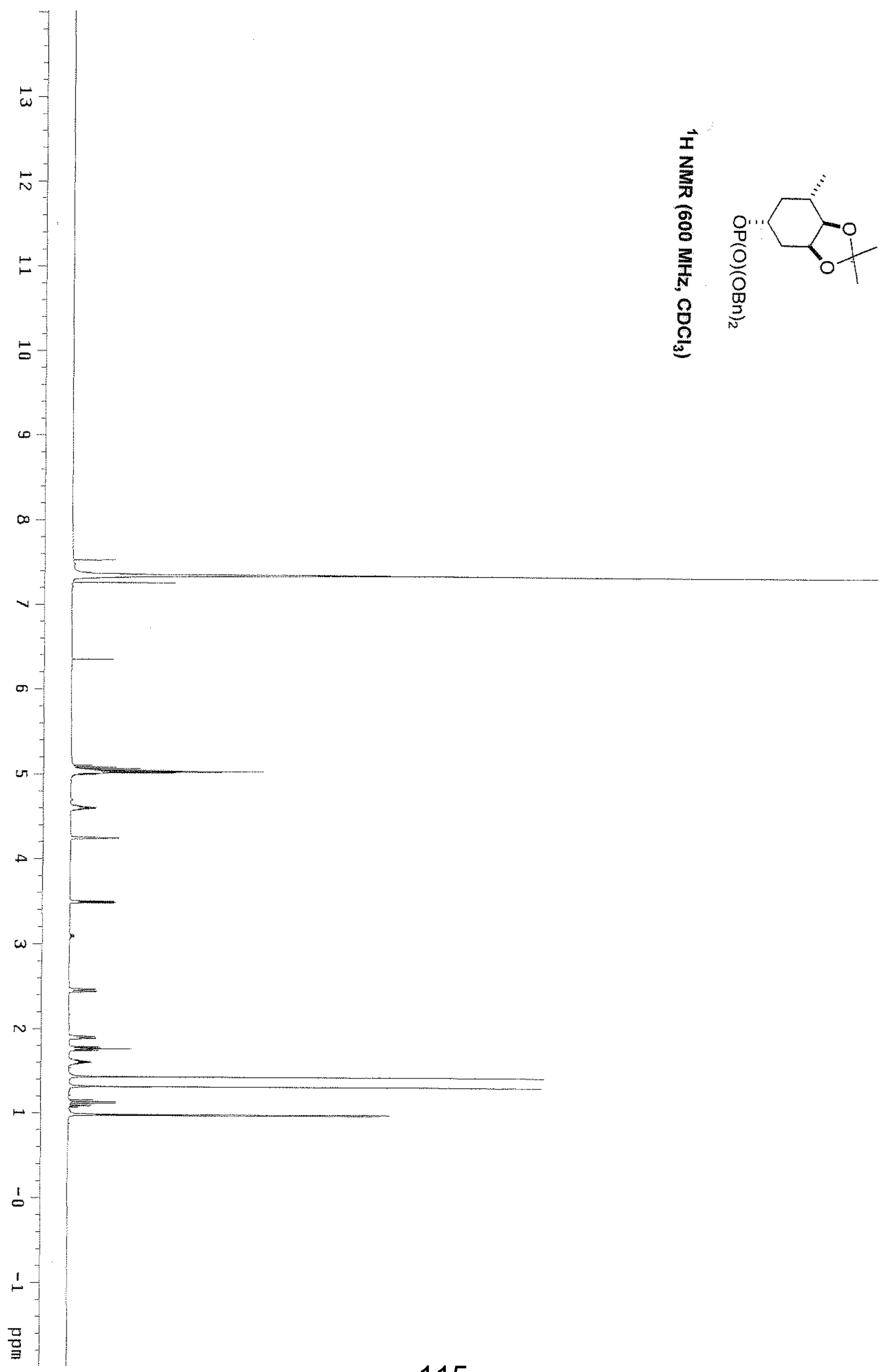



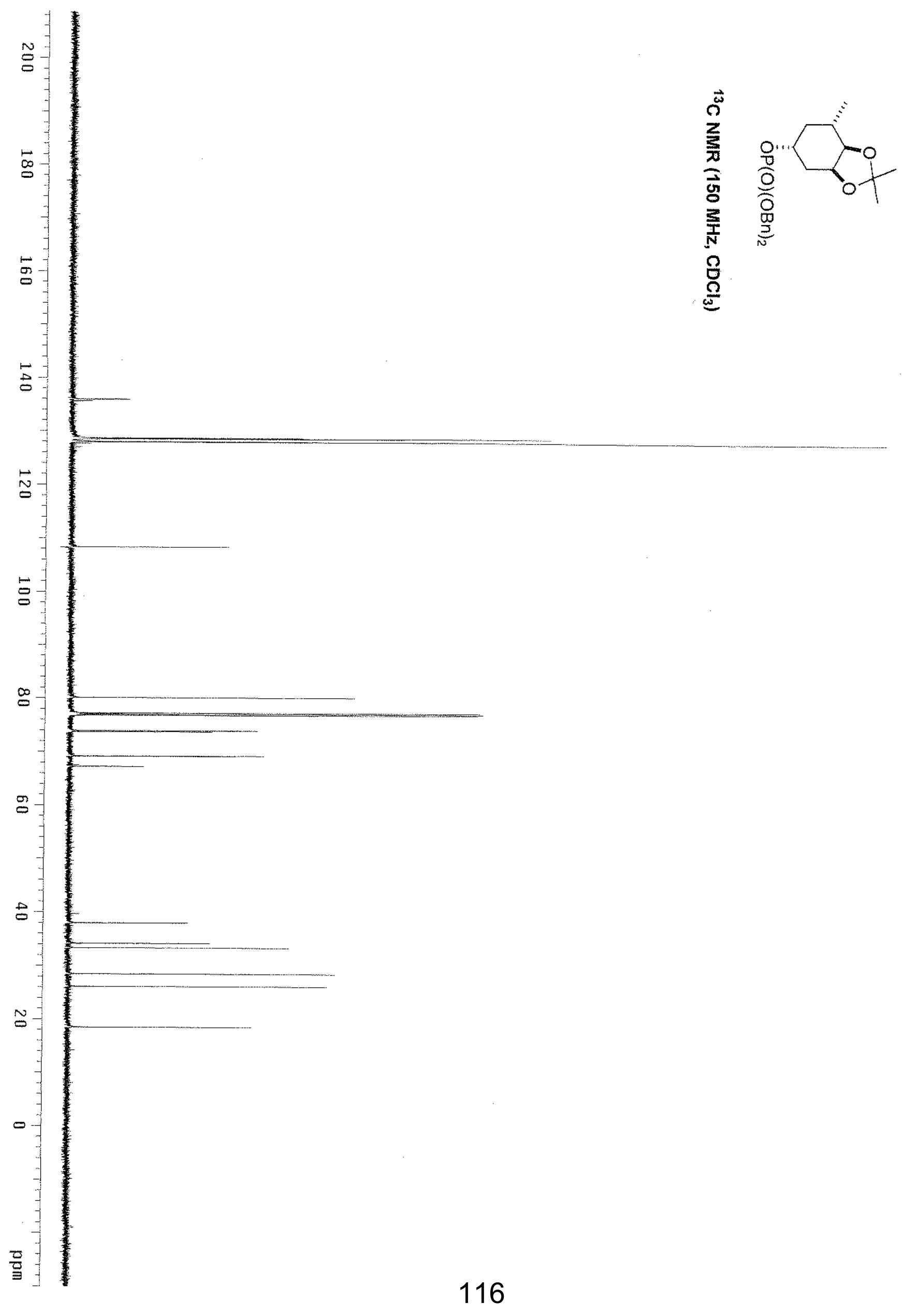


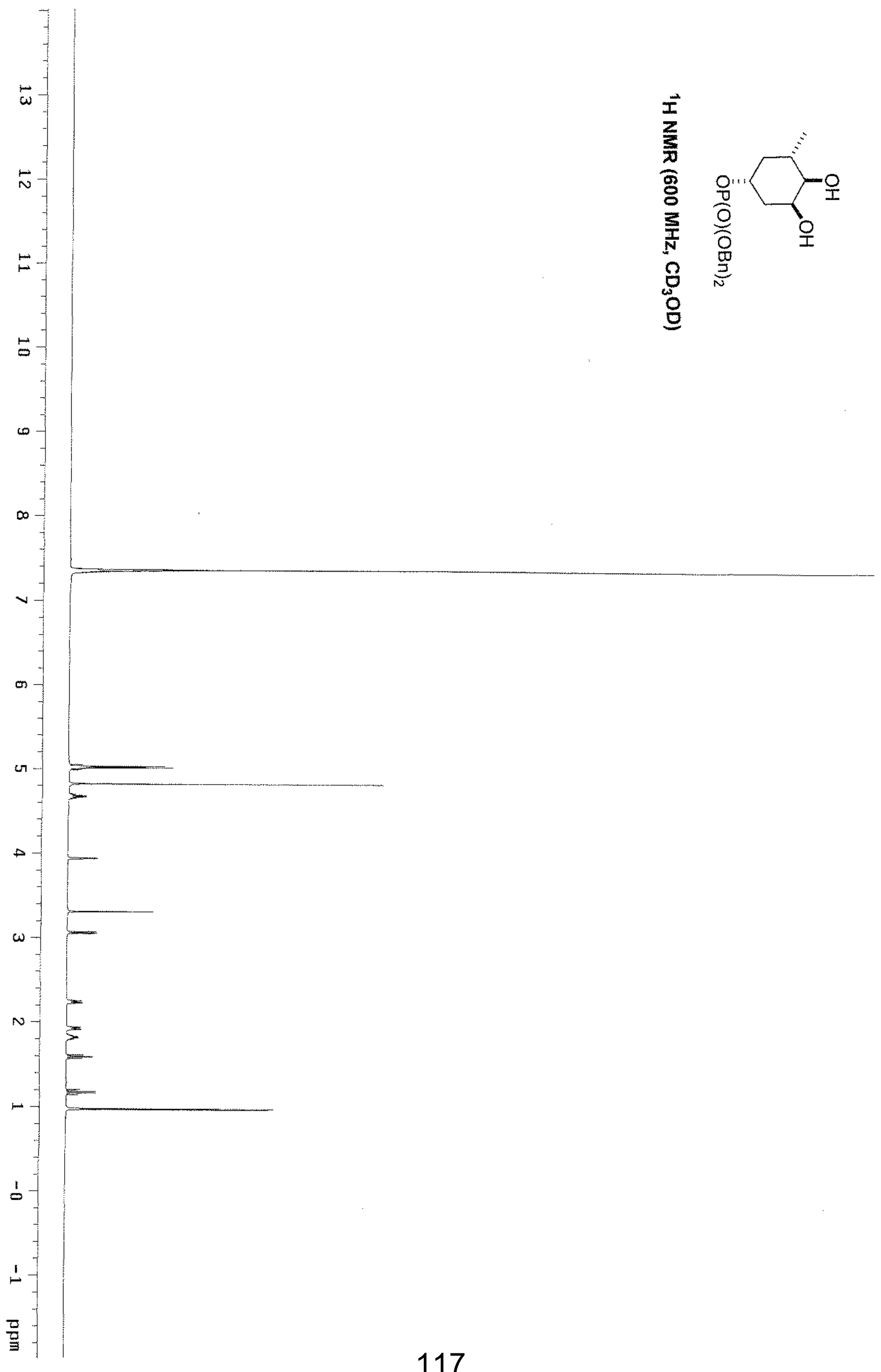




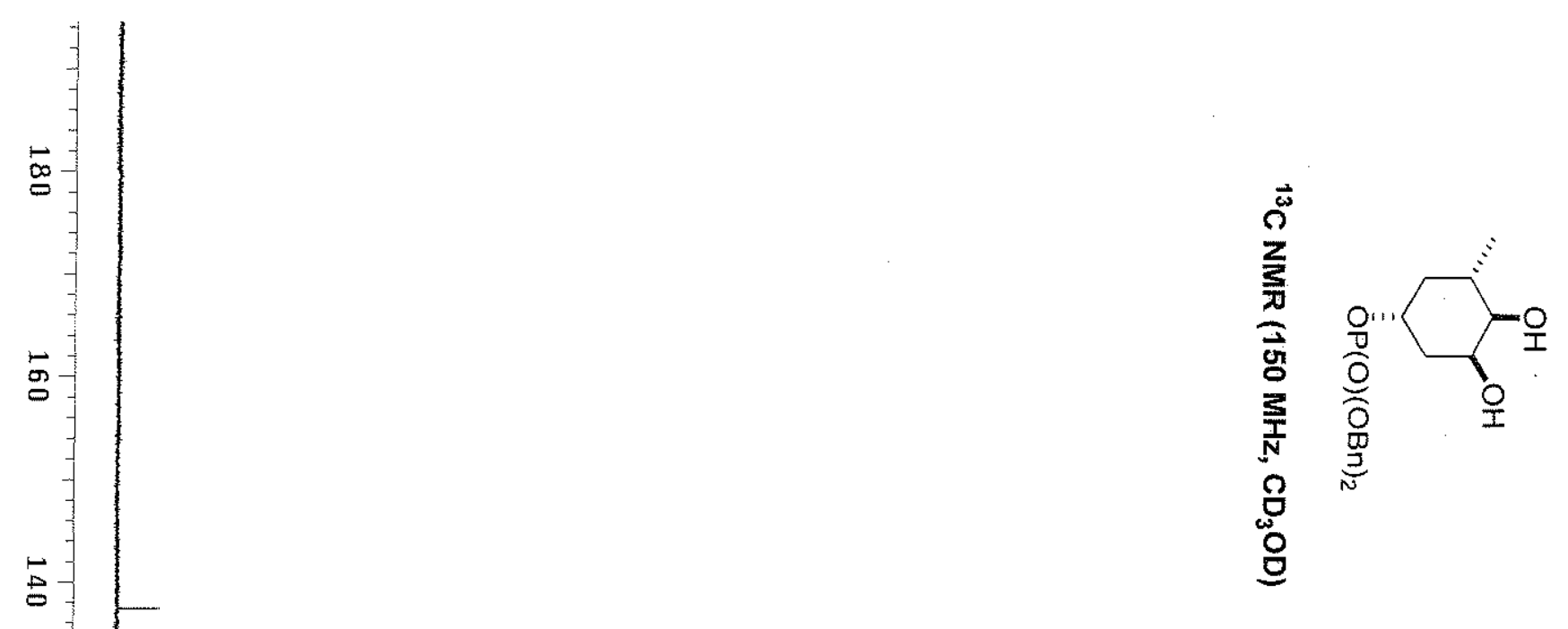

包

$\stackrel{\infty}{\circ}$

$\triangle$

ง.

。
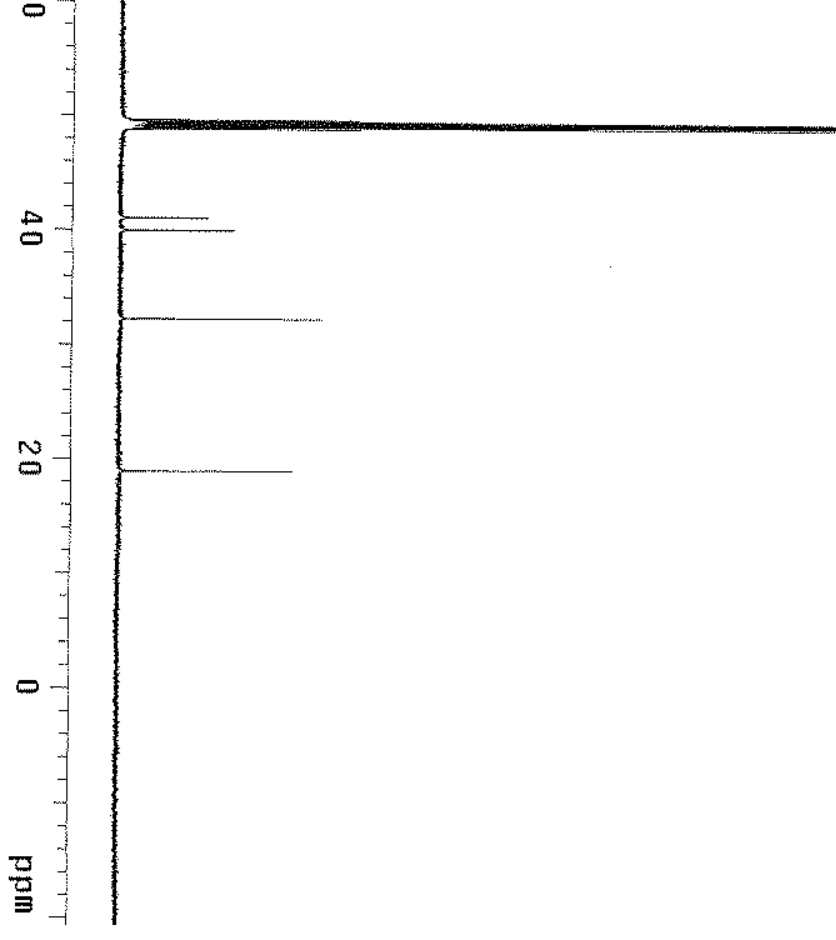


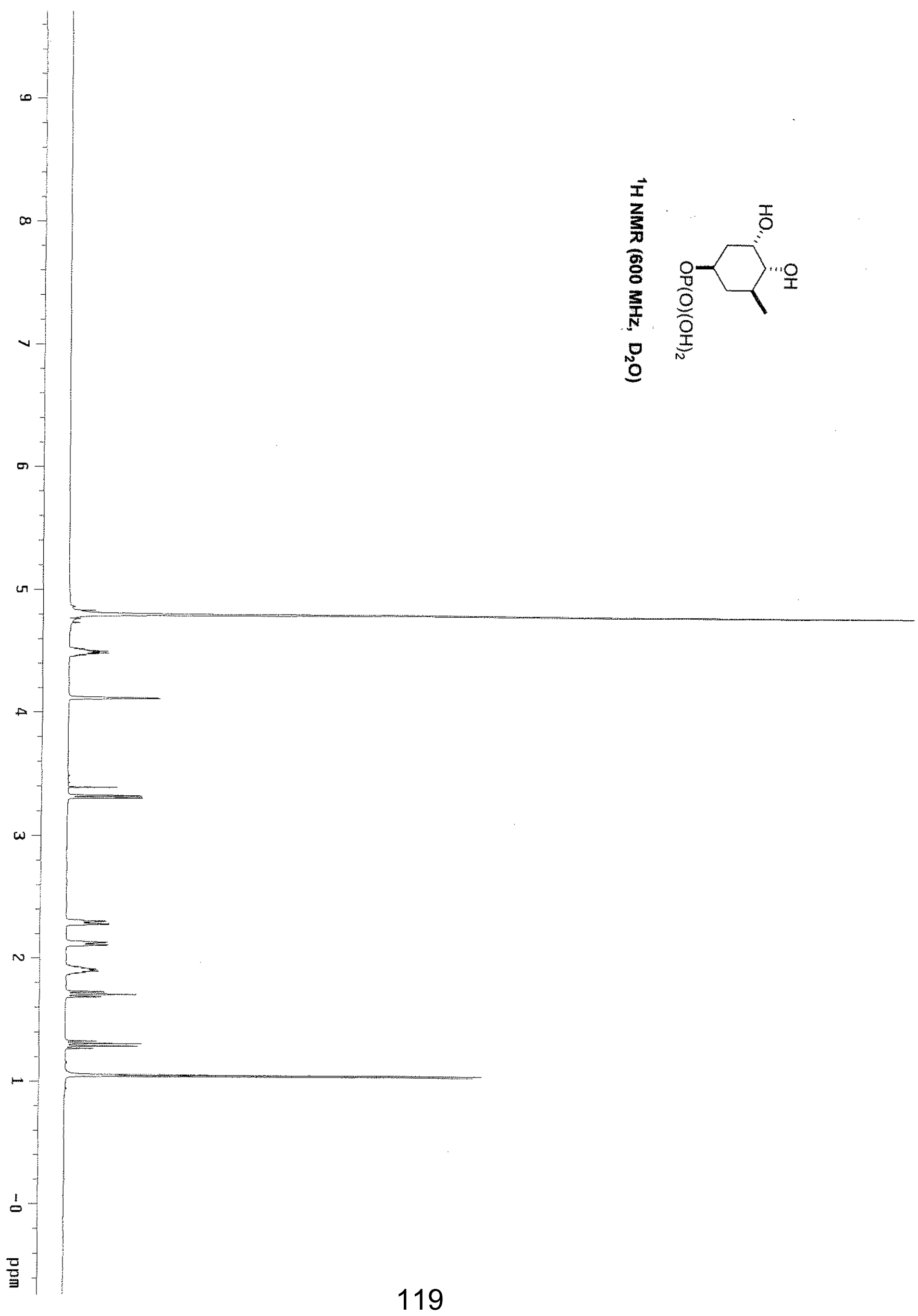




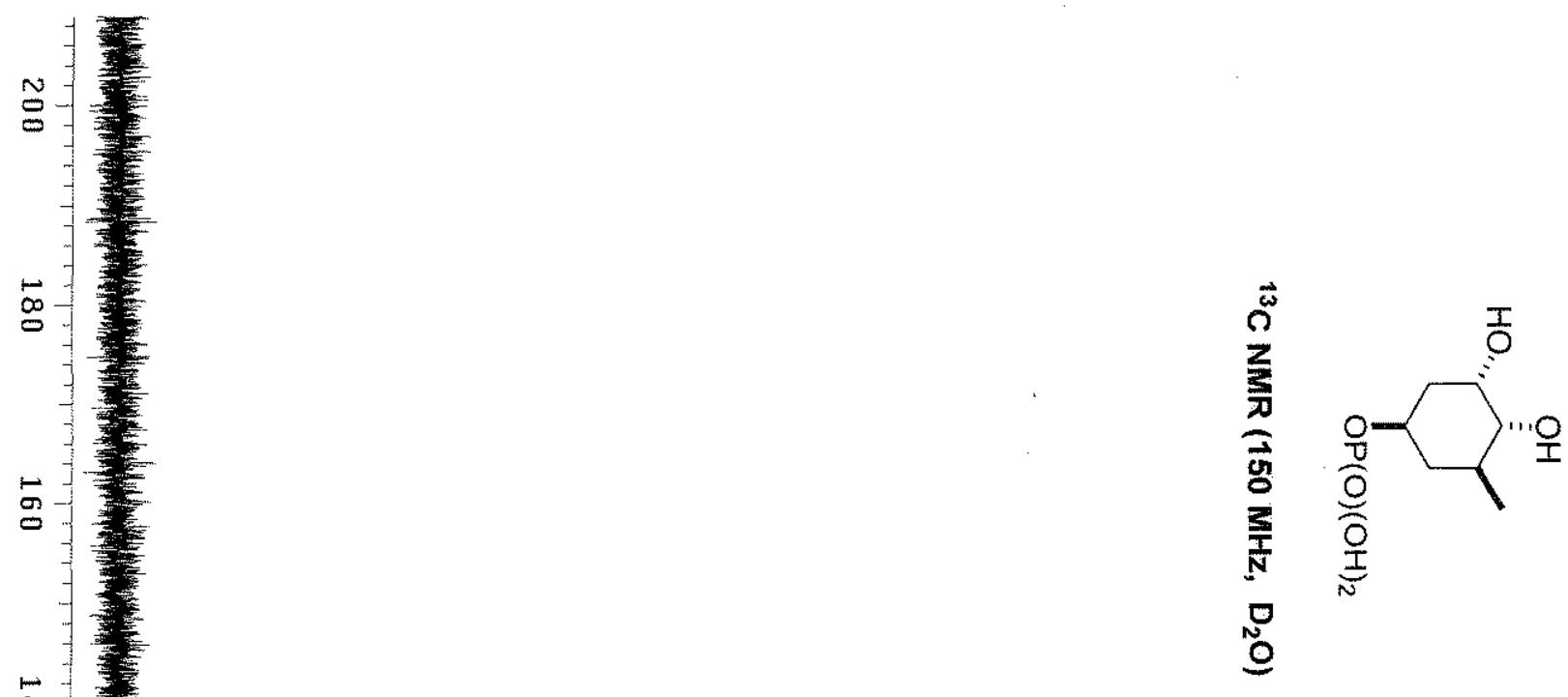

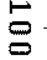

$\infty$

g -

8

N

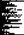




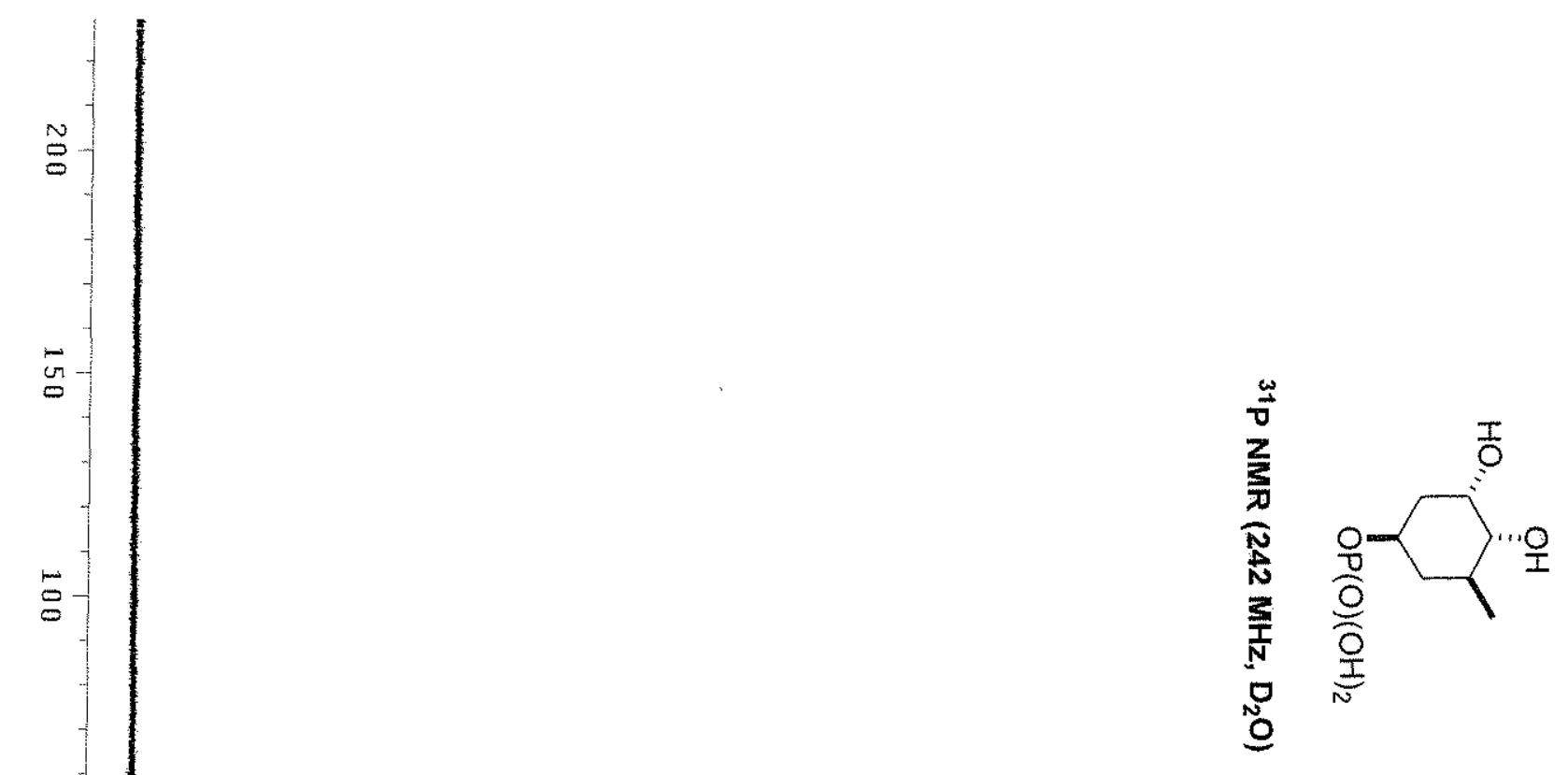

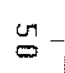

1
0
0

$\left.\begin{array}{r}1 \\ 0 \\ 0 \\ 0 \\ 1 \\ 1 \\ -5 \\ 0 \\ 0\end{array}\right]$

点

U

폼 


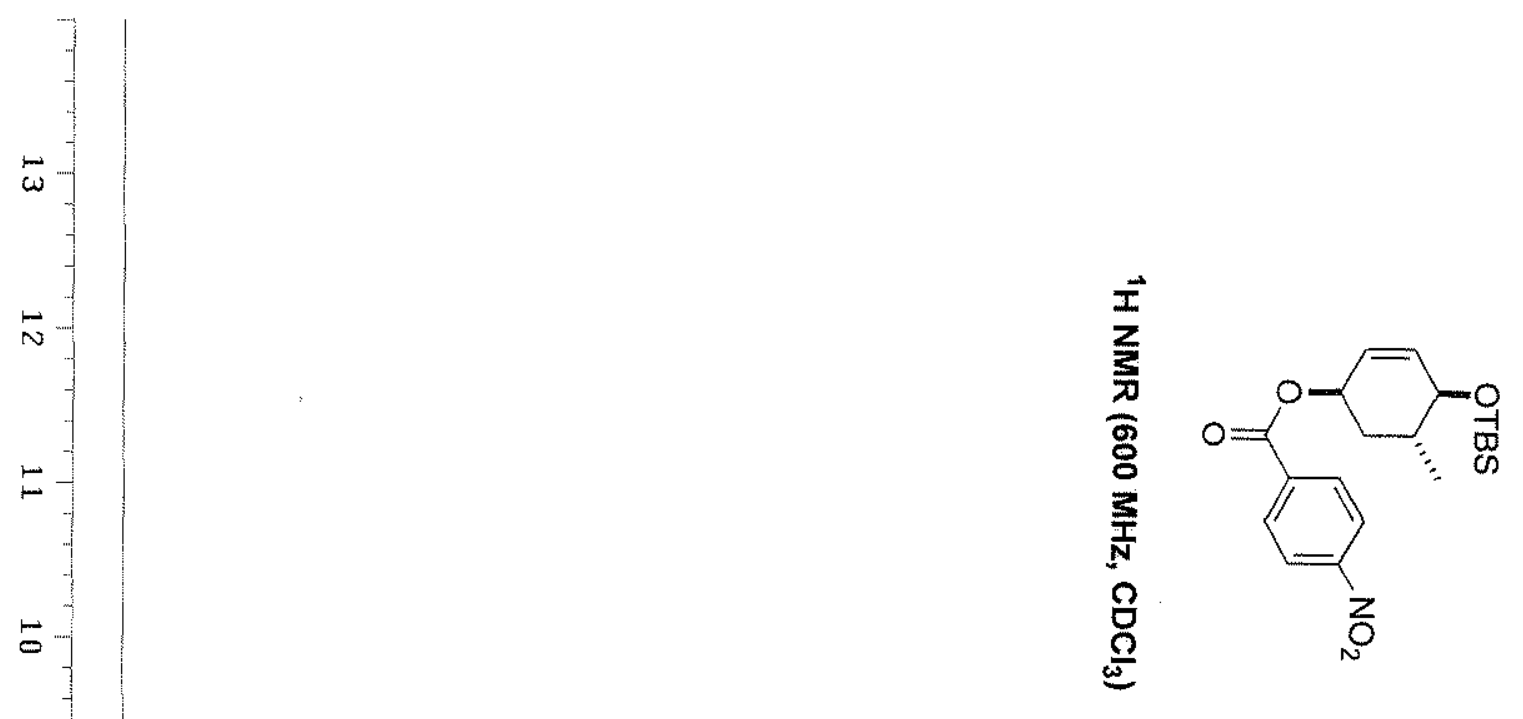

o 


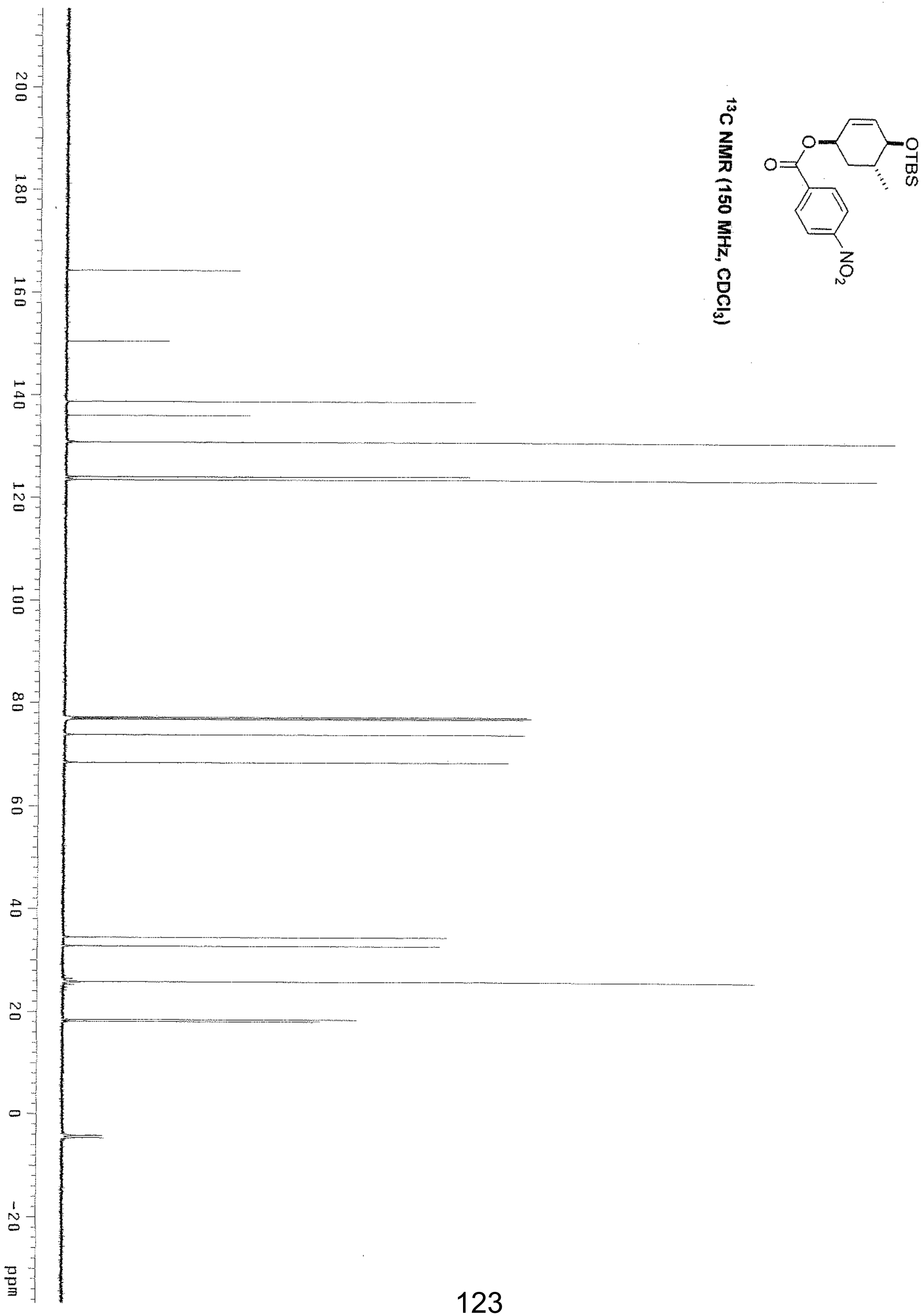




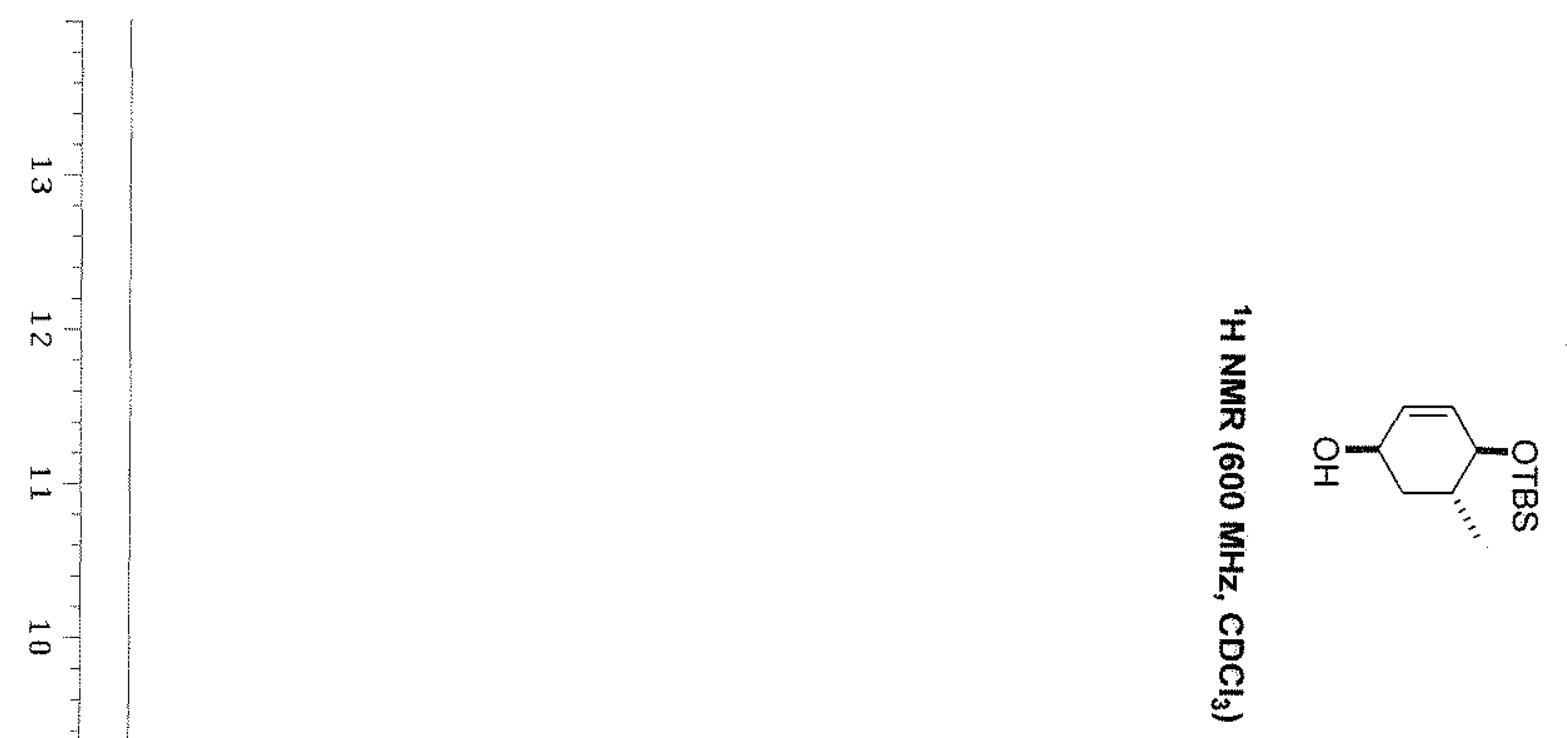

$\circ$

$\infty$ 


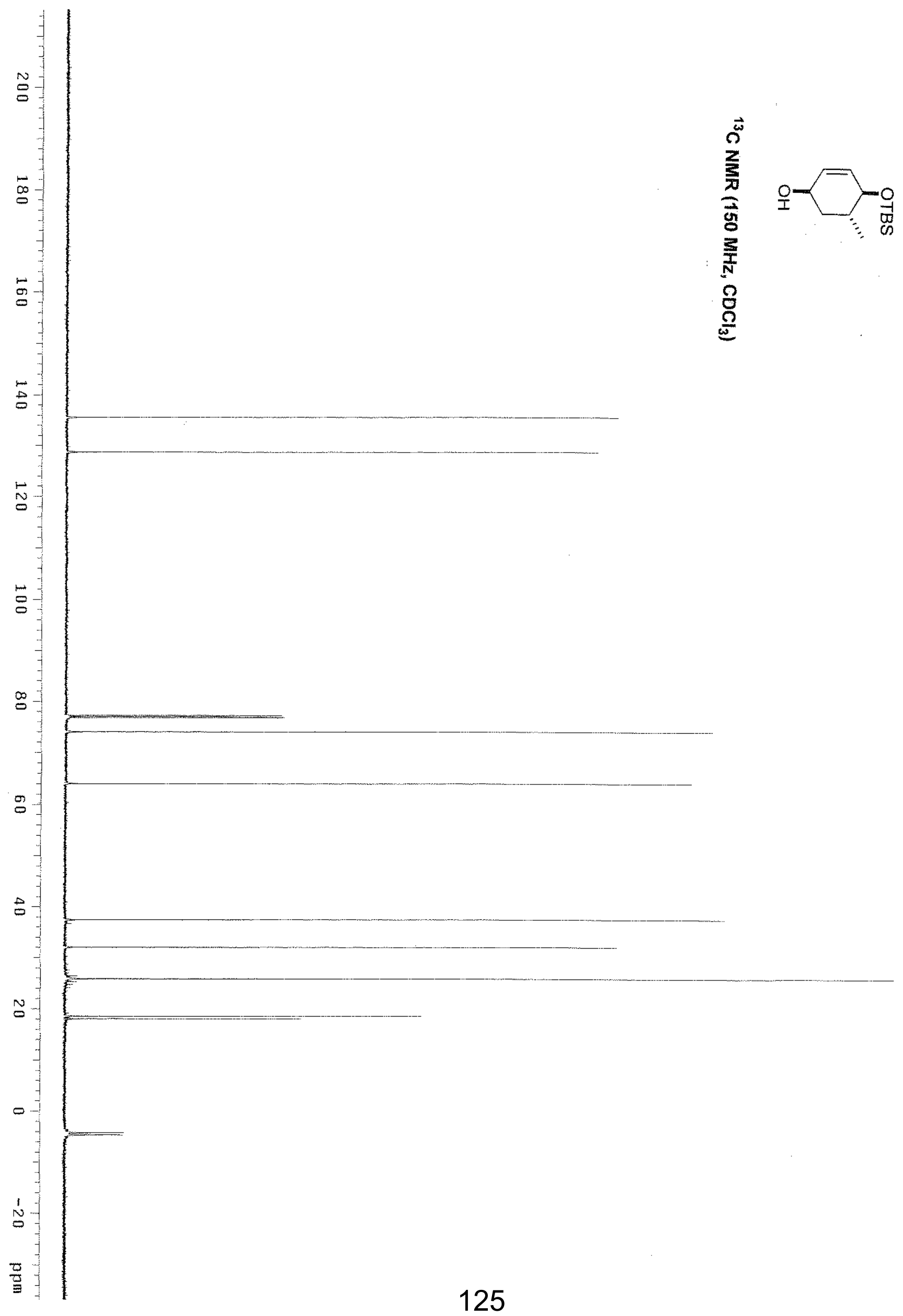



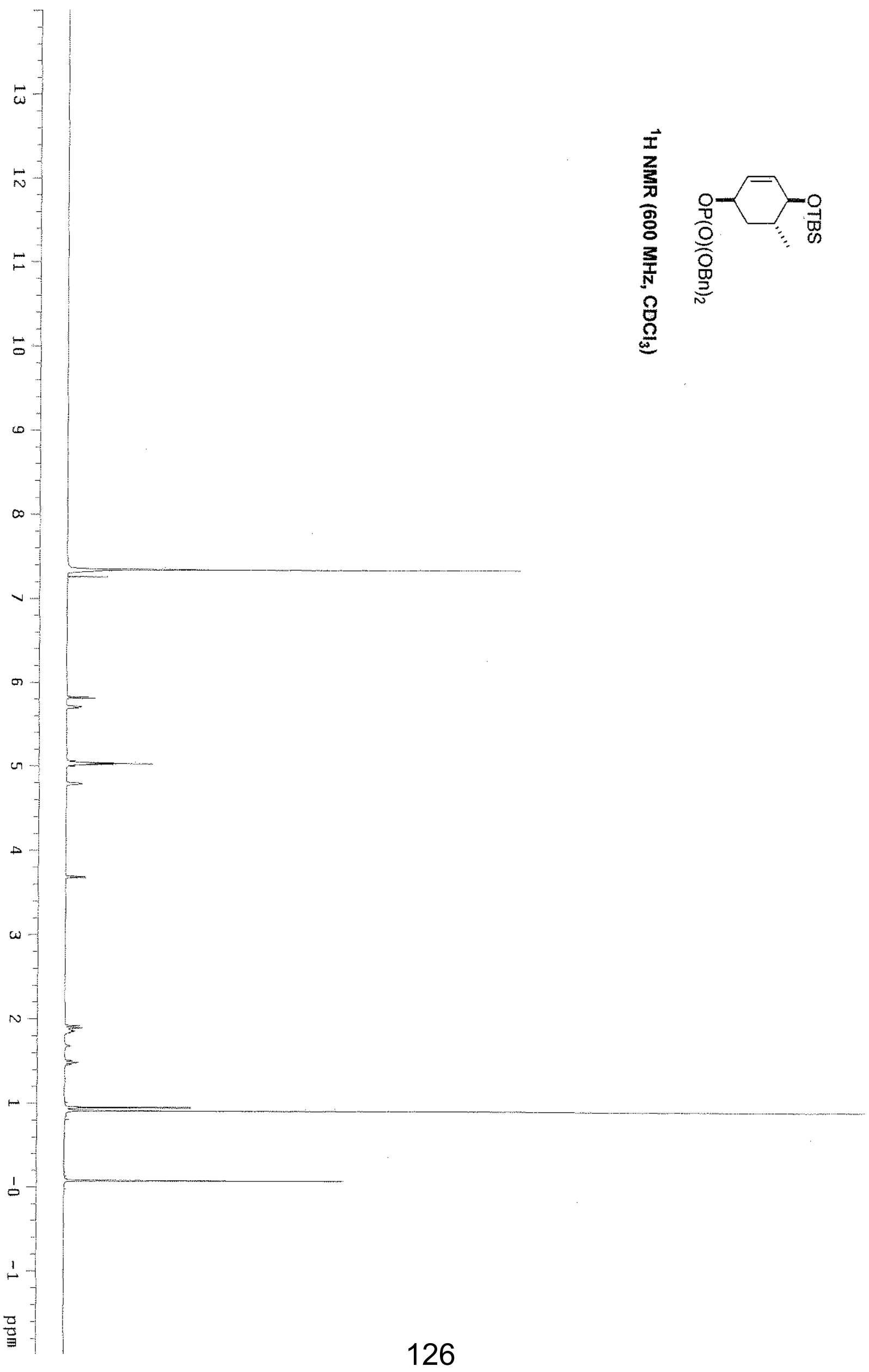


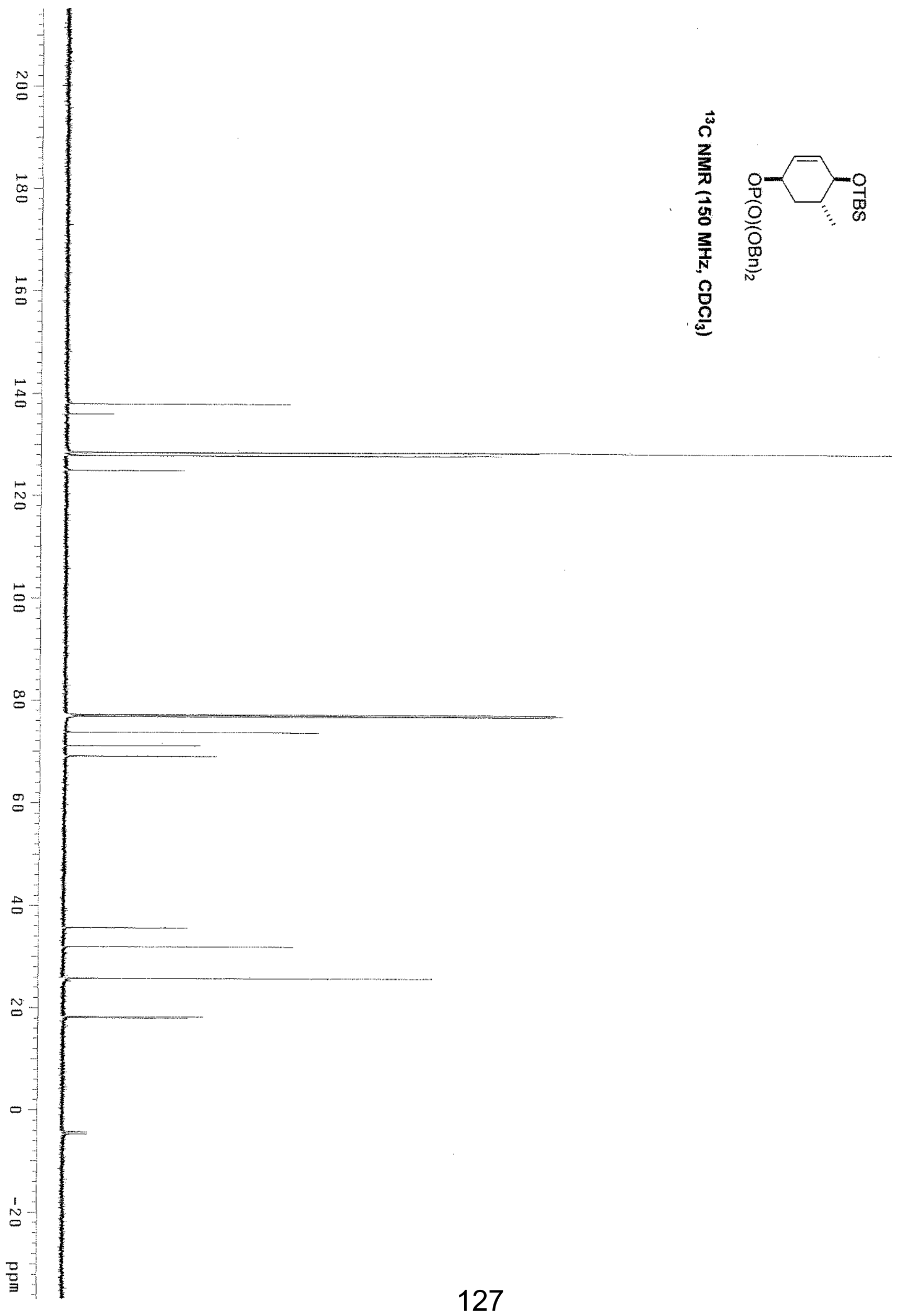




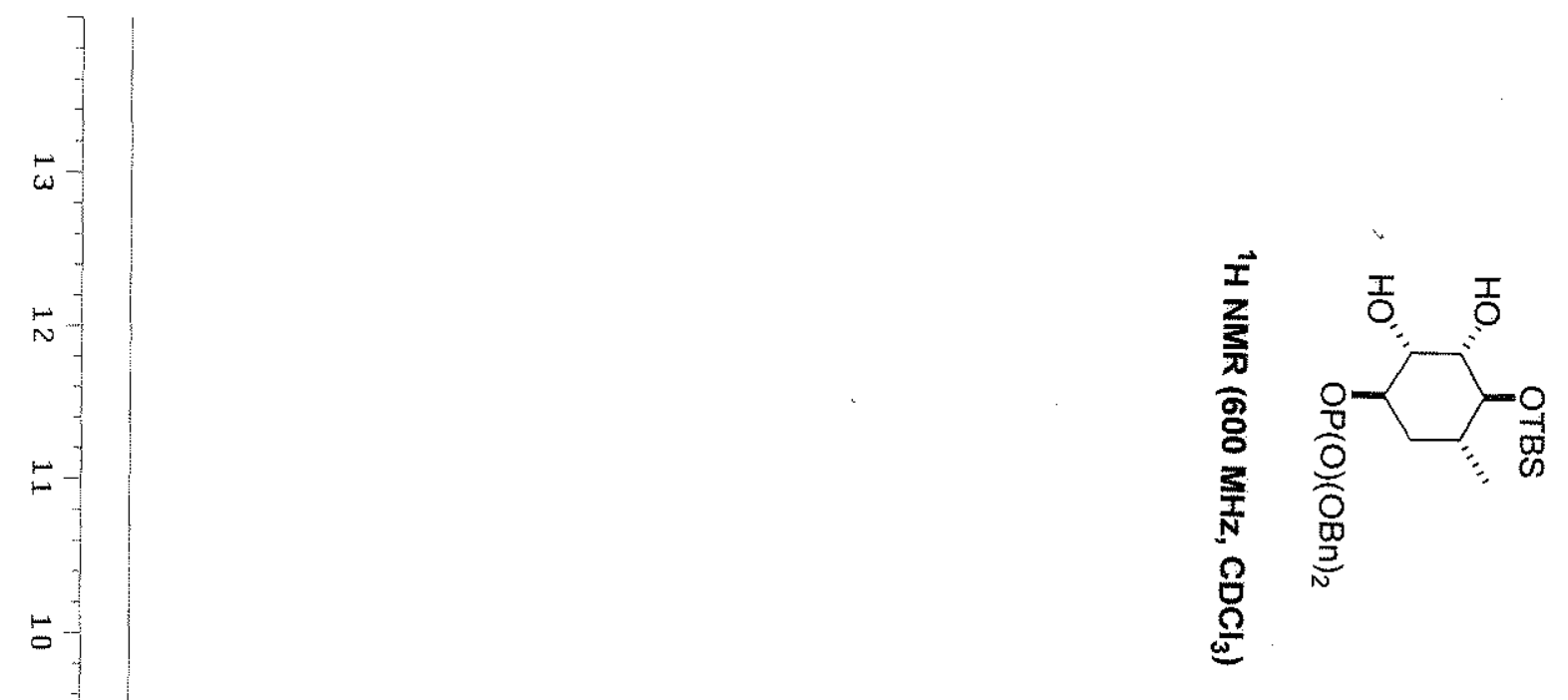

$\infty$
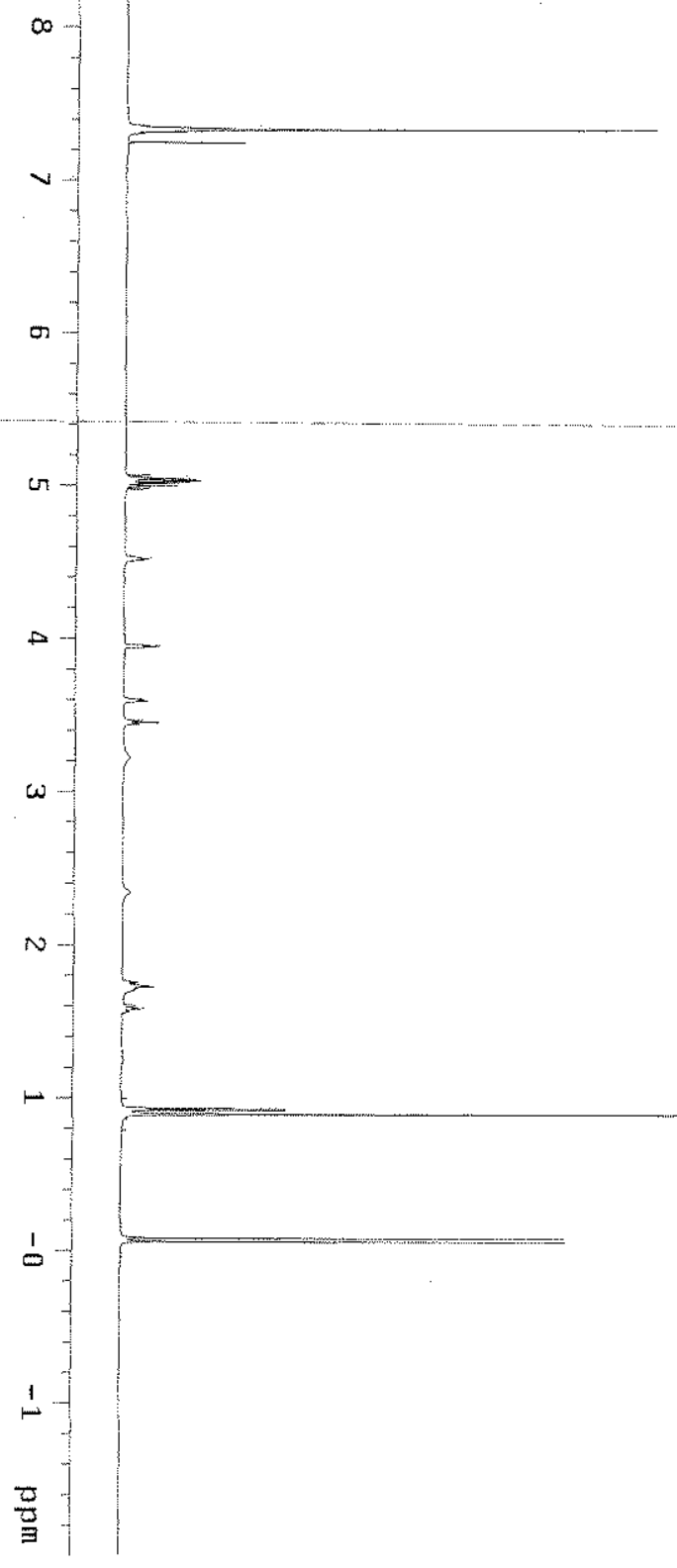


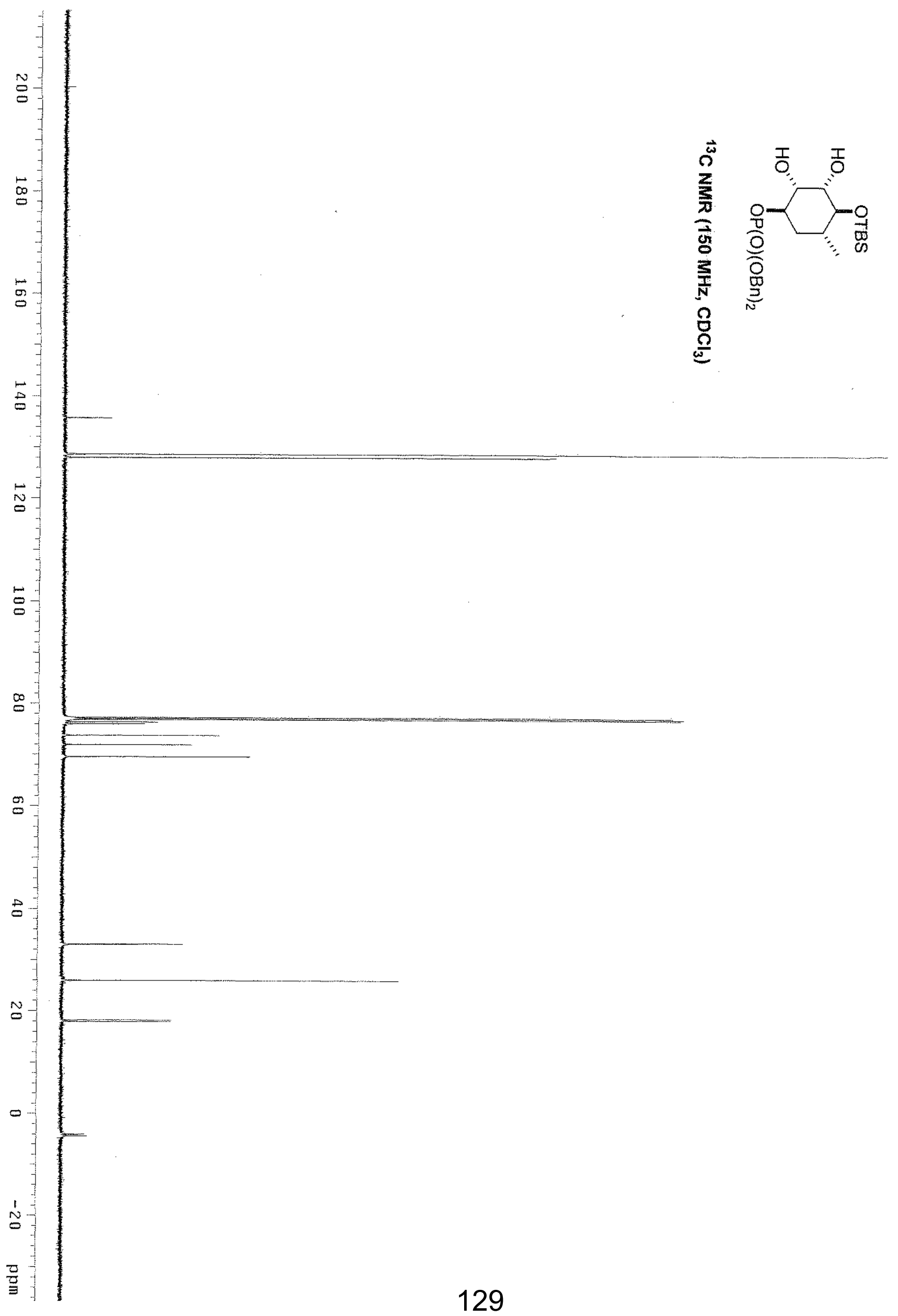




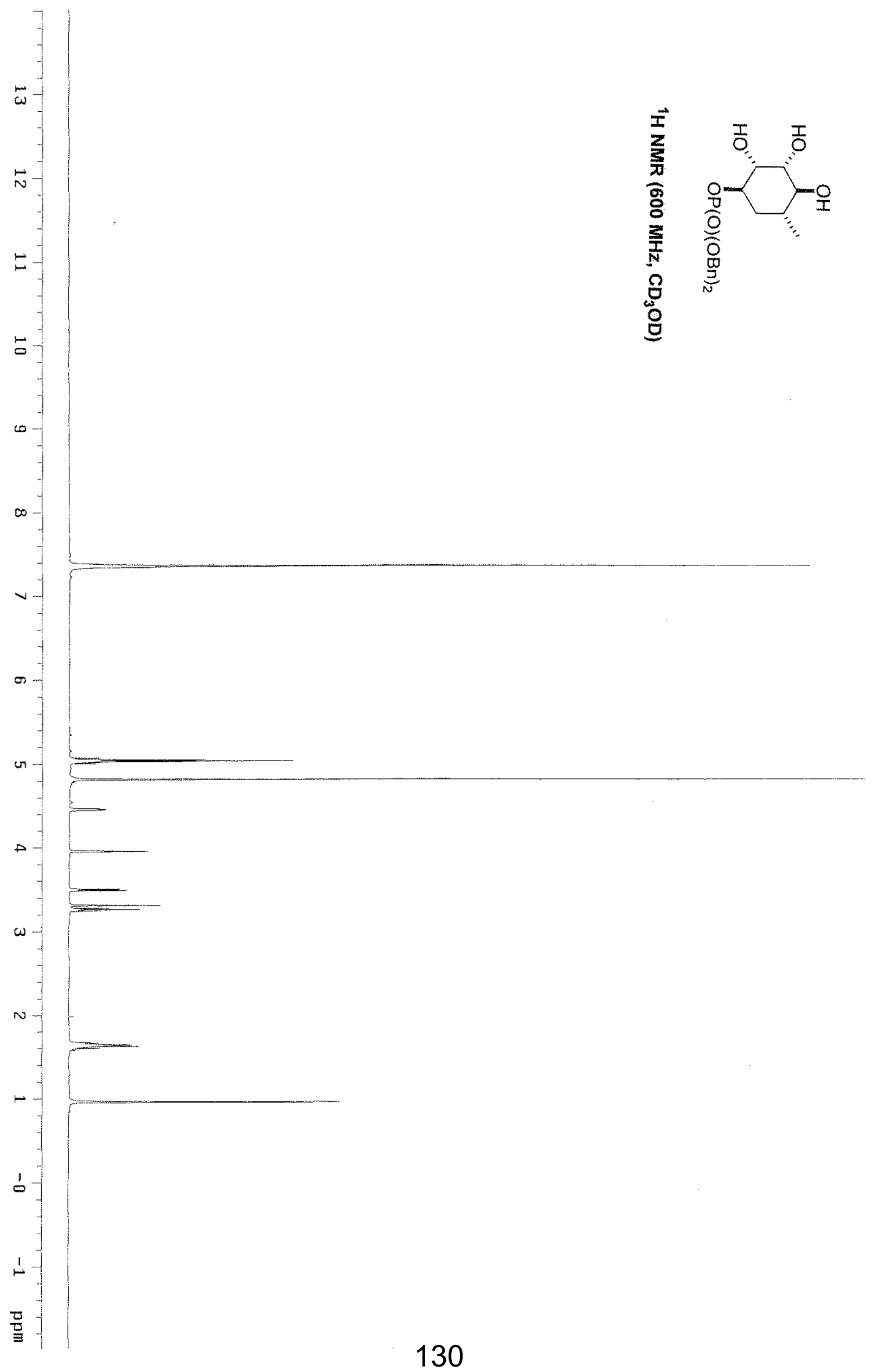




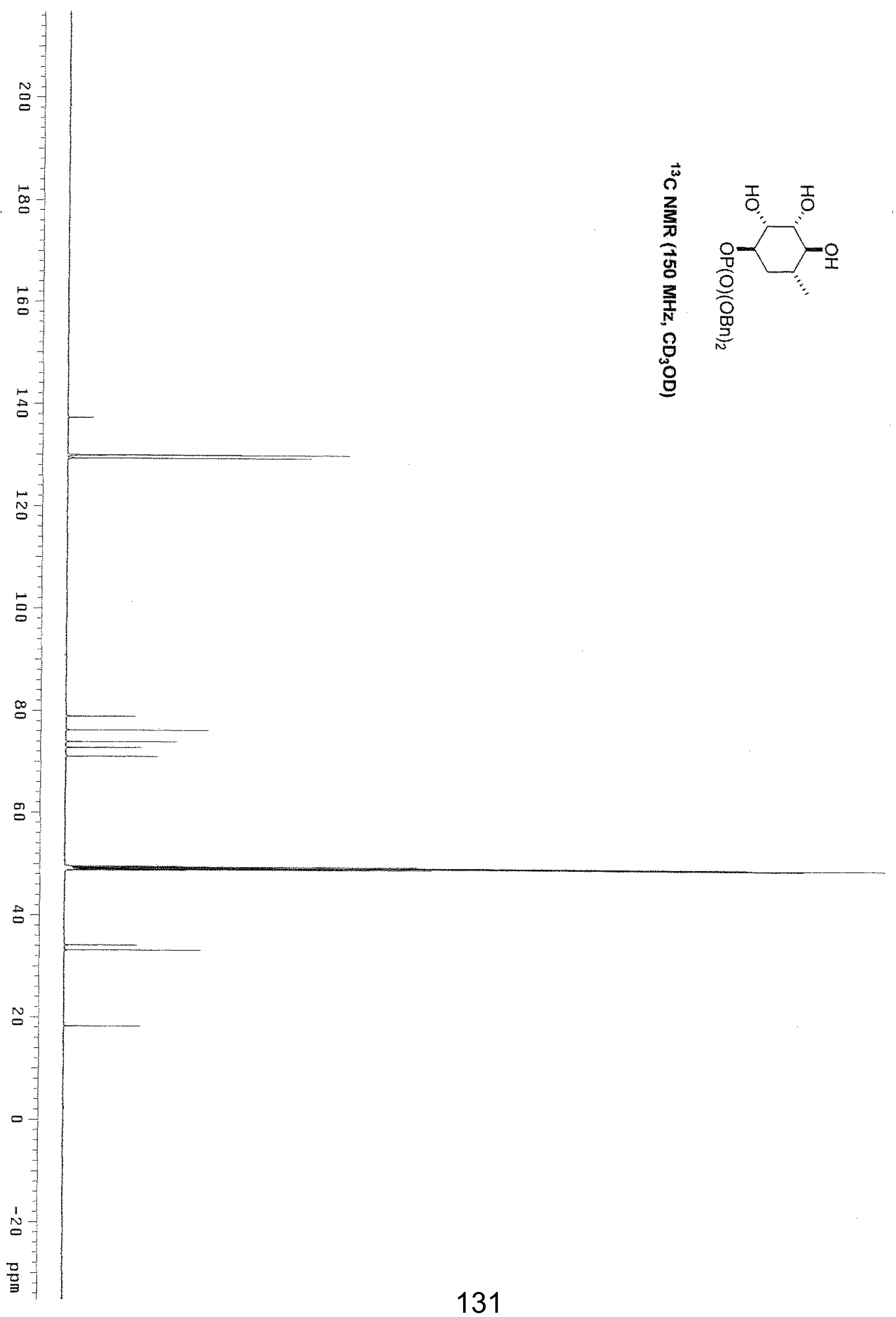




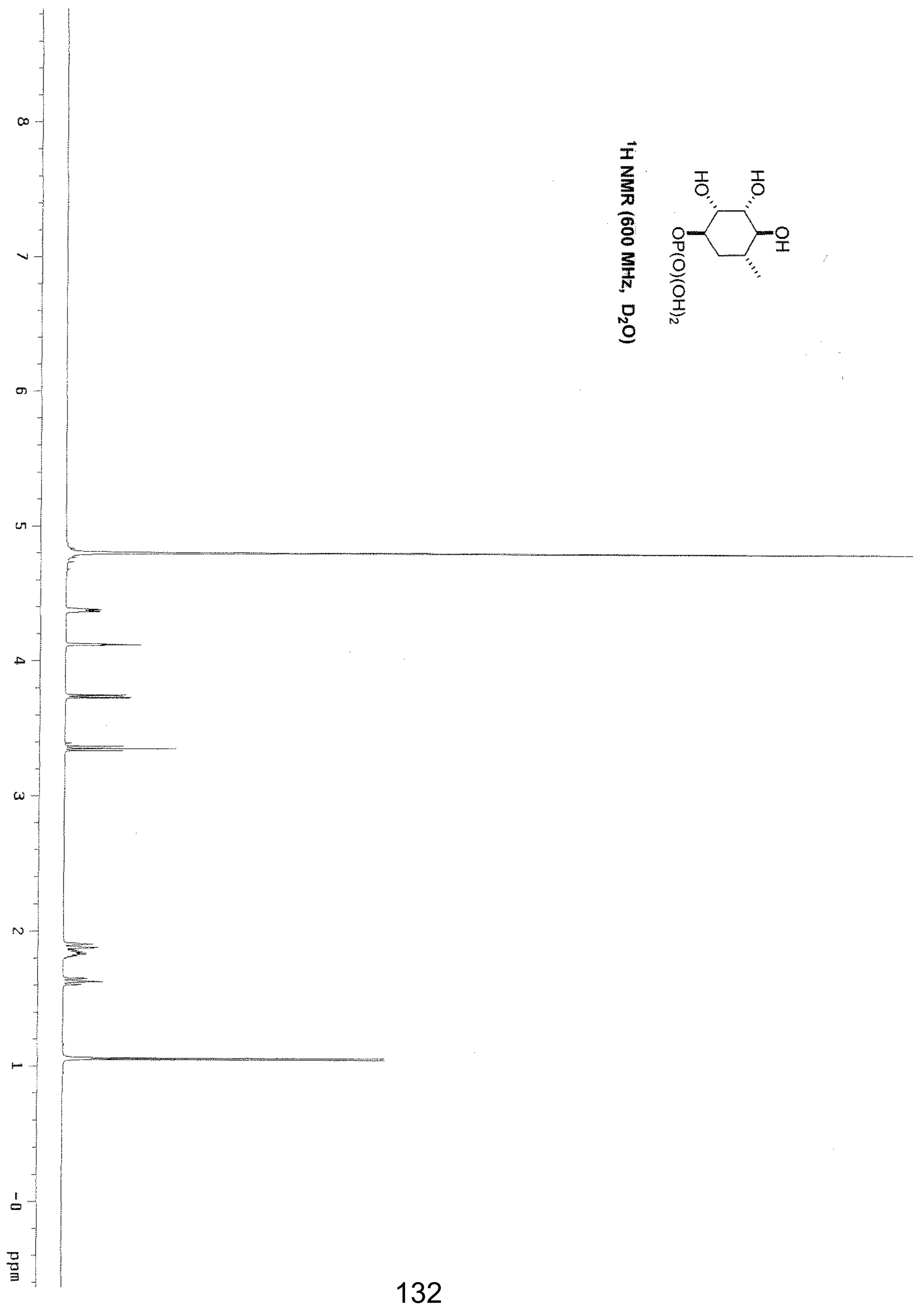




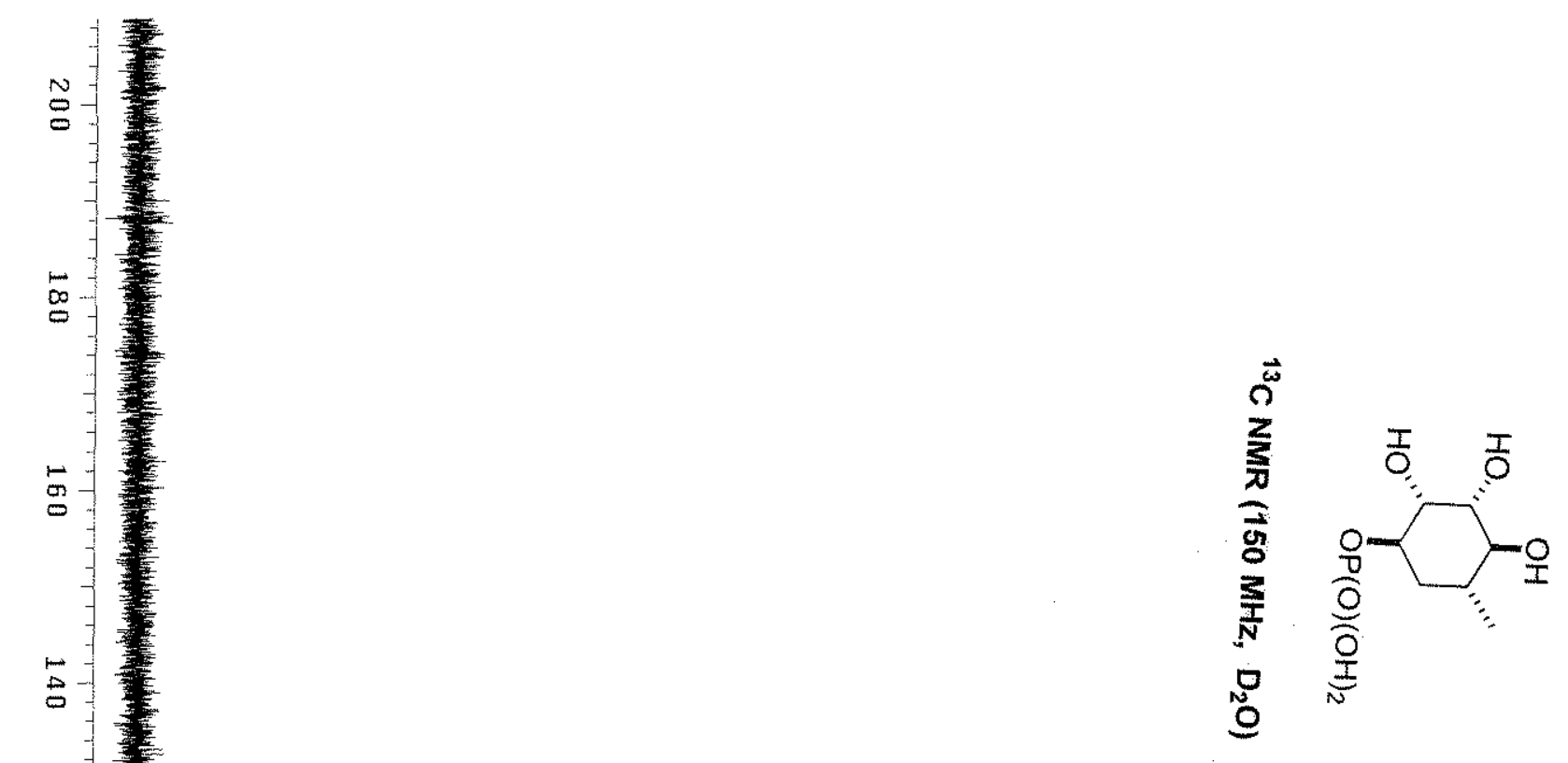

N

5

,

$\stackrel{\infty}{\circ}$

o

-

:

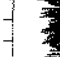

$\stackrel{v}{\circ}$

0

7

0.

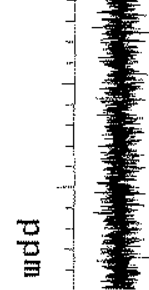

133 


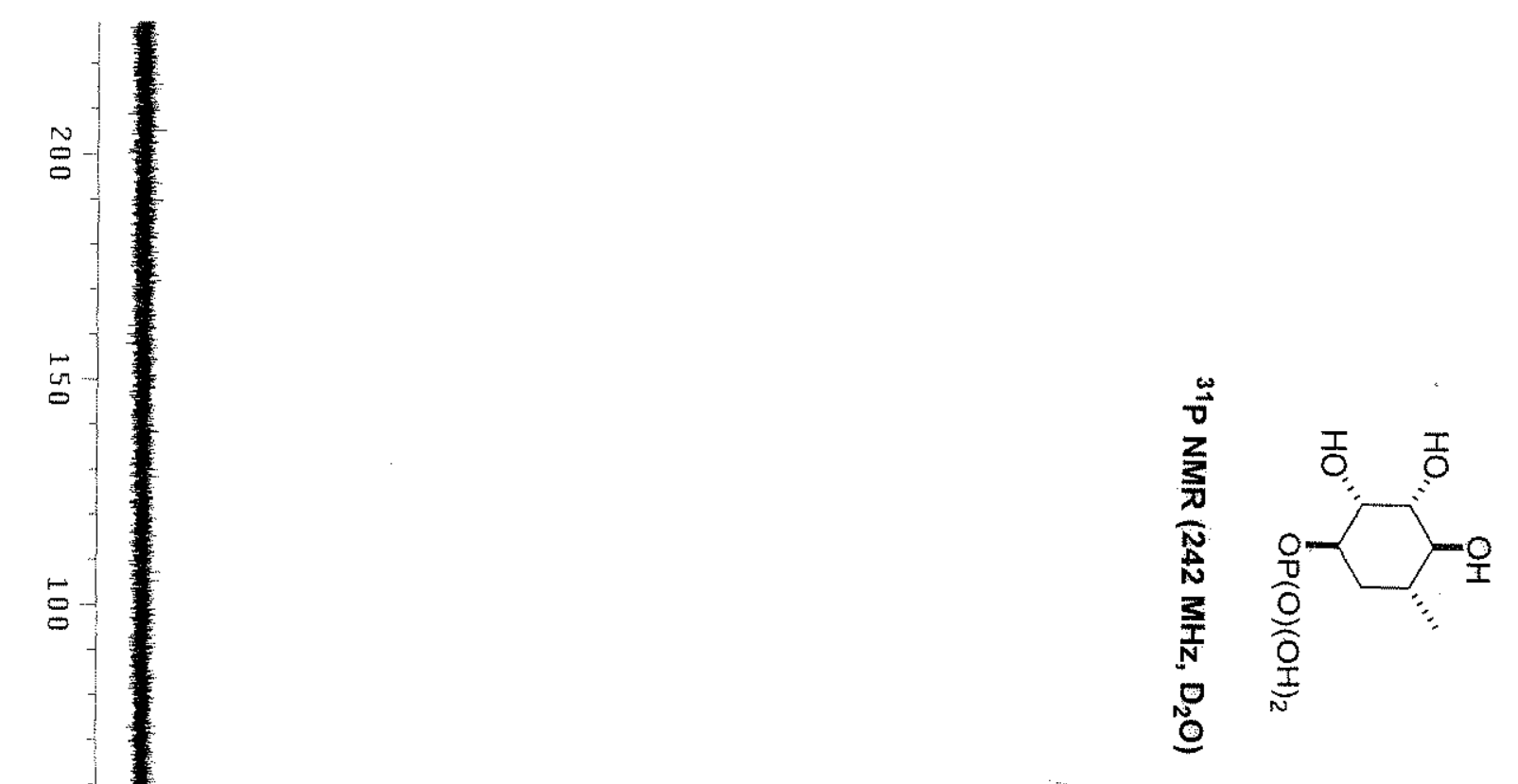

i

$\stackrel{1}{\circ}$

$\stackrel{n}{a}$

0

$\stackrel{5}{9}$

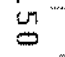

1
0
0

章- 


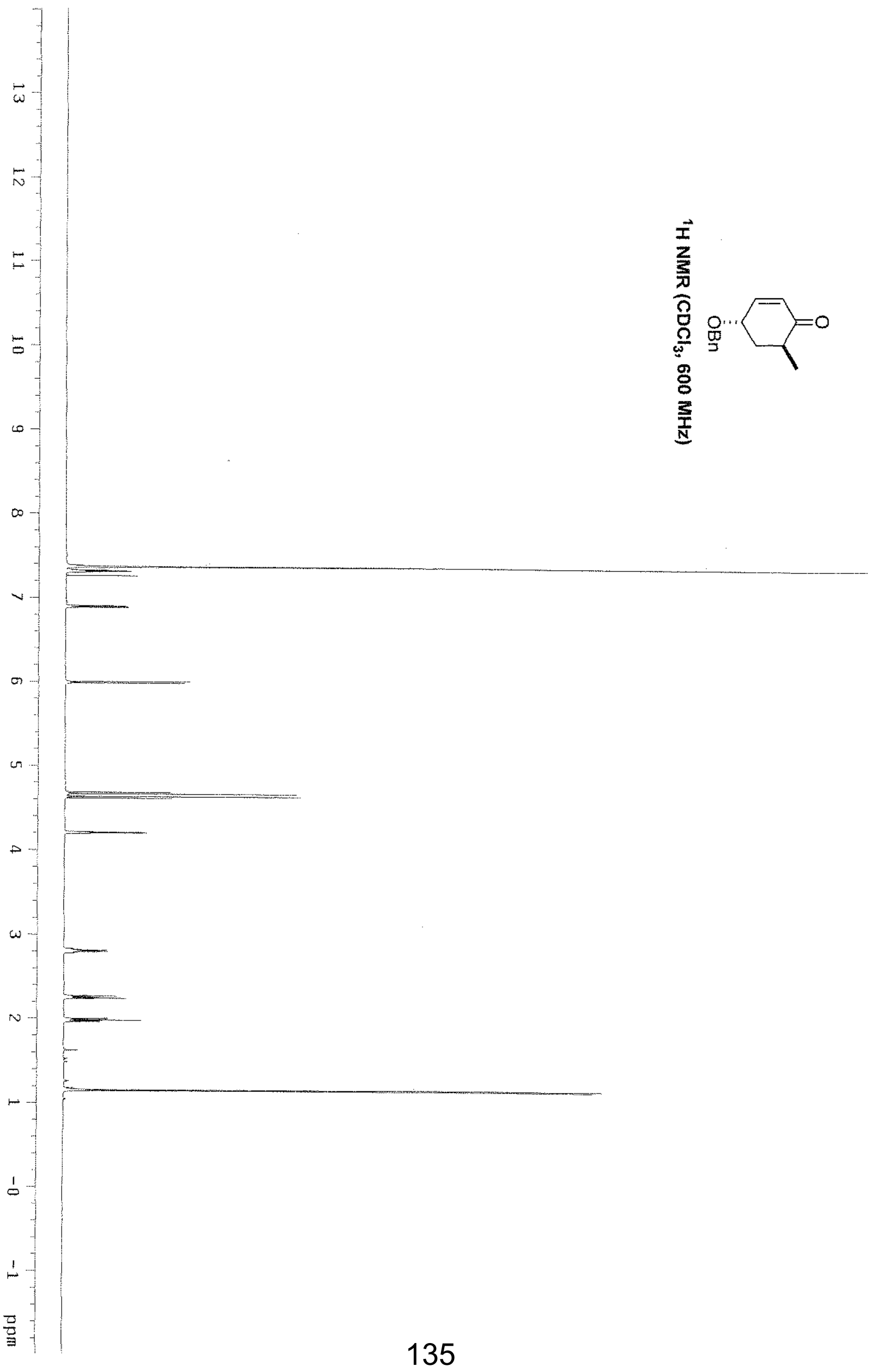




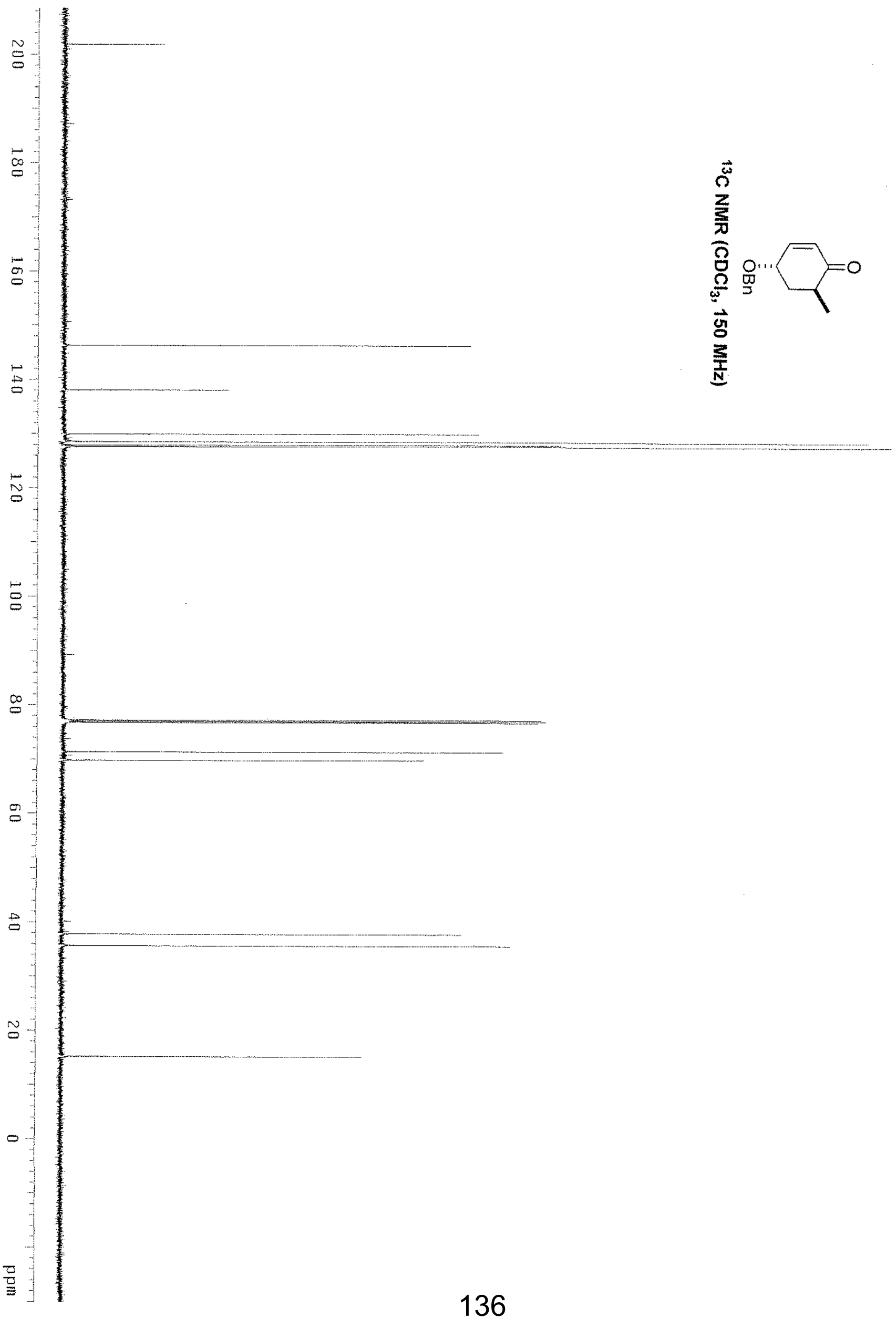




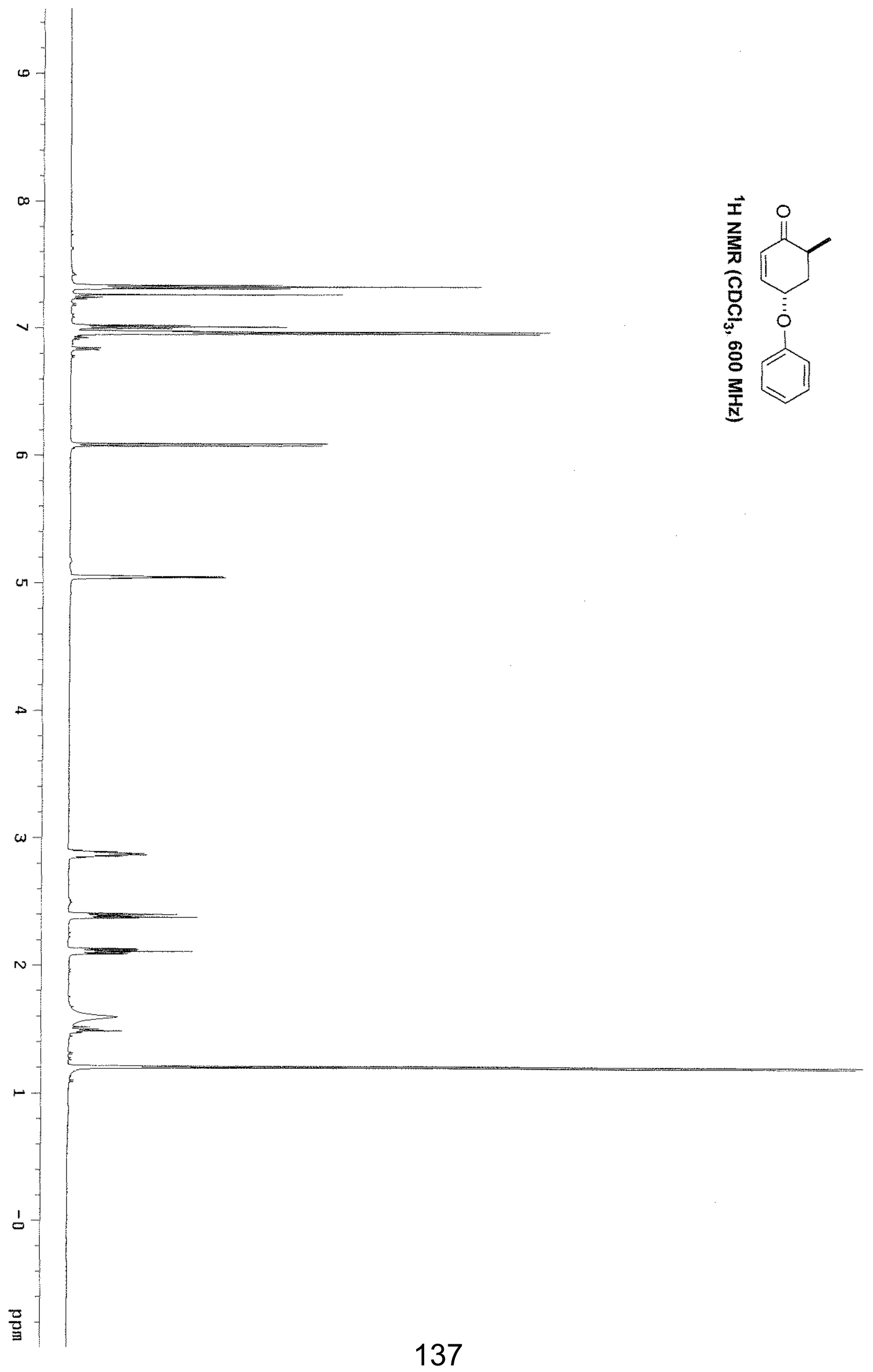




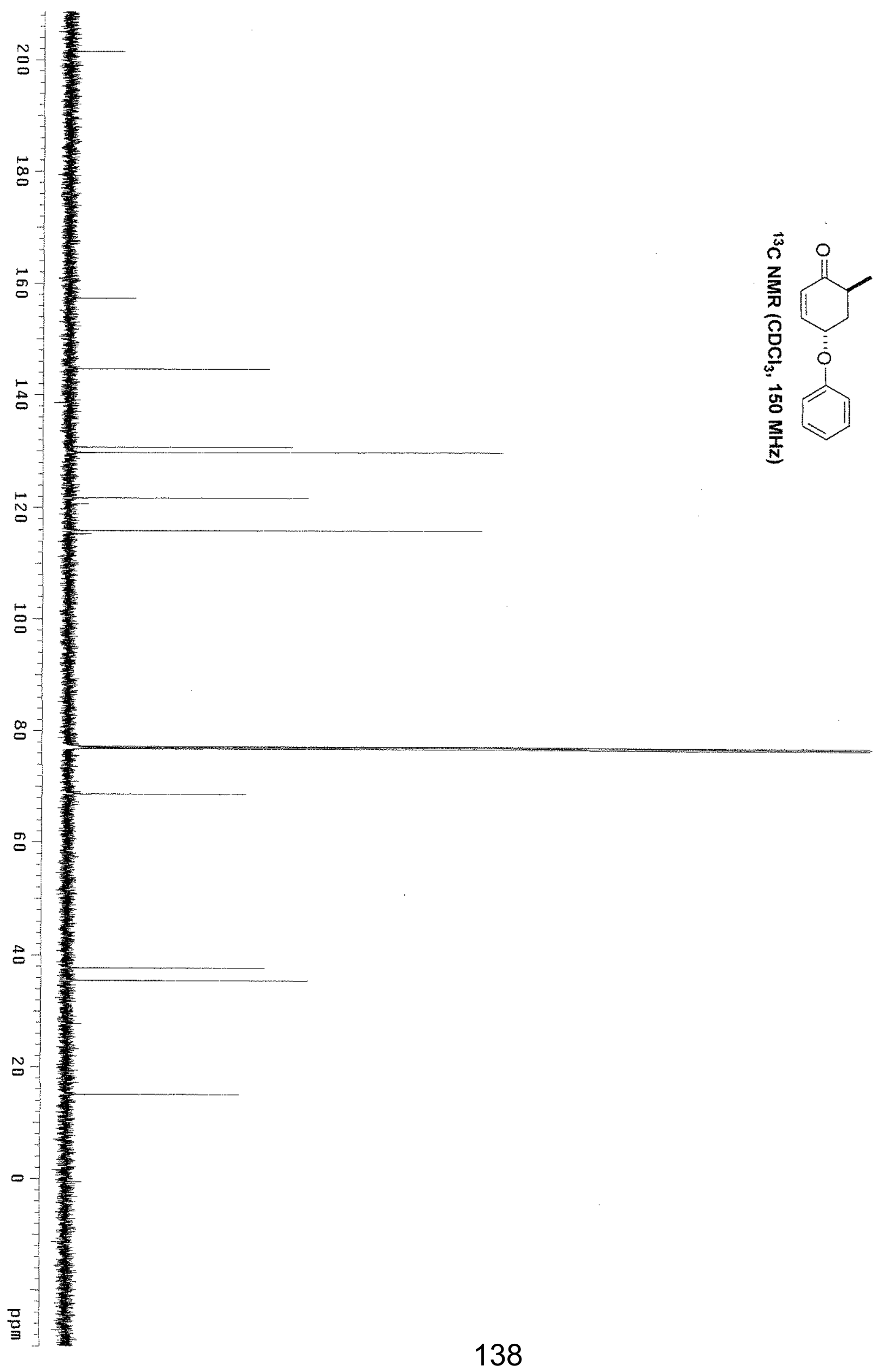




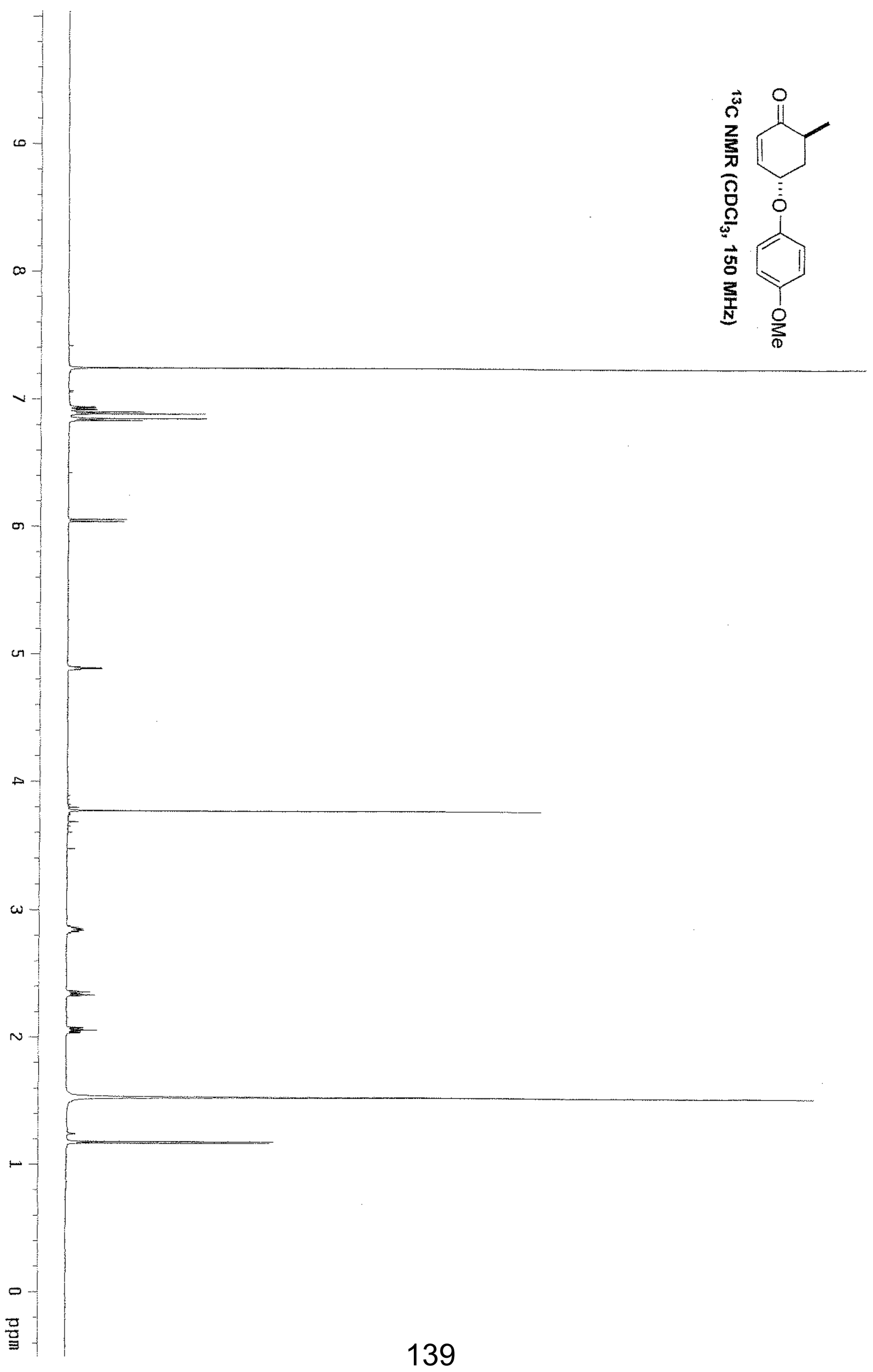




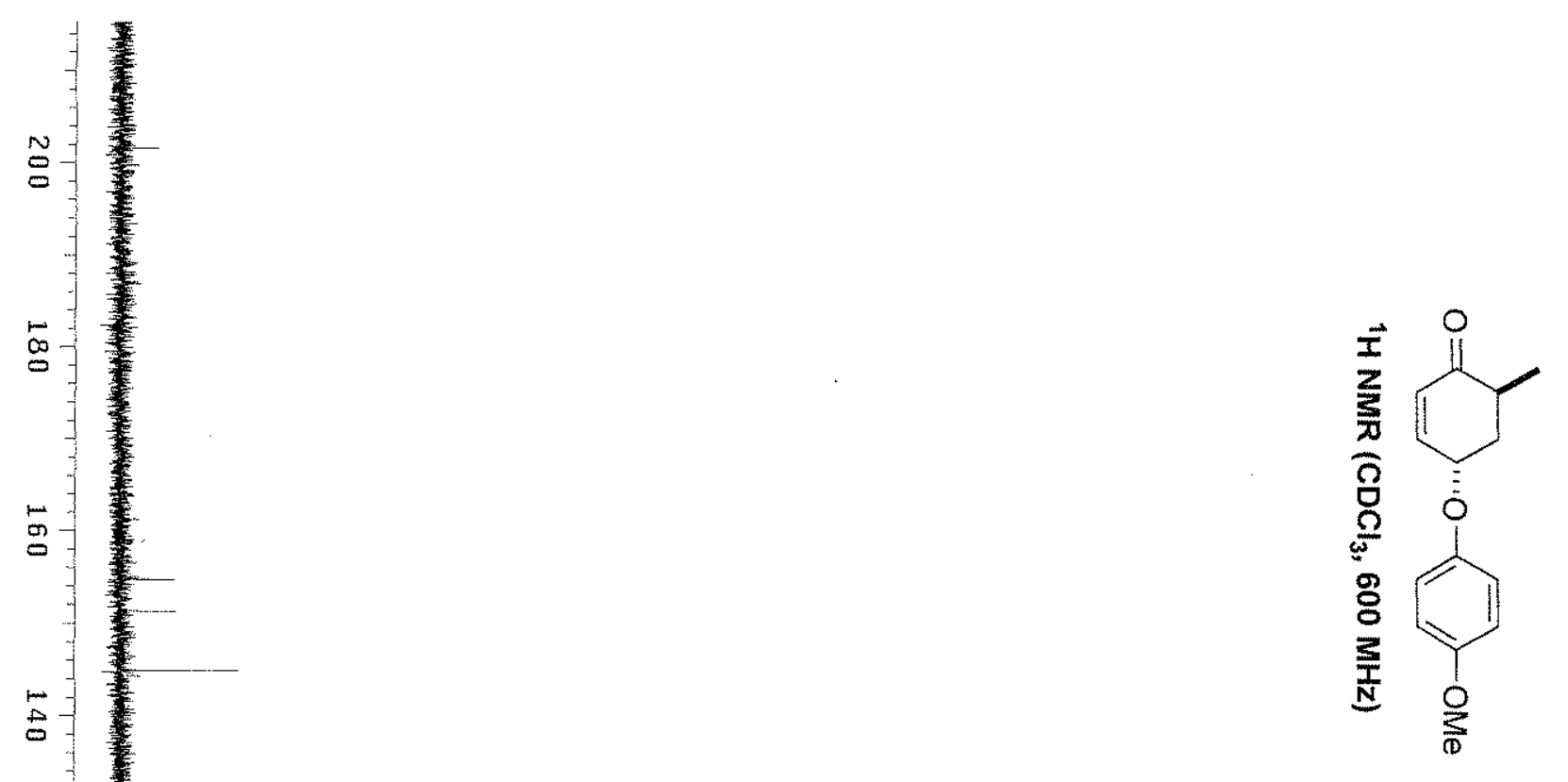

N

0

$\infty-1$

9

a

N.

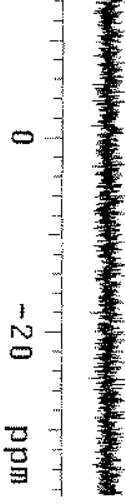




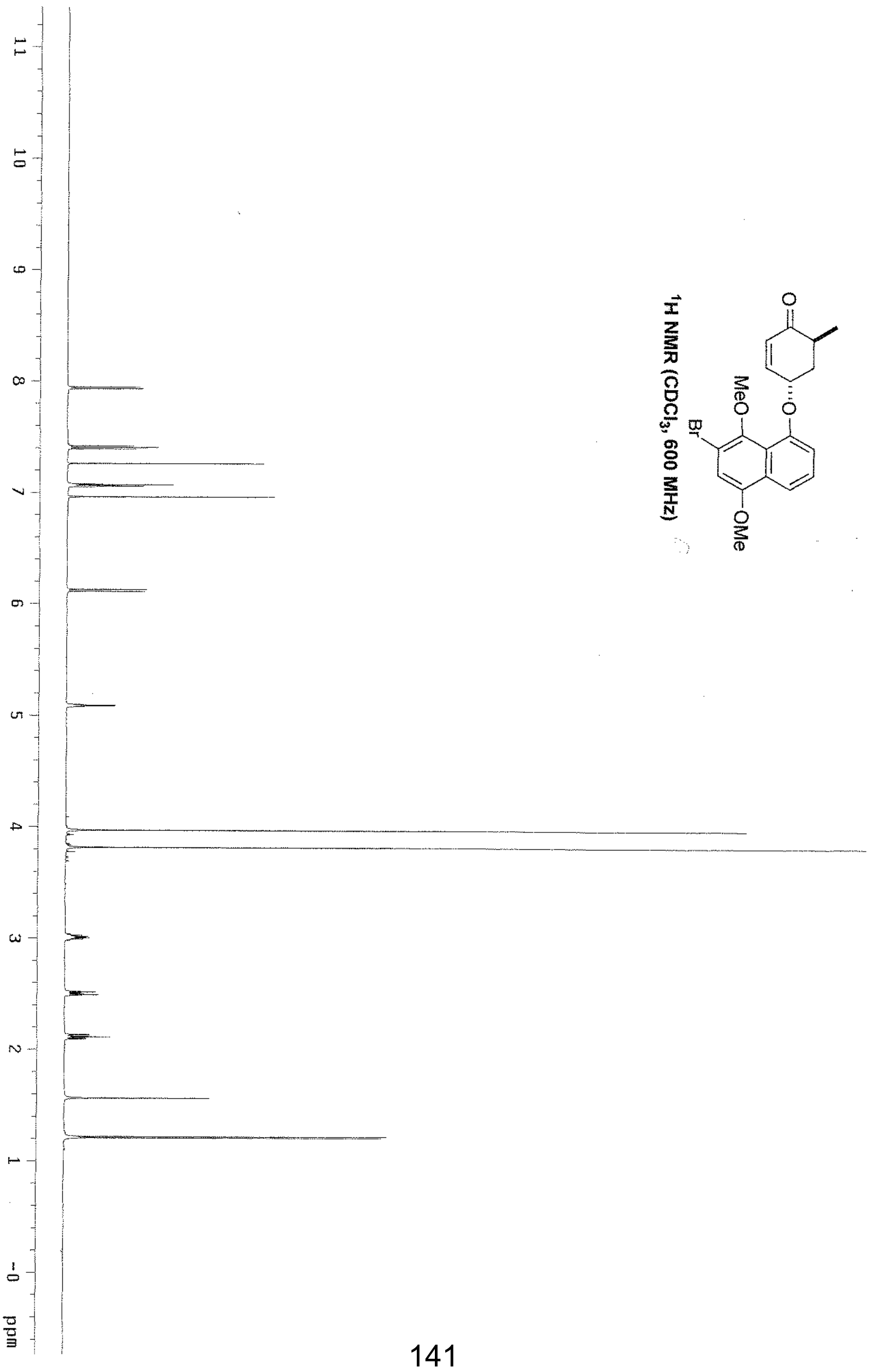




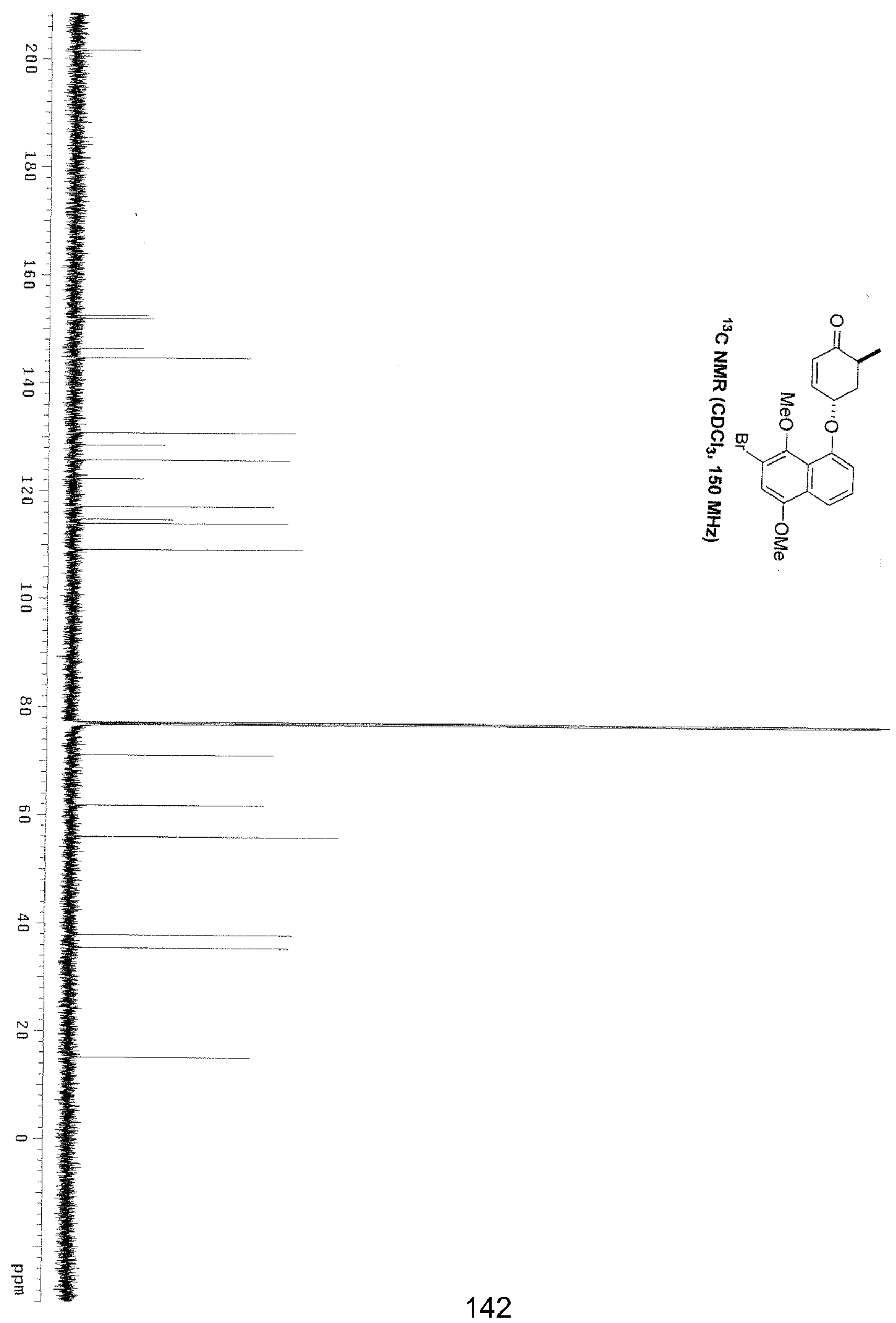




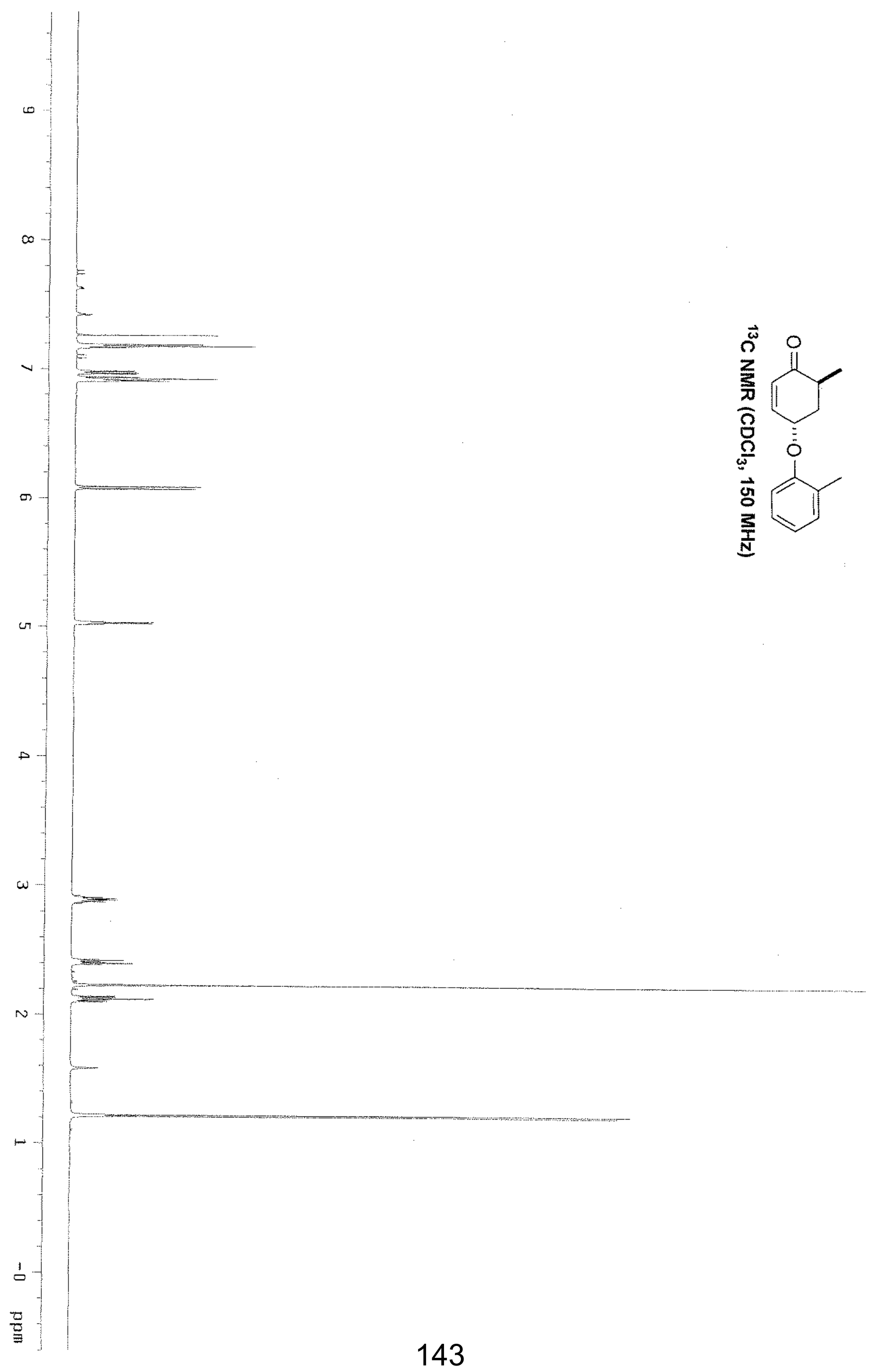




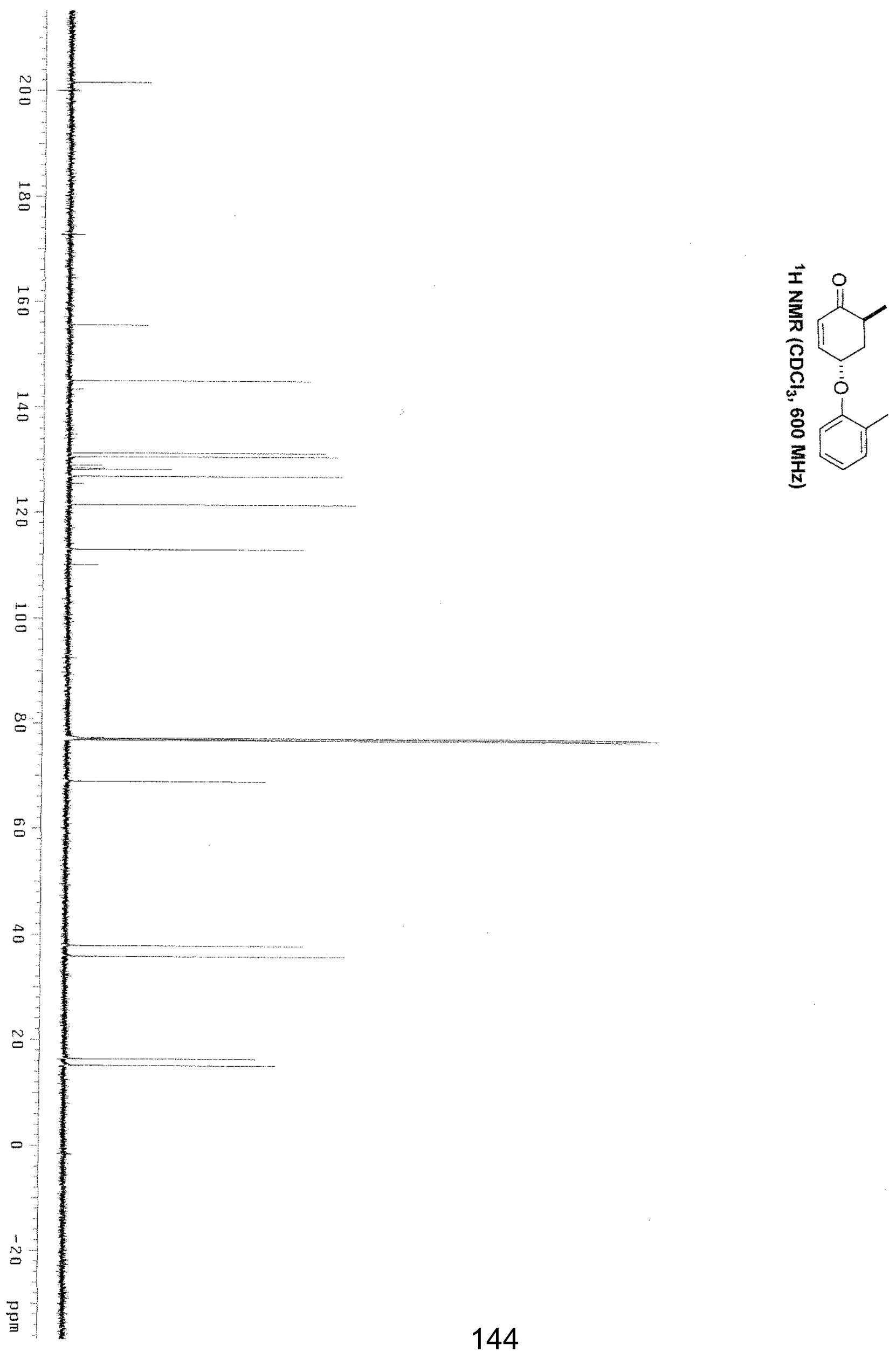




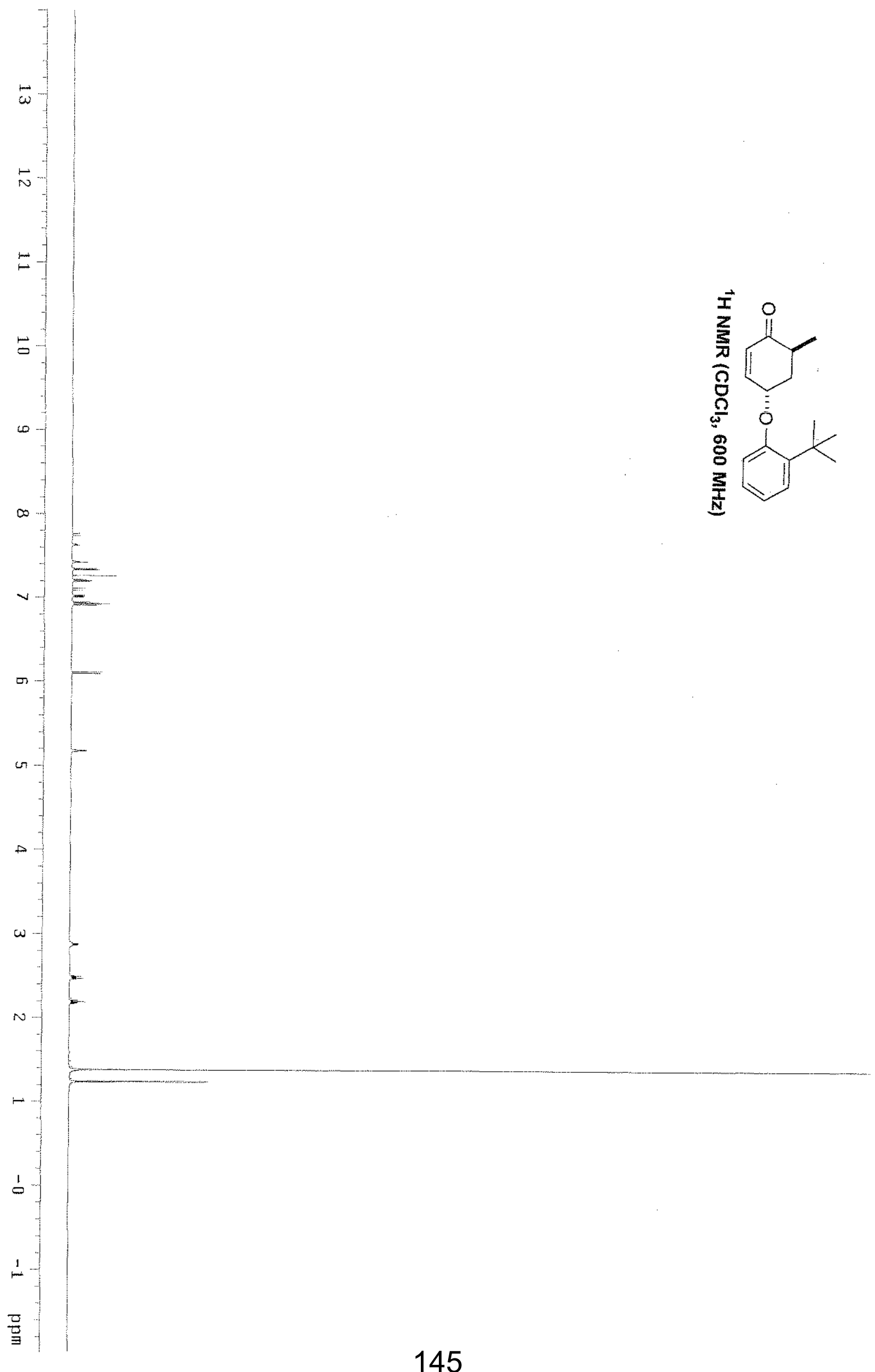




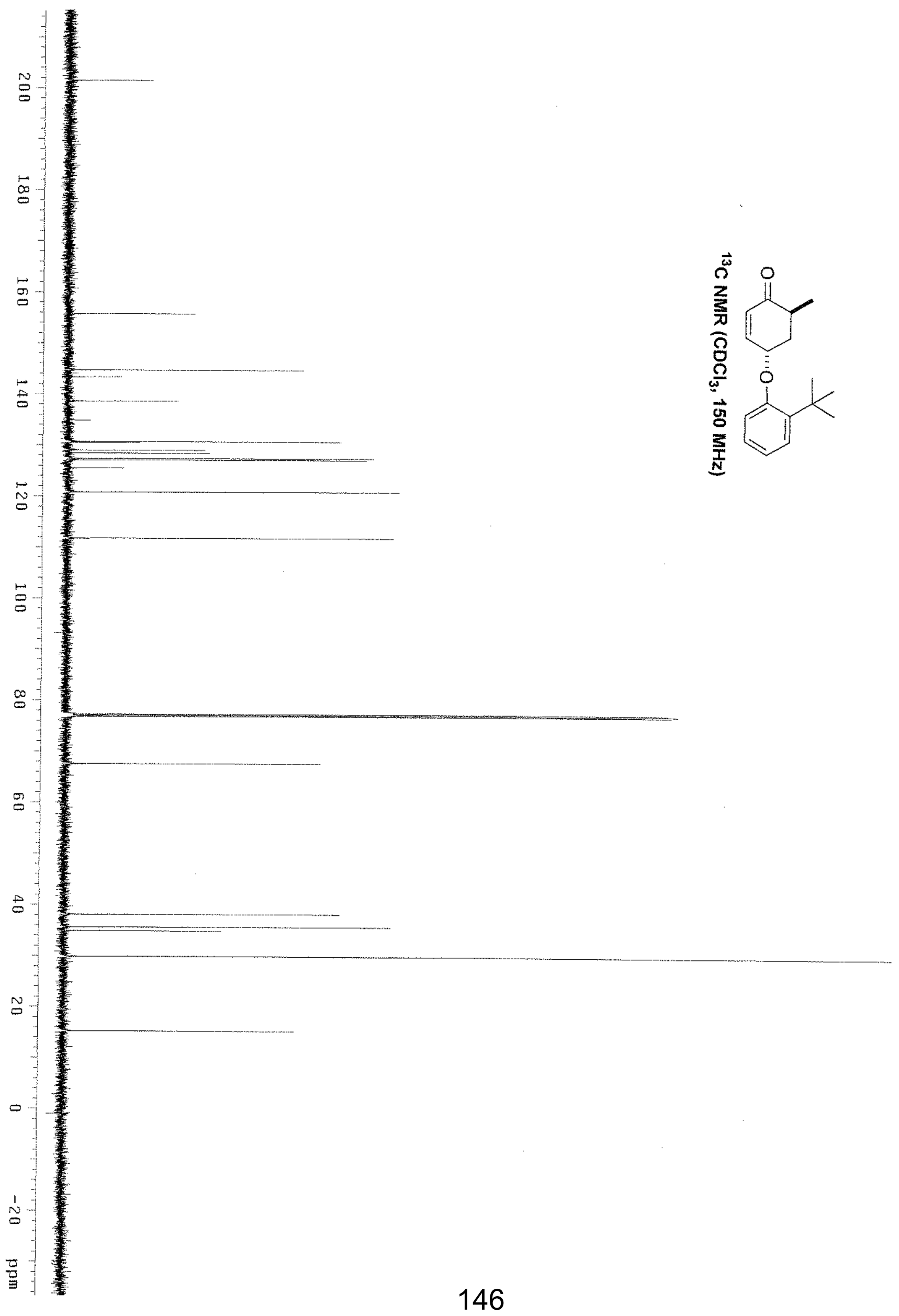




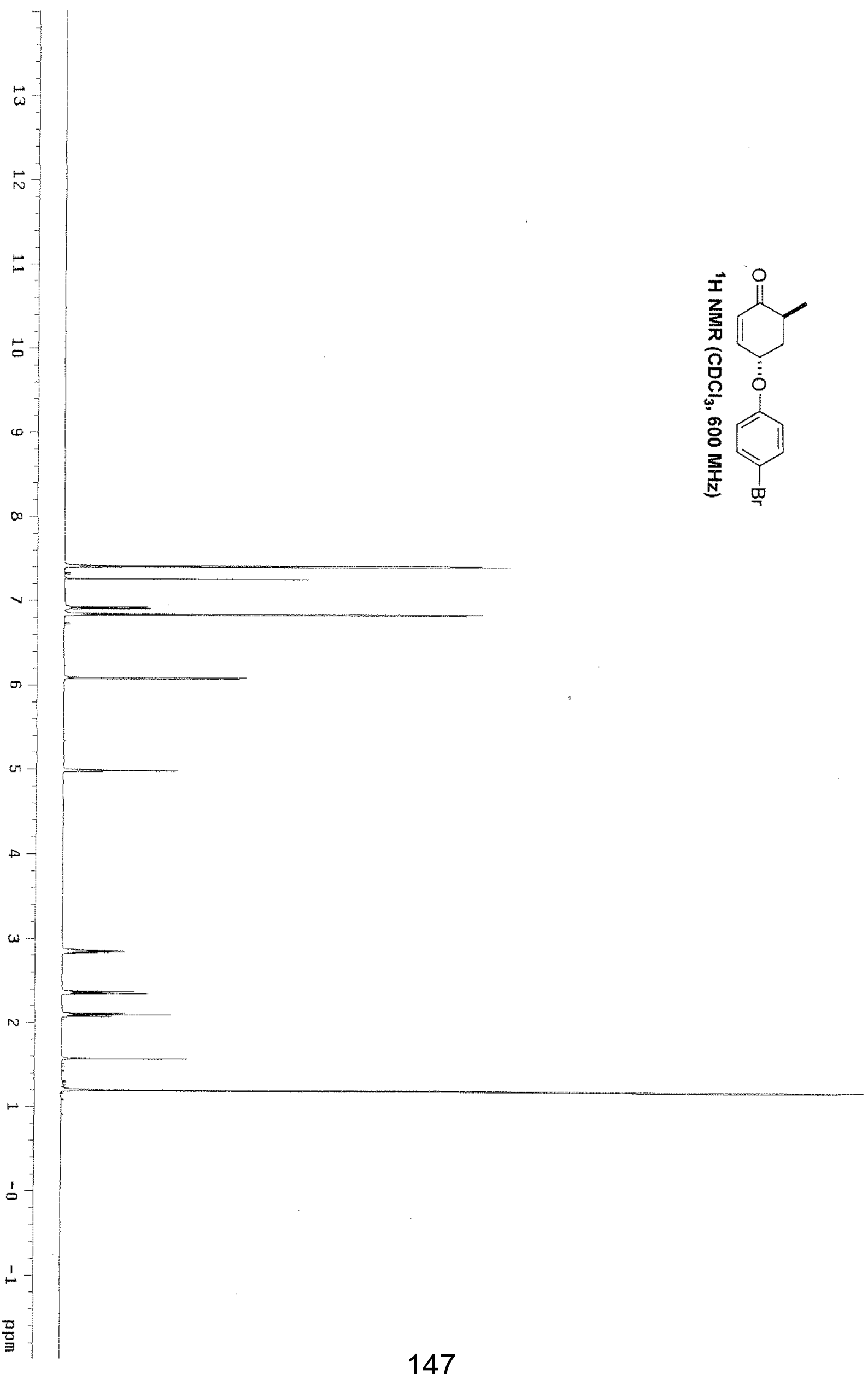




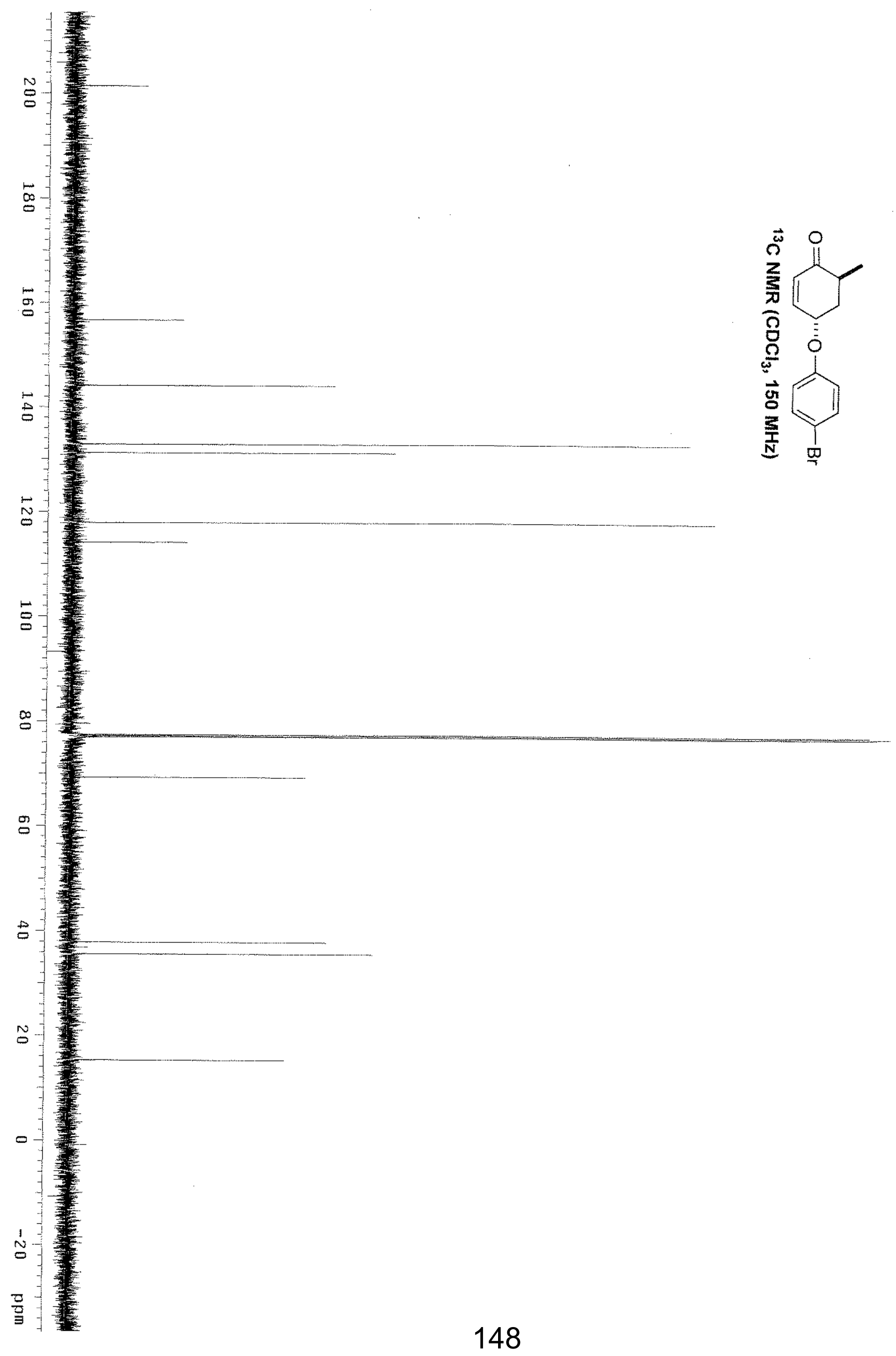




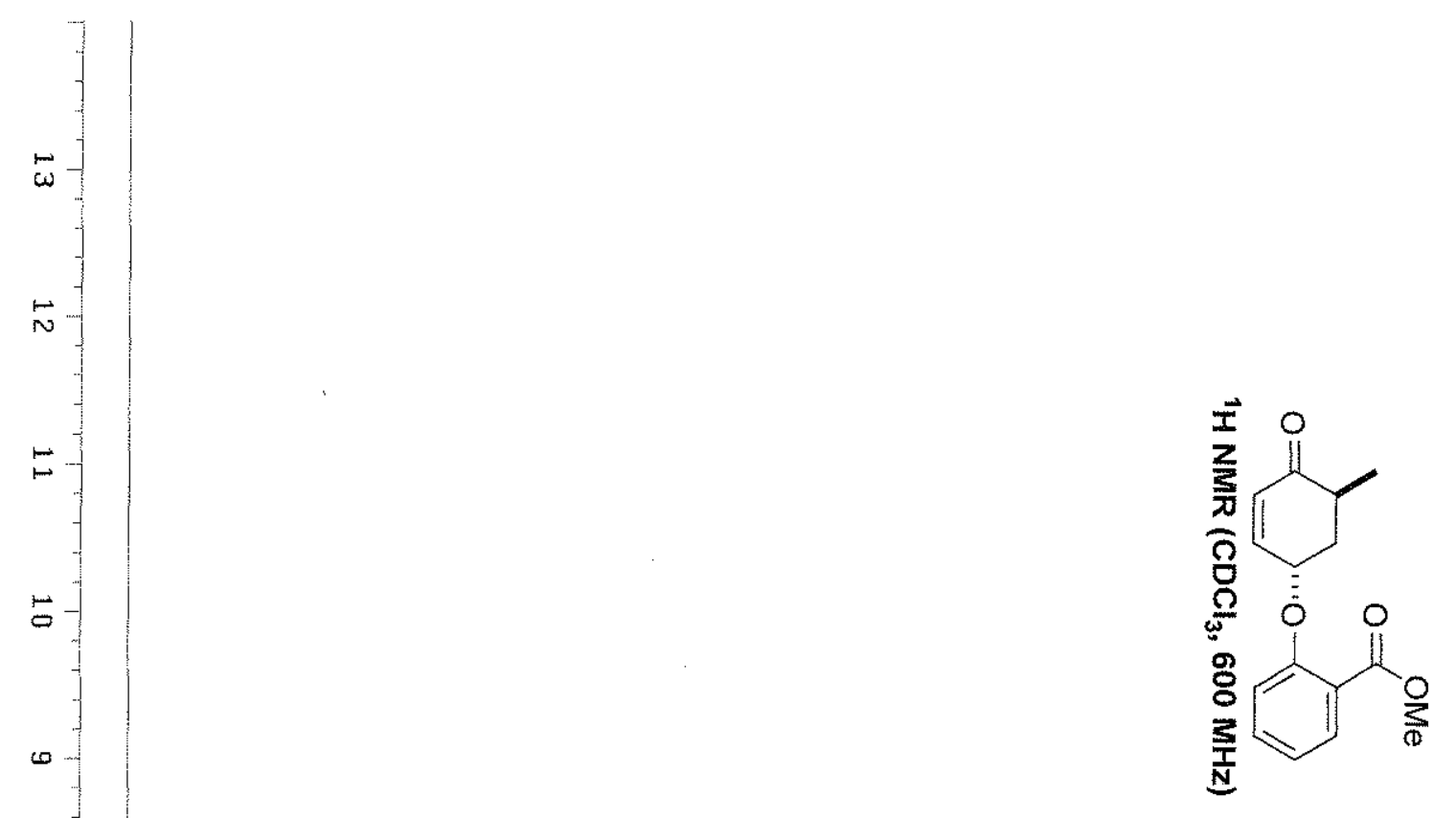

$\infty$
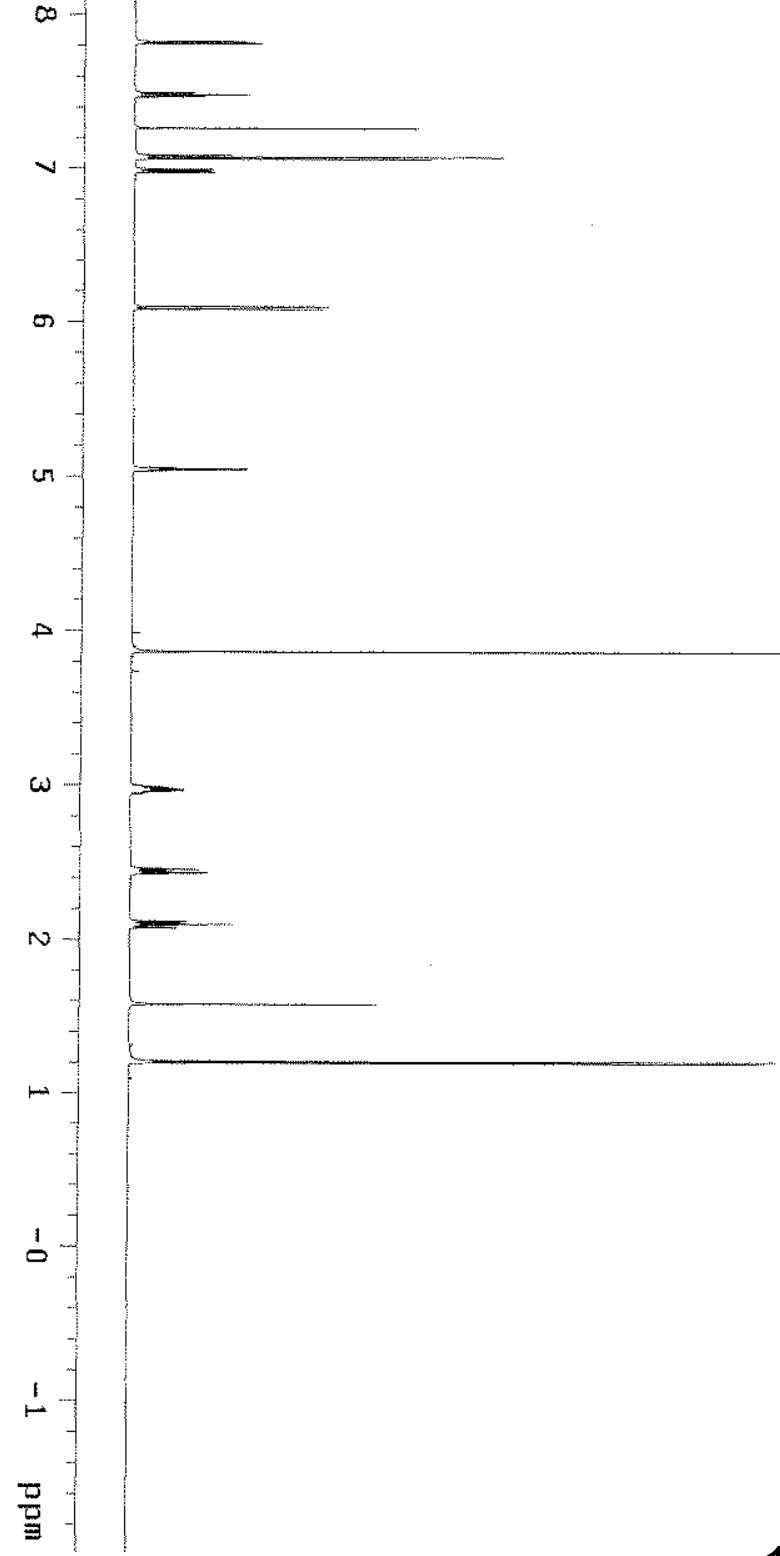


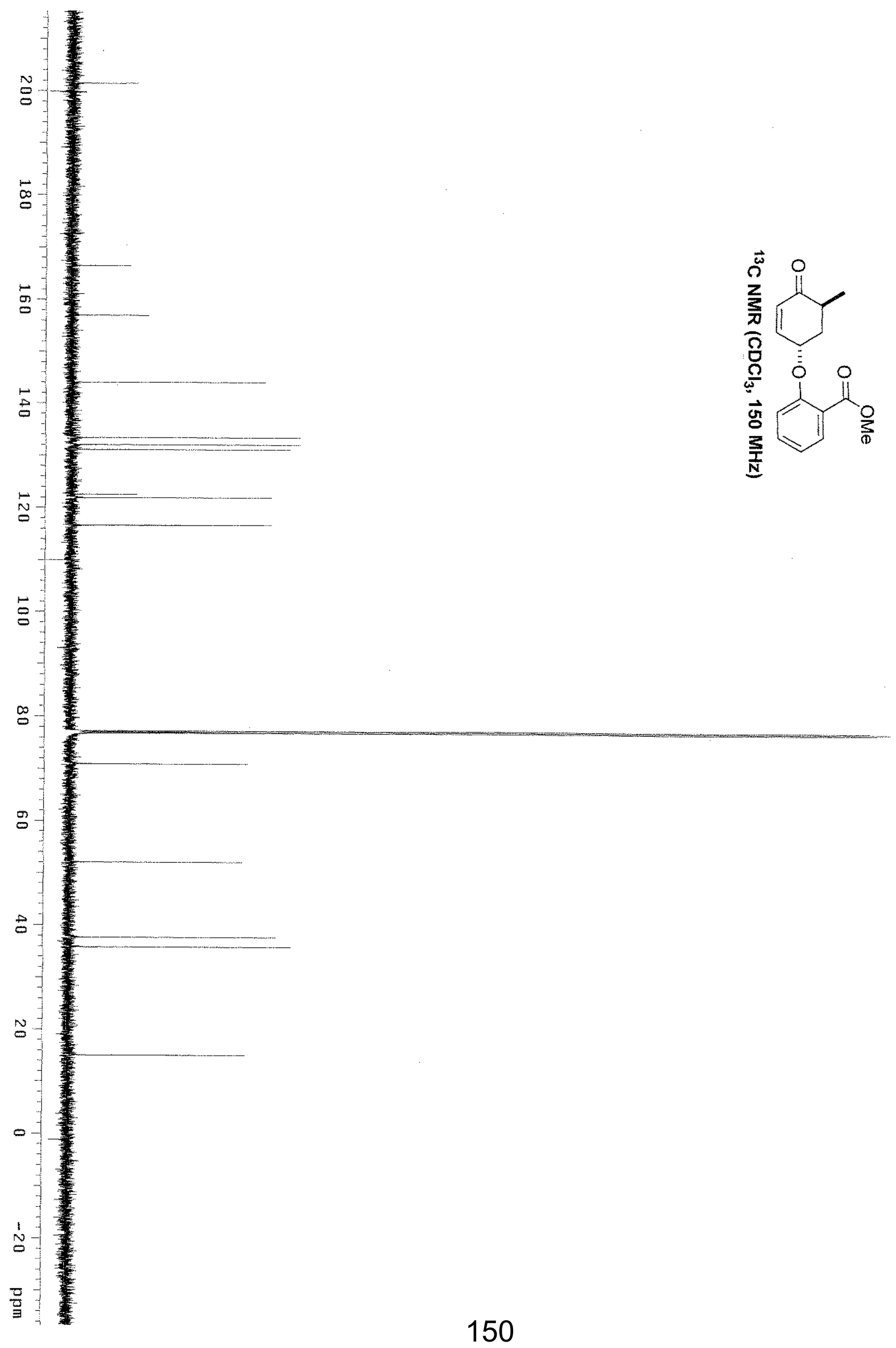




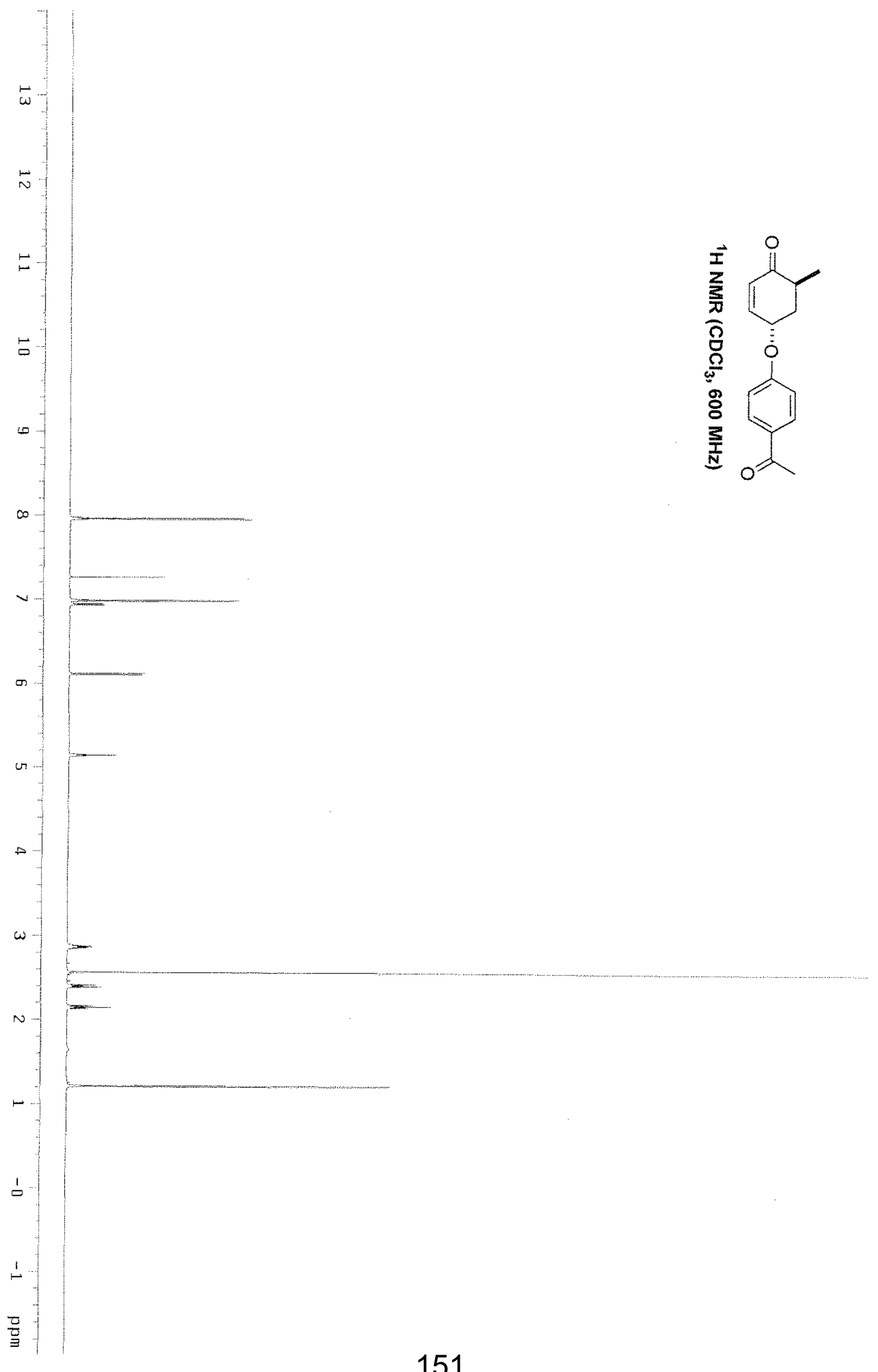




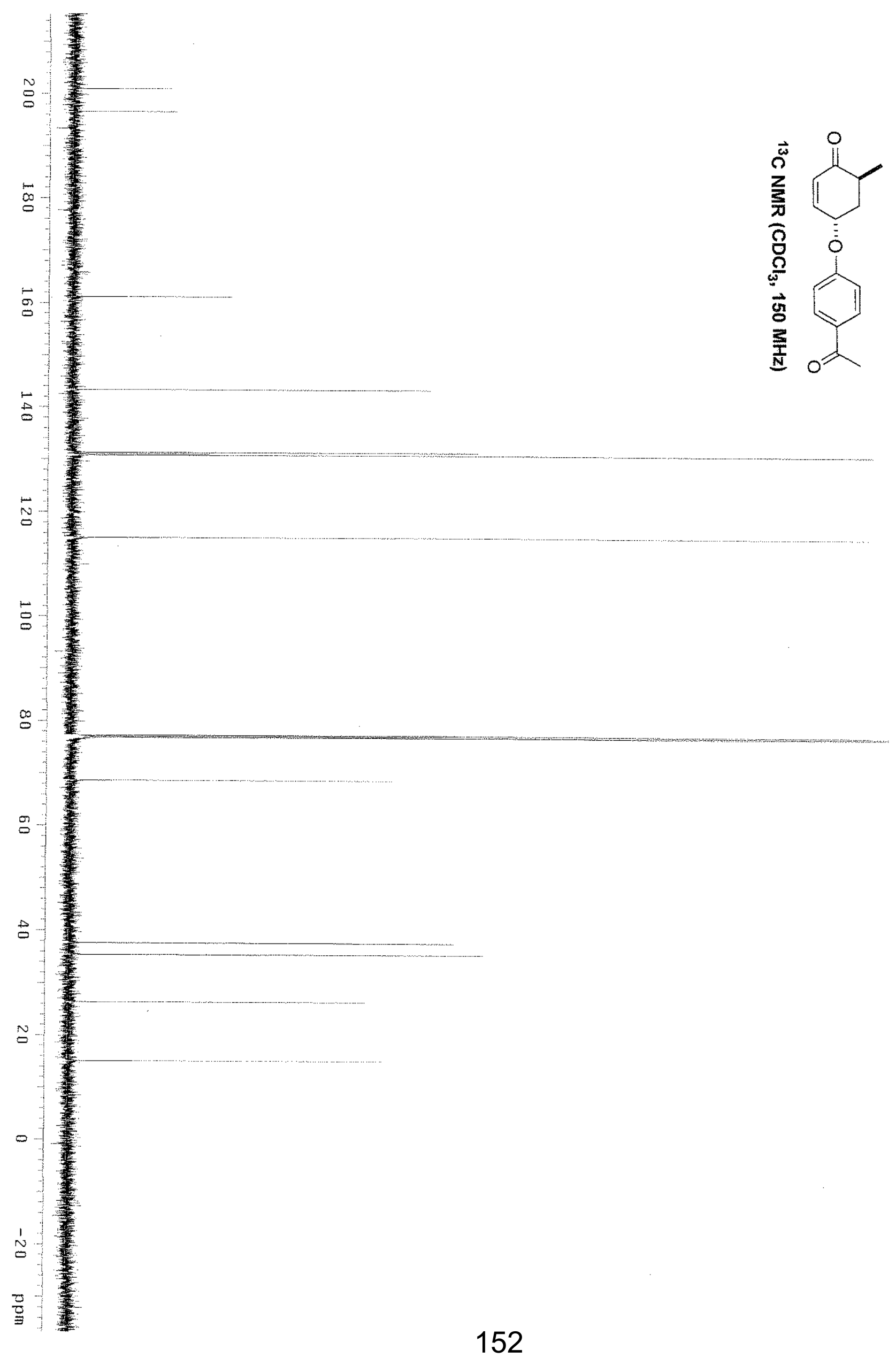



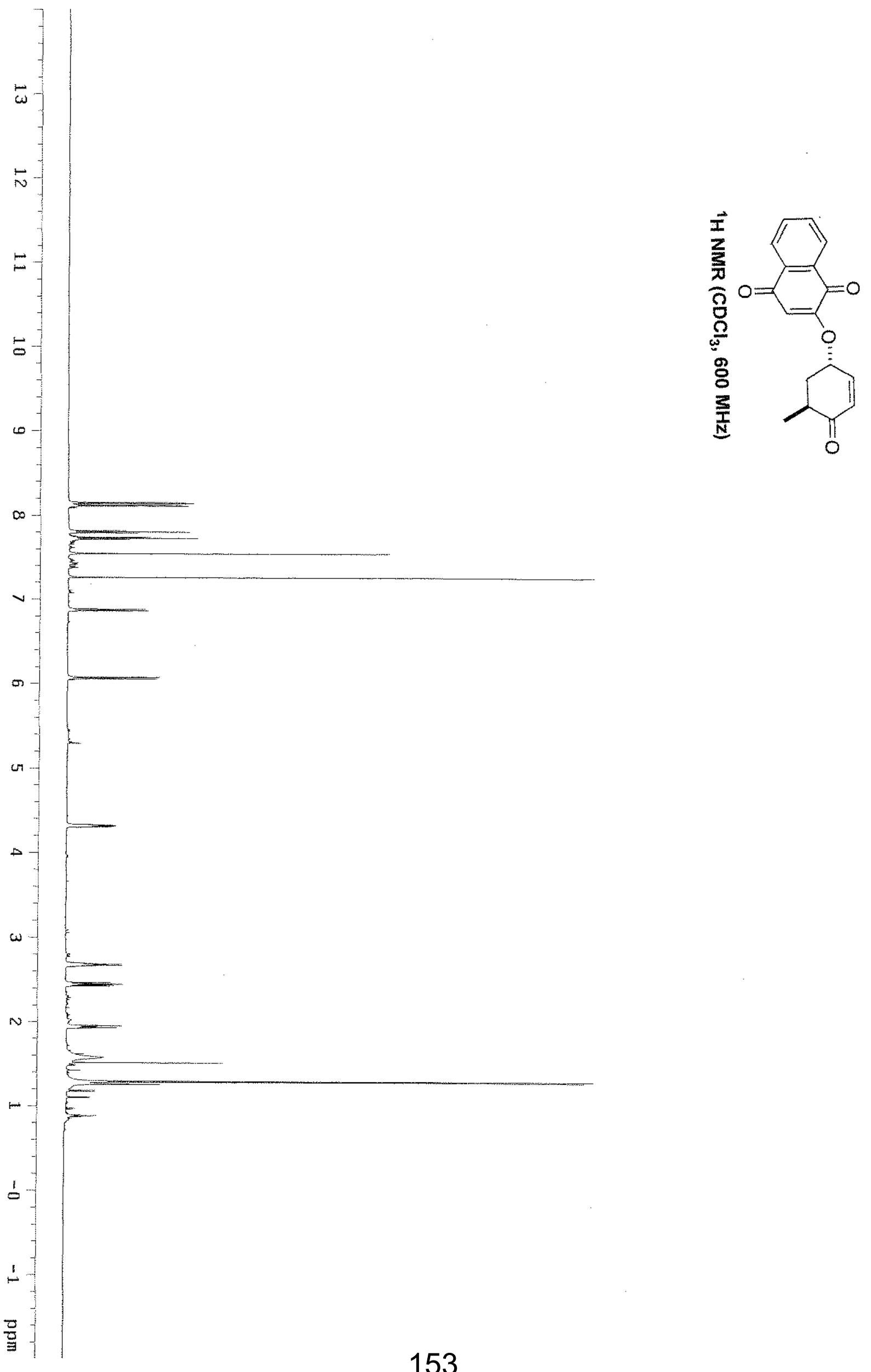


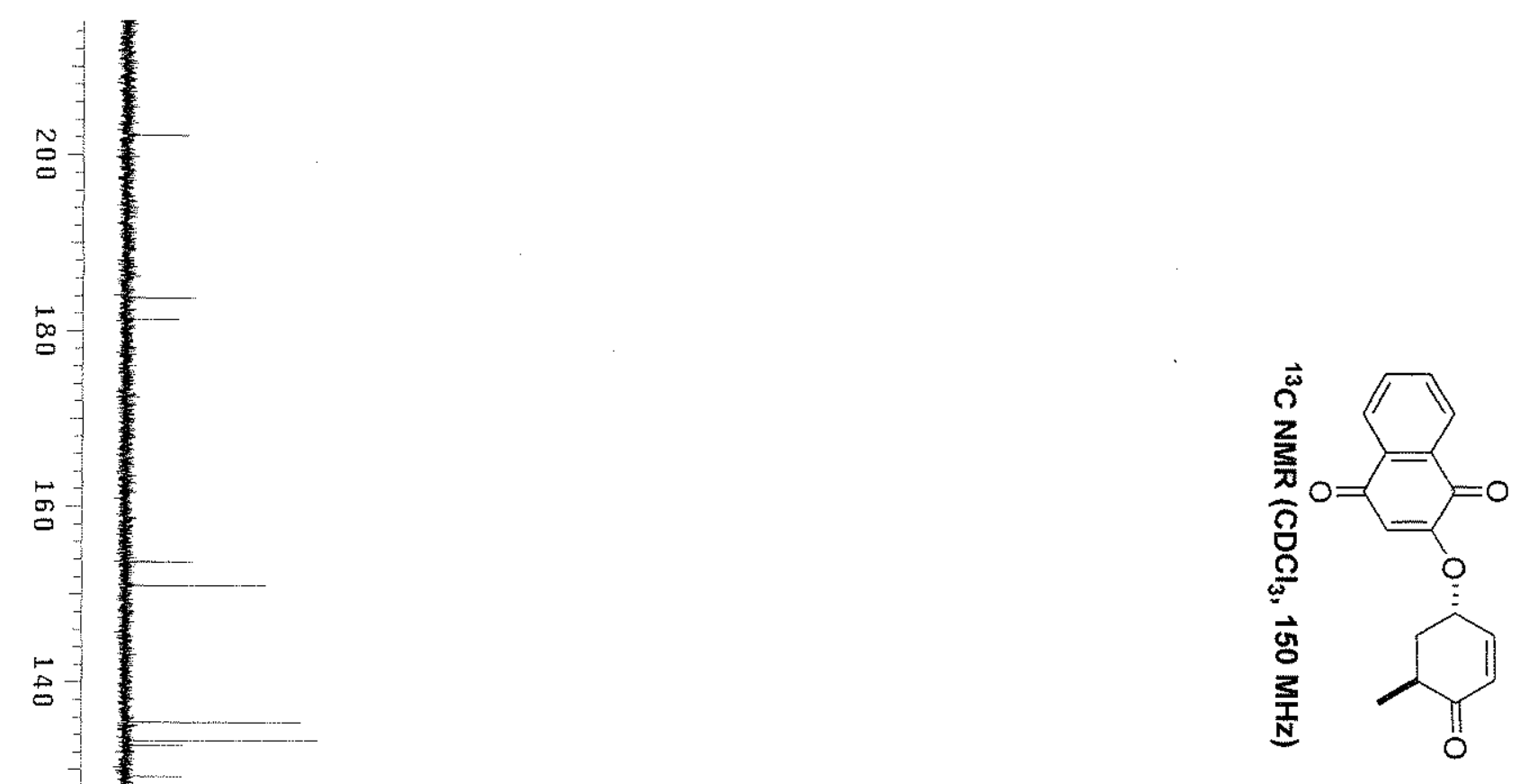

$\stackrel{\infty}{\circ}$

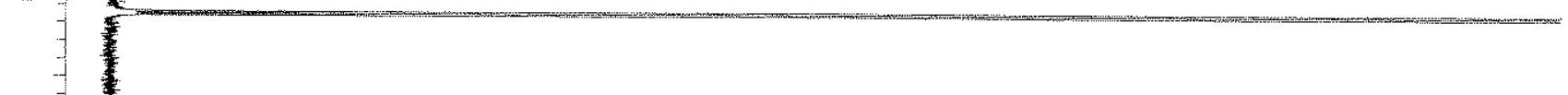

$g$

8

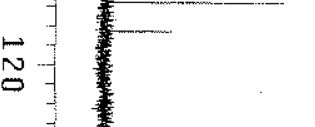

s

(

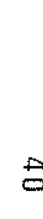

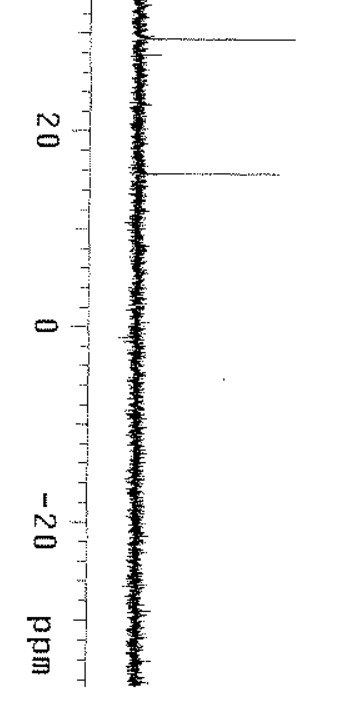




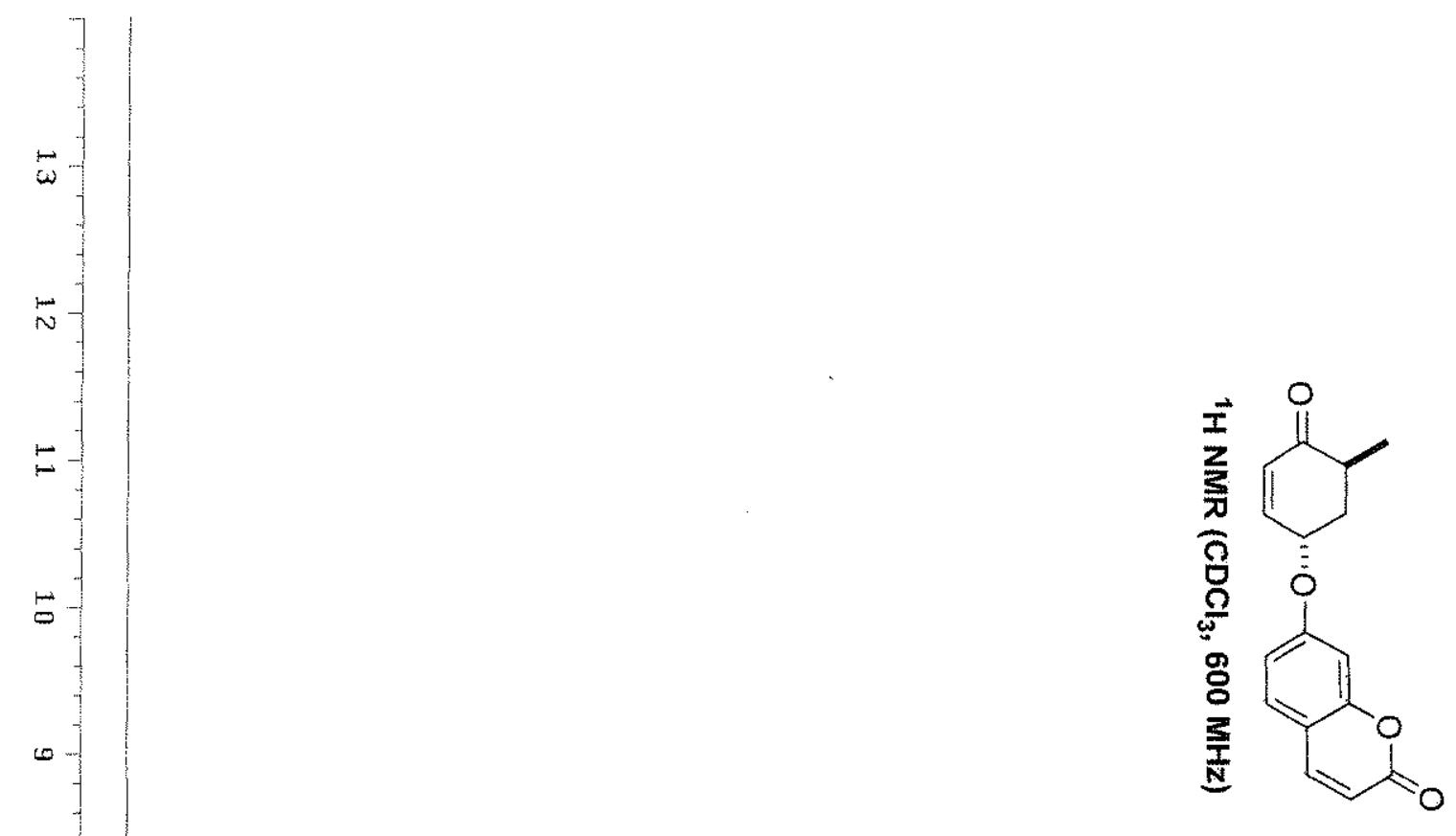

$\infty$

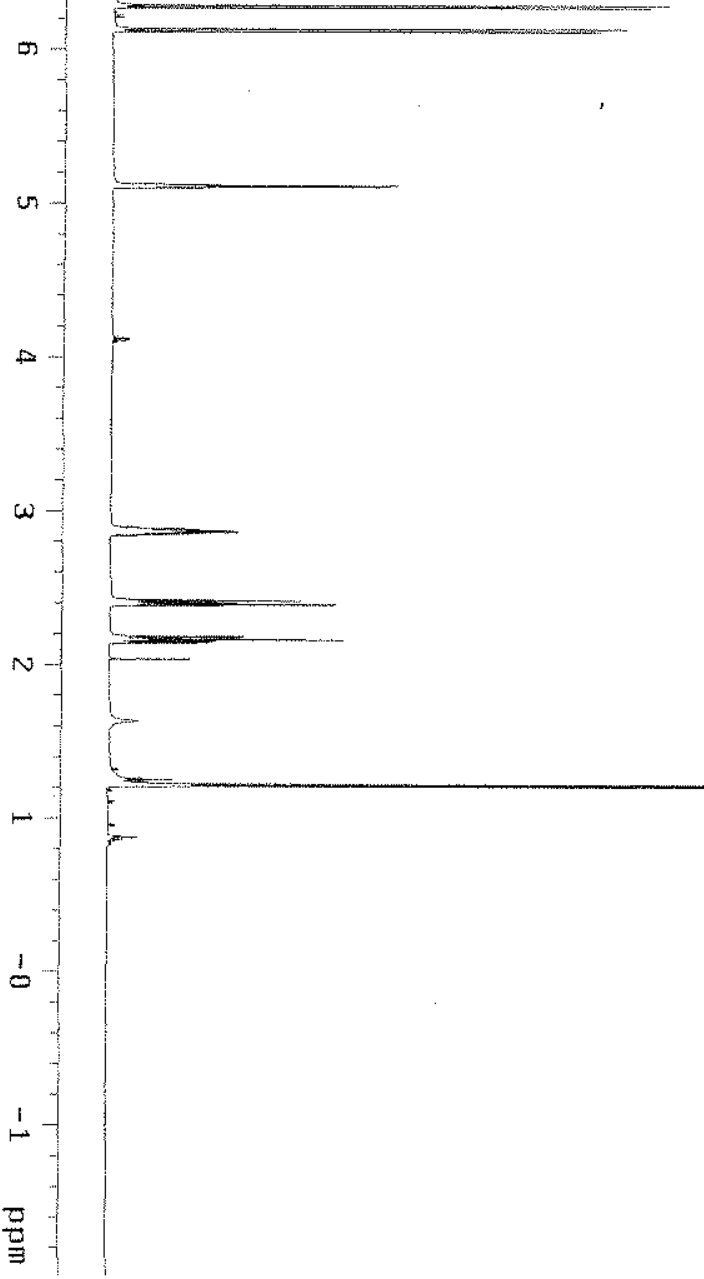




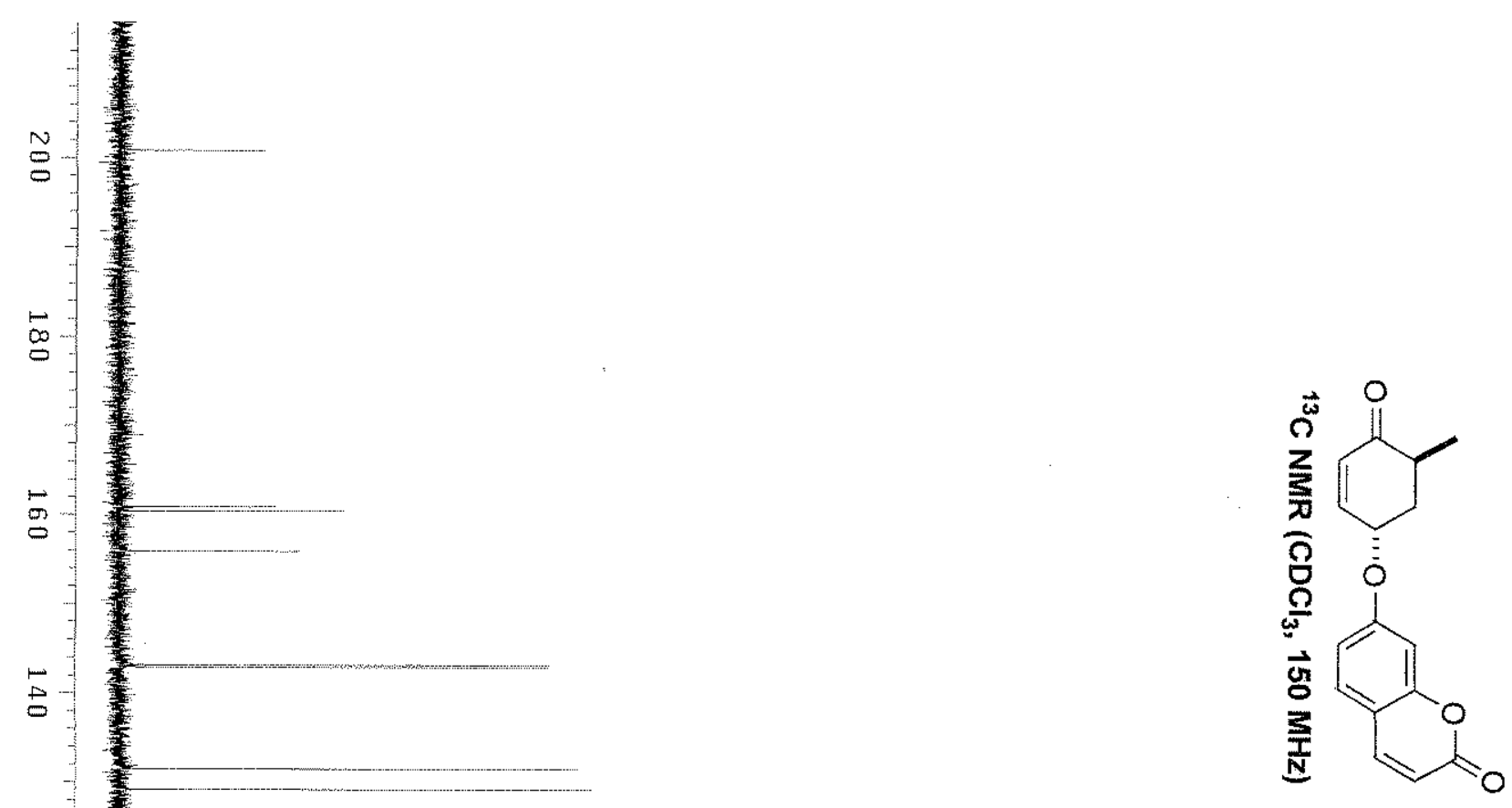

$\stackrel{\sim}{\Xi}$

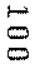

$\infty$

욤

ㅁ.

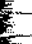

N

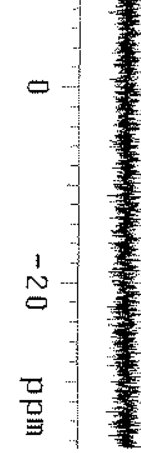




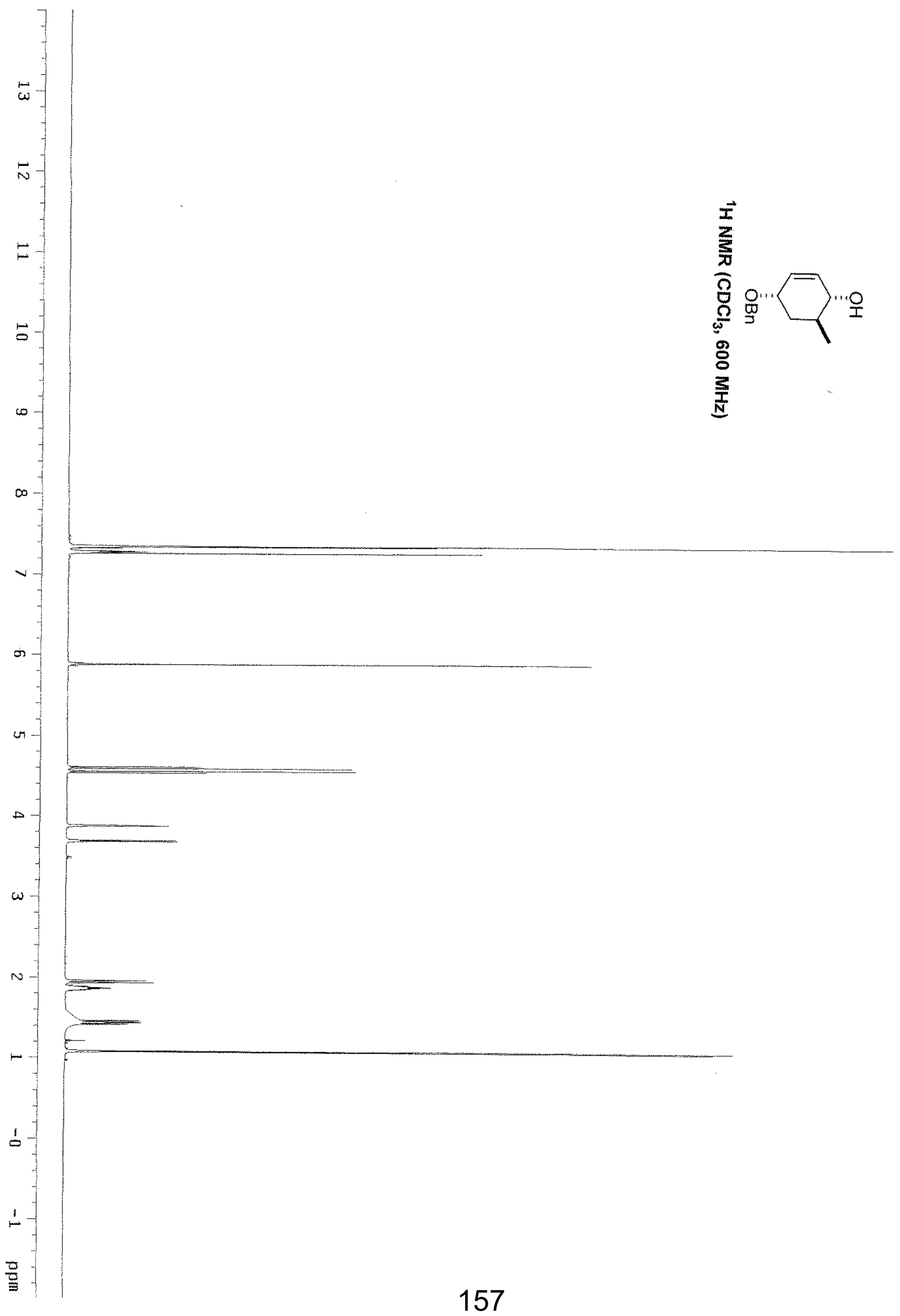




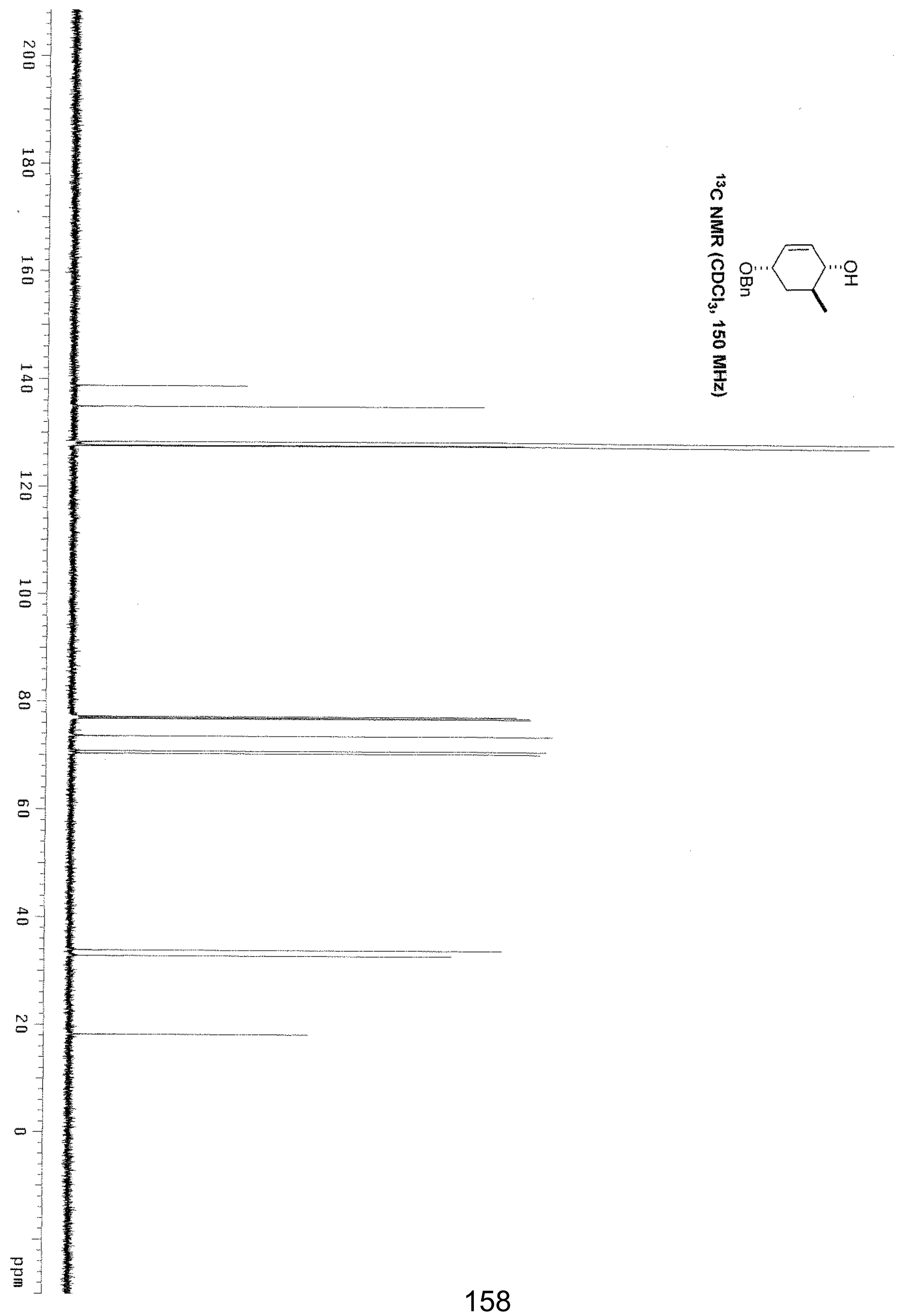




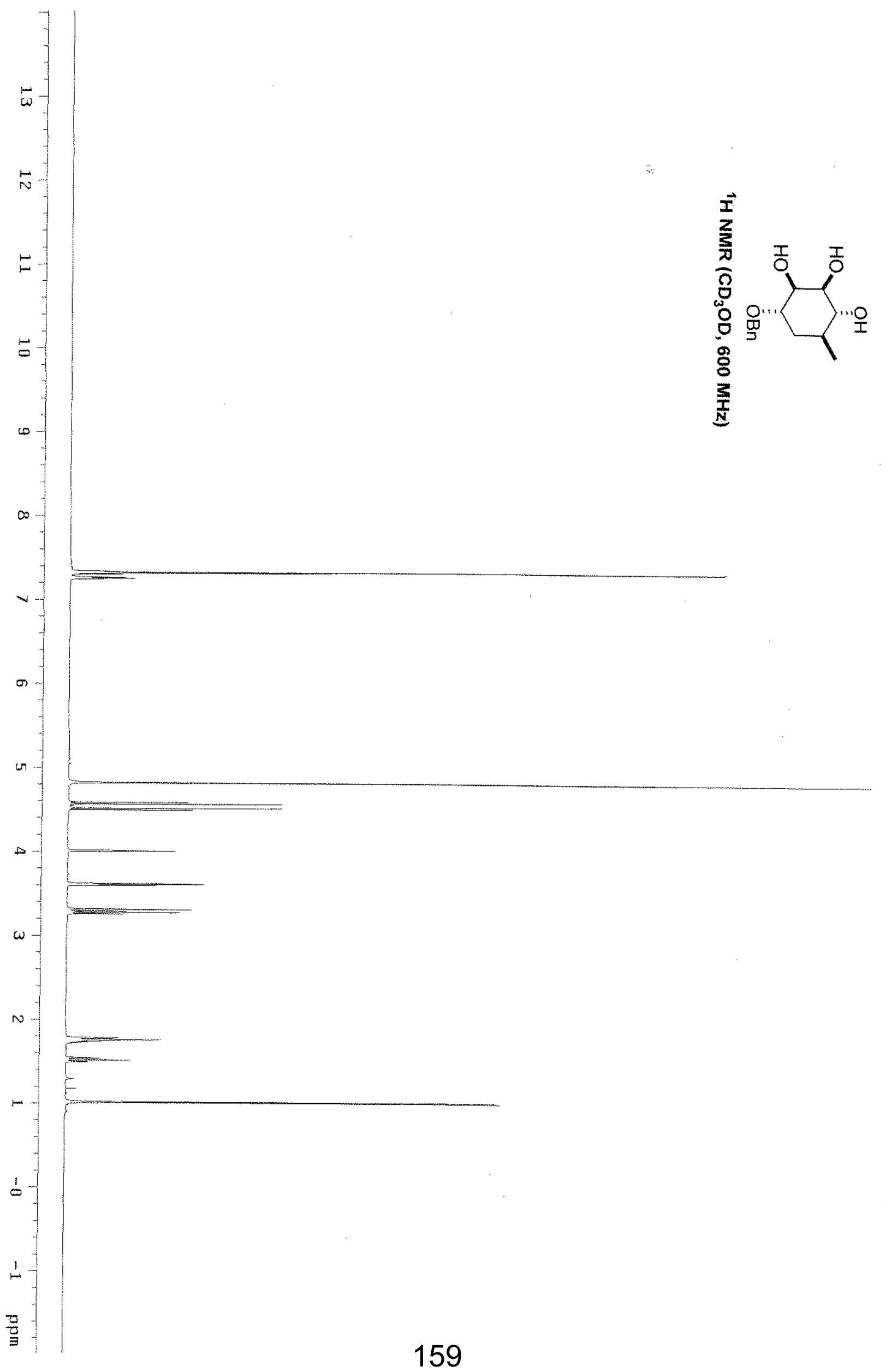




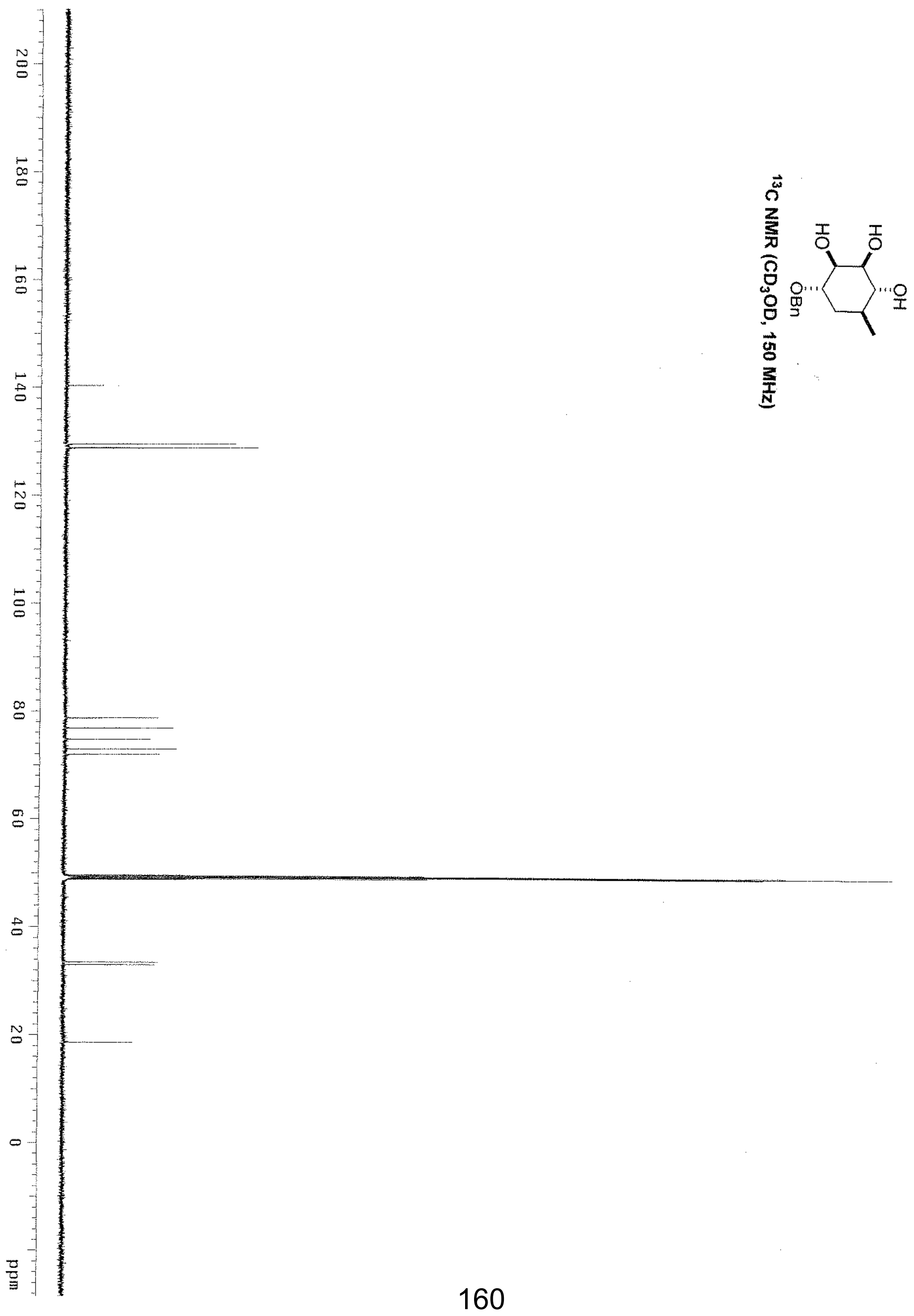




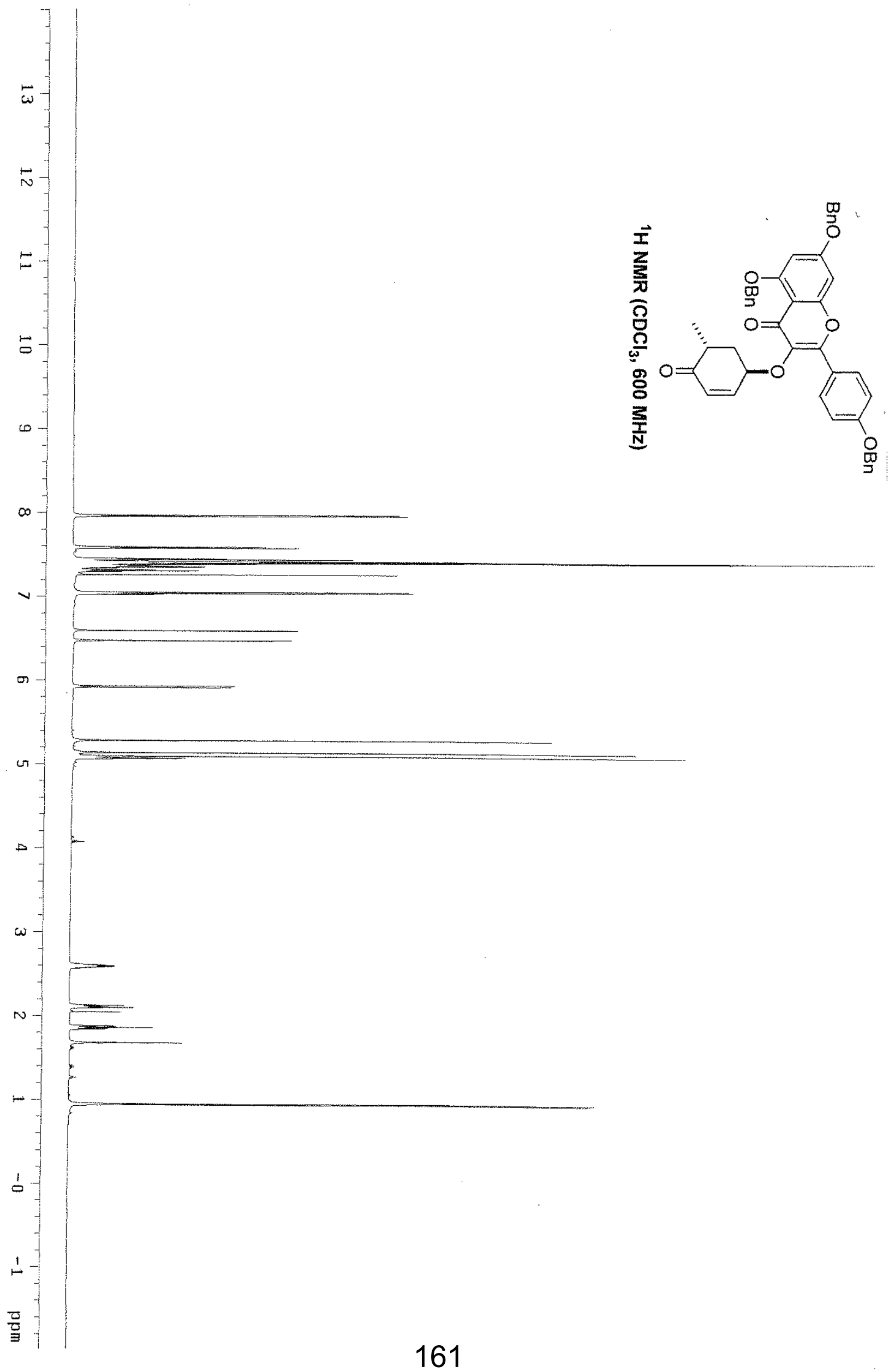




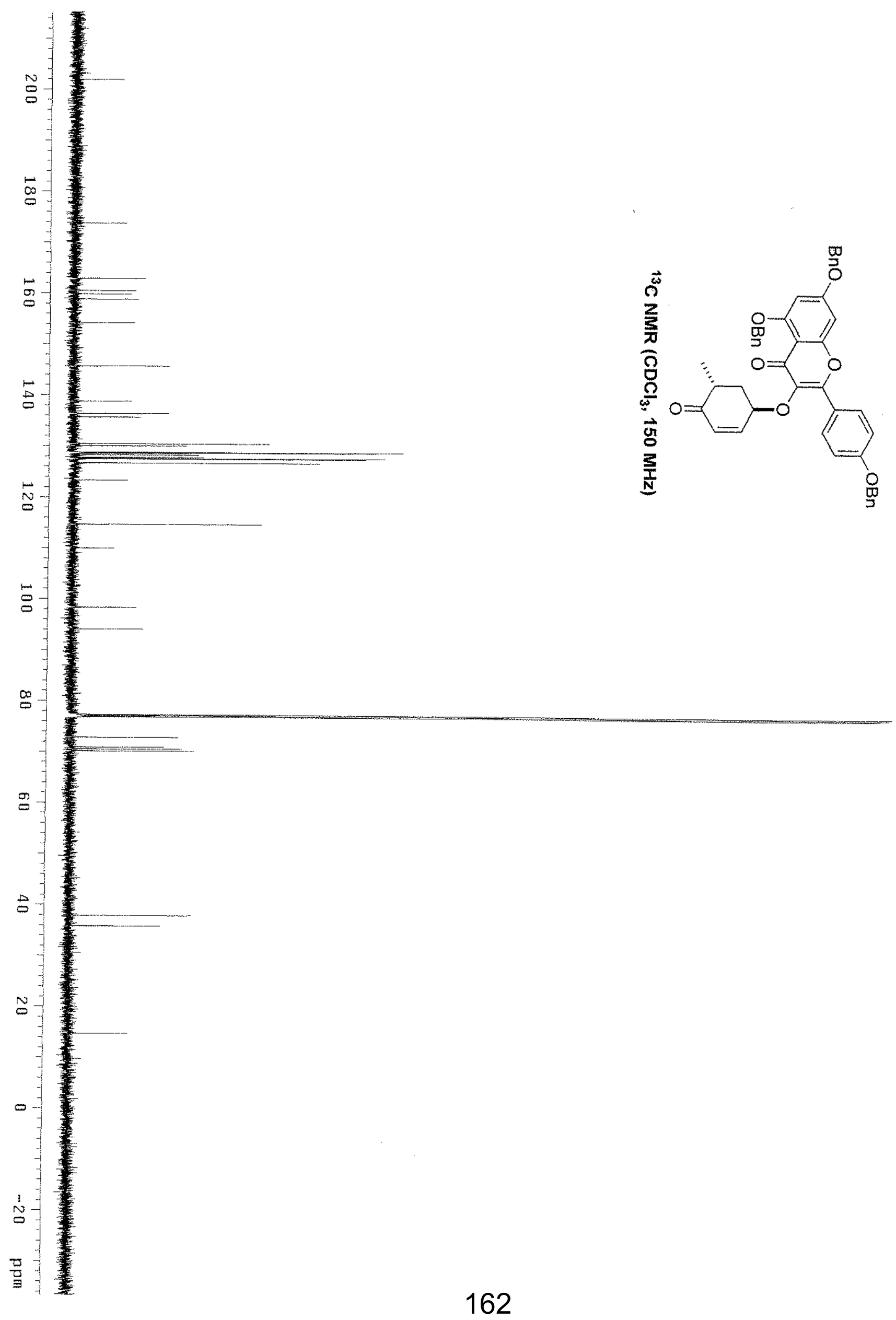




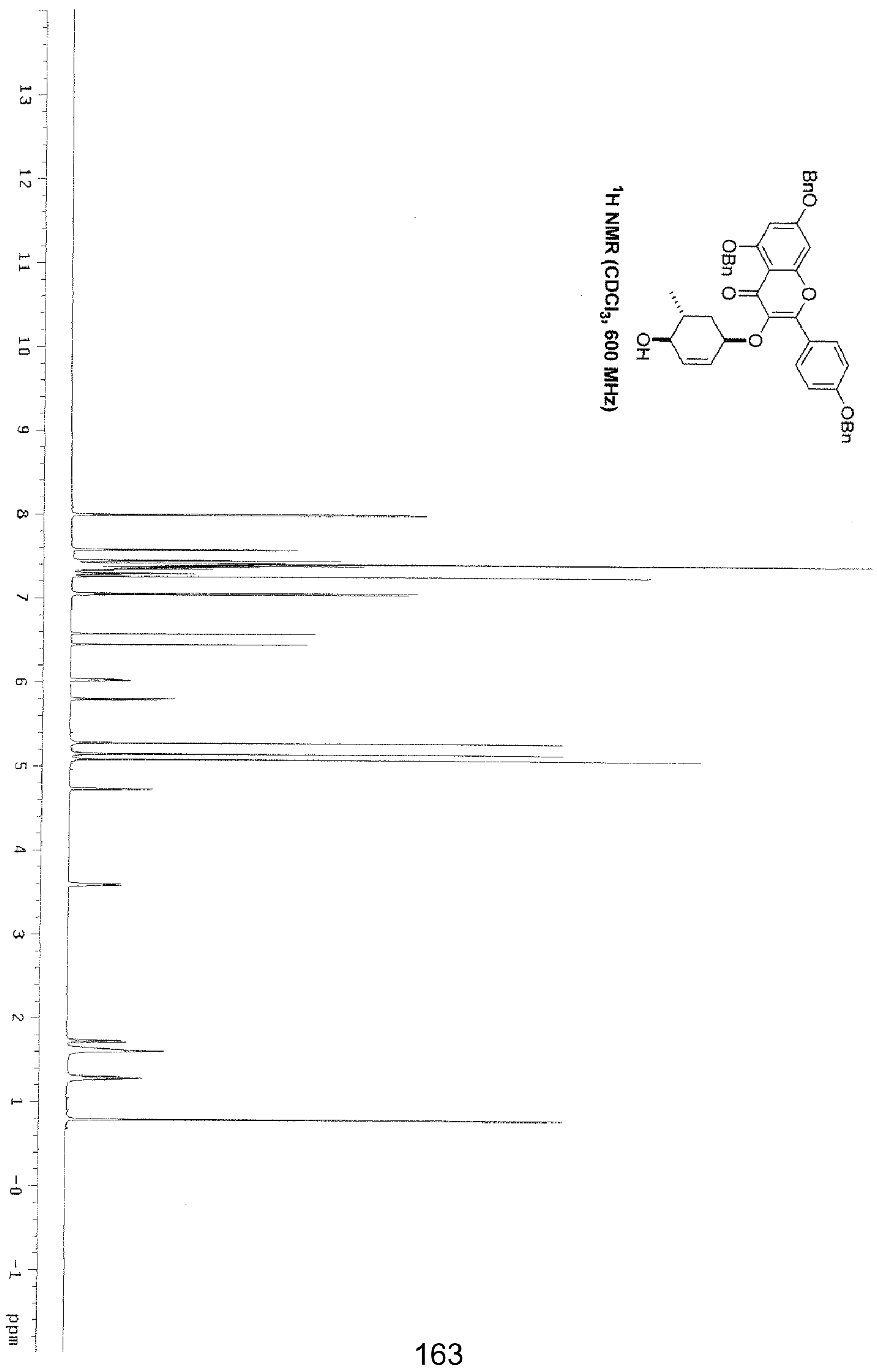




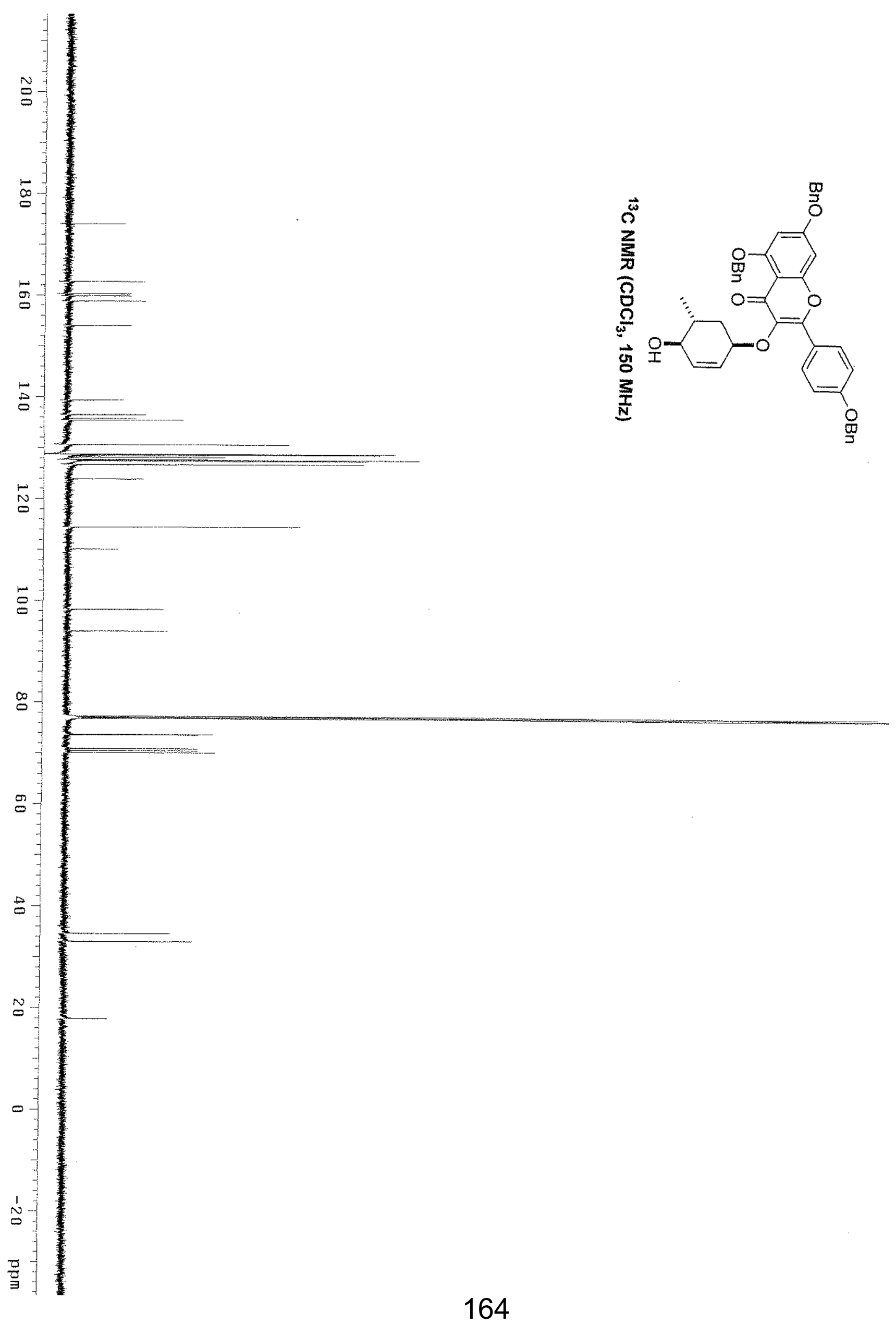




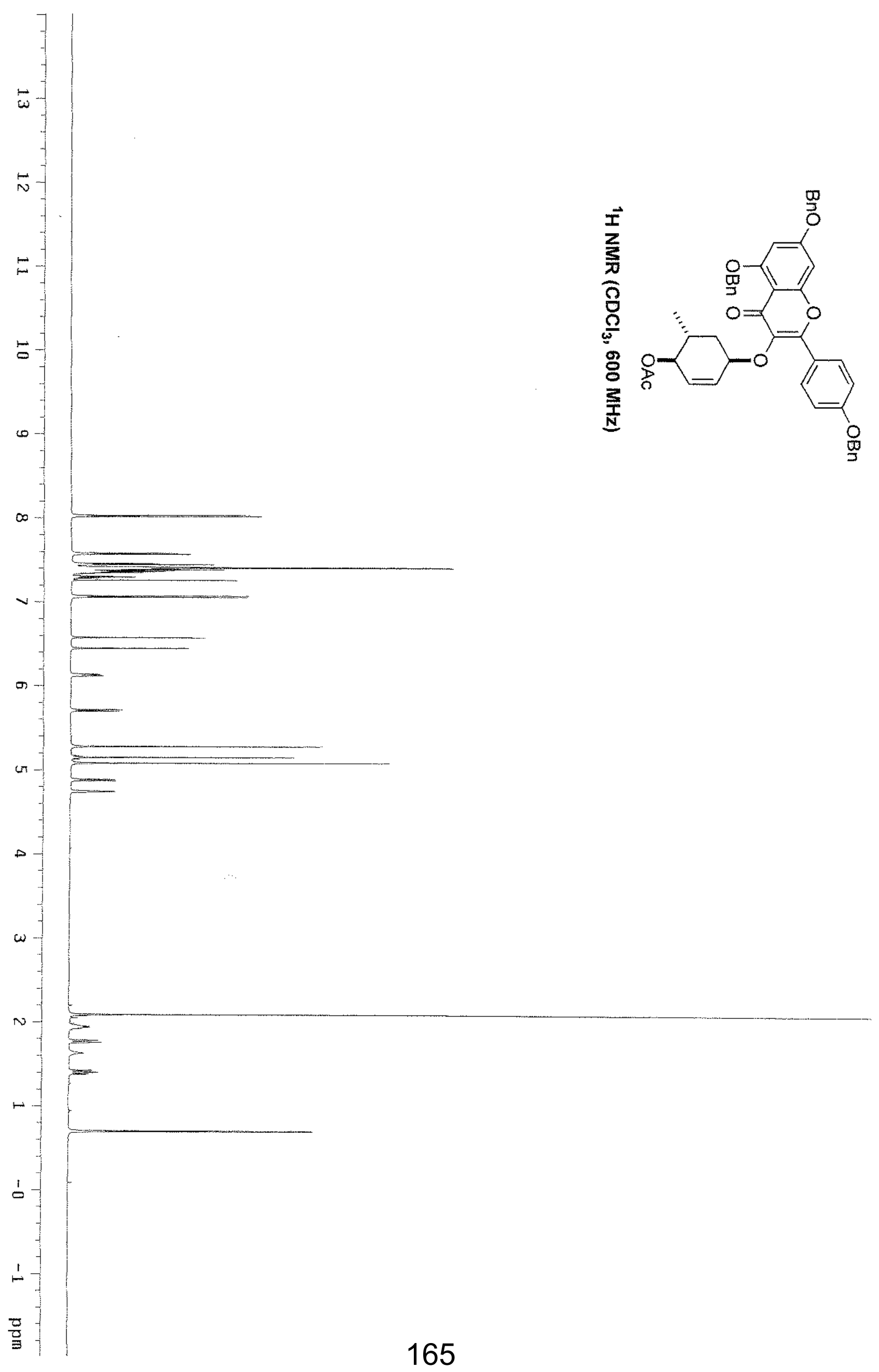




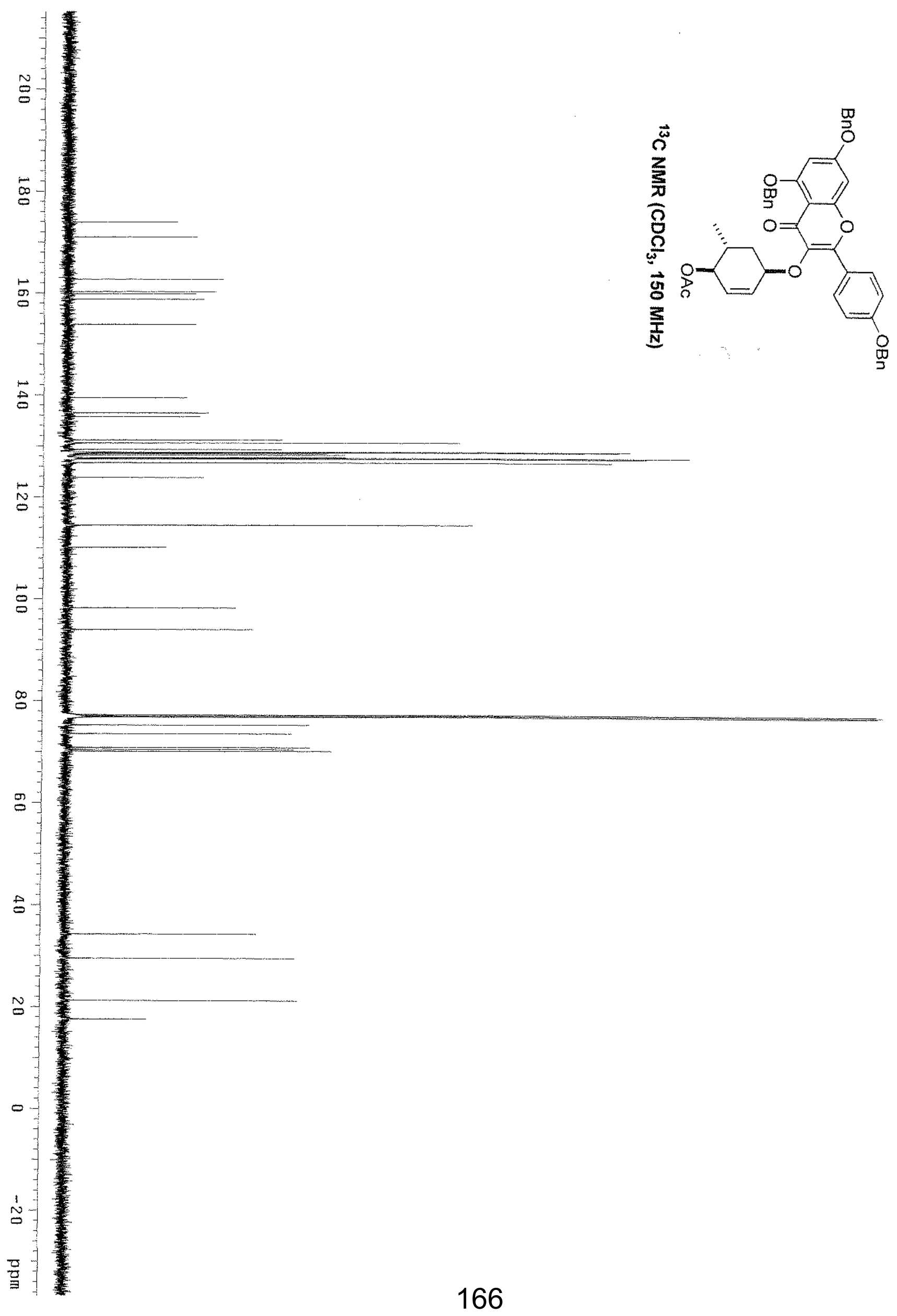




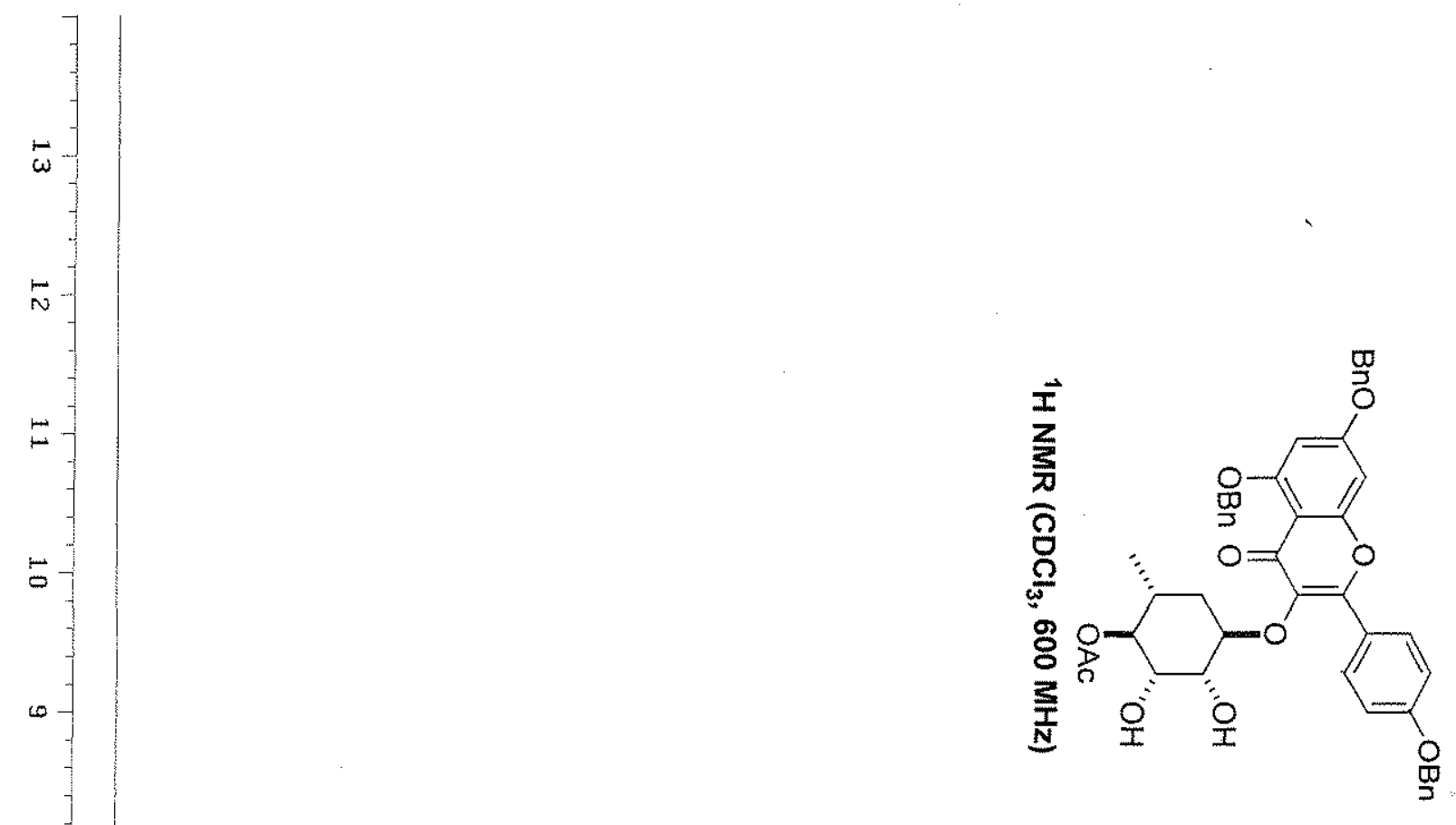




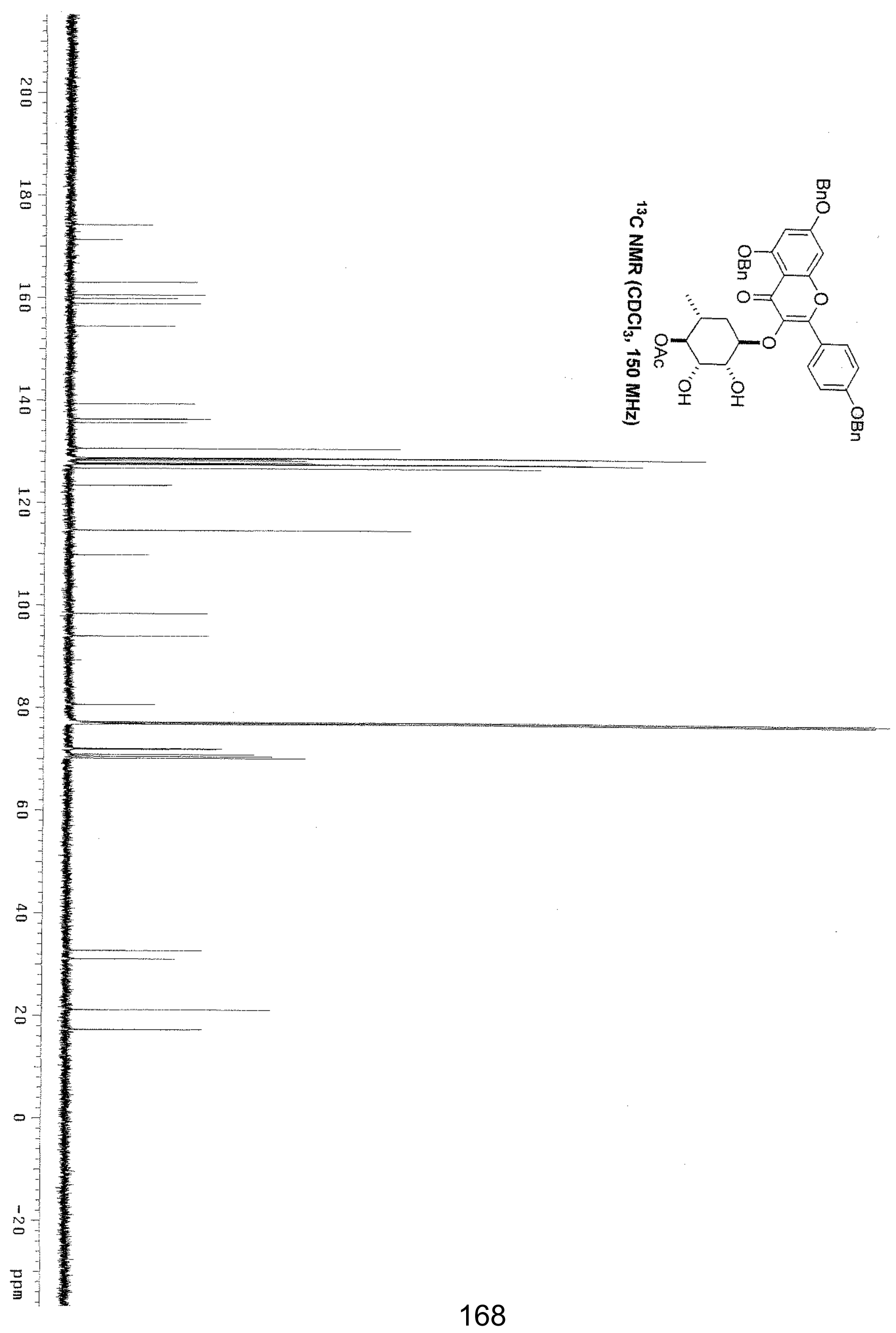




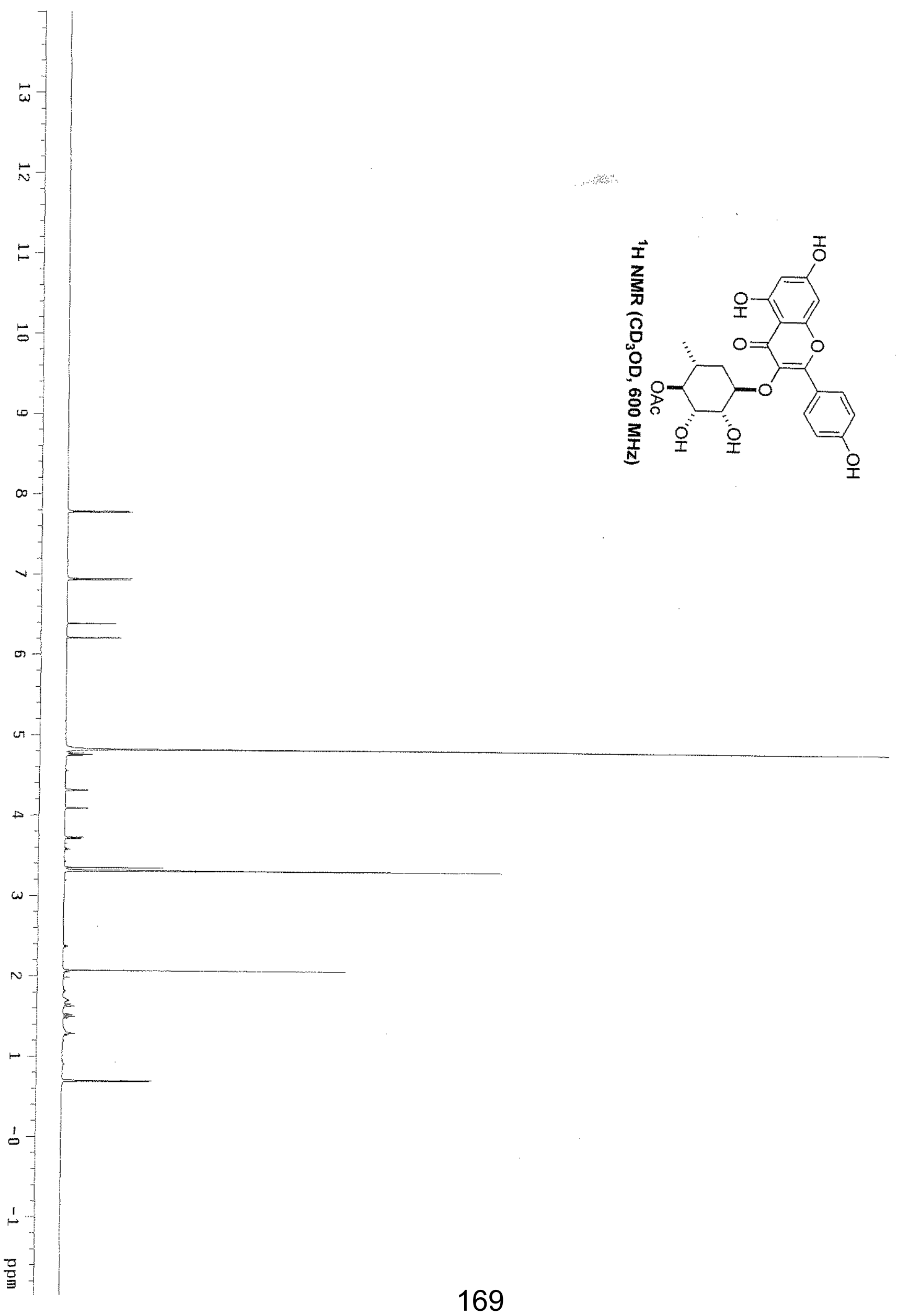




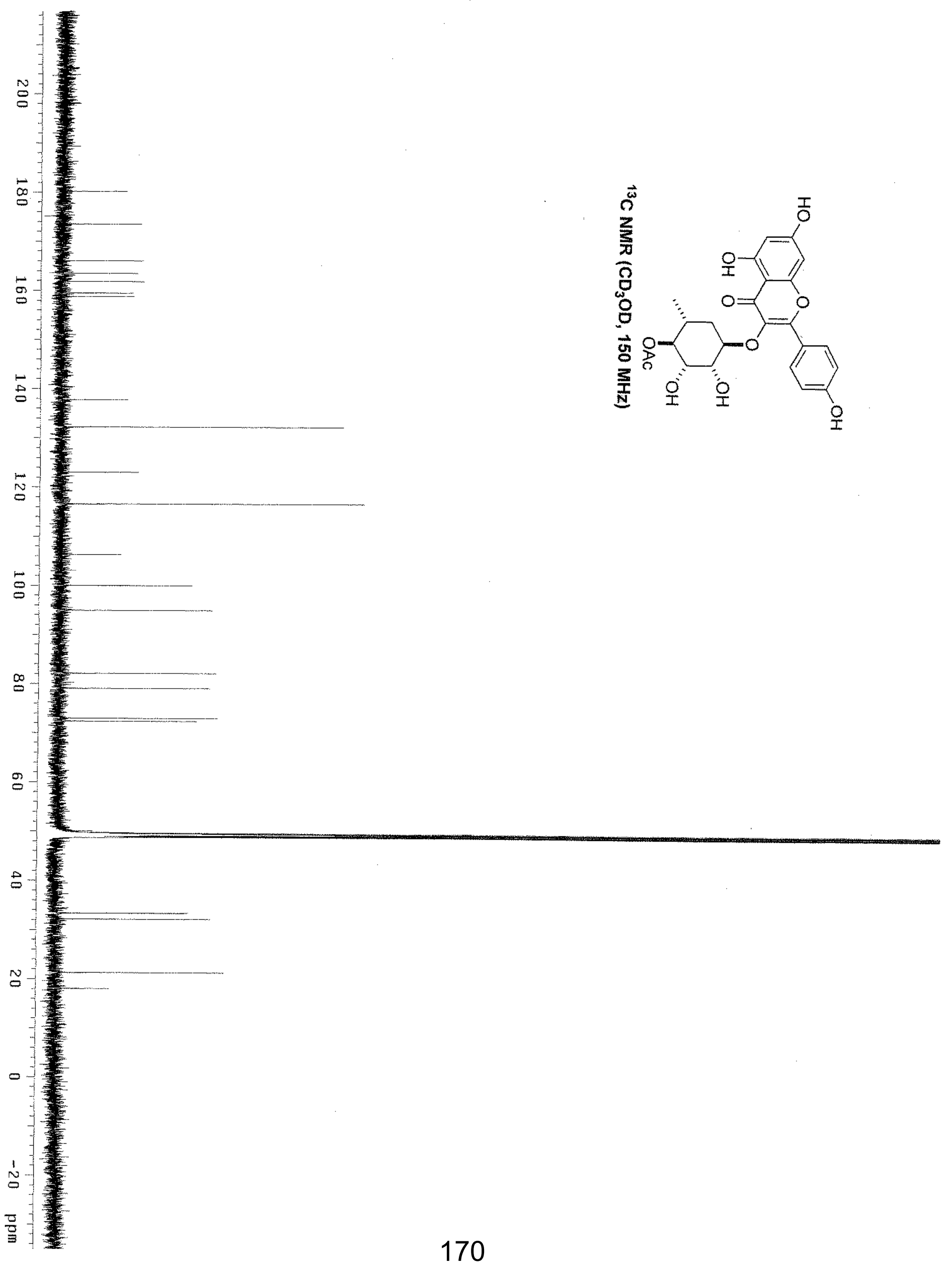




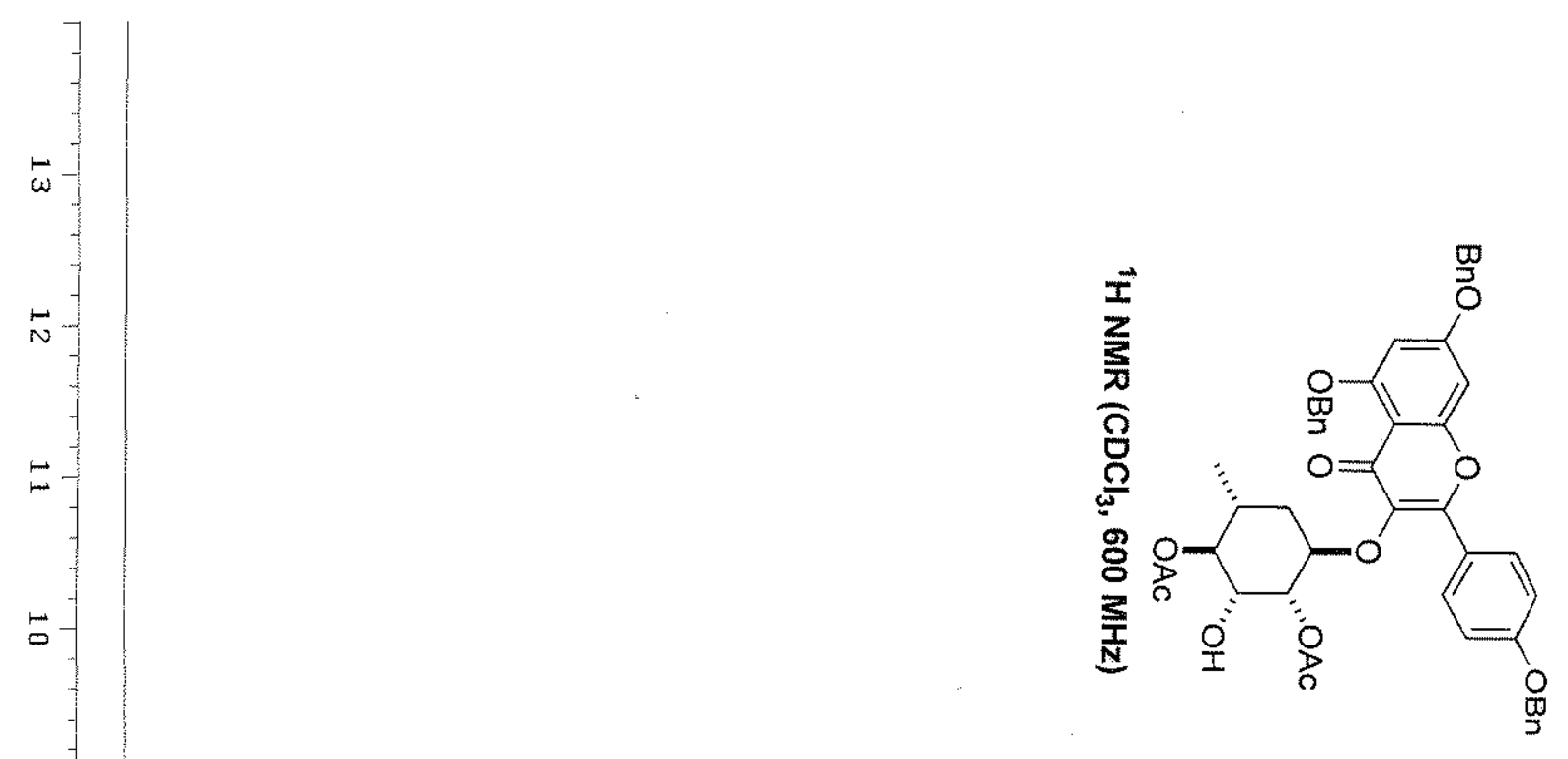

10

$\infty$

o

en

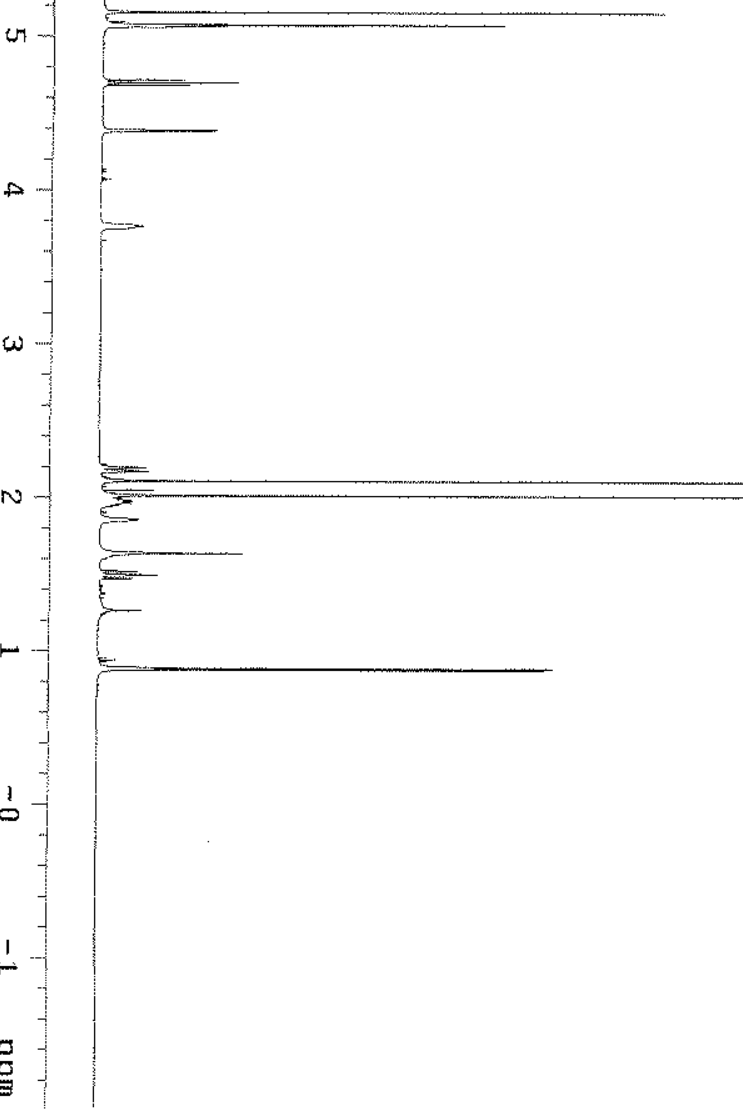




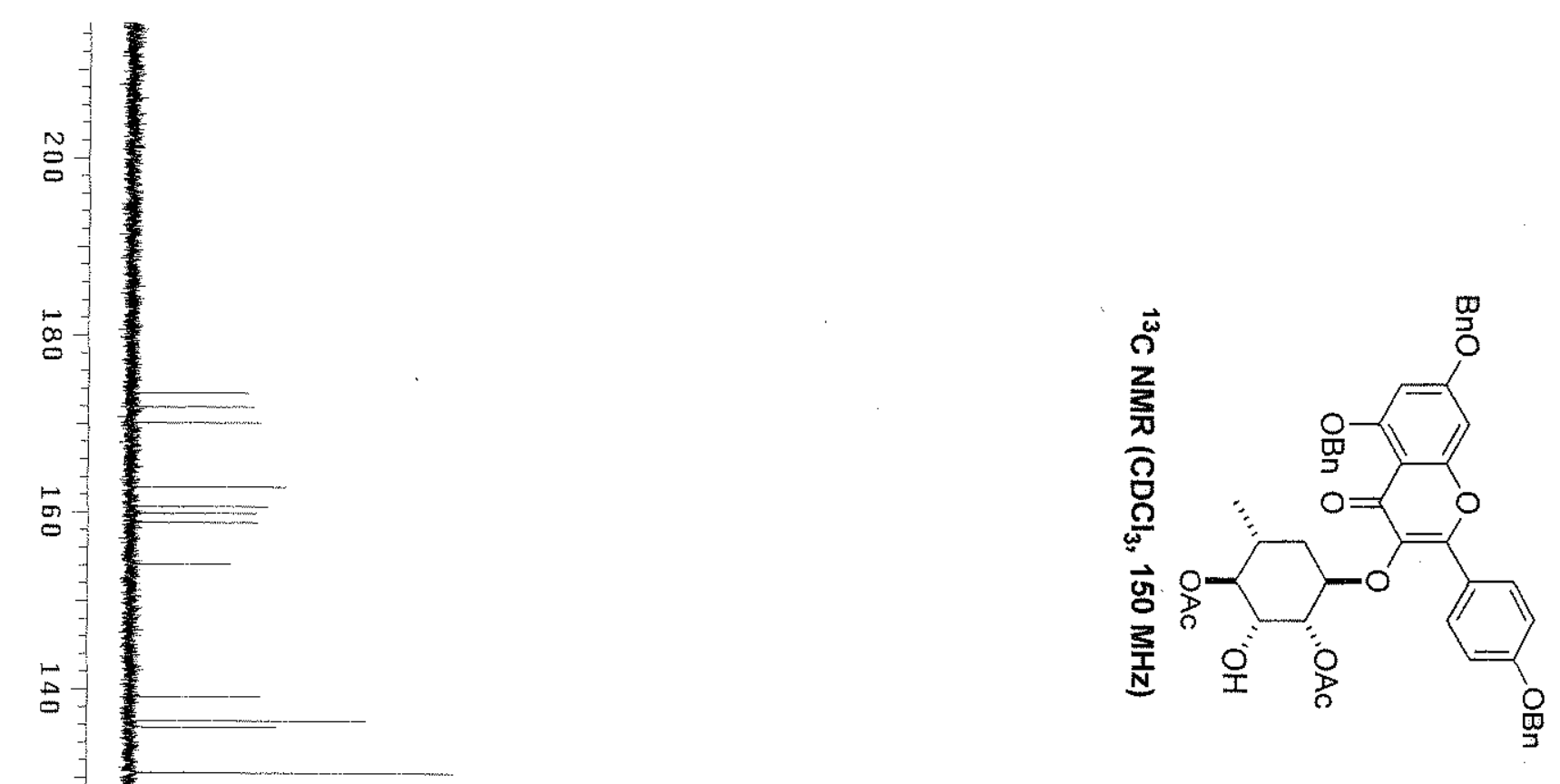



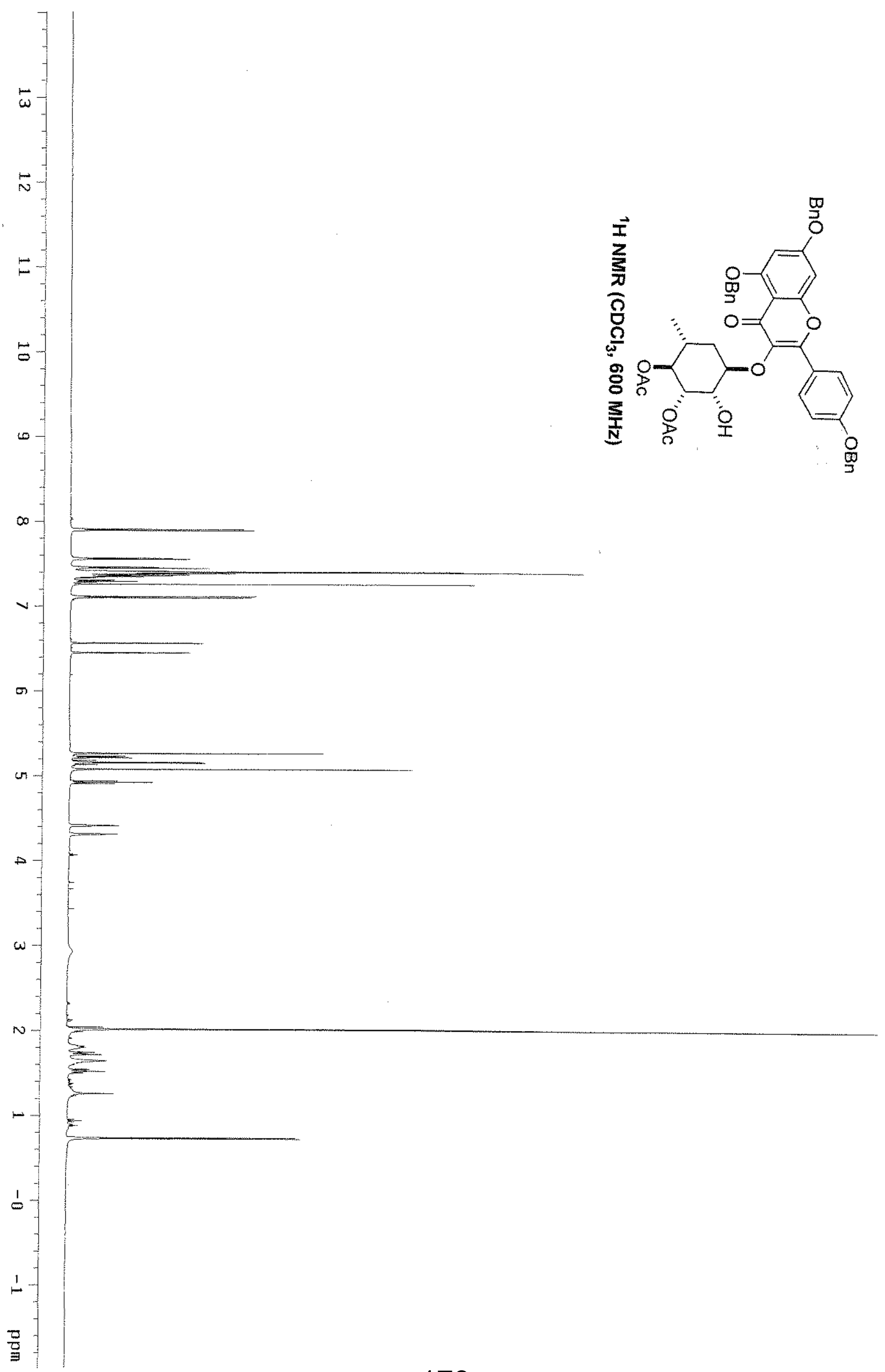


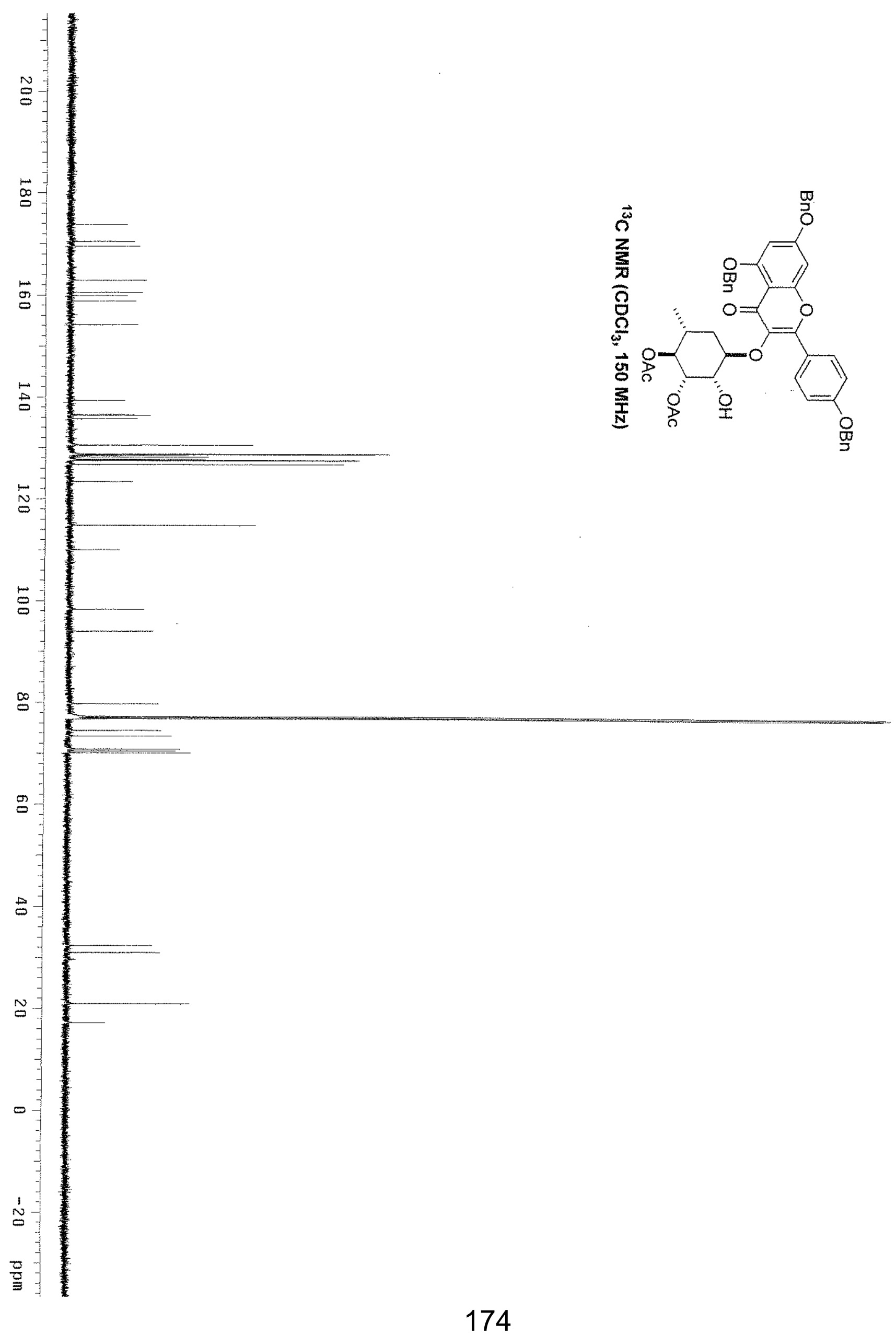




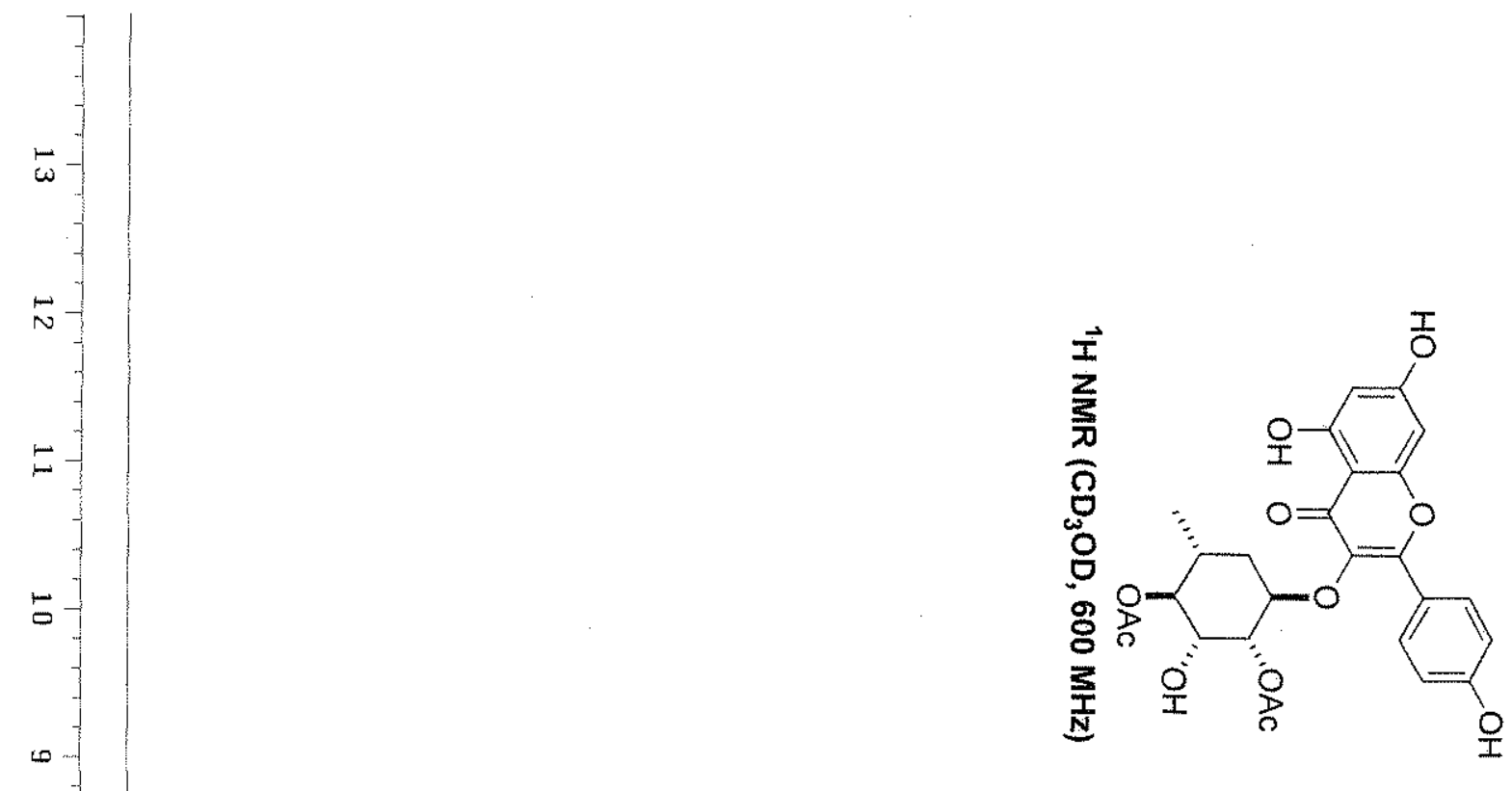

$\infty$

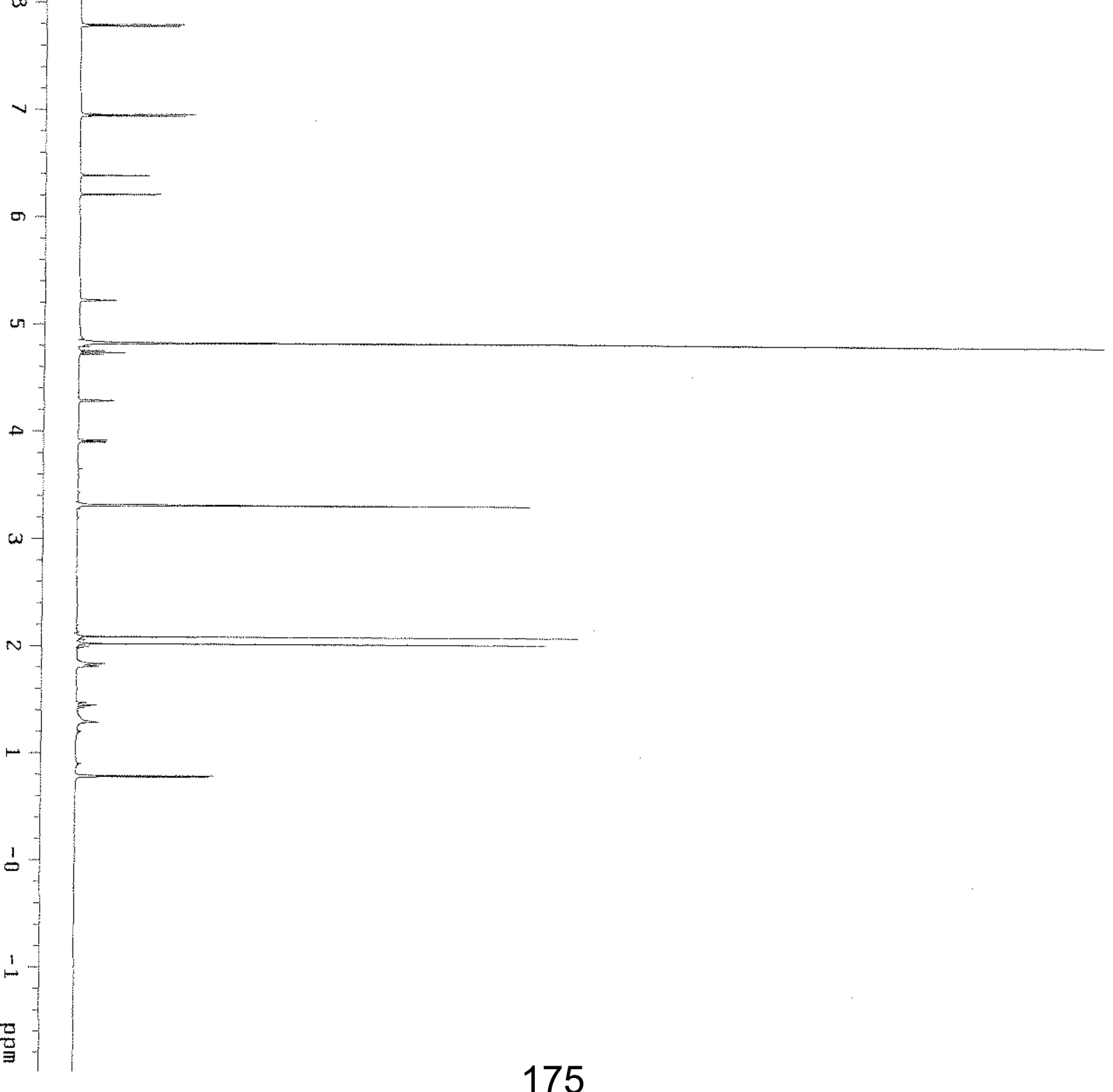




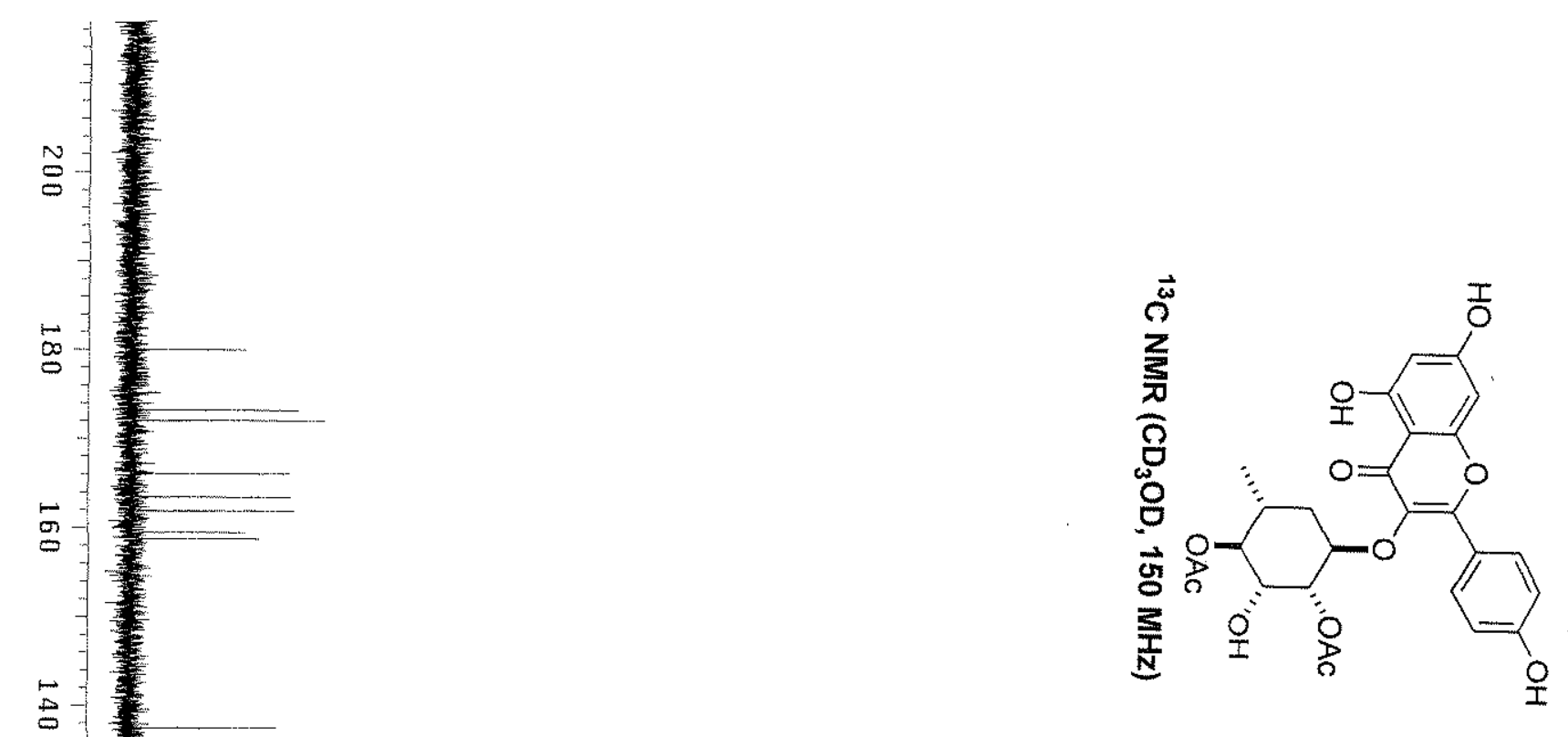




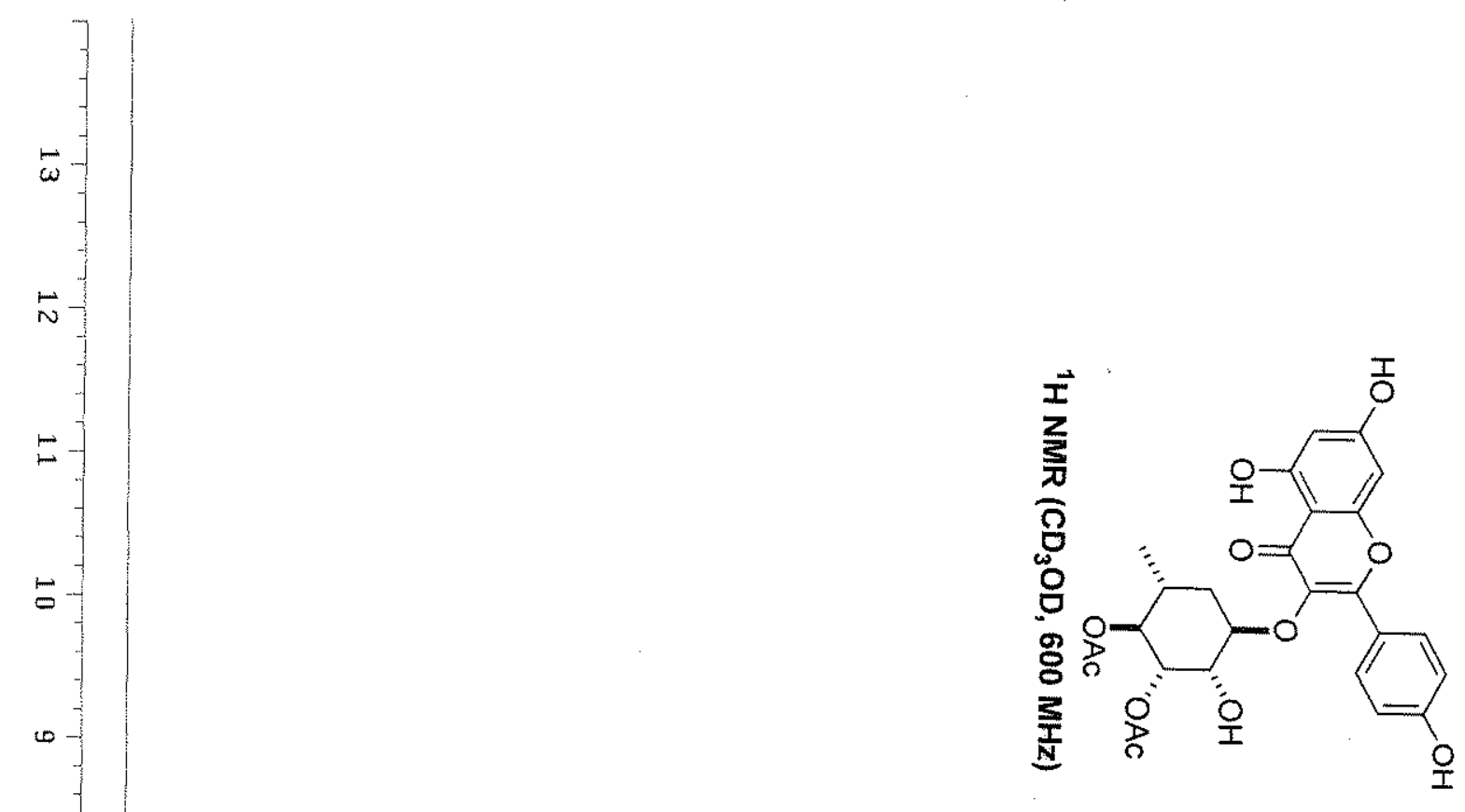

$\infty$

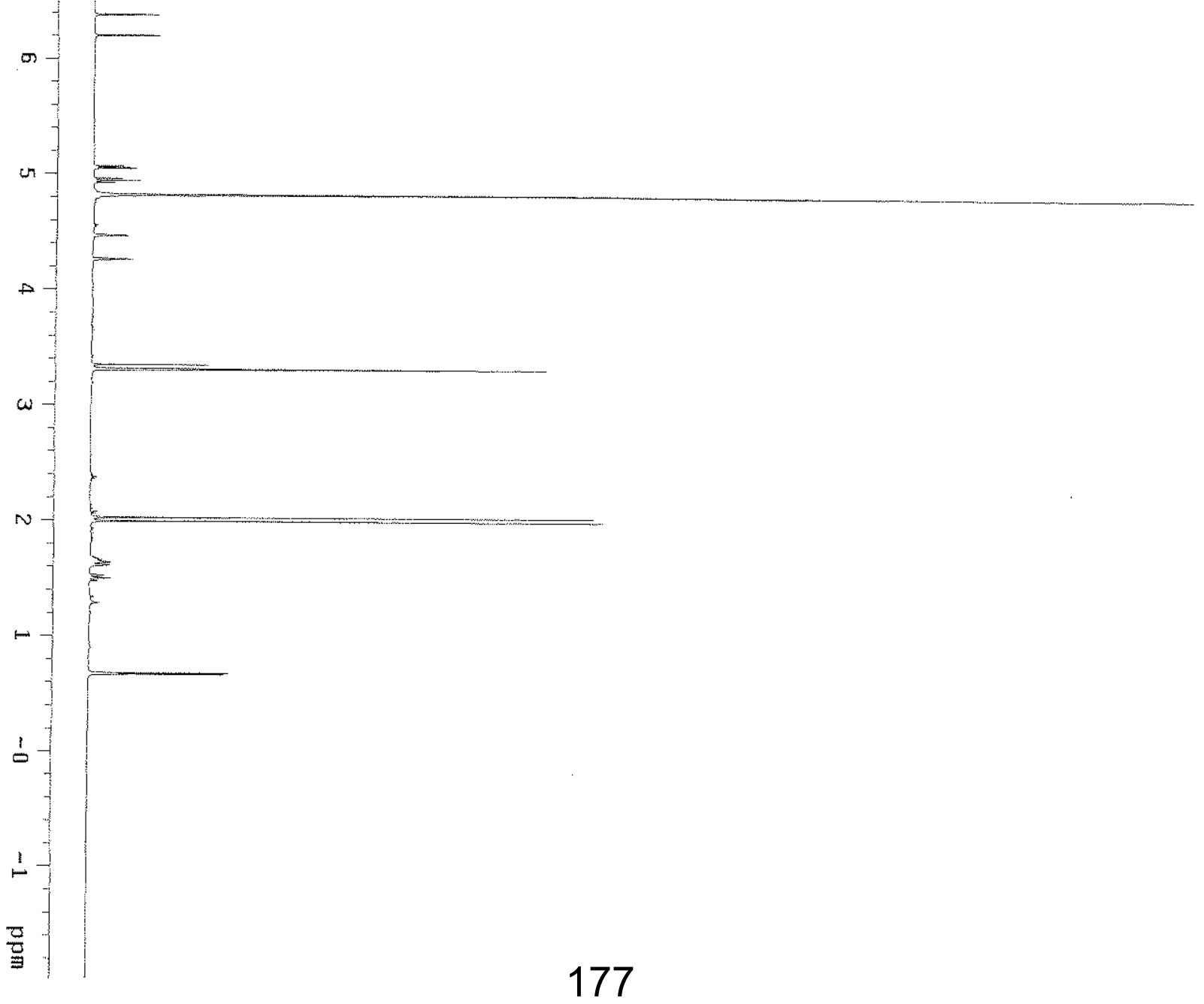




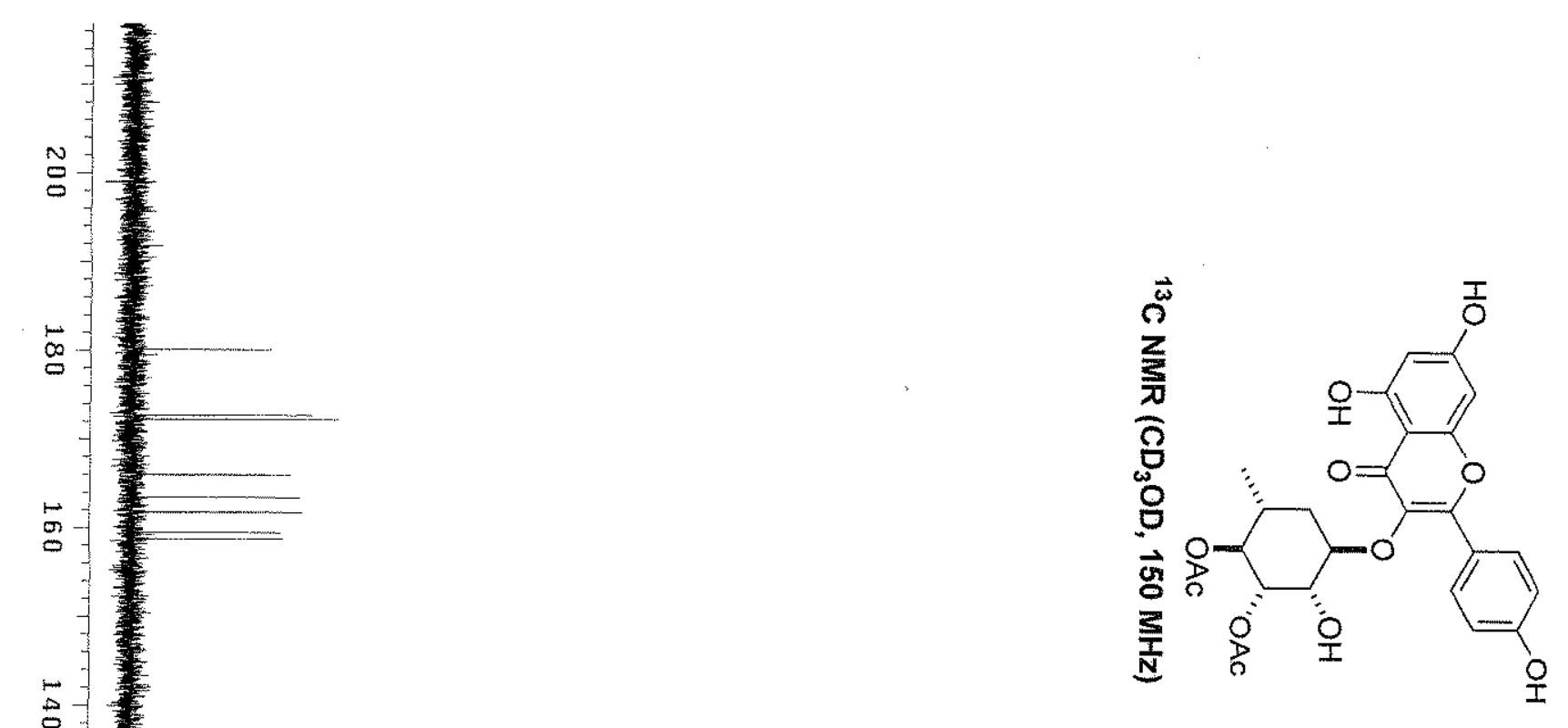

$\mathbb{v}$

5

$\infty$

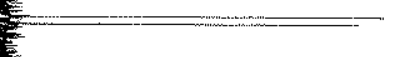

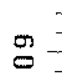

$B$

1
0
0

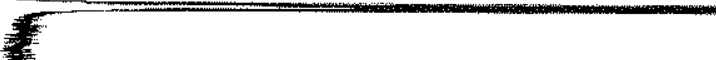




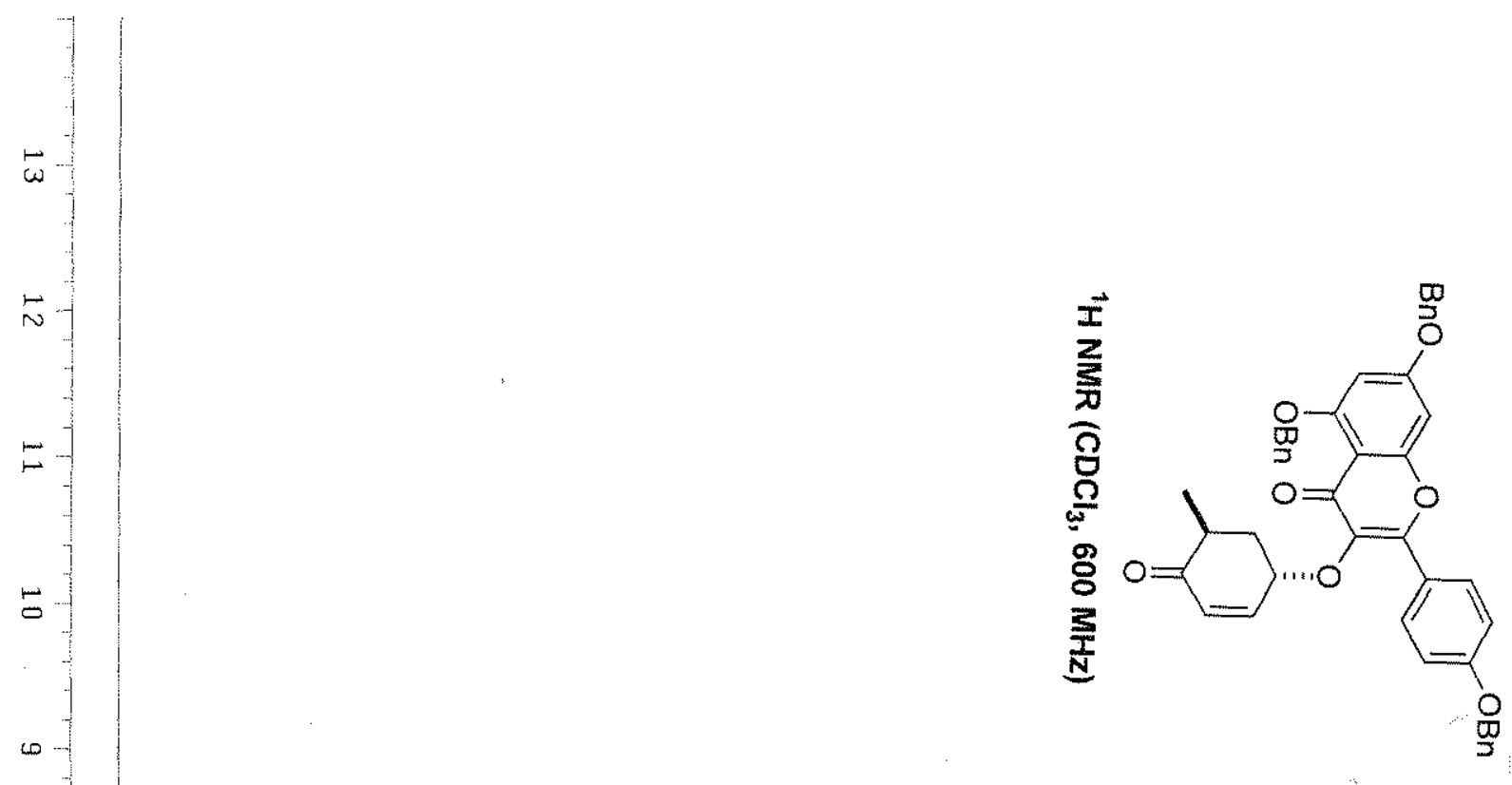

$\infty$

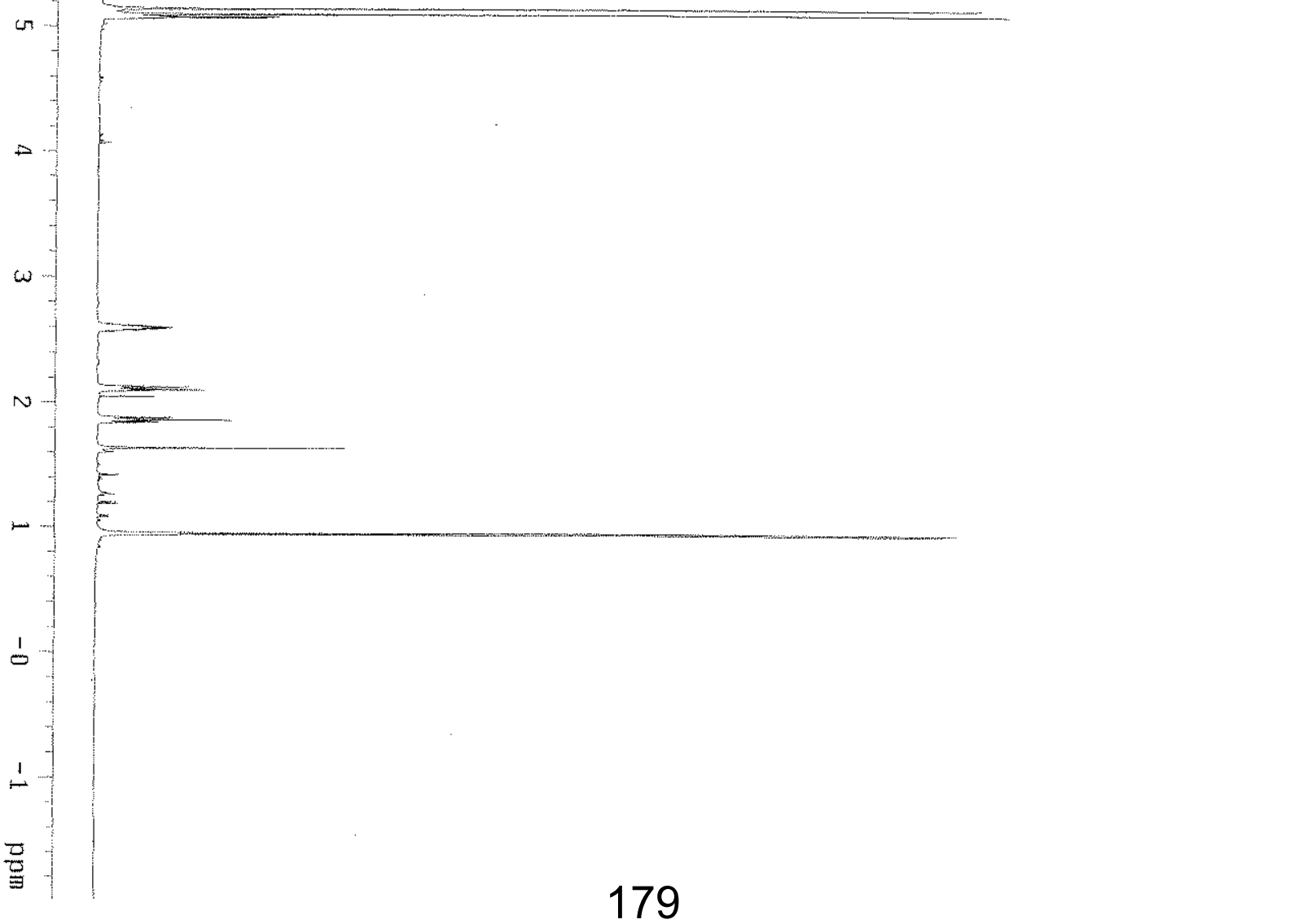




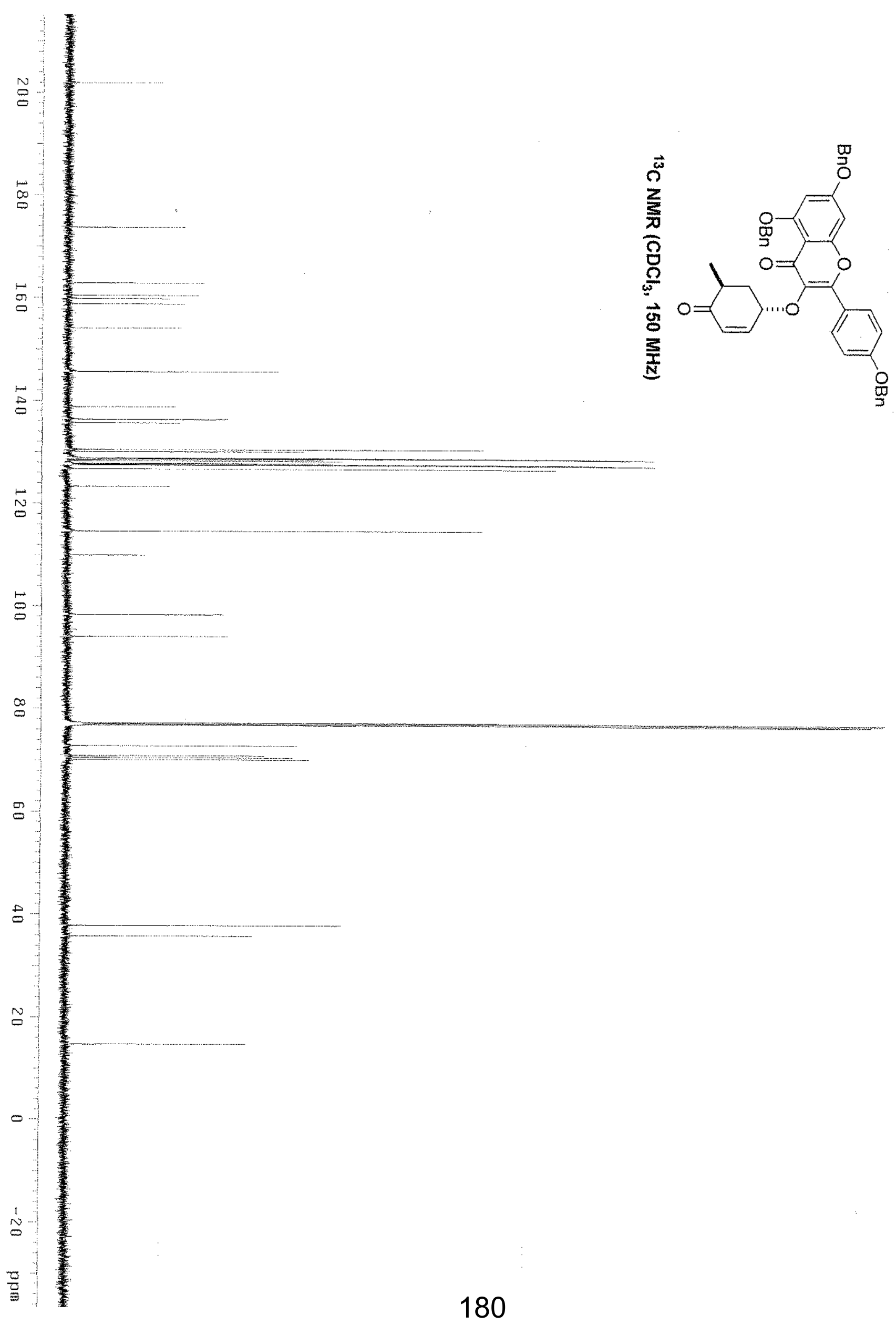




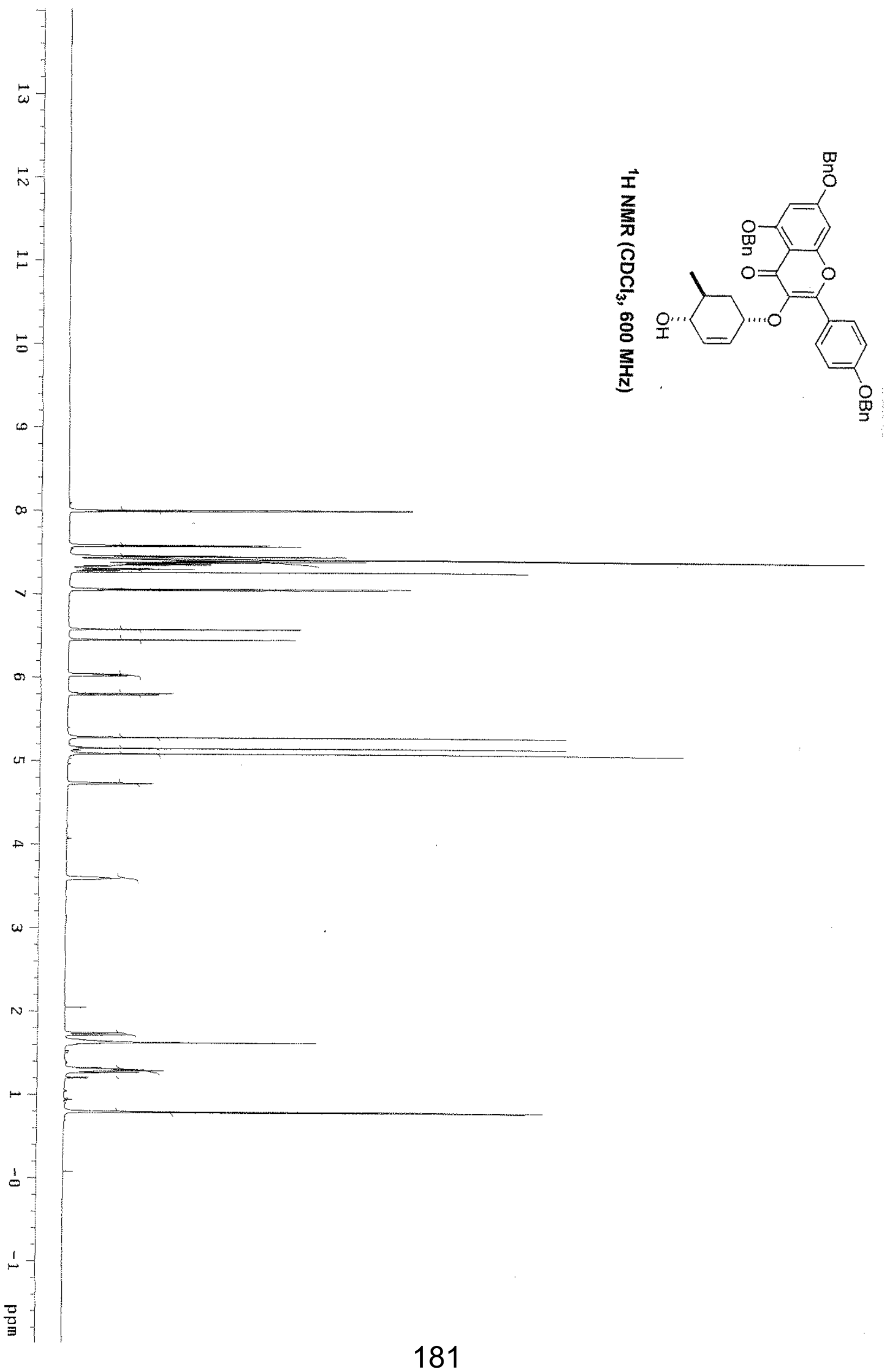




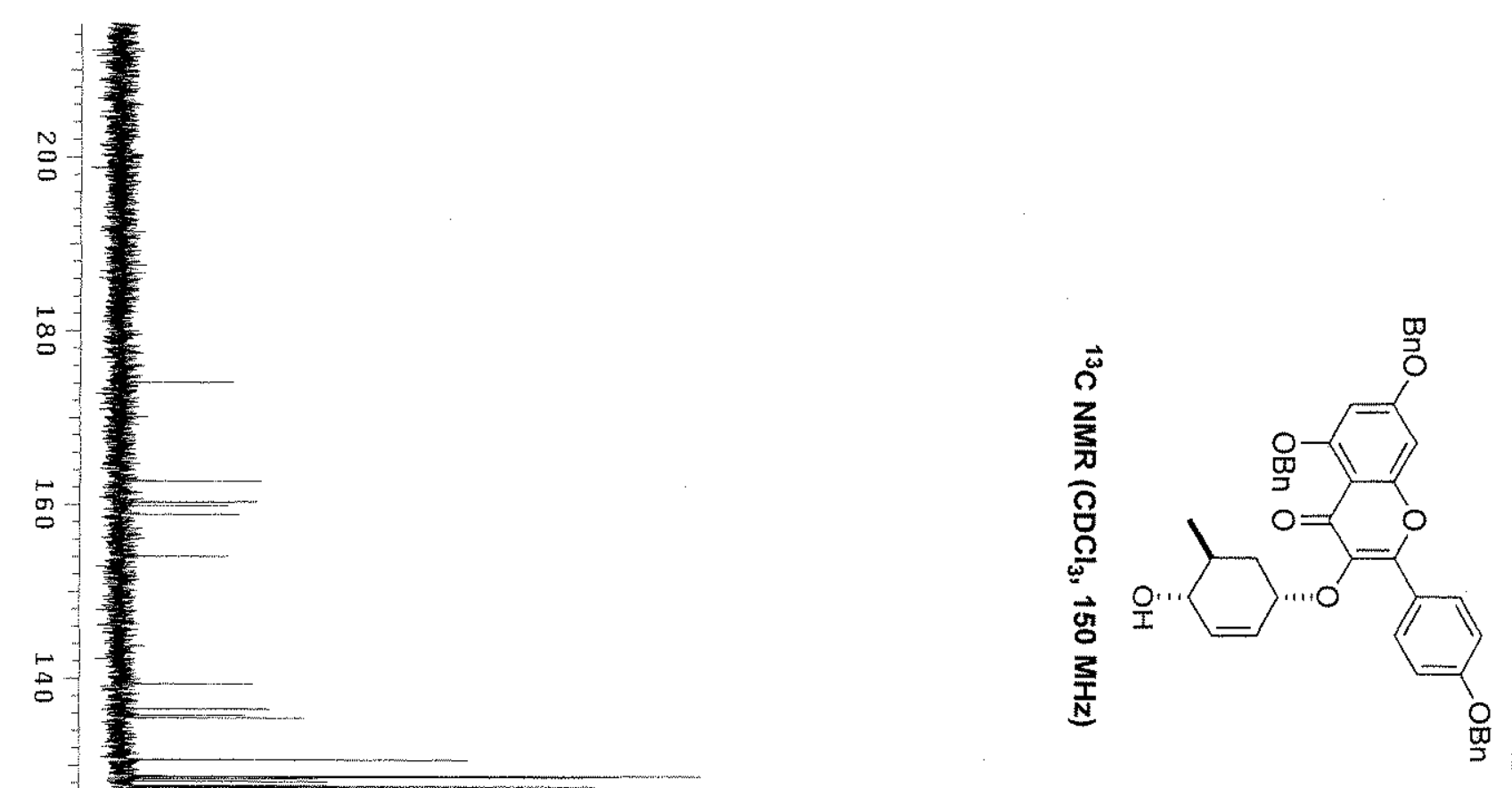




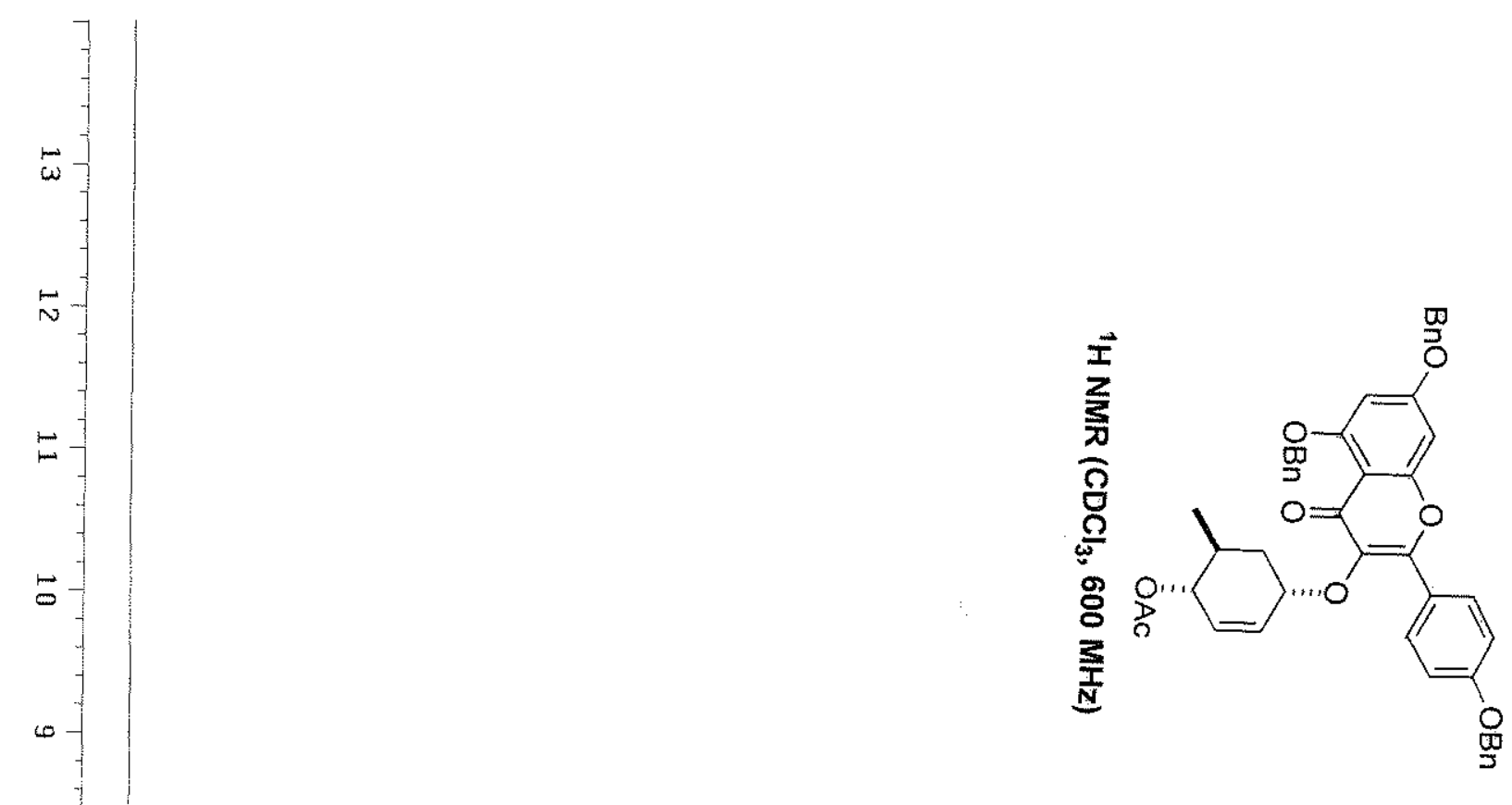




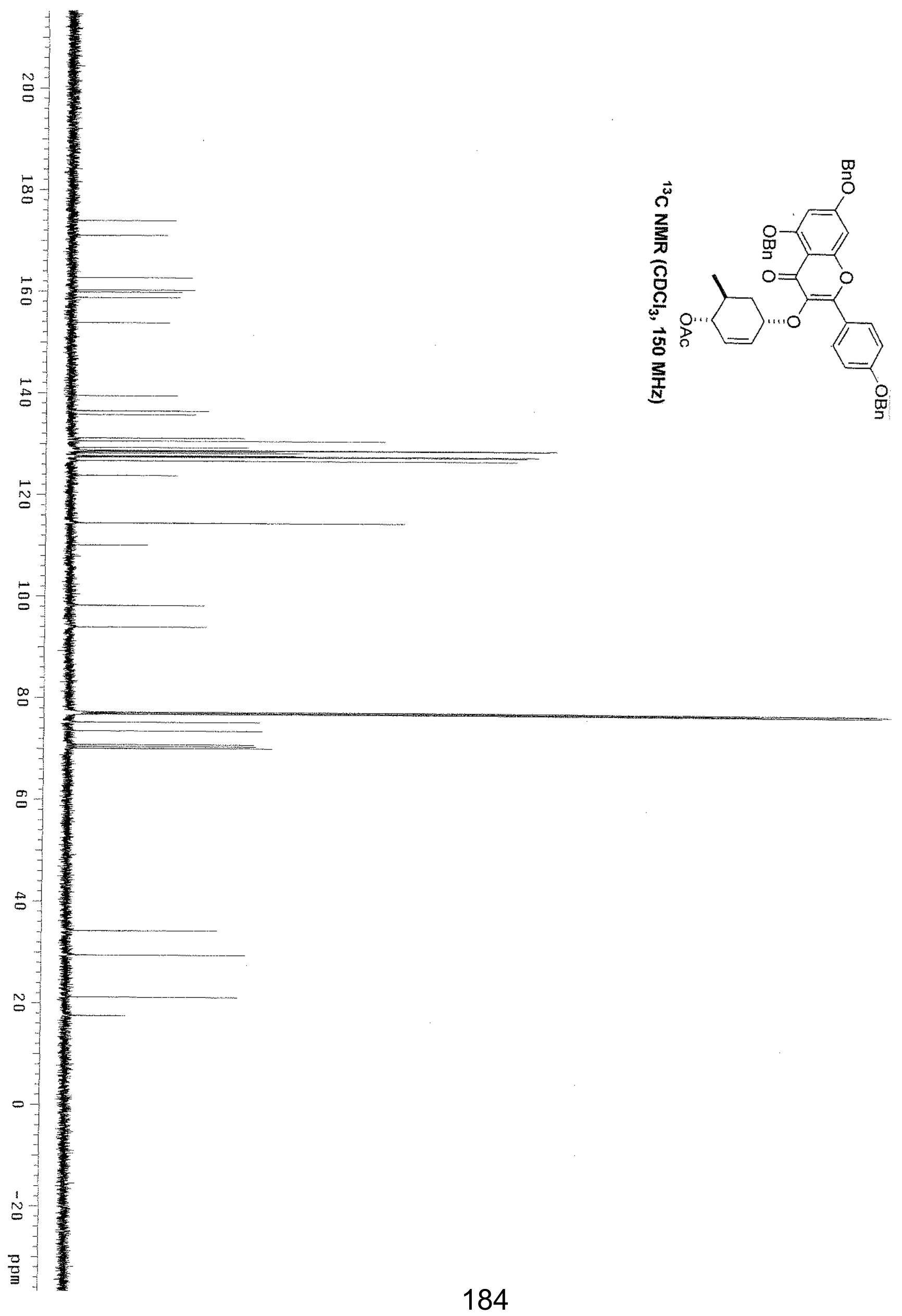



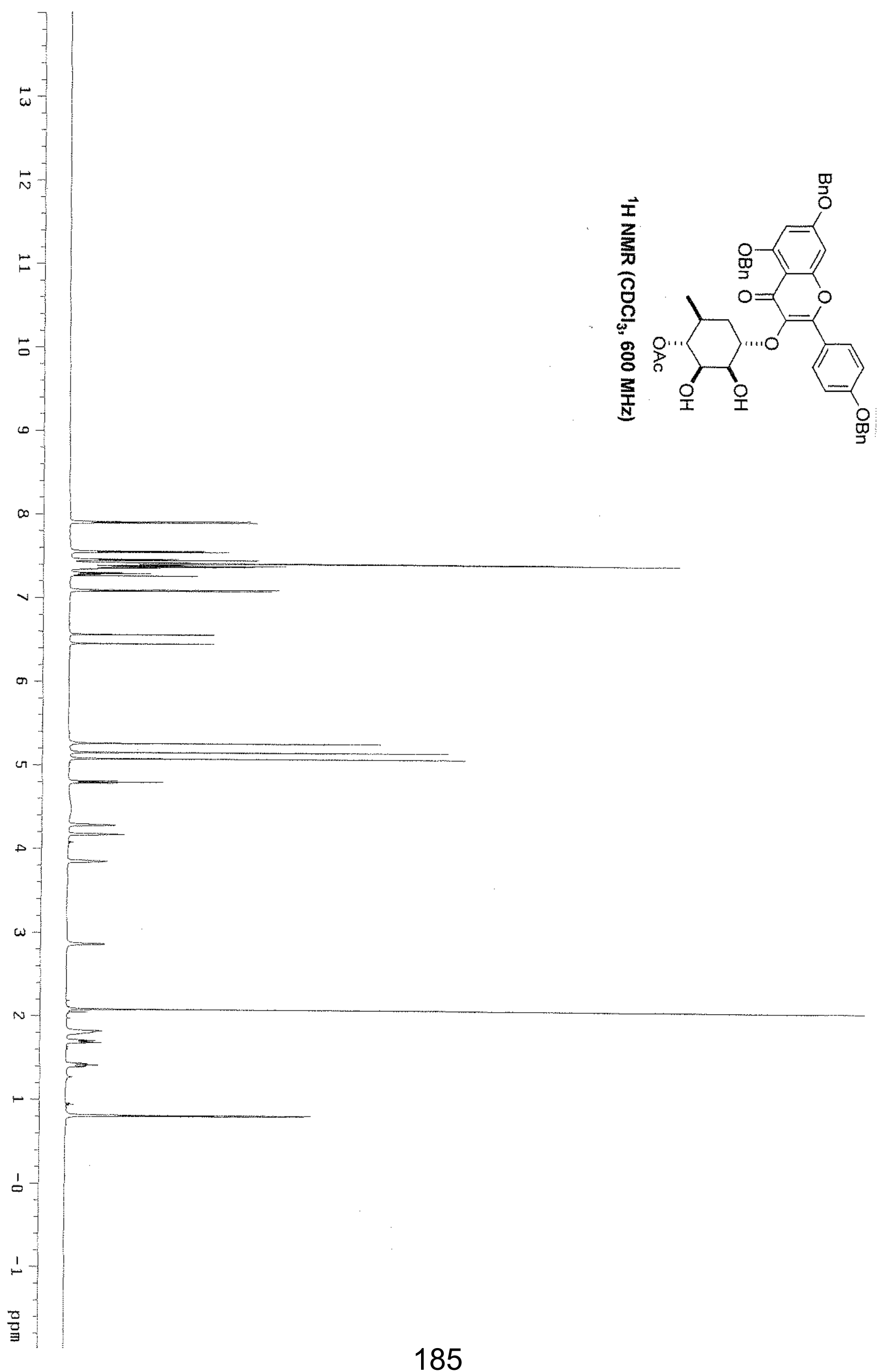


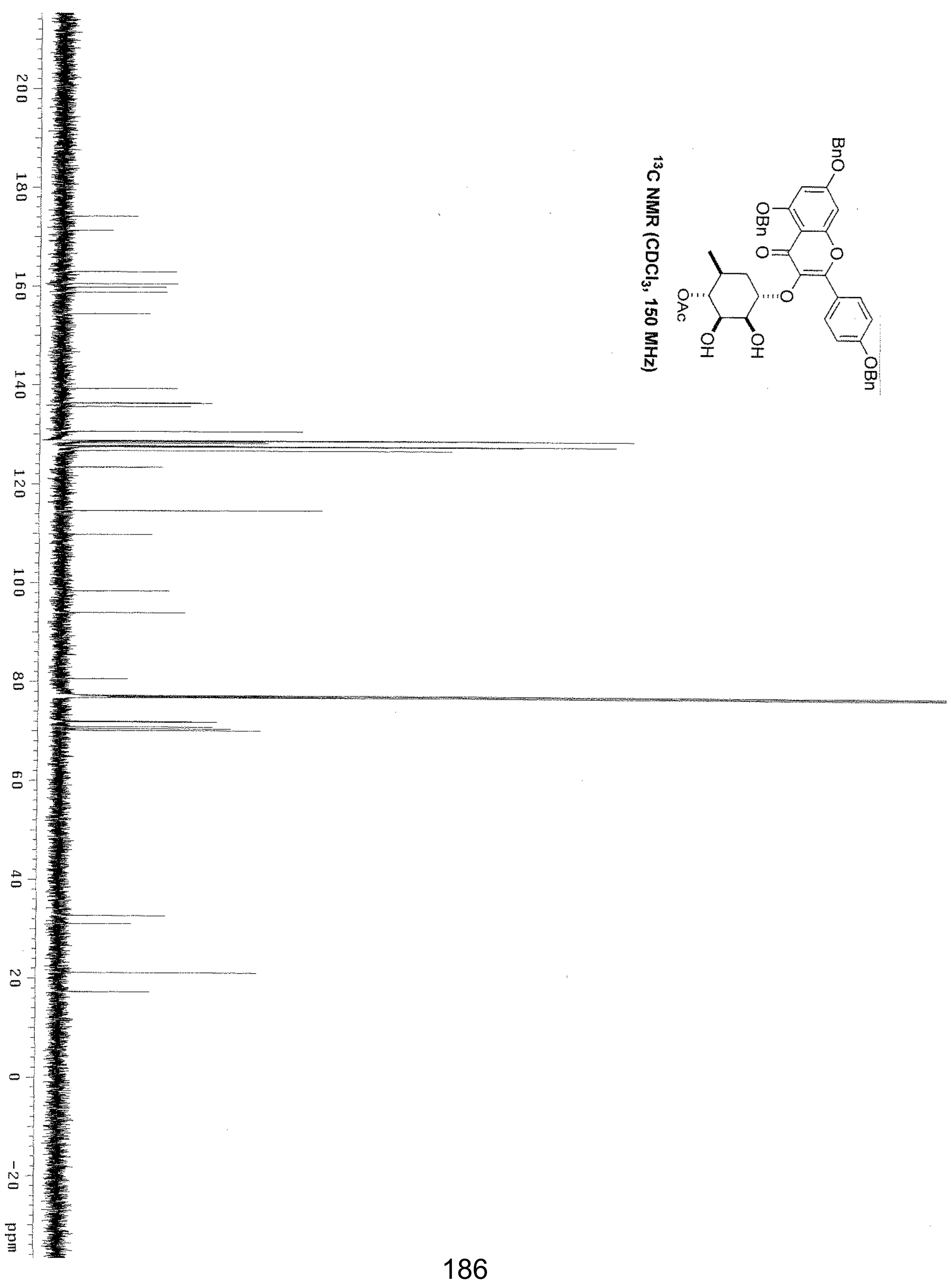




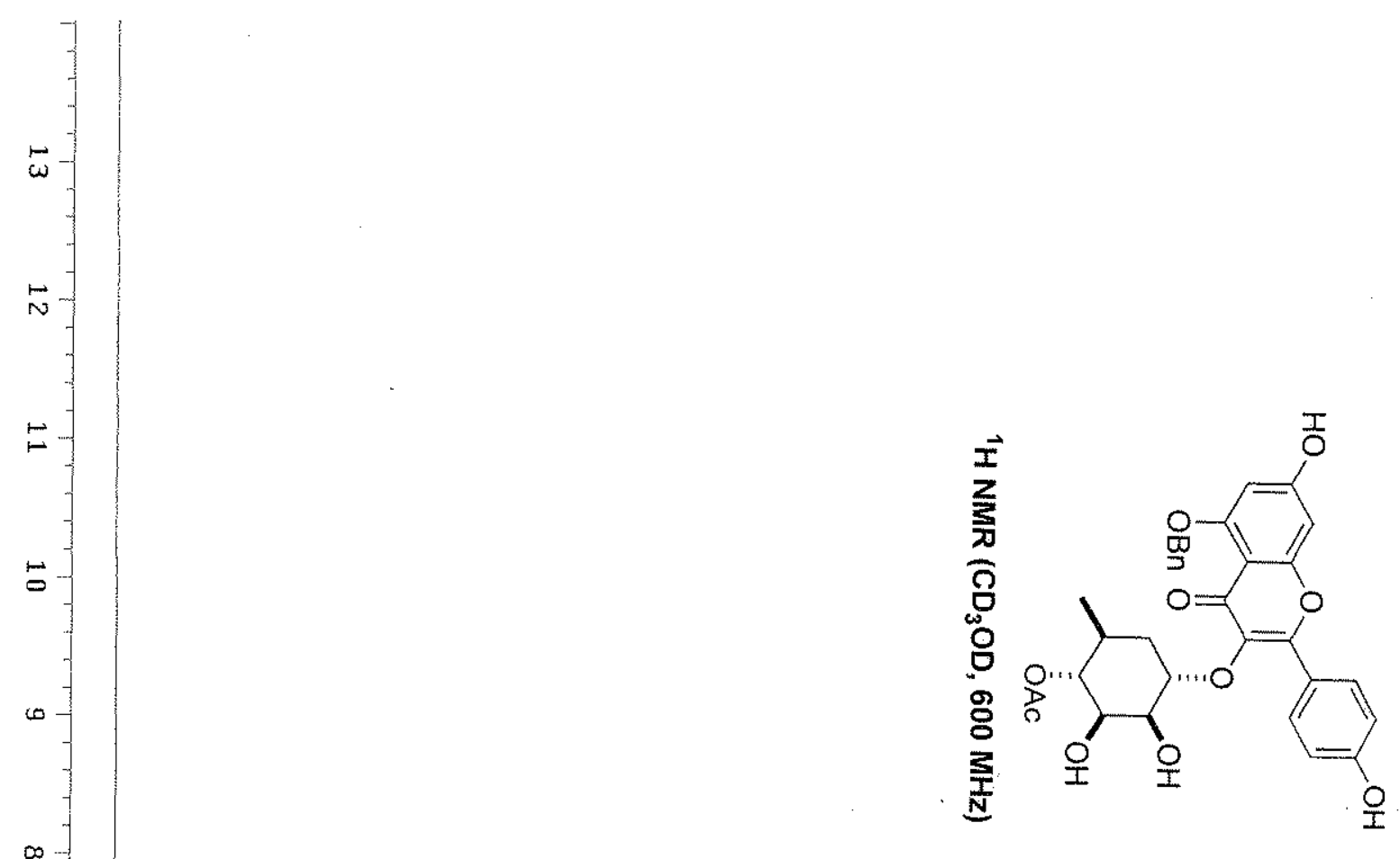




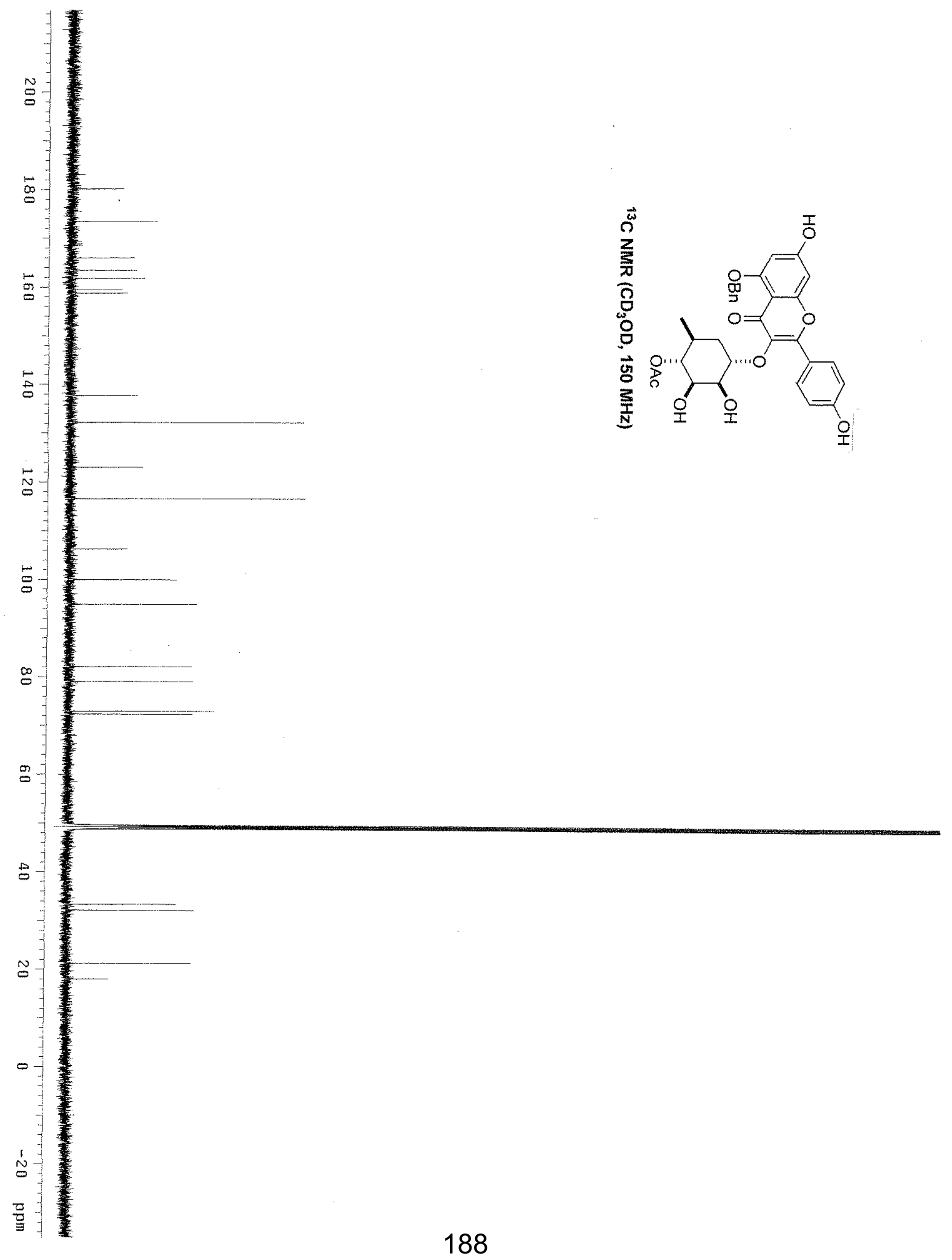



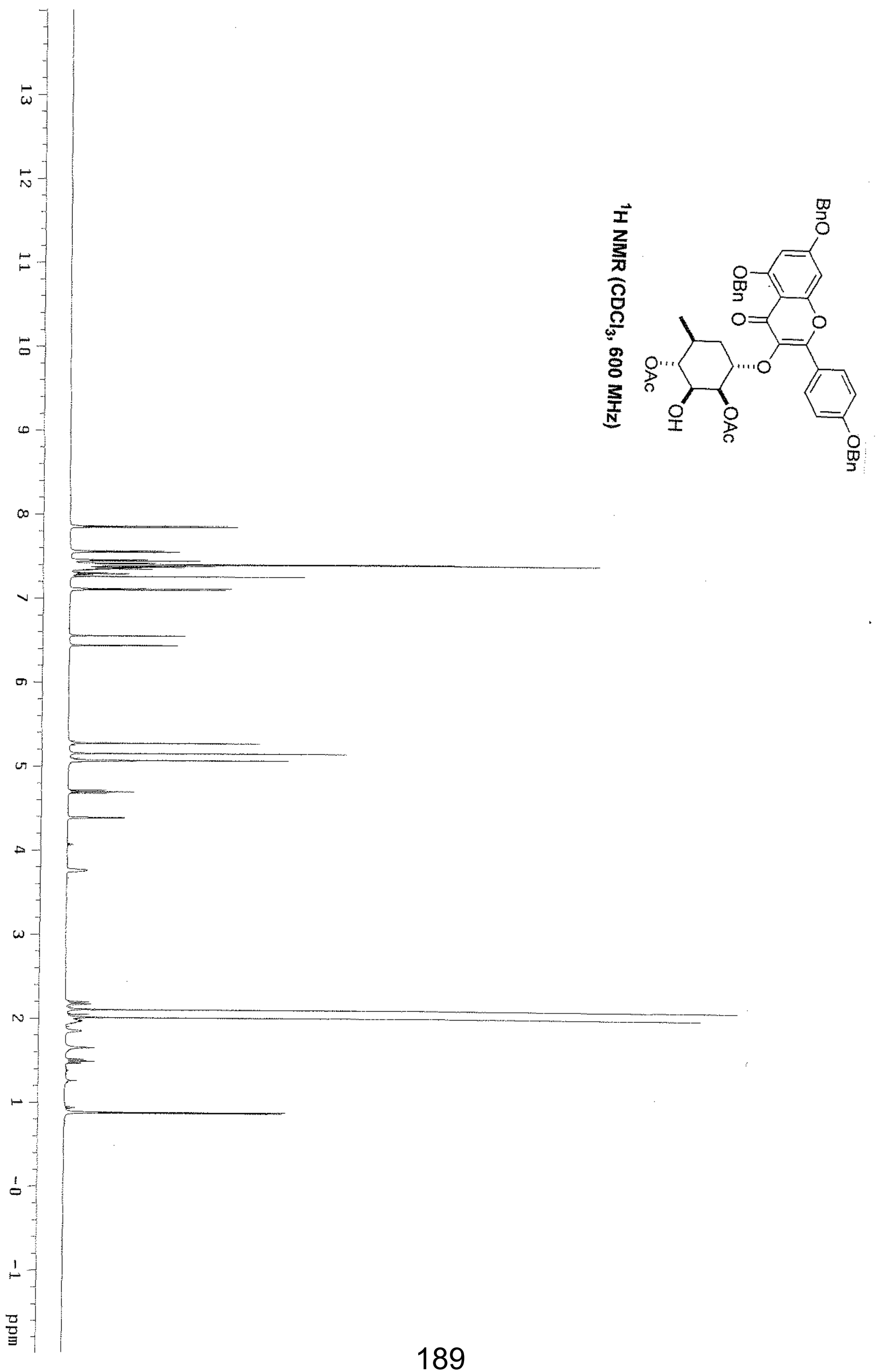


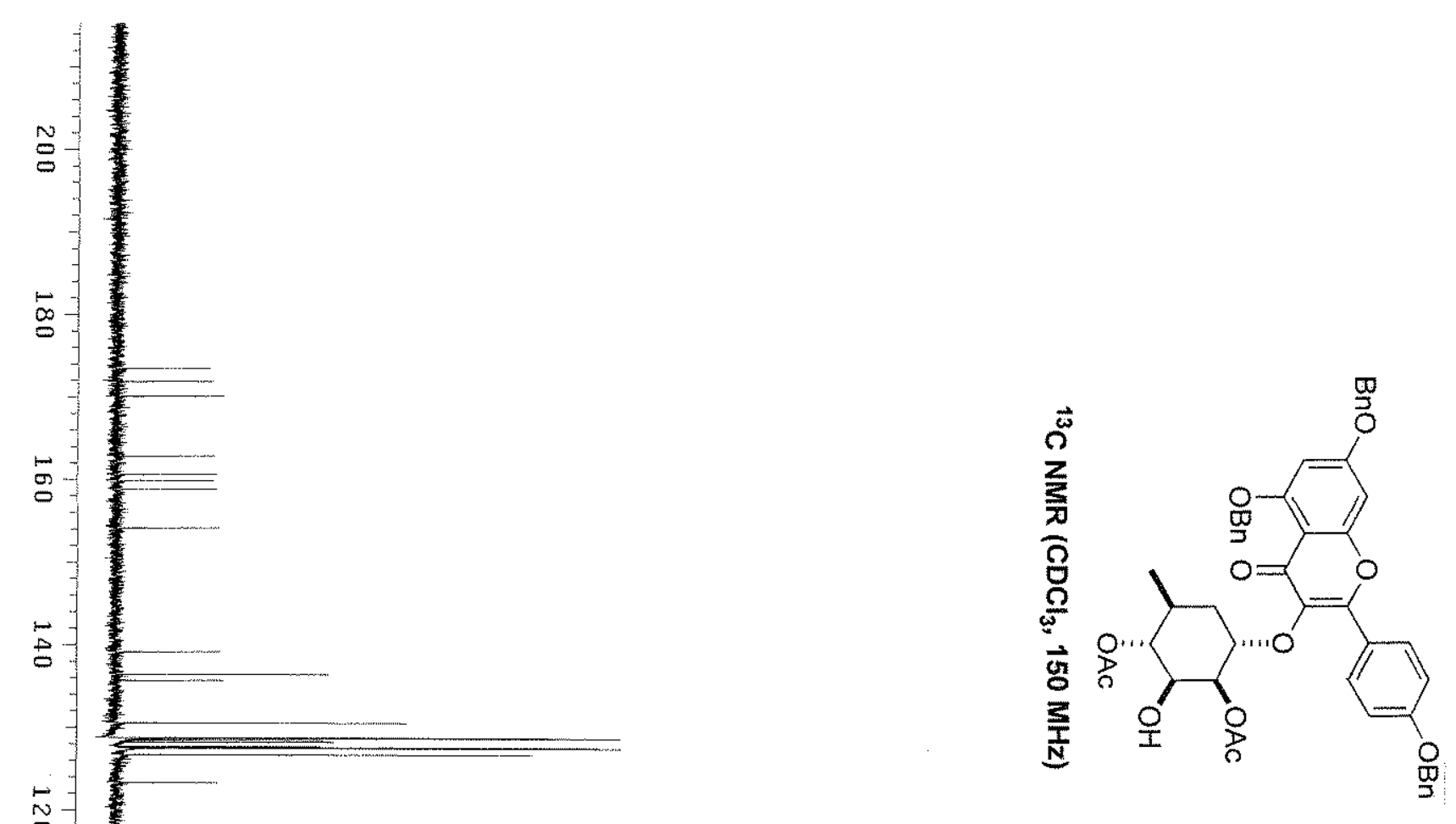



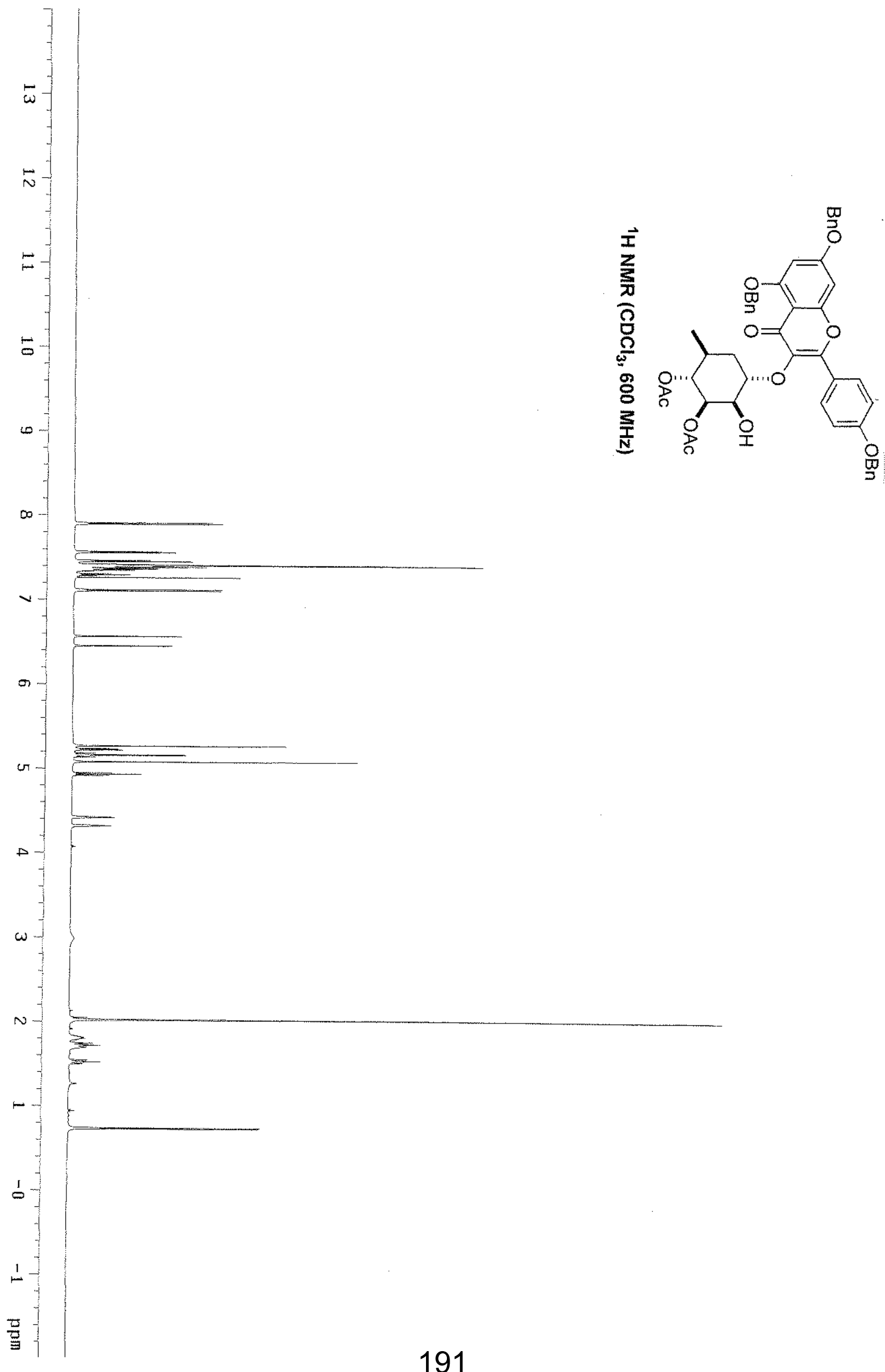


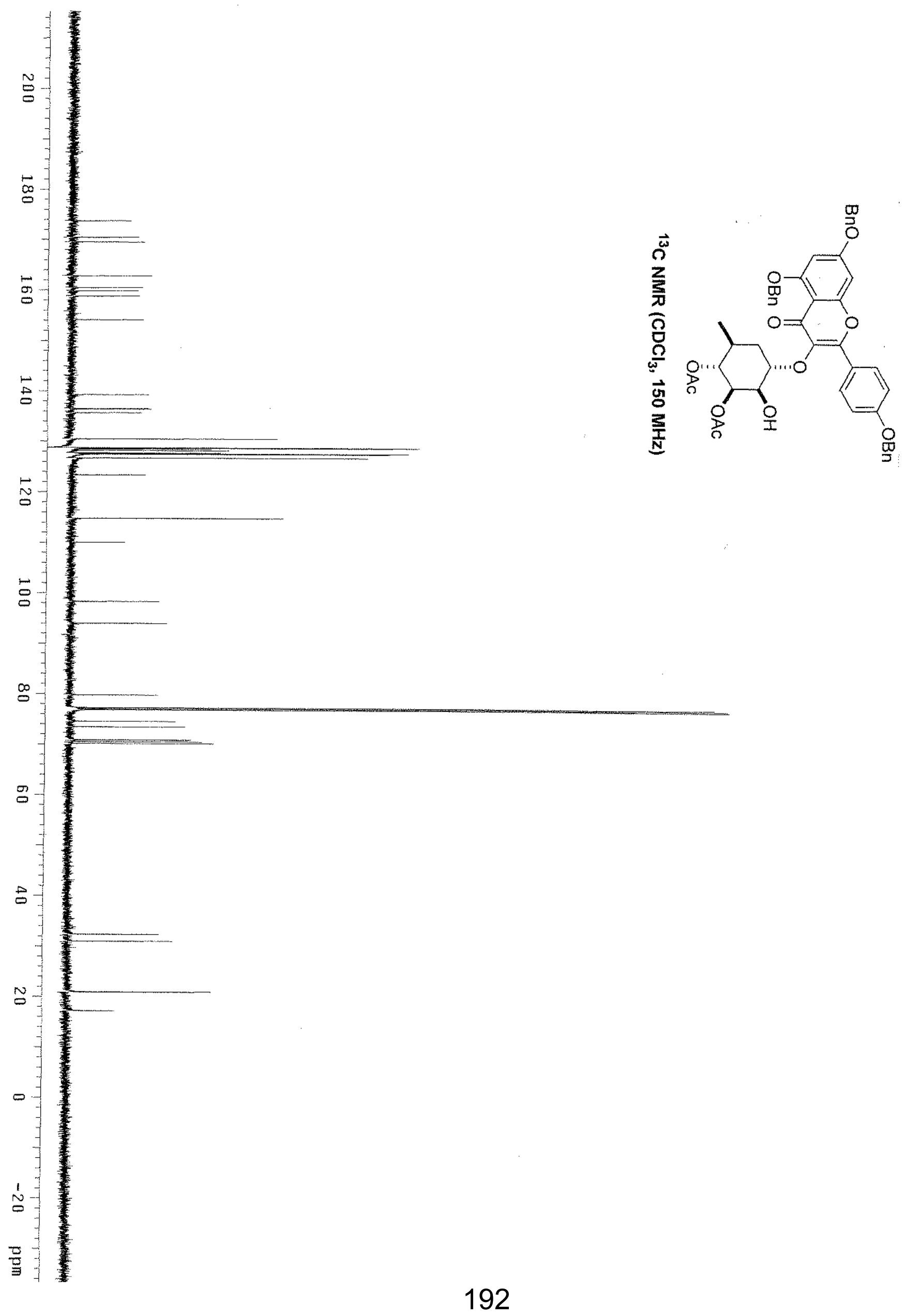




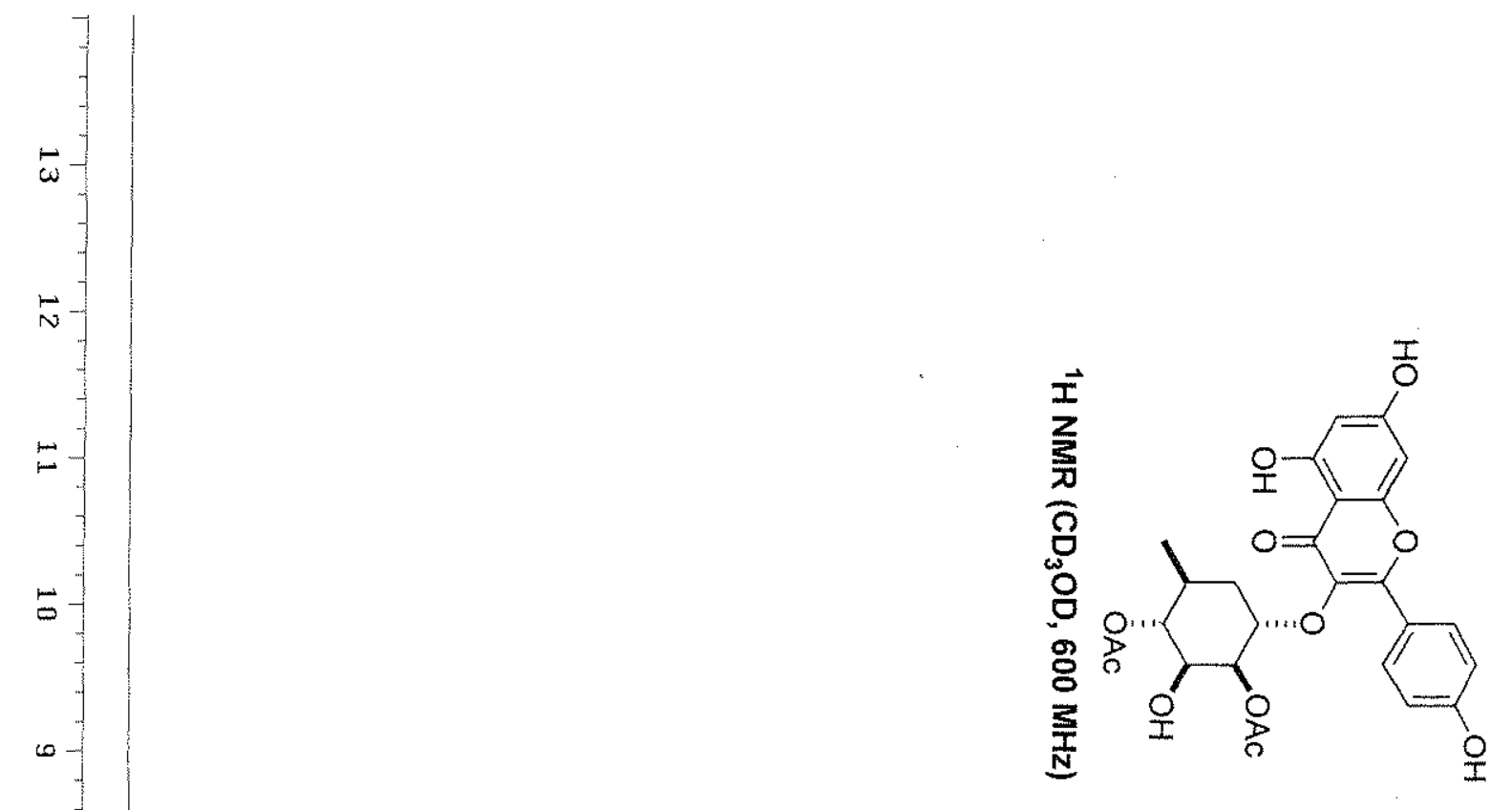




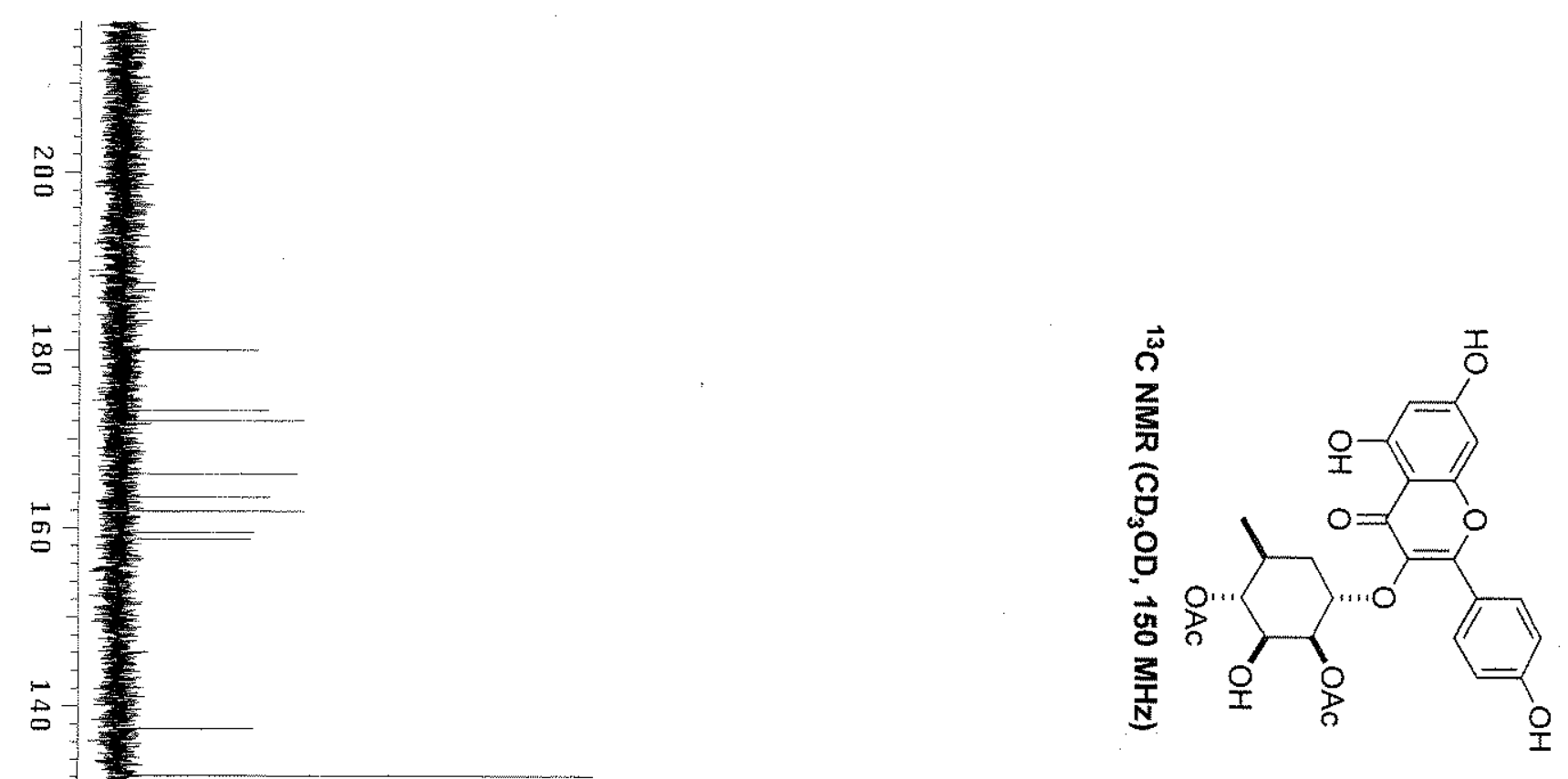



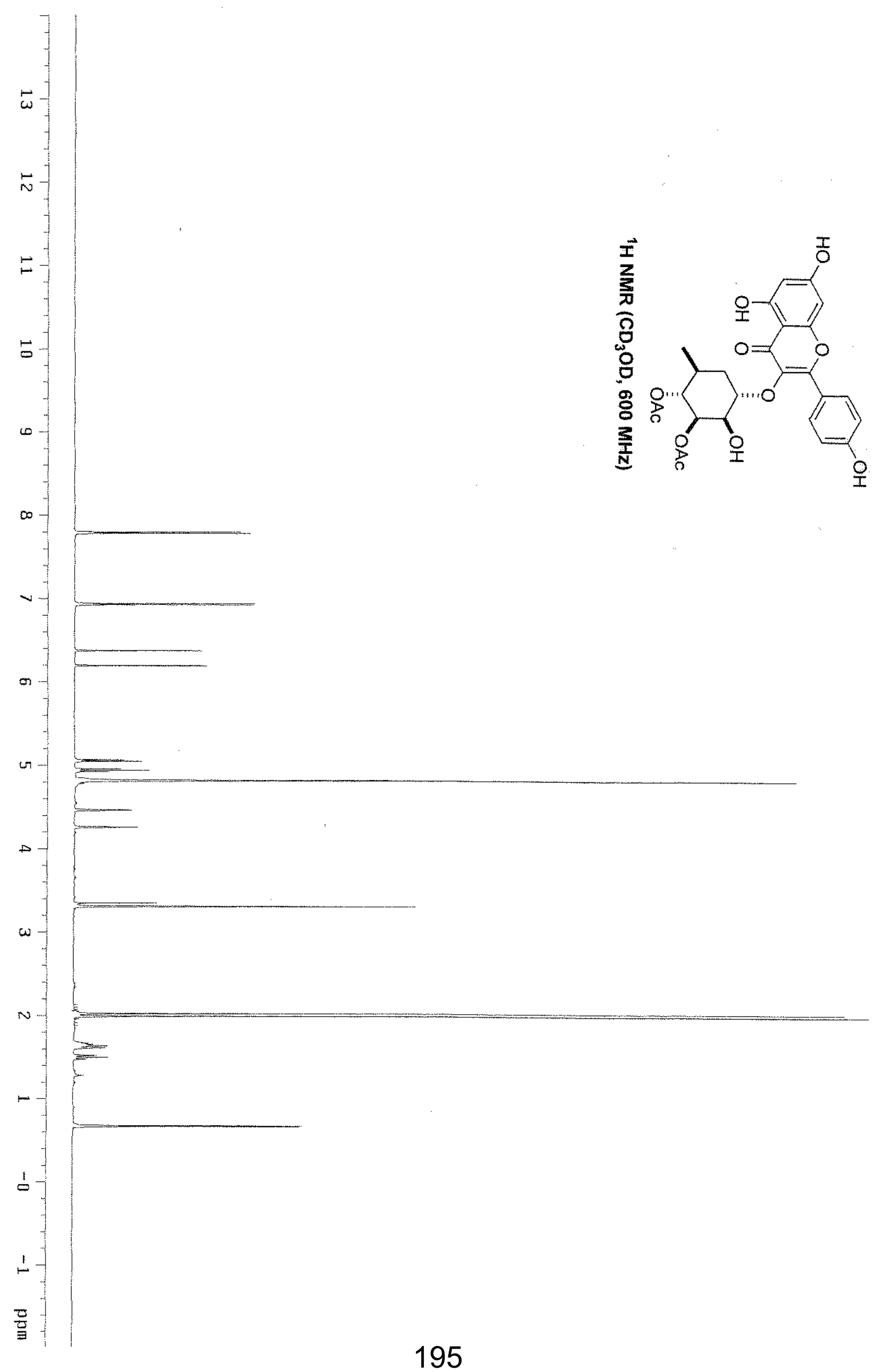


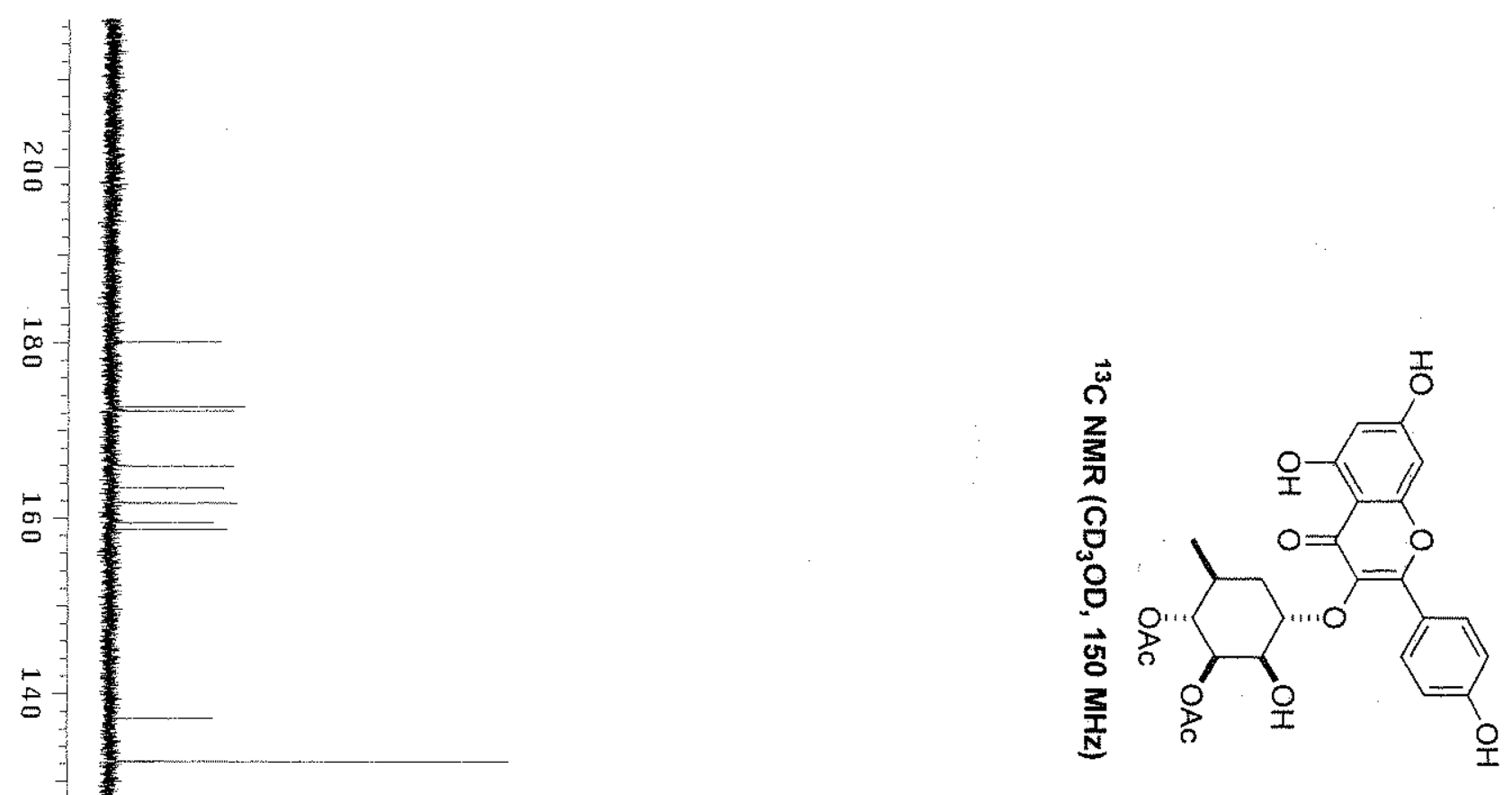

N.

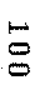

$\infty$

$\therefore=$

吕

$\approx-$

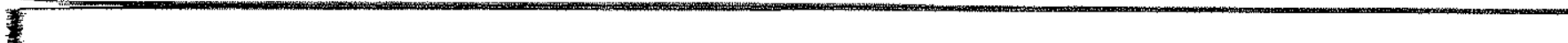

:

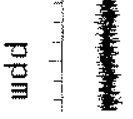




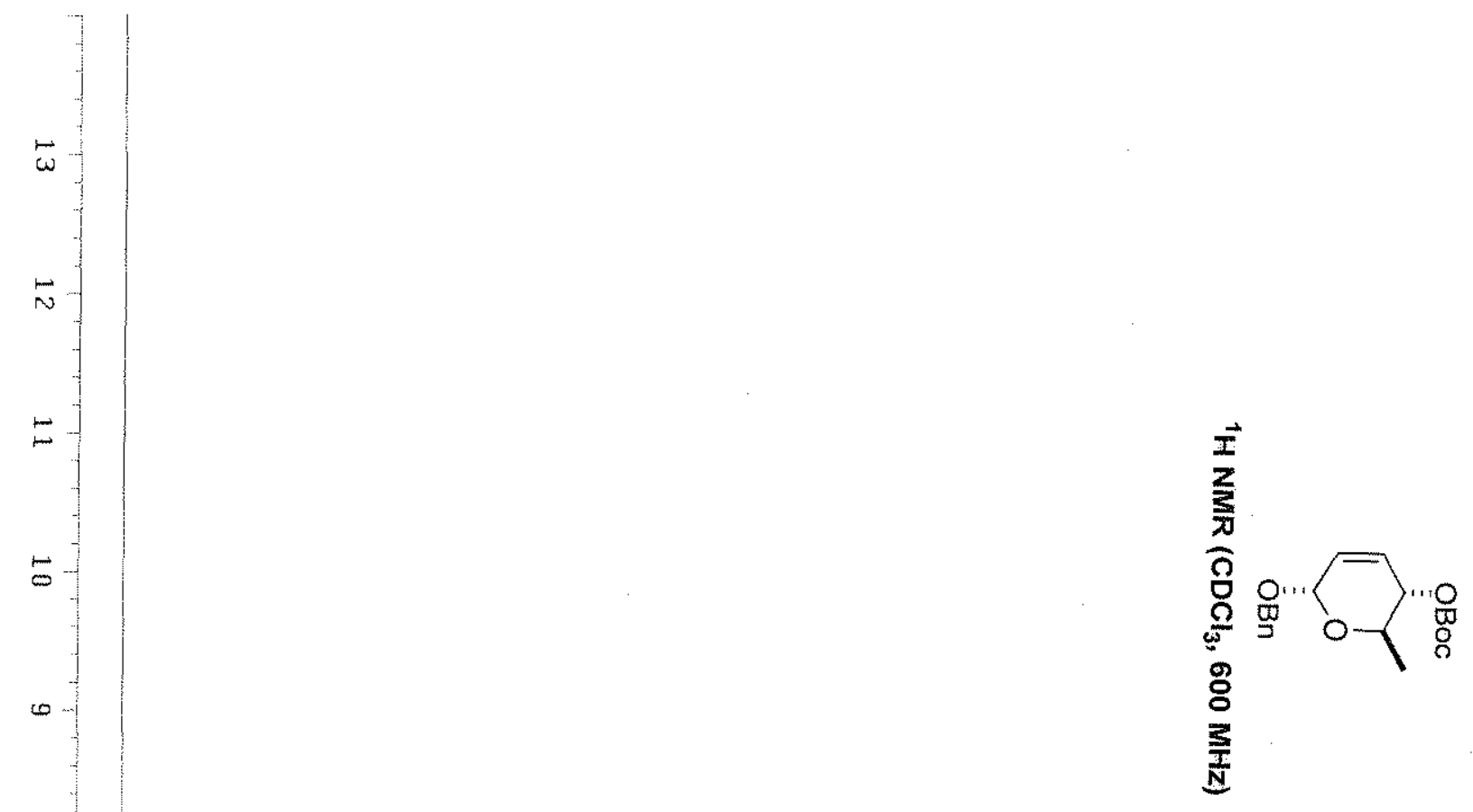

$\infty$
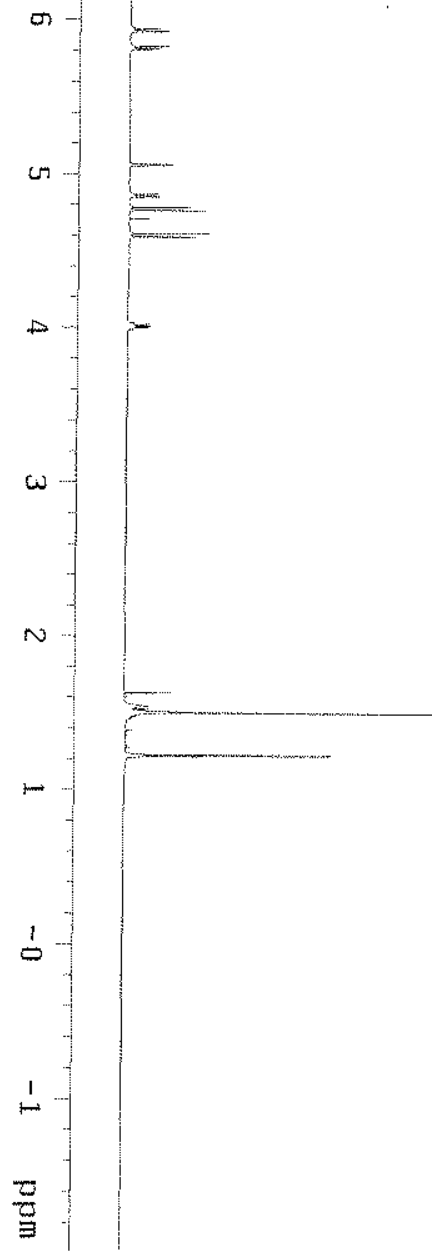


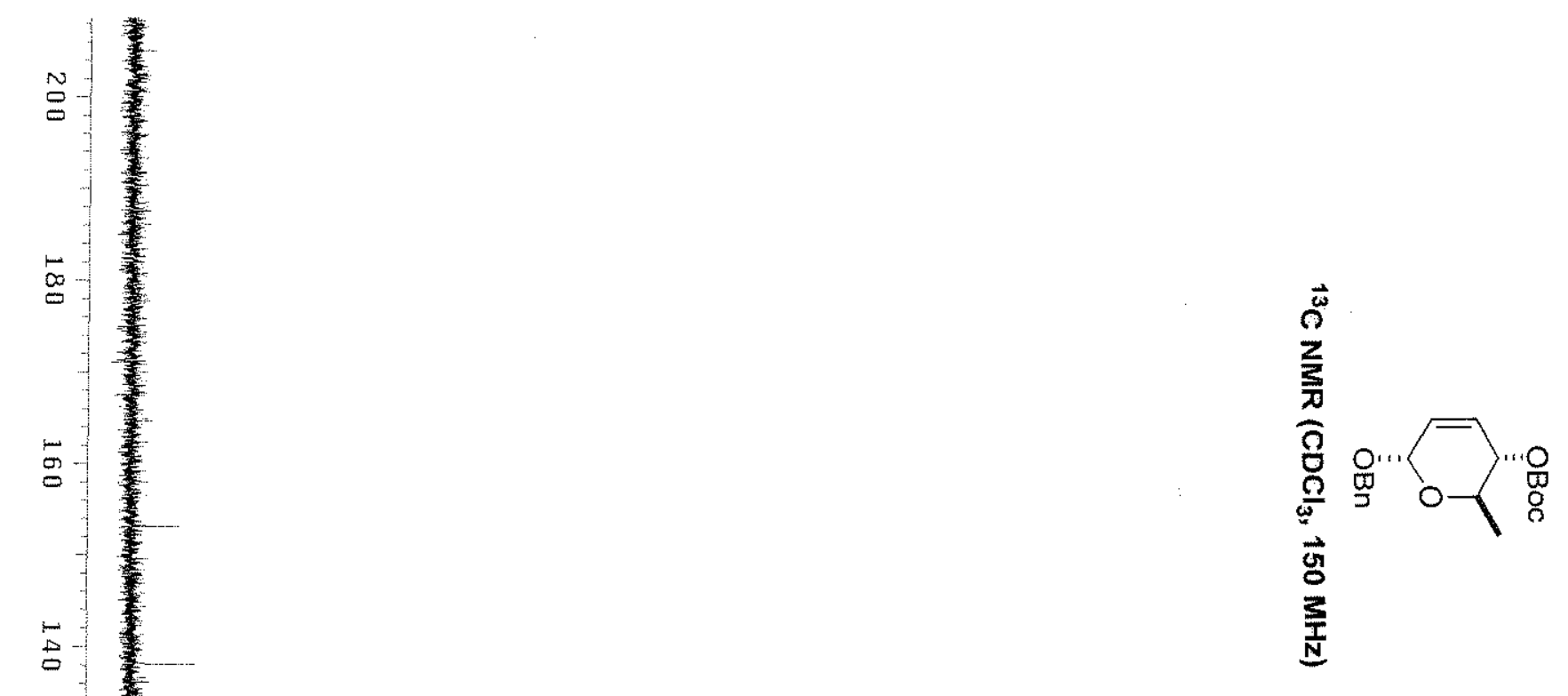

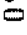

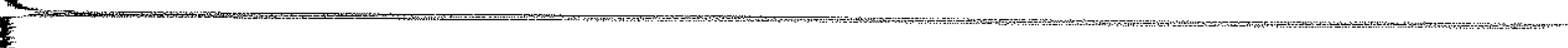

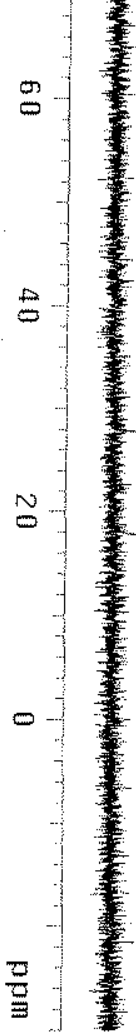




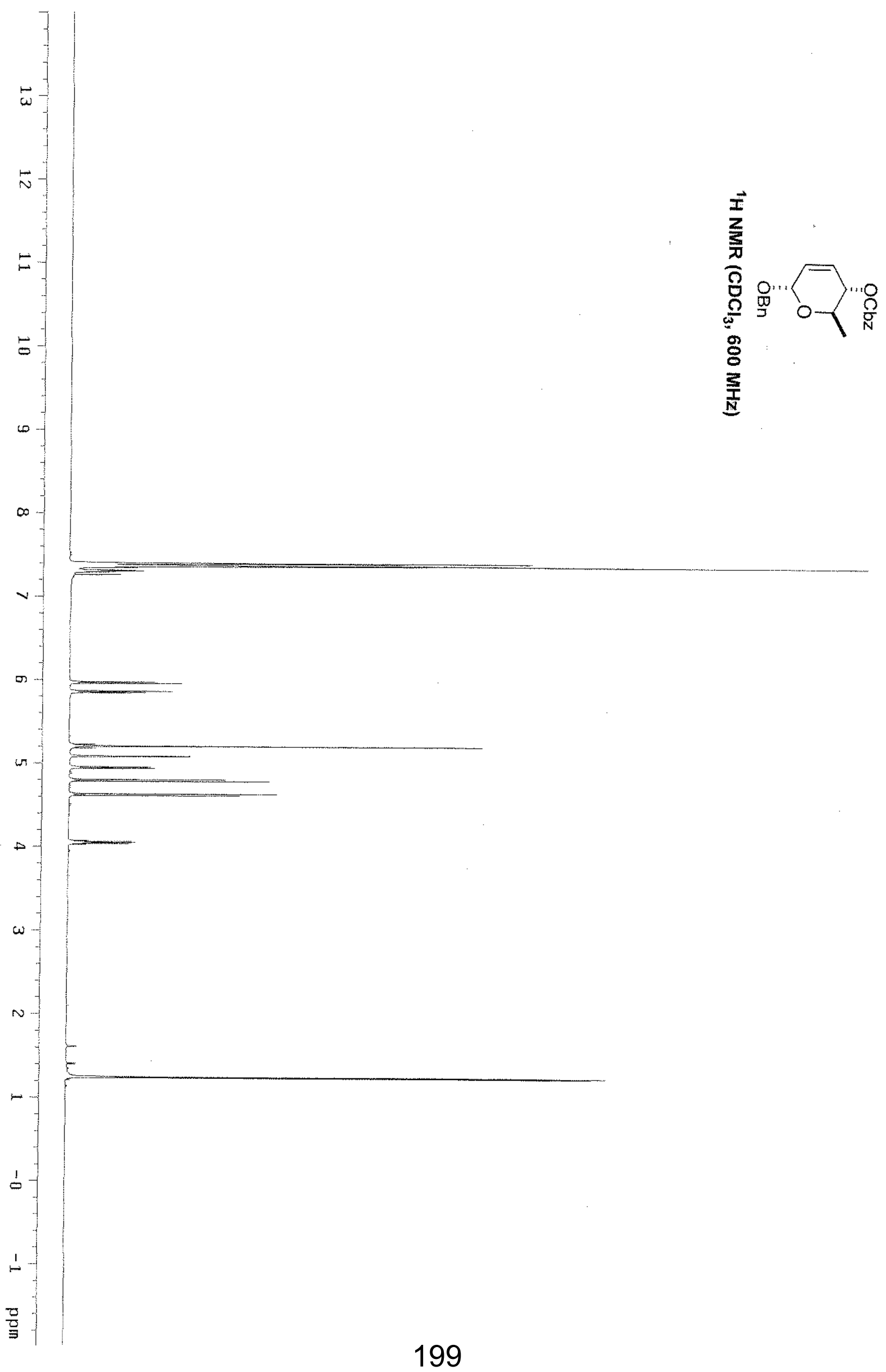




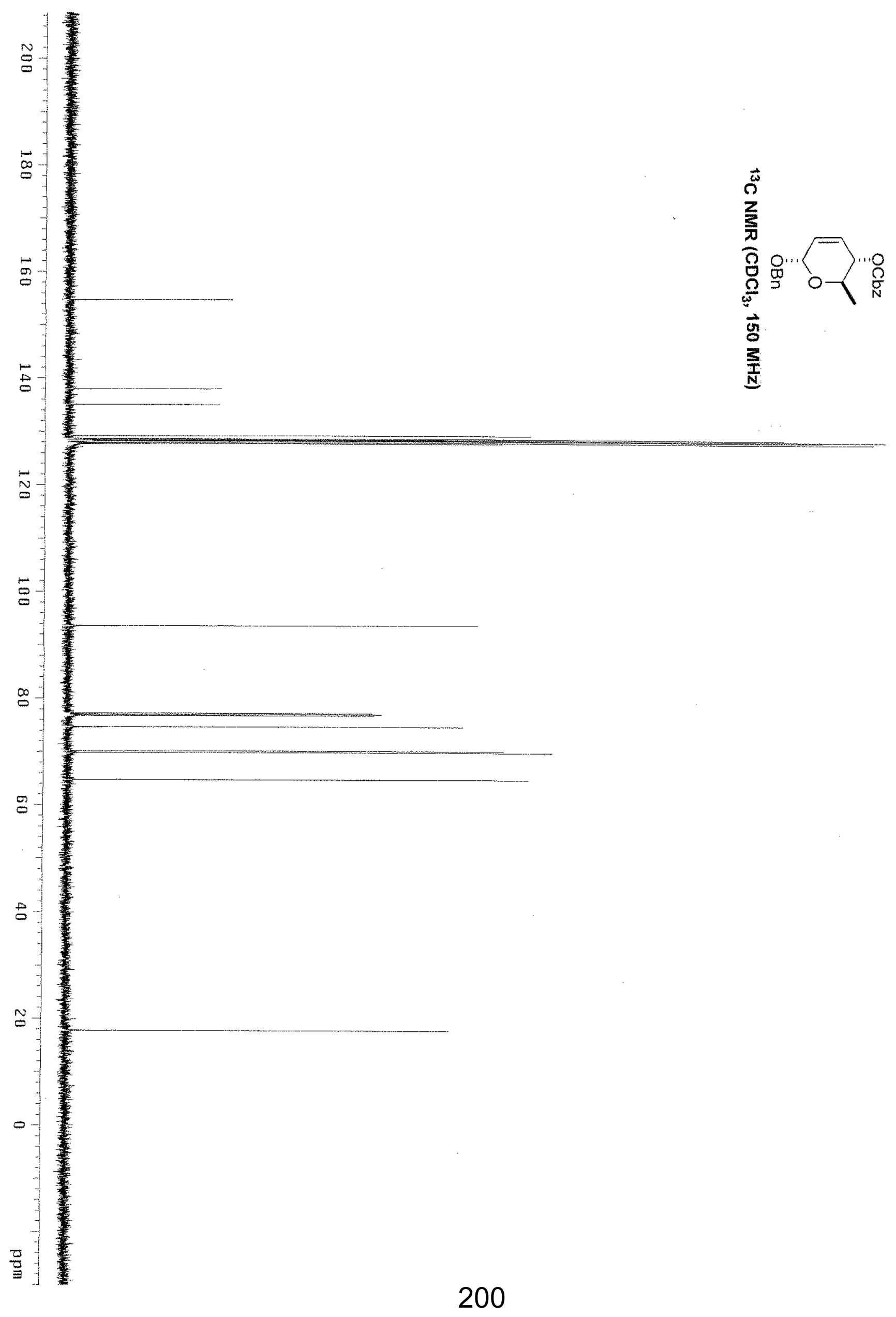




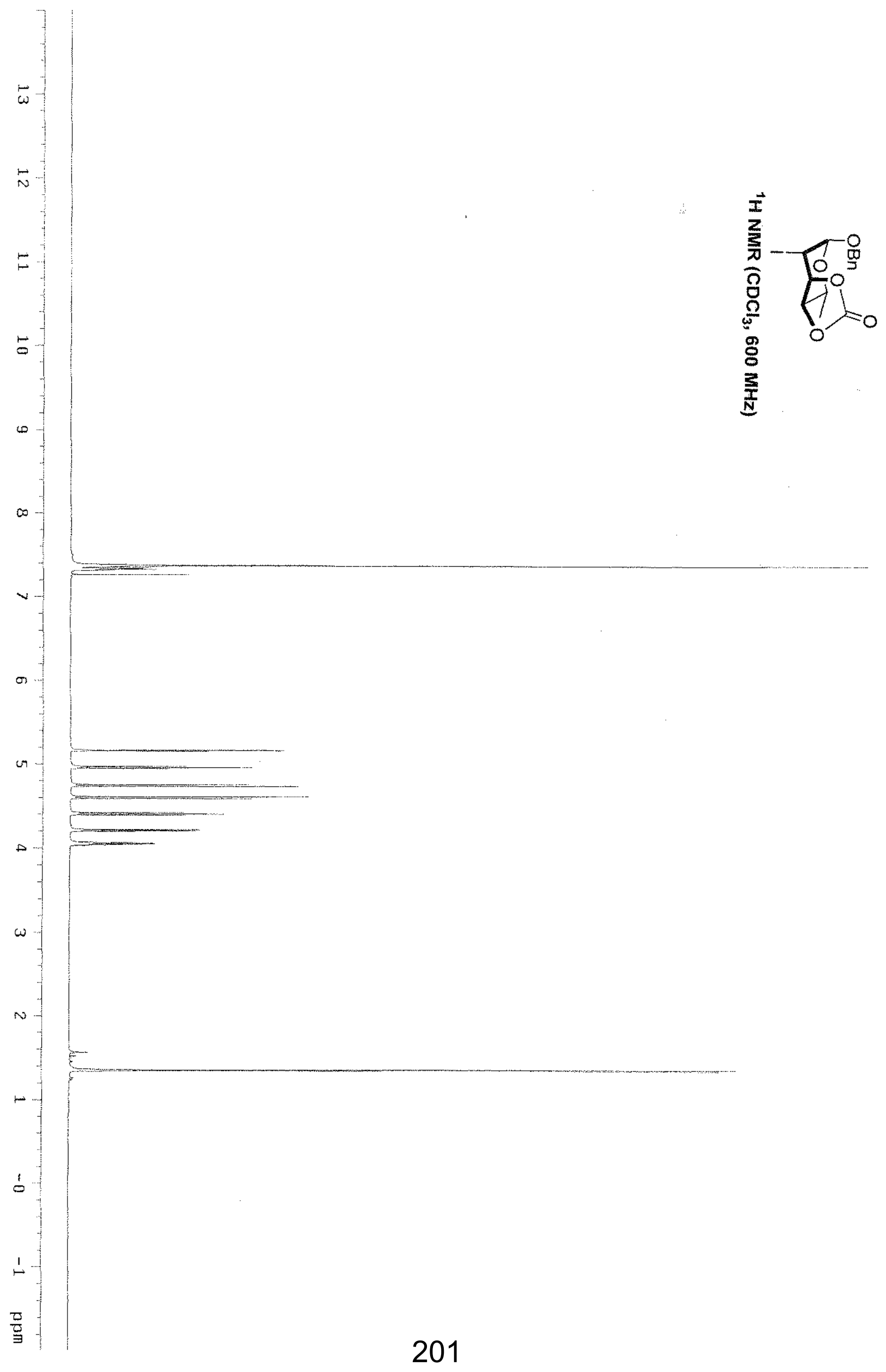




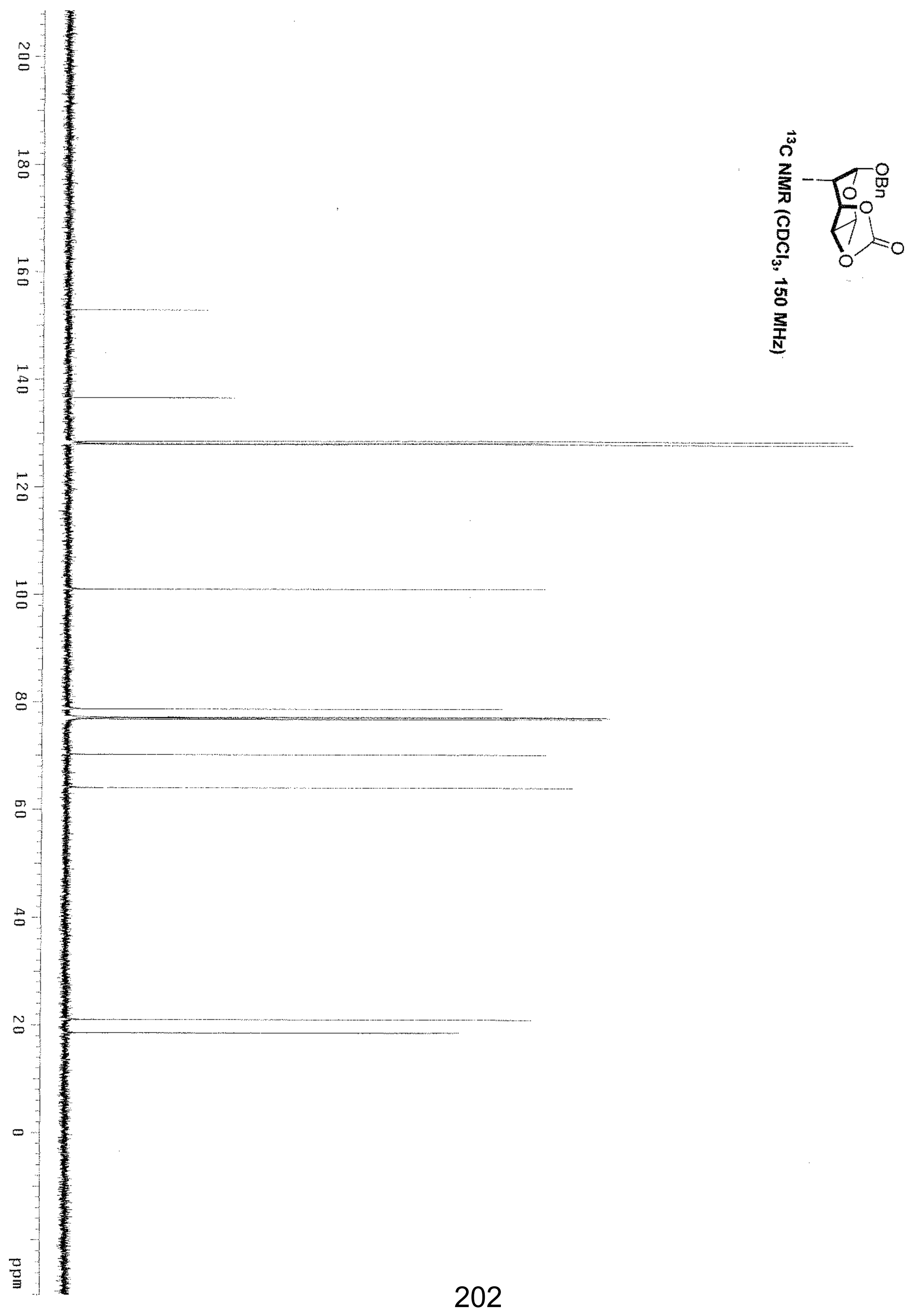




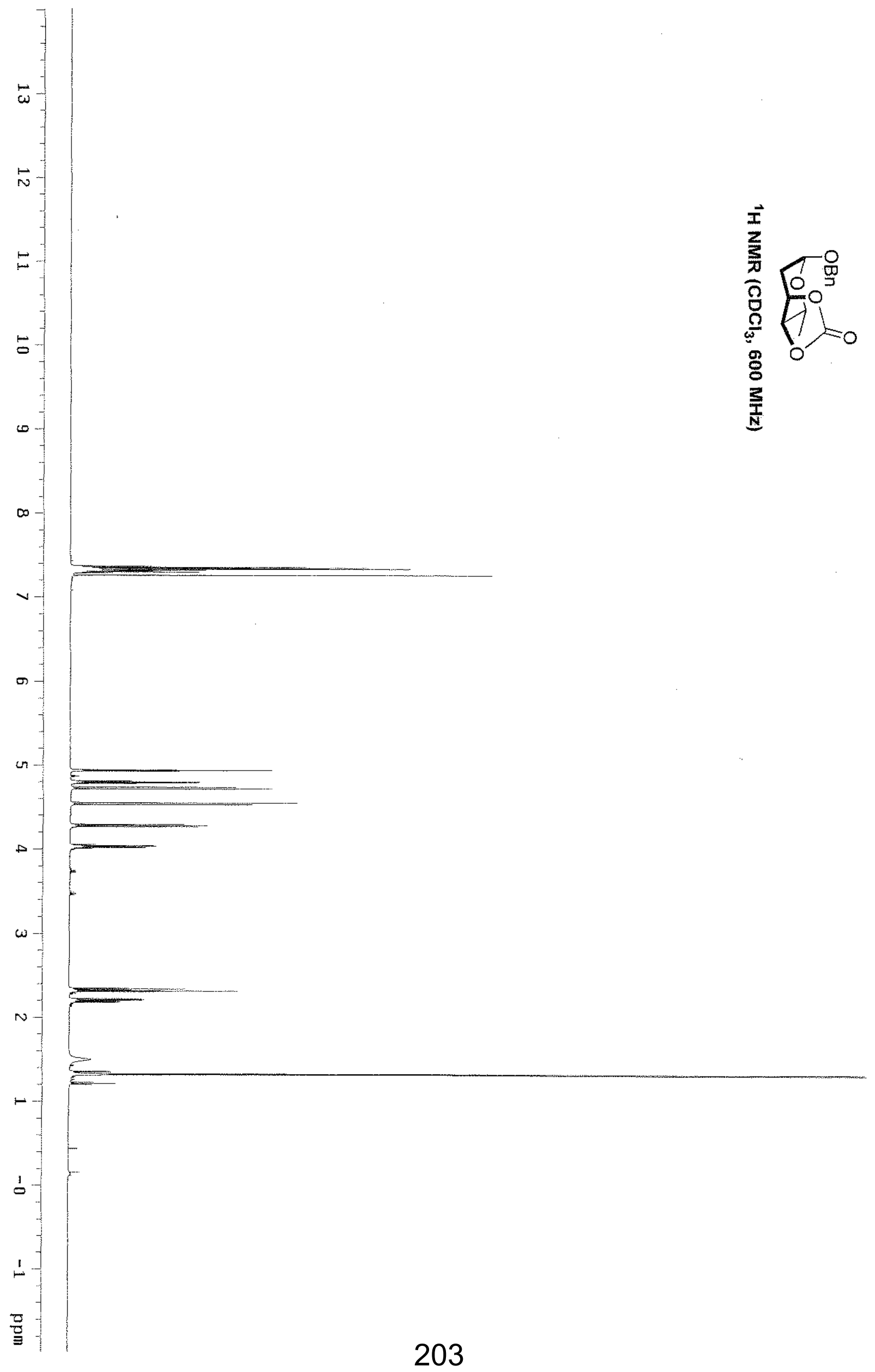



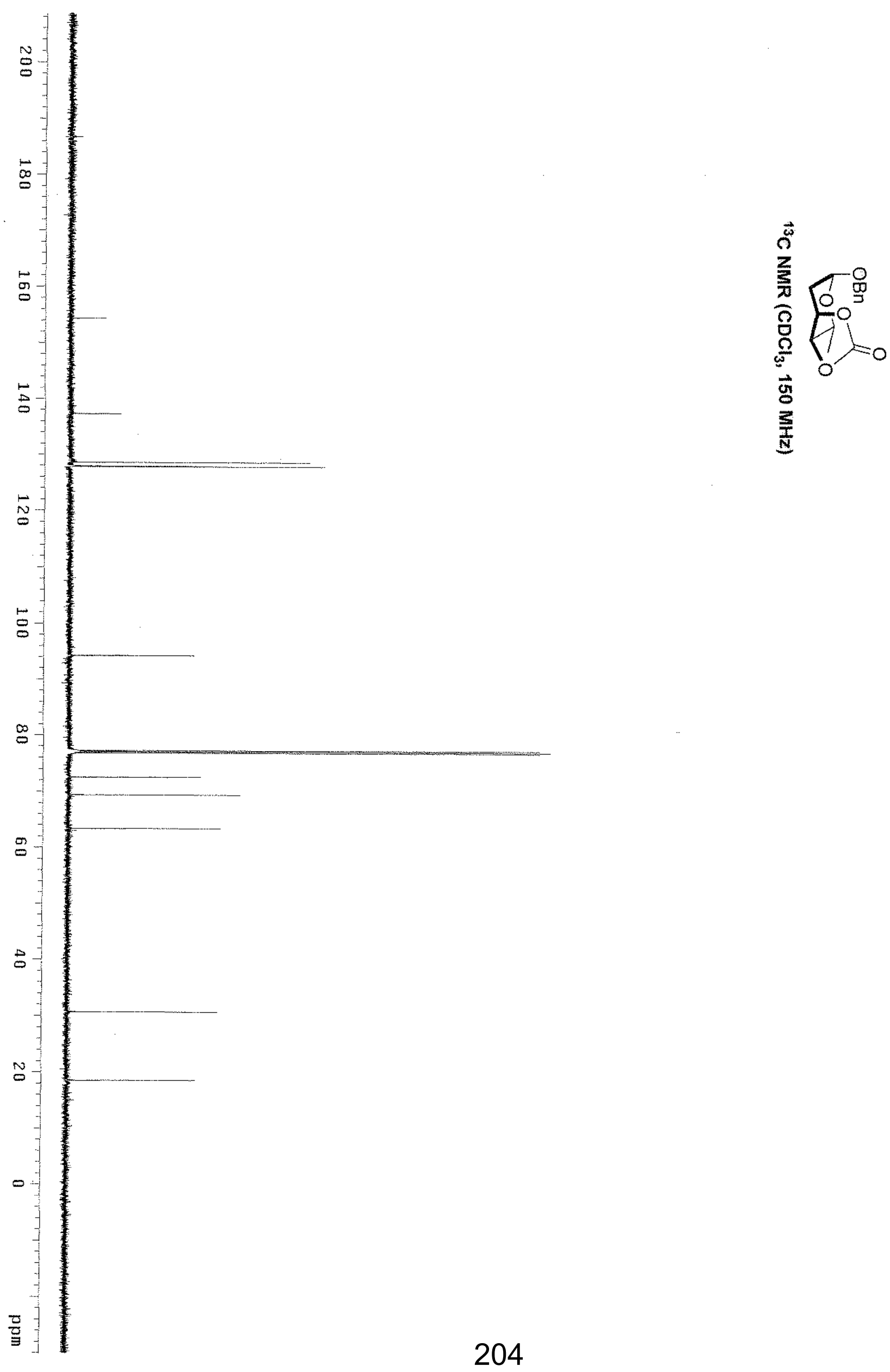


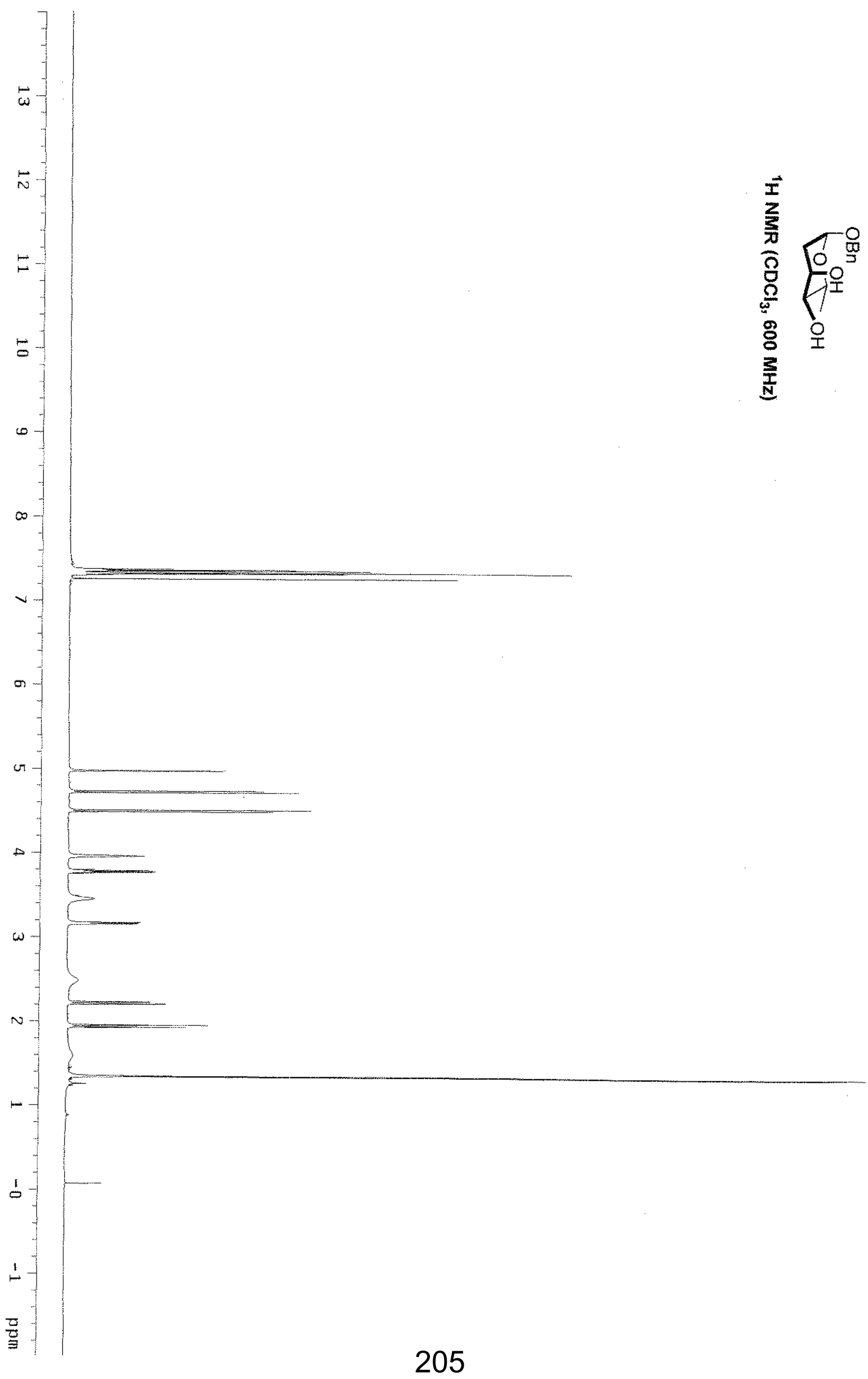




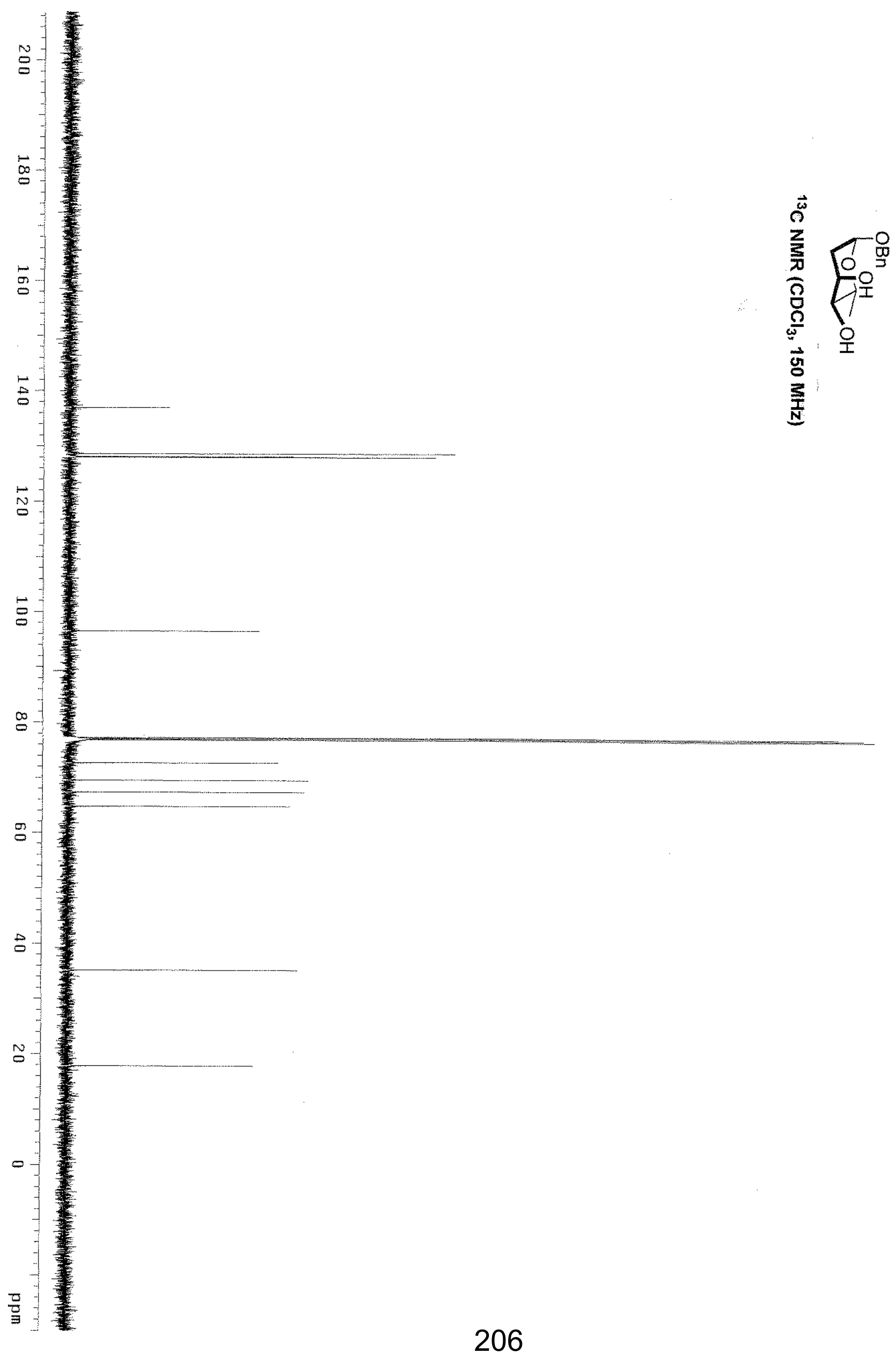




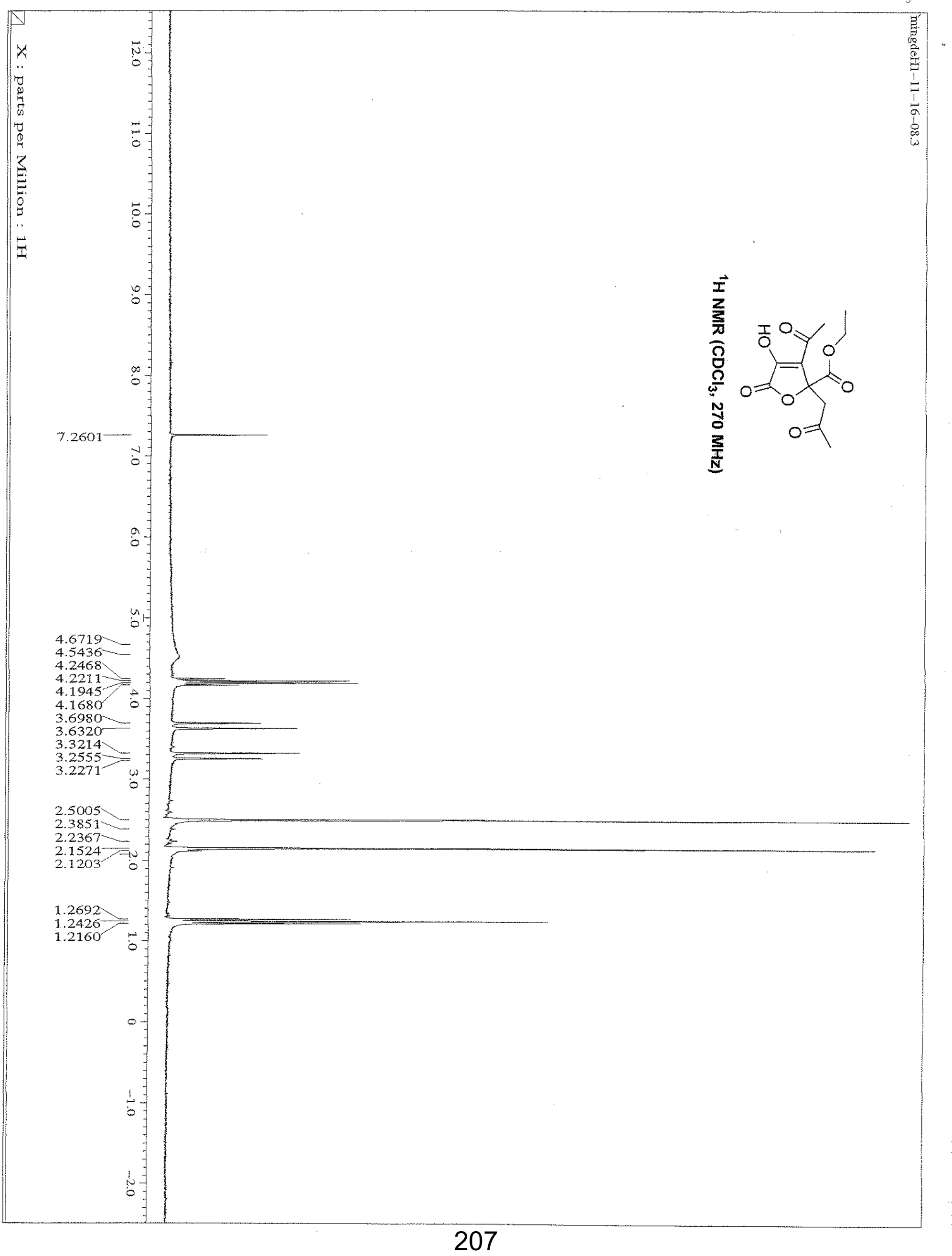




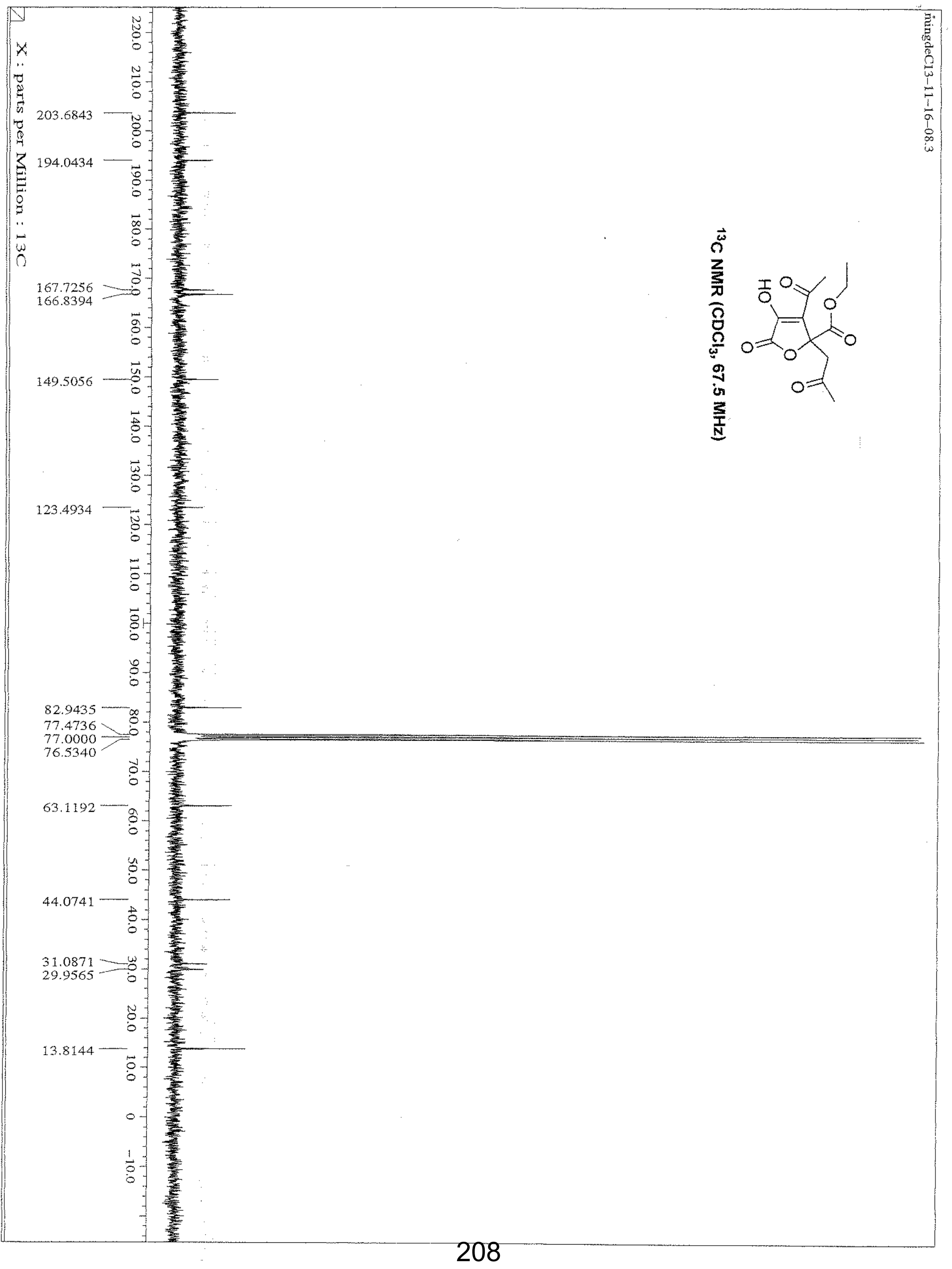




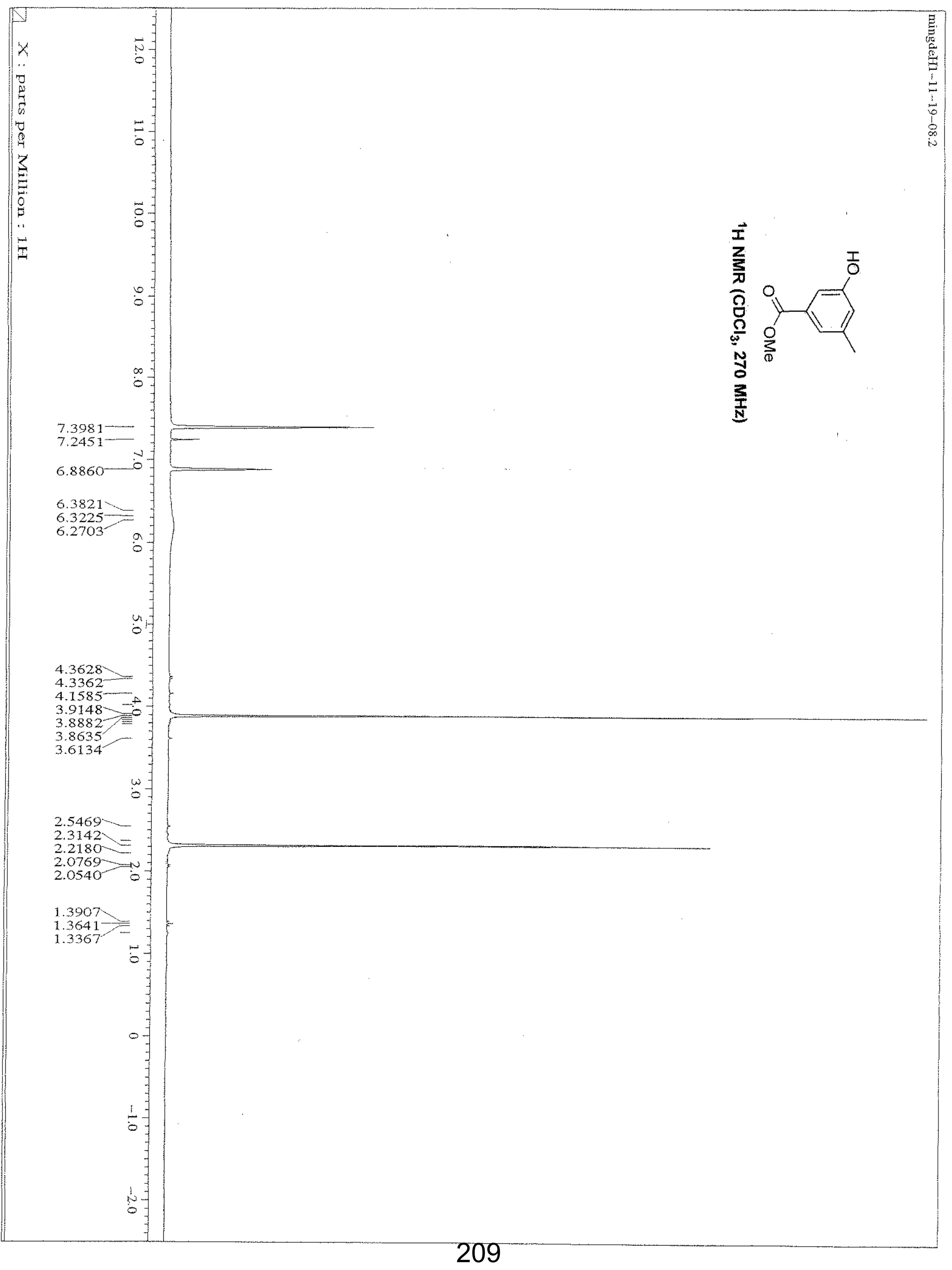




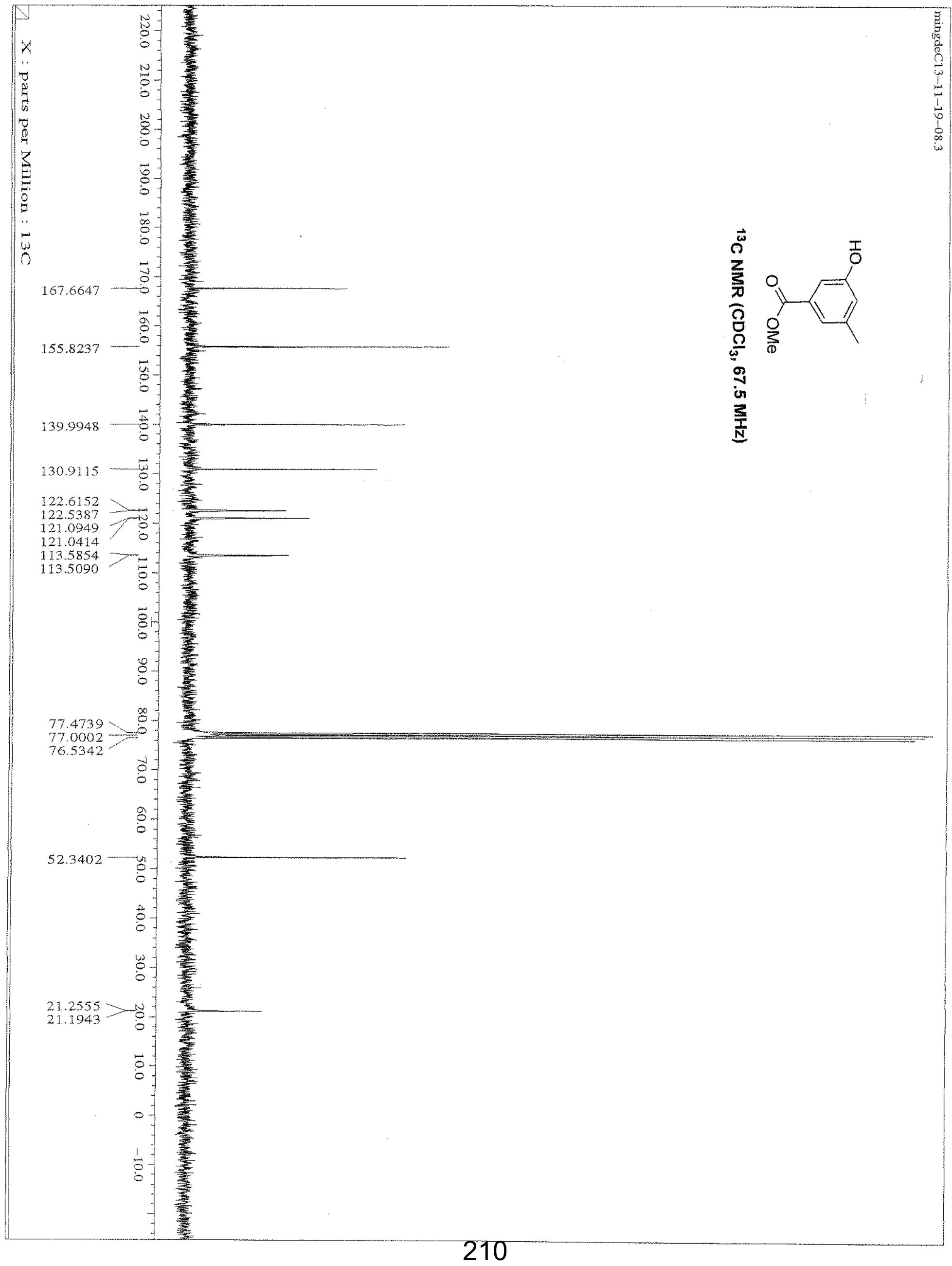




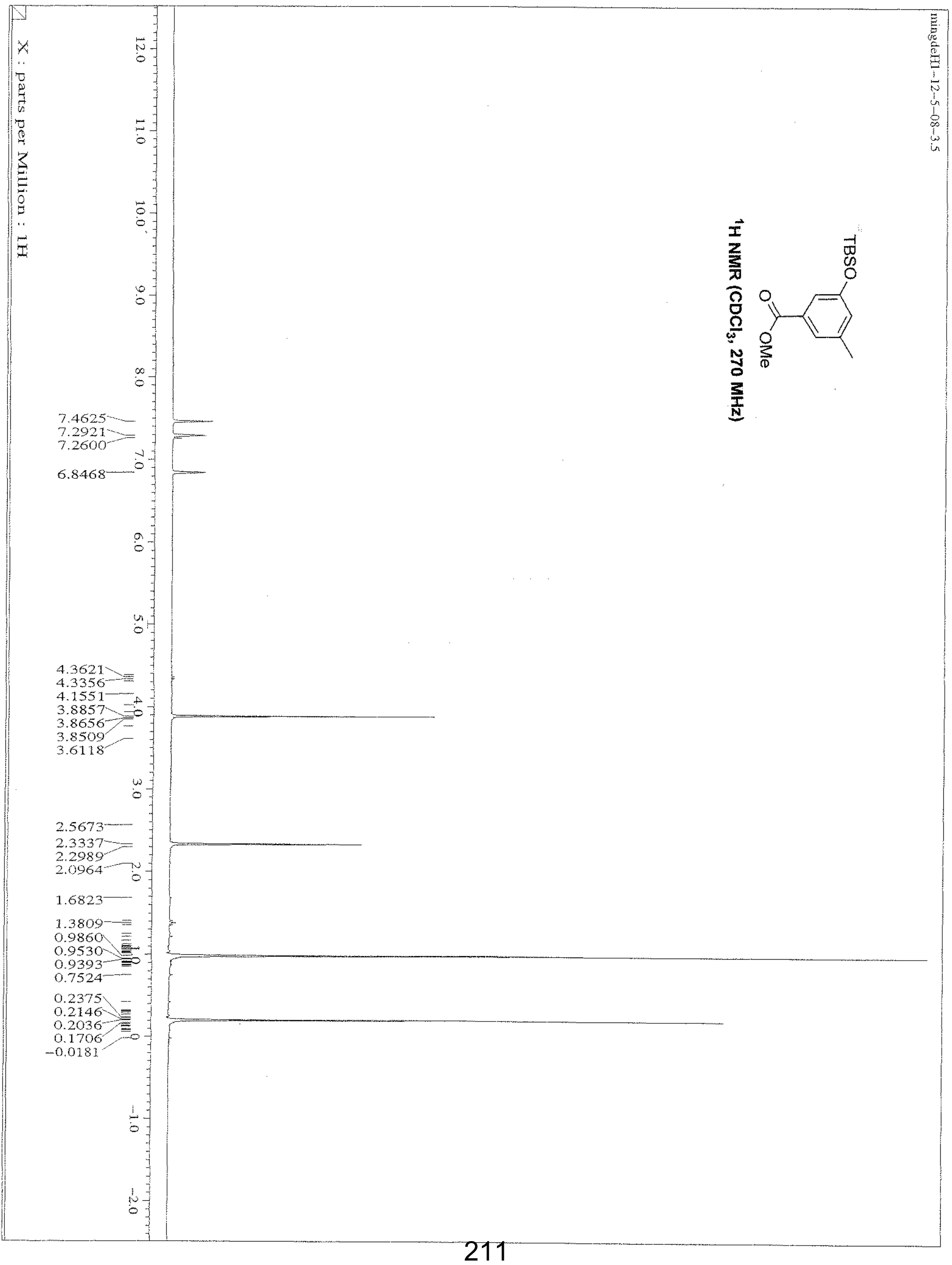




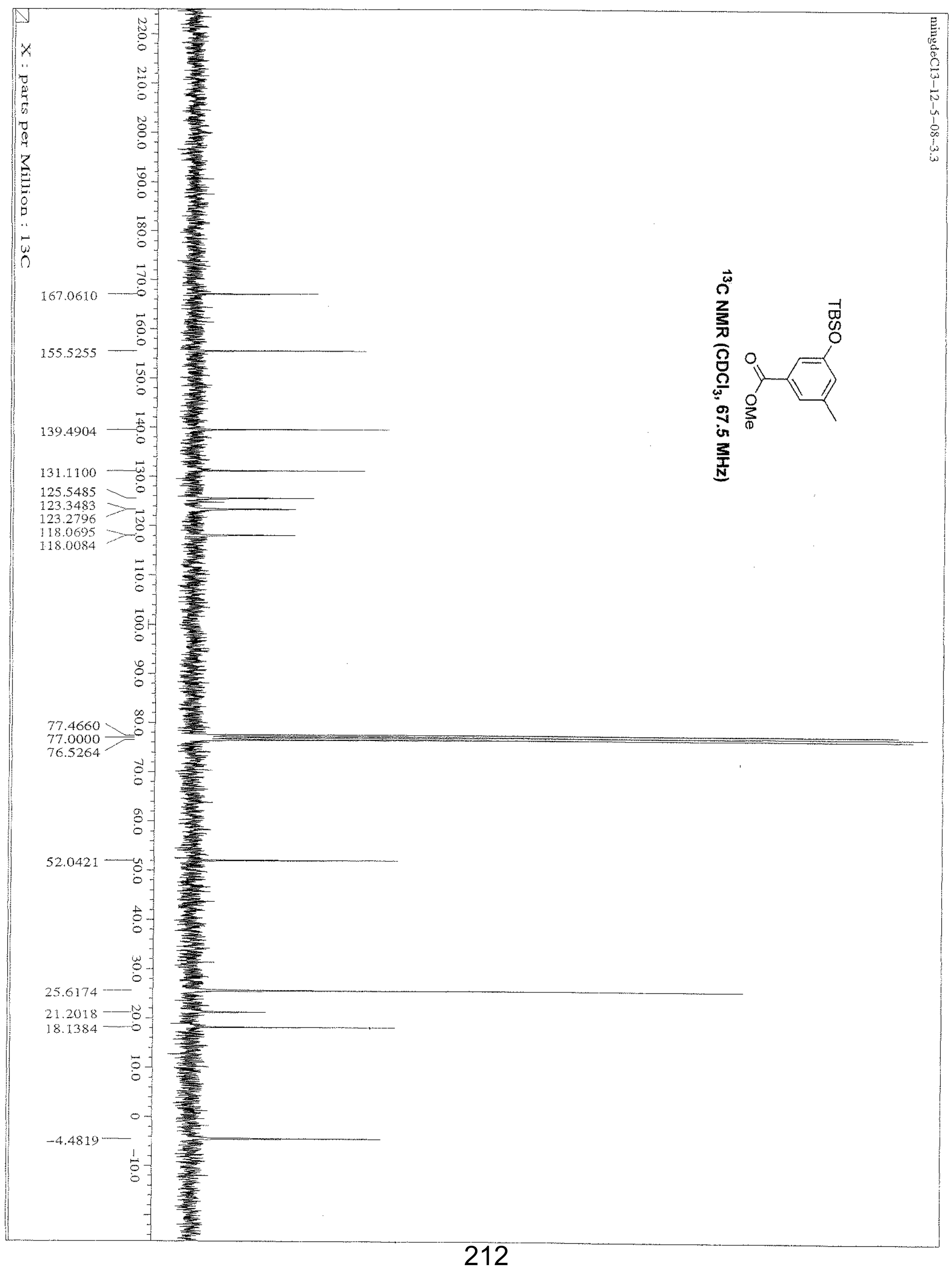




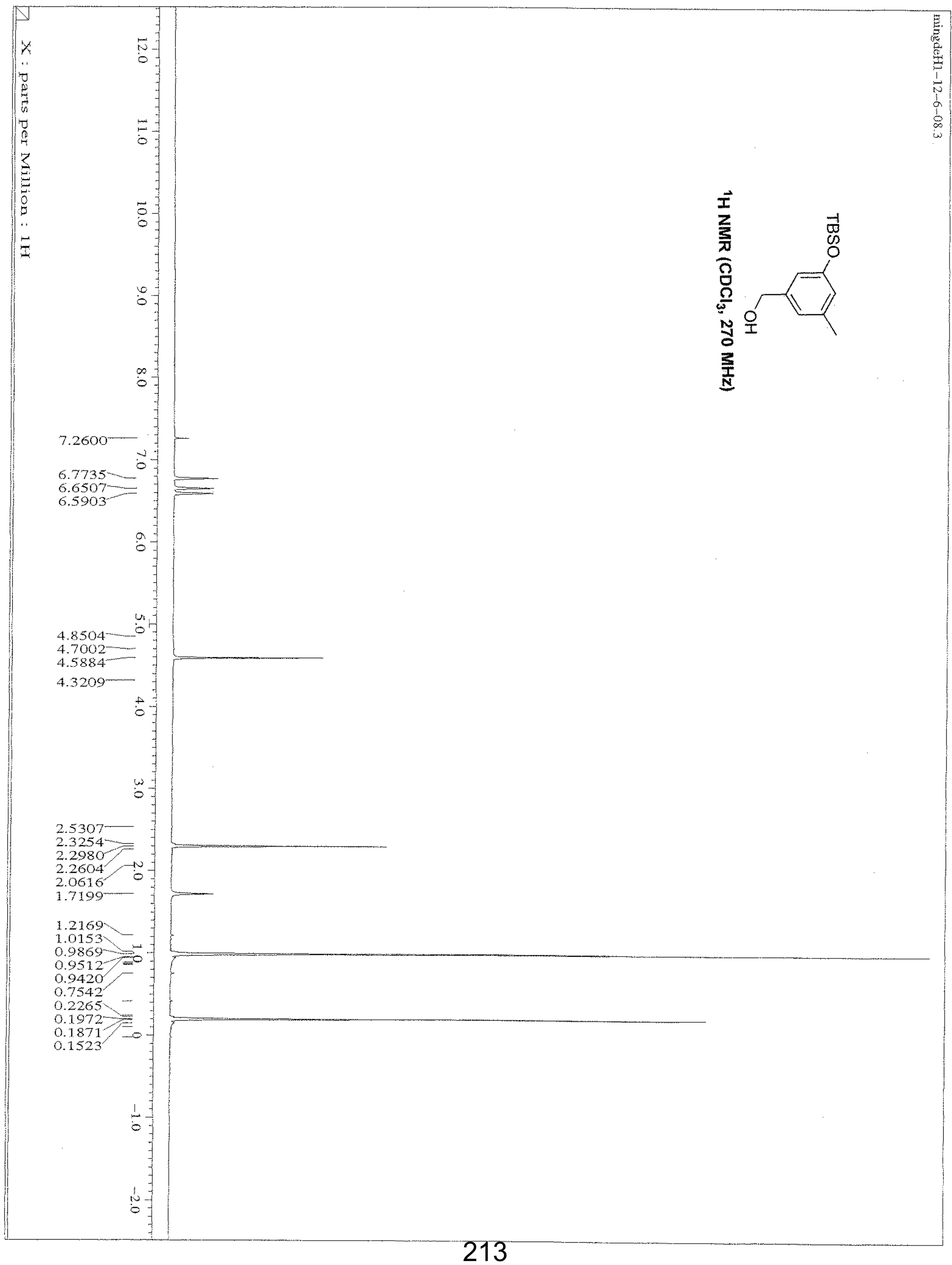




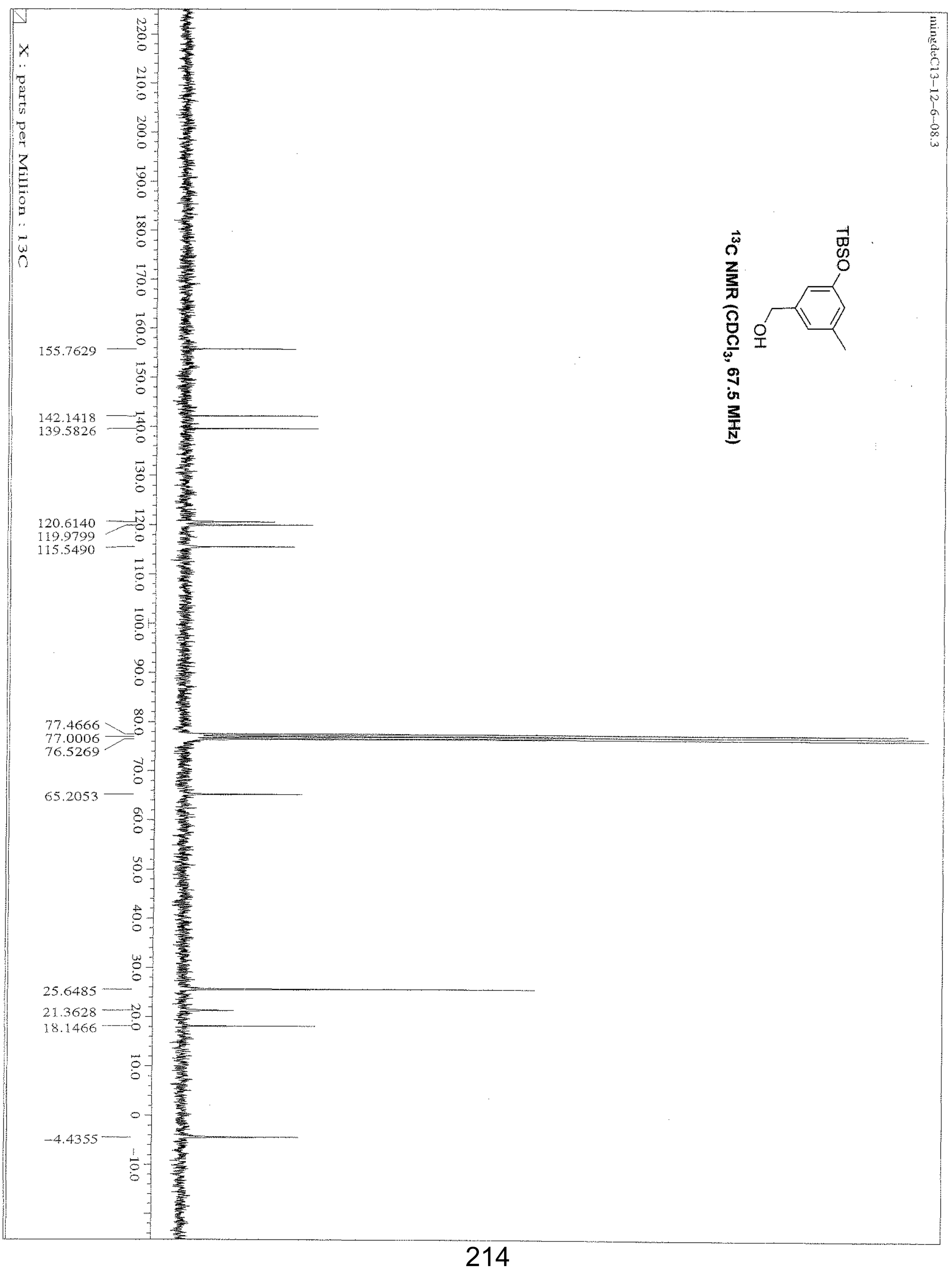




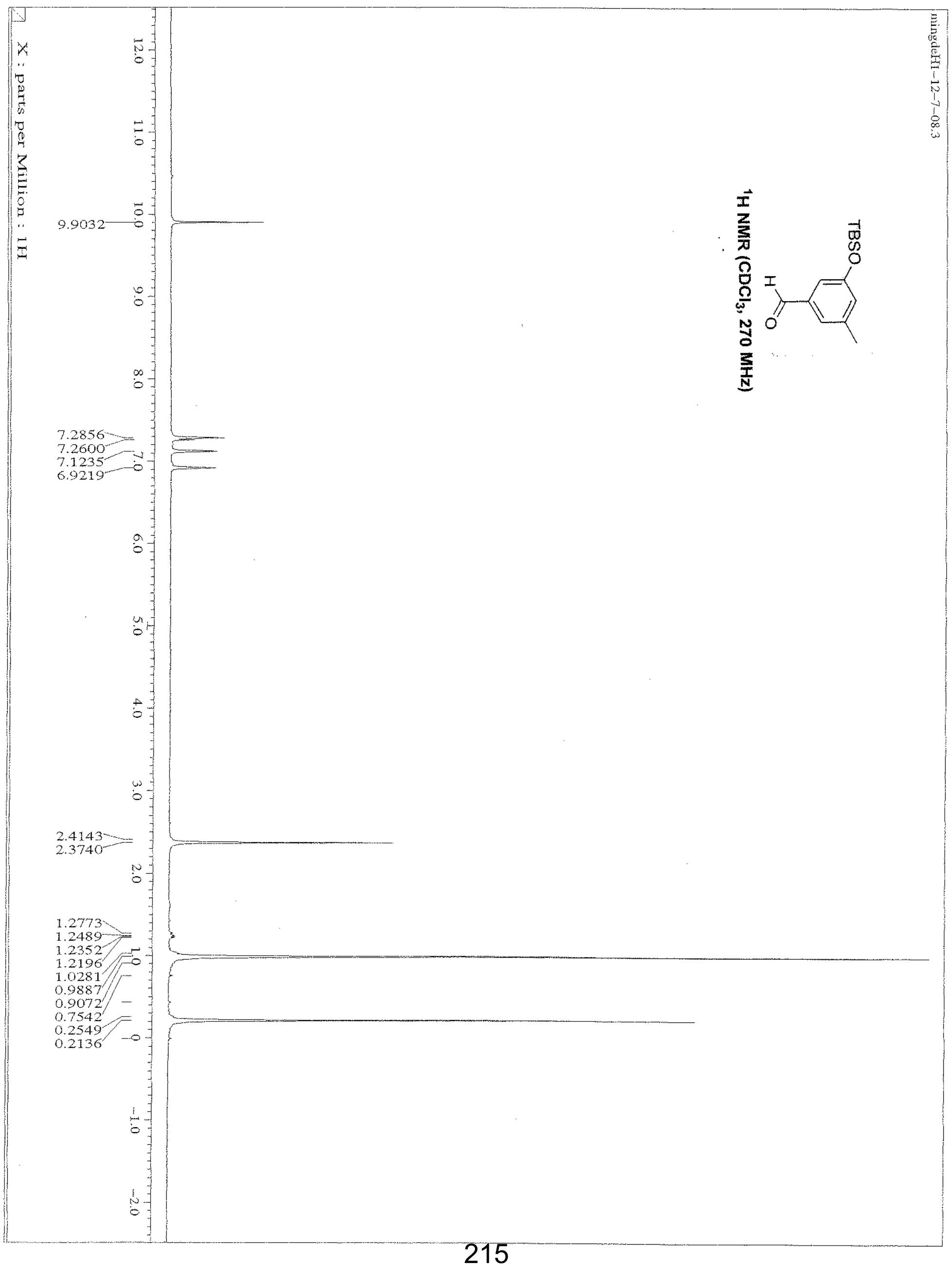




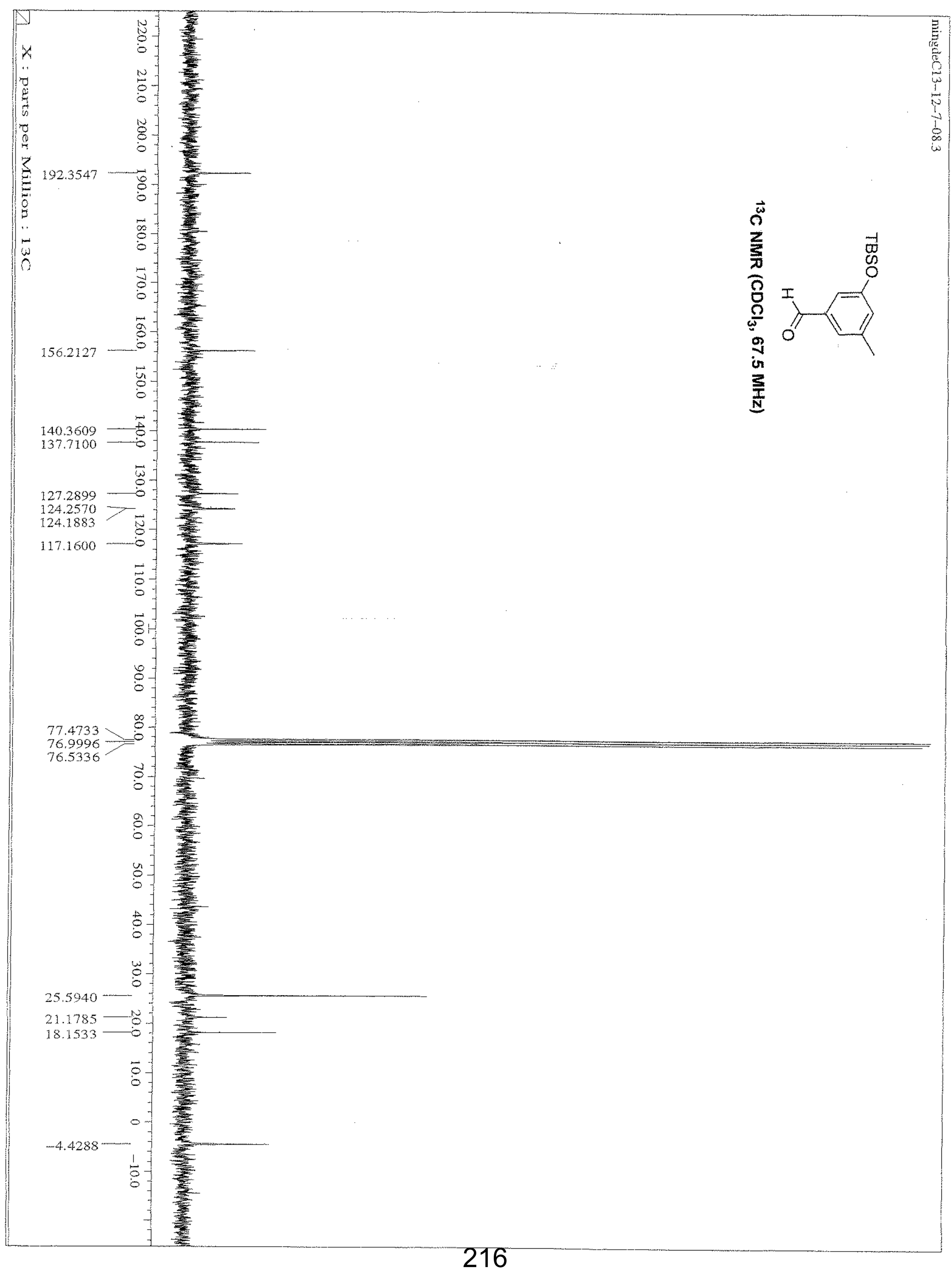




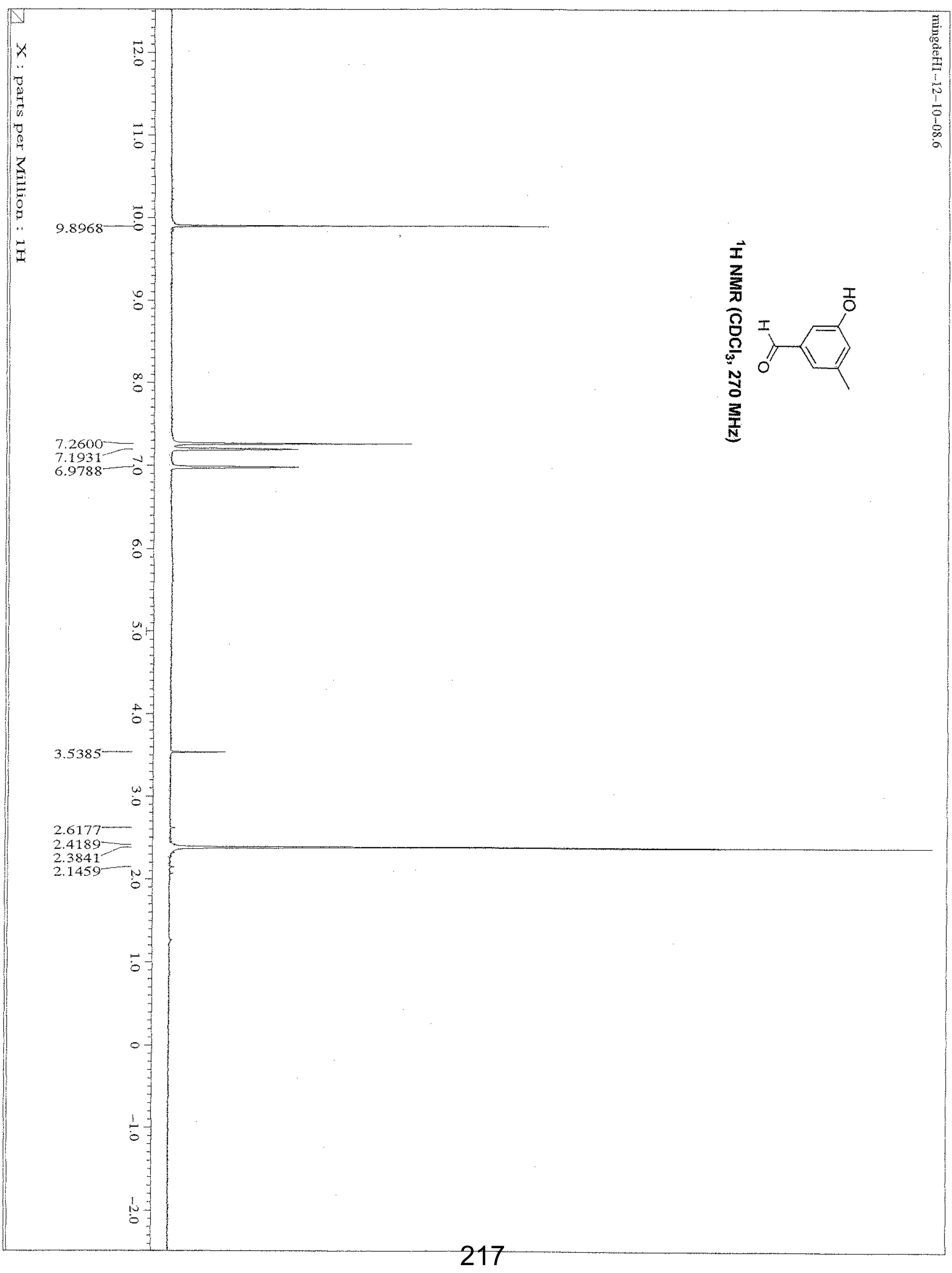




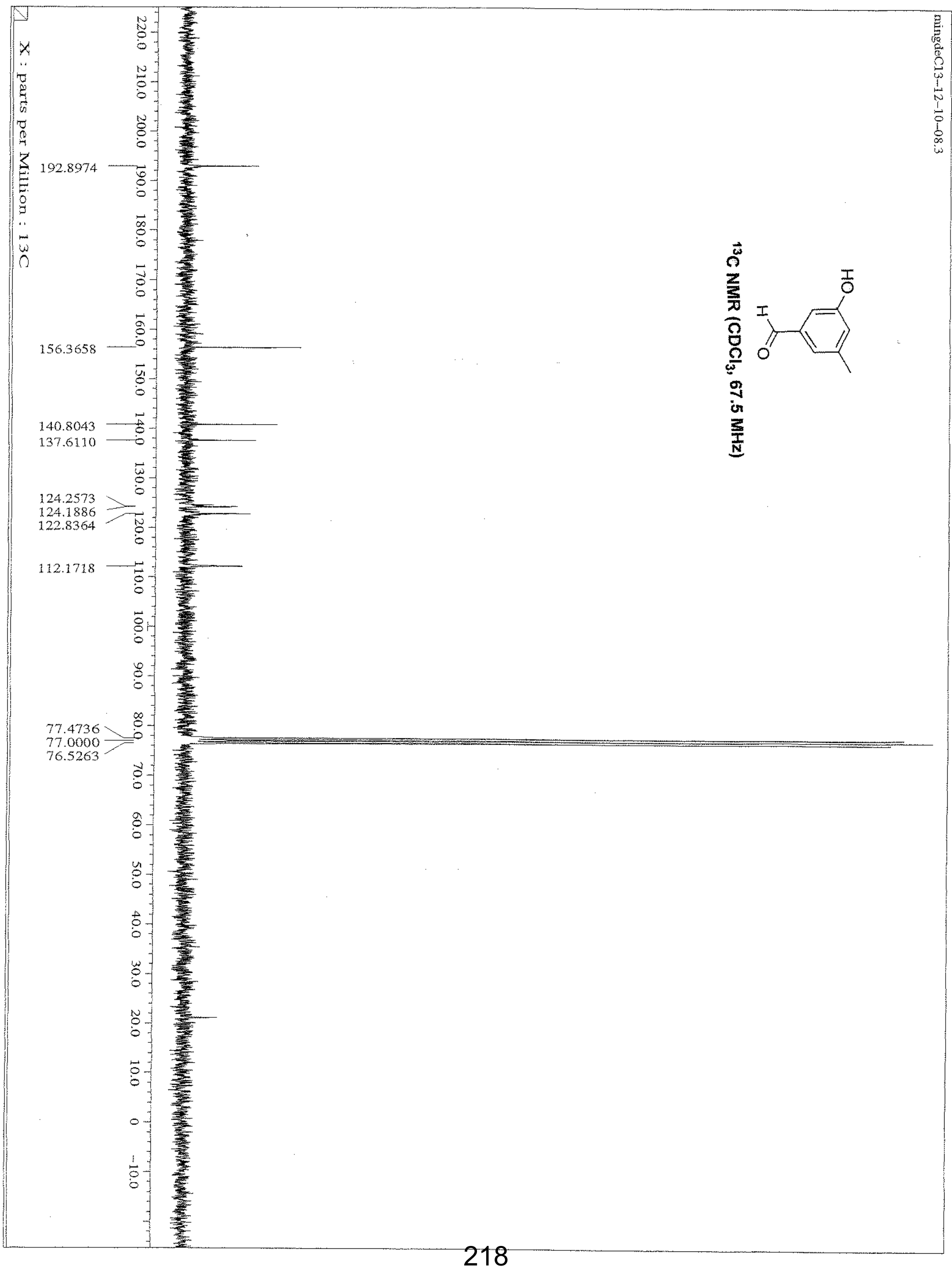




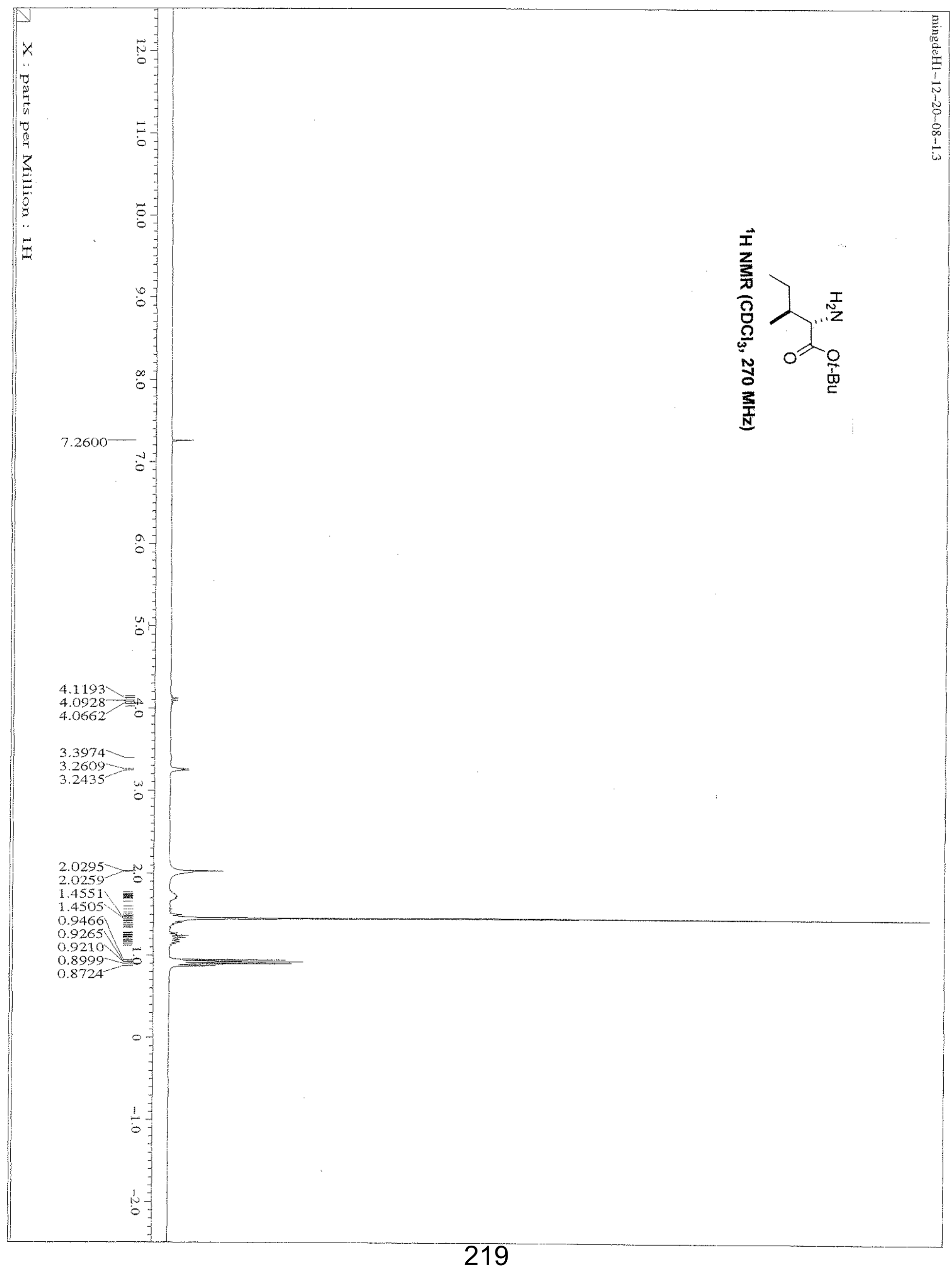




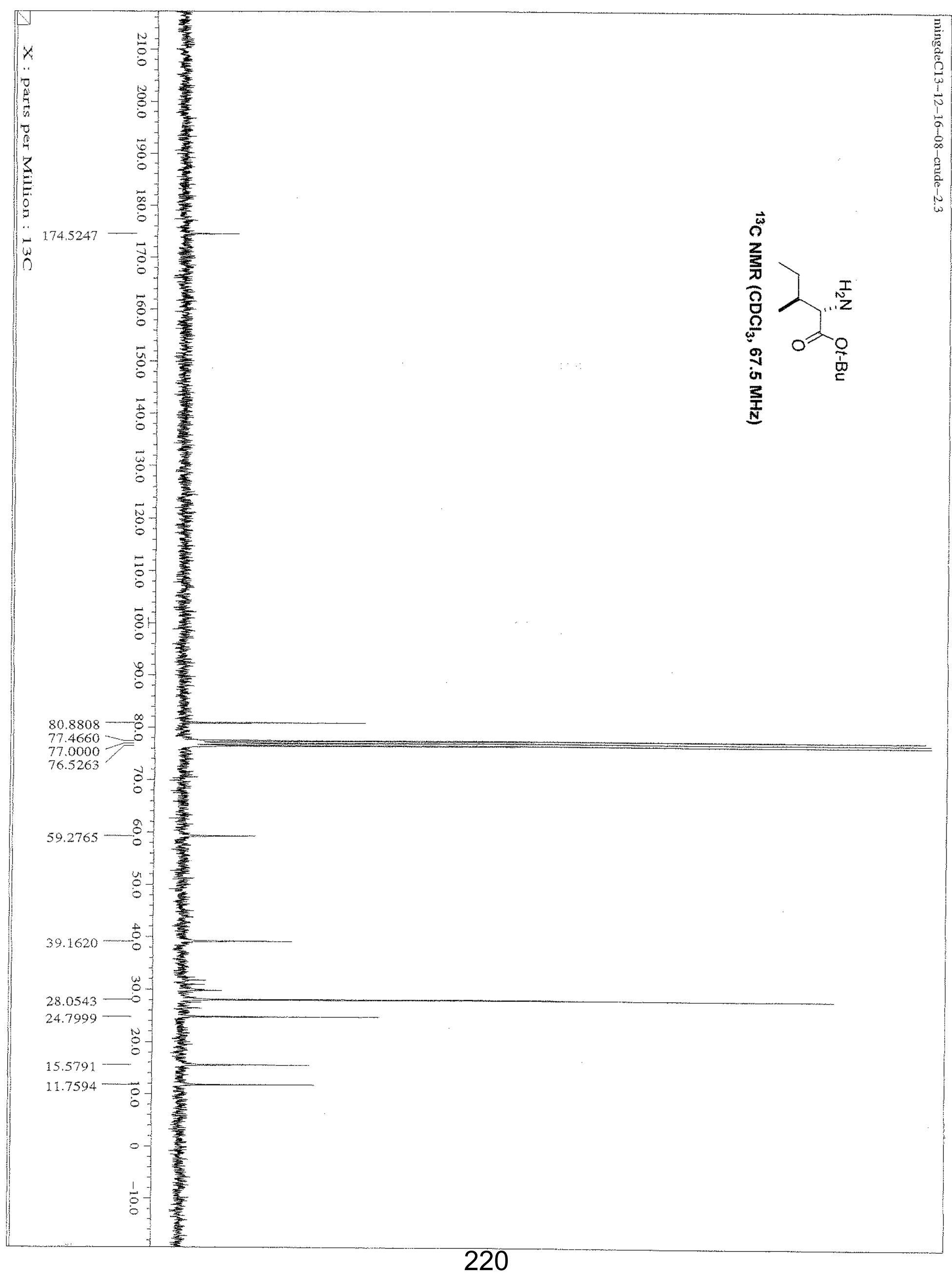




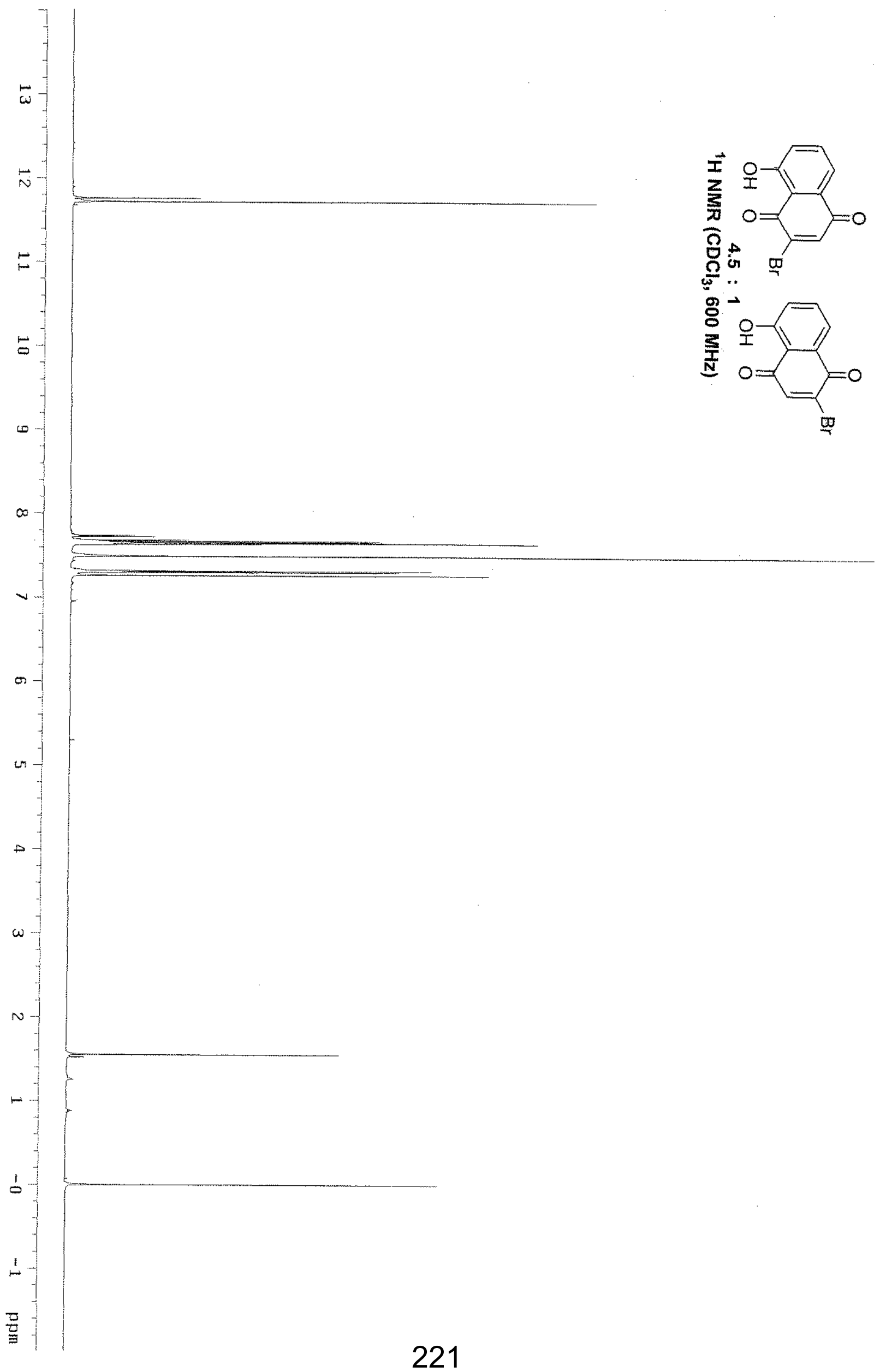




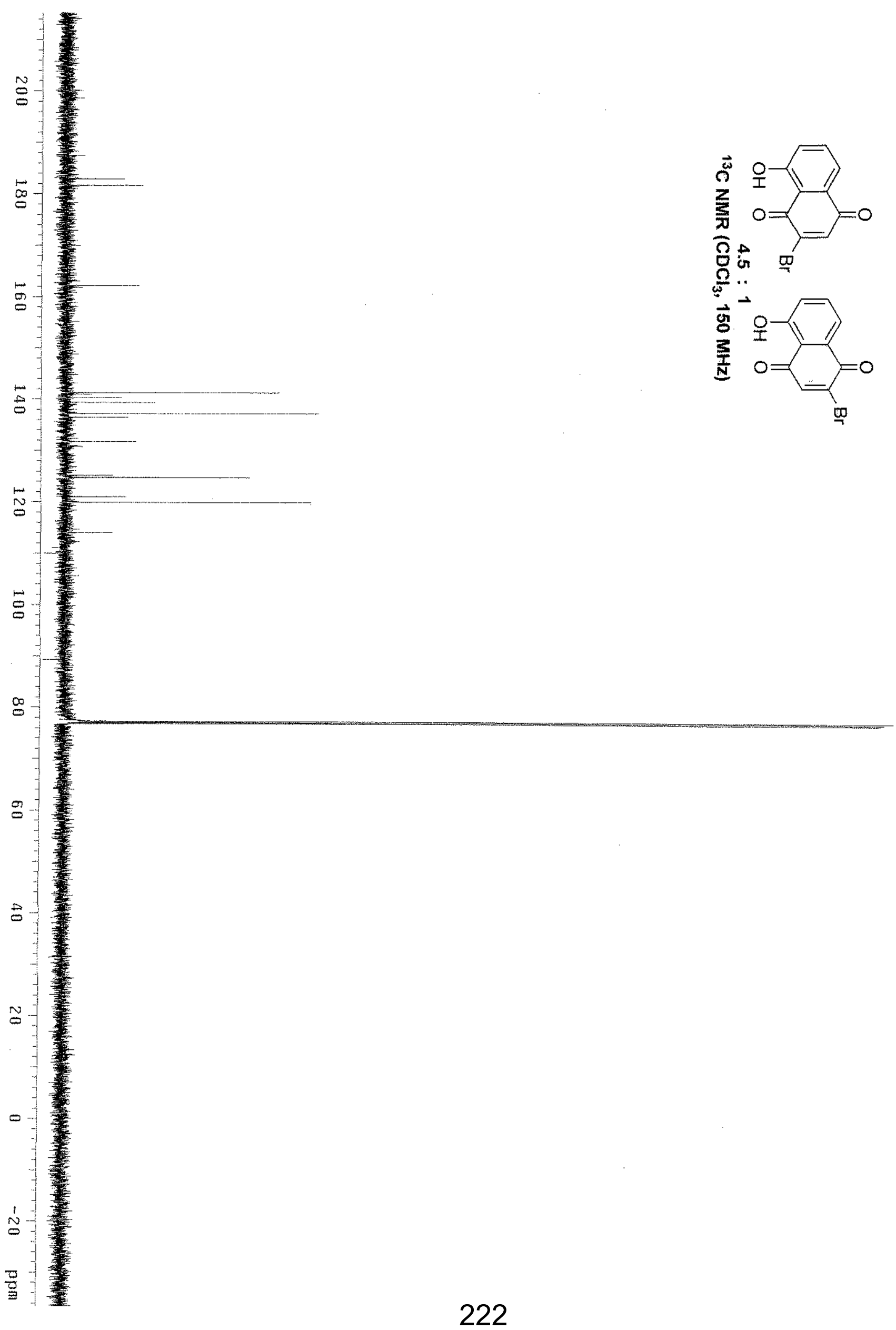




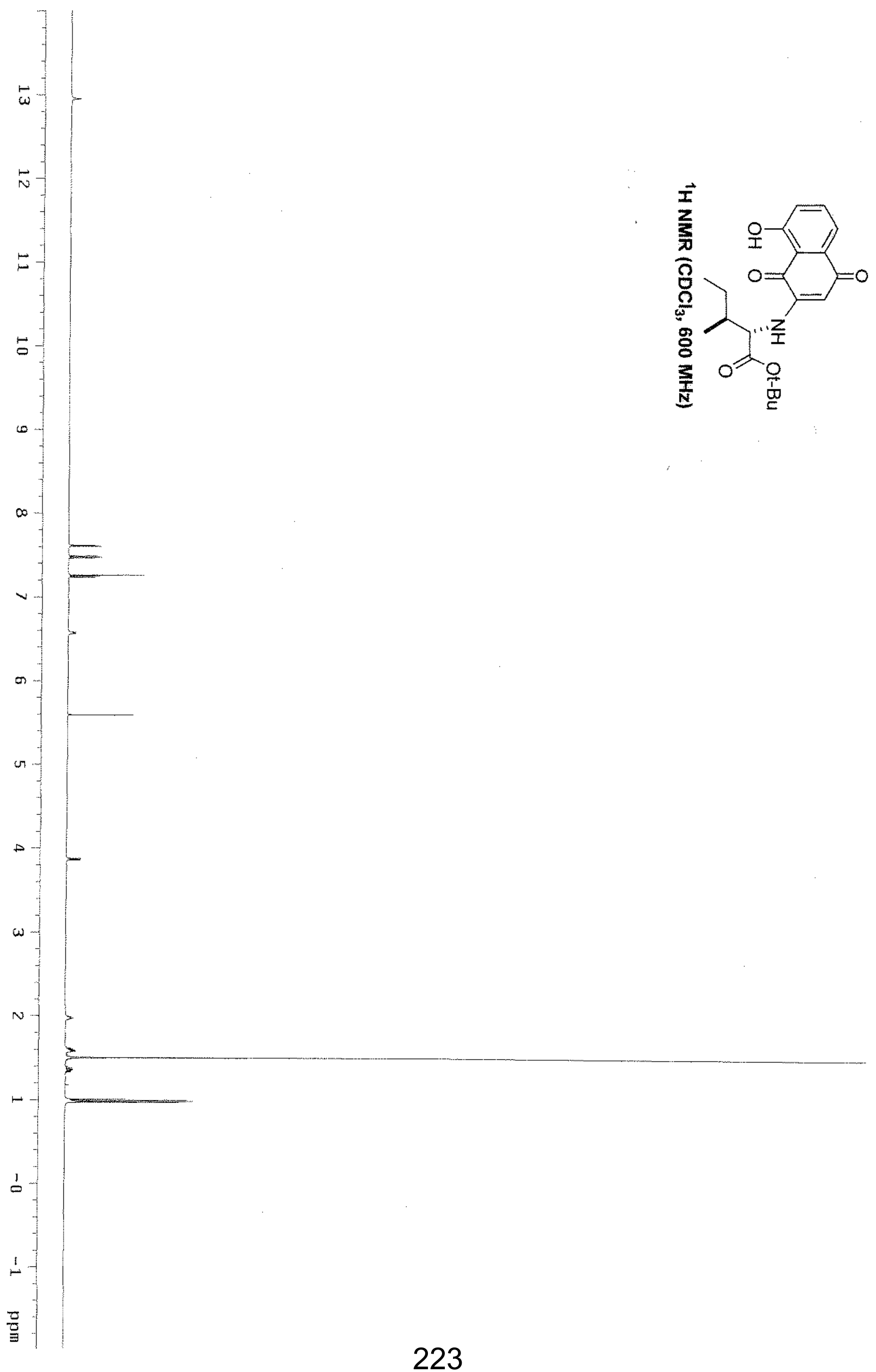




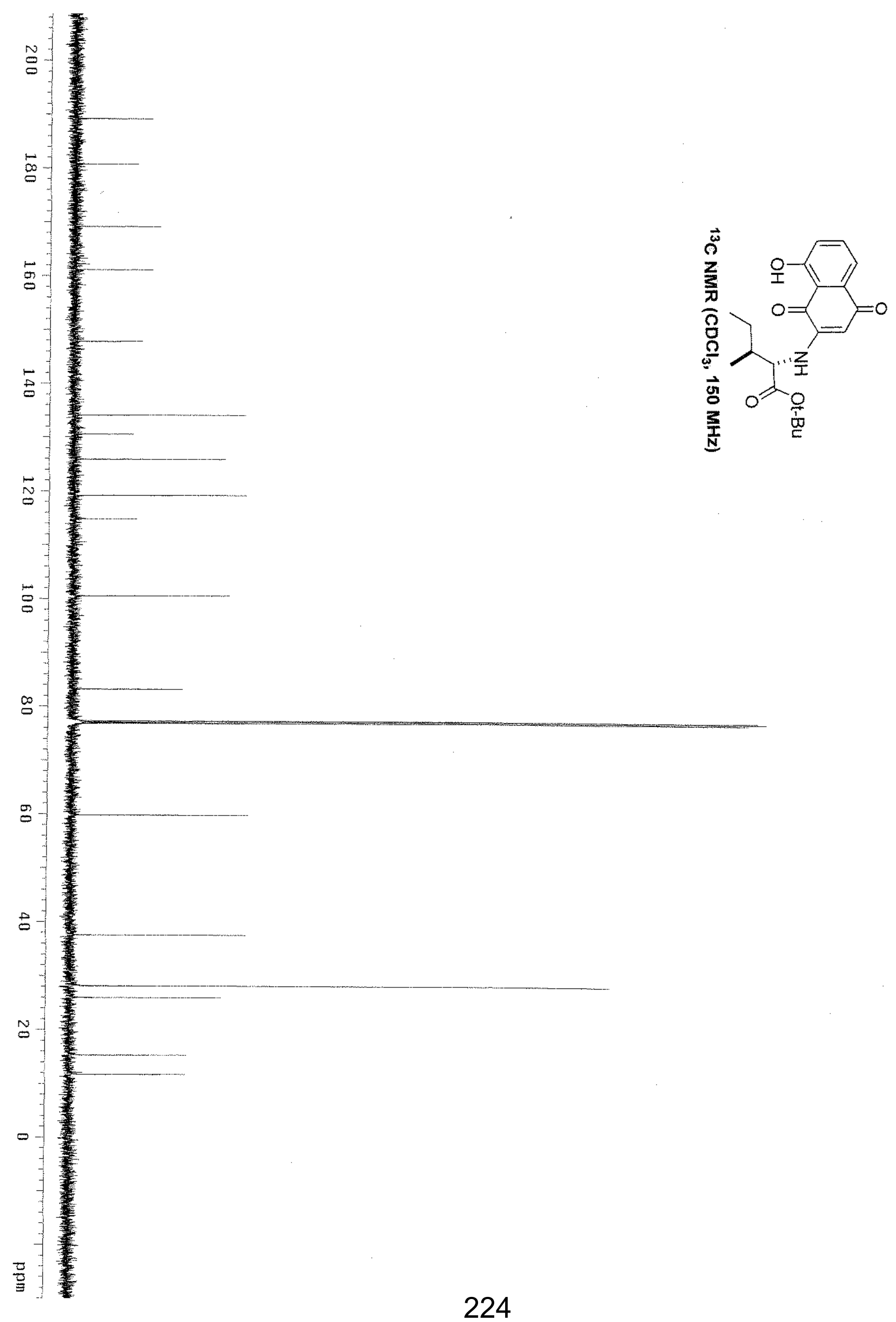




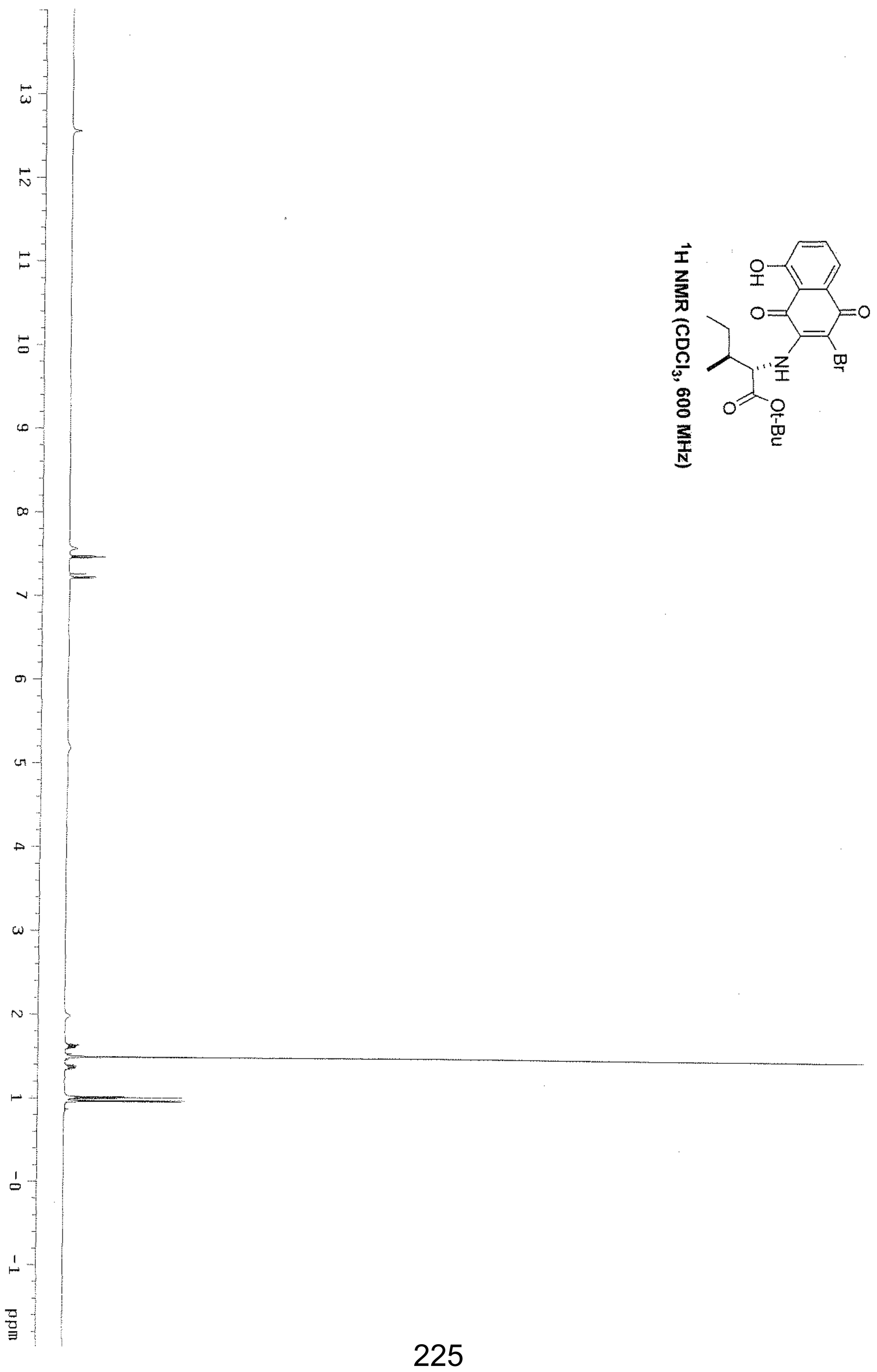




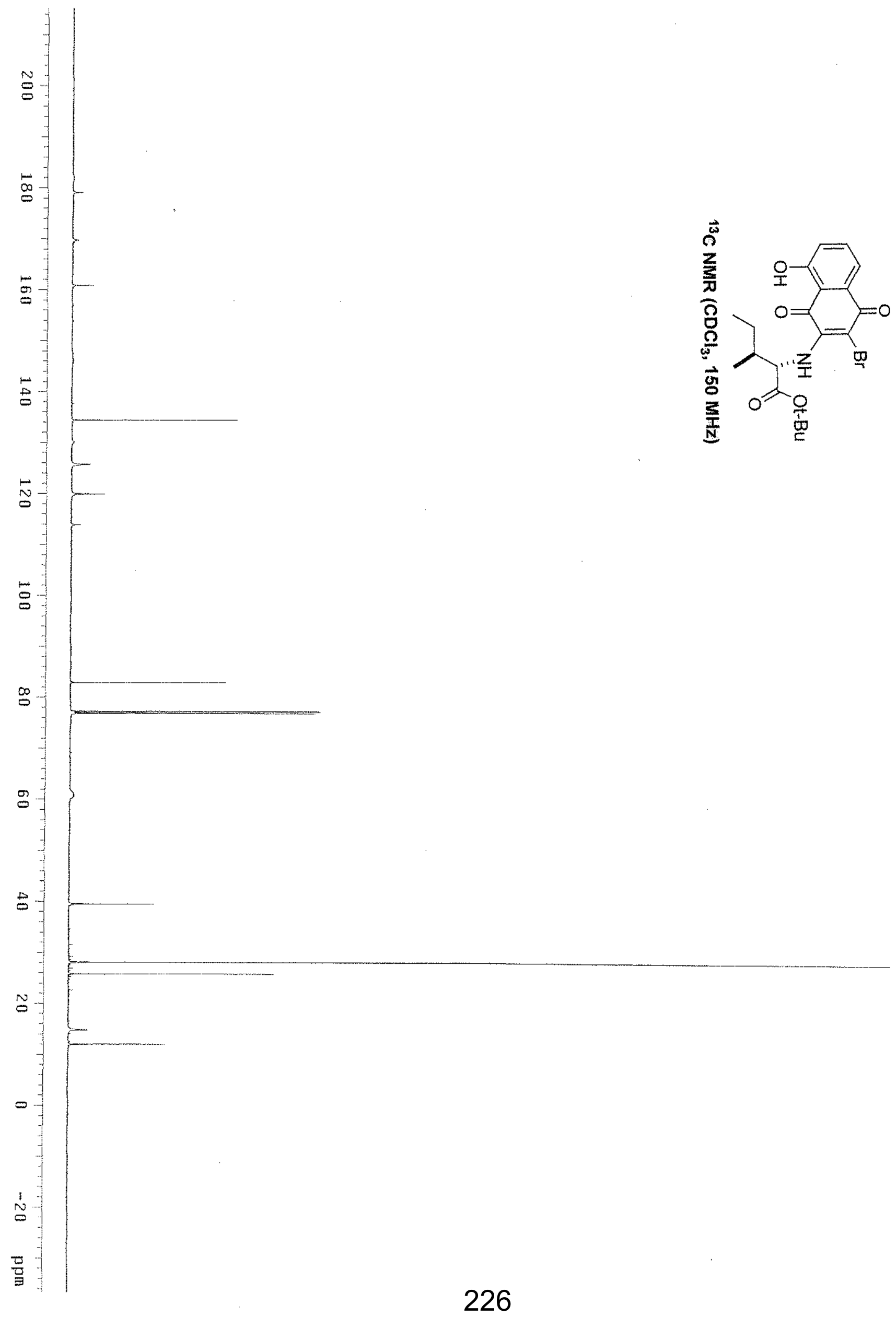




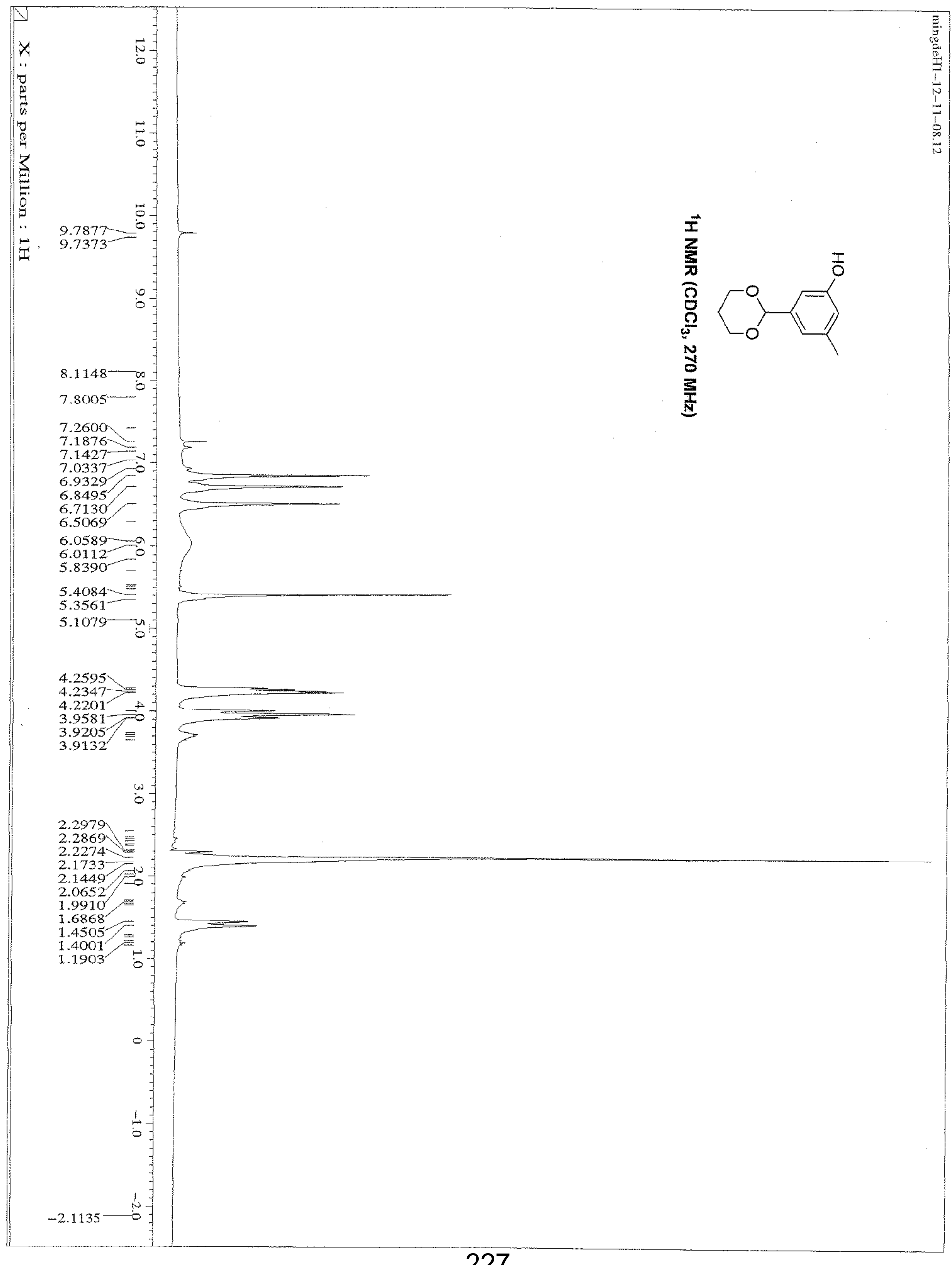




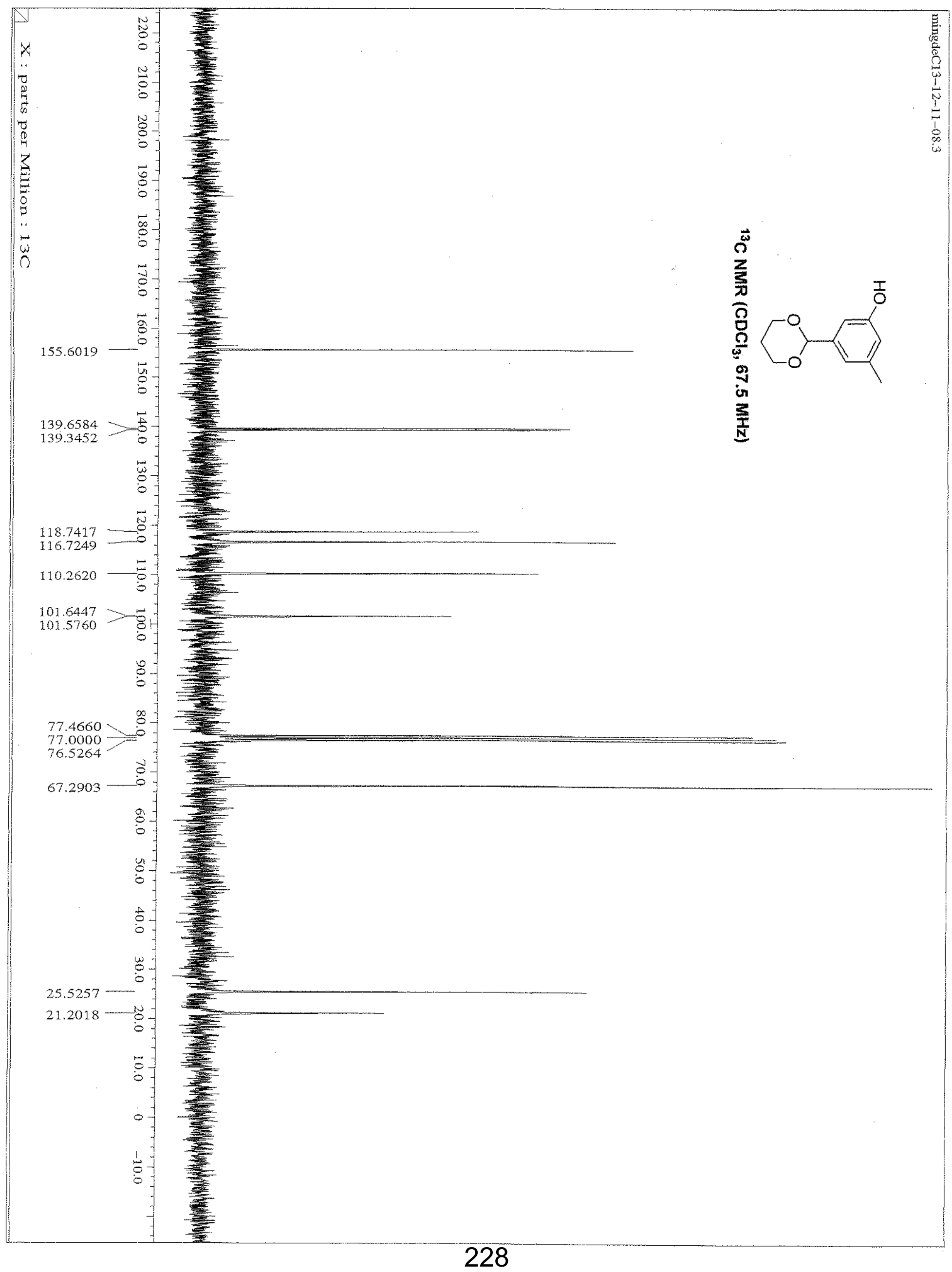




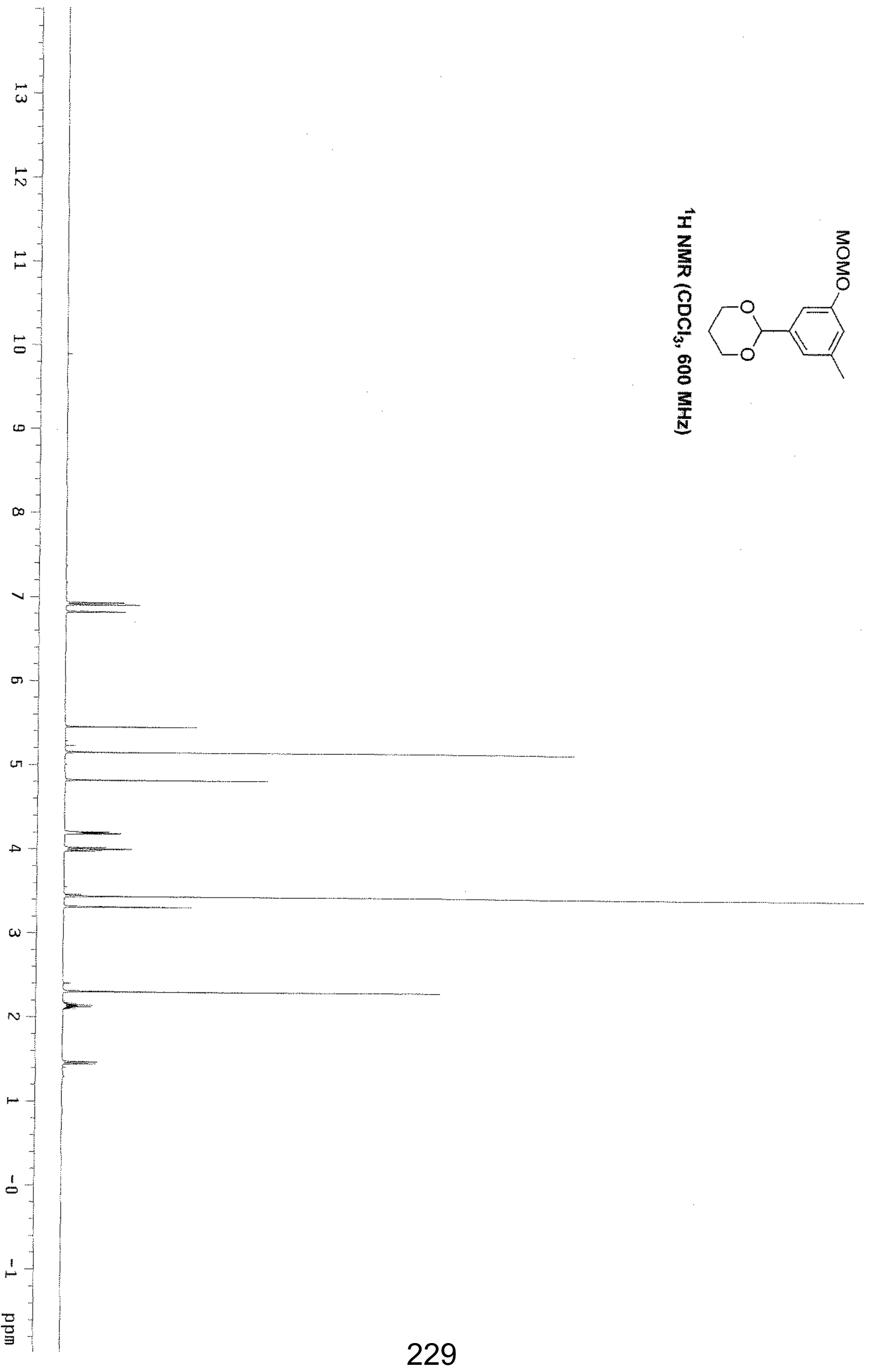




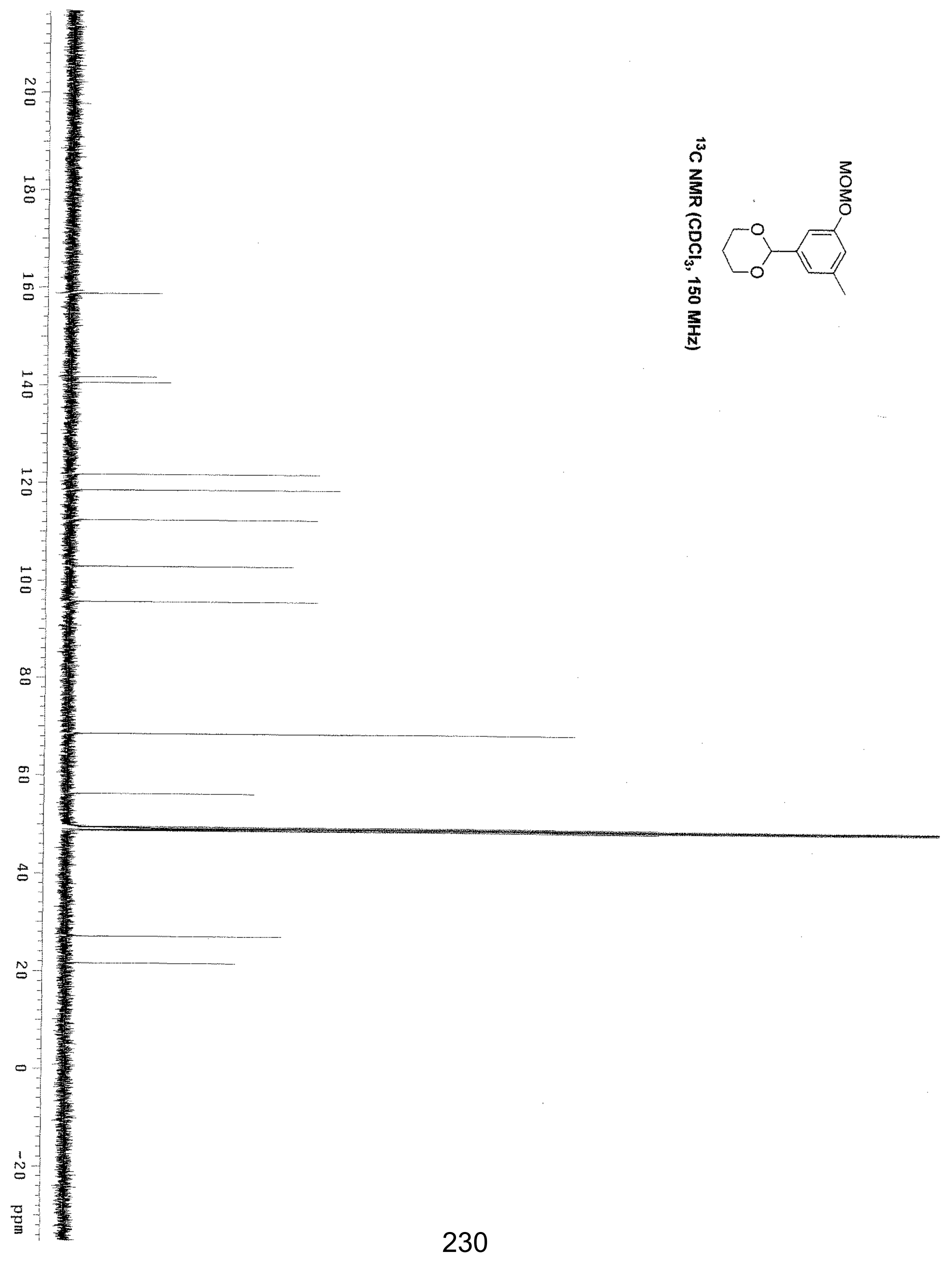




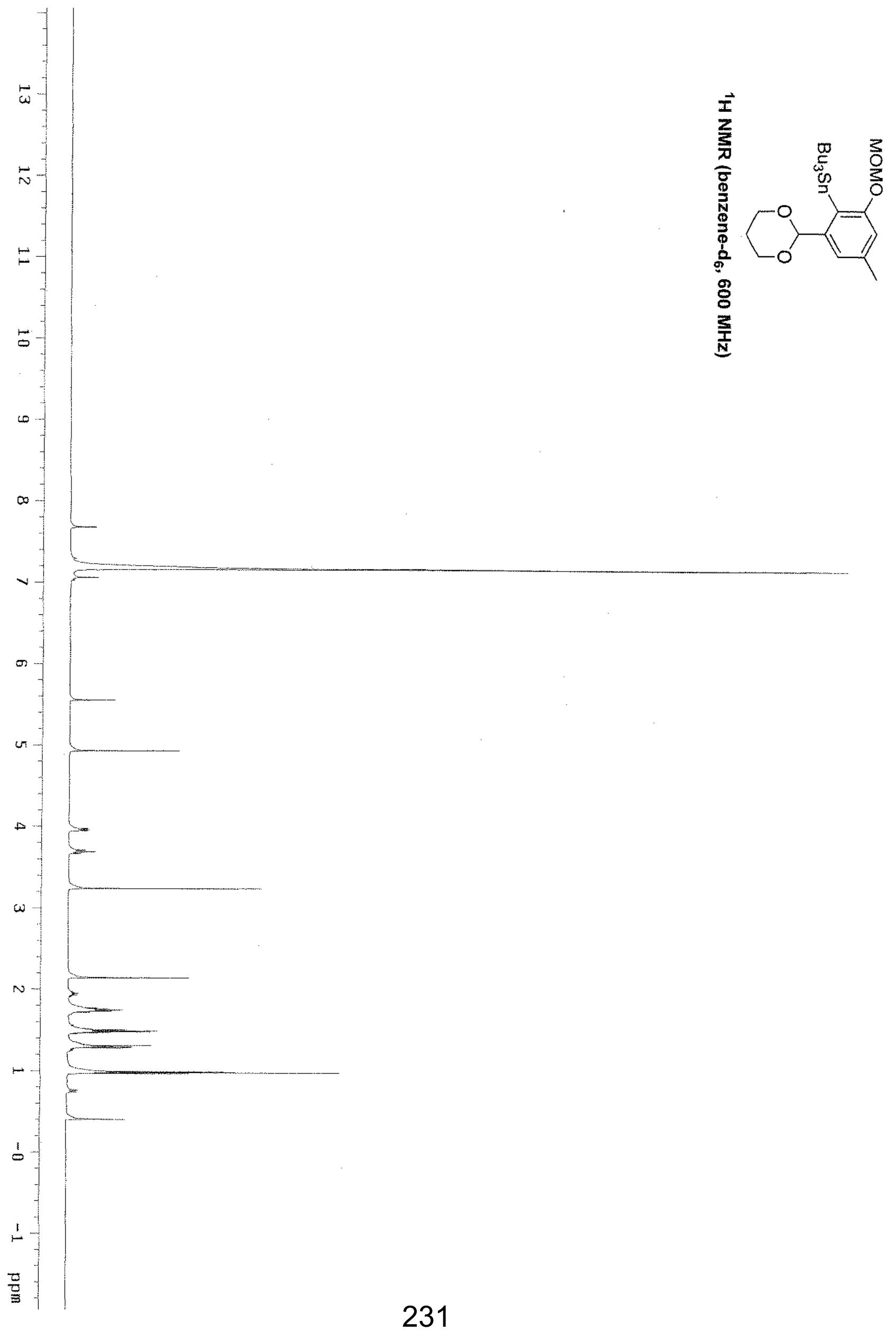




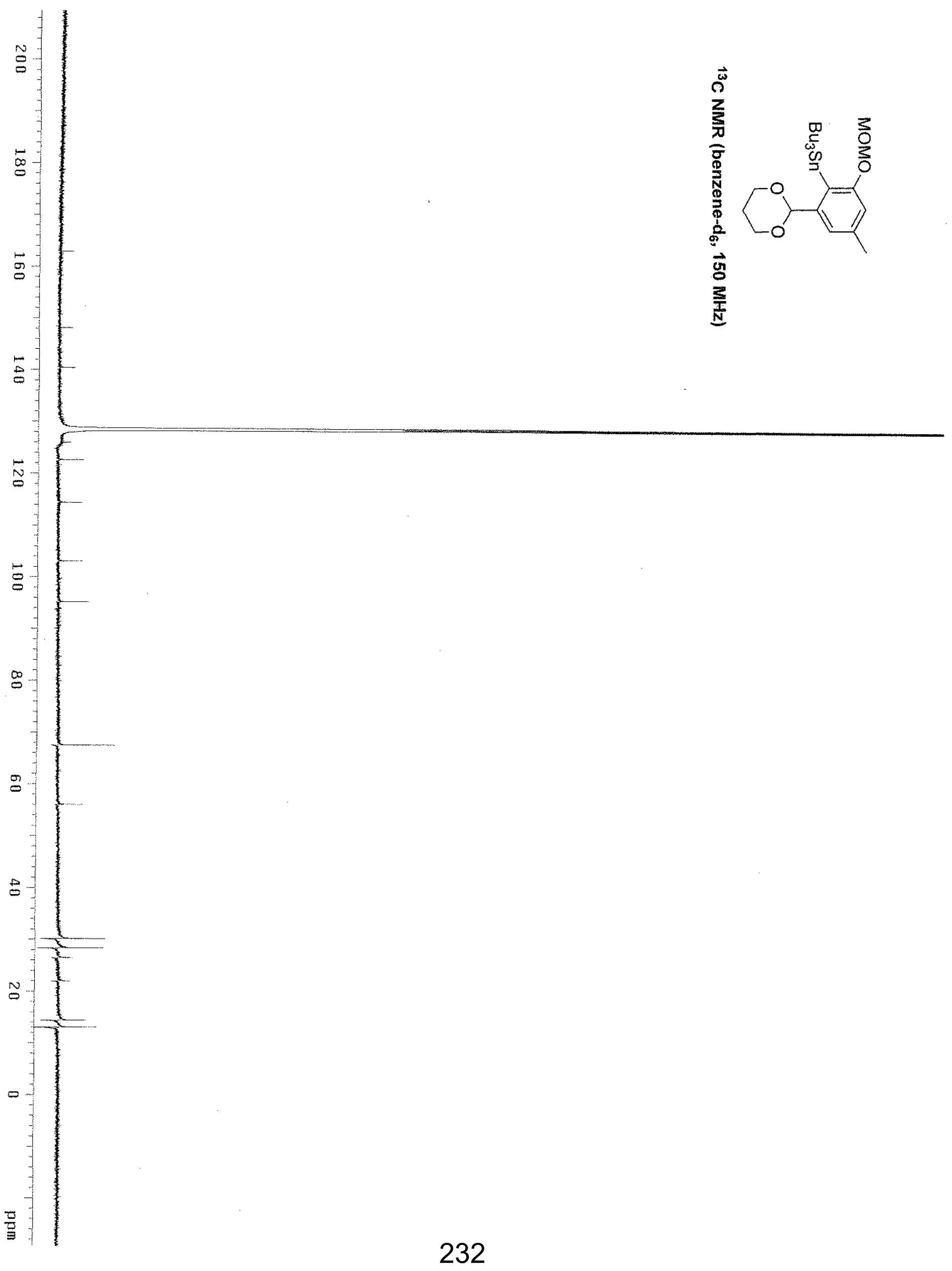




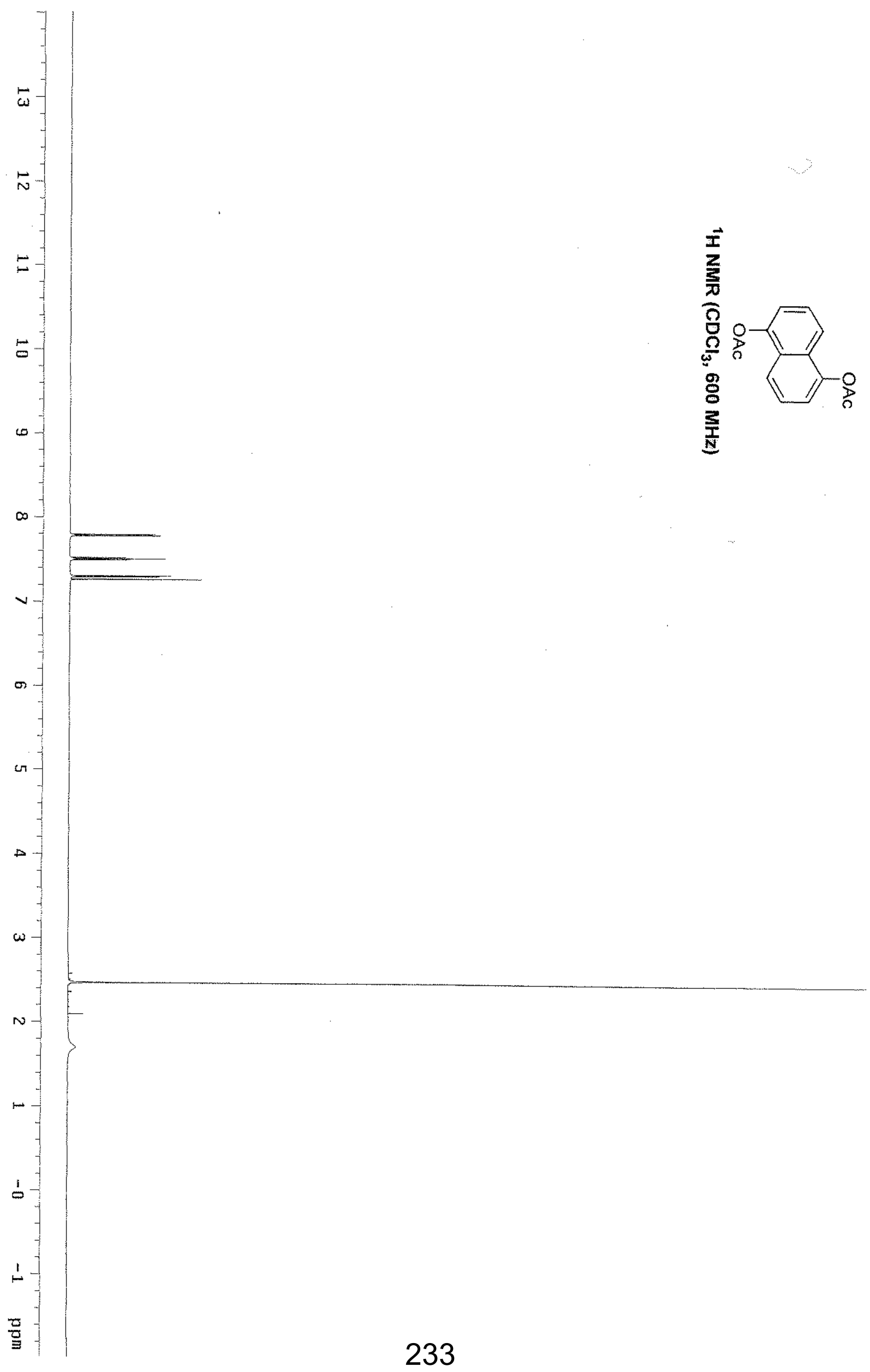




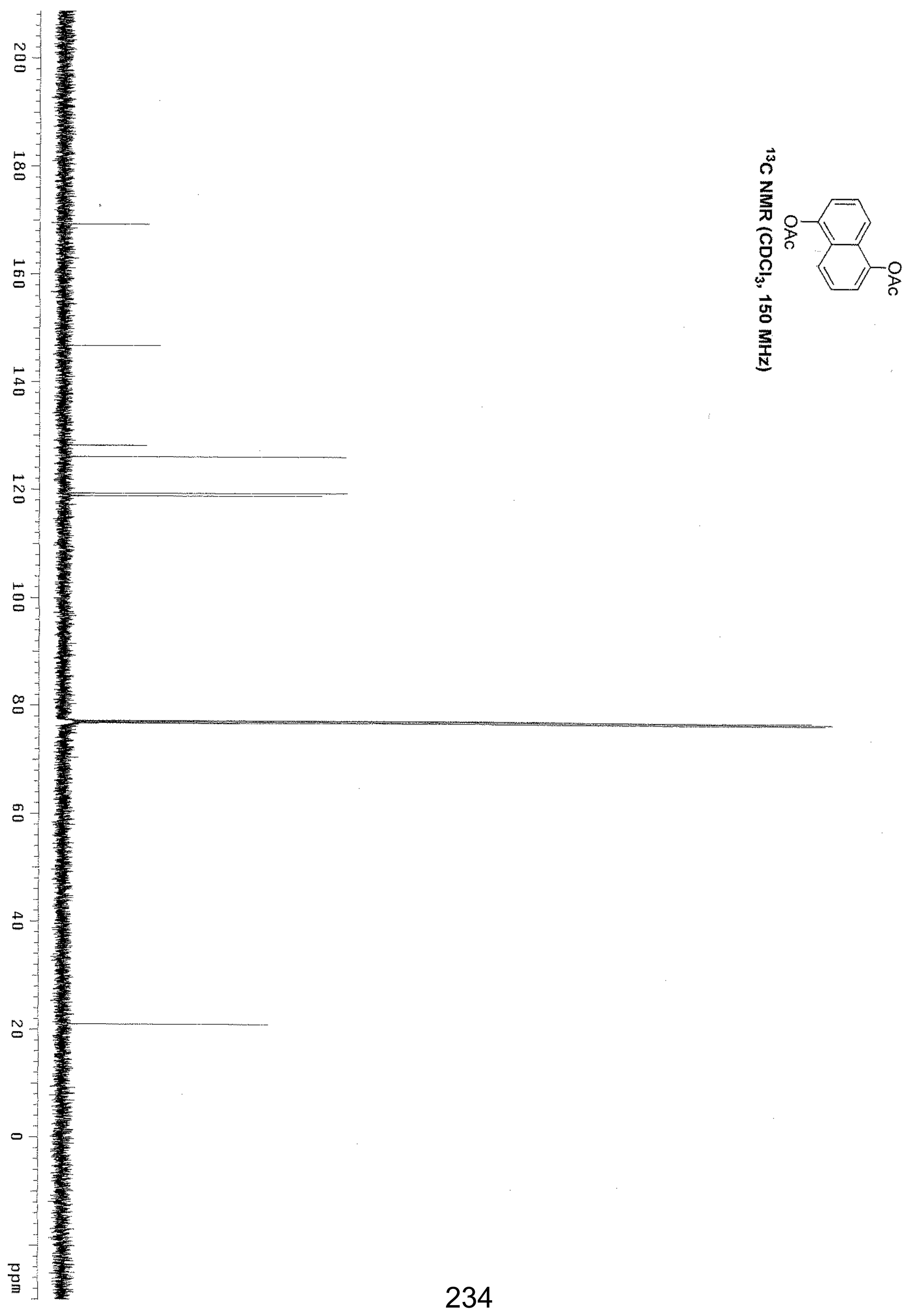




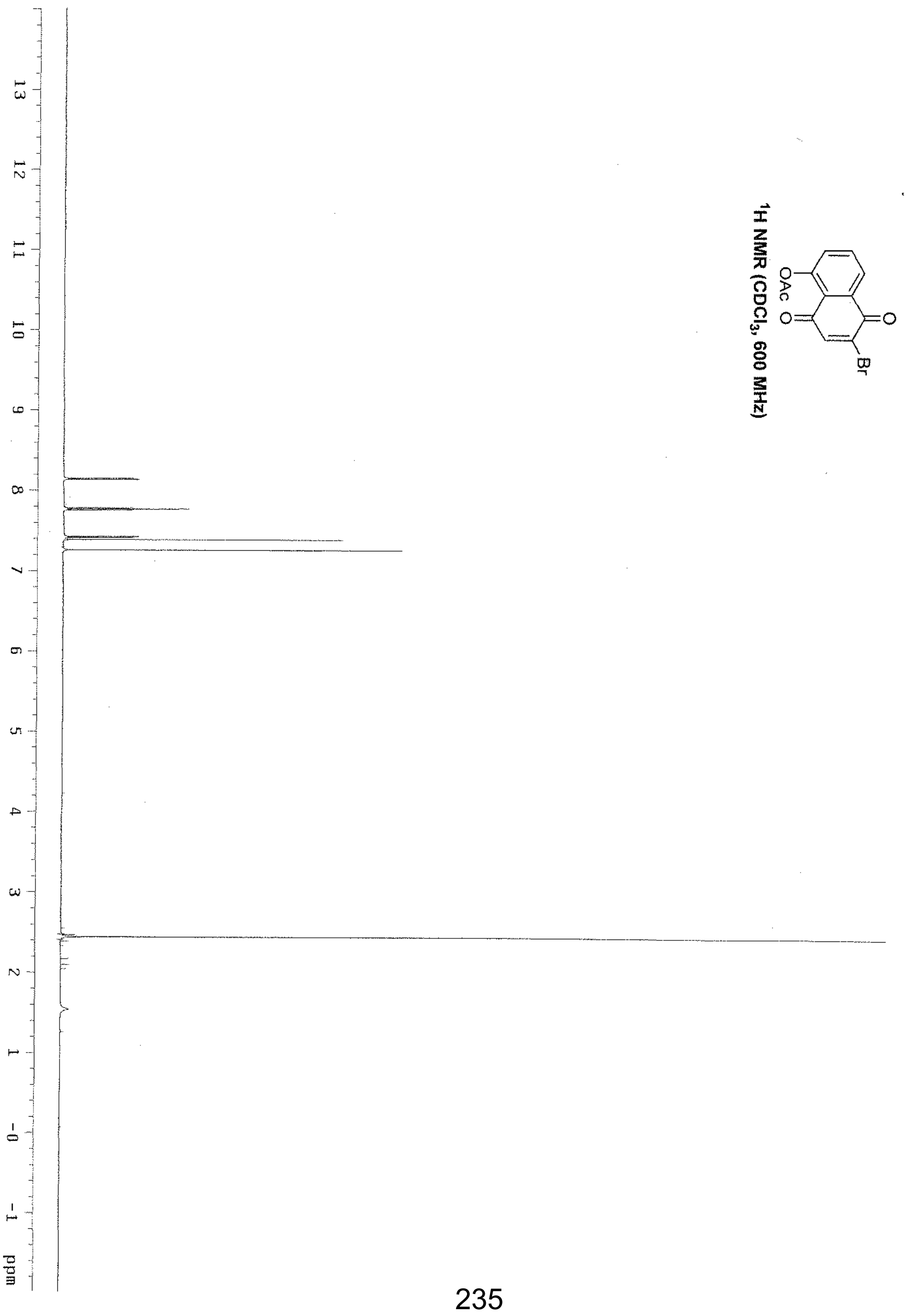




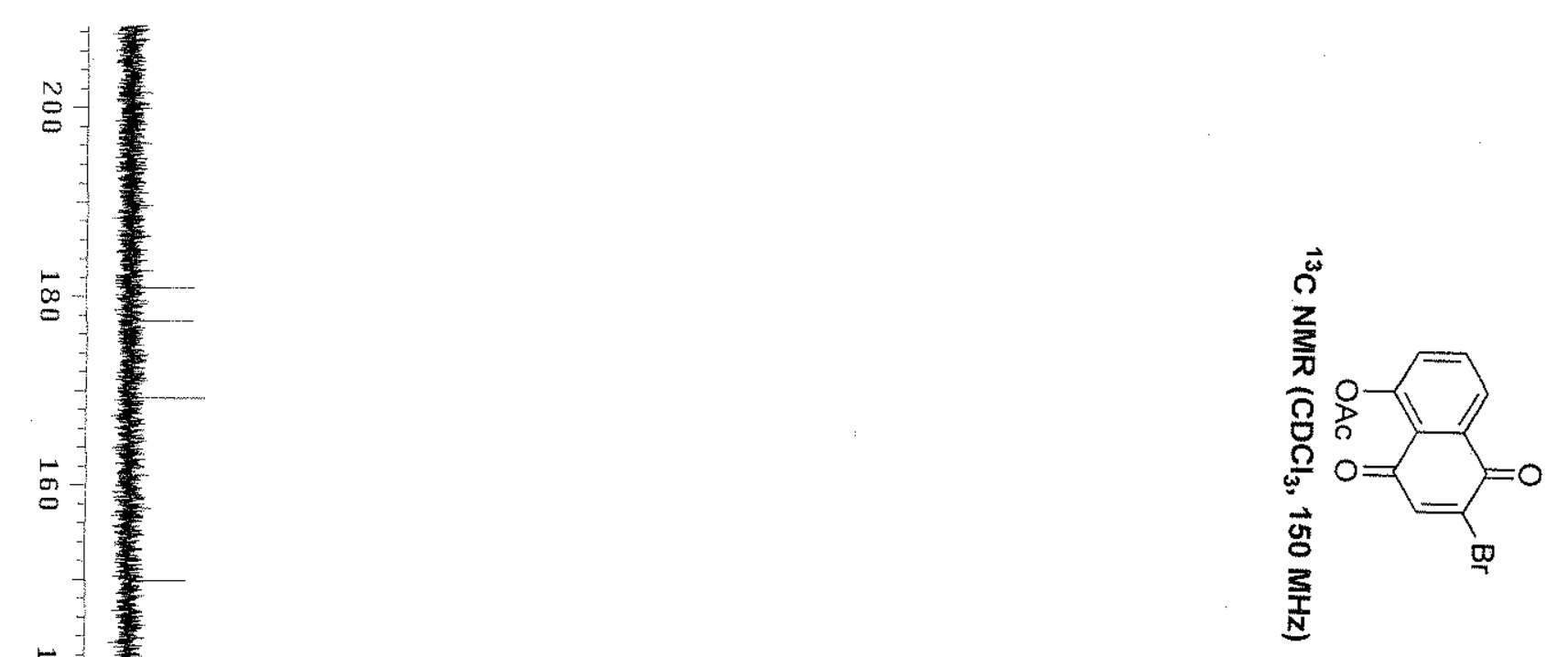

$\Xi$

5

列

$\stackrel{\infty}{\circ}$

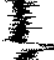




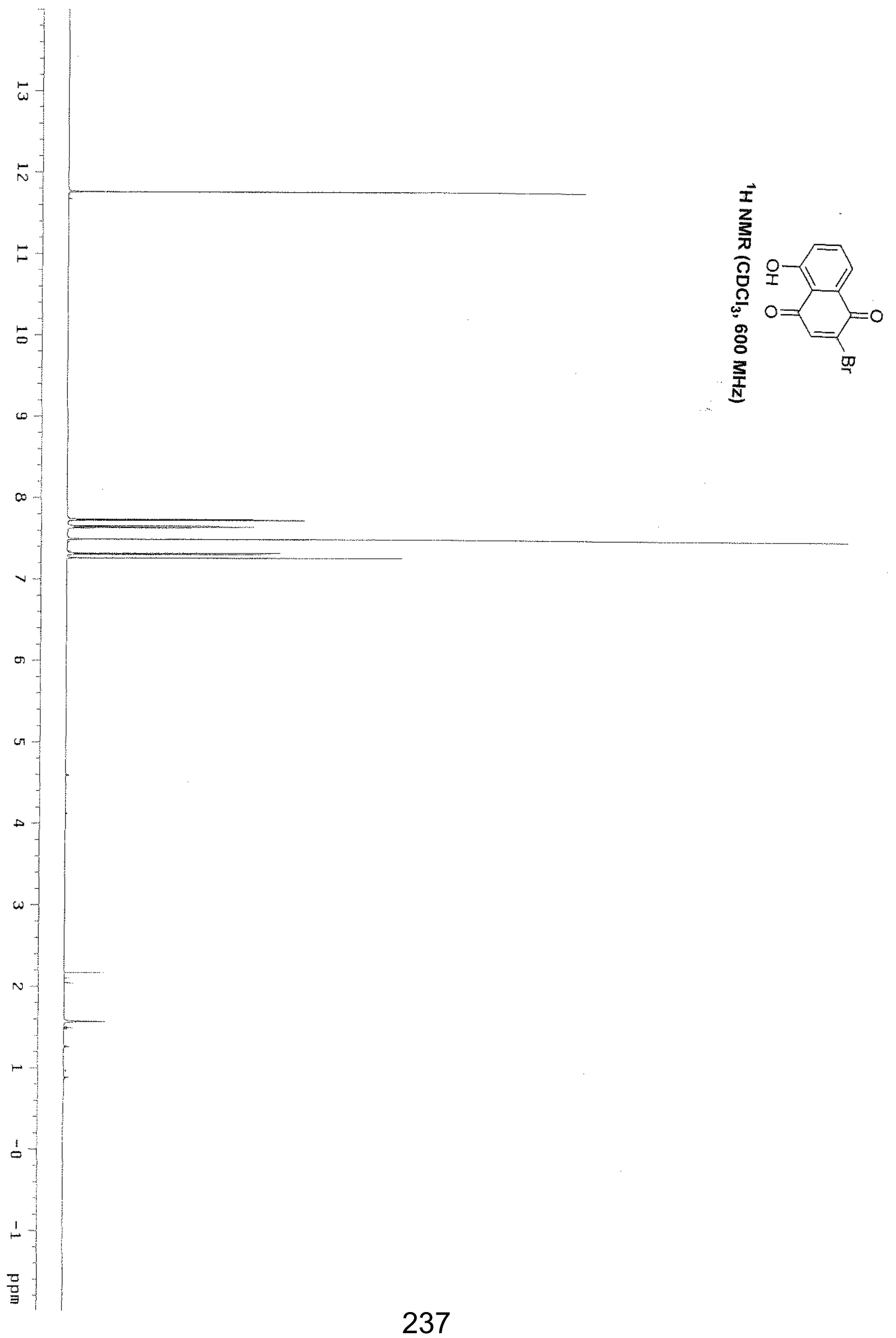




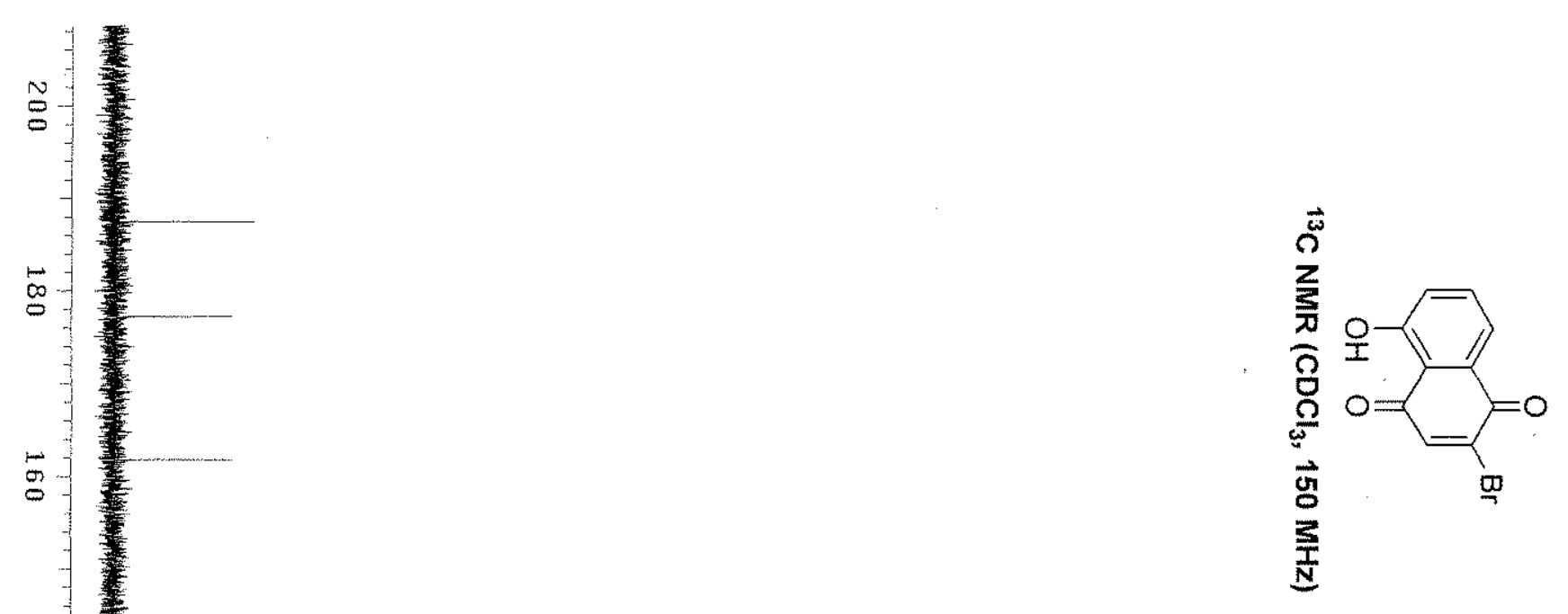

$\stackrel{\infty}{\circ}$

$\stackrel{\circ}{\circ}$

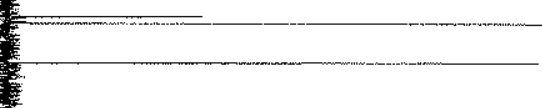

$\stackrel{N}{0}$

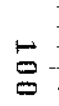

$-$

$\frac{}{4}$

$\infty$

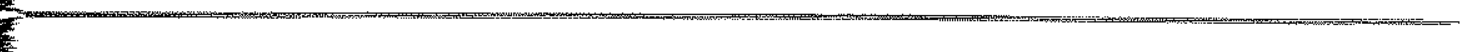

$g$

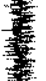

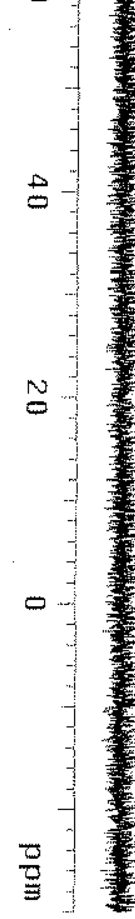




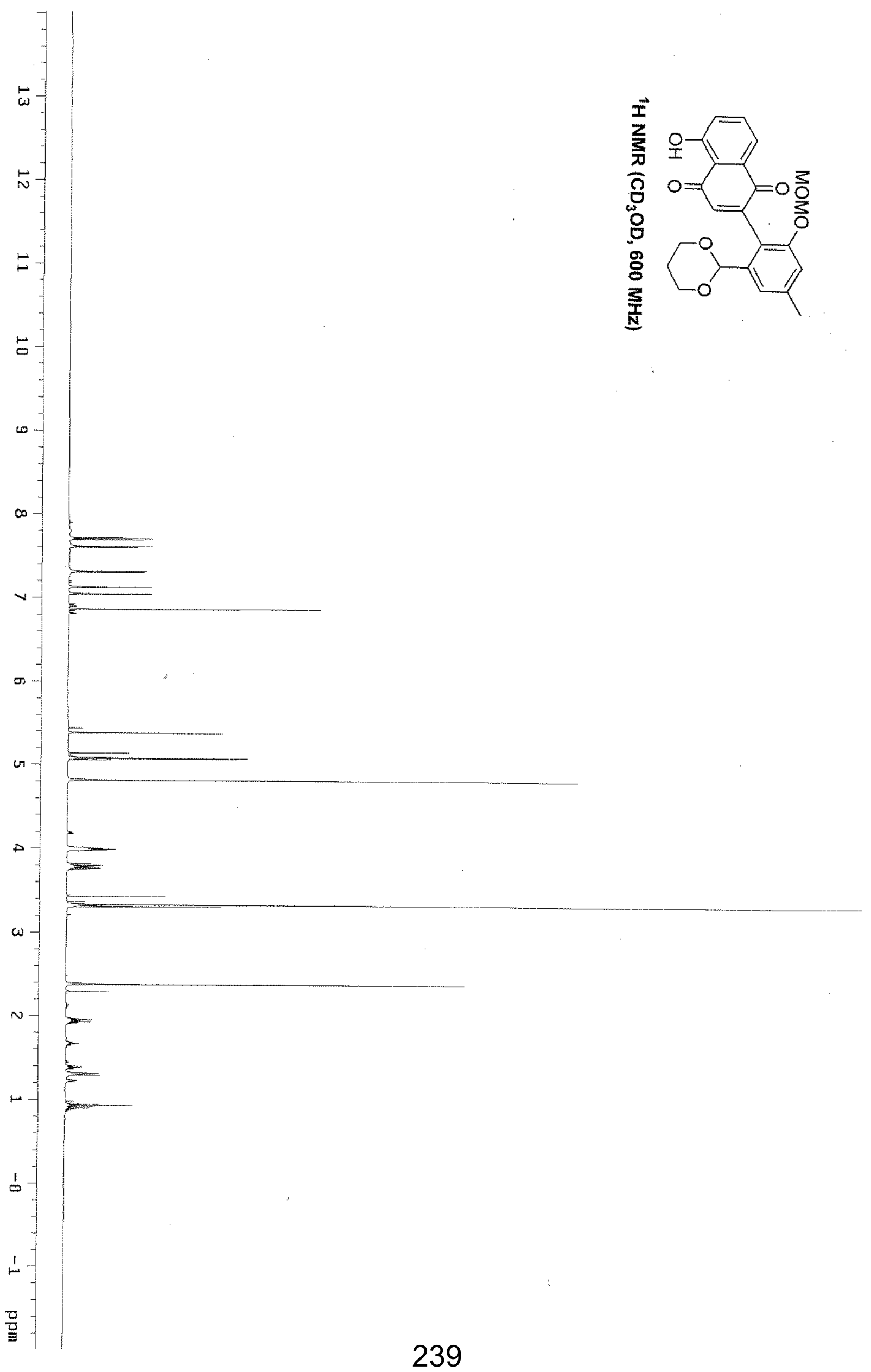




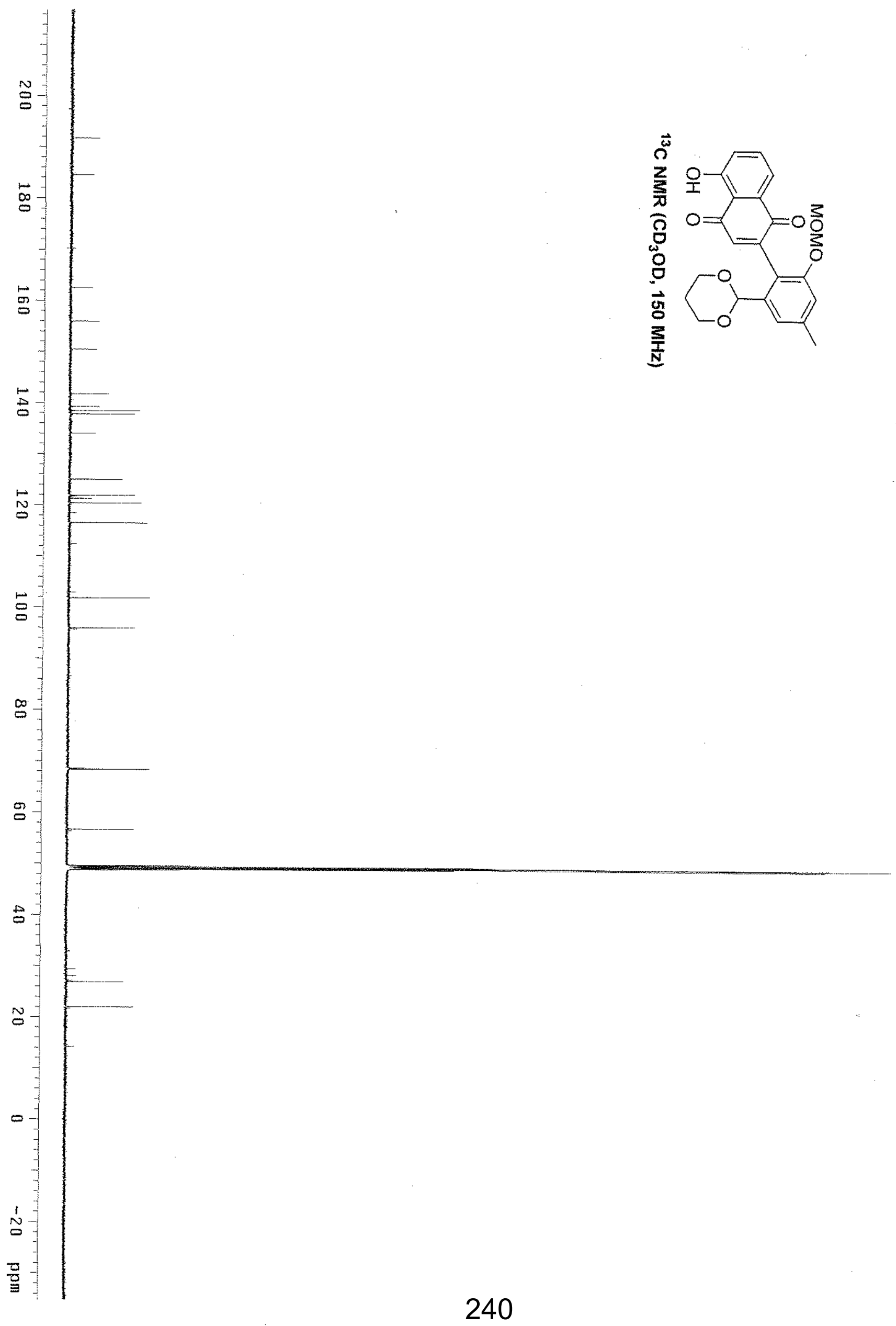




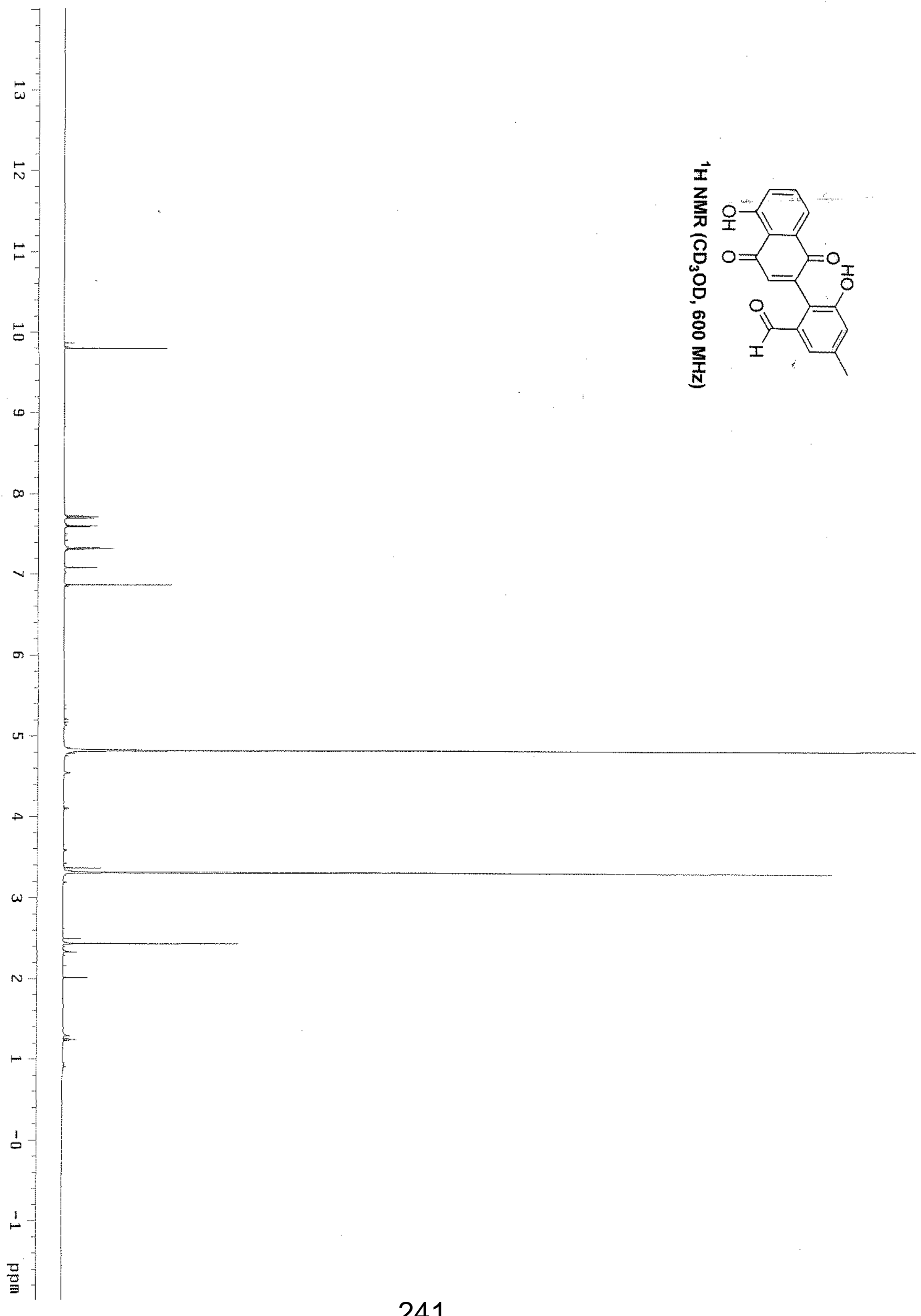



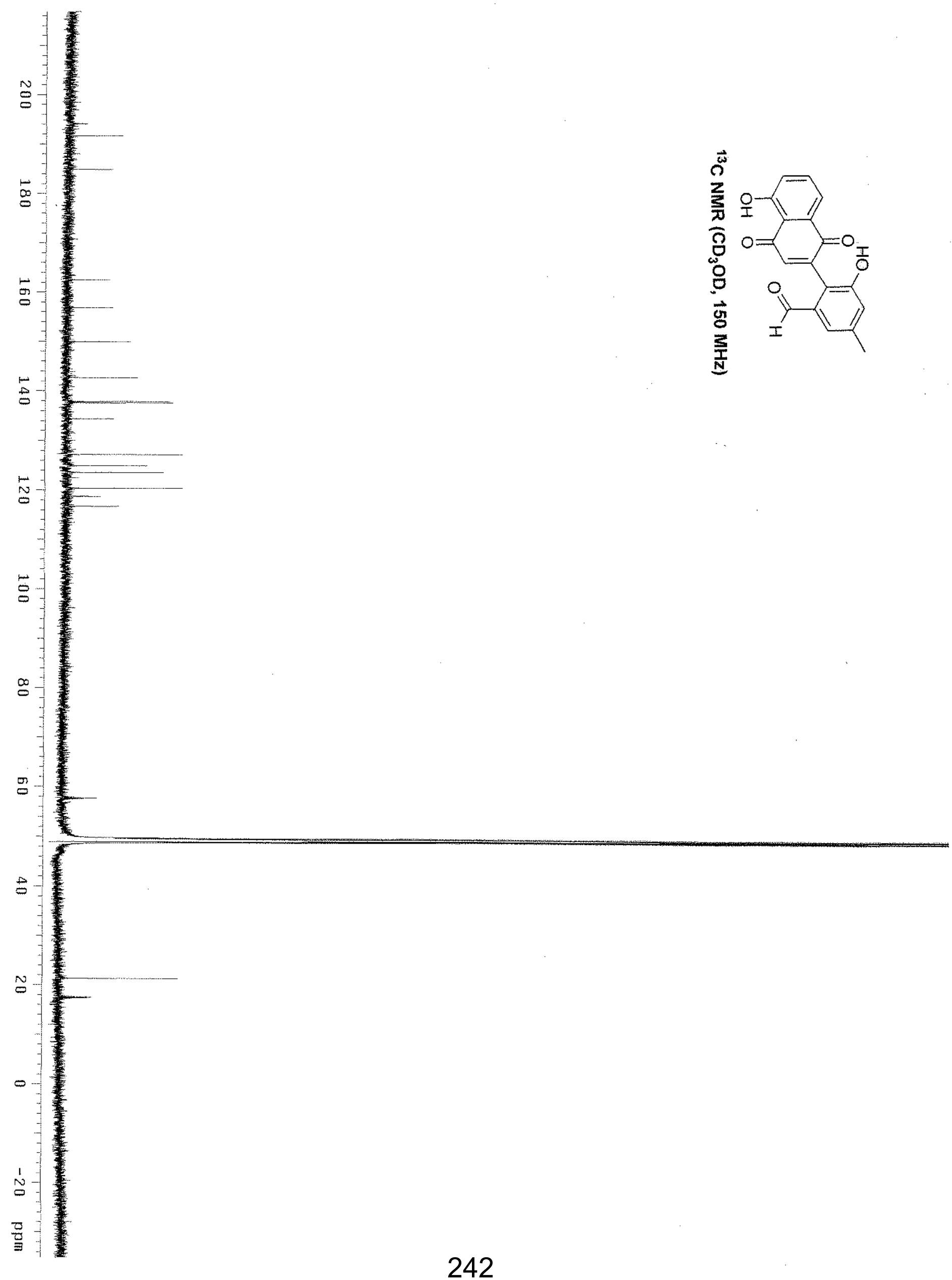


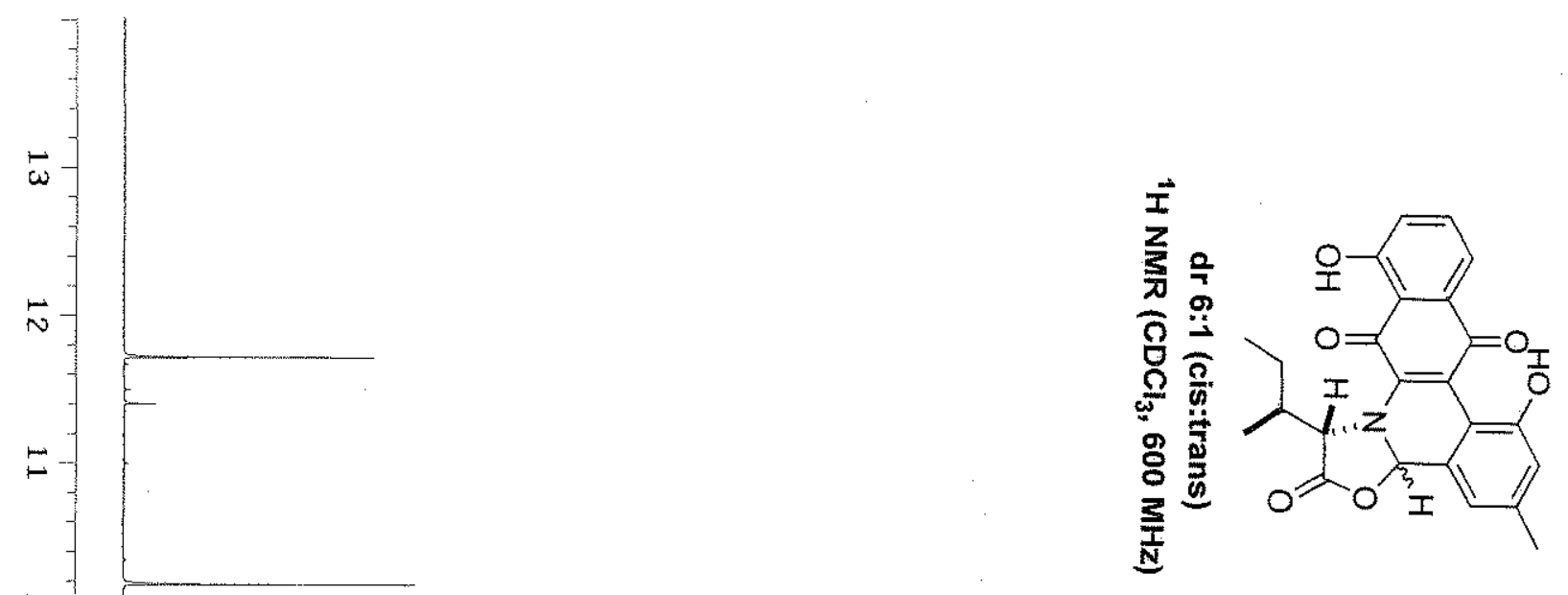

อ

$\infty$
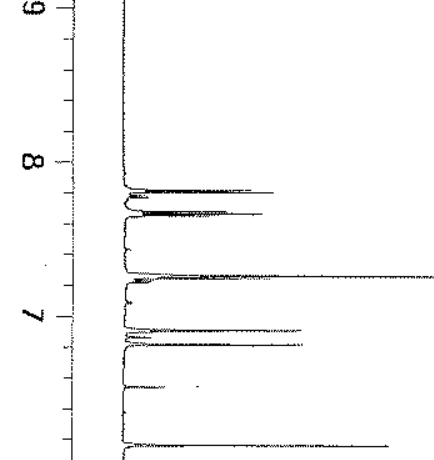

$\sigma$

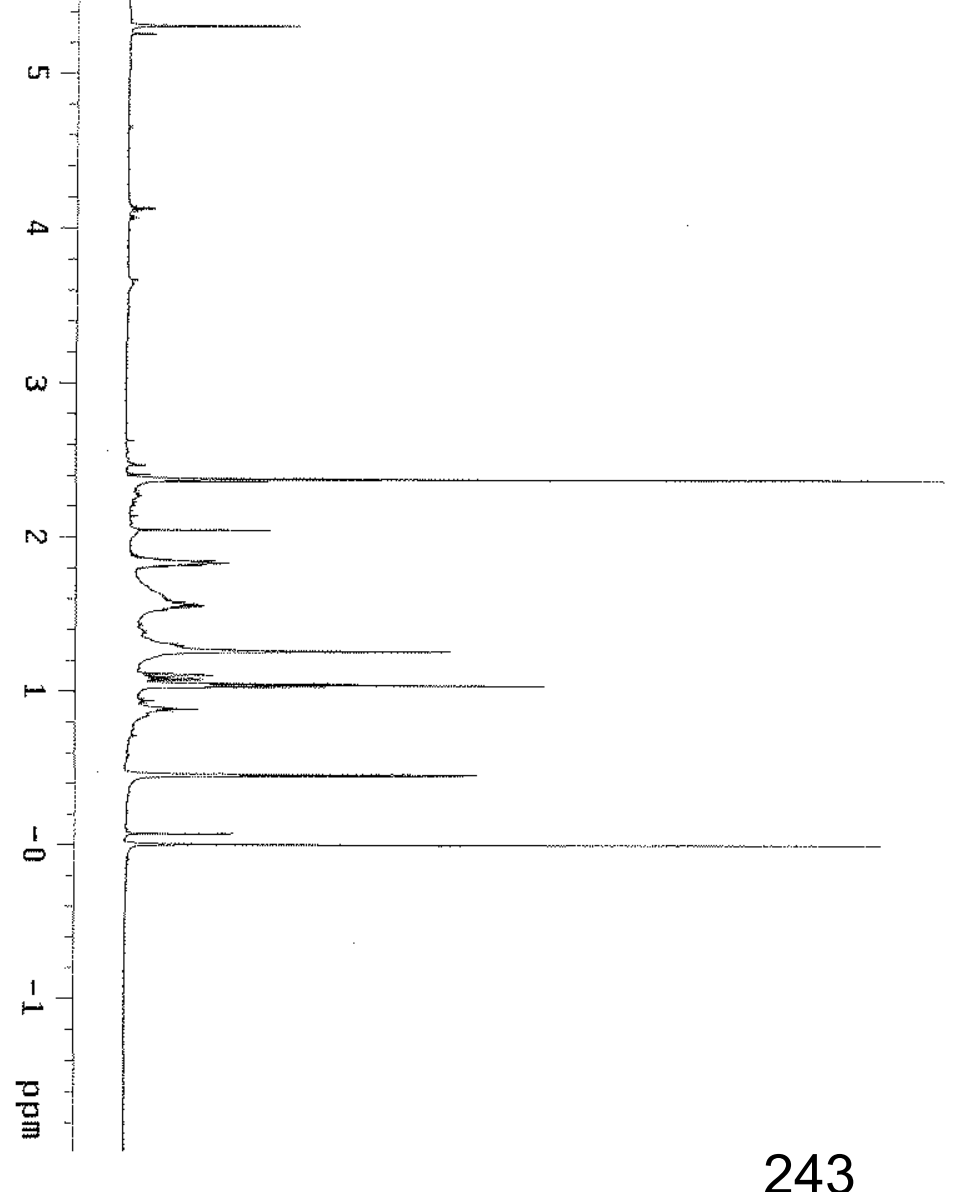




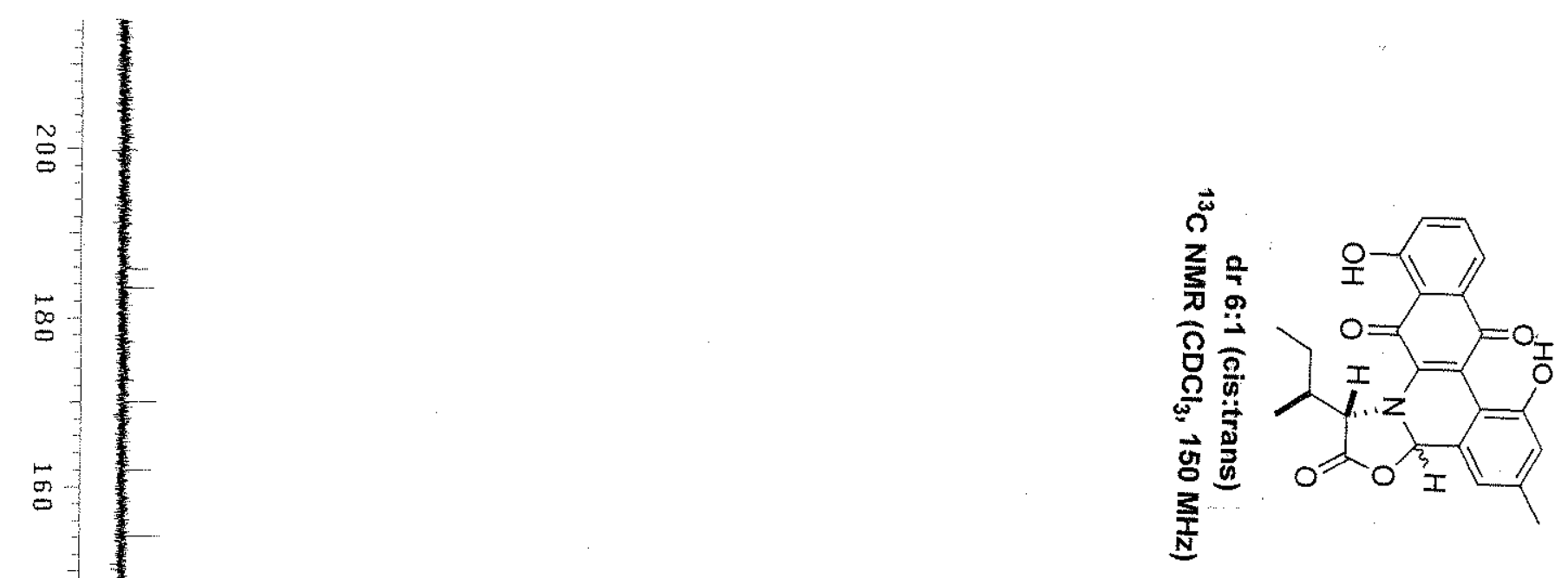

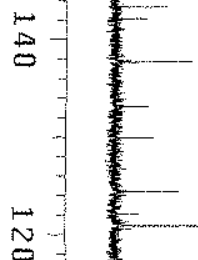

E

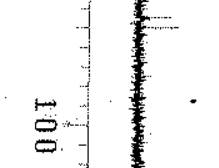

束

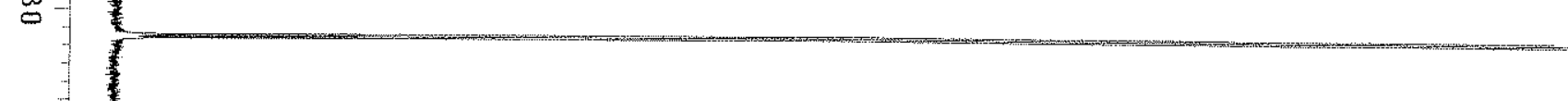

$9 \sqrt{1}$

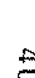

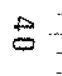

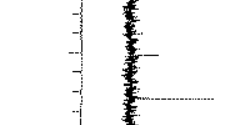

v

0

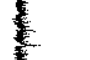

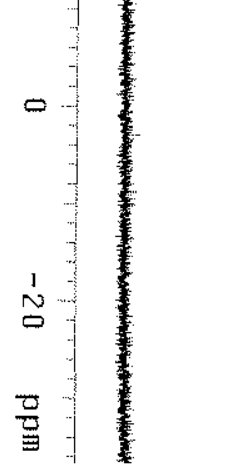

